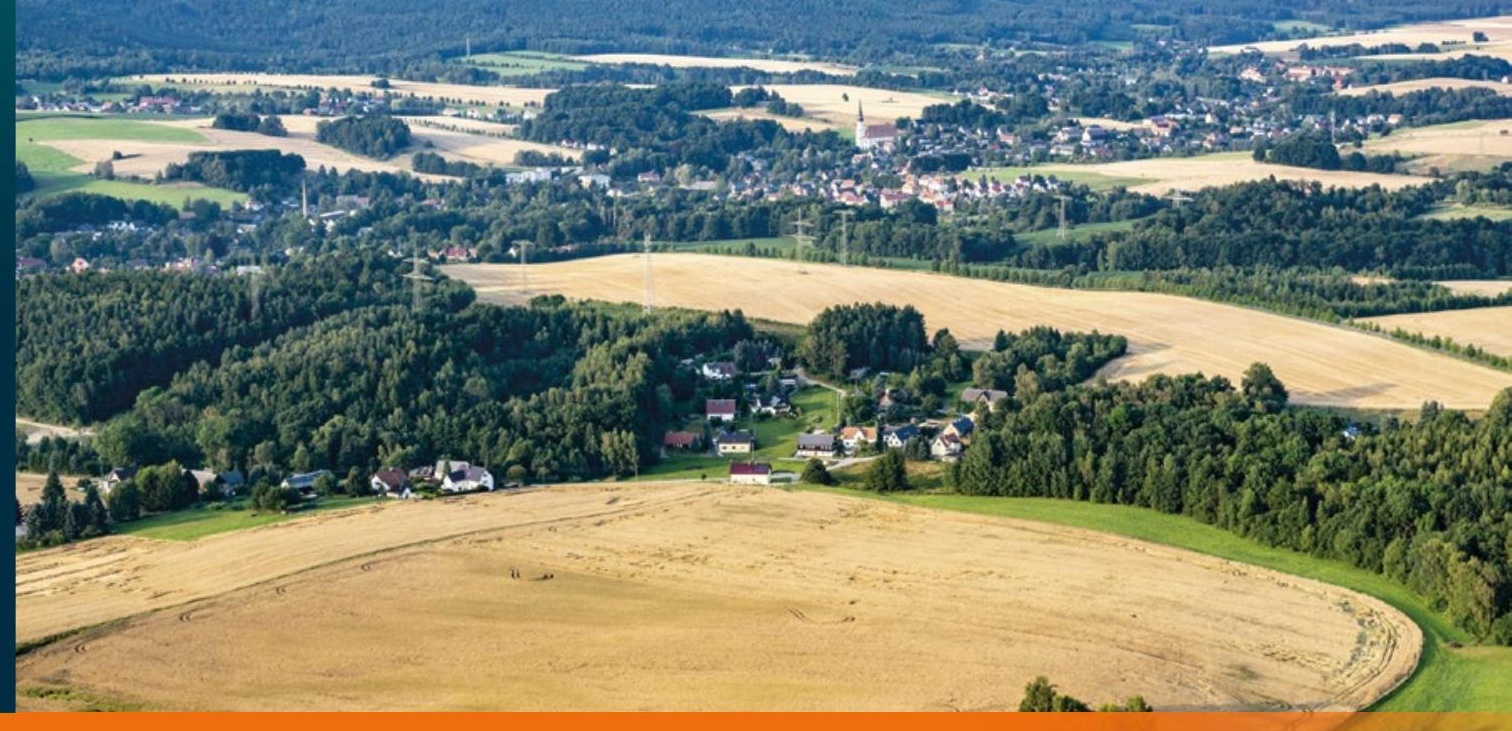

Peter H. Feindt, Christine Krämer, Andrea Früh-Müller, Alois Heißenhuber, Claudia Pahl-Wostl, Kai P. Purnhagen, Fabian Thomas, Caroline van Bers, Volkmar Wolters

\title{
Ein neuer \\ Gesellschaftsvertrag \\ für eine nachhaltige Landwirtschaft
}

Wege zu einer integrativen Politik für den Agrarsektor 
Ein neuer Gesellschaftsvertrag für eine nachhaltige Landwirtschaft 
Peter H. Feindt • Christine Krämer

Andrea Früh-Müller • Alois Heißenhuber

Claudia Pahl-Wostl • Kai P. Purnhagen

Fabian Thomas • Caroline van Bers

Volkmar Wolters

\section{Ein neuer Gesellschaftsvertrag für eine nachhaltige Landwirtschaft}

\section{Wege zu einer integrativen Politik für den Agrarsektor}

Das Projekt ZA-NExUS wurde gefördert durch das Bundesamt für Naturschutz mit Mitteln des Bundesministeriums für Umwelt, Naturschutz und nukleare Sicherheit, FKZ 3515880400. Der Bericht gibt die Auffassung und Meinung des Zuwendungsempfängers wieder und muss nicht mit der Meinung des Zuwendungsgebers übereinstimmen.

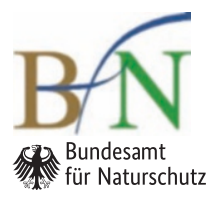

黛 Springer Open 
Peter H. Feindt

Fachgebiet Agrar- und Ernährungspolitik

Humboldt-Universität zu Berlin

Berlin, Deutschland

Andrea Früh-Müller

Forschungsgruppe Agrar- und

Regionalentwicklung Triesdorf (ART)

Weidenbach, Deutschland

Claudia Pahl-Wostl

Institut für Umweltsystemforschung

Universität Osnabrück

Osnabrück, Deutschland

Fabian Thomas

Institut für Umweltsystemforschung

Universität Osnabrück

Osnabrück, Deutschland

Volkmar Wolters

AG Tierökologie

Justus-Liebig-Universität Gießen

Gießen, Deutschland
Christine Krämer

Projektbüro Markt und Region (mareg)

Ippesheim, Deutschland

Alois Heißenhuber

Lehrstuhl für Produktions- und

Ressourcenökonomie

Technische Universität München

Freising, Deutschland

Kai P. Purnhagen

Law and Governance Group

Wageningen University

Wageningen, Niederlande

Caroline van Bers

Institut für Umweltsystemforschung

Universität Osnabrück

Osnabrück, Deutschland

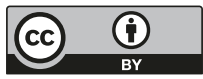

ISBN 978-3-662-58655-6

ISBN 978-3-662-58656-3 (eBook)

https://doi.org/10.1007/978-3-662-58656-3

Die Deutsche Nationalbibliothek verzeichnet diese Publikation in der Deutschen Nationalbibliografie; detaillierte bibliografische Daten sind im Internet über http://dnb.d-nb.de abrufbar.

Springer

(C) Der/die Herausgeber bzw. der/die Autor(en) 2019. Dieses Buch ist eine Open-Access-Publikation.

Open Access Dieses Buch wird unter der Creative Commons Namensnennung 4.0 International Lizenz (http://creativecommons.org/licenses/by/4.0/deed.de) veröffentlicht, welche die Nutzung, Vervielfältigung, Bearbeitung, Verbreitung und Wiedergabe in jeglichem Medium und Format erlaubt, sofern Sie den/die ursprünglichen Autor(en) und die Quelle ordnungsgemäß nennen, einen Link zur Creative Commons Lizenz beifügen und angeben, ob Änderungen vorgenommen wurden.

Die in diesem Buch enthaltenen Bilder und sonstiges Drittmaterial unterliegen ebenfalls der genannten Creative Commons Lizenz, sofern sich aus der Abbildungslegende nichts anderes ergibt. Sofern das betreffende Material nicht unter der genannten Creative Commons Lizenz steht und die betreffende Handlung nicht nach gesetzlichen Vorschriften erlaubt ist, ist für die oben aufgeführten Weiterverwendungen des Materials die Einwilligung des jeweiligen Rechteinhabers einzuholen.

Die Wiedergabe von allgemein beschreibenden Bezeichnungen, Marken, Unternehmensnamen etc. in diesem Werk bedeutet nicht, dass diese frei durch jedermann benutzt werden dürfen. Die Berechtigung zur Benutzung unterliegt, auch ohne gesonderten Hinweis hierzu, den Regeln des Markenrechts. Die Rechte des jeweiligen Zeicheninhabers sind zu beachten.

Der Verlag, die Autoren und die Herausgeber gehen davon aus, dass die Angaben und Informationen in diesem Werk zum Zeitpunkt der Veröffentlichung vollständig und korrekt sind. Weder der Verlag, noch die Autoren oder die Herausgeber übernehmen, ausdrücklich oder implizit, Gewähr für den Inhalt des Werkes, etwaige Fehler oder Äußerungen. Der Verlag bleibt im Hinblick auf geografische Zuordnungen und Gebietsbezeichnungen in veröffentlichten Karten und Institutionsadressen neutral.

Springer ist ein Imprint der eingetragenen Gesellschaft Springer-Verlag GmbH, DE und ist ein Teil von Springer Nature.

Die Anschrift der Gesellschaft ist: Heidelberger Platz 3, 14197 Berlin, Germany 


\section{Inhaltsverzeichnis}

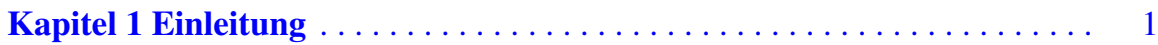

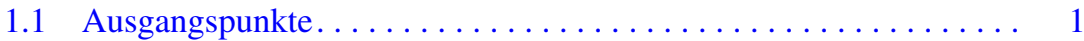

1.2 Das ZA-NExUS-Projekt: Ziele und Projektverbund ......... 4

1.3 Konzeptioneller Ansatz und Vorgehensweise ............. 6

1.4 Überblick über das Buch und Danksagung ............... 10

Kapitel 2 Policy-Paper: ,Ein zukunftsfähiger Gesellschaftsvertrag mit der Landwirtschaft: Plädoyer für eine neue Agrarpolitik“ . . . . 13

2.1 Zusammenfassung ............................ 13

2.2 Die aktuellen Probleme ....................... 14

2.3 Unser Vorschlag für einen zukunftsfähigen Gesellschaftsvertrag . . . 15

2.4 Anhang. . . . . . . . . . . . . . . . . . . . . . . 20

Kapitel 3 Kurzdarstellung der Ausgangssituation: Umwelteffekte der

Landwirtschaft ............................. 23

3.1 Zustand der Naturressourcen $\ldots \ldots \ldots \ldots \ldots \ldots \ldots \ldots \ldots .24$

3.1.1 Wirkungen der Landwirtschaft auf die Ressource Boden . . . 24

3.1.2 Wirkungen der Landwirtschaft auf die biologische Vielfalt . . . 26

3.1.3 Wirkungen der Landwirtschaft auf das Klima ......... . 29

3.1.4 Wirkungen der Landwirtschaft auf das Landschaftsbild .... 31

3.1.5 Wirkungen der Landwirtschaft auf die Ressource Luft .... 32

3.1.6 Wirkungen der Landwirtschaft auf die Ressource Wasser . . . 34

3.1 .7 Zusammenfassung ........................ 37

3.2 Landwirtschaftliche Wirkfaktoren ................... 37

3.2 .1 Stoffliche Einträge ..................... 37

3.2.1.1 Stickstoffverbindungen ................. 42

3.2.1.2 Phosphat ....................... 43

3.2.1.3 Pflanzenschutzmittel ................... 44

3.2.1.4 Schwermetalle ...................... 45

3.2.1.5 Kupfer........................... 45

3.2.1.6 Arzneimittel ...................... 46 


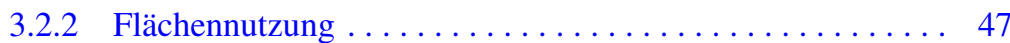

3.2.2.1 Grünlandverlust .................. 47

3.2.2.2 Beseitigung, Zersplitterung, Verkleinerung von Lebensräumen ................... 48

3.2.2.3 Aufgabe traditioneller, extensiver Nutzungsformen bzw. der Nutzung von Standorten mit speziellen Standortbedingungen ................ 51

3.2.2.4 Landwirtschaftliche Nutzung von Moorstandorten .................... 52

3.2.2.5 Fruchtfolgegestaltung ................ 53

3.2.2.6 Bodenbearbeitung und mechanische Belastungen...................... 54

3.2 .3 Zusammenfassung $\ldots \ldots \ldots \ldots \ldots \ldots \ldots \ldots \ldots \ldots \ldots$

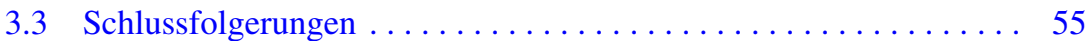

Kapitel 4 Agrarpolitische Rahmenbedingungen ................ 57

4.1 Die politische Logik der GAP: Zwischen Einkommenspolitik

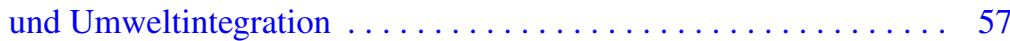

4.2 Die politische Logik des rechtlichen Rahmens ............. 65

4.2.1 Der Rahmen des Umweltordnungsrechts. . . . . . . . . . . 65

4.2.1.1 Der agrarrechtliche Kernbereich . . . . . . . . . 67

4.2.1.2 Das Rahmenrecht ................... 68

4.2.2 Die Veränderung des europarechtlichen Rahmens ....... . 69

4.2.2.1 Einbeziehung der GAP in das allgemeine

Beihilfen- und Wettbewerbsrecht und das „soziale“ Binnenmarktrecht .............. 70

4.2.2.2 Verbraucherschutzorientierung durch die Orientierung an der Regulierung der

Wertschöpfungskette ................. 74

4.2.3 Die politische Logik von Ko-Regulierung und

privatrechtlichem Rahmen...................... 74

4.3 Neuere Ansätze zur Integration von Umweltanliegen in den

Governance-Rahmen ......................... 76

4.3.1 Systemische Konzepte: Resilienz,

Ökosystemdienstleistungen,

Wasser-Energie-Nahrung-Nexus . . . . . . . . . . . . . . . 76

4.3.2 Vom theoretischen Konzept zur praktischen Umsetzung? . . 79

4.3.3 Verhaltenswissenschaftliche Ansätze ............ 83

4.3.3.1 Allgemeine verhaltenswissenschaftliche Befunde und mögliche Anwendungen zum Umweltverhalten von Landwirten . . . . . . . . . . . . . . . 83

4.3.3.2 Faktoren und Beeinflussung umweltbezogener Entscheidungen von Landwirten . . . . . . . . . . . . 
4.3.4 Design von Agrar- und Umweltpolitik aus der Perspektive der Zahlungen für Umweltleistungen . . . . . . . . . . 90 4.3.4.1 Das Konzept der Zahlungen für Umweltleistungen . . . . . . . . . . . . . . . 90

4.3.4.2 Zahlungen für Umweltleistungen in der GAP . . . 91

4.3.4.3 Gestaltungsansätze ................. 92

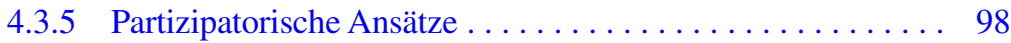

4.3.6 Instrumente auf der Konsumentenebene . . . . . . . . . . 101

4.4 Fazit . . . . . . . . . . . . . . . . . . . . . . . . . 107

Kapitel 5 SWOT-Analyse der derzeitigen Agrarpolitik aus Sicht des

Natur- und Umweltschutzes . . . . . . . . . . . . . . . . . . . . . 109

5.1 Vorgehen bei der SWOT-Analyse . . . . . . . . . . . . . . . . . . . . . 109

5.2 Stärken der derzeitigen europäischen Agrarpolitik . . . . . . . . . 110

5.3 Schwächen der derzeitigen europäischen Agrarpolitik . . . . . . . . . 111

5.4 Chancen aus Sicht des Natur- und Umweltschutzes . . . . . . . . . . . . 114

5.5 Risiken der derzeitigen Agrarpolitik aus Sicht des Natur- und Umweltschutzes . . . . . . . . . . . . . . . . . . . . . . . . . . 115

5.6 Zusammenfassung und Schlussfolgerungen . . . . . . . . . . . 117

5.6.1 Kernpunkte der SWOT-Analyse . . . . . . . . . . . . . 117

5.6.2 Fazit: Fünf Hypothesen zur Agrarpolitik . . . . . . . . . . . . . . 119

Kapitel 6 Ein zeitgemäßes Leitbild für eine nachhaltige Agrarpolitik . . . 123

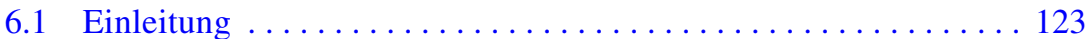

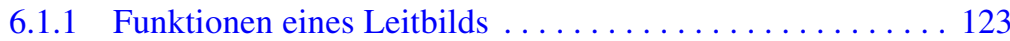

6.1.2 Gegenstand des Leitbilds: Das Was und das Wie . . . . . . . . 124

6.1.3 Einbettung in das agrarpolitische Leitbild der

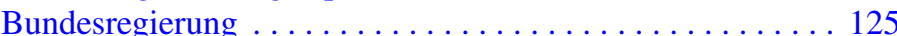

6.2 Leitbild für eine multifunktionale, natur- und umweltverträgliche

Landwirtschaft . . . . . . . . . . . . . . . . . . . . . . 126

6.3 Leitlinien einer zukunftsfähigen Agrarpolitik . . . . . . . . . . . 127

6.4 Dilemmata und Zielkonflikte . . . . . . . . . . . . . . . . . . 129

6.5 Qualitative und quantitative Zielwerte und Soll-Ist-Vergleich mit dem derzeitigen Zustand . . . . . . . . . . . . . . . . 132

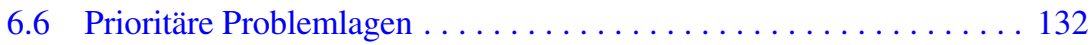

6.7 Abgleich der derzeitigen Agrarpolitik mit den agrarpolitischen Leitlinien . . . . . . . . . . . . . . . . . . . . . . . . . . . . 152

6.8 Fazit . . . . . . . . . . . . . . . . . . . . . . . 159

Kapitel 7 Bausteine zur Integration des Natur- und Umweltschutzes in

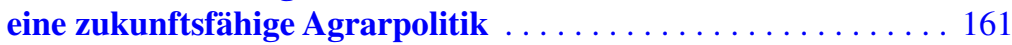

7.1 Baustein 1: Ordnungsrecht und gesetzliche Mindeststandards . . . . 162

7.2 Baustein 2: Budget . . . . . . . . . . . . . . . . . 169

7.2.1 Institutioneller Rahmen . . . . . . . . . . . . . . . . . . . 171

7.2.2 Systematische Aspekte . . . . . . . . . . . . . . 172 
7.2.3 Politische Aspekte . . . . . . . . . . . . . . . . . . . . . 173

7.2.4 Dilemma aus Sicht der Naturschutz- und Umweltpolitik . . 174

7.2.5 Handlungsalternativen ....................... 175

7.3 Baustein 3: Erste Säule der GAP - Flächenbezogene

Direktzahlungen ......................... 177

7.3.1 Bewertung des derzeitigen Systems der Direktzahlungen . . 177

7.3.2 Vorschläge zur Neustrukturierung der Ersten Säule ...... 182

7.3.3 Element 1: Gesetzlicher Mindeststandard zur

Aufrechterhaltung der natürlichen Produktivität der Standorte . . . . . . . . . . . . . . . . . . . . . 183

7.3.4 Element 2: Prämien zur Aufrechterhaltung der

Landbewirtschaftung in Gebietskulissen zum

Erhalt der Landschaftsvielfalt . . . . . . . . . . . . . . . . 184

7.3.5 Element 3: Honorierung der landschaftlichen Vielfalt ..... 185

7.3.6 Exkurs: Ansätze zur Honorierung der landschaftlichen Vielfalt ............................ 185

7.3.7 Exkurs: Praktikabilität einer GIS-gestützten Honorierung von Landschaftsvielfalt ....................... 189

7.3.8 Element 4: Honorierung besonderer Leistungen im landwirtschaftlichen Natur- und Umweltmanagement ... . 192

7.3.9 Diskussion . . . . . . . . . . . . . . . . . . . . . 193

7.4 Baustein 4: Regional und standörtlich ausgerichtete AUKM . . . . . 197

7.4.1 Ziele von Agrarumwelt- und Klimaschutzmaßnahmen . . . . . 197

7.4.2 Bewertung der gegenwärtigen Ausgestaltung der Agrarumwelt- und Klimaschutzmaßnahmen ........... 198

7.4.3 Ziele und Struktur zukünftiger regionaler und standörtlicher AUKM . .................... 200

7.4.3.1 Element 1: Betriebsübergreifende, langfristige Ansätze auf der Landschaftsebene zur Steigerung der Landschaftsvielfalt und zum Schutz der natürlichen Ressourcen ................ 200

7.4.3.2 Element 2: Zielgerichtete Maßnahmen des Arten- und Biotopschutzes ............... 201

7.4.3.3 Element 3: Förderung von lokalen oder regionalen Organisationsstrukturen zur gezielten Programmierung und Koordination von AUKM . . . . . . . . . . . . . . . . . . 202

7.4.4 Diskussion .............................. 203

7.5 Baustein 5: Nicht-staatliche Standards und Ko-Regulierung . . . . . 204

7.6 Baustein 6: Monitoring- und Sanktionssysteme ........... 207

7.6.1 Aufgaben, Zielkonflikte und Problemlagen von

Monitoring- und Sanktionssystemen . . . . . . . . . . . . . 207

7.6.2 Baustein 6.1: Investition in das Indikatorensystem . . . . . . . 208

7.6.3 Baustein 6.2: Digitale Informationssysteme und Fernerkundung zur automatischen Erhebung .......... 209 
7.6.4 Baustein 6.3: Monitoring stofflicher Einträge -

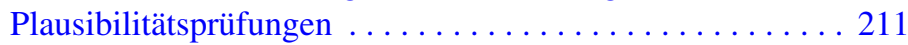

7.6.5 Weitere Bausteine . . . . . . . . . . . . . . . . . . . 211

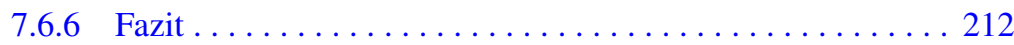

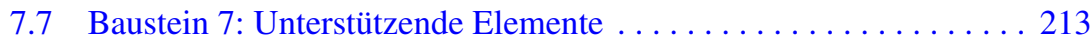

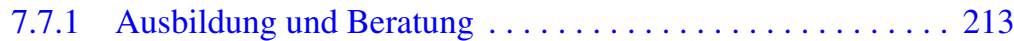

7.7.2 Verbraucherorientierte Maßnahmen .............. 215

7.7.2.1 Steuern und Regulation .............. 216

7.7.2.2 Produktkennzeichnung ............. 216

7.7.2.3 Bewusstseinsbildung und Verbindung zwischen

Konsumenten und Erzeugern . . . . . . . . . . . . . . . . . . . . . . 217

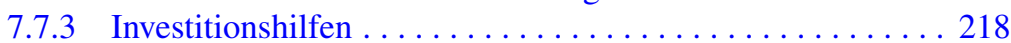

7.8 Fazit . . . . . . . . . . . . . . . . . . . . . . 219

Kapitel 8 Skizzierung und Bewertung umweltpolitischer Strategien für eine zukünftige Agrar- und Umweltpolitik . . . . . . . . . . . . 223

8.1 Einführung und Überblick . . . . . . . . . . . . . . . . . . . . 223

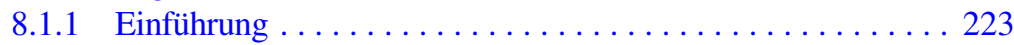

8.1.2 Überblick: Strategische Entwicklungsrichtungen für eine zukünftige Agrar- und Umweltpolitik . . . . . . . . . . . 223

8.1 .3 Gemeinsamkeiten aller Optionen . . . . . . . . . . . . . . 224

8.2 Option 1 - Weiterentwicklung des Greenings der Ersten Säule . . . 226

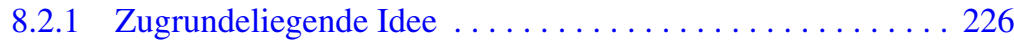

8.2.2 Einbettung in die agrarpolitische Diskussion . . . . . . . . 228

8.2 .3 Instrumentelle Ausgestaltung . . . . . . . . . . . . . . . . 229

8.2.4 Diskussion und Bewertung . . . . . . . . . . . . . . . 231

8.3 Option 2 - Stärkung der Zweiten Säule . . . . . . . . . . . 233

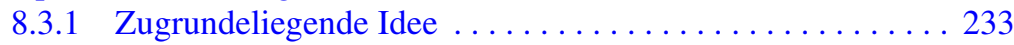

8.3.2 Einbettung in die agrarpolitische Diskussion ........ 233

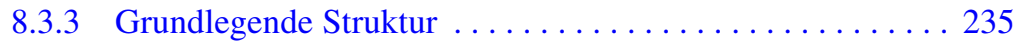

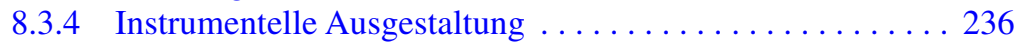

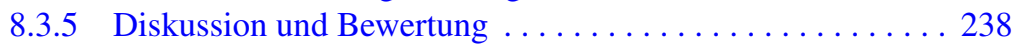

8.4 Option 3 - Integriertes Modell . . . . . . . . . . . . . . . . 242

8.4.1 Zugrundeliegende Idee . . . . . . . . . . . . . . . 242

8.4.2 Einbettung in die agrarpolitische Diskussion . . . . . . . . 243

8.4 .3 Grundlegende Struktur . . . . . . . . . . . . . . . 243

8.4 .4 Instrumentelle Ausgestaltung . . . . . . . . . . . . . 244

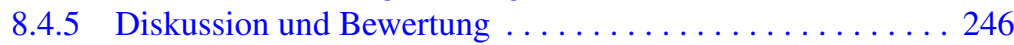

8.5 Exkurs: Umstellung der Regulierungslogik auf „prinzipienbasierte Regulation“. . . . . . . . . . . . . . . . . 248

8.5.1 Einleitung - Ein prinzipienbasierter „Neuer Ansatz“

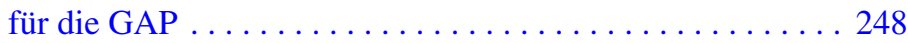

8.5.2 Ausgangspunkt: Der „Neue Ansatz“ im EU-Produktsicherheitsrecht . . . . . . . . . . . . . . 250

8.5.2.1 Der „Neue Ansatz“ als Alternative .......... 251 
8.5.2.2 Haftungsregeln als Sicherheitsnetz ......... 253

8.5.3 Übertragbarkeit aufgrund ähnlicher Voraussetzungen . . . . . 254

8.5.4 Vorschlag der Übertragung des prinzipienbasierten

„Neuen Ansatzes“ auf die GAP . . . . . . . . . . . . . . . 254

8.5.4.1 Rechtlich verbindliche Rahmenverordnung .... . . 255

8.5.4.2 Gesetzliche Minimalvorgaben und freiwilliges

Baukastensystem unterschiedlicher Standards . . . 255

8.5.4.3 Mögliche Verknüpfung mit dem System der

Agrarzahlungen ..................... 256

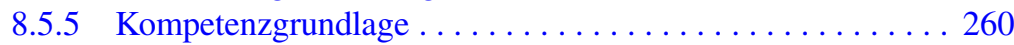

8.5.6 Schlussfolgerung und Ausblick . . . . . . . . . . . . . 260

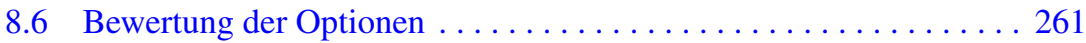

8.6.1 Machbarkeit ......................... 262

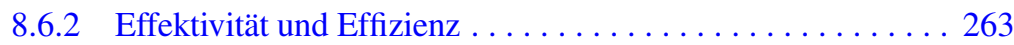

8.6 .3 Akteurskoalitionen ....................... 264

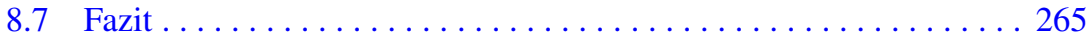

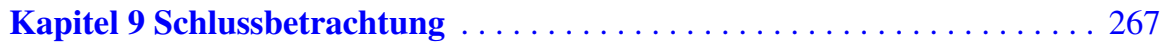

9.1 Verwirklichung des inter- und transdisziplinären Anspruchs . . . . . 267

9.2 Zusammenfassung der Aufgaben und Ergebnisse des

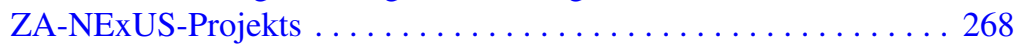

9.3 Reflexion und Ausblick . . . . . . . . . . . . . . . . 274

Anhänge . . . . . . . . . . . . . . . . . . . . . . . . . . . . . . . . . . 279

Literatur . . . . . . . . . . . . . . . . . . . . . 295 


\section{Autorenverzeichnis}

Prof. Dr. Peter H. Feindt Fachgebiet Agrar- und Ernährungspolitik, Humboldt-Universität zu Berlin, Berlin, Deutschland

Dr. Christine Krämer Projektbüro Markt und Region (mareg), Ippesheim, Deutschland

Dr. Andrea Früh-Müller Forschungsgruppe Agrar- und Regionalentwicklung Triesdorf (ART), Weidenbach, Deutschland

Prof. Dr. Dr. h.c. Alois Heißenhuber Lehrstuhl für Produktions- und Ressourcenökonomie, Technische Universität München, Freising, Deutschland

Prof. Dr. Claudia Pahl-Wostl Institut für Umweltsystemforschung, Universität Osnabrück, Osnabrück, Deutschland

Dr. Kai P. Purnhagen Universität Wageningen, Wageningen, Niederlande

Fabian Thomas, M.Sc. Universität Osnabrück, Institut für Umweltsystemforschung, Osnabrück, Deutschland

Caroline van Bers, M.Sc. Universität Osnabrück, Institut für Umweltsystemforschung, Osnabrück, Deutschland

Prof. Dr. Volkmar Wolters AG Tierökologie, Justus-Liebig-Universität Gießen, Gießen, Deutschland 


\section{Abkürzungsverzeichnis}

$\begin{array}{ll}\text { ABl } & \text { Amtsblatt der Europäischen Union } \\ \text { AEUV } & \text { Vertrag über die Arbeitsweise der Europäischen Union } \\ \text { AGB } & \text { Allgemeine Geschäftsbedingungen } \\ \text { Art. } & \text { Artikel } \\ \text { AUKM } & \text { Agrarumwelt- und Klimamaßnahmen (AUK-Maßnahmen) } \\ \text { BfN } & \text { Bundesamt für Naturschutz } \\ \text { BIP } & \text { Bruttoinlandsprodukt } \\ \text { BIT } & \text { Behavioral Insights Team } \\ \text { BMEL } & \text { Bundesministerium für Ernährung und Landwirtschaft } \\ \text { BMELV } & \text { Bundesministerium für Ernährung, Landwirtschaft und } \\ & \text { Verbraucherschutz } \\ \text { BMU } & \text { Bundesministerium für Umwelt, Naturschutz und nukleare Sicherheit } \\ \text { BMUB } & \text { Bundesministerium für Umwelt, Bau und Reaktorsicherheit } \\ \text { BNatSchG } & \text { Bundesnaturschutzgesetz (Gesetz über Naturschutz und } \\ & \text { Landschaftspflege) } \\ \text { BSE } & \text { Bovine Spongiforme Encephalopathie } \\ \text { BVL } & \text { Bundesamt für Verbraucherschutz und Lebensmittelsicherheit } \\ \text { CBD } & \text { Convention on Biological Diversity } \\ \text { CC } & \text { Cross Compliance } \\ \text { CE-Signatur } & \text { Communauté Européenne, Comunidad Europea, Comunidade } \\ & \text { Europeia und Comunità Europea } \\ \text { CO } & \text { Kohlendioxid } \\ \text { CSR } & \text { Corporate Social Responsibility (soziale } \\ \text { DG Agri } & \text { Unternehmensverantwortung) } \\ \text { DLG } & \text { Generaldirektion Landwirtschaft und ländliche Entwicklung } \\ \text { DLR } & \text { Deutsche Landwirtschafts-Gesellschaft } \\ \text { DüV } & \text { Deutsches Zentrum für Luft- und Raumfahrt } \\ \text { DVL } & \text { Düngeverordnung } \\ \text { DZ } & \text { Deutscher Verband für Landschaftspflege } \\ \text { EAST } & \text { Direktzahlung(en) } \\ & \text { Easy, Attractive, Social, and Timely } \\ & \end{array}$




$\begin{array}{ll}\text { EFSA } & \text { European Food Safety Authority (Europäische Behörde für } \\ & \text { Lebensmittelsicherheit) } \\ \text { EEA } & \text { European Environment Agency (Europäische Umweltagentur) } \\ \text { EEG } & \text { Erneuerbare-Energien-Gesetz } \\ \text { EG } & \text { Europäische Gemeinschaft } \\ \text { ELER } & \text { Europäischer Landwirtschaftsfonds für die Entwicklung des } \\ & \text { ländlichen Raums } \\ \text { ESAWADI } & \text { Utilising the Ecosystem Services Approach for Water Framework } \\ & \text { Directive Implementation } \\ \text { EU } & \text { Europäische Union } \\ \text { EuGH } & \text { Europäischer Gerichtshof } \\ \text { EU-VO } & \text { Verordnung der Europäischen Union } \\ \text { EUV } & \text { Vertrag über die Europäische Union } \\ \text { EWG } & \text { Europäische Wirtschaftsgemeinschaft } \\ \text { FAO } & \text { Food and Agricultural Organization of the United Nations } \\ \text { FFH } & \text { Flora-Fauna-Habitat } \\ \text { FH } & \text { Fachhochschule } \\ \text { GAB } & \text { Grundanforderungen an die Betriebsführung } \\ \text { GAP } & \text { Gemeinsame Agrarpolitik } \\ \text { GATT } & \text { General Agreement on Tariffs and Trade (Allgemeines Zoll- und } \\ & \text { Handelsabommen) } \\ \text { GBP } & \text { Britische Pfund (£) } \\ \text { gfP } & \text { gute fachliche Praxis } \\ \text { GIS } & \text { Geographische Informationssysteme } \\ \text { GLÖZ } & \text { Standards für die Erhaltung der Flächen in gutem } \\ & \text { landwirtschaftlichen und ökologischen Zustand } \\ \text { GMO } & \text { Gemeinsame Marktordnung } \\ \text { GV } & \text { Großvieheinheit } \\ \text { GVO } & \text { gentechnisch veränderter Organismus } \\ \text { ha } & \text { Hektar } \\ \text { HIT } & \text { Herkunftssicherungs- und Informationssystem für Tiere } \\ \text { HNV } & \text { High-Nature-Value Farmland (bezeichnet Landwirtschaftsflächen } \\ & \text { mit hohem Naturwert) } \\ \text { ICT } & \text { Information and Communication Technology } \\ \text { InVeKoS } & \text { Integriertes Verwaltungs- und Kontrollsystem } \\ \text { IT } & \text { Informationstechnologie } \\ \text { i. V. m. } & \text { in Verbindung mit } \\ \text { KBU } & \text { Kommission Bodenschutz beim Umweltbundesamt } \\ \text { LE } & \text { Landschaftselemente } \\ \text { LEADER } & \text { Liaison entre actions de développement de l'économie rurale } \\ & \text { (Verbindung zwischen Aktionen zur Entwicklung der ländlichen } \\ \text { LF } & \text { Wirtschaft) } \\ \text { LPIS } & \text { landwirtschaftlich genutzte Fläche } \\ \text { Mio. } & \text { Land Parcel Identification System } \\ & \text { Million(en) } \\ & \end{array}$




\begin{tabular}{|c|c|}
\hline Mrd. & Milliarde(n) \\
\hline $\mathrm{N}$ & Stickstoff \\
\hline NEC & national emission ceilings (nationale Emissionshöchstmengen) \\
\hline NMVOC & Non-Methane Volatile Organic Compounds (flüchtige \\
\hline & Nichtmethankohlenwasserstoffe) \\
\hline o. Ä. & oder Ähnliches \\
\hline ÖSL & Ökosystemleistungen \\
\hline ÖVF & ökologische Vorrangfläche \\
\hline $\mathrm{P}$ & Phosphor \\
\hline $\mathrm{P}_{2} \mathrm{O}_{5}$ & Diphosphorpentoxid \\
\hline PAG & Projektbegleitende Arbeitsgruppe \\
\hline PES & Payments for Environmental Service \\
\hline PSM & Pflanzenschutzmittel \\
\hline REACH & Registration, Evaluation, Authorisation and Restriction of \\
\hline & Chemicals (Registrierung, Bewertung, Zulassung und \\
\hline & Beschränkung chemischer Stoffe) \\
\hline RL & Richtlinie \\
\hline Rn. & Randnummer \\
\hline Rs. & Rechtssache \\
\hline SHARP & $\begin{array}{l}\text { Self-evaluation and Holistic Assessment of climate Resilience of } \\
\text { farmers and Pastoralists }\end{array}$ \\
\hline Slg. & Sammlung der Rechtsprechung des Gerichtshofes und des Gerichts \\
\hline & Erster Instanz \\
\hline $\operatorname{sog}$. & sogenannt \\
\hline SWOT & $\begin{array}{l}\text { Strengths (Stärken), Weaknesses (Schwächen), Opportunities } \\
\text { (Chancen) und Threats (Bedrohungen) }\end{array}$ \\
\hline $\mathrm{t}$ & Tonne(n) \\
\hline TÜV & Technischer Überwachungsverein \\
\hline UN & United Nations \\
\hline UNCBD & United Nations Convention on Biological Diversity \\
\hline UNESCO & United Nations Educational, Scientific and Cultural Organization \\
\hline UTZ & $\begin{array}{l}\text { Programm und Label für nachhaltigen Anbau von Kaffee, Kakao } \\
\text { und Tee (utz = „gut“ in der Sprache der Maya) }\end{array}$ \\
\hline v. a. & vor allem \\
\hline VDLUFA & Verband Deutscher Landwirtschaftlicher Untersuchungs- und \\
\hline & Forschungsanstalten \\
\hline vgl. & vergleiche \\
\hline WBA & Wissenschaftlicher Beirat für Agrarpolitik beim BMEL \\
\hline WEF & Water-Energy-Food \\
\hline WEFWI & World Economic Forum Water Initiative \\
\hline WRRL & Wasserrahmenrichtlinie \\
\hline WTO & World Trade Organization \\
\hline Z. B. & zum Beispiel \\
\hline
\end{tabular}




\section{Abbildungsverzeichnis}

Abb. 2.1 Architektur einer neuen Agrarpolitik.

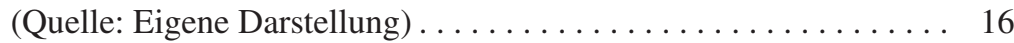

Abb. 7.1 Artenreichtum von Ackerwildkräutern in Abhängigkeit von der Landschaftsstruktur. (Quelle: Eigene Darstellung nach Tscharntke et al. 2005).

Abb. 7.2 Honorierung von Landschaftsvielfalt in Abhängigkeit von der Schlaggröße. (Quelle: Eigene Darstellung nach Engelhardt (2004)).

Abb. 7.3 Beispiel für honorierungswürdige Schlagformen, dargestellt am Schlagumfang in Abhängigkeit von der Schlaggröße. (Quelle: Eigene Darstellung). .

Abb. 7.4 Honorierung der Landschaftsvielfalt in Abhängigkeit von der Länge der Grenzlinien eines Schlages zu Landschaftselementen. (Quelle: Eigene Darstellung)

Abb. 7.5 Anteil Saumbiotope, Testregion 1. (Quelle: Orthophoto: geoservices.bayern.de; Lizenz: creativecommons.org/licenses/ by/3.0/deed.de (Analyse und Visualisierung: Thomas Machl (vgl. Machl 2016))

Abb. 7.6 Anteil Saumbiotope, Testregion 2. (Quelle: Orthophoto: geoservices.bayern.de; Lizenz: creativecommons.org/licenses/ by/3.0/deed.de (Analyse und Visualisierung: Thomas Machl (vgl. Machl 2016)) .

Abb. 7.7 Anteil Saumbiotope, Testregion 3. (Quelle: Orthophoto: geoservices.bayern.de; Lizenz: creativecommons.org/licenses/ by/3.0/deed.de (Analyse und Visualisierung: Thomas Machl (vgl. Machl 2016)) . 
Abb. 8.1 Option 1 - Weiterentwicklung des Greenings der Ersten Säule. (Quelle: Eigene Darstellung) ....................... 227

Abb. 8.2 Option 2 - Eine starke Zweite Säule. (Quelle: Eigene

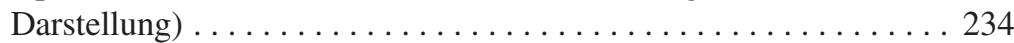

Abb. 8.3 Option 3 - Integriertes Modell. (Quelle: Eigene Darstellung) . . . . 242

Abb. 8.4 Mögliche Struktur einer prinzipienbasierten Regulierung für den Agrarsektor. (Quelle: Eigene Darstellung) . . . . . . . . . . . 257 


\section{Tabellenverzeichnis}

Tab. 2.1 Eine neue Architektur für die Agrarpolitik - Überblick.

(Diese Überblickstabelle war nicht Teil des im Januar 2017

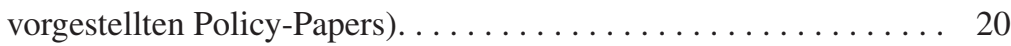

Tab. 3.1 Zielsetzungen und Zielerreichung für die Ressource Boden . . . . . . 26

Tab. 3.2 Zielsetzungen und Zielerreichung bezüglich der Ressource Biologische Vielfalt. (Zur Erläuterung der Zielwerte vergleiche Anhang) . . . . . . . . . . . . . . . . . . 29

Tab. 3.3 Zielsetzungen und Zielerreichung bezüglich der Ressource Luft . . . 33

Tab. 3.4 Zielsetzungen und Zielerreichung bezüglich der Ressource

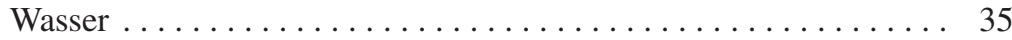

Tab. 3.5 Zielsetzungen und Zielerreichung bezüglich der Naturressourcen . . . 38

Tab. 3.6 Zielsetzung und Zielerreichung bezüglich der Stickstoff-Einträge . . . 43

Tab. 3.7 Zielsetzung und Zielerreichung bezüglich der Phosphor-Einträge . . . 44

Tab. 3.8 Zielsetzung und Zielerreichung bezüglich des Antibiotika-Einsatzes. . . . . . . . . . . . . . . . . . 46

Tab. 3.9 Zielsetzung und Zielerreichung bezüglich des Erhalts von

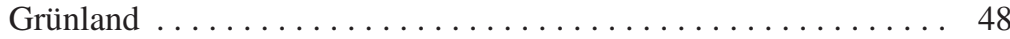

Tab. 3.10 Zielsetzung und Zielerreichung bezüglich der Beseitigung, Zersplitterung und Verkleinerung von Lebensräumen . . . . . . . . 50

Tab. 3.11 Zielsetzung und Zielerreichung bezüglich der Aufgabe traditioneller und extensiver Nutzungsformen . . . . . . . . . . . 51

Tab. 3.12 Zielsetzung und Zielerreichung bezüglich der landwirtschaftlichen

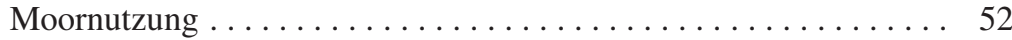

Tab. 3.13 Zielsetzung und Zielerreichung bezüglich der

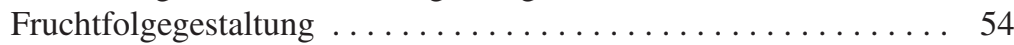

Tab. 4.1 Die zwei Säulen des Umweltordnungsrechts in der EU . . . . . . . . 67

Tab. 4.2 Die zwei Säulen des Rahmenrechts in der EU. . . . . . . . . . . . . . . 69

Tab. 5.1 SWOT-Analyse der derzeitigen GAP aus Sicht des Natur- und

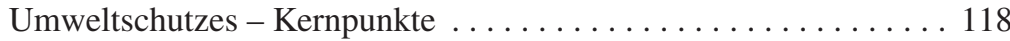


Tab. 6.1 Leitbildindikatoren, Zielwerte und Ist-Zustand . . . . . . . . . 133

Tab. 6.2 Abgleich der derzeitigen Agrarpolitik mit den agrarpolitischen Leitlinien . . . . . . . . . . . . . . . . . . . . . . . . 153

Tab. 7.1 Vorschriften für die landwirtschaftliche Bewirtschaftung ........ 163

Tab. 7.2 Bewertung von Umwelt- und Agrarrecht anhand ausgewählter Indikatoren . . . . . . . . . . . . . . . . . . . . . 165

Tab. 7.3 EU-Ausgaben aus dem Haushaltstitel 2 (Nachhaltiges Wachstum:

Natürliche Ressourcen) an deutsche Empfänger im Jahr 2015 . . . . 170

Tab. 7.4 Mehrjähriger Finanzrahmen der EU 2014-2020, Haushaltstitel 2 (Nachhaltiges Wachstum: Natürliche Ressourcen) . . . . . . . . 171

Tab. 7.5 Anteil landwirtschaftlicher Betriebe, die keine Direktzahlungen beziehen. (Quelle: Eigene Darstellnug, nach BMELV (2013a); BMEL (2014a, 2015d)) . . . . . . . . . . . . . . . . . . . 178

Tab. 7.6 Steigerung der betrieblichen Kosten für die Einhaltung der EU-Auflagen im Bereich Umwelt, Tierwohl und Verbraucherschutz (\%) nach Betriebstypen................ 180

Tab. 7.7 Einkommensstabilisierende Wirkung der Direktzahlungen in Deutschland ............................. 181

Tab. 7.8 „Baukasten“ zur Honorierung von Basisleistungen im landwirtschaftlichen Natur- und Umweltmanagement . . . . . . . . . . 194

Tab. 7.9 Überblick über die möglichen Elemente einer reformierten Ersten Säule . . . . . . . . . . . . . . . . . . . . . . . . . . 195

Tab. 7.10 Monitoring-Elemente und ihr Beitrag zur Problemlösung . . . . . . 214

Tab. 7.11 Zuordnung der Instrumenten-Bausteine zu den prioritären Problembereichen .............................. 220

Tab. 8.1 Überblick über die Elemente der strategischen Option 1 (Weiterentwicklung des Greenings der Ersten Säule) . . . . . . . . . 229

Tab. 8.2 Abgrenzung der strategischen Option 2 (Starke Zweite Säule) von Option 1. . . . . . . . . . . . . . . . . . . . . . . . . . 235

Tab. A.1 Auswahl von zielführenden, beispielhaften ManagementMaßnahmen auf unterschiedlichen räumlichen Skalen mit Wirkungen, Trade-offs und Einschränkungen zum Schutz der Umweltressourcen, der biologischen Vielfalt und der Ökosystemleistungen. . . . . . . . . . . . . . . . . . . . . . . . 281 


\section{Kapitel 1 \\ Einleitung}

\subsection{Ausgangspunkte}

Die Sicherung der Ernährungssicherheit und der ökologischen Lebensgrundlagen einer wachsenden Weltbevölkerung ist eine der zentralen gesellschaftlichen und politischen Aufgaben des 21. Jahrhunderts. Dies ist beispielsweise durch die Sustainable Development Goals und die Agenda 2030 der Vereinten Nationen weltweit anerkannt. Die landwirtschaftliche Produktion gerät dadurch in ein Spannungsfeld. Zum einen ist sie auf funktionierende ökologische Grundlagen wie ertragreiche Böden, sauberes Wasser, biologische Schädlingsregulierung oder Bestäuberleistungen angewiesen. Zugleich ist es unvermeidlich, dass landwirtschaftliche Produktion Umweltressourcen nutzt und verbraucht sowie in die ökologischen Kreisläufe eingreift. Wegen dieser wechselseitigen Abhängigkeit von agrarischer Erzeugung und intakten Naturressourcen sind flächendeckend umweltverträgliche Formen der Landwirtschaft auf Dauer unverzichtbar.

Global steigt der Druck, für eine wachsende Weltbevölkerung ausreichend Nahrungsmittel, Rohstoffe und Energie zu erzeugen (FAO 2008). Daher ist zu erwarten, dass zunehmende Knappheit, vermittelt über steigende Preise, zu einer intensiveren Nutzung der landwirtschaftlichen Flächen und zu einer übermäßigen Nutzung der Umwelt- und Naturressourcen führt.

In Deutschland und Europa stellt sich die Situation jedoch komplizierter dar. Auf der einen Seite finden sich hoch produktive Regionen, in denen unter intensivem Einsatz von Maschinen, Mineraldünger und chemischem Pflanzenschutz sehr hohe Erträge erzielt werden. Auf der anderen Seite droht in weniger produktiven Regionen die großflächige Aufgabe der Landwirtschaft (EEA - European Environment Agency 2010). Beide Entwicklungen haben bedenkliche Folgen für Umwelt und Natur. In den Intensivregionen kommt es zu einem weitgehenden Verlust von naturnahen Habitaten und biologischer Vielfalt, zu übermäßigen Einträgen von Stickstoff, 
Phosphor und Schadstoffen in Böden und Gewässer sowie zu hohen Emissionen von Feinstoffen und Klimagasen (siehe Kap. 3). In den weniger produktiven Regionen gehen dagegen durch die Nutzungsaufgabe offene Landschaften verloren und mit ihnen verschwinden mannigfaltige Habitate für viele Tier- und Pflanzenarten. Landschaftsvielfalt und biologische Vielfalt sind also sowohl durch die Intensivierung als auch durch die großflächige Aufgabe der Landbewirtschaftung bedroht (Tscharntke et al. 2005; Kleijn et al. 2009). Die Entwicklung der Agrarlandschaften stellt daher seit den 1980er-Jahren einen Schwerpunkt der Umwelt- und Naturschutzpolitik in Europa dar (SRU 1985; Heißenhuber et al. 2015).

Die sehr unterschiedlichen ökologischen Systeme, in denen in Europa Landwirtschaft betrieben wird, sind ökonomisch durch den gemeinsamen Binnenmarkt der EU eng miteinander verknüpft. Mit der Gründung der Europäischen Gemeinschaften 1957 wurde die Einrichtung eines gemeinsames Markts für Agrarerzeugnisse sowie einer Gemeinsamen Agrarpolitik (GAP) vereinbart. Unter dem Eindruck der Nahrungsmittelknappheit nach dem Zweiten Weltkrieg war die Landwirtschaftspolitik der Gründungsstaaten der EG vorranging darauf ausgerichtet, stabile Rahmenbedingungen für landwirtschaftliche Betriebe herzustellen und deren Produktivität und Einkommen zu erhöhen; dieses Paradigma wurde in den Gründungsverträgen der EG und damit in der GAP fest verankert (Tracy 1989). Seit Mitte der 1980er-Jahr wurde zunehmend deutlich, dass die modernen, produktionssteigernden Formen der Landbewirtschaftung oft negative Auswirkungen auf Landschaftsvielfalt, Gewässerqualität, biologische Vielfalt, Bodenfruchtbarkeit und natürliche Regulationsmechanismen haben. Unter dem Leitbegriff „Multifunktionalität“ verbreitete sich die Auffassung, dass auf den landwirtschaftlichen Flächen nicht nur marktgängige Erzeugnisse produziert werden, sondern auch öffentliche Güter (Lowe et al. 2010). Letztere wurden lange Zeit als unvermeidliche Kuppelprodukte der landwirtschaftlichen Produktion angesehen - bei zunehmender Intensivierung wurde aus dem Kopplungs- aber tendenziell ein Konkurrenzverhältnis. Das Ungleichgewicht zwischen entlohnten marktgängigen Erzeugnissen und nicht entlohnten öffentlichen Gütern führt dann zu einem Marktversagen, weil sich aus der einzelwirtschaftlichen Sicht der Betriebe und Konsumenten eigene Investitionen in öffentliche Güter, deren Nutzen breit verteilt ist oder erst langfristig anfällt, nicht lohnen. Diese ökonomische Analyse der ökologischen Konsequenzen der Landwirtschaft trug zu der Auffassung bei, dass umweltpolitische Anliegen nur durch eine Integration in die Agrarpolitik verwirklicht werden könnten (Lowe und Baldock 2000). Die GAP-Reform von 1992 führte Agrarumweltprogramme ein, mit denen seither Landwirte für die Bereitstellung öffentlicher Umweltgüter bzw. für besonders umweltfreundliche Praktiken honoriert werden.

Seit 1992 besteht auch ein Spannungsverhältnis innerhalb der GAP. Auf der einen Seite dient die Unterstützung der öffentlichen Leistungen der Landwirtschaft als Legitimation für die erheblichen Zahlungen der EU-Agrarpolitik. Auf der anderen Seite dient der größte Teil dieser Zahlungen nach wie vor dazu, die Einkommen der landwirtschaftlichen Betriebe zu erhöhen (Daugbjerg und Swinbank 2016). Das Erbe einer an den Interessen des Agrarsektors und an der Erhöhung der landwirtschaftlichen Produktion ausgerichteten Zielsetzung lebt in der Agrarpolitik der EU nicht nur fort, sondern stellt nach wie vor deren Kern dar (Daugbjerg und Swinbank 2016). Der Verweis 
auf die besonderen Umweltleistungen der Landwirtschaft dient vorwiegend dazu, den Liberalisierungsdruck abzuwehren, dem sich Wirtschaftssektoren und öffentliche Politiken allgemein ausgesetzt sehen (Daugbjerg und Feindt 2017).

Die Integration von Umweltanliegen in die Agrarpolitik war offensichtlich wenig erfolgreich. Der Zustand der Umwelt- und Naturressourcen in den Agrarlandschaften hat sich seit den 1980er-Jahren größtenteils weiter verschlechtert (EEA - European Environment Agency 2010; Nationale Akademie der Wissenschaften Leopoldina et al. 2018; Wissenschaftlicher Beirat für Biodiversität und Genetische Ressourcen beim BMELV 2018). Die Kritik an der unzureichenden Berücksichtigung von Umweltbelangen in der Agrarpolitik erfolgt dabei im Wesentlichen entlang von vier Linien: (i) unzureichende oder fehlende ordnungsrechtliche Mindeststandards (zum Beispiel zur Reinhaltung von Gewässern oder zur Emission von Feinstoffen), (ii) mangelhafte Umsetzung des bestehenden Ordnungsrechts (was beispielsweise zu erhöhten Nitratwerten im Grundwasser oder zu Feinstoffbelastungen oberhalb der Zielwerte führt), (iii) unzureichende Finanzierung von Maßnahmen des Natur- und Umweltschutzes sowie (iv) negative Umweltauswirkungen anderer agrarpolitischer Maßnahmen. Letzteres gilt etwa, wenn die flächenbezogenen Direktzahlungen zu einer Erhöhung der Preise für landwirtschaftliche Flächen und andere Produktionsinputs führen und davon Anreize ausgehen, die Böden noch intensiver zu bewirtschaften. Institutionell ist dabei nicht nur die Agrarpolitik im engeren Sinne angesprochen. Wichtige Teile des ordnungsrechtlichen Rahmens, wie etwa die Vogelschutz- oder die Wasserrahmenrichtlinie oder diverse Kennzeichnungsregeln, entstammen dem Umwelt- und Verbraucherschutzrecht und sind im allgemeinen Binnenmarktrecht der EU verankert.

Abgesehen von ordnungsrechtlichen Initiativen ist die agrarpolitische Reformdiskussion zeitlich von den siebenjährigen Haushaltszyklen der Europäischen Union geprägt. Die innerhalb der GAP möglichen Maßnahmen und der Rahmen für deren finanzielle Ausstattung werden jeweils für sieben Jahre festgelegt. Aufgrund der überragenden Bedeutung der GAP für die Umweltsituation in Agrarlandschaften konzentriert sich die umweltpolitische Kritik an der GAP häufig auf eine Kritik an der Verteilung des Budgets zwischen den flächenbezogenen Direktzahlungen in der sogenannten ersten Säule der GAP und den Natur-, Umwelt- und Klimaschutzmaßnahmen, die Teil der zweiten Säule sind. Unter dem Leitsatz „öffentliches Geld für öffentliche Güter“" werden die stärker ergebnis- und leistungsorientierten Maßnahmen der zweiten Säule den vorwiegend auf Einkommenstransfer dienenden Direktzahlungen in der ersten Säule gegenübergestellt.

Das hier vorliegende Buch verfolgt einen anderen Ansatz. Ausgehend von einer Besorgnis erregenden, teilweise sogar dramatischen Bestandsaufnahme der Umweltwirkungen der Landwirtschaft in Deutschland und Europa wird zunächst die Frage gestellt, warum die GAP und das Ordnungsrecht die Herausforderungen des Natur-, Umwelt- und Klimaschutz nur langsam und unzureichend aufgreifen und selbst die politisch vereinbarten und gesetzlich festgelegten Ziele nicht in angemessener Weise umsetzen. Daraus wird eine Stärken-Schwächen-Analyse des bestehenden Steuerungsrahmens abgeleitet, die Schwächen sowohl auf der Ebene der Ziele wie der Instrumente und ihrer Umsetzung aufzeigt. Als Beitrag zur Problemlösung 
wird ein Leitbild für eine nachhaltige Agrarpolitik entwickelt, die konsequent die Anforderungen des Natur-, Umwelt- und Klimaschutzes berücksichtigt. Mit dieser Zielorientierung werden Ansätze diskutiert, die agrarpolitischen und ordnungsrechtlichen Instrumente zu verbessern. Auf dieser Basis werden drei Optionen für eine Weiterentwicklung der GAP formuliert und zum Entwurf einer neuen Architektur für die Gemeinsame Agrarpolitik verdichtet.

Das Konzept eines Gesellschaftsvertrags für den Agrarsektor soll dabei einen Ansatzpunkt bieten um zu klären, was die Gesellschaft und die Landwirtschaft voneinander erwarten können. Der Begriff wurde vom Projektkoordinator im Laufe der Arbeiten eingeführt, um über ein angemessenes Verhältnis zwischen den Produktionsleistungen, den öffentlichen Leistungen und den Bedürfnissen der Landwirtschaft zu reflektieren. Die Grundidee ist, dass die Gesellschaft möglichst präzise formuliert, welche Leistungen sie von der Landwirtschaft erhalten möchte. Neben gesunden und preiswerten Nahrungsmitteln und Rohstoffen gehören hierzu die verschiedenen öffentlichen Leistungen wie biodiversitätsfreundliche Landschaften, Erhalt des agrarkulturellen Erbes und Tierwohl. Im Gegenzug ist zu klären, unter welchen Bedingungen die Landwirtschaft diese Leistungen erbringen kann - wie müssen Märkte, Ordnungsrecht sowie gesellschaftliche und finanzielle Unterstützung zusammenspielen? Eine solch umfassende Betrachtung soll eine realistische Perspektive für eine integrative Agrarpolitik eröffnen und letztlich die Entwicklung hin zu einem ökonomisch, ökologisch und sozial nachhaltigen Agrarsektor ermöglichen. Während die Natur-, Umwelt- und Klimawirkungen der Landwirtschaft also der Ausgangspunkt der hier vorgelegten Arbeiten sind, stellt das Konzept eines Gesellschaftsvertrags für die Landwirtschaft den Versuch dar, die Antwort auf die ökologischen Fragen des Agrarsektors in eine umfassendere Antwort auf die vielfältigen, komplexen und dringenden Herausforderungen der deutschen und europäischen Landwirtschaft im 21. Jahrhundert einzubetten.

\subsection{Das ZA-NExUS-Projekt: Ziele und Projektverbund}

Das hier vorgelegte Buch stellt die Ergebnisse des Projekts „Zukunftsfähige Agrarpolitik - Natur erhalten, Umwelt schützen (ZA-NExUS)“ vor. ZA-NExUS wurde von November 2015 bis März 2017 durch das Bundesamt für Naturschutz (BfN) in Kooperation mit dem Umweltbundesamt (UBA) mit Mitteln des Bundesministeriums für Umwelt, Naturschutz, Bau und Reaktorsicherheit (BMUB) gefördert. Ziel war es, wissenschaftlich basierte Optionen für die künftige Ausgestaltung der Agrarpolitik aus der Perspektive des Natur- und Umweltschutzes zu formulieren und in die öffentliche und politische Diskussion einzubringen.

$\mathrm{Zu}$ diesem Zweck wurden

- mittels eines systematischen Literaturberichts die für den Natur- und Umweltschutz relevanten systemischen Entwicklungen im Zusammenhang mit der landwirtschaftlichen Entwicklung aufbereitet und der Beitrag der bisherigen 
Agrarpolitik zur Ver- oder Entschärfung von Problemlagen sowie zur Ermutigung oder Behinderung positiver Ansätze und Entwicklungen aufgezeigt (siehe Kap. 3 und 4);

- Stärken und Schwächen der gegenwärtigen Agrarpolitik aus Sicht des Natur- und Umweltschutzes in Form einer SWOT-Analyse herausgearbeitet (siehe Kap. 5);

- ein Leitbild für eine multifunktionale, natur- und umweltverträgliche Landwirtschaft entwickelt, das eine politisch hinreichend breite Akteurskonstellation ansprechen kann, sowie Differenzen zwischen dem Leitbild und der gegenwärtigen Agrarpolitik herausgearbeitet (Kap. 6);

- politische Optionen (,Bausteine“) für das künftige Policy Design entwickelt und deren Vor- und Nachteile abgeschätzt (siehe Kap. 7);

- alternative agrarpolitische Strategieoptionen entwickelt und bewertet (siehe Kap. 8) sowie

- die Ergebnisse mittels eines Policy-Papers (Kap. 2) und eines Pressegesprächs mit der damaligen Bundesumweltministerin Barbara Hendricks in die öffentliche und politische Diskussion eingebracht.

Gegenstand des Projekts waren im Kern die Gemeinsame Agrarpolitik (GAP) der Europäischen Union (EU) sowie das deutsche und europäische Ordnungsrecht. Darüber hinaus wurde versucht, auch weitere relevante nationale und europäische Instrumente und Regulierungen, die den Agrarsektor betreffen, einzubeziehen. Die Handlungsempfehlungen sollen einen innovativen konzeptionellen Beitrag für die Transformation zu einer nachhaltigen und natur- und umweltverträglichen Agrarpolitik leisten.

Es wurde insbesondere angestrebt, Bausteine und Optionen zu erarbeiten, die neue, breitere Koalitionen ermöglichen, um die von der Landwirtschaft verursachten Natur- und Umweltschutzprobleme zu vermindern. Dazu sollten die zu erarbeitenden Optionen eine Vielzahl einflussreicher Akteure berücksichtigen (aus dem Bereich der Politik, Vertreter der Branche, relevante gesellschaftliche Gruppen), möglichst viele Brücken bauen und breite Expertise einbeziehen.

Im Vergleich zu früheren Arbeiten zur Agrarpolitik hat ZA-NExUS versucht, bei der Analyse und der Strategieentwicklung auch neuere integrative, systemische Ansätze wie Ökosystemdienstleistungen, Resilienz, Nexus-Ansätze und Adaptive Management/Adaptive Governance sowie verhaltenswissenschaftliche Ansätze (Behavioural Governance) mit einzubeziehen. Dies hat das Projekt gelegentlich in ein Spannungsverhältnis zum aktuellen Stand der agrarpolitischen Diskussion gebracht, in der diese Konzeptionen noch nicht durchgehend etabliert sind. Etwas zugespitzt könnte man von einem allgemeinen Dilemma zwischen politischer Anschlussfähigkeit der Projektergebnisse und der Innovationshöhe der im Projekt verfolgten Ansätze sprechen.

Das ZA-NExUS-Projekt wurde in einem Verbund von vier Partnern bearbeitet: Wageningen University (Strategic Communication und Law), Justus-LiebigUniversität Gießen (Professur für Tierökologie), Technische Universität MünchenWeihenstephan (Lehrstuhl für Produktions- und Ressourcenökonomie) sowie Universität Osnabrück (Institut für Umweltsystemforschung). Die Projektkoordination lag bei der Wageningen University. Die verschiedenen Teile in Kap. 3 und 4, 
welche eine Synthese der vorliegenden Literatur darstellen, wurden arbeitsteilig nach fachlicher Nähe, jedoch in enger Abstimmung erstellt. Die anderen Kapitel entstanden in intensiver Kooperation aller Autorinnen und Autoren.

\subsection{Konzeptioneller Ansatz und Vorgehensweise}

Dieses Buch verfolgt einen inter- und transdisziplinären Ansatz, der durch eine praxisbezogene Problemorientierung integriert wird. Die Praxisorientierung wird gleich im folgenden Kap. 2 sichtbar. Dieses enthält das Policy-Paper, welches das politisch-praktische Zielprodukt des ZA-NExUS-Projekts war und in einer Pressekonferenz mit der damaligen Bundesumweltministerin Barbara Hendricks einer breiten Öffentlichkeit vorgestellt wurde. Das Policy-Paper baut auf den Ergebnissen auf, die in den nachfolgenden Kapiteln dargestellt sind.

Die wissenschaftlichen Bestandsaufnahmen der Kap. 3 und 4 sind vorwiegend interdisziplinär ausgerichtet, indem sie den Kenntnisstand verschiedener Disziplinen zusammenführen. Die Synthese zu einer SWOT-Analyse der GAP (Kap. 5), die Entwicklung eines Leitbilds (Kap. 6) sowie die Ausarbeitung und Bewertung von agrarpolitischen „Bausteinen“ und Strategien (Kap. 7 und 8) folgen vorwiegend einer transdisziplinären Logik, welche neben den wissenschaftlichen Befunden die Problemwahrnehmungen im agrarpolitischen Handlungskontext einbezieht. Die inter- und transdiziplinäre Sichtweise wurde in allen Phasen des Projekts durch eine Projektbegleitende Arbeitsgruppe gestärkt. Dieser gehörten Vertreter des BfN, des UBA und des BMUB sowie fünf wissenschaftliche Peer Reviewer mit Expertise in Agrarökonomie, Agrarwissenschaften, Agrarlandschafts- und Agrarsystemforschung sowie Agrarumweltwissenschaften an.

Die Bestandsaufnahme der Wirkungen der Landwirtschaft auf Natur- und Umweltgüter in Kap. 3 erfolgte aus ökologischer und umweltwissenschaftlicher Sicht. In Kap. 4 werden die agrarpolitischen Rahmenbedingungen zum einen im Hinblick auf die institutionelle und ideelle Entwicklungslogik der GAP analysiert, wobei Ansätze des historischen Institutionalismus erkenntnisleitend waren (Abschn. 4.1). Zum anderen werden aus juristischer und rechtspolitischer Sicht neuere Veränderungen des europapolitischen Rahmens dargestellt (Abschn. 4.2). Weiterhin werden neuere Ansätze zur Integration von Umweltanliegen in den Governance-Rahmen eingeführt (Abschn. 4.3), die in der Agrarpolitik derzeit eher in Nischen implementiert werden, aber ein erhebliches Potenzial für einen stärker systemischen und integrativen Ansatz besitzen.

Auswahl und Auswertung der Literatur erfolgten nach den Regeln einer systematischen Literaturanalyse. Zunächst wurden für jeden Teilbereich von den jeweiligen Bearbeitern Suchbegriffe vorgeschlagen. Diese wurden im Projektteam diskutiert und gegebenenfalls revidiert. Mit Hilfe der Suchbegriffe wurde dann in einschlägigen Literaturdatenbanken (Web of Science, Scopus, Datenbanken der Universitätsbibliotheken) eine erste Abfrage durchgeführt. Diese ergab zumeist sehr hohe Trefferzahlen. Die Suche wurde dann mit Hilfe zusätzlicher Schlagworte verfeinert. 
Anschließend wurde eine manuelle Auswahl der Beiträge nach Relevanz für die Leitfragen des Projekts vorgenommen.

Zunächst war vorgesehen, die Literatur anhand der folgenden fünf Oberdimensionen aufzubereiten:

1. behandelte landwirtschaftliche Aktivitäten;

2. Aussagen zu Umweltauswirkungen in den verschiedenen Problemdimensionen;

3. Aussagen zur ökonomischen Vorteilhaftigkeit landwirtschaftlicher Aktivitäten, Betriebsformen usw.;

4. Aussagen zu Governance, Policy Design und Anreizmechanismen;

5. konzeptionelle Integrationsansätze und deren Anwendung.

Diese Gliederung wurde wegen unterschiedlicher Schwerpunkte in der Literatur zu den einzelnen Teilbereichen nicht strikt eingehalten, diente aber der erkenntnisleitenden Orientierung.

Weiterhin war zunächst geplant gewesen, die Veröffentlichungen getrennt nach sechs Publikationstypen auszuwerten:

1. wissenschaftliche Fachzeitschriften mit Peer Review;

2. Buchbeträge und Fachzeitschriften ohne Peer Review;

3. Projektberichte;

4. Gutachten von wissenschaftlichen Sachverständigenräten;

5. Arbeitspapiere, zum Beispiel aus der Ressortforschung;

6. Policy-Papers von NGOs, Interessengruppen und politischen Institutionen.

Aufgrund der hohen Trefferzahlen allein bei wissenschaftlichen Fachzeitschriften beschränkt sich die hier vorgestellte Analyse nun weitgehend auf wissenschaftliche Publikationen, und dabei vorwiegend auf solche mit Peer Review. Projektberichte und Gutachten von wissenschaftlichen Sachverständigenräten wurden v. a. dort berücksichtigt, wo sie einen guten und zuverlässigen Überblick zu einzelnen Problembereichen geben. Arbeitspapiere und Policy-Papiere von NGOs, Interessengruppen und politischen Institutionen wurden angesichts der Materialfülle und der beschränkten Projektlaufzeit in der Regel nicht berücksichtigt. Entsprechend dem Fortgang der Projektarbeiten sind Publikationen berücksichtigt, die bis zum Frühjahr 2016 erschienen sind.

Zur Strukturierung der Darstellung wurden zu Beginn des Projekts von den jeweils federführenden Bearbeitern erkenntnisleitende Hypothesen zu den verschiedenen Teilbereichen der Bestandsaufnahme formuliert. Die Hypothesen wurden im Projektteam und auf der ersten Sitzung der Projektbegleitenden Arbeitsgruppe im November 2015 diskutiert und anschließend teilweise modifiziert. Sie betrafen das Verhältnis von agrarpolitischem und ordnungsrechtlichem Rahmen, Umfang und Formen der Landbewirtschaftung sowie Auswirkungen auf Ziele des Natur- und Umweltschutzes. Eine kondensierte Version einiger dieser Hypothesen ist in Abschn. 5.6.2 enthalten.

Die auf die Literaturauswertung folgende SWOT-Analyse (Kap. 5) diente als heuristischer Ansatz zur Erarbeitung einer Synthese der Problembeschreibungen. Sie fasst die Stärken, Schwächen, Chancen und Gefährdungen der gegen- 
wärtigen Agrarpolitik aus Sicht des Natur- und Umweltschutzes zusammen. Das methodische Vorgehen entsprach dabei einem iterativen interdisziplinären Dialog. Die vorliegende SWOT-Analyse ist das Ergebnis intensiver Diskussionen im Projektteam mit dem Ziel einer verdichteten Beschreibung der vorrangigen Problemlagen aus Sicht des Natur- und Umweltschutzes. Auf Basis von schriftlichen Entwürfen wurde auf einem gemeinsamen Workshop des gesamten Projektteams im April 2016 eine erste Fassung erstellt. Diese wurde auf Grundlage der Rückmeldungen der Peer Reviewer und der Projektbegleitenden Arbeitsgruppe überarbeitet.

Bei der Formulierung eines Leitbilds für eine zukunftsfähige Agrarpolitik (Kap. 6) wurde zunächst eine Liste mit Leitprinzipien aus bestehenden, demokratisch legitimierten und international vereinbarten Rechtsnormen erstellt. Dadurch sollte sichergestellt werden, dass das Leitbild im bestehenden gesellschaftlichen und internationalen Normkonsens verankert ist. Anschließend wurden die Problemlagen aus Sicht des Natur- und Umweltschutzes priorisiert, um später prüfen zu können, inwiefern das vorgeschlagene Leitbild auch die wirklich relevanten Entwicklungen adressiert. Das hier nun vorgelegte Leitbild ist das Ergebnis intensiver Diskussionen im Projektteam. Dabei wurde insbesondere versucht, den Kern des Leitbilds auf eine kleine Anzahl greifbarer Leitlinien zu verdichten, die gewissermaßen als Kompass dienen können. Spezifische qualitative und quantitative Zielwerte wurden aus der umweltwissenschaftlichen Diskussion abgeleitet. Der Soll-Ist-Vergleich erfolgte durch Abgleich der Zielwerte mit der tatsächlichen Situation, die in der Literaturanalyse dargestellt ist.

Die im Projekt entwickelten strategischen Politik-Optionen sind ebenfalls das Ergebnis eines iterativen Deliberationsprozesses zwischen den Projektpartnern unter Einbeziehung der Rückmeldungen der Peer Reviewer und der Projektbegleitenden Arbeitsgruppe. Die Diskussionen basierten dabei auf den Ergebnissen des Literaturberichts sowie auf einer Bewertung laufender agrarpolitischer Debatten. In die Bewertung gingen auch die Erkenntnisse aus Hintergrundgesprächen mit agrarpolitischen Akteuren ein. Zunächst wurden von den Projektpartnern drei ,agrarpolitische Optionen" formuliert und im Projektteam sowie auf der zweiten Sitzung der Projektbegleitenden Arbeitsgruppe im Februar 2016 in Bonn zur Diskussion gestellt. Diese drei Optionen fokussierten auf ein weiteres „Greening“ der Ersten Säule der Gemeinsamen Agrarpolitik (Option 1), einen Ausbau der Zweiten Säule (Option 2) bzw. eine Anhebung der gesetzlichen Mindeststandards in Kombination mit zunehmend anspruchsvolleren privaten Standards (ursprüngliche Option 3). Diese Optionen wurden auf einem Workshop des Projektteams im April 2016 in Osnabrück intensiv diskutiert und anschließend in drei Richtungen weiterentwickelt: Zum einen wurden die unterschiedlichen politischen Handlungslogiken geschärft, zum zweiten wurde eine neue Option 3 mit einer neuen, integrierten Systematik entwickelt, und zum dritten wurden die jeweiligen Entwicklungsmöglichkeiten im Zeitablauf verdeutlicht. Weiterhin wurde eine vierte Option hinzugefügt, die eine Umstellung des Verhältnisses zwischen den landwirtschaftlichen Betrieben und den staatlichen Stellen auf eine ,prinzipienbasierte Regulierung" vorsieht (siehe dazu jetzt Purnhagen und Feindt 2017). Diese vier Optionen wurden im Ersten Zwischenbericht dargestellt und intensiv auf der dritten Sitzung der Projektbegleitenden Arbeitsgruppe unter Beteiligung der Peer 
Reviewer im April 2016 in Bonn diskutiert. Dabei wurde die Option einer radikalen Umstellung der umweltpolitischen Regulation des Agrarsektors auf eine ,prinzipienbasierte Regulation“ als interessant, aber nicht hinreichend anschlussfähig an die aktuelle agrarpolitische Diskussion eingeschätzt. Die weitere Ausarbeitung von strategischen Handlungsalternativen konzentrierte sich daher auf die drei in Kap. 8 dargestellten Optionen: Weiterentwicklung des Greenings in der Ersten Säule der GAP, Stärkung der Zweiten Säule der GAP sowie ein neues „Integriertes Modell“ der Agrarförderung. Weiterhin kam das Projektteam in Reaktion auf das Feedback zu dem Schluss, dass die agrarpolitischen Optionen besser auf zwei Ebenen dargestellt werden sollten:

- zum einen auf der Instrumentenebene alternativer Ansätze für die Weiterentwicklung der einzelnen Elemente der Agrarpolitik, die wir im Folgenden als „Bausteine“ bezeichnen;

- zum anderen auf der Ebene strategischer Entwicklungsrichtungen, die sich in ihren politischen Handlungslogiken unterscheiden und verschiedene Gewichtungen und Ausprägungen der einzelnen „Bausteine“ beinhalten. Diese strategischen Entwicklungsrichtungen bezeichnen wir als ,,agrarpolitische Optionen“.

Die „Bausteine“ wurden im Wesentlichen auf Basis einer Auswertung der wissenschaftlichen Literatur formuliert und dann im Hinblick auf die jeweiligen Stärken und Schwächen sowie mögliche Ansätze der Weiterentwicklung bewertet (siehe Kap. 7). Die weitere Diskussion der strategischen agrarpolitischen Optionen folgte in einem iterativen Prozess im Projektteam, der auf zwei ganztägigen TeamWorkshops im Mai und Juli 2016, jeweils in Frankfurt, kulminierte. Auf dem Workshop im Mai wurde die Diskussion zur Ausrichtung der strategischen Optionen weitgehend abgeschlossen. Der Workshop im Juli diente vor allem der Diskussion der Voraussetzungen und Implikationen dieser Optionen. Im Ergebnis stellen die Optionen eine konzeptionelle Konfiguration des agrarpolitischen Strategieraums dar, die nach Kriterien der politisch-praktischen Plausibilität und der Nützlichkeit für die Konzeption von Politik, zu beurteilen sind. Bei der Darstellung der Voraussetzungen und Implikationen der Optionen handelt es sich nicht um Szenarien oder Prognosen, sondern um eine qualitative Abschätzung auf Basis des Hintergrundwissens der Mitglieder des Projektteams. Diese Abschätzungen haben den Charakter von Hypothesen, die durch präzisere, aber auch wesentlich aufwändigere Verfahren, wie etwa Szenario-Analysen oder Modellrechnungen, und ggf. durch empirische Analysen zu validieren wären.

Die Optionen stellen verschiedene strategische Entwicklungslinien für die künftige Agrarpolitik aus Sicht des Natur- und Umweltschutzes dar, welche zum einen unterschiedliche Grade von Kontinuität mit der bisherigen Agrarpolitik repräsentieren und zum anderen jeweils verschiedene Aspekte der derzeitigen agrarpolitischen Architektur in den Mittelpunkt stellen. Die Optionen haben unterschiedliche Vorund Nachteile, die letztendlich Gegenstand einer politischen und gesellschaftlichen Bewertung sein müssen. Das Projektteam hat den Auftraggebern daher statt der im Projektantrag vorgesehenen einen Option mehrere Optionen angeboten, um eine umfassendere Bewertung des strategischen Optionenraums zu ermöglichen. 
Die in einer ersten Fassung des Projektberichts enthaltenen Optionen wurden auf einer Sitzung der Projektbegleitenden Arbeitsgruppe Anfang September 2016 in Bonn sowie auf einem Workshop mit den Peer Reviewern Mitte September 2016 in Hannover diskutiert. Seitens der Auftraggeber wurde Option 3 als die interessanteste Grundlage für das Policy-Paper ausgewählt.

Eine erste Fassung des Policy-Papers wurde auf der folgenden Sitzung der Projektbegleitenden Arbeitsgruppe Anfang Oktober 2016 in Bonn diskutiert. Dabei wurde beschlossen, die vorgelegte, mehr als 10 Seiten lange Fassung zu einem Hintergrundpapier zu machen, das ein möglichst kurzes Policy-Paper flankieren könnte. Weiterhin wurde der Vorschlag konkretisiert, die Ergebnisse des Projekts im Vorfeld der Grünen Woche im Rahmen eines großen Agrarkongresses in Berlin zu präsentieren.

Zwei Fassungen des ca. fünfseitigen Policy-Papers wurden auf zwei Sitzungen der Projektbegleitenden Arbeitsgruppe Anfang November und Anfang Dezember 2016 in Bonn diskutiert. Parallel dazu gaben drei der Peer Reviewer Rückmeldung zur Langfassung des Politik-Papiers.

Die endgültige Version des Policy-Papers (Kap. 2 dieses Berichts) wurde am 9. Januar 2017 den Auftraggebern übermittelt. Das Papier wurde am 17. Januar 2017 in Berlin im Rahmen eines Pressegesprächs mit der Bundesumweltministerin Dr. Barbara Hendricks der breiteren Öffentlichkeit vorgestellt und anschließend im Rahmen des vom BMUB veranstalteten Kongresses „Landwirtschaft mit Zukunft“ in Berlin der Fachöffentlichkeit zur Diskussion gestellt. Eine vom Projektteam ins Englische übertragene Version des Papiers wurde im Februar 2017 auf Research Gate eingestellt und ist dort frei zugänglich (Feindt et al. 2017). Das Papier sowie der Kongress fanden bundesweit ein breites und positives Medienecho.

\section{4 Überblick über das Buch und Danksagung}

Das hier vorgelegte Buch stellt eine redaktionell überarbeitete Fassung des Projektberichts dar, der im April 2017 den Auftraggebern vorgelegt wurde. Kap. 2 enthält das Hauptergebnis des Projekts: das im Januar 2017 vorgestellte Policy-Paper. Die weiteren Kapitel enthalten die wissenschaftlichen Arbeiten, auf denen das Policy-Paper aufbaut. Die Darstellung beginnt mit einer Übersicht über die gegenwärtige Ausgangssituation bezüglich der Umweltauswirkungen der Landwirtschaft (Kap. 3). Es folgt eine Analyse der Logik der derzeitigen agrarpolitischen Rahmenbedingungen (Kap. 4). Beide Teile basieren auf einer umfassenden Literaturanalyse. Die Darstellung der Ausgangslage bündeln wir in einer SWOT-Analyse der derzeitigen Agrarpolitik aus der Perspektive des Natur- und Umweltschutzes (Kap. 5). Anschließend stellen wir Grundlinien eines zeitgemäßen Leitbilds für die Agrarpolitik vor, welches neben der Produktionsfunktion der Landwirtschaft auch die Natur- und Umweltauswirkungen effektiv in den Blick nimmt (Kap. 6). Darauf aufbauend präsentieren wir Möglichkeiten zur Weiterentwicklung der verschiedenen Elemente der Agrarpolitik, die wir als „Bausteine“ 
einer zukunftsfähigen Agrar-Umweltpolitik verstehen (Kap. 7). Es folgt die Entwicklung und Bewertung von alternativen politischen Optionen zur besseren Integration des Natur- und Umweltschutzes in die zukünftige Agrarpolitik (Kap. 8). Eine Schlussbetrachtung rundet die Darstellung ab (Kap. 9). Zwei Anhänge enthalten ergänzende Informationen, deren Ausführlichkeit den Gang der Argumentation im Haupttext nicht unnötig unterbrechen soll.

Die Autorinnen und Autoren bedanken sich für die konstruktive und offene $\mathrm{Zu}$ sammenarbeit mit den Auftraggebern, die hervorragenden Rückmeldungen der Peer Reviewer sowie die wichtigen Beiträge und kritischen Kommentare der Teilnehmerinnen und Teilnehmer an den Projektworkshops. Ebenfalls danken wir Kerstin Oertel und Dr. Astrid Häger für die unermüdliche Unterstützung bei der Überarbeitung und Fertigstellung des Manuskripts. All dies hat wesentlich zur Qualität des vorliegenden Buches beigetragen. Die Verantwortung für alle verbliebenen Fehler liegt selbstverständlich bei den Autorinnen und Autoren.

Open Access Dieses Kapitel wird unter der Creative Commons Namensnennung 4.0 International Lizenz (http://creativecommons.org/licenses/by/4.0/deed.de) veröffentlicht, welche die Nutzung, Vervielfältigung, Bearbeitung, Verbreitung und Wiedergabe in jeglichem Medium und Format erlaubt, sofern Sie den/die ursprünglichen Autor(en) und die Quelle ordnungsgemäß nennen, einen Link zur Creative Commons Lizenz beifügen und angeben, ob Änderungen vorgenommen wurden.

Die in diesem Kapitel enthaltenen Bilder und sonstiges Drittmaterial unterliegen ebenfalls der genannten Creative Commons Lizenz, sofern sich aus der Abbildungslegende nichts anderes ergibt. Sofern das betreffende Material nicht unter der genannten Creative Commons Lizenz steht und die betreffende Handlung nicht nach gesetzlichen Vorschriften erlaubt ist, ist für die oben aufgeführten Weiterverwendungen des Materials die Einwilligung des jeweiligen Rechteinhabers einzuholen.

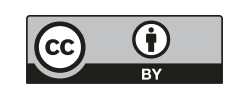




\section{Kapitel 2 \\ Policy-Paper: ,Ein zukunftsfähiger \\ Gesellschaftsvertrag mit der \\ Landwirtschaft: Plädoyer für eine neue Agrarpolitik66}

Das nun folgende Policy-Paper stellt das wichtigste Ergebnis des ZA-NEXUSProjekts dar, dessen Ziel es war, wissenschaftlich basierte Optionen für die künftige Ausgestaltung der Agrarpolitik aus der Perspektive des Natur- und Umweltschutzes $\mathrm{zu}$ formulieren und in die öffentliche und politische Diskussion einzubringen. Das Policy-Papier wurde im Herbst 2016 in enger Abstimmung zwischen den Projektnehmern und Projektgebern auf Basis eines längeren Reflexionspapiers erstellt. Die Verantwortung für die Inhalte sowie eventuelle Unzulänglichkeiten liegt selbstverständlich allein bei den Autorinnen und Autoren.

Das Policy-Paper wurde am 17. Januar 2017 vom Projektkoordinator in einer Pressekonferenz mit Bundesministerin Dr. Barbara Hendricks sowie auf dem BMU-Kongress „Landwirtschaft mit Zukunft“ in Berlin der Öffentlichkeit vorgestellt. ${ }^{1}$

\subsection{Zusammenfassung}

Was können Landwirtschaft, Politik und Gesellschaft voneinander erwarten? Zur Beantwortung dieser Frage plädieren wir für eine neue Agrarpolitik auf der Basis eines zukunftsfähigen Gesellschaftsvertrags mit der Landwirtschaft. Dieser Vertrag sieht vor, dass weiterhin erhebliche öffentliche Mittel für den Agrarsektor aufgewendet werden. Diese Gelder sollen aber in Zukunft die Landwirtinnen und Landwirte unterstützen, die qualitativ hochwertige Lebensmittel herstellen und zugleich Gemeinwohlleistungen erbringen, die vom Markt nicht honoriert werden. Dazu

\footnotetext{
${ }^{1}$ Zitierweise des Policy-Papers: Peter H. Feindt, Christine Krämer, Andrea Früh-Müller, Volkmar Wolters, Claudia Pahl-Wostl, Alois Heißenhuber, Caroline van Bers, Fabian Thomas, Kai Purnhagen: Ein zukunftsfähiger Gesellschaftsvertrag mit der Landwirtschaft: Plädoyer für eine neue Agrarpolitik. Policy-Paper des Forschungs- und Entwicklungs-Verbundvorhabens „ZA-NExUS: Zukunftsfähige Agrarpolitik - Natur erhalten, Umwelt sichern“, gefördert vom Bundesamt für Naturschutz und dem Umweltbundesamt, FKZ 3515880 400, Bonn/Berlin, Januar 2017.
} 
gehören etwa Beiträge zum Natur-, Umwelt- und Klimaschutz, die Bewirtschaftung besonders vielfältiger Landschaften oder die Erhaltung der Landschaftsvielfalt durch Bewirtschaftung unter schwierigen natürlichen Bedingungen. Eine neue Architektur der Agrarpolitik soll es den Landwirtinnen und Landwirten ermöglichen, mit nachhaltig erzeugten Produkten und mit ihren Leistungen für das Gemeinwohl trotz starker internationaler Konkurrenz ein angemessenes Einkommen zu erzielen. Effiziente und flexible Instrumente wie Prämien für Landschaftsvielfalt und für Basismaßnahmen im Agrarumwelt- und Klimaschutz verringern den Bürokratieaufwand für Landwirtschaft und Verwaltung. Darauf aufbauende regionale Agrarumwelt- und Klimaschutzprogramme sowie ein neues Kooperationsprogramm Natur und Landwirtschaft sichern wichtige Ökosystemleistungen und fördern die Vernetzung und Partizipation. Daneben sichert ein effektives Ordnungsrecht die Einhaltung von erweiterten Mindeststandards. Ein Innovationsprogramm, Verbraucherkommunikation, praxisorientierte Forschung und Beratung helfen bei der Entwicklung von Mehrwertmärkten für umweltfreundliche Produkte. Denn zu einem zukunftsfähigen Gesellschaftsvertrag gehört auch, dass kompetente Verbraucherinnen und Verbraucher die Gemeinwohlleistungen der Landwirtschaft kennen, wertschätzen und dafür angemessene Preise bezahlen.

\subsection{Die aktuellen Probleme}

Die Landwirtschaft in Deutschland befindet sich im Umbruch. Liberalisierung und Marktöffnung, neue Technologien sowie die steigende Nachfrage einer wachsenden Weltbevölkerung eröffnen enorme Entwicklungsmöglichkeiten. Gleichzeitig wachsen die Herausforderungen. Landwirtinnen und Landwirte sehen sich angesichts steigender Konkurrenz zu immer neuem betrieblichem Wachstum mit hohen Investitionsrisiken gezwungen. Weiterhin hat Deutschland schon heute Schwierigkeiten, europäische Vorgaben für den Natur- und Umweltschutz im Agrarbereich einzuhalten. Die Belastung des Grundwassers mit Nitrat ist in vielen landwirtschaftlich geprägten Regionen zu hoch. Gleichzeitig steigen die gesellschaftlichen Ansprüche an Transparenz, Tierwohl, Umweltschutz und Erhalt der biologischen Vielfalt. Hinzu kommen die Auswirkungen des internationalen Agrarhandels auf Entwicklungs- und Schwellenländer mit oft problematischen Folgen für Landnutzung, Umwelt, Ernährungssicherheit und kleinbäuerliche Strukturen.

So verändert sich die Rolle der bäuerlichen Betriebe für die ländliche Entwicklung - als Einkommensquelle, soziales Rückgrat und Landschaftsgestalter.

Angesichts der Problemlagen braucht die Agrarpolitik in Deutschland und Europa einen neuen Ansatz, der sowohl Arbeitsplätze in der Landwirtschaft sichert, als auch die ökologische Basis der Agrarproduktion erhält und die Erwartungen der Verbraucherinnen und Verbraucher an eine nachhaltige und gesunde Landwirtschaft erfüllt. Mit ihren alljährlichen Milliardenausgaben ist die aktuelle Förderpolitik jedoch vorwiegend als sektorale Einkommenspolitik angelegt - mit oft negativen Folgen für Umwelt- und Naturschutz. Ein wesentlicher Teil der Mittel verbleibt 
dabei gar nicht bei den Landwirtinnen und Landwirten, sondern fließt über steigende Land- und Pachtpreise an außerlandwirtschaftliche Grundbesitzer. Nur ein kleiner Teil der staatlichen Agrarzahlungen dient der Vergütung von Gemeinwohlleistungen. Den negativen Auswirkungen der gängigen Produktionsverfahren auf Boden, Wasser, Klima, Landschaftsbild, Luft und biologische Vielfalt wird damit nicht hinreichend entgegengewirkt. Es fehlen konsequente Strategien, um die Gemeinwohlleistungen der Landwirtschaft unter den Bedingungen offener Märkte und des schnellen technologischen und gesellschaftlichen Wandels zu sichern.

Daher brauchen wir in Deutschland dringend eine zukunftsweisende Verständigung darüber, was die Gesellschaft von der Landwirtschaft erwartet und welche Unterstützung die Landwirtinnen und Landwirte im Gegenzug dafür erwarten dürfen.

\subsection{Unser Vorschlag für einen zukunftsfähigen Gesellschaftsvertrag}

Das vorliegende Papier soll die Diskussion um einen zukunftsfähigen Gesellschaftsvertrag mit der Landwirtschaft in Deutschland und Europa anstoßen. Ausgangspunkt sind zwei Prämissen. Erstens benötigen wir eine Landwirtschaft, die marktfähige Produkte erzeugt und die Menschen mit vielfältigen und hochwertigen Lebensmitteln versorgt. Zweitens erwartet die Gesellschaft Gemeinwohlleistungen, für die es oft keinen Markt gibt. So soll die Landwirtschaft insbesondere

- die Attraktivität und damit die Vitalität ländlicher Räume durch vielfältig strukturierte Landschaften erhöhen;

- Lebensräume für zahlreiche Arten bereitstellen und damit die biologische Vielfalt fördern bzw. den dramatischen Artenschwund verringern;

- Ökosystemleistungen erhalten (z. B. die Bestäubung der Blütenpflanzen durch Insekten oder die Grundwasserneubildung in offenen Landschaften);

- zum Klimaschutz beitragen (etwa durch eine standortgerechte Bodennutzung);

- Stoffeinträge in Boden, Luft und Wasser durch umweltfreundliche Produktionsverfahren stärker reduzieren als gesetzlich mindestens vorgeschrieben;

- besondere Leistungen im Tierwohl erbringen.

All dies können die Landwirtinnen und Landwirte jedoch nur leisten, wenn sie damit ein angemessenes Einkommen erzielen - generiert entweder durch Verbrauchernachfrage oder durch öffentliche Mittel. Wenn die Gesellschaft eine flächendeckende, multifunktionale, natur- und umweltverträgliche Landwirtschaft wünscht, muss sie auch für die entsprechenden Rahmenbedingungen sorgen. Deshalb muss sich die Agrarpolitik dringend neu orientieren. Drei Grundsätze sind zentral:

- die Sicherung der ökologischen Lebens- und Produktionsgrundlagen;

- die konsequente Ausrichtung der Vergabe öffentlicher Mittel an der Bereitstellung von öffentlichen Gütern, die vom Markt nicht honoriert werden;

- die Garantie eines fairen Wettbewerbs durch wirksame soziale und ökologische Standards für alle Produzenten (inklusive Importware). 
Außerdem sollte die Agrarpolitik den Einsatz öffentlicher Mittel besser mit Trends wie Verbrauchersensibilisierung, Digitalisierung und Vernetzung, gesellschaftlicher Partizipation, integrierten Wertschöpfungsketten oder transnationalen privaten Standards verknüpfen.

Um dieses Ziel zu erreichen, schlagen wir eine neue Architektur der Agrarpolitik vor (siehe Abb. 2.1, Tab. 2.1). Sie kann durch gleitende Umschichtung der derzeitigen Mittel ab 2020 schrittweise bis zum Jahr 2027 eingeführt werden.

Im Kern besteht die neue Architektur aus fünf Elementen:

A. Mehr Umwelt- und Klimaschutz in allen Betrieben: Mit voll von der EU finanzierten Basismaßnahmen Agrarumwelt- und Klimaschutz sollen umweltund klimafreundliche Bewirtschaftungsformen unbürokratisch honoriert werden. Die Landwirte und Landwirtinnen können dabei die für sie passenden Maßnahmen aus einer Liste auswählen. Dies wird durch eine betriebliche Beratung unterstützt. Alle Maßnahmen haben unabhängig von den standortspezifischen Gegebenheiten einen Nutzen für Natur- und Umweltschutz und sind relativ leicht in die Produktion

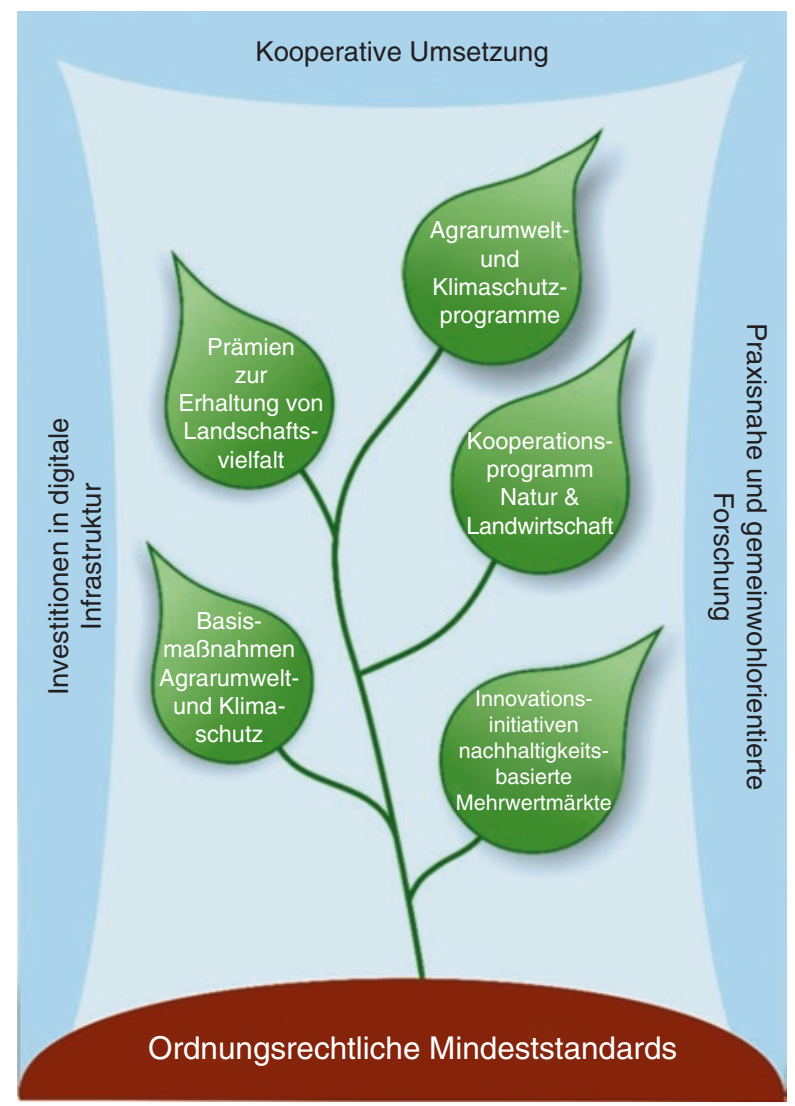

Abb. 2.1 Architektur einer neuen Agrarpolitik. (Quelle: Eigene Darstellung) 
zu integrieren. Sie umfassen z. B. vielgliedrige Fruchtfolgen, Bereitstellung von Habitaten, Landschaftselementen oder Gewässerrandstreifen, Verminderung von Düngung, Pflanzenschutzmitteln sowie Bewirtschaftungsmaßnahmen (Striegeln, Pflügen, Walzen, Schleppen etc.), (mehrjährige) Brache, Raufutterprämie und nachhaltige Verfahren der Tierhaltung (Weidegang, Begrenzung des Tierbesatzes). Jede Maßnahme erhält eine pauschale Wertigkeit (Punkte), die sich am typischen Aufwand orientiert. Um die Umsetzung auch an ertragsstarken Standorten attraktiv zu machen, werden dort zusätzlich Punkte vergeben (z. B. gemessen an der Bodengüte). Da von der Teilnahme aller landwirtschaftlichen Betriebe ausgegangen wird, steht pro Hektar Agrarfläche ein einheitlicher Finanzbetrag zur Verfügung. Um diese Mittel zu bekommen, muss ein Betrieb im Durchschnitt der bewirtschafteten Fläche eine Mindestpunktzahl erreichen. Dies ist auch Voraussetzung für die Auszahlung der Prämie zur Förderung der Landschaftsvielfalt (siehe Element B). Über die Mindestpunktzahl hinaus können die Betriebe weitere Maßnahmen aus der Liste wählen, die dann gesondert honoriert werden.

B. Zusätzliche Prämien für den Erhalt der Landschaftsvielfalt: Auch diese Prämien werden voll aus EU-Mitteln finanziert und gelten EU-weit. Sie dienen der Sicherung der bestehenden, reich strukturierten Landschaften und damit dem Erhalt der biologischen Vielfalt. Ihre Vergabe ist unbürokratisch, weil sie automatisiert berechnet und gewährt werden. Ihre Auszahlung ist an die Erbringung von Basismaßnahmen Agrarumwelt- und Klimaschutz geknüpft. Es gibt zwei Prämientypen:

- Eine Landschaftserhaltungsprämie wird für relativ strukturreiche Standorte unabhängig von Gebietskulissen und Betriebsgrößen gewährt. Sie belohnt z. B. kleinstrukturierte Landschaften, Randstreifen entlang von Strukturelementen oder Flächen mit Landschaftselementen, wie z. B. Streuobstwiesen. Die Prämienhöhe wird flächenbezogen auf der Basis von Fernerkundungsdaten berechnet. Sie ist nach Dichte und ökologischer Wertigkeit der Strukturelemente gestaffelt.

- Eine Bewirtschaftungsprämie wird an auf der Schlagebene definierten Standorten gewährt, an denen aufgrund erschwerter Bewirtschaftungsbedingungen eine Landbewirtschaftung typischerweise nicht kostendeckend ist. Die Prämienhöhe ist gestaffelt nach dem Grad der Erschwernis an dem jeweiligen Standort. Der Erschwernisgrad hängt z. B. von Hangneigung, Bodenqualität oder der Länge der Vegetationsperiode ab. Diese Prämie ist nur in Gebietskulissen erhältlich, in denen eine großflächige Nutzungsaufgabe abgewendet werden muss, um die Aufrechterhaltung einer vielgestaltigen Landschaft zu gewährleisten.

C. Anreize für Agrarumwelt- und Klimaschutz: Agrarumwelt- und Klimaschutzprogramme honorieren gezielte betriebliche Maßnahmen des Natur- und Umweltschutzes (z. B. spezieller Artenschutz, Management von Schutzgebieten, Wiedervernässung von Mooren oder Aufbau von Biotopverbünden). Der Erfolg solcher Maßnahmen erfordert in der Regel eine regionale Ausgestaltung der Programme unter natur- oder umweltfachlicher Leitung, welche die standörtlichen Gegebenheiten umfassend berücksichtigt. Ihr Beitrag zu EU-weiten Politikzielen begründet eine Kofinanzierung durch EU und Mitgliedstaaten. Zur Erhöhung der Attraktivität sollten die 
Transaktionskosten bei der Prämienhöhe berücksichtigt sowie eine Anreizkomponente im Einklang mit dem WTO-Recht vorgesehen werden. Dadurch besteht ein finanzieller Anreiz zur Teilnahme über die Erstattung des Ertragsausfalls hinaus.

D. Mehr Geld für innovative Kooperationen. Mit einem Kooperationsprogramm Natur und Landwirtschaft werden regionale Kooperationen gefördert, die ein integriertes Landschafts- und Ressourcenmanagement entwickeln und umsetzen. Das sind insbesondere überbetriebliche Maßnahmen zum Management von Wasser, Boden, Luft und Klima sowie zum Arten- und Biotopschutz. Dazu gehören auch nicht-produktionsorientierte Investitionen (,grüne und blaue Infrastruktur“). Im Gegensatz zu den vorgegebenen Agrarumwelt- und Klimaschutzprogrammen (siehe Element A) sind lernorientierte Ansätze und innovative Kooperationsformen erwünscht. Deshalb sollten $80 \%$ der Mittel dieses Programms für die eigentliche Durchführung der Maßnahmen und bis zu $20 \%$ für deren Planung, Monitoring sowie die Akteursvernetzung zur Verfügung stehen. Die Mittel werden in einem Wettbewerbsverfahren vergeben - so wie derzeit im LEADER-Programm der EU. Dabei können alle Beteiligten (Landwirtschaft, Forstwirtschaft, Fischerei, Naturschutzorganisationen, Kommunen usw.) für kooperative Leistungen im Umwelt- und Naturschutz honoriert werden. Das Kooperationsprogramm sollte EU-weit eingeführt und von EU und Mitgliedstaaten kofinanziert werden. In einem ersten Schritt kann auf nationaler Ebene ein Bundesprogramm für Modell- und Demonstrationsvorhaben aufgelegt werden.

E. Mehr Geld für nachhaltige Märkte. Wer eine nachhaltige Landwirtschaft will, der braucht auch Marktteilnehmer, die nachhaltig handeln. Eine Innovationsinitiative für nachhaltigkeitsbasierte Mehrwertmärkte könnte national umgesetzt und als Bundesprogramm oder im Rahmen der Gemeinschaftsaufgabe ,Verbesserung der Agrarstruktur und des Küstenschutzes“ (GAK) finanziert werden. Die Initiative umfasst drei Bereiche:

a) Stärkung der Verbraucherkompetenz zum Beispiel durch Maßnahmen zur Förderung eines gesunden und nachhaltigen Ernährungsverhaltens. Die Maßnahmen reichen von der Ausgestaltung des Entscheidungsumfelds über klassische Kommunikationskampagnen durch Bund, Länder oder gesellschaftliche Gruppen bis zur Erstellung von Lehrmaterialien für allgemeinbildende Schulen.

b) Förderung nachhaltigkeitsbasierter Wertschöpfungsketten, vor allem regionaler Vermarktungsstrukturen, die den Umweltschutz besonders berücksichtigen. Dazu zählt aber auch die Nutzung digitaler Technologien für die Vernetzung und Transparenz des gesamten Produktionssystems. Neue Konzepte der Finanzierung und der Beziehungen zwischen Produktion, Verteilung und Konsum (wie etwa bei der Solidarischen Landwirtschaft) gehören ebenfalls hierher.

c) Einführung eines europaweit einheitlichen Kennzeichnungssystems für die Naturschutz- und Umweltleistungen der Landwirtschaft. Dies macht u. a. die Teilnahme an den Maßnahmen A und C für die Konsumenten nachvollziehbar. 
Möglich ist auch die Verknüpfung mit gesamtbetrieblichen Zertifizierungssystemen für Nachhaltigkeitsleistungen, sofern diese anspruchsvoll ausgestaltet und staatlich akkreditiert sind. Die Kennzeichnung hoher Nachhaltigkeits- und Tierwohlstandards sollte in transnationalen privatrechtlichen Systemen wie GlobalGAP verankert und damit auch für Importprodukte verbindlich werden.

Die fünf Kernelemente der neuen Agrarpolitik werden durch Investitionen in die folgenden Rahmenbedingungen flankiert:

1. Investitionen in die digitale Infrastruktur im ländlichen Raum sind die Voraussetzung für Vernetzung, neue Geschäftsmodelle und die interaktive Nutzung digitaler Daten.

2. Eine praxisnahe und gemeinwohlorientierte, staatlich finanzierte Forschung und ein verbesserter Wissenstransfer, Beratungs-, Aus- und Fortbildungsangebote sowie betriebliche Investitions- und Innovationsförderung unterstützen die schnelle Entwicklung und Verbreitung neuer nachhaltigkeitsorientierter Praktiken.

Neben allen Fördermaßnahmen sichern ordnungsrechtliche Mindeststandards die Durchsetzung des Verursacherprinzips (Nitratrichtlinie/Düngeverordnung, Verordnungen zur Tierkennzeichnung usw.). Ihre konsequente Anwendung verhindert, dass Umweltkosten auf Dritte oder die Allgemeinheit abgewälzt werden. Zusätzlich $\mathrm{zu}$ den bestehenden Grundanforderungen an die Betriebsführung sollten künftig auch der Erosionsschutz, der Humuserhalt und das Dauergrünlandumwandlungsverbot in das Ordnungsrecht aufgenommen werden.

Eine kooperative Umsetzung in enger Abstimmung zwischen der Agrar- und der Umwelt- und Naturschutzpolitik füllt den Gesellschaftsvertrag mit Leben. Gemäß der jeweiligen Sachnähe sollte die Federführung für Programmteile mit überwiegend umwelt- und naturschutzfachlicher Zielsetzung bei der Umwelt- und Naturschutzpolitik bzw. -verwaltung liegen. Bei Teilen mit vorwiegend agrarwirtschaftlichen und einkommenspolitischen Zielen sollten Agrarpolitik und -verwaltung federführend sein. Die umwelt- und naturschutzfachlichen Programmteile sollten es nach Umfang und Ausgestaltung insbesondere ermöglichen, die Ziele der Natura 2000-Richtlinien umzusetzen. Sektorenübergreifende Auswirkungen und Folgen für Natur- und Umweltschutz müssen bei der Ausgestaltung der Politik konsequent berücksichtigt werden. Flexible, ergebnisorientierte Planungs- und Implementierungsprozesse erleichtern Kooperations- und Lernprozesse zwischen den Akteuren und erhöhen die Adaptivität. Auf der Basis des wissenschaftlichen Erkenntnisfortschritts und der praktischen Erfahrungen der Landwirtschaft sollten alle Maßnahmen regelmäßig hinsichtlich der Notwendigkeit zur Anpassung an die durch den globalen Wandel veränderten Bedingungen geprüft werden.

Die hier vorgeschlagene neue Agrarpolitik nimmt die wechselseitigen Erwartungen von Gesellschaft und Agrarsektor auf. Als Ausdruck eines zukunftsfähigen Gesellschaftsvertrags mit der Landwirtschaft sollte sie es ermöglichen, den enormen ökonomischen, ökologischen und sozialen Herausforderungen im ländlichen Raum wirksam zu begegnen. 


\subsection{Anhang (Tab. 2.1)}

Tab. 2.1 Eine neue Architektur für die Agrarpolitik - Überblick. (Diese Überblickstabelle war nicht Teil des im Januar 2017 vorgestellten Policy-Papers)

\begin{tabular}{|c|c|c|c|}
\hline Element & Mechanismus & Finanzierung & $\begin{array}{l}\text { anstrebte } \\
\text { Wirkungsziele }\end{array}$ \\
\hline $\begin{array}{l}\text { Basismaß- } \\
\text { nahmen } \\
\text { Agrarum- } \\
\text { welt- und } \\
\text { Klimaschutz }\end{array}$ & $\begin{array}{l}\text { Punktebasierte Honorierung } \\
\text { von Maßnahmen mit } \\
\text { standortunabhängigem } \\
\text { Mehrwert für Naturschutz und } \\
\text { Umwelt; Auswahl aus } \\
\text { Maßnahmenliste; Mindest- } \\
\text { punktzahl für Prämienerhalt }\end{array}$ & Voll EU-finanziert & $\begin{array}{l}\text { Mehr Umwelt- und } \\
\text { Klimaschutz in allen } \\
\text { Betrieben }\end{array}$ \\
\hline $\begin{array}{l}\text { Landschafts- } \\
\text { erhaltungs- } \\
\text { prämie }\end{array}$ & $\begin{array}{l}\text { Flächenbezogen Prämie auf } \\
\text { Basis von Fernerkundungs- } \\
\text { und InVeKoS-Daten, nach } \\
\text { Dichte und ökologischer } \\
\text { Wertigkeit der Strukturele- } \\
\text { mente gestaffelt }\end{array}$ & Voll EU-finanziert & $\begin{array}{l}\text { Sicherung der } \\
\text { bestehenden, reich } \\
\text { strukturierten } \\
\text { Landschaften und } \\
\text { damit den Erhalt der } \\
\text { biologischen Vielfalt }\end{array}$ \\
\hline $\begin{array}{l}\text { Bewirtschaf- } \\
\text { tungsprämie }\end{array}$ & $\begin{array}{l}\text { Auf der Schlagebene } \\
\text { definierte Prämie an Standor- } \\
\text { ten, an denen aufgrund } \\
\text { erschwerter Bewirtschaftungs- } \\
\text { bedingungen eine Landbewirt- } \\
\text { schaftung typischerweise nicht } \\
\text { kostendeckend ist. Prämien- } \\
\text { höhe gestaffelt nach dem Grad } \\
\text { der Erschwernis am jeweiligen } \\
\text { Standort. }\end{array}$ & Voll EU-finanziert & $\begin{array}{l}\text { Aufrechterhaltung } \\
\text { einer vielgestaltigen } \\
\text { Landschaft in } \\
\text { definierten Gebietsku- } \\
\text { lissen }\end{array}$ \\
\hline $\begin{array}{l}\text { Agrarum- } \\
\text { welt- und } \\
\text { Klimaschutz- } \\
\text { programme }\end{array}$ & $\begin{array}{l}\text { Honorierung freiwilliger } \\
\text { betrieblicher Maßnahmen des } \\
\text { Natur- und Umweltschutzes }\end{array}$ & $\begin{array}{l}\text { Kofinanzierung von } \\
\text { EU und Mitglied- } \\
\text { staaten }\end{array}$ & $\begin{array}{l}\text { Gezielte Verbesserung } \\
\text { des Natur-, Umwelt- } \\
\text { und Klimaschutz }\end{array}$ \\
\hline $\begin{array}{l}\text { Koopera- } \\
\text { tionspro- } \\
\text { gramm Natur } \\
\text { und } \\
\text { Landwirt- } \\
\text { schaft }\end{array}$ & $\begin{array}{l}\text { Förderung regionaler } \\
\text { Kooperationen, die ein } \\
\text { integriertes Landschafts- und } \\
\text { Ressourcenmanagement } \\
\text { entwickeln und umsetzen; } \\
\text { Vergabe im Wettbewerbsver- } \\
\text { fahren }\end{array}$ & $\begin{array}{l}\text { Zunächst national, } \\
\text { etwa als Bundespro- } \\
\text { gramm für Modell- } \\
\text { und Demonstra- } \\
\text { tionsvorhaben; } \\
\text { später ggf. } \\
\text { Kofinanzierung von } \\
\text { EU und Mitglied- } \\
\text { staaten }\end{array}$ & $\begin{array}{l}\text { Entwicklung und } \\
\text { Implementierung eines } \\
\text { integrierten Land- } \\
\text { schafts- und Ressour- } \\
\text { cenmanagement auf } \\
\text { regionaler Ebene; } \\
\text { Förderung von } \\
\text { Vernetzung, Lernpro- } \\
\text { zessen und Kooperation } \\
\text { zwischen den Akteuren }\end{array}$ \\
\hline $\begin{array}{l}\text { Innovations- } \\
\text { initiative für } \\
\text { nachhaltig- } \\
\text { keitsbasierte } \\
\text { Mehrwert- } \\
\text { märkte }\end{array}$ & $\begin{array}{l}\text { U. a. Kommunikationskampa- } \\
\text { gnen für Verbraucher, } \\
\text { Förderung nachhaltigkeitsba- } \\
\text { sierter Wertschöpfungsketten; } \\
\text { Einführung eines europaweit } \\
\text { einheitlichen Kennzeichnungs- } \\
\text { systems }\end{array}$ & $\begin{array}{l}\text { National: Bundes- } \\
\text { programm oder im } \\
\text { Rahmen der } \\
\text { Gemeinschaftsauf- } \\
\text { gabe „Verbesserung } \\
\text { der Agrarstruktur } \\
\text { und des Küsten- } \\
\text { schutzes“(GAK) }\end{array}$ & $\begin{array}{l}\text { Verbesserung der } \\
\text { Rahmenbedingungen } \\
\text { für nachhaltigkeitsba- } \\
\text { sierte Geschäftsmo- } \\
\text { delle }\end{array}$ \\
\hline
\end{tabular}


Tab. 2.1 (Fortsetzung)

\begin{tabular}{|c|c|c|c|}
\hline $\begin{array}{l}\text { Ordnungs- } \\
\text { rechtliche } \\
\text { Mindeststan- } \\
\text { dards }\end{array}$ & $\begin{array}{l}\text { Operationalisierung unklarer } \\
\text { Rechtsbegriffe, verbindliche } \\
\text { Zielwerte, effektive Kontrol- } \\
\text { len, wirksame Sanktionierung } \\
\text { bei Übertretung }\end{array}$ & $\begin{array}{l}\text { Im Rahmen der } \\
\text { üblichen Finanzie- } \\
\text { rung der Adminis- } \\
\text { tration von Bund } \\
\text { und Ländern }\end{array}$ & $\begin{array}{l}\text { Konsequente Durchset- } \\
\text { zung des Verursacher- } \\
\text { prinzips, Behebung von } \\
\text { Implementationsdefizi- } \\
\text { ten }\end{array}$ \\
\hline $\begin{array}{l}\text { Flankierende } \\
\text { Maßnahmen }\end{array}$ & $\begin{array}{l}\text { Investitionen in die digitale } \\
\text { Infrastruktur }\end{array}$ & GAK & $\begin{array}{l}\text { Erleichterung des } \\
\text { Monitoring; Verringe- } \\
\text { rung des administrati- } \\
\text { ven Aufwands }\end{array}$ \\
\hline $\begin{array}{l}\text { Flankierende } \\
\text { Maßnahmen }\end{array}$ & $\begin{array}{l}\text { praxisnahe und gemeinwohl- } \\
\text { orientierte, staatlich finan- } \\
\text { zierte Forschung und ein } \\
\text { verbesserter Wissenstransfer; } \\
\text { Beratungs-, Aus- und } \\
\text { Fortbildungsangebote sowie } \\
\text { betriebliche Investitions- und } \\
\text { Innovationsförderung }\end{array}$ & Bund, Länder, GAK & $\begin{array}{l}\text { Verbesserter Wissens- } \\
\text { transfer }\end{array}$ \\
\hline $\begin{array}{l}\text { Kooperative } \\
\text { Umsetzung in } \\
\text { enger } \\
\text { Abstimmung } \\
\text { zwischen der } \\
\text { Agrar-, der } \\
\text { Umwelt- und } \\
\text { Naturschutz- } \\
\text { politik }\end{array}$ & $\begin{array}{l}\text { Federführung nach Sachnähe; } \\
\text { ergebnisorientierte Planungs- } \\
\text { und Implementierungspro- } \\
\text { zesse; }\end{array}$ & $\begin{array}{l}\text { Im Rahmen der } \\
\text { üblichen Finanzie- } \\
\text { rung der Adminis- } \\
\text { tration von Bund } \\
\text { und Ländern }\end{array}$ & $\begin{array}{l}\text { Verbesserte Kooperati- } \\
\text { ons- und Lernprozesse } \\
\text { zwischen den } \\
\text { Akteuren; erhöhte } \\
\text { Adaptivität }\end{array}$ \\
\hline
\end{tabular}

Open Access Dieses Kapitel wird unter der Creative Commons Namensnennung 4.0 International Lizenz (http://creativecommons.org/licenses/by/4.0/deed.de) veröffentlicht, welche die Nutzung, Vervielfältigung, Bearbeitung, Verbreitung und Wiedergabe in jeglichem Medium und Format erlaubt, sofern Sie den/die ursprünglichen Autor(en) und die Quelle ordnungsgemäß nennen, einen Link zur Creative Commons Lizenz beifügen und angeben, ob Änderungen vorgenommen wurden.

Die in diesem Kapitel enthaltenen Bilder und sonstiges Drittmaterial unterliegen ebenfalls der genannten Creative Commons Lizenz, sofern sich aus der Abbildungslegende nichts anderes ergibt. Sofern das betreffende Material nicht unter der genannten Creative Commons Lizenz steht und die betreffende Handlung nicht nach gesetzlichen Vorschriften erlaubt ist, ist für die oben aufgeführten Weiterverwendungen des Materials die Einwilligung des jeweiligen Rechteinhabers einzuholen. 


\section{Kapitel 3 \\ Kurzdarstellung der Ausgangssituation: Umwelteffekte der Landwirtschaft}

Landwirtschaftliche Produktion ist unweigerlich mit Eingriffen in die Natur und damit in unsere Umwelt verbunden. Je nach Art der menschlichen Aktivitäten können diese Eingriffe zu vielfältigen oder zu monotonen Landschaften führen und die Umweltqualität verbessern oder verschlechtern. In den vergangenen Jahrzehnten sind in vielen Regionen Deutschlands (wie auch andernorts) besorgniserregende Veränderungen zu beobachten. Der Zustand der Naturressourcen entwickelt sich auf großer Fläche negativ: Verlust an biologischer Vielfalt, Belastungen von Boden, Wasser und Luft sowie Veränderung des Klimas und Homogenisierung der Landschaft. Dabei bestehen oft enge Wechselbeziehungen zwischen dem Zustand der Naturressourcen und der landwirtschaftlichen Produktivität.

Der folgende Abschnitt gibt einen knappen Überblick über den aktuellen ${ }^{1}$ Zustand der natürlichen Ressourcen, die Ausprägung der landwirtschaftlichen Wirkfaktoren, über das Erreichen politischer Ziele und die genannten Wechselwirkungen. Die Darstellung fokussiert überwiegend auf die nationale Ebene. Auf Wirkungen, die in Drittländern entstehen, z. B. durch indirekte Landnutzungsänderungen, wird nicht näher eingegangen. Zudem ist eine erschöpfende Darstellung der Umweltwirkungen der Landwirtschaft in diesem Kontext nicht beabsichtigt. Dafür sei auf weitergehende Fachliteratur verwiesen (siehe Literaturverzeichnis).

\footnotetext{
${ }^{1}$ Da dieser Abschnitt die Grundlage für die späteren Überlegungen des ZA-NExUS-Konsortiums bildete, endet die Literaturaufarbeitung Mitte 2016. Auf die Berücksichtigung neuerer Publikationen wurde verzichtet, um die Bezugspunkte der nachfolgenden Kapitel nicht zu verschieben.
} 


\subsection{Zustand der Naturressourcen}

\subsubsection{Wirkungen der Landwirtschaft auf die Ressource Boden}

Der Standortfaktor „Boden“ ist eine entscheidende Grundlage der landwirtschaftlichen Produktion. Böden erbringen darüber hinaus bedeutende ökosystemare Leistungen wie Filter-, Puffer- und Stoffumwandlungsfunktionen. Diese Leistungen sind auch im Bundes-Bodenschutzgesetz als schützenswert aufgeführt. ${ }^{2}$ Alle werden von der Landwirtschaft durch Stoffeinträge und mechanische Bearbeitung sowie durch die Verursachung von Erosion und Humusab- bzw. -aufbau beeinflusst.

Stoffeinträge durch die Landwirtschaft sind die Folge der Stickstoff- und Phosphordüngung, der Anwendung von Pflanzenschutzmitteln sowie der Ausbringung von Schwermetallen und der Anwendung von Tierarzneimitteln, deren Rückstände im organischen Dünger enthalten sind (Umweltbundesamt 2014a, S. 29). Die Belastungsgrenzen der Böden werden durch sogenannte Critical Loads festgelegt. Bereits im Jahre 2010 wurden die Grenzwerte für Blei, Quecksilber, Eutrophierung (auf $54 \%$ der empfindlichen Ökosysteme) und Versauerung (auf $18 \%$ der empfindlichen Ökosysteme) überschritten. Beim Cadmium gab es dagegen kaum Überschreitungen (Umweltbundesamt 2013a). Als politisches Ziel wurde formuliert, dass bis 2020 die Critical Loads für Eutrophierung und Versauerung nicht mehr überschritten werden sollen (Umweltbundesamt 2015b, c).

Bodenschadverdichtungen entstehen durch mechanische Belastungen und durch das Überschreiten der natürlichen Traglast des Bodens. Von Schadverdichtungen wird gesprochen, wenn der Fluss von Luft, Wasser und Nährstoffen im Porensystem so stark behindert ist, dass negative Wirkungen auf die Bodenfunktionen auftreten (Sommer und Hutchings 2001; Lebert 2004). Solche Verdichtungen können in der Folge auch zu vermehrtem Oberflächenabfluss, verstärkter Erosion, erhöhter Denitrifikation und damit zu Stickstoff-Verlusten führen. In Deutschland ist die Produktivität auf $50 \%$ der Ackerflächen durch Bodenverdichtung beeinträchtigt (Umweltbundesamt 2010a).

Steigende Nutzungs- und Überrollhäufigkeit belastet den Boden und damit die unterirdischen Nahrungsnetze (Thiele-Bruhn et al. 2012). Durch regelmäßiges Pflügen wird beispielsweise das Hyphensystem von Pilzen geschädigt. Dies kann langfristig die Nahrungsnetze zugunsten von Bakterien verschieben, deren Dominanz zu schnellen Umsätzen und geringer Speicherkapazität führt (Thiele-Bruhn et al. 2012; Nielsen et al. 2015). Solche Veränderungen haben erhebliche Auswirkungen auf wichtige ökologische Funktionen wie die Nährstoffversorgung der Pflanzen, die Unterdrückung von Schädlingen und Pathogenen oder die Resilienz gegenüber Störungen und Extremereignissen (Nielsen et al. 2015). Eine große Zahl von Untersuchungen weist auf einen positiven Zusammenhang zwischen der Vielfalt der Lebensgemeinschaften im Boden und wichtigen Bodenprozessen, wie der Zersetzung

${ }^{2}$ Gesetz zum Schutz vor schädlichen Bodenveränderungen und zur Sanierung von Altlasten (BBodSchG), Stand 2017, § 2, Abs. 2. 
von organischem Material, der Bodenbildung oder den Nährstoffkreisläufen, hin (van Capelle et al. 2012; Nielsen et al. 2015).

Erosion bezeichnet die Verlagerung von Bodenmaterial durch Wind oder Wasser. Dadurch wird der ertragreiche Oberboden abgetragen und die Bodenfunktionen werden beeinträchtigt. Das Erosionsgeschehen nimmt mit dem Verlust an Grünland (Osterburg et al. 2009; Bundesamt für Naturschutz 2014), der Beseitigung von Hecken, Terrassen und weiteren Landschaftselementen, dem Anbau von Hackfrüchten, der Schlaggröße und -neigung sowie der Art der Bodenbearbeitung zu. So zeigt beispielsweise eine Untersuchung aus Brandenburg, dass die vorhandenen Landschaftselemente den Anteil stark durch Winderosion gefährdeter Flächen von ca. $41 \%$ auf $18 \%$ reduzieren können (Funk et al. 2013). Die Erosionsrate auf landwirtschaftlichen Flächen ist um ein bis zwei Größenordnungen höher als die natürliche Neubildung des Bodens (Montgomery 2007). In Europa beträgt die jährliche Bodenneubildung ca. 0,5 t/ha (Alexandrovskiy 2007; KBU 2008; Sauer et al. 2008). Allein die mittlere Jahresrate der Wassererosion in Europa übersteigt mit einem Wert von $2 \mathrm{t} / \mathrm{ha}$ bereits die Rate der Bodenneubildung (EEA - European Environment Agency 1999). In Deutschland liegt der jährliche Bodenabtrag auf $14 \%$ der Flächen im Mittel bei mehr als 3 t/ha. Diese Flächen sind stark erosionsgefährdet. Auf weiteren $36 \%$ der Flächen ist die Bodenfurchtbarkeit langfristig durch Erosion gefährdet (Umweltbundesamt 2011a). Die Ablagerungen der Bodenteilchen an Hangsohlen oder in Gewässern verursacht darüber hinaus weitere Schäden - z. B. durch die Eutrophierung sensibler Ökosysteme aber auch durch die Verfrachtung von Pflanzenschutzmitteln (Clay et al. 2001).

Humus ist der Überbegriff für die organischen Bodenbestandteile. Er beeinflusst die Bodenfunktionen in vielfältiger Weise $-z$. B. die Speicherung von Nährstoffen und Wasser, die Stabilisierung des Bodengefüges sowie die Steuerung des Luft- und Wasserhaushalts. Darüber hinaus bindet der Humus große Mengen an Kohlenstoff im Boden, dessen Mineralisierung zu Treibhausgasen andernfalls zum Klimawandel beitragen würde (siehe auch Abschn. 3.1.3). Global ist die Kohlenstoffspeicherkapazität des Bodens drei- bis viermal höher als die der Vegetationsdecke (Jering et al. 2013). Da der Humusgehalt durch Faktoren wie Temperatur, Niederschlag, Bodenart, pH-Wert und die Bewirtschaftung beeinflusst wird, kann die jeweilige Humusanreicherung nur im Vergleich mit den standorttypischen Gehalten bewertet werden. Letztere schwanken zwischen $<1 \%$ und $>30 \%$, wobei die Humusvorräte unter Wald und Grünland generell höher sind als auf Äckern (Düwel et al. 2007). Das Bodenschutzgesetz fordert den Erhalt standorttypischer Humusgehalte durch eine ausreichende Zufuhr an organischer Substanz oder durch die Reduzierung der Bearbeitungsintensität (BBodschG Stand 2017, § 17, Abs. 2, Ziff. 7). In Bayern wurde dennoch bei Acker- und Grünlandböden für den Zeitraum zwischen 1986 und 2007 eine Abnahme der mittleren organischen Kohlenstoffgehalte um etwa $3 \%$ festgestellt (LfL - Bayerische Landesanstalt für Landwirtschaft 2010). Bundesweit liegen entsprechende Daten bisher nicht vor.

Tab. 3.1 gibt einen Überblick über die wichtigsten umweltpolitischen Zielsetzungen und die jeweilige Zielerreichung in Bezug auf den Boden. 
Tab. 3.1 Zielsetzungen und Zielerreichung für die Ressource Boden

\begin{tabular}{|c|c|}
\hline Zielsetzungen & Zielerreichung \\
\hline \multicolumn{2}{|l|}{ Immissionsbelastungen } \\
\hline $\begin{array}{l}\text { Critical Loads für Blei, Quecksilber, } \\
\text { Cadmium, Eutrophierung, Versauerung; } \\
\text { keine Überschreitung der Critical Loads für } \\
\text { Eutrophierung und Versauerung bis } 2020\end{array}$ & $\begin{array}{l}\text { Critical Loads für Blei, Quecksilber, } \\
\text { Eutrophierung und Versauerung } \\
\text { überschritten; kaum Überschreitungen } \\
\text { bei Cadmium }\end{array}$ \\
\hline $\begin{array}{l}\text { - Grenzwerte für die Belastung von Klär- } \\
\text { schlamm und die Ausbringung von } \\
\text { Klärschlamm (Klärschlammverordnung, } \\
\text { Bioabfallverordnung) }\end{array}$ & $\begin{array}{l}\text { Abnahme der Klärschlammmenge } 1998 \\
\text { und 2009, Rückgang der Schwermetall- } \\
\text { gehalte, aber weiterhin der Nährstoffträ- } \\
\text { ger mit höchsten Schadstoffbelastungen }\end{array}$ \\
\hline - Tierarzneimittel - keine Zielsetzung & - Unklar, da kein Zielwert definiert \\
\hline - Uran in Phosphatdüngern - keine Zielsetzung & - Unklar, da kein Zielwert definiert \\
\hline \multicolumn{2}{|c|}{ Bodenschadverdichtungen und mechanische Belastung } \\
\hline $\begin{array}{l}\text { - Allgemeine Formulierung im Bodenschutz- } \\
\text { gesetz }\end{array}$ & - Kein Zielwert definiert \\
\hline \multicolumn{2}{|l|}{ Erosion } \\
\hline $\begin{array}{l}\text { Bewirtschaftung entsprechend der Einteilung } \\
\text { nach dem Grad der Wasser- und Windero- } \\
\text { sionsgefährdung (GLÖZ) }\end{array}$ & - Keine entsprechende Bewirtschaftung \\
\hline $\begin{array}{l}\text { - Allgemeine Formulierung im Bodenschutz- } \\
\text { gesetz }\end{array}$ & - Unklar, da kein Zielwert definiert \\
\hline $\begin{array}{l}\text { Wissenschaft: } 0,5 \text { t/ha und Jahr (Alexan- } \\
\text { drovskiy 2007; KBU 2008; Sauer et al. 2008) }\end{array}$ & - Nein: 2 t/ha und Jahr durch Wassererosion \\
\hline \multicolumn{2}{|l|}{ Humuserhalt/-aufbau } \\
\hline $\begin{array}{l}\text { - Allgemeine Formulierung im Bodenschutz- } \\
\text { gesetz }\end{array}$ & - Unklar, da kein Zielwert definiert \\
\hline $\begin{array}{l}\text { Verbot des Abbrennens von Stoppelfeldern, } \\
\text { Dauergrünlanderhalt, Anbaudiversifizierung, } \\
\text { Zwischenfruchtanbau }\end{array}$ & $\begin{array}{l}\text { - Nein: vielfach Humusabbau zu } \\
\text { beobachten, Angaben zu Verstößen }\end{array}$ \\
\hline
\end{tabular}

\subsubsection{Wirkungen der Landwirtschaft auf die biologische Vielfalt}

Die biologische Vielfalt (oder Biodiversität) umfasst sowohl den Reichtum an Arten und Lebensräumen als auch die genetische Variabilität wildlebender sowie domestizierter Tier- und Pflanzenarten (BMUB 2007). Diese Vielfalt erbringt eine große Zahl an ökosystemaren Leistungen, die für den Menschen grundlegend sind (z. B. Erhalt der Bodenfruchtbarkeit, Bestäubung durch Insekten oder biologische Schädlingsbekämpfung; TEEB DE 2012; Soliveres et al. 2016). Die landwirtschaftliche Produktion kann die biologische Vielfalt und deren Ökosystemleistungen sowohl positiv als auch negativ beeinflussen (Tscharntke et al. 2005). Dabei wird die Biodiversitätsänderung in der Agrarlandschaft im Wesentlichen durch zwei Entwicklungen beeinflusst: Intensivierung und übermäßige Extensivierung bzw. Aufgabe der landwirtschaftlichen Flächennutzung.

Von Intensivierung wird im Allgemeinen gesprochen, wenn der Einsatz der Produktionsfaktoren zunimmt. Dazu zählen z. B. vermehrter Eintrag von Dünge- und 
Pflanzenschutzmitteln, Einengung von Fruchtfolgen, steigender Einsatz von schweren Maschinen oder die Vergrößerung der Schläge durch die Beseitigung von natürlichen und halbnatürlichen Strukturelementen. Diese Zunahme bewirkt eine Vereinheitlichung der Standortbedingungen. Der damit verbundene Verlust von geeigneten Habitaten und die Fragmentierung der Lebensräume gehören zu den wesentlichen Treibern des weltweiten Rückgangs der biologischen Vielfalt (Benton et al. 2003; Petit et al. 2011; Leuschner et al. 2014; Richner et al. 2015; Tsiafouli et al. 2015). Als Folge der seit Mitte des letzten Jahrhunderts wachsenden Schlaggröße (siehe auch Abschn. 3.2.2.2) hat sich beispielsweise der mittlere Flächenanteil der Ackerrandstreifen halbiert (Leuschner et al. 2014). Dies führte zu einer starken Abnahme der Diversität von Ackerwildkräutern (Petit et al. 2011). Entsprechend nahmen z. B. die regionalen Artenpools der Ackerbegleitflora in Mittel- und Norddeutschland durchschnittlich um $23 \%$ ab (Meyer et al. 2015b) und etwa ein Drittel der typischen Ackerwildkräuter Deutschlands sind gefährdet (Bundesamt für Naturschutz 2015a). In Thüringen wurden $86 \%$ der Arten (Blütenpflanzen, Laufkäfer, Brutvögel, Tagfalter, Säugetiere, Amphibien, Kriechtiere) in naturnahen Randstrukturen nachgewiesen, in Ackerschlägen waren es dagegen nur $14 \%$ (Hoffmann und Kretschmer 2001). Die biologische Vielfalt der Ackerschläge wird auch dadurch negativ beeinflusst, dass mit der Flächenvergrößerung und durch einheitliche Gewannbewirtschaftung in der Regel die Vielfalt angebauter Kulturen in der Landschaft abnimmt (Mohn et al. 2003; Rodríguez und Wiegand 2009). Die fortschreitende Verarmung der Pflanzenwelt in der Agrarlandschaft verändert die Nahrungsnetze mit direkten und indirekten Auswirkungen auf Wildtiere. Als Beispiel sei die Verringerung des Nahrungsangebots (z. B. an Blüten, Samen) für Honig- und Wildbienen, Schmetterlinge, andere Gliederfüßer und Wirbeltiere (z. B. Vögel) genannt. Dies beeinträchtigt u. a. deren Fortpflanzungserfolg und führt zu einem Rückgang der Bestände (Biesmeijer et al. 2006; Potts et al. 2010; Hadley und Betts 2012; Shackelford et al. 2013; van Swaay et al. 2013; Jahn et al. 2014). In intensiv genutzten Landschaften sind zudem geeignete Nisthabitate (z. B. in Totholz, Heckenstrukturen) nur begrenzt verfügbar und häufig weit von Nahrungshabitaten entfernt (Hadley und Betts 2012). Die Diversität der Pflanzen und damit die Vielfalt des Blütenangebotes wird auch durch einseitige Fruchtfolgeglieder reduziert (Kluser und Peduzzi 2007; Hadley und Betts 2012) oder ist zeitlich durch Massentrachten wie bei Raps stark begrenzt (Nicholls und Altieri 2013). Durch frühe Erntetermine (z. B. bei Ganzpflanzensilage) und enge Bestände verlieren bodenbrütende Vogelarten die Nistmöglichkeit (Hart et al. 2006). Die Eintönigkeit homogener und großflächiger Bestände wirkt sich darüber hinaus auch aus Sicht des Menschen negativ auf das Landschaftsbild aus (siehe hierzu Abschn. 3.1.4).

Der Einsatz von Pflanzenschutzmitteln hat erhebliche Auswirkungen auf die biologische Vielfalt, denn viele Organismen werden dadurch entweder direkt (durch toxische Einwirkung) oder indirekt (durch Reduktion von Nahrung und Deckung) beeinflusst (Frische et al. 2016). So vergiften Herbizide beispielsweise entsprechend ihres Wirkbereichs auch Nicht-Zielpflanzen. Zudem begünstigt deren einseitige Anwendung die Selektion von herbizidresistenten Unkrautpopulationen. So sind in Deutschland unter anderem Resistenzen bei Acker-Fuchsschwanzgras 
(Alopecurus myosuroides) und dem Gemeinen Windhalm (Apera spica-venti) regional bereits so stark ausgeprägt, dass erhebliche Bekämpfungsprobleme auftreten (Petit et al. 2011; JKI 2015). Insektizide können für viele Insekten (Honig- und Wildbienen, Schmetterlinge etc.), andere Gliederfüßer (Spinnen, Asseln etc.) und Wirbeltiere (Amphibien) ähnlich toxisch sein wie für die Schadinsekten. Bei Amphibien haben Pflanzenschutzmittel Auswirkungen auf alle Entwicklungsstadien, führen zu Verhaltens-, Geschlechts- und Wachstumsänderungen und erhöhen die Sterberate (Schmidt 2007). Deshalb gilt der Einsatz von Pflanzenschutzmitteln auch als eine wesentliche Ursache für den Rückgang der biologischen Vielfalt in Agrarlandschaften (Schmidt 2007; Simon et al. 2010; Petit et al. 2011; Jahn et al. 2014; Nielsen et al. 2015). Eine negative Rückwirkung auf die Landwirtschaft ist, dass der Verlust an natürlichen Feinden zu einer Verringerung der biologischen Kontrolle von Schädlingen und Krankheitserregern führt (Simon et al. 2010; Nielsen et al. 2015).

Verstärkte Nährstoffzufuhr wird in zahlreichen Studien als wichtiger Grund für den Verlust der Pflanzenvielfalt auf landwirtschaftlichen Flächen genannt (Kleijn et al. 2009; Spiertz 2010; Gaujour et al. 2012; Krause et al. 2014; Meyer et al. 2015b). Der erhöhte Einsatz von Düngemitteln führt zu einem starken Rückgang von jenen Gruppen der Ackerbegleitflora, die an nährstoffarme Bedingungen angepasst sind, während Generalisten gefördert werden (Meyer et al. 2014). Durch Oberflächenabfluss, Versickerung oder über die Luft können Nährstoffe auch in Gewässer gelangen und diese eutrophieren. Dies steigert unter anderem die Biomasseproduktion aquatischer Pflanzen und Algen mit der negativen Folge, dass die Zersetzung der absinkenden toten organischen Substanz eine Sauerstoffzehrung bewirkt. Dies kann beispielsweise in Amphibienlaichgewässern zum Absterben der Eier und zum Erstickungstod der Larven oder der im Wasser überwinternden geschlechtsreifen Tiere führen (Baker et al. 2013). Insgesamt hat die Intensivierung der Landwirtschaft zu drastischen Bestandseinbrüchen bei Amphibien und Reptilien beigetragen. Durch die starke Bindung dieser Tiergruppen an selten gewordene Habitate wie störungsfreie Zonen an Gewässern oder an Felsen und Trockenhängen sind über $60 \%$ der heimischen Amphibien- und Reptilienarten gefährdet (Haupt et al. 2009).

Neben der Intensivierung ist die Nutzungsaufgabe in Gebieten mit ungünstigen Produktionsbedingungen, eine wesentliche Ursache für den Verlust an biologischer Vielfalt. Dieser Prozess ist durch die Abnahme des Einsatzes der Produktionsfaktoren gekennzeichnet und findet in Deutschland insbesondere in Mittelgebirgslagen statt. Landnutzungsformen, wie z. B. die Bewirtschaftung von Streuobstwiesen oder die Aufrechterhaltung extensiver Beweidungssysteme, werden unrentabel und in Folge unterliegen die aufgegebenen Flächen Sukzessionsprozessen, durch die häufig wichtige Offenlandlebensräume für viele Arten verlorengehen (Bundesamt für Naturschutz 2014). Von der Nutzungsaufgabe sind besonders artenreiche extensive Mager- und Trockenrasen sowie Heiden, Hutewälder und Streuobstwiesen betroffen, die nur durch angepasste Landbewirtschaftung erhalten werden können (Bundesamt für Naturschutz 2014; Lomba et al. 2014). Das ist fatal, denn ein bedeutender Teil der europäischen Flora und Fauna hängt von solchen Nutzungsformen ab (Kleijn et al. 2009). 
Tab. 3.2 Zielsetzungen und Zielerreichung bezüglich der Ressource Biologische Vielfalt. (Zur Erläuterung der Zielwerte vergleiche Anhang)

\begin{tabular}{|c|c|}
\hline Zielsetzungen & Zielerreichung \\
\hline $\begin{array}{l}\text { Artenvielfalt und Landschaftsqualität: Index } \\
\text { (Maßzahl in \%) über die bundesweiten } \\
\text { Bestandsgrößen von } 59 \text { repräsentativen } \\
\text { Vogelarten in sechs Hauptlebensraum- und } \\
\text { Landschaftstypen: } 100 \% \text { im Jahr } 2015\end{array}$ & $\begin{array}{l}\text { - Artenvielfalt und Landschaftsqualität: } \\
63 \% \text { (Stand: 2011), signifikanter Trend } \\
\text { weg vom Zielwert (BMUB 2015a), } \\
\text { Teilindikator Agrarland } 56 \% \\
\text { (BMUB 2015a) }\end{array}$ \\
\hline $\begin{array}{l}\text { - Gefährdete-Arten-Index: (Maßzahl in \%) } \\
\text { Indikator bilanziert das Ausmaß der bundeswei- } \\
\text { ten Gefährdung ausgewählter Artengruppen, } \\
\text { Zielwert } 15 \% \text { im Jahr } 2020\end{array}$ & $\begin{array}{l}\text { - Gefährdete Arten: } 23 \% \text { Stand } 2013 \\
\text { (BMUB 2015a) }\end{array}$ \\
\hline $\begin{array}{l}\text { Erhaltungszustand der FFH-Arten: Index } \\
\text { (Maßzahl in \%) über die Bewertung des } \\
\text { Erhaltungszustandes der Arten der Anhänge II, } \\
\text { IV und V der FFH-Richtlinie in den biogeogra- } \\
\text { phischen Regionen in Deutschland, Zielwert } \\
80 \% \text { im Jahr } 2020\end{array}$ & $\begin{array}{l}\text { - Erhaltungszustand der FFH-Arten: } \\
46 \% \text { Stand } 2013 \text { (BMUB 2015a) }\end{array}$ \\
\hline $\begin{array}{l}\text { FFH-Lebensräume: Index (Maßzahl in \%) über } \\
\text { die Bewertung des Erhaltungszustandes der } \\
\text { Lebensraumtypen des Anhangs I in den } \\
\text { biogeographischen Regionen in Deutschland, } \\
\text { Zielwert } 80 \% \text { im Jahr } 2020\end{array}$ & $\begin{array}{l}\text { FFH-Lebensräume: } 46 \% \text { (Stand } \\
\text { 2007-2012), insbesondere Erhal- } \\
\text { tungszustand landwirtschaftlich } \\
\text { geprägter Lebensraumtypen als } \\
\text { ungünstig bewertet (BMUB 2015a) }\end{array}$ \\
\hline $\begin{array}{l}\text { Anteil gefährdeter heimischer Nutztierrassen: } \\
\text { Prozentualer Anteil gefährdeter einheimischer } \\
\text { Nutztierrassen, Ziel: Ausmaß der Gefährdung } \\
\text { soll verringert werden }\end{array}$ & $\begin{array}{l}\text { Anteil gefährdeter heimischer } \\
\text { Nutztierrassen: } 2013 \text { etwas mehr als } \\
70 \% \text { (BMUB 2015a) }\end{array}$ \\
\hline
\end{tabular}

Um dem dramatischen Verlust der weltweiten Biodiversität zu begegnen, wurde im Jahre 1992 das UN-Übereinkommen über die biologische Vielfalt (Convention on Biological Diversity, CBD) verabschiedet, das Deutschland 1993 ratifiziert und in der Nationalen Strategie zur biologischen Vielfalt aufgegriffen hat (BMUB 2007). Das Ziel, den Biodiversitätsverlust bis $2010 \mathrm{zu}$ stoppen, wurde allerdings nicht erreicht. Der Zustand der biologischen Vielfalt soll durch bundesweite Monitoring-Programme erfasst und bewertet werden (BMUB 2015a). Eine Reihe von Indikatoren zum Erhalt der biologischen Vielfalt weist auf eine zum Teil deutlich negative Entwicklung hin (vgl. Tab. 3.2; siehe auch die Erläuterungen zu den Indikatoren für biologische Vielfalt im Anhang).

\subsubsection{Wirkungen der Landwirtschaft auf das Klima}

Die vom Menschen verursachten Änderungen des Klimas beruhen in erster Linie auf der Freisetzung von Treibhausgasen. Hierzu zählen vorwiegend Kohlendioxid $\left(\mathrm{CO}_{2}\right)$, Methan $\left(\mathrm{CH}_{4}\right)$, Lachgas $\left(\mathrm{N}_{2} \mathrm{O}\right)$ und halogenierte Kohlenwasserstoffe, die jeweils ein unterschiedliches Treibhauspotenzial und eine unterschiedliche Verweildauer in der Atmosphäre haben (IPCC 1996). So sind z. B. Methan und Lachgas 
wesentlich klimawirksamer als Kohlendioxid. Die Konzentration der Treibhausgase in der Atmosphäre nimmt seit Beginn der Industrialisierung beständig zu (IPCC 2014). Bis 2012 stiegen die globalen Land- und Ozean-Oberflächentemperaturen im Mittel um $0,85^{\circ} \mathrm{C}$ an (IPCC 2014), wobei die Klimaänderungen regional sehr unterschiedlich ausfallen (Umweltbundesamt 2014b). Die Landwirtschaft ist sowohl Verursacherin der Freisetzung von Treibhausgasen als auch Betroffene des Klimawandels. Sie kann aber auch durch den Anbau von nachwachsenden Rohstoffen oder durch Bewirtschaftungsformen, welche die Speicherung von Kohlenstoff in Böden und Vegetation fördern, einen Beitrag zum Klimaschutz leisten. In Deutschland war die Landwirtschaft nach den Verursachergruppen „Energie“ und „Industrieprozesse“ lange die drittgrößte Quelle von Treibhausgasen. Gemäß einem Bericht aus dem Jahre 2014 hatte sie zu diesem Zeitpunkt allerdings die „Industrieprozesse“ bereits überholt (Umweltbundesamt 2014b). Grund ist, dass die Reduzierung der Emissionen in der Landwirtschaft geringer war als bei den anderen Quellgruppen.

Treibhausgase entstehen zum einen im vorgelagerten Bereich der Landwirtschaft, zum anderen bei der Tierhaltung durch die Fermentation während der Verdauung, bei der Nutzung landwirtschaftlicher Böden und durch die Düngewirtschaft (Umweltbundesamt 2014b). Im vorgelagerten Bereich der Landwirtschaft werden Treibhausgase erzeugt, die einem Äquivalent von etwa 45,3 Mio. $t$ $\mathrm{CO}_{2}$ entsprechen (Heißenhuber et al. 2015). Diese werden jedoch in der Klimaberichterstattung nicht dem Sektor Landwirtschaft zugeordnet. Im Magendarmtrakt von Wiederkäuern entsteht durch mikrobielle Gärung in erheblichen Mengen Methan. Durch die Nutzung landwirtschaftlicher Böden entsteht vor allem Lachgas, wobei die Bewirtschaftung von Mooren die größte Treibhausgas-Einzelquelle im Sektor Landwirtschaft ist (Drösler et al. 2011). Emissionen der Düngewirtschaft sind die Folge der Lagerung und Ausbringung organischer Dünger. Dabei werden vor allem die Treibhausgase Methan und Lachgas freigesetzt. Die Quellgruppen Landnutzung, Landnutzungsänderung und Forstwirtschaft werden bisher nicht dem Sektor Landwirtschaft zugerechnet, obgleich die Abnahme der weltweiten Waldflächen und der Verlust an Grünland in Deutschland wesentlich durch die Flächennachfrage der Landwirtschaft bedingt werden. Die Freisetzung von Treibhausgasen durch landwirtschaftliche Nutzung wird durch eine Vielzahl von Faktoren beeinflusst, die auch innerhalb eines Produktionsverfahrens sehr unterschiedlich gestaltet werden können. Dies betrifft z. B. die Tierhaltung. In der Milchviehhaltung sinken die Treibhausgas-Emissionen pro erzeugter Produkteinheit, wenn hohe Milchmengen pro Tier erzielt werden, da die Anzahl der gehaltenen Tiere pro Produktionseinheit zurückgeht. Dieser Zusammenhang gilt jedoch nur bis zu einer Milchleistung von ca. $11.000 \mathrm{~kg}$ Milch/Jahr (Flachowsky und Lebzien 2005) und unter der Voraussetzung, dass ausreichend Kälber für die Fleischproduktion vorhanden sind und nicht durch die Haltung von Mutterkühen produziert werden müssen (Zehetmeier et al. 2012). ${ }^{3}$ Eine umfassendere Darstellung

\footnotetext{
${ }^{3}$ Allerdings sind Mutterkühe in der Lage, extensive Weidestandorte zu nutzen und stehen damit zum einen nicht in Nahrungskonkurrenz mit dem Menschen und können zum anderen Leistungen für die Biodiversität auf diesen Standorten erbringen (Heißenhuber et al. 2015, S. 163).
} 
der Einflüsse unterschiedlicher Produktionsmerkmale der Landwirtschaft auf die Entstehung von Treibhausgasen findet sich bei Schuler et al. (2015).

Die Bundesregierung hat sich im „Klimaschutzplan 2050“ (BMUB 2016) das Ziel gesetzt, bis zur Mitte des Jahrhunderts den Ausstoß von Treibhausgasen um 80 bis $95 \%$ gegenüber 1990 zu reduzieren. Der Klimaschutzplan von 2016 legt erstmals auch Treibhausgasminderungsziele für die Landwirtschaft fest. Bisher existierten Vorgaben für das Management organischer Dünger (max. Einsatz von organischem Dünger $170 \mathrm{~kg} \mathrm{~N} / \mathrm{ha}$, zeitnahe Einarbeitung), für den Erhalt von Grünland und für den Schutz von Mooren (siehe Abschn. 3.2.2), die ebenfalls Auswirkungen auf den Klimaschutz haben.

\subsubsection{Wirkungen der Landwirtschaft auf das Landschaftsbild}

Im Bundesnaturschutzgesetz wird Landschaft neben der Natur als Schutzgut genannt. Sie ist ,so zu schützen, dass [...] Vielfalt, Eigenart und Schönheit sowie der Erholungswert von Natur und Landschaft auf Dauer gesichert sind" (BNatschG 2009, § 1, Abs. 1). Das ,Landschaftsbild“ wird nicht explizit genannt, sondern durch die Merkmale Vielfalt, Eigenart und Schönheit umschrieben. Insbesondere Schönheit kann jedoch nur subjektiv bewertet werden (Trepl 2012). Da sie aber gewöhnlich das Ergebnis von „Vielfalt und Eigenart des Naturraums“ ist (Ratzbor 2011), werden Landschaften im Allgemeinen umso positiver bewertet, ,je mehr (visuell) deutlich unterscheidbare Elemente" bzw. für die Landschaft typische Strukturen vorhanden sind (Nohl 1993). Abwechslungsreiche Landschaften mit einem ausgewogenen Verhältnis von Wald und Offenland steigern die Erholungs- und Erlebnisqualität (Bundesamt für Naturschutz 2014). Dabei wird dem Grünland meist ein besonders hoher ästhetischer Wert zugeordnet und dessen Bedeutung als Erholungsraum betont (Osterburg et al. 2009).

Die Landwirtschaft hat über Jahrhunderte dazu beigetragen, dass in Europa und Deutschland vielfältige Landschaften entstanden, die durch einen Wechsel an Forst-, Grünland-, Acker- und Dauerkulturflächen, durch das Vorkommen von natürlichen und halbnatürlichen Habitaten sowie durch standorttypische Ausprägungen der Nutzungsformen (z. B. Fruchtfolgen, extensiven Weideflächen) gekennzeichnet sind. Zunehmende Intensivierung auf der einen und vermehrte Nutzungsaufgabe auf der anderen Seite gefährden mittlerweile viele prägende Elemente des Landschaftsbildes. Das ist auch deswegen besonders kritisch, weil ein enger Zusammenhang zwischen dem Erhalt des Landschaftsbildes und dem Schutz der biologischen Vielfalt besteht, denn viele Strukturen und Elemente haben auch einen positiven Einfluss auf die Verfügbarkeit von Habitaten.

Die Ziele für den Erhalt der Vielfalt, Eigenheit und Schönheit der Landschaft sind meist qualitativ formuliert. So sieht die Nationale Biodiversitätsstrategie vor, „regionaltypische[n] Bewirtschaftungsformen“, die zur Erhaltung und Entwicklung von Kulturlandschaften und ihren Elementen beitragen, zu fördern und das Landschaftsbild vor Beeinträchtigungen zu schützen (BMUB 2007, S. 41). 
Messbare Ziele in Bezug auf das Landschaftsbild wurden jedoch nicht etabliert. Folgende Indikatoren können dennoch für die Beschreibung und teilweise Quantifizierung des Einflusses der Landwirtschaft auf das Landschaftsbild herangezogen werden:

- Erhalt traditioneller, naturverträglicher Nutzungsformen (als Ziel in der Biodiversitätsstrategie formuliert) (Deimer 2005):

- Traditionelle Nutzungsformen, z. B. Streuobstwiesen, extensives Grünland

- High-Nature-Value: Anteil von Flächen mit hohem Naturschutzwert an der Landwirtschaftsfläche, Zielwert $19 \%$ im Jahr 2015

- Erhalt und Pflege der Kulturlandschaft (Deimer 2005), abwechslungsreiche Landschaft (Bundesamt für Naturschutz 2014):

- Erhalt von Grünland

- Aufrechterhaltung der Bewirtschaftung auf Grenzertragsstandorten

- Vermeidung von Sukzession als Ausdehnung der Waldfläche

- Vielfältige Fruchtfolgen (Heißenhuber et al. 2004; Deimer 2005; Heißenhuber et al. 2015)

- Prägung durch kleinräumige, natürliche und naturnahe Biotope (Deimer 2005):

- High-Nature-Value: s. o.

- Erhalt von Landschaftselementen (Heißenhuber et al. 2004)

- Vielfalt

- Schlaggröße

- Vielfältige Fruchtfolge

- Ökologische Vorrangfläche

Die genannten Indikatoren werden in Abschn. 6.5 ausführlicher dargestellt.

\subsubsection{Wirkungen der Landwirtschaft auf die Ressource Luft}

Die Luftqualität wird durch die Konzentration einzelner Gase und durch Aerosole beeinflusst. Zu den wichtigsten Luftschadstoffen zählen Ammoniak, Benzol, Blei, Feinstaub, Kohlenmonoxid, Ozon, Schwefeldioxide und Stickoxide (Umweltbundesamt 2016). Luftschadstoffe können Menschen, Pflanzen und Tiere sowohl direkt negativ beeinflussen, als auch die abiotischen Schutzgüter z. B. über die Ablagerung von Schwermetallen nachteilig verändern. Sie wirken durch Änderungen der Zusammensetzung des Gasgemisches, durch feste Bestandteile in der Luft und durch Geruchsbelästigungen.

Luftschadstoffe entstehen in der Landwirtschaft - wie bereits erwähnt - durch Tierhaltung, Düngung, Ausbringung von Pflanzenschutzmitteln und Bodenbearbeitung. Dabei trägt die intensive Tierhaltung zunehmend zur Entstehung von 
Ammoniak (Schieß1 et al. 2015), Feinstaub und Geruchsbelästigungen bei (Umweltbundesamt 2011a). Die Landwirtschaft ist jedoch nicht nur Erzeugerin von Luftschadstoffen, sie ist auch von deren schädigenden Wirkungen negativ betroffen. Als Beispiel seien die Effekte von Schwefel- und Stickoxiden sowie von Ammoniak genannt. Schwefel- und Stickoxide wirken versauernd (Baumgarten 2006) und beeinträchtigen so die Bodenfunktionen. Sie können sich durch indirekte Düngewirkungen jedoch auch positiv auf das Pflanzenwachstum auswirken. Stickoxide tragen darüber hinaus zur bodennahen Ozonbildung bei. Sie werden aus landwirtschaftlichen Böden freigesetzt (etwa $17 \%$ der Stickoxidbelastungen der Luft), wobei bei gedüngten Böden höhere Freisetzungsraten gemessen werden. Auch Ammoniak wirkt versauernd und eutrophierend. Es beeinflusst so ebenfalls Bodenfunktionen, Biodiversität sowie Wasserqualität und trägt zur Entstehung von Treibhausgasen bei. Außerdem bildet Ammoniak durch die Reaktion mit anderen Gasen gesundheitsschädlichem Feinstaub in der Atmosphäre. Ammoniak kann beim Menschen neben Reizungen der Augen und Schleimhäute auch Asthma, Husten und Atemnot hervorrufen. Zusätzlich kann es Schäden an Pflanzen verursachen (LfU - Bayrisches Landesamt für Umwelt 2004).

Die NEC-Richtlinie (Richtlinie über nationale Emissionshöchstmengen) der EU sieht eine Senkung des gemittelten Ausstoßes von Schwefeldioxid, Ammoniak, NMVOC und Stickstoffoxiden bis 2010 gegenüber 1999 um $70 \%$ vor. Dies entspricht z. B. einer Begrenzung der Ammoniak-Emissionen auf max. 550 kt/ Jahr bis 2010 (NEC-RL). Dieser Wert konnte nicht erreicht werden. Nach dem fortgeschriebenen Multikomponenten-Protokoll sind die Ammoniak-Emissionen bis 2020 gegenüber 2005 um $5 \%$ zu senken und die Stickstoffoxid-Emissionen um $39 \%$. Ein Vorschlag der EU-Kommission zur Fortentwicklung der NEC-Richtlinie sieht eine weitere Reduzierung der Ammoniak-Emissionen um $39 \%$ bis $2030 \mathrm{im}$ Vergleich zu 2005 vor. Tab. 3.3 fasst bezüglich der Luft die Ziele und deren Erreichung zusammen.

Tab. 3.3 Zielsetzungen und Zielerreichung bezüglich der Ressource Luft

\begin{tabular}{|c|c|}
\hline Zielsetzung & Zielerreichung \\
\hline $\begin{array}{l}\text { NEC-Richtlinie: Grenzwerte für die } \\
\text { Emissionen von Schwefeldioxid, } \\
\text { Ammoniak, NMVOC und Stickstoff- } \\
\text { oxiden }\end{array}$ & $\begin{array}{l}\text { Nein: Grenzwerte von drei der vier geregelten } \\
\text { Luftschadstoffe werden nicht eingehalten }\end{array}$ \\
\hline $\begin{array}{l}\text { - Ammoniak: Begrenzung der } \\
\text { Ammoniak-Emissionen auf max. } \\
550 \mathrm{kt} / \mathrm{Jahr} \text { bis } 2010 \text { (NEC-RL) }\end{array}$ & $\begin{array}{l}\text { - Nein: 2013: } 671 \text { kt Ammoniak-Emissionen } \\
\text { (Umweltbundesamt 2013b) } \\
\text { - keine Reduktion seit } 1994\end{array}$ \\
\hline $\begin{array}{l}\text { Ammoniak: Senkung des gemittelten } \\
\text { Ausstoßes von Schwefeldioxid, } \\
\text { Ammoniak, NMVOC und Stickstoff- } \\
\text { oxiden bis } 2010 \text { gegenüber } 1999 \text { um } \\
70 \% \text { (Umweltbundesamt 2015a. S. 60) }\end{array}$ & $\begin{array}{l}\text { Nein: bis } 2013 \text { sanken die gemittelten } \\
\text { Emissionen von Schwefeldioxid, Ammoniak, } \\
\text { NMVOC und Stickstoffoxiden (Umweltbun- } \\
\text { desamt 2015a. S. 60) um 42,5\%; 2015: } \\
\text { 1186 kt Stickoxid-Emissionen (Umweltbun- } \\
\text { desamt 2016) }\end{array}$ \\
\hline
\end{tabular}




\subsubsection{Wirkungen der Landwirtschaft auf die Ressource Wasser}

Der Gewässerschutz wird auf europäischer Ebene u. a. durch die Wasserrahmenrichtlinie (WRRL), die Grundwasserrichtlinie, die Nitratrichtlinie und die Meeresstrategie-Rahmenrichtlinie geregelt. Die Wasserrahmenrichtlinie sieht vor, bis 2015 für alle Gewässer einen guten Zustand zu erreichen. Die MeeresstrategieRahmenrichtlinie zielt darauf, bis 2020 die Meeresgewässer in einen guten Umweltzustand zu versetzen bzw. diesen zu erhalten. Auf nationaler Ebene werden die Wasserrahmenrichtlinie und die Meeresstrategie-Rahmenrichtlinie durch das Wasserhaushaltsgesetz umgesetzt. Die Grundwasserverordnung dient der Umsetzung der Grundwasserrichtlinie in nationales Recht und die Düngeverordnung der Umsetzung der Nitratrichtlinie. Die Richtlinien und Verordnungen zielen auf eine Verbesserung - oder mindestens auf die Vermeidung einer Verschlechterung - der Wasserqualität im Grundwasser, in Flüssen, Seen und Meeren.

Die Landwirtschaft beeinflusst das Wasser durch Entnahmen für die Bewässerung zum einen quantitativ, zum anderen qualitativ durch den Eintrag von Schwemm- und Schadstoffen. Auch Landnutzungsänderungen (z. B. die Umwandlung von Wald und Grünland in Acker) haben einen Einfluss auf den Wasserhaushalt. Dadurch verändern sich Grundwasserneubildung und Retentionsraum (Osterburg et al. 2009; Bundesamt für Naturschutz 2014), gleichzeitig wird die Belastung von Grund ${ }^{4}$ - und Oberflächenwasser ${ }^{5}$ durch zunehmende Einträge erhöht. Darüber hinaus können Bodenverdichtungen durch den Einsatz schwerer Maschinen die Erosion erhöhen, die Versickerungsrate verringern und den Oberflächenabfluss verstärken. Und schließlich kann ein durch Bodenbearbeitung bedingter Humusabbau das Wasserhaltevermögen reduzieren.

Die heimische Landwirtschaft hat durch den Eintrag von schädlichen Stoffen oder über Depositionen einen direkten Einfluss auf die Qualität der Oberflächengewässer und des Grundwassers. Dabei wird das Grundwasser meist durch diffuse Einträge belastet. Dazu gehören z. B. Nitratauswaschungen. Aber auch Schadstoffe wie Pflanzenschutzmittel, Schwermetalle (z. B. Uran) und Arzneimittel sind im Grundwasser zu finden (Abschn. 3.2.1). Oberflächengewässer werden hingegen auch durch punktuelle Einträge belastet. Hinsichtlich des Schutzes und des Erhalts eines guten chemischen Zustandes des Wassers gibt es Grenzwerte für Nitrat und Pflanzenschutzmittel. ${ }^{6}$

\footnotetext{
${ }^{4}$ Nur bei $7 \%$ der Grundwassermessstellen im Umfeld von Grünland wurden hohe Nitratbelastungen nachgewiesen (Umweltbundesamt 2010c, S. 19), Messstellen im Einzugsbereich von Ackernutzung zeigen signifikant höhere Nitratbelastungen des Grundwassers (Umweltbundesamt 2010b, S. 13).

${ }^{5}$ Höhere Erosionsraten auf Acker- als auf Grünlandflächen.

${ }^{6}$ Richtlinie 91/676/EWG des Rates vom 12. Dezember 1991 zum Schutz der Gewässer vor Verunreinigung durch Nitrat aus landwirtschaftlichen Quellen; Richtlinie 2000/60/EG des Europäischen Parlaments und des Rates vom 23. Oktober 2000 zur Schaffung eines Ordnungsrahmens für Maßnahmen der Gemeinschaft im Bereich der Wasserpolitik; Richtlinie 2006/118/EG des Europäischen Parlaments und des Rates vom 12. Dezember 2006 zum Schutz des Grundwassers vor Verschmutzung und Verschlechterung.
} 
Diese werden jedoch in einigen Regionen - bei Nitrat insbesondere in Landstrichen mit hoher Viehdichte - nicht eingehalten. Die EU-Kommission hat im April 2016 wegen Nicht-Einhaltung der Nitratrichtlinie Klage vor dem europäischen Gerichtshof gegen die Bundesrepublik Deutschland eingereicht. ${ }^{7}$

Obwohl die Landwirtschaft weltweit die größte Nutzerin von Wasser ist, spielt sie in Europa und Deutschland bei der Wasserentnahme quantitativ nur eine geringe Rolle (Umweltbundesamt 2011b). Durch den Import von Lebens- und Futtermitteln kann Deutschland jedoch in den Erzeugerländern zu einer (zum Teil übermäßigen) Nutzung von Wasser beitragen (Heißenhuber und Krämer 2013). Eine Übersicht über wasserbezogene Zielsetzungen und die Zielerreichung findet sich in Tab. 3.4.

Tab. 3.4 Zielsetzungen und Zielerreichung bezüglich der Ressource Wasser

\begin{tabular}{|c|c|}
\hline Zielsetzung & Zielerreichung \\
\hline $\begin{array}{l}\text { Guter ökologischer Zustand der } \\
\text { Oberflächengewässer (WRRL): geringe } \\
\text { anthropogene Abweichungen, d. h. } \\
\text { geringe Abweichungen von den Werten, } \\
\text { die sich normalerweise bei Abwesenheit } \\
\text { störender Einflüsse einstellen würden }\end{array}$ & $\begin{array}{l}\text { - Sehr guter und guter Zustand bei ca. } 14 \% \text { der } \\
\text { Fließgewässer und } 39 \% \text { der Seen (Umwelt- } \\
\text { bundesamt 2010c) }\end{array}$ \\
\hline $\begin{array}{l}\text { Guter chemischer Zustand der } \\
\text { Oberflächengewässer (WRRL) }\end{array}$ & $\begin{array}{l}\text { - Fließgewässer: guter chemischer Zustand bei } \\
88 \% \text { der Wasserkörper } 2010 \text { erreicht; bei } \\
\text { Anwendung der Umweltqualitätsnormricht- } \\
\text { linie ab } 2018 \text { werden voraussichtlich } 100 \% \\
\text { der Wasserkörper das Ziel ,guter chemischer } \\
\text { Zustand“ verfehlen } \\
\text { - Seen: guter chemischer Zustand bei } 92 \% \text { der } \\
\text { Wasserkörper } 2010 \text { erreicht; einzelne Über- } \\
\text { schreitungen der Grenzwerte bei Schwermetal- } \\
\text { len, Pflanzenschutzmitteln und Polyzyklischen } \\
\text { Aromatischen Kohlenwasserstoffen; bei } \\
\text { Anwendung der Umweltqualitätsnormrichtlinie } \\
\text { ab } 2018 \text { werden voraussichtlich } 100 \% \text { der } \\
\text { Wasserkörper das Ziel,,guter chemischer } \\
\text { Zustand“ verfehlen (Umweltbundesamt 2010c) }\end{array}$ \\
\hline $\begin{array}{l}\text { - Guter mengenmäßiger Zustand des } \\
\text { Grundwassers (WRRL) }\end{array}$ & $\begin{array}{l}\text { Kaum Wassermengenprobleme, } 2010 \text { wiesen } \\
\text { nur } 4 \% \text { der Grundwasserkörper keinen guten } \\
\text { mengenmäßigen Zustand auf (Umweltbundes- } \\
\text { amt 2010c) }\end{array}$ \\
\hline $\begin{array}{l}\text { Guter chemischer Zustand des } \\
\text { Grundwassers (WRRL) - siehe } \\
\text { Stickstoff und Pflanzenschutzmittel }\end{array}$ & $\begin{array}{l}\text { - } 37 \% \text { der Grundwasserkörper in einem } \\
\text { schlechten chemischen Zustand (2010); } 27 \% \\
\text { der Grundwasserkörper übersteigen die } \\
\text { Qualitätsnorm für Nitrat, } 4 \% \text { der Grundwasser- } \\
\text { körper übersteigen die Qualitätsnorm für } \\
\text { Pflanzenschutzmittel (Umweltbundesamt 2010c) }\end{array}$ \\
\hline
\end{tabular}

(Fortsetzung)

\footnotetext{
${ }^{7}$ Europäische Kommission: Nitratbelastung in Gewässern: EU-Kommission verklagt Deutschland, abrufbar unter: https://ec.europa.eu/germany/news/nitratbelastung-gew\%C3\%A4ssern-eu-kommission-verklagt-deutschland_de. Zugegriffen am 18.05.2017.
} 
Tab. 3.4 (Fortsetzung)

\begin{tabular}{|c|c|}
\hline Zielsetzung & Zielerreichung \\
\hline $\begin{array}{l}\text { - Gesamt-Umweltzustand der deutschen } \\
\text { Meeresgewässer gemäß Meeresstrate- } \\
\text { gierahmenrichtlinie }\end{array}$ & $\begin{array}{l}\text { Der zusammenfassende Umweltzustand für } \\
\text { Nord- und Ostsee wird } 2012 \text { mit „,nicht gut““ } \\
\text { bewertet, dabei spielt die Landwirtschaft } \\
\text { insbesondere beim Kriterium „Anreicherung } \\
\text { mit Nährstoffen und organischem Material““ } \\
\text { eine entscheidende Rolle, welches ebenfalls } \\
\text { mit „nicht gut“ bewertet wird (Umweltbundes- } \\
\text { amt 2010c) }\end{array}$ \\
\hline $\begin{array}{l}\text { - Stickstoff: max. } 50 \mathrm{mg} \text { N/l Trinkwasser } \\
\text { (Nitratrichtlinie) }\end{array}$ & $\begin{array}{l}\text { 2010: zu hohe Nitratkonzentrationen bei } \\
14 \% \text { der Messstellen, starke regionale } \\
\text { Unterschiede } \\
\text { - Zunahme der Nitratkonzentrationen an } 40 \% \\
\text { der Messstellen zwischen 2004/2006 und } \\
\text { 2008/2010 } \\
\text { Klage der Europäischen Kommission gegen } \\
\text { Deutschland wegen Nicht-Einhaltung der } \\
\text { Nitratrichtlinie }\end{array}$ \\
\hline - Phosphor & $\begin{array}{l}\text { - Abnahme der Konzentrationen an den } \\
\text { Messstellen }\end{array}$ \\
\hline $\begin{array}{l}\text { Pflanzenschutzmittel: } 0,1 \mu \mathrm{g} / 1 \\
\text { Trinkwasser für einzelne Stoffe; } \\
0,5 \mu \mathrm{g} / \mathrm{l} \text { für mehrere Stoffe }\end{array}$ & $\begin{array}{l}\text { Vereinzelte Überschreitungen bei } 13 \\
\text { Pestiziden (2008-2010), bei } 24 \text { Pestiziden } \\
\text { keine Überschreitungen (Umweltbundesamt } \\
2012 a \text { ) } \\
\text { - Überschreitungen an } 4,7 \% \text { der Messstellen } \\
\text { (2006-2008) (Umweltbundesamt 2010c) }\end{array}$ \\
\hline
\end{tabular}

- Schwermetalle:

- Kein Zielwert für Belastung der Gewässer definiert

- Grenzwerte für die Belastung des Klärschlamms mit Schwermetallen und anderen Stoffen

- Grenzwerte für die Ausbringung von Klärschlamm

- Arzneimittel: kein Zielwert definiert
- Uran: in oberflächennahen Grundwässern unter Acker 2-fach höhere Konzentration als unter Wald (<10 $\mu \mathrm{g}$ Uran/l) (Utermann et al. 2009); keine definierten Ziele

- Rückstände nahezu ganzjährig und flächendeckend in Fließgewässern zu finden

- 150 Wirkstoffe in Umwelt (meist Gewässern)

- Antibiotika-Monitoring:

- ab bestimmter Bestandsgröße Meldungen von Angaben zum Antibiotikaeinsatz

- Betriebe, die mehr Arzneimittel anwenden als Vergleichsbetriebe, müssen Maßnahmen ergreifen, um diesen Einsatz zu reduzieren

- Arzneimittel dürfen nur zur Behandlung kranker Tiere eingesetzt werden

- Antibiotika dürfen nur von einem Tierarzt oder einer Apotheke auf Verschreibung abgegeben werden; nachgewiesen in Konzentrationen von 0,1 bis $1 \mu \mathrm{g} / \mathrm{l}$ (Umweltbundesamt 2014a) 


\subsubsection{Zusammenfassung}

Ein guter Zustand der schützenswerten Naturressourcen wurde bisher vielfach nicht erreicht. Auch die in verschiedenen Gesetzen, Richtlinien und Strategien definierten Zielwerte werden häufig verfehlt. Der Anteil der Landwirtschaft an der negativen Beeinflussung der Ressourcen ist dabei oft groß und hat in der Vergangenheit weiter zugenommen, während in vielen anderen Bereichen größere Erfolge bei der Reduzierung von Belastungen erreicht werden konnten (Heißenhuber et al. 2015).

Festzuhalten ist, dass sich bestimmte landwirtschaftliche Wirkfaktoren gleichzeitig auf mehrere Ressourcen auswirken, wobei diese Faktoren im Wesentlichen zwei Mechanismen zugeordnet werden können: stoffliche Einträge und Flächennutzung (siehe Tab. 3.5 für eine Übersicht). ${ }^{8}$

\subsection{Landwirtschaftliche Wirkfaktoren}

In diesem Abschnitt wird der in Abschn. 3.1 behandelte Zustand der Naturressourcen den beiden landwirtschaftlich Wirkfaktoren „Stoffliche Einträge“ und ,Flächennutzung“" zugeordnet. Der Fokus liegt auf der Beeinflussung des Bodens, der biologischen Vielfalt, des Klimas, des Landschaftsbilds, der Luft und des Wassers. Weiterhin werden die für diese Naturressourcen definierten Zielsetzungen behandelt.

\subsubsection{Stoffliche Einträge}

Stoffliche Einträge gelangen zum einen direkt durch den Einsatz landwirtschaftlicher Produktionsmittel in die Umwelt, zum anderen können sie auch die indirekte Folge bestimmter Formen der landwirtschaftlichen Flächennutzung sein (z. B. Stickstofffreisetzung bei Grünlandumwandlung). Hier fokussieren wir auf die Einträge von Stickstoff, Phosphat, Pflanzenschutzmitteln, Kupfer und Arzneimitteln. Zusätzlich

\footnotetext{
${ }^{8}$ Nicht aufgeführt sind dabei Wirkfaktoren, die insbesondere der Tierhaltung zuzuordnen sind. Dies betrifft bspw. den Viehbesatz pro Hektar, die Formen der Düngerwirtschaft, technische Verfahren der Tierhaltung (z. B. Stalltechnik), die Art der eingesetzten Futtermittel und deren Erzeugung sowie die mit der Tierhaltung verbundenen extensiven Nutzungsverfahren. Dabei wird der Viehbesatz implizit dem Punkt ,stoffliche Einträge von Stickstoff und Phosphor“" zugerechnet, die Erzeugung von Futter erscheint auch unter dem Punkt „Fruchtfolgegestaltung“ und extensive Tierhaltungsverfahren unter dem Punkte „Aufgabe traditioneller, extensiver Nutzungsformen“. Nicht weiter eingegangen wird jedoch auf die Verfahren der Düngewirtschaft (Festmist, Gülle, Jauche), wobei diese über die Entstehung von Treibhausgasen oder die Zufuhr von organischer Substanz in den Boden durchaus Wirkungen auf die genannten Ressourcen haben kann. Auch technische Aspekte des Stallbaus finden keine Berücksichtigung, diese können jedoch auf die Luftqualität und die Emission von Treibhausgasen wirken.
} 


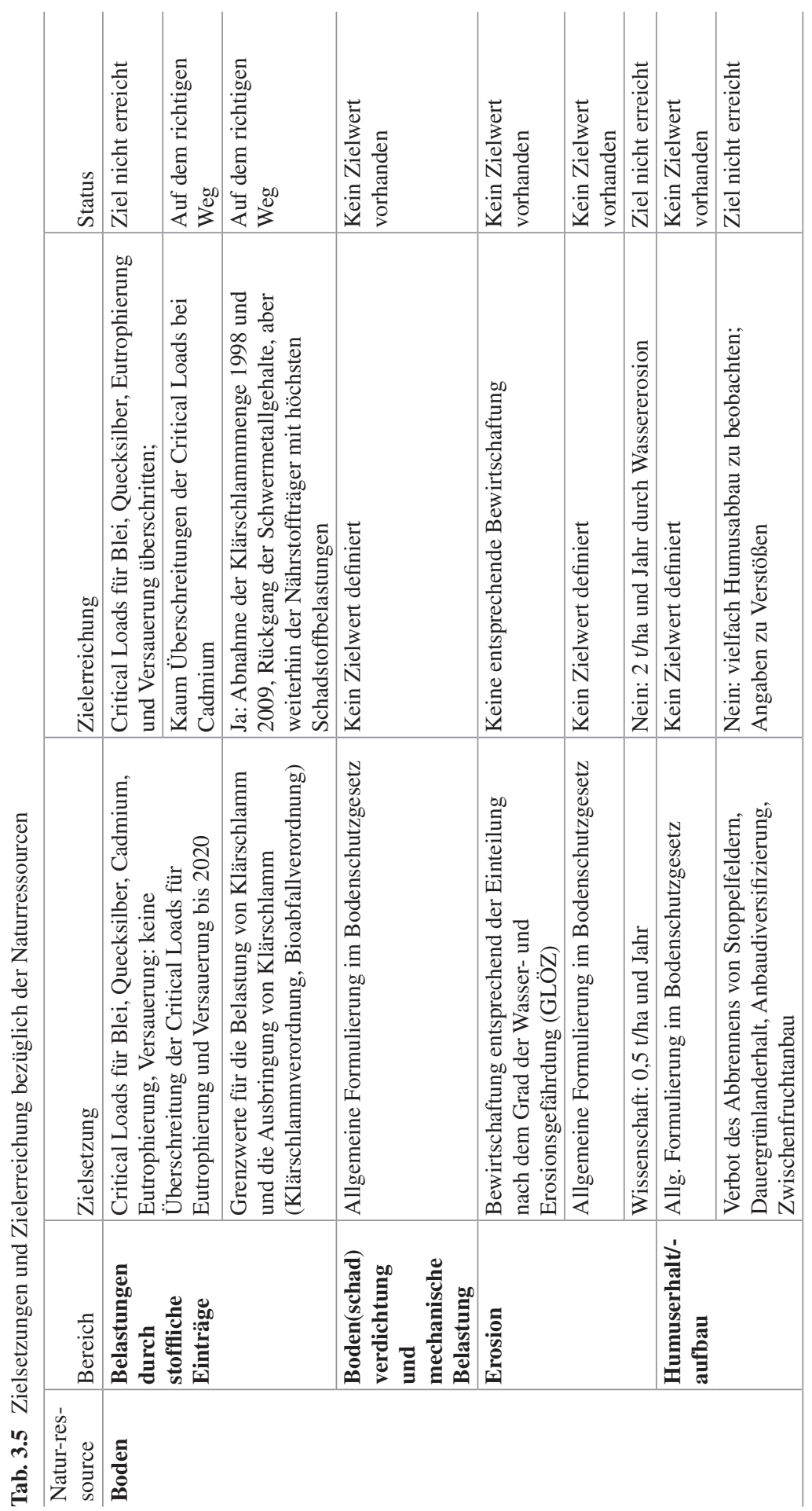




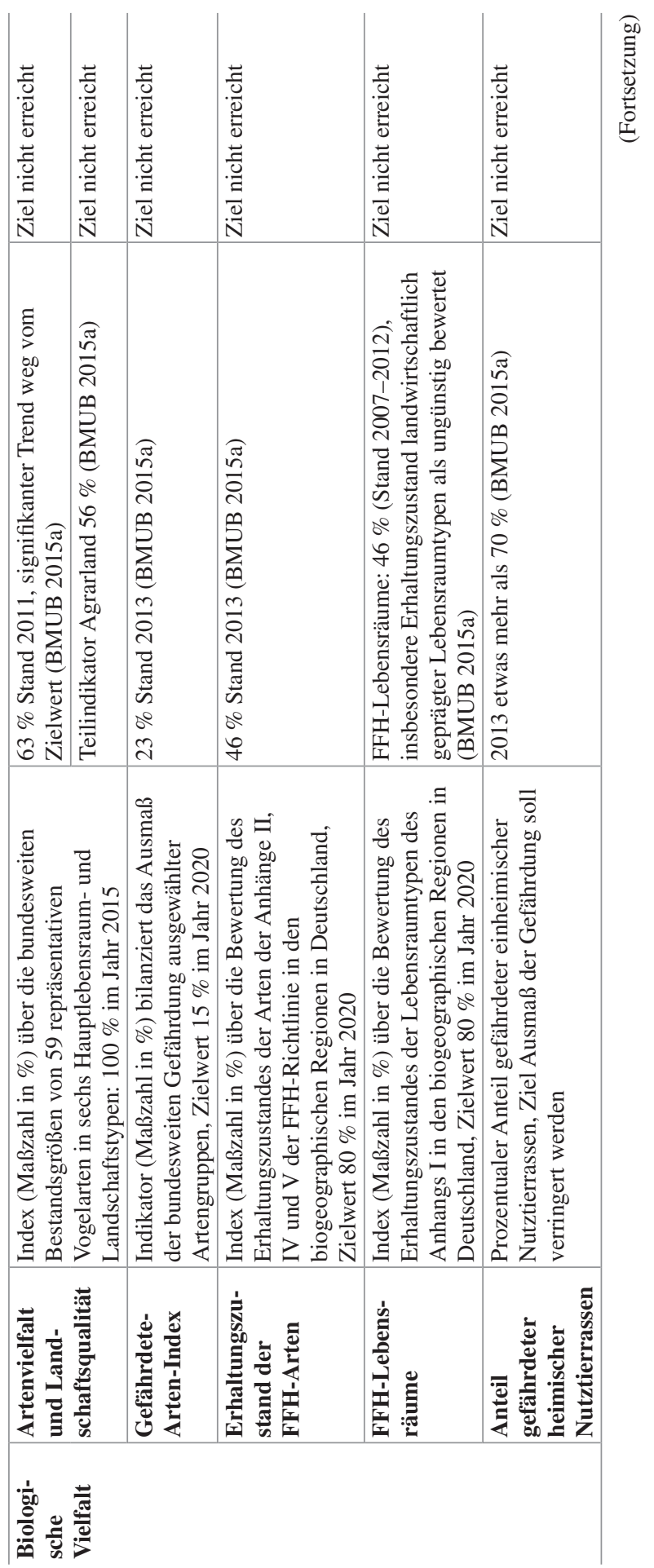




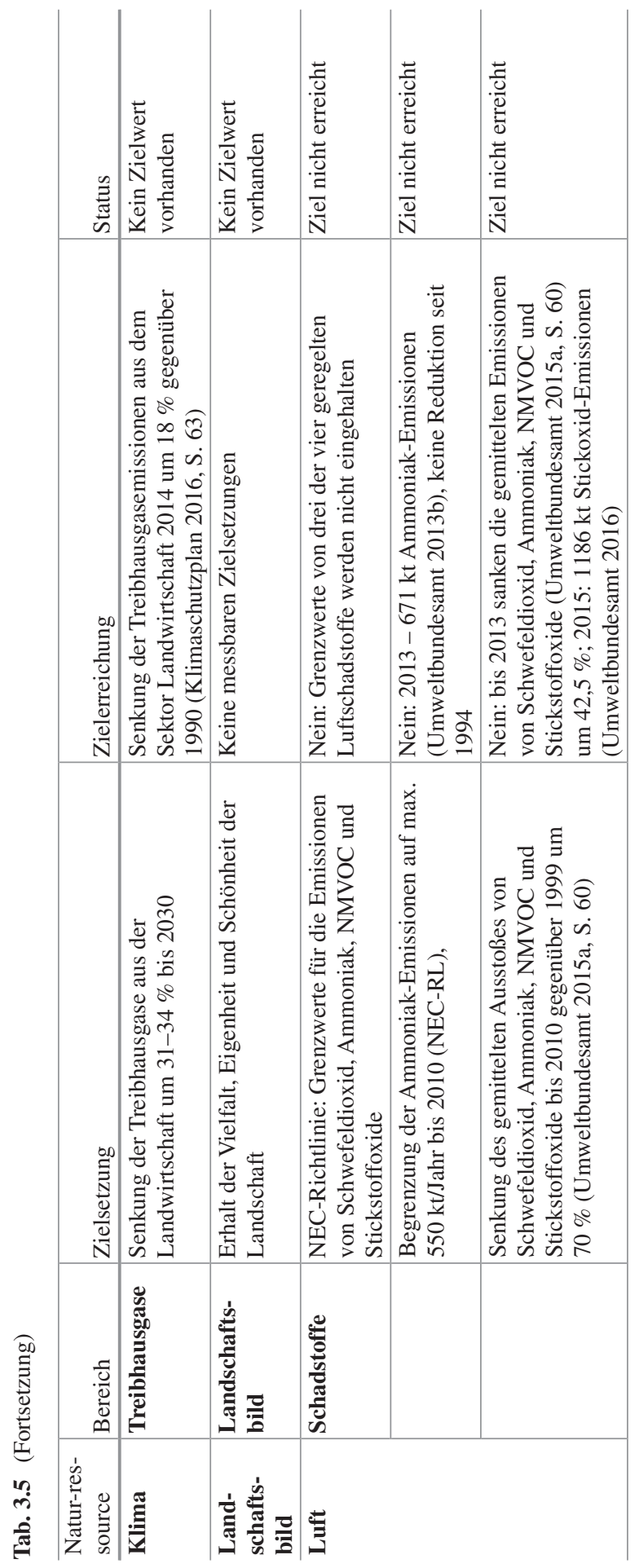




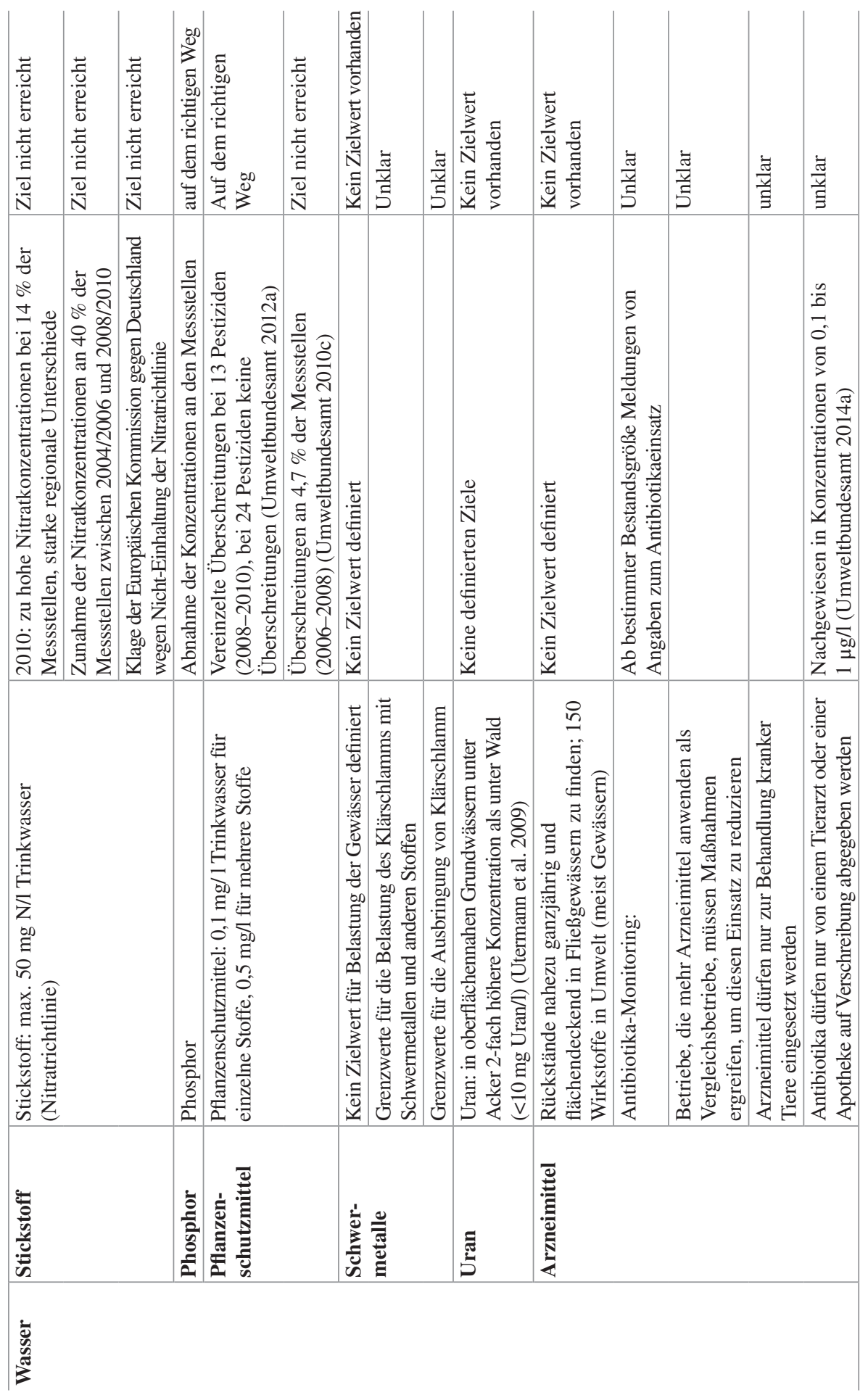


werden Schwermetalle betrachtet, die nicht gezielt ausgebracht werden, sondern die als „Verunreinigungen“ (z. B. von Dünge- und Pflanzenschutzmitteln) in die Umwelt gelangen. Über die reinen Mengen hinaus spielen Ausbringungs- und Einarbeitungszeitpunkte, die Art der Ausbringungstechnik, die Gestaltung von Fruchtfolgen, die Einhaltung von Bearbeitungsabständen zu Gewässern und die Durchführung von humusaufbauenden Maßnahmen eine wichtige Rolle (Heißenhuber und Krämer 2013).

\subsubsection{Stickstoffverbindungen}

Stickstoffverbindungen aus der Landwirtschaft werden durch mineralische und organische Düngemittel eingetragen, können aber auch durch den Umbruch von Grünland und den Abbau von Humus freigesetzt werden. Bei organischen Düngemitteln hat darüber hinaus die Art der Lagerung, Ausbringung und Einarbeitung einen Einfluss auf die Entstehung von Stickstoffverbindungen. Die deutsche Nachhaltigkeits- und die deutsche Biodiversitätsstrategie (BMUB 2007; Statistisches Bundesamt 2014) formulierten für die jährliche Stickstoffbilanz einen Zielwert von $80 \mathrm{~kg} \mathrm{~N} / \mathrm{ha}$, der bis 2010 erreicht werden sollte, was jedoch nicht gelang (siehe Tab. 3.6). Allerdings gibt es starke regionale Unterschiede. In der jüngsten Vergangenheit haben - neben der Tierhaltung - die Förderung der nachwachsenden Rohstoffe durch das Erneuerbare-Energien-Gesetz, der Güllebonus und die fehlende Berücksichtigung der Gärreste in der Düngebilanz zu erhöhten Werten in der Stickstoffbilanz geführt (Taube 2016). Die Ausbringung von Stickstoff durch Düngung ist in der Düngeverordnung geregelt. Sie schreibt beispielsweise für jeden Landwirt, der mehr als 10 ha bewirtschaftet, die Erstellung einer Nährstoffbilanz (Feld-Stall) vor und beschränkt die Ausbringung von organischen Düngemitteln auf max. $170 \mathrm{~kg} \mathrm{~N} / \mathrm{ha}$ und Jahr. Entsprechend der regionalen Verteilung der genutzten Großvieheinheiten ist das Aufkommen an tierischem Wirtschaftsdünger - vor allem mit hohen Konzentrationen von über $170 \mathrm{~kg} \mathrm{~N} / \mathrm{ha}$ - in Gebieten mit intensiver Tierhaltung besonders hoch (z. B. Westniedersachsen, nördliches Nordrhein-Westfalen). Weitere Konzentrationsgebiete liegen im Allgäu und im Alpenvorland (BLAG 2012). Der Stickstoffüberschuss nach Flächenbilanz darf einen gesetzlichen Höchstwert nicht überschreiten. Dieser Wert konnte seit 2007 schrittweise von 90 auf $60 \mathrm{~kg} / \mathrm{ha}$ gesenkt werden (Umweltbundesamt 2015a, S. 85). ${ }^{9}$

Auch Ammoniak-Emissionen entstehen vorwiegend im Zusammenhang mit der Tierhaltung, bei der Lagerung und Ausbringung organischer Dünger und aus landwirtschaftlich genutzten Böden (Flessa et al. 2012). Das Ausmaß der Freisetzung durch die Tierhaltung variiert mit Tierkategorie, Stalltyp, Einstreu und Art der Wirtschaftsdünger sowie Tierleistung und -ernährung. Etwa $95 \%$ des Luftschadstoffes Ammoniak werden von der Landwirtschaft freigesetzt.

\footnotetext{
${ }^{9}$ In dem UBA-Bericht wird ausgeführt, dass Werte der Flächenbilanz um etwa $30 \mathrm{~kg} / \mathrm{ha}$ geringer sind als Werte der Gesamt- oder Hoftorbilanz, weil sie nicht den gasförmigen Stickstoff enthalten, der aus den Ställen sowie bei der Lagerung von Gülle und Mist entweicht.
} 
Tab. 3.6 Zielsetzung und Zielerreichung bezüglich der Stickstoff-Einträge

\begin{tabular}{|c|c|}
\hline Zielsetzung für die Landwirtschaft & Zielerreichung \\
\hline $\begin{array}{l}\text { - Stickstoffbilanz: } 80 \mathrm{~kg} \mathrm{~N} / \mathrm{ha} \text { und Jahr bis } \\
2010 \text { (Umweltbundesamt 2015a, S. 84) }\end{array}$ & $\begin{array}{l}\text { - Nein: Stickstoffbilanz 2012: } 98 \mathrm{~kg} \text { N/ha und } \\
\text { Jahr (Umweltbundesamt 2015a, S. 84) }\end{array}$ \\
\hline $\begin{array}{l}\text { - Organische Düngung: } 170 \mathrm{~kg} \text { N/ha und } \\
\text { Jahr, bisher nur organischer Dünger aus } \\
\text { tierischer Herkunft berücksichtigt, } \\
\text { zukünftig sollen alle organischen Dünger } \\
\text { berücksichtigt werden }\end{array}$ & $\begin{array}{l}\text { - Nordrhein-Westfalen: mit Ausnahme eines } \\
\text { Landkreises wird der Grenzwert von } 170 \mathrm{~kg} \\
\text { N/ha und Jahr organischer Dünger aus } \\
\text { tierischer Herkunft in allen Landkreisen } \\
\text { eingehalten (Landwirtschaftskammer } \\
\text { Nordrhein-Westfalen 2014). } \\
\text { - Niedersachsen: mit Ausnahme eines Landkrei- } \\
\text { ses wird der Grenzwert von } 170 \mathrm{~kg} \text { N/ha und } \\
\text { Jahr organischer Dünger aus tierischer } \\
\text { Herkunft in allen Landkreisen eingehalten } \\
\text { (Landwirtschaftskammer Niedersachsen 2016) }\end{array}$ \\
\hline $\begin{array}{l}\text { Nährstoffbilanz (Feld-Stall): } 60 \mathrm{~kg} \text { N/ha } \\
\text { (über drei Jahre gemittelt) (Umweltbun- } \\
\text { desamt 2015a, S. 85), in der Novellie- } \\
\text { rung der DüV wird die Anwendung der } \\
\text { Hoftorbilanz gefordert, um die „Nähr- } \\
\text { stoffsituation und Nährstoffeffizienz des } \\
\text { Gesamtbetriebes valide ab(zu)bilden“ } \\
\text { (Taube et al. 2015) }\end{array}$ & $\begin{array}{l}\text { - Nein: Flächenbilanzüberschüsse 2010: } 68 \text { kg } \\
\text { N/ha (BMU und BMELV 2012, S. 43) } \\
\text { - Schleswig-Holstein: je nach Modell } 51 \text { bis } \\
80 \mathrm{~kg} \text { N/ha und Jahr (Taube et al. 2015) }\end{array}$ \\
\hline
\end{tabular}

In Europa verursacht der übermäßige Eintrag reaktiver Stickstoffverbindungen in die Umwelt externe Kosten von 70-320 Mrd. Euro (entsprechend 0,5-3 \% des europäischen Bruttoinlandproduktes). Dagegen beträgt der durch den Stickstoffeinsatz erhöhte Wert der produzierten Agrarprodukte lediglich 45-180 Mrd. Euro (Sutton et al. 2011). Zur Minderung der Stickstoffproblematik sieht die Naturschutzoffensive 2020 des Bundesumweltministerium daher die Erarbeitung einer umfassenden Stickstoffstrategie vor (BMUB 2015b).

\subsubsection{Phosphat}

Phosphat wird mit der organischen oder mineralischen Düngung ausgebracht. Dabei schreibt die Düngeverordnung einen maximalen betrieblichen Nährstoffüberschuss pro Hektar von $20 \mathrm{~kg}$ im Mittel der letzten 6 Jahre vor (Tab. 3.7). Bei Düngungen von mehr als $30 \mathrm{~kg} / \mathrm{ha}$ und Jahr (mineralisch und/oder organisch) muss für jeden Schlag ab der Größe von 1 ha eine repräsentative Bodenuntersuchung vorliegen, die am Tag der Ausbringung nicht älter als 6 Jahre sein darf. Die Zielsetzungen für den Eintrag von Phosphor konnten in den letzten Jahren erreicht werden. Dennoch sieht die Novellierung der Düngeverordnung eine Senkung des maximalen Bilanzwertes von 20 auf $10 \mathrm{~kg} / \mathrm{ha}$ und Jahr vor. Die Folge der neuen Regelung wird aber wahrscheinlich sein, dass zum Beispiel die landwirtschaftlichen Betriebe Schleswig-Holsteins in ca. $66 \%$ der Landkreise einem Anpassungsdruck hinsichtlich der zu reduzierenden Phosphorbilanzen ausgesetzt sind (Taube et al. 2015). 
Tab. 3.7 Zielsetzung und Zielerreichung bezüglich der Phosphor-Einträge

\begin{tabular}{|c|c|}
\hline Zielsetzung für die Landwirtschaft & Zielerreichung \\
\hline $\begin{array}{l}\text { Phosphatbilanz: } 20 \mathrm{~kg} \text { P2O5/; in der Novellie- } \\
\text { rung der DüV wird ein Bilanzwert von max. } \\
10 \mathrm{~kg} \text { P/ha und Jahr ab dem Jahr } 2023 \text { gefordert } \\
\text { sowie eine Absenkung der maximalen Phosphat- } \\
\text { zufuhr auf hoch und sehr hoch versorgten Böden } \\
\text { auf die Höhe der Abfuhr im Dreijahresmittel = } \\
\text { Saldo von } 0 \mathrm{~kg} \text { P2O5/ha (BMELV 2015) }\end{array}$ & $\begin{array}{l}\text { - Phosphor-Hoftorbilanz seit } \\
\text { 1980er-Jahren rückläufig, } \\
\text { - Flächenbilanz: 1,4 kg P/ha LF } \\
\text { (Lütke-Entrup und Schneider 2003) } \\
\text { - Hoftorbilanz 2009: } 8 \mathrm{~kg} \text { P/ha*a } \\
\text { (Umweltbundesamt 2010b) } \\
\text { - Abnahme der Konzentrationen an den } \\
\text { Messstellen } \\
\text { - Schleswig-Holstein: Flächenbilanz- } \\
\text { werte zwischen -2 und +26 kg } \mathrm{P}_{2} \mathrm{O}_{5} / \\
\text { ha*a (Taube et al. 2015) }\end{array}$ \\
\hline
\end{tabular}

Der Verband deutscher landwirtschaftlicher Untersuchungs- und Forschungsanstalten befürwortet zur Reduktion der Austräge über Erosion und Sickerwasser sowie der dadurch verursachten negativen Umweltwirkungen eine Senkung der Richtwerte der Gehaltsklassen für Phosphor (VDLUFA 2015).

Phosphatdünger können Verunreinigungen mit Uran enthalten. Dieses reichert sich ebenfalls im Boden an. So gelangen jährlich 0,1 bis $0,7 \mathrm{~kg}$ Uran/ha auf Acker und Grünland (KBU 2012). Auf Ackerflächen wurde eine mittlere Anreicherung von $0,15 \mathrm{mg}$ Uran/ $\mathrm{kg}$ Trockensubstanz des Bodens nachgewiesen (Dienemann und Utermann 2012).

\subsubsection{Pflanzenschutzmittel}

Seit 2005 hat der Absatz von Pflanzenschutzmitteln (PSM) leicht zugenommen (BVL 2015). Dabei entspricht der Absatz jedoch nicht unbedingt der ausgebrachten Menge, da Mittel bei günstigen Preisen auch auf Vorrat gekauft werden (Umweltbundesamt 2011a). Im Jahr 2014 wurden in Deutschland 106.155 t PSM bzw. 34.515 t PSM-Wirkstoffe (ohne inerte Gase) verkauft (BVL 2015). Daraus lässt sich ein durchschnittlicher Einsatz von 8,8 kg PSM bzw. 2,8 kg PSM-Wirkstoffen auf jedem Hektar Anbaufläche errechnen (Frische et al. 2016). Bei der Diskussion um die Beschränkung der eingesetzten Mittel muss bedacht werden, dass die Mengenreduktion bei einer Verbesserung der Wirksamkeit nicht unbedingt die negativen Folgen für die Umwelt vermindert (Gay et al. 2004). So erlaubt die Höhe des Mitteleinsatzes keine Aussagen über die Umweltwirkungen, solange die Ökotoxizität der Mittel nicht berücksichtigt wird.

Unbeabsichtigte Wirkungen auf Natur und Umwelt werden durch Abdrift, Erosion, Verdunstung, Austrag über Dränagen sowie übermäßigen und unsachgemäßen Pflanzenschutzmitteleinsatz verursacht. So gelangen etwa ein Promille (30 t) der Aufwandmenge über Abschwemmung, Abdrift, Drainagen und Hofabläufe in Oberflächengewässer Deutschlands (Gay et al. 2004). Bei der Abdrift sind Werte der Frühjahrsspritzung von deutlich über $10 \%$ in Obstkulturen 
und unter $1 \%$ in Getreide- und Gemüsekulturen möglich (LfU - Bayrisches Landesamt für Umwelt 2008). In benachbarten Flächen konnten bis zu $10 \%$ der in den behandelten Kulturpflanzen nachgewiesenen Konzentration enthalten sein (LfU - Bayrisches Landesamt für Umwelt 2008). Die Naturschutzoffensive 2020 formuliert daher das Ziel einer angemessenen Berücksichtigung der Auswirkungen auf die biologische Vielfalt bei der Zulassung von Pflanzenschutzmitteln (BMUB 2015b).

Die Anwendung von Pflanzenschutzmitteln wird in der EU-Verordnung 2009/128/ EG geregelt und in Deutschland im Pflanzenschutzgesetz sowie in nationalen Verordnungen umgesetzt. Das Ordnungsrecht fordert die Vorlage eines Sachkundenachweises, der ab 2015 Voraussetzung für den Kauf von Pflanzenschutzmitteln ist, und den Einsatz entsprechend geprüfter Geräte für die Ausbringung (z. B. abdriftarme Düsen). Darüber hinaus müssen Anwendungsgebiete und -bestimmungen des jeweiligen Pflanzenschutzmittels und - im Rahmen der Auflagenbindung der EU (Cross Compliance) - die Vorgaben zur guten fachlichen Praxis eingehalten werden. Über die Anwendungen muss Buch geführt werden.

\subsubsection{Schwermetalle}

Schwermetalle können in landwirtschaftlichen Produktionsmitteln wie Futterzusätzen, Dünge- und Pflanzenschutzmitteln und besonders Klärschlämmen enthalten sein. Der Einsatz von Klärschlamm und die darin enthaltenen Schwermetalle sind mittlerweile in einer eigenen Verordnung geregelt (Klärschlammverordnung). Etwa $30 \%$ des anfallenden Klärschlamms werden in der Landwirtschaft für die Düngung von 2,1\% der landwirtschaftlichen Nutzfläche eingesetzt (BMU 2012). Allerdings hat die zu entsorgende Klärschlammmenge durch das Verfahren der Schlammfaulung (anaerobe Behandlung) zwischen 1998 und 2009 kontinuierlich abgenommen (Umweltbundesamt 2012b). Obgleich auch die Schwermetallgehalte rückläufig sind, ist Klärschlamm weiterhin der Nährstoffträger mit der höchsten Schadstoffbelastung. Bodenvorsorgewerte für die Belastung mit Schwermetallen sind in der Bundes-Bodenschutz- und Altlastenverordnung definiert. Schwermetalle werden im Boden fest gebunden und sind nur wenig auswaschungsgefährdet. In landwirtschaftlichen Böden kann es jedoch zu Anreicherungen kommen, die über Erosion und Drainageabfluss verfrachtet werden können (Fuchs et al. 2010). Diese Pfade sind mittlerweile für 20 bis $40 \%$ der Schwermetalleinträge in Oberflächengewässer verantwortlich (Umweltbundesamt 2011a).

\subsubsection{Kupfer}

Kupfer wird in der Landwirtschaft als Düngemittel (gezielt oder als Nebenbestandteil) und für den Schutz der Pflanzen gegen Pilzkrankheiten eingesetzt. Dabei kann es zu Anreicherungen im Boden mit negativen Auswirkungen auf die Bodenorganismen 
kommen. Die EU-Kommission nahm Kupfer im Jahre 2009 in den Anhang I der Richtlinie über das Inverkehrbringen von Pflanzenschutzmitteln (91/414/EWG) auf. Damit war die Auflage verbunden, dass die Mitgliedsländer bis Ende 2016 Maßnahmen zur Reduzierung der Anwendung ergreifen. In Deutschland kamen Behörden und Verbände zu der Übereinkunft, sich um die Verringerung der jährliche Kupfermenge bei der Anwendung kupferhaltiger Pflanzenschutzmittel auf $3 \mathrm{~kg} / \mathrm{ha}$ (bei Hopfen $4 \mathrm{~kg} / \mathrm{ha}$ ) zu bemühen. Im ökologischen Landbau existieren Grenzwerte für den Einsatz von Klärschlämmen und kupferbasierten Pflanzenschutzmitteln. Weitere Grenzwerte existieren nicht.

\subsubsection{Arzneimittel}

Insbesondere Antibiotika, die in der Tierhaltung (aber auch in der Humanmedizin) angewendet werden, gelangen mit den Abwässern von Kläranlagen (Humanarzneimittelwirkstoffe) und organischen Düngern (Tierarzneimittelwirkstoffe) in die Umwelt (Umweltbundesamt 2014a). Mittlerweile werden Arzneimittelrückstände nahezu flächendeckend und ganzjährig in Fließgewässern sowie Bodenund Grundwasserproben gefunden (Umweltbundesamt 2014a). Dabei ist seit 2006 der Einsatz von Antibiotika als Wachstumsbeschleuniger für die Tiermast in der EU verboten. Antibiotika dürfen nur zur Behandlung erkrankter Tiere und nicht zur prophylaktischen Behandlung verwendet werden (Tab. 3.8). Darüber hinaus dürfen Antibiotika nur von einem Tierarzt oder einer Apotheke auf Verschreibung abgeben werden. Die ersten Daten zum Antibiotikaeinsatz in der Tierhaltung wurden 2011 erhoben. Damals wurden ca. 1700 t Antibiotika abgegeben. 2014 lag der Absatz bei 1232 t. Seit 2014 ist das Antibiotika-Minimierungskonzept in Kraft, welches die Reduzierung des Antibiotikaeinsatzes in der Tierhaltung und die Erhebung entsprechender Daten vorsieht. Hierfür wurde ein Antibiotika-Monitoring eingeführt. Ab bestimmten Bestandsgrößen sind entsprechende Meldungen über den Antibiotikaeinsatz zu machen.

Tab. 3.8 Zielsetzung und Zielerreichung bezüglich des Antibiotika-Einsatzes

\begin{tabular}{l|l}
\hline Zielsetzung für die Landwirtschaft & Zielerreichung \\
\hline - $\begin{array}{l}\text { Einsatz von Tierarzneimitteln: keine } \\
\text { quantitativen Zielwerte }\end{array}$ & $\begin{array}{l}\text { 2014 wurden in der Tiermedizin 214 t (ca. 15\%) } \\
\text { weniger Antibiotika abgegeben als im Vorjahr; } \\
\text { das sind rund 468 t (ca. 27 \%) weniger gegenüber } \\
\text { der ersten Erfassung 2011 (BMEL 2015c) }\end{array}$ \\
$\begin{array}{l}\text { - Betriebe, die mehr Arzneimittel } \\
\text { anwenden als Vergleichsbetriebe, } \\
\text { müssen Maßnahmen ergreifen, um } \\
\text { diesen Einsatz zu reduzieren }\end{array}$ & $\begin{array}{l}\text { 98 } \% \text { der antibiotischen Wirkstoffe werden für } \\
\text { die Behandlung von Schweinen und Geflügel } \\
\text { eingesetzt (SRU 2007) }\end{array}$ \\
$\begin{array}{l}\text { Arzneimittel dürfen nur zur Behand- } \\
\text { lung kranker Tiere eingesetzt } \\
\text { werden } \\
\text { Antibiotika dürfen nur von einem } \\
\text { Tierarzt oder einer Apotheke auf } \\
\text { Verschreibung abgegeben werden }\end{array}$ & \\
\hline
\end{tabular}




\subsubsection{Flächennutzung}

Der heutige Reichtum Mitteleuropas an Arten und Lebensräumen ist ganz wesentlich das Ergebnis der Schaffung von Offenlandschaften durch Ackerbau und Tierhaltung. Änderungen der Flächennutzung können jedoch zu einer Verringerung von geeigneten Habitaten führen und andere schädliche Wirkungen auf die natürliche Umwelt haben. Im Folgenden werden kurz die Formen der Flächennutzung dargestellt, deren Entwicklung in der jüngeren Vergangenheit mit negativen Folgen für die Naturressourcen verbunden war.

\subsubsection{Grünlandverlust}

Artenreiches Grünland gehört aus Sicht des Naturschutzes zu den bedeutendsten Lebensräumen Deutschlands (NABU 2012) und trägt entscheidend zur ästhetischen Qualität von Landschaften bei (siehe Abschn. 3.1.4). Mehr als die Hälfte des vorhandenen Grünlands werden als besonders wichtig für den Naturhaushalt (Klima-, Boden-, Gewässer-, Biodiversitätsschutz) angesehen (Röder et al. 2015). Regional kann aber der Grünlandanteil mit einem besonders hohen Naturwert auch deutlich geringer sein. Aufgrund der zunehmenden Nutzung auch in FFH-Gebieten (NABU 2012) ist ein Verlust der Lebensraumqualität des Grünlands zu beobachten. Die Intensivierung erfolgt durch häufigere und frühere Schnitte sowie durch höhere Düngegaben. Der Anteil des intensiv genutzten Grünlands lag im Jahre 2000 bei $75 \%$ (Dierschke et al. 2002) und nimmt weiter zu (Bundesamt für Naturschutz 2014).

In der Vergangenheit war die Dauergrünlandfläche in Deutschland rückläufig (Nitsch, Osterburg et al. 2009; Umweltbundesamt 2011a; BMEL 2015g). An einigen Gunststandorten lässt sich inzwischen allerdings eine leichte Zunahme der Dauergrünlandflächen feststellen (DAFA 2015; Statistisches Bundesamt 2016). Dennoch ging bundesweit der Anteil des Dauergrünlands zwischen 2003 und 2012 um ca. 5 \% zurück (Bundesamt für Naturschutz 2014). Dies liegt sowohl am Umbruch von ackerfähigem Grünland als auch an Nutzungsaufgabe und Wiederbewaldung. Bei der räumlichen Verteilung von Grünlandverlusten gibt es starke regionale Unterschiede. Besonders hohe Verluste sind in Schleswig-Holstein, der Westhälfte Niedersachsens und im Süden Bayerns zu verzeichnen (Bundesamt für Naturschutz 2014; Schmidt et al. 2014). Die regionalen Unterschiede sind offenbar auch durch den Ausbau der Bioenergieerzeugung zu erklären (Bundesamt für Naturschutz 2014). So werden etwa $50 \%$ der umgebrochenen Grünlandflächen für den Anbau von Mais genutzt (Nitsch et al. 2010), dessen Erzeugung wegen der durch das EEG garantierten hohen Einspeisevergütung wirtschaftlich vorteilhaft war (Osterburg et al. 2009; Bundesamt für Naturschutz 2014).

Erhalt und Schutz von Grünland sind in mehreren Gesetzen und Richtlinien geregelt. So sind vor allem artenreiche Grünlandbestände, die eine herausragende Bedeutung als Biotope haben, durch $\S 30$ des Bundesnaturschutzgesetzes geschützt. 
Einen besonderen Schutzstatus genießen ebenfalls Grünländer auf erosionsgefährdeten Hängen, in Überschwemmungsgebieten, auf Standorten mit hohem Grundwasserstand und auf Moorstandorten ( $\$ 5$ des Bundesnaturschutzgesetzes). Ergänzend zu den gesetzlichen Regelungen sieht zum Beispiel die Biodiversitätsstrategie den Schutz von hochwertigem Grünland ebenfalls vor. Darüber hinaus ermöglichen die FFH-Richtlinie und die Vogelschutzrichtlinie den strengen Schutz der genannten Grünlandstandorte. Ein strenges Umwandlungsverbot gilt jedoch nur in FFH-Gebieten. Der Erhalt von Dauergrünland ist in den Greening-Auflagen der Ersten Säule festgeschrieben. Demnach darf der Grünlandanteil in den einzelnen Bundesländern nur um maximal $5 \%$ gegenüber 2012 abnehmen. Es besteht eine Genehmigungspflicht für die Umwandlung von Grünland und die Erlaubnis darf nur erteilt werden, wenn an anderer Stelle wieder Grünland angelegt wird. Allerdings wurde die maximal erlaubte Grünlandumwandlung in einigen Bundesländern schon während der vorherigen Förderperiode erreicht (Bundesamt für Naturschutz 2014). Eine Antrags- und Genehmigungspflicht für Grünlandumwandlung bzw. ein grundsätzliches Grünland-Erhaltungsgebot ist in sechs Bundesländern und zwei Stadtstaaten erlassen (Bundesamt für Naturschutz 2014). Grünland von Ökobetrieben und Betrieben, die keine Direktzahlungen beziehen, unterliegt ab 2017 nicht mehr dem Umbruchverbot (Bundesamt für Naturschutz 2014). Es lässt sich somit zusammenfassen, dass es „trotz (...) ordnungsrechtlichen Vorgaben (...) selbst in gesetzlich geschützten Gebieten zu einem Grünlandverlust (kommt), da zum Teil die entsprechenden rechtssicheren Kulissen, also amtlich ausgewiesene Naturräume, fehlen" (Röder et al. 2015). In der Naturschutzoffensive 2020 wird daher u. a. eine Grünland-Initiative mit der Extensivierung intensiv genutzter Moore gefordert (BMUB 2015b) (Tab. 3.9).

\subsubsection{Beseitigung, Zersplitterung, Verkleinerung von Lebensräumen}

In den letzten Jahrzehnten führten Intensivierung und Mechanisierung der Landwirtschaft zu einer Vergrößerung der Bewirtschaftungseinheiten durch die Zusammenlegung von einzelnen kleinen Schlägen. Dies dezimierte charakteristische Ökosysteme der landwirtschaftlichen Nutzflächen und die damit verbundene strukturelle Vielfalt (Plieninger und Bieling 2013; Haber 2014; Leuschner et al. 2014). Natürliche und halb-natürliche Habitate (z. B. Hecken, Feldraine, Saumstreifen) wurden beseitigt und in die Acker- oder Grünlandnutzung überführt. Durch

Tab. 3.9 Zielsetzung und Zielerreichung bezüglich des Erhalts von Grünland

\begin{tabular}{|c|c|}
\hline $\begin{array}{l}\text { Zielsetzung für die } \\
\text { Landwirtschaft }\end{array}$ & Zielerreichung \\
\hline $\begin{array}{l}\text { Cross Compli- } \\
\text { ance: Grünland- } \\
\text { erhalt }\end{array}$ & $\begin{array}{l}\text { - Grünland-Verlust: von } 2003 \text { bis } 2012 \text { ca. } 5 \text { \% Verlust des Dauergrün- } \\
\text { landanteils (Bundesamt für Naturschutz 2014), Grünland bedeckt } \\
\text { noch } 30 \% \text { der landw. Nutzfläche (Nitsch et al. 2009) bzw. } \\
\text { 4,6 Mio. ha (BMEL 2012) }\end{array}$ \\
\hline
\end{tabular}


den Eintrag von Nährstoffen und Pflanzenschutzmitteln wird darüber hinaus die Qualität der halb-natürlichen Habitate beeinträchtigt. Hier konzentrieren wir uns insbesondere auf Flächennutzungsänderungen, die Größe von Nutzungseinheiten und die Zersplitterung von Lebensräumen (siehe Abschn. 3.2.1 für stoffliche Einträge). Kriterien sind die Bereitstellung ökologischer Vorrangflächen, der Anteil und die Entwicklung von Cross-Compliance-relevanten Landschaftselementen und HNV-Flächen, die Schlaggrößen sowie die Landschaftszerschneidung.

Seit der Agrarreform 2013/14 sind Landwirte, die mehr als 15 ha bewirtschaften, im Rahmen der Greening-Auflagen dazu verpflichtet, $5 \%$ der Ackerfläche als ökologische Vorrangfläche (ÖVF) bereitzustellen, sofern sie die Greening-Prämie beziehen. Allerdings ist auch eine Nutzung der Flächen für den Anbau von Kurzumtriebsplantagen, Leguminosen und Zwischenfrüchten möglich. Auf solchen Flächen ist teilweise sogar der Einsatz von Pflanzenschutz- und Düngemitteln erlaubt. Im Jahre 2015 wurden 57 \% der in diesem Rahmen beantragten Flächen als produzierende ÖVF beantragt (BMEL 2015f.). Dabei erfolgte die Bereitstellung der Flächen meist nach ökonomischen und nicht nach ökologischen ${ }^{10}$ Kriterien. Wichtige Gesichtspunkte waren z. B. der räumliche Zusammenhang (Plieninger et al. 2012; Heinrich et al. 2013) oder das entsprechende Management der Flächen (Matzdorf 2011; Oppermann et al. 2012). Der ökologische Mehrwert, den diese Flächen erbringen, ist folglich gering (Oppermann et al. 2012; NABU 2013; Isermeyer et al. 2014). Er dürfte sich in erster Linie auf den Schutz vor Erosion und Nitratauswaschungen sowie auf den Humusaufbau beschränken. Auch die Effekte auf die Flächenkonkurrenz (Heißenhuber et al. 2015) und den Pachtmarkt werden als unerheblich erachtet, da kaum zusätzliche Flächen ausgewiesen werden. Vor allem in strukturreichen und von Grünland geprägten Landschaften mit extensiver Bewirtschaftung gibt es ausreichend Flächen, die als ÖVF gemeldet werden können (Isermeyer et al. 2014).

Für Landschaftselemente, die innerhalb der landwirtschaftlichen Fläche liegen oder an diese angrenzen und eine maximale Größe nicht überschreiten, werden seit der Reform von 2003 Direktzahlungen gewährt. Der Anteil dieser Elemente an der landwirtschaftlichen Fläche wurde 2009 mit 0,3 bis 0,4 \% angegeben, wobei sie meist Cross Compliance relevant sind, d. h. sie unterliegen einem Beseitigungsverbot (Nitsch et al. 2009). Wegen fehlender Daten sind Angaben zur Entwicklung des Anteils und Bestandes an Landschaftselementen für die Zeit vor 2005 nicht möglich; es ist jedoch davon auszugehen, dass es zur Beseitigung von Landschaftselementen vor deren Registrierung kam (Nitsch et al. 2009).

High-Nature-Value (HNV) Farmland bezeichnet Landwirtschaftsflächen mit hohem Naturwert (Oppermann et al. 2013). Als solche gelten extensiv genutzte, artenreiche Grünland-, Acker-, Streuobst- und Weinbergflächen sowie Brachen

\footnotetext{
${ }^{10}$ In der Schweiz erfolgt eine zusätzliche Honorierung in Abhängigkeit der Qualität und Lage ökologischer Ausgleichsflächen (Birrer et al. 2010).
} 
und Landschaftselemente, wie z. B. Hecken, Raine, Feldgehölze und Kleingewässer (BMUB 2015a). Im Jahr 2013 betrug der Anteil der HNV-Flächen mit äußerst hohem Naturwert 2,2\%, mit sehr hohem Naturwert 4,3\% und mit mäßig hohem Naturwert 5,3\% der landwirtschaftlichen Nutzfläche. Im Vergleich zu dem vorhergehenden Berichtsjahr 2009 bedeutet dies eine Verschlechterung des Gesamtindikatorwerts von $13,1 \%$ auf 11,8 \%. Somit hatte sich dieser Indikator noch weiter von dem bis $2015 \mathrm{zu}$ erreichenden Zielwert von $19 \%$ Anteil entfernt. Es zeigten sich allerdings große Unterschiede in der räumlichen Verteilung (Oppermann et al. 2013). Einige Mittelgebirgsregionen und Regionen des Nordostdeutschen Tieflands hatten HNV-Anteile von über $15 \%$, während viele Regionen nicht einmal den vom BNatSchG geforderten Mindestanteil von $10 \%$ erreichten (Oppermann et al. 2013).

In Deutschland sind nur noch 23,2 \% der Landesfläche als unzerschnittene verkehrsarme Räume zu bewerten (BMUB 2015a). Die Nationale Biodiversitätsstrategie sieht jedoch einen Wert von 25,4 \% als Zielwert vor, ohne einen Zeitpunkt für die Erreichung dieses Ziels zu nennen. Der Grad der Zerschneidung wirkt sich negativ auf die biologische Vielfalt und das Landschaftsbild aus, beeinträchtigt aber auch durch Bodenversiegelung Boden und Wasser. Die Landwirtschaft trägt im Rahmen von Flurbereinigungsverfahren durch Wegebau zur Landschaftszerschneidung bei (BMELV 2013b) (Tab. 3.10).

Tab. 3.10 Zielsetzung und Zielerreichung bezüglich der Beseitigung, Zersplitterung und Verkleinerung von Lebensräumen

\begin{tabular}{|c|c|}
\hline Zielsetzung & Zielerreichung \\
\hline $\begin{array}{l}\text { Bereitstellung von } 5 \% \text { ökologi- } \\
\text { scher Vorrangfläche; ökologische } \\
\text { Wertigkeit abhängig von Art und } \\
\text { Nutzung der Flächen }\end{array}$ & $\begin{array}{l}\text { - Ja: jedoch wenige positive Effekte auf die } \\
\text { Ressourcen des Naturschutzes }\end{array}$ \\
\hline - Erhalt von Landschaftselementen & $\begin{array}{l}\text { Keine Zielwerte über Anteil, Fläche und ökologi- } \\
\text { sche Qualität definiert } \\
\text { - Landschaftselemente auf 0,3 bis 0,4 \% der } \\
\text { landwirtschaftlichen Fläche, meist Cross } \\
\text { Compliance-relevante Landschaftselemente } \\
\text { (Nitsch et al. 2009) } \\
\text { - Keine Aussage zum Erhalt aufgrund fehlender } \\
\text { Daten }\end{array}$ \\
\hline $\begin{array}{l}\text { High-Nature-Value: Anteil von } \\
\text { Flächen mit hohem Naturschutz- } \\
\text { wert an der Landwirtschaftsfläche, } \\
\text { Zielwert: } 19 \%(2015)\end{array}$ & $\begin{array}{l}\text { High-Nature-Value: } 11,8 \% \text { (2013), davon 2,2 \% } \\
\text { mit äußerst hohem und 4,3\% der Flächen mit } \\
\text { hohem Naturschutzwert; HNV zu einem Drittel LE, } \\
\text { zu zwei Dritteln artenreiches Grünland, Äcker, } \\
\text { Brachen und Weinberge (Bundesamt für Natur- } \\
\text { schutz 2014) }\end{array}$ \\
\hline - Schlaggröße & $\begin{array}{l}\text { - Kein Zielwert definiert } \\
\text { - Zunahme der Schlaggröße in der Vergangenheit }\end{array}$ \\
\hline $\begin{array}{l}\text { - Zerschneidung: } 25,4 \% \text { Flächenan- } \\
\text { teil der unzerschnittenen verkehrs- } \\
\text { armen Räume mit einer Flächen- } \\
\text { größe von mindestens } 100 \mathrm{~km}^{2}\end{array}$ & - Nein: erreichter Wert ist 23,2 \% (2010). \\
\hline
\end{tabular}




\subsubsection{Aufgabe traditioneller, extensiver Nutzungsformen bzw. der Nutzung von Standorten mit speziellen Standortbedingungen}

Viele Arten sind besonders an extensive Nutzungsformen und nährstoffarme Standorte angepasst (Abschn. 3.1.2). Mit der Aufgabe dieser Nutzungsformen und Standorte gehen auch die Lebensräume für diese Arten verloren. Streuobstflächen wie auch Hutewälder und viele FFH-Lebensraumtypen sind auf extensive Nutzung und Beweidung angewiesen (Bundesamt für Naturschutz 2014). Die Entwicklung des Flächenumfangs von Streuobstflächen und extensiv genutzter Grünlandstandorte (Beweidung, Heunutzung) kann also Hinweise auf die Aufgabe traditioneller, extensiver Nutzungsformen geben. Gleiches gilt für den Umfang der Waldfläche, da brachfallende Flächen mit der Zeit verwalden. Streuobstflächen erstreckten sich 1950 auf rund 1,5 Mio. ha, während 1990 nur noch 300.000 ha registriert wurden (BUND ohne Datum). So nahmen etwa die Streuobstflächen Nordrhein-Westfalens in den letzten vier Jahrzehnten um $74 \%$ ab (Umweltbundesamt 2011a). Die deutsche Waldfläche wuchs dagegen in den vergangenen Jahren um 1 Mio. ha.

Ungünstige Standortbedingungen, wie z. B. Hangneigung und flachgründige Böden, bedingen häufig eine unrentable Produktion, da der Ertrag gering, aber z. B. der arbeitswirtschaftliche Aufwand hoch ist. Solche Gebiete werden als Grenzertragsstandorte bezeichnet. Die Aufrechterhaltung der Bewirtschaftung ist in diesen „landwirtschaftlichen Rückzugsgebieten“ nicht gesichert (Heißenhuber et al. 2004). Allerdings hat die hohe Flächennachfrage in der jüngsten Vergangenheit auch dazu geführt, dass die Bedeutung des Brachfallens abgenommen hat (Bundesamt für Naturschutz 2014). Statistische Daten über Grenzertragsstandorte liegen leider nicht vor. Es wird jedoch davon ausgegangen, dass es sich um ca. 4,87 Mio. ha handelt (Landwirtschaftskammern 2010). Für die Aktivierung von Zahlungsansprüchen der Direktzahlungen wird die Durchführung einer Mindesttätigkeit auf solchen Flächen verlangt: sie müssen mindestens einmal pro Jahr gemäht werden und das Mähgut muss abgefahren oder gehäckselt auf der Fläche verteilt werden (Tab. 3.11).

Tab. 3.11 Zielsetzung und Zielerreichung bezüglich der Aufgabe traditioneller und extensiver Nutzungsformen

\begin{tabular}{|c|c|}
\hline Zielsetzungen & Zielerreichung \\
\hline $\begin{array}{l}\text { - Traditionelle Nutzungsfor- } \\
\text { men, z. B. Streuobstwie- } \\
\text { sen, extensives Grünland }\end{array}$ & $\begin{array}{l}\text { Nein: Verlust an Streuobstwiesen (Umweltbundesamt } \\
\text { 2011a), Anteil intensiven Grünlands } 75 \% \text { (2000) nimmt zu } \\
\text { (Dierschke et al. 2002; Bundesamt für Naturschutz 2014) }\end{array}$ \\
\hline $\begin{array}{l}\text { Aufrechterhaltung der } \\
\text { Bewirtschaftung auf } \\
\text { Grenzertragsstandorten }\end{array}$ & $\begin{array}{l}\text { - Nein: Aufrechterhaltung der Landwirtschaft in „landwirt- } \\
\text { schaftlichen Rückzugsgebieten“ nicht gesichert (Heißenhu- } \\
\text { ber et al. 2004), aber durch gestiegene Flächennachfrage ist } \\
\text { die Aufgabe der Nutzung nicht mehr so von Bedeutung } \\
\text { (Bundesamt für Naturschutz 2014) bzw. die Nachfrage nach } \\
\text { Agrarprodukten und Gewährung von Direktzahlung hat auch } \\
\text { wieder zur Inkulturnahme von Grenzertragsstandorten } \\
\text { geführt (Lefebvre et al. 2012), Umfang der Grenzertrags- } \\
\text { standorte ca. 4,87 Mio. ha (Landwirtschaftskammern 2010) }\end{array}$ \\
\hline $\begin{array}{l}\text { - Vermeidung von } \\
\text { Sukzession }\end{array}$ & $\begin{array}{l}\text { - Nein: Ausdehnung der Waldfläche (2011: 11,1 Mio. ha, } \\
\text { Zunahme um } 1 \text { Mio. ha in den letzten } 40 \text { Jahren) }\end{array}$ \\
\hline
\end{tabular}




\subsubsection{Landwirtschaftliche Nutzung von Moorstandorten}

Moorstandorte können einen wesentlichen Beitrag zum Schutz von Biodiversität, Landschaft, Wasser und insbesondere Klima leisten. In Deutschland sind rund $4 \%$ der Landesfläche mit Mooren bedeckt (Bundesamt für Naturschutz 2016c). Etwa $90 \%$ der Moorfläche werden derzeit land- oder forstwirtschaftlich genutzt - davon $50 \%$ als Grünland, 25-30\% als Acker und $13 \%$ als Forst. Etwa $8 \%$ der landwirtschaftlichen Nutzfläche befinden sich auf Mooren (Drösler et al. 2011). Nur ca. ein Zwölftel der Moorflächen werden als naturnah eingestuft (Schäfer 2009). Intakte, Torf akkumulierende Hochmoore sind in der Bundesrepublik auf $1 \%$ ihrer ehemaligen Ausdehnung zurückgedrängt worden (Ellenberg und Leuschner 2009; Joosten 2012). Für die landwirtschaftliche Nutzung wird der Grundwasserspiegel durch Drainierung abgesenkt. In der Folge wird der in den Böden gespeicherte organische Kohlenstoff durch den Kontakt mit Sauerstoff mineralisiert. Hierdurch werden zum einen Treibhausgase freigesetzt (siehe auch Abschn. 3.1.3), zum anderen wird der Moorkörper abgebaut und vermindert. Die landwirtschaftliche Moornutzung ist als die größte einzelne Quelle von Treibhausgasen im Sektor Landwirtschaft anzusehen (Wegener et al. 2006; Drösler et al. 2011; Joosten et al. 2016). Durch die Absenkung des Grundwassers verlieren darüber hinaus viele auf Feuchtflächen angewiesene Arten - wie z. B. die dort brütenden Vogelarten Kiebitz und Uferschnepfe - ihren Lebensraum (Bundesamt für Naturschutz 2014). Auch der regionale Wasserhaushalt wird beeinflusst. Durch den Eintrag von Drainageflüssigkeiten in die Gewässer können weitere Belastungen entstehen (Fuchs et al. 2010, S. 13 f.). Zudem kommt es auch auf Moorstandorten zu weiteren Grünlandumwandlungen, durch welche die negativen Wirkungen der Moornutzung noch verstärkt werden. Zwischen 2005 und 2007 wurden 6000 ha Grünland auf Moorstandorten umgebrochen (Nitsch et al. 2010).

Ein Ziel der Nationalen Strategie zur biologischen Vielfalt war es, bis 2010 natürlich wachsende Hochmoore zu sichern und eine natürliche Entwicklung zu ermöglichen (BMUB 2007). Darüber hinaus sollten alle Bundesländer bis zu diesem Jahr Moorentwicklungskonzepte erstellen und bis 2015 umsetzen. Besonders in den moorreichen Bundesländern wurden solche Konzepte bereits erstellt (Bundesamt für Naturschutz 2016b), über die Zielerreichung gibt es jedoch noch keine bundesweiten Angaben (Tab. 3.12).

Tab. 3.12 Zielsetzung und Zielerreichung bezüglich der landwirtschaftlichen Moornutzung

\begin{tabular}{|c|c|}
\hline Zielsetzung & Zielerreichung \\
\hline $\begin{array}{l}\text { - Schutz und natürliche Entwicklung der Moore: } \\
\text { bestehende, natürlich wachsende Hochmoore bis } 2010 \\
\text { sichern, Erarbeitung von Moorentwicklungskonzepten } \\
\text { (BMUB 2007) }\end{array}$ & $\begin{array}{l}\text { Schutz der Moore: keine } \\
\text { Angaben zur Zielerreichung }\end{array}$ \\
\hline
\end{tabular}




\subsubsection{Fruchtfolgegestaltung}

Gezielte und vielfältige Fruchtfolgen können eine Reihe von positiven Wirkungen auf die Ressourcen des Naturschutzes haben. Beispielsweise kann die Gefahr der Nährstoffauswaschung durch die Integration gut deckender Pflanzenbestände in die Fruchtfolge und durch den Anbau von Zwischen- und Untersaaten reduziert werden (SRU 1985; Nitsch et al. 2008). Mittlerweile beschränkt sich die Fruchtfolge allerdings nur noch auf wenige Arten. So werden auf $85 \%$ der landwirtschaftlichen Nutzfläche gerade mal neun Fruchtglieder angebaut und auch das Sortenspektrum wird immer geringer; die „Generosion“ ist im Getreideanbau besonders hoch (Wissenschaftlicher Beirat für Biodiversität und Genetische Ressourcen beim BMELV 2007). Auch ist eine zunehmende regionale Spezialisierung zu beobachten, wobei Weizen in Ackerbauregionen und Mais überwiegend in Viehhaltungsregionen angebaut wird (Wissenschaftlicher Beirat für Agrarpolitik beim BMEL 2015). Die regionale Spezialisierung ermöglicht kurzfristig zwar eine Senkung der Produktionskosten, lässt mittelfristig aber negative Effekte erwarten (Fruchtfolge-Krankheiten, Resistenzproblematik, erhöhter Einsatz von Pflanzenschutzmitteln; (Schmidt et al. 2003; Wissenschaftlicher Beirat für Agrarpolitik beim BMEL 2015). Der Anbau von Hackfrüchten verstärkt beispielsweise den Humusabbau (mit allen negativen Folgen; (KBU 2008) und erhöht auch die Gefahr der Bodenverdichtung (SRU 1985). Letzteres hängt auch mit den ungünstigen Bodenbedingungen zusammen, die an den späten Ernteterminen häufig als Folge von Niederschlägen herrschen. Zudem steigt die Erosionsgefahr aufgrund von jahreszeitlich später und unvollständiger Bodenbedeckung. Zu Problemen kommt es, wenn der Boden seine Filter- und Pufferfunktionen nicht ausreichend erfüllen kann. So nahm die Anbaufläche für die Hackfrucht Mais zwischen 2001 und 2011 um $80 \%$ zu (Heißenhuber et al. 2015). In einigen Regionen beträgt der Anteil der Maisfläche bereits mehr als 50 \% (Deutsches Maiskomitee e.V. 2016). Ein Großteil des Maises wird - gefördert durch das Erneuerbare-Energien-Gesetz ${ }^{11}$ - für die Erzeugung von Biogas verwendet. Mittlerweile dienen ca. $12 \%$ der landwirtschaftlichen Nutzfläche Deutschlands dem Anbau von Energiepflanzen. Im Jahr 2000 betrug der Anteil lediglich 2,5\% (Bundesamt für Naturschutz 2014). Der Nationale Biomasseaktionsplan der Bundesregierung sieht einen Korridor von 2,5 bis 4,0 Mio. ha (20-30 \% der Ackerfläche) für den Anbau von nachwachsenden Rohstoffen vor. Neben der Einengung der Fruchtfolgen ist im Zusammenhang mit nachwachsenden Rohstoffen auch zu berücksichtigen, dass wegen der ökonomischen Vorteilhaftigkeit des Anbaus und der damit verbundenen Möglichkeit, höhere Pachten zu zahlen, insbesondere extensivere Verfahren der Flächennutzung an

\footnotetext{
${ }^{11}$ Das EEG hat durch feste Einspeisevergütungen über einen Zeitraum von 20 Jahren dazu geführt, dass Landwirte nicht mehr auf den volatilen Märkten für Agrarprodukte agieren mussten, sondern mit festen Einnahmen über einen langen Zeitraum planen konnten.
} 
Tab. 3.13 Zielsetzung und Zielerreichung bezüglich der Fruchtfolgegestaltung

\begin{tabular}{|c|c|}
\hline Zielsetzung & Zielerreichung \\
\hline $\begin{array}{l}\text { - Anbaudiversifizierung im Rahmen des } \\
\text { Greenings } \\
\text { - Einzelbetriebliche Vorgaben, keine auf die } \\
\text { Landschaft bezogenen Zielwerte }\end{array}$ & $\begin{array}{l}\text { - Keine Daten vorhanden (z. B. über } \\
\text { Verstöße gegen die Auflage) }\end{array}$ \\
\hline
\end{tabular}

Konkurrenzfähigkeit verloren haben (Bundesamt für Naturschutz 2014). In der Naturschutzoffensive 2020 wurde daher formuliert, dass es zu keinen weiteren Flächenausweitungen für den Anbau von Biomasse für die Energie-Erzeugung kommen soll, wenn die Anbaugrenze von 2,5 Mio. ha erreicht ist.

Die mit den betrieblichen Direktzahlungen verknüpften Greening-Auflagen sehen seit 2014 vor, dass Betriebe Maßnahmen der Anbaudiversifizierung umsetzen. Ausgenommen sind Betriebe mit weniger als 10 ha Ackerfläche. Betriebe zwischen 10 und 30 ha müssen mindestens zwei Kulturen anbauen, wobei die Hauptkultur auf maximal $75 \%$ der Fläche angebaut werden darf. Werden mehr als 30 ha Ackerfläche bewirtschaftet, müssen mindesten drei Kulturen angebaut werden, und der Anteil der Hauptkultur muss auf maximal $75 \%$ und der der beiden Hauptkulturen auf maximal $95 \%$ der Ackerfläche beschränkt sein. Die Maßnahmen bringen jedoch nur geringe Vorteile für den Schutz der Ressourcen des Naturschutzes. So wurde prognostiziert, dass nur $38 \%$ der Betriebe Anpassungen vornehmen müssten und die Maisfläche um 4,7\% reduziert werden müsste (Forstner et al. 2012). Große homogene Bewirtschaftungseinheiten sind weiterhin möglich, da auch der Anbau verschiedener Getreidesorten die Auflage erfüllt (Plieninger et al. 2012) (Tab. 3.13).

\subsubsection{Bodenbearbeitung und mechanische Belastungen}

Die landwirtschaftliche Flächenbewirtschaftung umfasst eine Reihe von Arbeitsgängen mit unterschiedlichen Maschinen. Der Maschineneinsatz macht die Bewirtschaftung einiger Standorte überhaupt erst möglich bzw. erleichtert die Arbeiten und reduziert die Arbeitszeiten sowie die Kosten. Die Arbeitsgänge und die Verwendung von Maschinen beeinflussen immer die Naturressourcen. Doch insbesondere die wendende Bodenbearbeitung und die Zunahme der Maschinengewichte haben zunehmend negative Wirkungen. So führt die wendende Bodenbearbeitung zu einem verstärkten Abbau von Humus (vgl. Abschn. 3.1.1). Hohe Maschinengewichte können insbesondere bei häufigem Befahren und bei ungünstigen Witterungsbedingungen zu Bodenschadverdichtungen führen (Heißenhuber et al. 2015). All dies verursacht Schäden am Bodengefüge mit negativen Folgen für die Wasser- und Luftführung in den Bodenporen sowie für die Bodenlebewesen (siehe Abschn. 3.1.1). Auch die Bodenscherung, bei der das Bodengefüge seitlich verschoben wird, verändert die Porenführung (Heißenhuber et al. 2015). Allerdings haben größere und damit meist schwerere Maschinen in der Regel eine höhere Schlagkraft und ermöglichen so eine Reduzierung der Überfahrten. Zudem ist eine 
Reduzierung der negativen Wirkungen erhöhter Maschinengewichte durch angepasste Reifeninnendrücke, die Reduzierung von Schlupf und die Anlage von Fruchtfolgen, die die Notwendigkeit der Befahrung zu ungünstigen Zeitpunkten reduzieren, möglich.

Heutzutage wird der überwiegende Anteil der Ackerflächen gepflügt (Statistisches Bundesamt 2011). Dadurch kann es zum Abbau von Humus und zu verstärkter Erosion kommen (siehe Abschn. 3.1.1). Durch Vertiefungen der Pflugfurche in der zweiten Hälfte des 20. Jahrhunderts konnte aber auch der durchwurzelte Bodenhorizont vergrößert und damit mehr Humus angereichert werden (Taube 2016). Allerdings haben auch nicht-wendende Verfahren negative Wirkungen, da dabei z. B. bedingt durch Direktsaatverfahren - häufig mehr Herbizide eingesetzt werden (van Capelle et al. 2012; Lal 2013). Vorgaben zum Einsatz der wendenden Bodenbearbeitung sowie zu Maschinengewichten bestehen nicht.

\subsubsection{Zusammenfassung}

Die Landwirtschaft wirkt in vielfältiger Art und Weise auf die Naturressourcen ein. Dies kann deren Zustand negativ aber auch positiv beeinflussen. Negativ wirken sowohl die Intensivierung der Flächennutzung als auch der Rückzug der landwirtschaftlichen Produktion aus Grenzertragsstandorten. Die zunehmende räumliche Konzentration der Tierhaltung verursacht in diesen Regionen besonders hohe Belastungen durch stoffliche Einträge. Bei der Bewertung der Wirkungen von Flächennutzungen muss insbesondere zwischen intensiv genutzten Regionen und Grenzertragsstandorten unterschieden werden. Zielsetzungen für den Schutz der Naturressourcen werden überwiegend nicht eingehalten bzw. Aussagen über die Zielerreichung können wegen der mangelnden Datenlage nicht getroffen werden. Zudem sind einige Zielsetzungen nicht ausreichend operationalisiert oder nicht ambitioniert genug, um die intendierten Ziele zu erreichen. Für wichtige Teilbereiche gibt es bislang keine Zielwerte.

\subsection{Schlussfolgerungen}

Dieser Überblick dient nicht der erschöpfenden Behandlung der Wechselwirkungen zwischen Landwirtschaft und Naturressourcen, sondern er soll vor allem den diesbezüglichen Sachstand als Ausgangspunkt für die Entwicklung einer zukunftsfähigen Agrarpolitik umreißen. Trotz aller Kürze wird deutlich, dass die Politik in Gesetzen und Strategien eine ganze Reihe von Zielwerten und Vorgaben für den Zustand der Ressourcen und für die Belastungen durch die Landwirtschaft formuliert hat. Diese werden allerdings vielfach nicht erreicht. Das liegt zum einen an der fehlenden Operationalisierung, d. h. es existieren keine Vorgaben für die Art der Landbewirtschaftung, mit der die gesetzten Ziele erreicht werden sollen (Regelungsdefizit). 
Zum andern sind die Kontroll- und Sanktionsmechanismen oft unzureichend (Vollzugsdefizit).

Einige Vorgaben für die Landbewirtschaftung sind nicht ambitioniert genug, um die mit ihrer Umsetzung intendierten Zustände der Naturressourcen zu erreichen. Als Beispiel sei die Bereitstellung ökologischer Vorrangflächen genannt. Da diese Flächen in erster Linie unter ökonomischen und nicht unter ökologischen Gesichtspunkten ausgewählt werden, sind allenfalls geringe positive Effekte auf die biologische Vielfalt zu erwarten. Die Gestaltung der Anreizmechanismen führt außerdem dazu, dass die Betriebe nicht hinreichend motiviert werden, Schutzmaßnahmen über die geforderten Auflagen hinaus umzusetzen.

Das folgende Kapitel wendet sich daher den agrarpolitische Rahmenbedingungen zu. Es untersucht die politische Logik der Entwicklung der Gemeinsamen Agrarpolitik der Europäischen Union sowie des bestehenden rechtlichen Rahmens. Dem werden neuere Governance-Ansätze gegenübergestellt, die zu einer besseren Integration der Belange des Natur- und Umweltschutzes in die Agrarpolitik und die landwirtschaftliche Praxis beitragen könnten.

Open Access Dieses Kapitel wird unter der Creative Commons Namensnennung 4.0 International Lizenz (http://creativecommons.org/licenses/by/4.0/deed.de) veröffentlicht, welche die Nutzung, Vervielfältigung, Bearbeitung, Verbreitung und Wiedergabe in jeglichem Medium und Format erlaubt, sofern Sie den/die ursprünglichen Autor(en) und die Quelle ordnungsgemäß nennen, einen Link zur Creative Commons Lizenz beifügen und angeben, ob Änderungen vorgenommen wurden.

Die in diesem Kapitel enthaltenen Bilder und sonstiges Drittmaterial unterliegen ebenfalls der genannten Creative Commons Lizenz, sofern sich aus der Abbildungslegende nichts anderes ergibt. Sofern das betreffende Material nicht unter der genannten Creative Commons Lizenz steht und die betreffende Handlung nicht nach gesetzlichen Vorschriften erlaubt ist, ist für die oben aufgeführten Weiterverwendungen des Materials die Einwilligung des jeweiligen Rechteinhabers einzuholen.

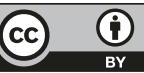




\section{Kapitel 4 \\ Agrarpolitische Rahmenbedingungen}

Das vorangegangene Kap. 3 hat dargestellt, dass viele Natur- und Umweltressourcen in keinem guten Zustand sind und sich oft weiter negativ entwickeln. Weiterhin wurde dargestellt, wie die vorherrschenden Methoden der Landbewirtschaftung wesentlich zu den beschriebenen Problemlagen beitragen. Diese Zustandsbeschreibung lässt einen deutlichen politischen Handlungsbedarf zum Schutz der Naturressourcen erkennen. Dieser wird derzeit offenbar unzureichend erfüllt. Aus Sicht des Natur- und Umweltschutzes ist daher ein Politikversagen zu diagnostizieren. In diesem Kapitel werden daher die agrarpolitischen Rahmenbedingungen analysiert. Dabei wird die Entwicklungslogik der Gemeinsamen Agrarpolitik (GAP) der EU und des ordnungsrechtlichen Rahmens in den Mittelpunkt gestellt, um die Ursachen des Politikversagens zu erkennen. Anschließend werden neue, innovative Ansätze für eine bessere Integration von Belangen des Natur- und Umweltschutzes in die landwirtschaftliche Praxis und in die Agrarpolitik vorgestellt. Insgesamt ist es das Ziel dieses Kapitels, mögliche Ansatzpunkte für Reformen zur Verbesserung des Natur- und Umweltschutzes zu identifizieren.

\subsection{Die politische Logik der GAP: Zwischen Einkommenspolitik und Umweltintegration}

Die politikwissenschaftliche Diskussion der Agrarpolitik kreist um drei Grundfragen: Warum konnte sich die Agrarpolitik als relativ autonomes und abgeschottetes Politikfeld außerhalb der allgemeinen Wirtschaftspolitik etablieren und stabilisieren? Wie verändert sich die Agrarpolitik unter dem Einfluss zunehmender Wechselwirkungen mit anderen Politikbereichen, insbesondere Naturschutz-, Umwelt- und Handelspolitik? Wie können Wandel und Stabilität der Agrarpolitik erklärt werden? 
Wesentliche Arbeiten zur Agrarpolitik sind aus dem Ansatz des historischen Institutionalismus entstanden, der die Bedeutung von Policy-Ideen und Policy-Paradigmen betont. Hier wurde herausgearbeitet, dass die Gemeinsame Agrarpolitik (GAP) der Europäischen Union ihre historischen Wurzeln in den Preisstützungsmaßnahmen hat, die seit Ende des 19. Jahrhunderts in Deutschland und anderen westlichen Ländern zum Schutz der Produzenten in einigen landwirtschaftlichen Teilsektoren ergriffen wurden und mit der besonderen nationalen Bedeutung der landwirtschaftlichen Produktion sowie den Besonderheiten agrarischer Märkte begründet wurden (Tracy 1989).

Als in den 1950er-Jahren die Verhandlungen über die Bildung der Europäischen Wirtschaftsgemeinschaft aufgenommen wurden, bestand einerseits Einigkeit darüber, dass die Märkte für agrarische Produkte Teil des gemeinsamen Marktes sein sollten (Knudsen 2009; Fietz 2012). Andererseits hatten alle sechs Gründungsmitglieder komplizierte Systeme zur Einkommensstützung der landwirtschaftlichen Bevölkerung etabliert, die als Äquivalent der sozialstaatlichen Sicherungssysteme für den landwirtschaftlichen Sektor fungierten und daher treffend als „landwirtschaftlicher Wohlfahrtsstaat" (,agricultural welfare state") bezeichnet worden sind (Sheingate 2003). Im Vertrag zur Gründung der Europäischen Wirtschaftsgemeinschaft (EWG-Vertrag, weitgehend bekannt als Teil der Römischen Verträge) wurde daher in Art. 34 (jetzt Art. 40 AEUV) die Etablierung eines Gemeinsamen Markts für landwirtschaftliche Produkte und in Art. 32 (jetzt Art. 38 AEUV) die Errichtung einer Gemeinsamen Agrarpolitik (GAP) festgeschrieben, welche im Nachgang weitgehend von den Regeln des sonstigen gemeinsamen Markts abgekoppelt wurde (Oppermann et al. 2011: § 24, Rn. 11). Aus dem historischen Kontext wird dabei deutlich, dass mit der GAP im Wesentlichen eine Einkommenspolitik für die landwirtschaftliche Bevölkerung gemeint ist (Knudsen 2009). Dementsprechend wird auch in Art. 39 Abs. 1 des AEUV die Erhöhung des Einkommens für die „landwirtschaftliche Bevölkerung“ als eines der fünf Ziele der GAP festgeschrieben. ${ }^{1}$ In Deutschland ist zudem im Landwirtschaftsgesetz ein Einkommensziel für die landwirtschaftliche Bevölkerung festgeschrieben, wenn auch mit unbestimmten Rechtsbegriffen.

Das Policy Design, mit dem die Gemeinsame Agrarpolitik die im EWG-Vertrag von 1957 festgeschriebenen Ziele verfolgt, hat sich grundlegend verändert. Im Zuge der Etablierung der GAP wurden in den 1960er-Jahren sektoral unterschiedliche Systeme zur Sicherung eines relativ hohen Preisniveaus etabliert, die von Marktinterventionen zur Preisstützung bis Mengenregulierung durch Quoten reichen. Investitionsförderungsprogramme zur „Modernisierung“ der Landwirtschaft wurden unter dem Einfluss des höchst kontrovers aufgenommenen Mansholt-Plans erst 1972 in die Gemeinsame Agrarpolitik eingefügt (Grant 1997; Knudsen 2009). Die Kombination von Produktionsanreizen durch Marktstützung und Effizienzsteigerung durch ,Modernisierung ' führte seit den späten

\footnotetext{
${ }^{1}$ Entgegen einem häufig vorgebrachten Argument hat der EuGH in der Rs. 71/74, Frubo, Slg. 1975, 563, Rn. 25/26 das Einkommensziel gegenüber den anderen Zielen der GAP jedoch nicht rechtlich priorisiert (Purnhagen und Schebesta 2017).
} 
1970er-Jahren zu chronischer Überproduktion, Preisverfall, Kritik im Rahmen der Welthandelsordnung sowie wiederholten Haushaltskrisen in der Europäischen Gemeinschaft (Grant 1997). Zugleich wurde massive Kritik an den negativen Umweltauswirkungen der GAP laut (SRU 1985), auf die seit 1989 mit der Einführung von freiwilligen Agrarumweltprogrammen reagiert wurde (Buller et al. 2000). Die sogenannte MacSharry-Reform von 1992 brachte einen radikalen Umbau der Instrumente der GAP mit der Einführung einer teilweise von der Produktion entkoppelten, flächenbezogenen Direktzahlung, die als Kompensation für erhebliche Senkungen der Garantiepreise begründet wurde. Durch diese Reform sollte die Einkommensstützung für die Landwirtschaft in Einklang mit den WTO-Regeln gebracht werden, die dann 1994 vereinbart wurden. Die Direktzahlungen waren verpflichtend an die Auflage gebunden, dass $15 \%$ der Flächen aus der Produktion genommen werden.

Mit der 1999 beschlossenen Agenda 2000 wurden die Programme zur Unterstützung von Modernisierungsinvestitionen, Agrarumweltmaßnahmen und regionaler ländlicher Entwicklung (u. a. LEADER) zu einer ländlichen Entwicklungspolitik, der jetzigen „Zweiten Säule“ zusammengefasst. Die sogenannte Fischler-Reform von 2003 brachte die weitgehende Entkopplung der Direktzahlungen von der Produktion und deren verpflichtende Verknüpfung mit der Einhaltung einer Reihe von ordnungsrechtlichen Auflagen (Cross Compliance, in der Agenda 2000 eine wenig populäre Option für die Mitgliedstaaten). Die jüngste GAP-Reform von 2013 verknüpft die Zahlung von $30 \%$ der Direktzahlungen mit Auflagen zur Bereitstellung von $5 \%$ der betrieblichen Fläche als ökologische Vorrangfläche, zur Fruchtfolgegestaltung und dem Dauergrünlanderhalt (sog. Greening der Ersten Säule).

Das Ausmaß, in dem sich die vielfach als dysfunktional kritisierte GAP verändert hat, ist umstritten und abhängig von der konzeptionellen Rahmung der Diagnose. Einerseits zeigt die historisch-institutionelle Analyse der langfristigen Entwicklung der GAP eine hohe Pfadabhängigkeit auf der Ebene der Policy-Ideen und Politik-Instrumente (Kay 2003; Greer 2005). Andererseits kommt es in größeren Abständen zu erheblichem Politikwandel in der Form der Einführung neuer Politik-Instrumente - insbesondere die Agrarumweltprogramme, die Einheitliche Betriebsprämie, die Cross-Compliance- und die Greening-Verpflichtungen - deren Ausgestaltung und Begründung in späteren Reformen dann oft erheblich variiert werden.

Die Diskussion zum Wandel der Agrarpolitik hat sich in der Diagnose eines kumulativen Paradigmenwechsels, der über eine Abfolge von kleineren Reformschritten zu einem grundlegenden Wandel der Agrarpolitik führte, verdichtet (Coleman et al. 1997). Gegen diesen Befund gibt es zwei Einwände, einerseits gegen die Diagnose einer Verschiebung der ideellen Grundlagen der GAP, andererseits gegen die Diagnose einer substanziellen Veränderung der politischen Logik der GAP.

Bezüglich des ersten Einwands sehen andere Autoren weniger eine Verschiebung des Politik-Paradigmas der GAP als eine Konkurrenz zwischen vier divergierenden Paradigmen bzw. Leitbildern (Josling 2002; Moyer und Josling 2002): 
- Agrarexzeptionalismus basiert auf der Annahme, dass die Landwirtschaft zu wesentlichen öffentlichen Politikzielen beiträgt, dass freie Marktmechanismen aber nicht der beste Weg sind, eine effiziente und produktive Landwirtschaft sicherzustellen (Coleman et al. 1997; Grant 1997; Skogstad 1998). Dieses Paradigma hat eine produktivistische Orientierung und geht davon aus, dass die Landwirtschaft staatliche Unterstützung braucht.

- Das marktliberale Paradigma geht davon aus, dass die Landwirtschaft mit anderen Sektoren und Ländern auf dem Markt um knappe Ressourcen konkurrieren kann und daher weder Außenprotektion noch sektorale Sonderregelungen benötigt. Übergangsregelungen - etwa zeitlich begrenzte Direktzahlungen können aus sozialen oder politischen Erwägungen Härten bei der Marktliberalisierung abfedern.

- Das Paradigma einer multifunktionellen Landwirtschaft geht davon aus, dass die Landwirte sowohl marktfähige Produkte wie auch öffentliche Güter (Landschaften, Ökosystemleistungen) als Koppelprodukte erzeugen. Da für letztere freie Marktmechanismen nicht funktionieren, sind staatliche Marktinterventionen und Entgeltmechanismen notwendig. Das Multifunktionalitätsparadigma teilt mit dem Paradigma der staatsgestützten Landwirtschaft die exzeptionalistische Annahme, dass Landwirtschaft besondere Bedingungen aufweist, diese werden jedoch vor allem im Bereich der Allokationsprobleme für öffentliche Güter gesehen und weniger in den Einkommensproblemen der Landwirtschaft. Auch wird die produktivistische Orientierung nicht geteilt.

- Das Paradigma einer globalisierten Landwirtschaft lässt sich als Weiterentwicklung des marktliberalen Paradigmas unter Bedingungen global integrierter Wertschöpfungsketten verstehen. Angesichts der Proliferation von staatlichen und privaten Standards steht hier die regulative Harmonisierung von Sozial- und Umweltstandards im Vordergrund (Josling 2002). Dabei können innerhalb des Paradigmas unterschiedliche Akzente gesetzt werden, beispielsweise wenn entweder die Aufrechterhaltung und Verbreitung hoher Standards oder der Ruf nach evidenzbasieren Regeln und Maßnahmen im Vordergrund steht. Der Streit um den Stellenwert des Vorsorgeprinzips etwa bei der Regulierung von Nahrungsmitteln mit gentechnisch veränderten Bestandteilen ist hier ein gutes Beispiel.

Eine umfassende Befragung von agrarpolitischen Akteuren in Deutschland hat die Präsenz aller vier Paradigmen aufgezeigt, deren Elemente jedoch von den Akteuren oft kombiniert werden (Feindt 2008). Im Ergebnis besteht im Politikfeld einerseits keine ideelle Homogenität oder Hegemonie mehr, sondern die agrarpolitischen Akteure bewegen sich in einem vielfältigen Diskursfeld, in dem je nach Situation unterschiedliche Aspekte in den Vordergrund gestellt werden können. In eine ähnliche Richtung weisen die Befunde von Alons und Zwaan (2015). Sie zeigen in einer Analyse der agrarpolitischen Diskurse in Brüssel sowie in Deutschland, Frankreich und Großbritannien, dass die sehr vielfältigen Begründungen für die GAP von den Regierungsvertretern strategisch genutzt werden, um einerseits in gemeinsamen Verhandlungen zu einer Übereinstimmung zu kommen (koordinative Funktion des Diskurses), und andererseits die GAP den jeweiligen heimischen Adressaten zu 
vermitteln (kommunikative Funktion des Diskurses). Diese Studien zeigen, dass es vielfältig überlappende Diskurse zur europäischen Agrarpolitik gibt, die sich jeweils zu Recht auf bestimmte Aspekte des ideellen Rahmens der GAP beziehen können und dabei andere Aspekte strategisch ausblenden.

Weitere Studien zeigen, dass insbesondere die Europäische Kommission neue Diskurse durchaus aufnimmt, um Reformen der GAP bzw. die Fortsetzung der erheblichen Zahlungen zu begründen, ohne dass dies den Kern der Politik wesentlich berührt. Garzon (2006) zeichnet nach, wie das Konzept der Multifunktionalität in den 1990er-Jahren zunächst adoptiert wurde, um im Rahmen der GATT/WTOVerhandlungen die Stützungszahlungen für das ,,europäische Modell der Landwirtschaft“ zu verteidigen. Nachdem die Direktzahlungen durch die Fischler-Reform von der Produktion entkoppelt und damit in die Green Box der nur gering produktionsverzerrenden Maßnahmen verschoben worden waren, verschwand das Multifunktionalitätskonzept jedoch schnell aus dem Zentrum der Argumentation. Candel et al. (2014) zeigen, dass unter dem Einfluss der Nahrungsmittelpreiskrise ab 2008 die Kommission der Ernährungssicherheit einen prominenten Stellenwert in der Begründung der GAP zuwies, ohne dass dies jedoch zu wesentlichen politischen Initiativen geführt habe - abgesehen von einem Fonds mit einer Milliarde Euro sowie einem neuen Mechanismus zur Überprüfung von Politik auf ihre Konsistenz mit dem Ziel der Ernährungssicherheit. Candel et al. (2014) zeigen weiterhin, dass das Thema der Ernährungssicherheit in alle verschiedenen agrarpolitischen Paradigmen eingebaut wurde. Im Ergebnis bestehen bei oberflächlichem Konsens sehr unterschiedliche Annahmen über die Ursachen und Problemlösungen - etwa ob eine massive Produktionssteigerung oder eine langfristige Sicherung der Biodiversität Priorität haben soll. Insgesamt ergibt sich damit das Bild einer Diskursdiversifizierung mit oft marginalem Einfluss auf den Policy-Kern der GAP.

Der zweite Einwand gegen die These eines kumulativen Paradigmenwechsels stellt in Frage, ob die ideelle Verschiebung beziehungsweise Pluralisierung der Agrarpolitik die GAP in der Substanz verändert hat. Auf der einen Seite hat infolge von AUKM, Cross Compliance und Greening durchaus eine Integration von Umweltbelangen in die GAP stattgefunden, diese ist jedoch nicht allein und nicht vorrangig umweltpolitischen Motiven zu verdanken (Feindt 2010). Daugbjerg und Swinbank (2016) argumentieren, dass die Reformschritte der GAP seit 1992 einen Fall von Policy-Layering darstellen, bei dem um einen stabilen Policy-Kern Zusatzaspekte angelagert werden, um den Kern gegenüber veränderten Herausforderungen im Zeitablauf zu verteidigen und zu legitimieren. Den ,harten Kern“ der GAP stellt demnach die Einkommenspolitik für den landwirtschaftlichen Sektor dar. Der redistributive Charakter der GAP war angesichts der komplizierten Marktmechanismen jedoch wenig sichtbar. Die schrittweise Umstellung der Einkommensstützung von Marktintervention hin zu steuerfinanzierten Direktzahlungen seit 1992 hat die Sichtbarkeit der Einkommenstransfers und damit den Legitimationsbedarf erhöht. Die Addition von Cross Compliance und Modulation sowie später dem Greening dient dazu, gegenüber der Öffentlichkeit und auch gegenüber anderen Politikfeldern, die um knappe Mittel konkurrieren, einen öffentlichen Nutzen der erheblichen öffentlichen Zahlungen darzustellen und die Politik damit argumentativ zu verteidigen 
(vgl. Majone 1989). Die Anforderungen an die Landwirte sind jedoch jeweils so ausgestaltet, dass sie - abgesehen von Dokumentationspflichten - relativ geringe zusätzliche Erbringungskosten erfahren und damit die Einkommenskomponente der Direktzahlungen möglichst weitgehend unangetastet bleibt. Daugbjerg und Swinbank (2016) kommen daher zu dem Ergebnis, dass von einem Paradigmenwechsel in der GAP nicht die Rede sein könne, da es sich im Kern seit Jahrzehnten um Einkommenspolitik für den Sektor handele.

Diese Argumentation bestärkt den Befund einer ausgeprägten Pfadabhängigkeit der Gemeinsamen Agrarpolitik. Diese wird durch verschiedene Faktoren erklärt. Zunächst ging die Europäisierung der Agrarpolitik mit einer starken institutionellen Abschottung der GAP einher, die bis zur jüngsten institutionellen Reform der EU im Wesentlichen zwischen der Generaldirektion Landwirtschaft (DG Agri) der EU-Kommission und dem Rat der Agrarminister bestimmt und auch von den europarechtlichen Rahmenbedingungen materiell entsprechend flankiert wurde (siehe auch Oppermann et al. 2011: § 24, Rn. 11; Purnhagen und Schebesta 2017). Von der institutionellen Reform der EU, welche die GAP in den Bereich der Mitentscheidung des Parlaments verschoben hat, wurde vielfach eine stärkere Öffnung der Beratungen für nicht-agrarische Interessen erwartet. Tatsächlich hat diese institutionelle Reform jedoch eher zu einer Stärkung der agrarischen Interessen über den federführenden Agrarausschuss des EU-Parlaments geführt (Greer und Hind 2012).

Stärker politökonomisch angeregte Untersuchungen zeigen, dass die bestehende Agrarpolitik von den Gruppen, die von ihr in erheblichem Maße profitieren, mit großem Aufwand verteidigt wird. Die agrarpolitischen Netzwerke, die sich in den Mitgliedstaaten sowie erst in der EWG, dann in der EG und schließlich in der EU etablierten und sich wechselseitig mit den institutionellen Strukturen stabilisieren, gelten als eng geknüpft und relativ geschlossen (Jordan et al. 1994; Daugbjerg 1997, 1999). Seit Mitte der 1990er-Jahre wird zwar vielfach ein Verlust des exklusiv agrarischen Charakters der agrarpolitischen Netzwerke beobachtet, was auch als ein Erklärungsfaktor für die Verschiebung der agrarpolitischen Paradigmen vorgeschlagen wird (Coleman et al. 1997). Den Interessenorganisationen der Agrarwirtschaft wurde aber selbst zu Zeiten der Fischler-Reform und unter dem Eindruck der BSE-Krise das höchste Einflusspotenzial zugeschrieben, wie eine eigene Befragung 2003 unter agrarpolitischen Akteuren in Deutschland und in Brüssel ergab (Feindt 2008).

Größere Reformen werden jedoch dadurch erschwert, dass bei den Verhandlungen über den Finanzrahmen der GAP eine Verteilungslogik dominiert, in der die Mitgliedstaaten vor allem auf ihre Nettozahlerposition achten. Dies wirkt insgesamt ebenfalls strukturerhaltend, da neue Instrumente oder Veränderungen des Finanzierungsschlüssels entweder nicht verteilungsneutral sind oder die Verteilungskonsequenzen schwer abzusehen sind (Ackrill 2005; Ackrill und Kay 2006). Dieser Mechanismus steht insbesondere einer Verschiebung von Mitteln aus der zu $100 \%$ EU-finanzierten Ersten Säule in die lediglich ko-finanzierte Zweite Säule entgegen, solange nicht besondere Vereinbarungen für eine solche „Modulation“ getroffen werden. 
Insgesamt ist die GAP also so konstruiert, dass starke wirtschaftliche und gesellschaftliche Gruppen sowie wichtige Kräfte in den Parlamenten und Regierungen der Mitgliedsländer der EU ein erhebliches Interesse an ihrer Fortführung haben ein selbstverstärkender Effekt, der auch als positives Policy-Feedback bezeichnet wird (Pierson 1993; Daugbjerg 2003).

Größere Reformen der GAP kommen nur unter erheblichem Druck aus anderen Politikbereichen, insbesondere der Handels- und Finanzpolitik zustande (Ackrill et al. 2008). Der Reform von 1992 ging der Abbruch der Verhandlungen über die Gründung einer Welthandelsorganisation 1990 in Brüssel voraus. Ohne eine grundlegende Reform der GAP hätte daher ein Scheitern der Uruguay-Runde gedroht (Swinbank 1993, 1999; Swinbank und Tanner 1996). Daneben stand die MacSharry-Reform im Kontext des neuen mittelfristigen Finanzrahmens (19931999), der das Wachstum der Agrarausgaben begrenzte, sowie der bevorstehenden Norderweiterung der EU von 1995. Bei der Diskussion um die Agenda $2000 \mathrm{im}$ Jahr 1999 fehlte ein solch starker Problemdruck und es blieb bei kleineren Reformen. Bei der Fischler-Reform von 2003 wirkte hingegen eine Kombination von handelspolitischem Druck (Doha-Runde des GATT), umwelt- und verbraucherpolitischem Druck im Nachgang der BSE-Krise (Purnhagen 2013a, S. 24-42), und finanzpolitischem Druck im Vorfeld der EU-Osterweiterung um zehn neue Mitglieder mit zum Teil bedeutenden Agrarsektoren und erheblichen Strukturproblemen (Daugbjerg und Swinbank 2007).

Dennoch bleibt erklärungsbedürftig, warum die GAP 2015 sich im Hinblick auf die Instrumente und die Begründung erheblich von der GAP 1990 unterscheidet. Neuere Arbeiten zur Agrarpolitik nehmen verstärkt eine Prozessperspektive auf den Wandel der GAP ein. Daugbjerg (2009) zeigt, dass bei einer Betrachtung über mehrere GAP-Reformrunden sichtbar wird, wie „reaktive Sequenzen“ - zeitlich geordnete Ketten kausal miteinander verknüpfter Ereignisse (Mahoney 2000, S. 526) - substanziellen Politikwandel über Zeit erklären können. Anders als selbst-verstärkende Sequenzen, die eine einmal etablierte Politik oder Institution über lange Zeiträume stabilisieren, sind reaktive Sequenzen durch interaktive und oft transformative Prozesse gekennzeichnet: „Whereas self-reinforcing sequences are characterized by processes of reproduction that reinforce early events, reactive sequences are marked by backlash processes that transform and perhaps reverse early events. In a reactive sequence, early events trigger subsequent development not by reproducing a given pattern, but by setting in motion a chain of tightly linked reactions and counterreactions“ (Daugbjerg 2009, S. 398). Die von MacSharry vorgeschlagenen Direktzahlungen wurden von den Bauernverbänden zunächst scharf abgelehnt, wurden bei den Empfängern dann aber wegen der einkommensstabilisierenden Wirkung durchaus populär. Die Überwachung der Stilllegungsverpflichtungen erforderte jedoch den Aufbau einer neuen und komplexen Bürokratie. Kommissar Fischler reagierte darauf mit dem Vorschlag einer vollständig entkoppelten Direktzahlung für Kleinlandwirte, die 2001 eingeführt wurde und offenbar als Testballon für die Akzeptanz einer von der Produktion entkoppelten Zahlung diente. Anfang 2002 schlug Fischler dann für die osteuropäischen Beitrittsländer einen „,vereinfachten Ansatz“ vor, nämlich eine rein flächenbezogene, produktionsentkoppelte 
Flatrate. Nachdem dieser Ansatz für ein Drittel der Farmer in der künftigen erweiterten EU akzeptiert war, fiel es den widerstrebenden Agrarministern schwer, Fischlers Vorschlag zurückzuweisen, das Prinzip im Midterm-Review auf die gesamte EU auszudehnen (wobei Mitgliedstaaten die Möglichkeit erhielten, weiterhin einen Teil der Direktzahlungen an die Produktion zu koppeln und zwischen einer betriebsindividuell historischen und einer regionalen Flächenprämie zu wählen).

Im Hinblick auf die Verhandlungsprozesse und die Ergebnisse ist die Sequenz der Verhandlungen über den Finanzrahmen und die Politikgestaltung der GAP von erheblicher Bedeutung. Bei der Einrichtung der Marktpolitiken in den 1960er-Jahren war davon ausgegangen worden, dass sich die Marktinterventionen und Exportsubventionen durch die Einnahmen aus den Importabgaben finanzieren lassen würden, die GAP sich also selbst tragen würde. Der GAP wurde daher kein finanzieller Rahmen vorgegeben. Im Ergebnis kam es wiederholt zu massiven Budgetkrisen und einer kontinuierlichen Ausweitung der Finanzmittel für die GAP. Dies ändert sich erst 1988 mit dem ersten mittelfristigen Finanzrahmen, eine Konsequenz der Verabschiedung der Einheitlichen Europäischen Akte von 1987. Hatten sich bis dahin die Agrarminister (oder bei deren Uneinigkeit die Staats- und Regierungschefs) auf agrarpolitische Maßnahmen geeinigt, für die dann eine Finanzierung gefunden werden musste, hat sich seitdem die Reihenfolge der Verhandlungen geändert: Zunächst wird ein finanzieller Rahmen festgelegt, innerhalb dessen sich die GAP zu bewegen hat. Die Einigung über den Finanzrahmen kann dabei mit der Festlegung von politischen Grundlinien einhergehen. So wurde etwa im Vorfeld der GAP-Reform 2013 in der Einigung über den Mehrjährigen Finanzplan festgeschrieben, dass es ein Greening der Ersten Säule geben soll (Swinnen 2015).

Vor dem Hintergrund der erheblichen Pfadabhängigkeit der Agrarpolitik ist es nicht überraschend, dass für den Agrarsektor wesentliche Reformen außerhalb der GAP stattfinden. Hier sind drei Entwicklungslinien zu nennen:

1. Entwicklung eines ordnungsrechtlichen Rahmens, der die naturschutz-, umweltschutz- und verbraucherrechtlich relevanten Auswirkungen landwirtschaftlicher Tätigkeiten reguliert;

2. Bemühungen, die GAP stärker dem allgemeinen Rahmen der Binnenmarktregulierung zu unterwerfen;

3. Eine Reihe von weiteren neue Politiken, die erhebliche Auswirkungen auf den Agrarsektor haben, ohne dass dies das vorrangige Interventionsziel ist, wie etwa das Erneuerbare Energien Gesetz (EEG) mit der Förderung der Biomasse (Delzeit et al. 2012; Herbes et al. 2014). Der Einfluss von anderen Politikfeldern auf die Agrarpolitik wird etwa als Policy-Spillover oder Policy-Stretching diskutiert (Feindt und Flynn 2009).

Hinzu kommt die wachsende Bedeutung von staatlichen und nicht-staatlichen Standards. Erklärungsansätze verweisen insbesondere auf den Einfluss der Globalisierung auf die veränderten Regulationsformen im Agrarbereich (Higgins und Lawrence 2005). Aus Sicht des Natur- und Umweltschutzes sowie von Akteuren, die an regionalen Produkten interessiert sind, wird dabei ein Dilemma deutlich. Standards etwa für den ökologischen Landbau schaffen zwar einerseits Vertrauen in die gekennzeichneten Produkte und unterstützen damit die Etablierung „grüner“ 
Märkte, andererseits führen sie aber auch zu einer Standardisierung von Produkten und Prozessen, die nicht immer im Einklang mit den Akteuren im ,grünen“ Teilsektor steht (Boström und Klintman 2006, 2009).

Insgesamt ergibt sich aus der Forschung das Bild, dass sich die Agrarpolitik in einem langfristigen Wandlungsprozess befindet, der als kumulativer Paradigmenwechsel beschrieben werden kann und bei dem die folgenden Elemente von Bedeutung sind:

- Veränderung des institutionellen Rahmens durch internationale Einbettung (GATT etc.), Mitentscheidungsverfahren für die GAP, ordnungsrechtlicher Rahmen;

- Einführung neuer Politik-Instrumente, z. B. Cross Compliance und Greening, neue Umwelt- und Verbraucherschutzstandards und -labels;

- zunehmende Wechselwirkungen mit anderen Politikfeldern, insbesondere Handels-, Umwelt- und Energiepolitik;

- Veränderung der agrarpolitischen Netzwerke;

- wachsende Bedeutung von Ideen- und Paradigmenstreit in der Agrarpolitik.

Zugleich bestehen erhebliche Barrieren für eine bessere Berücksichtigung des Natur- und Umweltschutzes:

- die fortbestehende Dominanz des Verständnisses der GAP als Einkommenspolitik für den Sektor;

- die Dominanz der Verteilungslogik zwischen den Mitgliedstaaten, die größeren Reformen mit unklaren Verteilungswirkungen entgegen steht;

- die institutionelle Verankerung der GAP bei der DG Agri und den Agrarministerien, die Ziele jenseits der Einkommenslogik, wie Verbraucherschutz, Naturschutz- und Umweltanliegen, eher als Zusatzaspekte denn als Kern der Agrarpolitik verstehen;

- das Fehlen von externem Handlungsdruck, der sich für naturschutz- und umweltpolitische Ziele nutzen lässt (die mögliche Ausnahme bildet hier eine finanzpolitische Drohkulisse in Verbindung mit Zweifeln an der Effektivität und Effizienz der in der GAP eingesetzten Mittel);

- der mit zielgenauen Maßnahmen oft verbundene Bürokratieaufwand, der solche Maßnahmen unpopulär macht.

Im nächsten Abschnitt stellen wir dar, welche Handlungsspielräume und Handlungsansätze sich aus Veränderungen des rechtlichen Rahmens ergeben können.

\subsection{Die politische Logik des rechtlichen Rahmens}

\subsubsection{Der Rahmen des Umweltordnungsrechts}

Ausgehend von der Annahme, dass landwirtschaftliche Tätigkeiten in den seltensten Fällen ausschließlich nur Kernbereiche der Landwirtschaft tangieren, sondern gleichzeitig häufig Tätigkeiten auf anderen Politikfeldern wie dem Umwelt- oder Verbraucherschutz darstellen, sehen sich landwirtschaftliche Tätigkeiten zunehmend 
Regelungen aus diesen Bereichen ausgesetzt. Diese sollen im Folgenden als „Umweltordnungsrecht" bezeichnet werden. Das Umweltordnungsrecht kann in zwei Bereiche aufgeteilt werden: Zum einen enthält das Ordnungsrecht einen agrarrechtlichen „Kernbereich“, der direkt die Agrarwirtschaft regelt und oft nur mittelbar zum Schutz von Ökosystemen beiträgt (Möckel 2016). Hierher gehören das Pflanzenschutzrecht, ${ }^{2}$ das Recht zum ökologischen Landbau, ${ }^{3}$ das Agrarbeihilfenrecht, ${ }^{4}$ sowie das Recht zur Bioenergie. ${ }^{5}$ Zum anderen gibt es Regelungen, die originär aus anderen Bereichen des Binnenmarktes stammen (wie beispielsweise direkt aus dem Umwelt-, klassischen Binnenmarkt- oder Verbraucherrecht) und landwirtschaftliche Tätigkeit „,von den Rändern“ flankieren. Hierzu gehören Regelungen zum Klimawandel, zur Biotechnologie (insbesondere die Regelungen zu den genetisch veränderten Organismen, siehe als Übersicht Purnhagen und Wesseler 2016), zum Schutz der Waldressourcen, ${ }^{6}$ zu Forschung und Innovation, zur Lebensmittelsicherheit, ${ }^{7}$

\footnotetext{
${ }^{2}$ Verordnung (EG) Nr. 1107/2009 des Europäischen Parlaments und des Rates vom 21. Oktober 2009 über das Inverkehrbringen von Pflanzenschutzmitteln und zur Aufhebung der Richtlinien 79/117/EWG und 91/414/EWG des Rates, ABl. L 309 vom 24.11.2009, S. 1-50.

${ }^{3}$ Verordnung (EG) Nr. 834/2007 des Rates vom 28. Juni 2007 über die ökologische/biologische Produktion und die Kennzeichnung von ökologischen/biologischen Erzeugnissen und zur Aufhebung der Verordnung (EWG) Nr. 2092/91, AB1. L 189 vom 20.07.2007, S. 1-23 sowie Verordnung (EG) Nr. 2003/2003 des Europäischen Parlaments und des Rates vom 13. Oktober 2003 über Düngemittel, ABl. L 304 vom 21.11.2013, S. 1-194.

${ }^{4}$ „Cross Compliance“ und „Greening“: Verordnung (EU) Nr. 1306/2013 des Europäischen Parlaments und des Rates vom 17. Dezember 2013 über die Finanzierung, die Verwaltung und das Kontrollsystem der Gemeinsamen Agrarpolitik und zur Aufhebung der Verordnungen (EWG) Nr. 352/78, (EG) Nr. 165/94, (EG) Nr. 2799/98, (EG) Nr. 814/2000, (EG) Nr. 1290/2005 und (EG) Nr. 485/2008 des Rates, ABl. L 347 vom 20.12.2013, S. 549-607; Verordnung (EU) Nr. 1307/2013 des Europäischen Parlaments und des Rates vom 17. Dezember 2013 mit Vorschriften über Direktzahlungen an Inhaber landwirtschaftlicher Betriebe im Rahmen von Stützungsregelungen der Gemeinsamen Agrarpolitik und zur Aufhebung der Verordnung (EG) Nr. 637/2008 des Rates und der Verordnung (EG) Nr. 73/2009 des Rates, ABl. L 347 vom 20.12.2013, S. 608-670; Verordnung (EU) Nr. 702/2014 der Kommission vom 25. Juni 2014 zur Feststellung der Vereinbarkeit bestimmter Arten von Beihilfen im Agrar- und Forstsektor und in ländlichen Gebieten mit dem Binnenmarkt in Anwendung der Artikel 107 und 108 des Vertrags über die Arbeitsweise der Europäischen Union, ABl. L 193 vom 01.07.2014, S. 1-193; Verordnung (EU) Nr. 1408/2013 der Kommission vom 18. Dezember 2013 über die Anwendung der Artikel 107 und 108 des Vertrags über die Arbeitsweise der Europäischen Union auf De-minimis-Beihilfen im Agrarsektor, ABl. 352,vom 24.12.2013, S. 9-17.

${ }^{5}$ Richtlinie 2009/28/EG des Europäischen Parlaments und des Rates vom 23. April 2009 zur Förderung der Nutzung von Energie aus erneuerbaren Quellen und zur Änderung und anschließenden Aufhebung der Richtlinien 2001/77/EG und 2003/30/EG, AB1. L140 vom 05.06.2009, S. 16-62; Richtlinie 2009/30/EG des Europäischen Parlaments und des Rates vom 23. April 2009 zur Änderung der Richtlinie 98/70/EG im Hinblick auf die Spezifikationen für Otto-, Diesel- und Gasölkraftstoffe und die Einführung eines Systems zur Überwachung und Verringerung der Treibhausgasemissionen sowie zur Änderung der Richtlinie 1999/32/EG des Rates im Hinblick auf die Spezifikationen für von Binnenschiffen gebrauchte Kraftstoffe und zur Aufhebung der Richtlinie 93/12/EWG, ABl. L 140 vom 05-06-2009, S. 88-113.

${ }^{6}$ Eine neue EU-Waldstrategie: für Wälder und den forstbasierten Sektor, $\operatorname{COM(2013)~} 659$ final/2.

${ }^{7}$ Siehe insbesondere die Lebensmittelbasisverordnung: Verordnung (EG) Nr. 178/2002 des Europäischen Parlaments und des Rates vom 28. Januar 2002 zur Festlegung der allgemeinen
} 
Tab. 4.1 Die zwei Säulen des Umweltordnungsrechts in der EU

\begin{tabular}{|c|c|}
\hline Agrarrechtlicher Kernbereich & Rahmenrecht \\
\hline $\begin{array}{ll}\text { - } & \text { Agrarbeihilfenrecht } \\
\text { - } & \text { Düngemittelrecht } \\
\text { - } & \text { Beslanzenschutzrecht (agrarrechtliche } \\
\text { - } & \text { Regelungen zu Tiergesundheit und zum } \\
\text { Schutz landwirtschaftlicher Nutztiere } \\
\text { - Recht zum ökologischen Landbau } \\
\text { Recht zur Bioenergie }\end{array}$ & 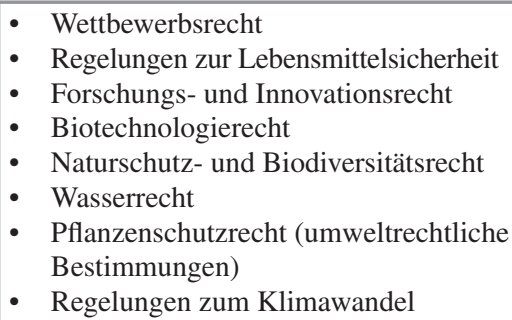 \\
\hline
\end{tabular}

zur Tiergesundheit und zum Tierschutz ${ }^{8}$ sowie dem Pflanzenschutz ${ }^{9}$ und der Biodiversität (Tab. 4.1). ${ }^{10}$

Im Folgenden soll zunächst der agrarrechtliche Kernbereich dargestellt werden, bevor auf das Rahmenrecht eingegangen wird.

\subsubsection{Der agrarrechtliche Kernbereich}

Der agrarrechtliche Kernbereich folgt in seiner Regulierungslogik der europäischen Agrarpolitik, wie sie in den Art. 38-44 AEUV festgeschrieben wurde (zum Inhalt dieser Agrarpolitik und ihrer Ausrichtung auf die Einkommenssicherung für Landwirte siehe Abschn. 4.1). Das heißt vor allem, dass selbst wenn diese Regelungen umweltrechtlichen Charakter haben, diese Regelungen stets auch im Lichte der Verwirklichung der in Art. 38-44 AEUV festgelegten Ziele zu erfolgen hat.

Das Pflanzenschutzrecht soll in erster Linie der Verbreitung von Schadorganismen vorbeugen. Demnach sind nicht-chemischen Methoden wie geeigneten Fruchtfolgen, Kultivierungsverfahren, die Verwendung resistenter Sorten, ausgewogener Düngung und Bewässerung sowie der Förderung wichtiger Nutzorganismen stets der Vorzug vor dem Verwenden von Pestiziden zu geben. Ein Pestizideinsatz ist erst ab der Überschreitung eines wissenschaftlich begründeten Schwellenwertes möglich. Darüber hinaus werden strenge formelle Anforderungen an die Verwendung von Pestiziden wie etwa Aufzeichnung und Meldung der verwendeten Menge, der behandelten Fläche und Kulturpflanzen gestellt. Die Regelungen zum ökologischen Landbau sollen einen europaweiten Mindeststandard für aus ökologischem Landbau erzeugte Produkte sicherstellen. Dabei müssen im Herstellungsverfahren für solche Produkte insbesondere hohe Anforderungen an den Schutz der Fruchtbarkeit

Grundsätze und Anforderungen des Lebensmittelrechts, zur Errichtung der Europäischen Behörde für Lebensmittelsicherheit und zur Festlegung von Verfahren zur Lebensmittelsicherheit, AB1. L 31 vom 01.02.2002, S. 1-24.

${ }^{8}$ Siehe insbesondere die Webseite http://ec.europa.eu/food/animals_en.

${ }^{9}$ Siehe insbesondere die Webseite http://ec.europa.eu/food/plant_en.

${ }^{10}$ Richtlinie 92/43/EWG des Rates vom 21. Mai 1992 zur Erhaltung der natürlichen Lebensräume sowie der wildlebenden Tiere und Pflanzen, ABl. L 206 vom 22.07.1992, S. 7-50. 
der Böden und der Verringerung der Stoffausträge beachtet werden. Insbesondere wird in diesem Zusammenhang die Verwendung chemisch-synthetischer Produktionsmittel reguliert. Das Agrarbeihilferecht, vor allem als Cross Compliance oder Greening bekannt, enthält Mindestanforderungen für Landwirte, um Direktzahlungen zu erhalten. Ähnlich den ordnungsrechtlichen Regeln zur guten fachlichen Praxis beinhalten die Cross-Compliance- und Greening-Anforderungen Mindestanforderungen für Landwirte. Diese Mindestanforderungen im Rahmen der Cross Compliance wiederum verweisen vor allem auf das später darzustellende Rahmenrecht. Die Greening-Anforderungen und die Maßnahmen aus der ersten Säule statuieren betriebsbezogene Pflichten zum Erhalt der Dauergrünlandflächen, der Bereitstellung ökologischer Vorrangflächen sowie zur Vielfalt des Anbaus von Kulturen auf Ackerflächen. Die Überprüfung dieser Vorgaben ist für mindestens $5 \%$ der Empfänger verbindlich. Die Regulierung der Bioenergie soll sicherstellen, dass der Anbau und der Import von Biomasse, aus der Energie gewonnen wird, nachhaltig erfolgen. Insgesamt soll die Nutzung von Bioenergie eine positive Treibhausgasbilanz aufweisen und die biologische Vielfalt nicht gefährden.

Die Umsetzung oder Ausgestaltung dieser Regelungen in nationales Recht ist angesichts der Ausgestaltung der meisten dieser Rechtsakte als Verordnungen mit Maximalharmonisierungscharakter in den meisten Fällen nicht notwendig. Soweit und sofern nur Mindeststandards europarechtlich vorgegeben sind, kann der Gesetzgeber jedoch darüber hinausgehen. Hier kann nicht in Gänze auf das umfangreiche deutsche Ordnungsrecht eingegangen werden. Da es in vielen Bereichen das EU-Recht widerspiegelt, ist dies auch nicht unbedingt angezeigt. Für eine umfangreichere Untersuchung wird insoweit auf Möckel et al. (2014) verwiesen.

\subsubsection{Das Rahmenrecht}

Das Rahmenrecht entspringt einer anderen Regulierungslogik, die jeweils in Bezug auf die entsprechenden Politiken zu verstehen ist. Gemein haben diese, dass sie damit in erster Linie nicht der Verwirklichung der in Art. 38-44 AEUV festgelegten Agrarpolitik dienen, sondern hauptsächlich an ihrem jeweiligen verfolgten Ziel zu messen sind. Da sie dennoch Auswirkungen auf die Agrarwirtschaft haben können, sind Art. 38-44 AEUV jedoch zumindest zu berücksichtigen, vor allem bei den diversen zu treffenden normativen Entscheidungen im Rahmen des Verhältnismäßigkeitsgrundsatzes. Gemäß dem zugrundeliegenden regulatorischen Ziel können die Regelungen grob eingeteilt werden in solche, die das klassische Binnenmarktziel verwirklichen, und solche, die dem Schutz der Umwelt dienen (Tab. 4.2).

Das Wettbewerbsrecht sowie das Forschungs- und Innovationsrecht beziehen sich auf alle Sektoren und finden entsprechend auch auf den Agrarbereich Anwendung. Sektorspezifischer ist das Lebensmittelsicherheitsrecht, das jedoch seiner Genese nach im Wesentlichen als Verbraucherrecht entstanden ist. Als solches ist das Lebensmittelsicherheitsrecht dem Binnenmarktziel des Art. 114 AEUV verpflichtet, der seinerseits auf wirtschaftliche Binnenmarktintegration zugeschnitten ist. Das Lebensmittelsicherheitsrecht bezweckt, innerhalb der gesamten Lieferkette 
Tab. 4.2 Die zwei Säulen des Rahmenrechts in der EU

\begin{tabular}{|c|c|}
\hline Klassisches Binnenmarktrecht & Umweltrecht \\
\hline $\begin{array}{l}\text { - Biotechnologierecht (binnenmarktrechtliche } \\
\text { Bestimmungen) } \\
\text { - Forschungs- und Innovationsrecht } \\
\text { - Regelungen zur Lebensmittelsicherheit } \\
\text { Wettbewerbsrecht }\end{array}$ & $\begin{array}{l}\text { - Naturschutz- und Biodiversitätsrecht } \\
\text { - Wasserrecht } \\
\text { - Pflanzenschutzrecht (umweltrechtliche } \\
\text { Bestimmungen) } \\
\text { - Biotechnologierecht (umweltrechtliche } \\
\text { Bestimmungen) } \\
\text { - Regelungen zum Klimawandel }\end{array}$ \\
\hline
\end{tabular}

sicherzustellen, dass keine unsicheren Lebensmittel auf den Markt gelangen. Da auch die Lebensmittelproduktion unter das Lebensmittelrecht fällt, sind auch Primärproduzenten wie Landwirte daran gebunden und können damit, wenn sie unsichere Lebensmittel herstellen, im Rahmen des Lebensmittelrechts dafür haftbar gemacht werden. Die Haftung ist als solche relativ weit, da als „nicht sicher" nicht nur gesundheitsschädliche Lebensmittel gelten, sondern auch solche, die für den Verzehr für den Menschen ungeeignet sind. Gleichzeitig wird die Haftung teilweise durch ein verschuldensunabhängiges Haftungsrecht flankiert. Das Recht der Biotechnologie statuiert u. a. eine grundsätzliche Koexistenz von genetisch veränderten und genetisch nicht veränderten Organismen. Hierfür ist zum einen eine Zulassungspflicht für alle genetisch veränderten Organismen vorgesehen, die für den Verzehr gedacht sind oder in die Umwelt verbracht werden sollen. Daneben statuiert das Europarecht auch die Notwendigkeit der verschuldensunabhängigen Haftung für eine Vermengung von genetisch veränderten und nicht genetisch veränderten Organismen.

Zum umweltrechtlichen Rahmen gehören das Naturschutz- und Biodiversitätsrecht, das Wasserrecht, Regelungen zum Klimawandel sowie die umweltrechtlichen Bestimmungen im Pflanzenschutzrecht und im Biotechnologierecht. Diese Regelungen betreffen zwar nicht ausschließlich die Landwirtschaft, haben aber zum Teil wichtige Auswirkungen auf die landwirtschaftliche Praxis.

\subsubsection{Die Veränderung des europarechtlichen Rahmens}

Die deutsche Agrarpolitik ist eingebettet in einen rechtlichen Rahmen, der sich mittlerweile nahezu ausschließlich am EU-Recht orientiert. Die Rolle, die das EURecht der Agrarpolitik beimisst, hat sich auch mit der fortschreitenden europäischen Integration verändert. Dies ist im Wesentlichen auf zwei voneinander unabhängige Entwicklungen zurückzuführen, die sich mittlerweile immer mehr annähern: Zum einen reagiert das Recht auf Entwicklungen, die die veränderte politische Rolle der GAP von einem System zur Einkommenssicherung von Landwirten hin zu einem wettbewerbsorientierten Wirtschaftszweig reflektieren (hierzu Abschn. 4.2.2.1). Zum anderen hat der „From farm to table“-Ansatz dazu geführt, dass sich landwirtschaftliche Produktionszweige immer mehr an der Vermeidung von Haftungsrisiken orientieren müssen, die aus verbraucherschützenden Normen resultieren (hierzu Abschn. 4.2.2.2). 


\subsubsection{Einbeziehung der GAP in das allgemeine Beihilfen- und Wettbewerbsrecht und das ,soziale“ Binnenmarktrecht}

Die GAP wurde im Jahr 2007 durch die Einführung der Gemeinsamen Organisation der Agrarmärkte (Verordnung (EG) Nr. 1234/2007) ${ }^{11}$ ergänzt. Damit reagierte die EU auf den mit der Lissabon-Strategie eingeschlagenen Konsolidierungskurs, der auch zum Ziel hat, die EU durch Wettbewerbsorientierung der EU-Politiken international wettbewerbsfähiger zu machen. EU-rechtlich heißt dies, dass die GAP, welche primärrechtlich in der Vergangenheit faktisch weitgehend von den üblichen Wettbewerbsregeln abgekoppelt war, nunmehr vermehrt den allgemeinen Regeln unterstellt werden kann. Das heißt nicht nur, dass die allgemein gültigen Regeln des Wettbewerbsrechts wie die Grundfreiheiten (insbesondere die Freiheit des Warenverkehrs nach Art. 34 AEUV) - wie bei anderen Produktionszweigen auch - regulär Anwendung finden könnten. Mit der Implementierung dieser allgemeinen unionsrechtlichen Wettbewerbsregeln finden auch deren Ausnahmen, d. $h$. der Umweltschutz, der Verbraucherschutz und der Gesundheitsschutz, eine ebensolche Anwendung in der GAP. Dieser „Mehr-Wettbewerb-Ansatz“ hat im Wesentlichen drei Folgen: Er gibt Unternehmen und Mitgliedstaaten mehr Spielraum bei der Preisbildung von landwirtschaftlichen Produkten, er unterwirft mitgliedstaatliche und private Maßnahmen der Kontrolle des Wettbewerbsrechts und der Grundfreiheiten und führt schließlich das ,Soziale“ Binnenmarktrecht (insbesondere Umwelt- und Verbraucherschutz) auch in die GAP ein. Der Beginn eines solchen Schwenks zu einem „Mehr-Wettbewerb-Ansatz“ wurde zuletzt durch das EuGH-Urteil zur Preisbindung bei schottischem Whisky bestätigt.

Die Agrarmärkte waren von Beginn an ein wesentlicher Bestandteil des gemeinsamen Marktes. Zwar wurden sie stets formell auch als Teil des Binnenmarktes gesehen, so dass die Wettbewerbsregeln, vor allem die Grundfreiheiten und das Beihilfenrecht, prinzipiell volle Anwendung auch im Bereich der Agrarwirtschaft finden und fanden (Oppermann et al. 2011: § 24, Rn. 1). Allerdings ist die historische Bedeutung des Agrarsektors für die EU von solch fundamentaler Bedeutung, dass die GAP gem. Art. 38 Abs. 2 AEUV speziellere Regeln für die Grundfreiheiten aufstellen kann, die sodann den allgemeinen Regeln der Grundfreiheiten vorgehen. Während im Zentrum der Grundfreiheiten die Verwirklichung der Freihandelstheorie und die damit verbundenen positiven Effekte für Verbraucherinnen und Verbraucher stehen (Purnhagen 2013b), entwickelte sich die GAP unter dem Schirm der Spezialregelung des Art. 38 Abs. 2 AEUV in der Praxis des EuGH und der Kommission - entgegen dem allgemeinen Grundsatz, dass spezielle Regelungen eng auszulegen sind - zu einem Sonderregime, welches besondere Schutzwirkungen für landwirtschaftliche Produkte beinhaltete (Oppermann et al. 2011: § 24, Rn. 11). Zentrales Regelungsziel dieses Sonderregimes war zum einen die Steigerung der Produktivität und zum anderen die Gewährleistung einer angemessenen Lebenshaltung

\footnotetext{
${ }^{11}$ Aktuell gültig ist die Verordnung (EU) Nr. 1308/2013 des Europäischen Parlaments und des Rates vom 17. Dezember 2013 über eine gemeinsame Marktorganisation für landwirtschaftliche Erzeugnisse und zur Aufhebung der Verordnungen (EWG) Nr. 922/72, (EWG) Nr. 234/79, (EG) Nr. 1037/2001 und (EG) Nr. 1234/2007, AB1. L 347 vom 20.12.2013, S. 671-854.
} 
für Landwirte, ${ }^{12}$ welches sich heute noch in Art. 39 Abs. 1 lit. a) und b) AEUV niederschlägt (Bittner 2012: Art. 39, Rn. 6). Faktisch hat sich dies dahingehend entwickelt, dass traditionell die Einkommenssicherung der Landwirte als wesentliches Ziel der GAP angesehen wurde. ${ }^{13}$ Gemäß Art. 39 AEUV verfolgt die GAP allerdings noch eine Reihe weiterer Ziele, welchen, je nach den Umständen und Bedürfnissen der Zeit, mehr oder weniger Gewicht beigemessen wird (Bittner 2012: Art. 39, Rn. 7). Diese unterschiedlichen Gewichtungen finden allerdings innerhalb der GAP statt, eine Neuausrichtung der GAP im Verhältnis zu den Grundfreiheiten, und damit die Auslegung von Art. 38 Abs. 2 AEUV, wurde bislang nicht thematisiert.

Um diese Ziele zu erreichen, folgte die GAP dem Prinzip der Markteinheit und der Marktordnung. Das Prinzip der Markteinheit galt auf Produktions- und Großhandelsebene, auf der unilateral die Preise im Binnenmarkt abgesprochen wurden. ${ }^{14}$ Das Prinzip der Marktordnung galt auf Verbraucher- und Einzelhandelsebene, auf der Preise für landwirtschaftliche Produkte prinzipiell frei, das heißt nur eingerahmt durch mitgliedstaatliche Regelungen, geformt wurden. ${ }^{15}$ Dieses System hatte und hat noch immer erhebliche Auswirkungen auf den globalen Handel (Trebilcock 2015, S. 110-111): Zum einen hat es dazu geführt, dass billigere Anbieter aus dem EU-Ausland, die die abgesprochenen Preise unterbieten würden, durch Einfuhrzölle und -tarife auf das höhere Binnenpreisniveau gebracht werden mussten (Marktabschottung); und zum anderen mussten die Produktionsüberschüsse, die durch die hohen Garantiepreise ermutigt, aber von Verbrauchern nicht nachgefragt wurden, durch subventionierte Exporte abgesetzt oder durch Intervention vom Markt genommen und vernichtet wurden. Durch die Exportsubventionierung hat die EU-Agrarpolitik dazu beigetragen, dass vielerorts der lokale Handel und die lokale Produktion in Drittländern verdrängt wurden (Trebilcock 2015, S. 110-111).

Innerhalb der GAP haben mittlerweile beide Prinzipien, die Markteinheit und auch die Marktordnung, an Bedeutung verloren. Unilaterale Preisabsprachen bilden nach vielen Reformen der GAP mittlerweile nicht mehr eine Grundbasis der GAP. ${ }^{16}$ Dies unterstellt allerdings Agrarprodukte nicht unbedingt den Mechanismen des Marktes, es schafft vielmehr auch mehr Spielraum für Mitgliedstaaten, Preise und preisbildende Faktoren auch auf Produktions- und Großhandelsebene zu bestimmen. ${ }^{17}$ Damit verlieren die Sonderbestimmungen der GAP hinsichtlich des Prinzips der Markteinheit immer mehr an Bedeutung. In der Konsequenz nähern sich damit landwirtschaftliche Produkte herkömmlichen Waren an, die, wie alle anderen Unionswaren auch, unter die Warenverkehrsfreiheit fallen. ${ }^{18}$

\footnotetext{
${ }^{12}$ EuGH, Rs. 71/74, Frubo, Slg. 1975, 563, Rn. 25/26 „Hauptziele“, in der englischen Fassung aber nur: ,the first two objectives“; in der italienischen Fassung auch nur ,le due finalita principali““.

${ }^{13}$ Generalanwalt Bot, EuGH C-333/14, Scotch Whisky Association, at 31.

${ }^{14}$ Generalanwalt Bot, EuGH C-333/14, Scotch Whisky Association, Rn. 30.

${ }^{15}$ GA Bot, EuGH C-333/14, Scotch Whisky Association, 30.

${ }^{16}$ GA Bot, EuGH C-333/14, Scotch Whisky Association, 32.

${ }^{17}$ GA Bot, EuGH C-333/14, Scotch Whisky Association, 33 ff.

${ }^{18}$ GA Bot, EuGH C-333/14, Scotch Whisky Association, 37.
} 
Das Prinzip der Marktordnung wird durch vermehrte Direktzahlungen an Landwirte verändert (Oppermann et al. 2011: § 24, Rn. 24). Grund hierfür ist ein Verständnis, dass der Binnenmarkt nicht nur auf Effizienzgewinne zugeschnitten sein, sondern auch ,sozialen“ Zielen wie dem Umwelt- und Verbraucherschutz dienen soll. Im Recht findet dies vor allem Ausdruck in Querschnittsklauseln wie dem Art. 11 AEUV, dem zufolge ,die Erfordernisse des Umweltschutzes (...) bei der Festlegung und Durchführung der Unionspolitiken und -maßnahmen insbesondere zur Förderung einer nachhaltigen Entwicklung einbezogen werden“ müssen. Ähnliches gilt für den Verbraucherschutz, ${ }^{19}$ die Regionalpolitik, ${ }^{20}$ die Zusammenarbeit mit Drittländern ${ }^{21}$ und im Bereich der Landwirtschaft auch für den Tierschutz. ${ }^{22}$ Damit muss sich nunmehr die GAP auch an diesen Zielen orientieren, auch wenn diese nicht ausdrücklich in Art. 39 AEUV erwähnt sind. Dies hat Auswirkungen auf das rechtliche Verständnis der Rolle des Landwirts bzw. der Landwirtin, die ihr Einkommen nun nicht mehr nur durch möglichst effiziente Produktion landwirtschaftlicher Güter zu garantierten Preisen erzielen sollen, sondern von den zuständigen Behörden auch mit Aufgaben der Landschaftspflege beauftragt werden können. ${ }^{23}$

Beide Entwicklungen machen deutlich, dass sich der Markt, der von der GAP umfasst ist, von einer sektorspezifischen Markteinheit mit eigenen ausschließenden Regeln zu einem regulären Teil des Binnenmarktes entwickelt. „Waren“ sind ebenfalls den Querschnittsklauseln, insbesondere der Art. 11 und 12 AEUV, unterworfen. Im Rahmen der Prüfung der Rechtfertigung und Verhältnismäßigkeit einer in die Warenverkehrsfreiheit eingreifenden Maßnahme kommen diese Rechtsgüter (und noch viele mehr!) ohnehin zum Tragen. Wenn Mitgliedstaaten im Rahmen der GAP nunmehr auch auf allen Ebenen preisbildende Faktoren bestimmen können, so hat dies zwei Konsequenzen: Erstens haben Mitgliedstaaten nunmehr einen deutlich weiteren Spielraum, im Rahmen der GAP eigene Maßnahmen zu ergreifen, die GAP-fremden Zielen wie beispielsweise der Gesundheit der Verbraucherinnen und Verbraucher dienen (so können Mitgliedstaaten beispielsweise trotz anderslautender Formulierung in der Gemeinsamen Marktordnung (GMO) Mindestpreisregelungen für Alkohol bestimmen, um den Alkoholkonsum zugunsten der Gesundheit der Bevölkerung einzuschränken). Zum anderen haben diese Eingriffe allerdings auch das Potenzial, den Binnenmarkt zu behindern, was wiederum die Grundfreiheiten betreffen würde. ${ }^{24}$ Damit müssen sich preisbildende Eingriffe der Mitgliedstaaten in den Agrarsektor, wie beispielsweise die Einführung eines Mindestpreises

\footnotetext{
${ }^{19}$ Art. 12 AEUV.

${ }^{20}$ Art. 174 ff. AEUV.

${ }^{21}$ Art. 208 ff. AEUV.

${ }^{22}$ Art. 13 AEUV.

${ }^{23} \S 3$ (4) i. V. m. § 1(1) BNatSchG.

${ }^{24}$ So auch zu Recht GA Bot, EuGH C-333/14, Scotch Whisky Association, 45 ff.
} 
für Alkohol, nunmehr auch an den allgemeinen Grundsätzen der Marktfreiheiten, vor allem am Verhältnismäßigkeitsgrundsatz, messen lassen. ${ }^{25}$

In diesem Sinne hat Generalanwalt Bot seine Schlussanträge zum Urteil Scotch Whisky Association $^{26}$ jüngst zu einer Generalanalyse der GAP anhand der GMO Wein genutzt, um deren Reformbedarf im Hinblick auf ihr Verhältnis zum allgemeinen Binnenmarktrecht zu unterstreichen: In ausdrücklicher Abgrenzung zur Rechtsprechung des EuGH in den 1980er-Jahren, die die GMO Wein als ,,abschließende Regelung“" angesehen hat, habe sich die GMO ,(i)n den letzten 20 Jahren (...) tiefgreifend verändert. Ursprünglich von der Idee ausgehend, dass die Einkommen der betreffenden Landwirte mit einer Preis- und Interventionsregelung gewährleistet werden sollten, wurde die GMO für Wein vielfältigen Änderungen unterzogen, die, wie die Kommission betont, allmählich dazu geführt haben, dass die klassischen Interventionssysteme zugunsten einer Liberalisierung des Weinmarkts, bei dem die Preise frei nach Angebot und Nachfrage bestimmt werden, aufgegeben wurden."27 Daraus ließe sich insbesondere ableiten, dass die Sonderstellung der GAP in Art. 39 AEUV nicht in der Weise zu verstehen sei, dass sie nicht „Ausdruck des Grundsatzes des freien Warenverkehrs unter den Bedingungen eines wirksamen Wettbewerbs sei. “28 Infolgedessen prüft der Generalanwalt folgerichtig die entsprechende, unter die GMO fallende Maßnahme direkt anhand der Warenverkehrsfreiheit des Art. 34 AEUV. ${ }^{29}$ Der EuGH ist dieser Sicht im Ergebnis gefolgt und hat auch den Prüfungsmaßstab der unter die GAP fallenden Maßnahmen dem der Grundfreiheiten angepasst. Damit hat der EuGH im Ergebnis der GAP den Sonderstatus entzogen und sie dem allgemeinen Binnenmarktrecht angepasst (Purnhagen und Schebesta 2017).

Damit wird die Sonderstellung, welche die GAP im Rahmen der Verträge im Hinblick auf die Grundfreiheiten hat, in Frage gestellt. Vor diesem Hintergrund könnte auch Art. 38 Abs. 2 AEUV neu zu verstehen sein. Zwar bleiben die Vorschriften der GAP lex specialis im Verhältnis zu den Grundfreiheiten; die Maßnahmen der Mitgliedstaaten, die innerhalb der von der GAP erfassten Märkte erlassen worden sind, sind nunmehr jedoch auch direkt an den Grundfreiheiten zu überprüfen. Das heißt im Umkehrschluss auch, dass den Mitgliedstaaten nunmehr auch im Rahmen der GAP vermehrt umweltpolitisch motivierte Ziele im Anwendungsbereich der GAP ermöglicht werden, sofern diese sich an den Grundfreiheiten messen lassen können. Mitgliedstaaten können daher eigene Akzente in der Umweltpolitik, auch im besonderen Rahmen der Vorschriften der GAP, setzen, sofern sie dafür gute und gerichtsfeste Gründe vorbringen können.

\footnotetext{
${ }^{25}$ EuGH C-333/14, Scotch Whisky Association, Purnhagen EuZW 2016, im Druck.

${ }^{26}$ C-333/14, The Scotch Whisky Association ECLI:EU:C:2015:845.

${ }^{27}$ AG Bot, C-333/14, The Scotch Whisky Association ECLI:EU:C:2015:845, Rn. 31.

${ }^{28}$ AG Bot, C-333/14, The Scotch Whisky Association ECLI:EU:C:2015:845, Rn. 37.

${ }^{29}$ AG Bot, C-333/14, The Scotch Whisky Association ECLI:EU:C:2015:845, Rn. 47 ff.
} 


\subsubsection{Verbraucherschutzorientierung durch die Orientierung an der Regulierung der Wertschöpfungskette}

Der rechtliche Rahmen der Agrarpolitik hat sich nicht nur ,aus sich heraus“ verändert, sondern musste mit den veränderten Rahmenbedingungen im sonstigen Binnenmarktrecht, insbesondere im Lebensmittel- und Umweltrecht, kompatibel gehalten werden. Je mehr sich die GAP der Ratio dieser Bereiche annähert (siehe oben), desto weniger entstehen Reibungen zwischen diesen Bereichen.

Das übrige Binnenmarktrecht ist in der Vergangenheit zum einen maßgeblich durch die verbraucherschutzorientierte Auslegung des Art. 114 AEUV geprägt worden. Das Lebensmittelrecht hat mit der Schaffung eines einheitlichen Regulierungsrahmens durch die Lebensmittelbasisverordnung und immer speziellere Regulierungswerke, welche verbraucherschützenden Charakter haben, einen zunehmend engmaschigeren Teppich der Regulierung des Lebensmittelsektors auf EU-Ebene geschaffen. Zwar dienen diese Regeln dem Verbraucherschutz, Regelungsgegenstand ist jedoch nach dem, ,vom Bauernhof zur Gabel“-Ansatz die gesamte Wertschöpfungskette. Damit muss sich gerade auch die Landwirtschaft an diese verbraucherschutzorientierte Regulierung halten, um das Haftungsrisiko zu vermeiden.

Zum anderen ist das übrige Binnenmarktrecht durch die Gesetzgebung zum Umweltrecht geprägt. Zwar besteht ein eigenes Kompetenzregime zur Umweltgesetzgebung in der Union, faktisch sind in der Vergangenheit die meisten Umweltschutzregeln jedoch als Binnenmarktrecht ergangen. Diese Gesetzgebung ist geprägt von vielen übergeordneten Zielen wie dem Kampf gegen den Klimawandel und die Bereitstellung und Erhaltung der Naturressourcen.

\subsubsection{Die politische Logik von Ko-Regulierung und privatrechtlichem Rahmen}

Neben dem klassischen, nationalstaatsgebundenen Recht gewinnt das Regulierungsregime der privaten Regulierung immer mehr an Bedeutung (Purnhagen 2015). Private Regulierung beschreibt ein Rechtsregime, welches durch Standardsetzung, Auditing und Rechtsdurchsetzung gekennzeichnet ist. In kompletten Systemen der privaten Regulierung sind alle drei Bereiche einem privaten Regime untergeordnet, das heißt sie sind, zumindest dem Anspruch nach, dem staatlichen Einfluss entzogen.

Im Agrarsektor nimmt die Bedeutung solcher privater Regulierung zu. Die exportorientierte Landwirtschaft der ersten Welt und der aufstrebenden zweiten und dritten Welt, die durch immer bessere Spezialisierung und technologische Fortschritte auf den Weltmarkt drängen, sind maßgeblich abhängig von globalen Wertschöpfungsketten. Private Regulierung schafft in einem solchen Umfeld idealerweise eine weltweit einheitliche Standardisierung für Produkte und senkt so die Transaktionskosten für Produzenten, Verarbeiter und Vermarkter. Davon profitieren im Allgemeinen auch die Verbraucherinnen und Verbraucher durch niedrigere 
Verbraucherpreise. Produkte und Arbeitsbedingungen können aber auch sicherer werden, wenn Standardisierung höhere Standards eines Endverbraucherlandes (in der Regel in der ersten Welt) auch auf das Produktionsland (dann meist in weniger entwickelten Gebieten) anwenden kann. Diese Vorteile kommen allerdings nicht ohne mögliche Nachteile: Mehr Standardisierung kann auch dazu führen, dass die Vielfalt der Produkte eingeschränkt wird und damit die Verbraucherinnen und Verbraucher weniger Wahlmöglichkeiten haben. Standards können ferner ausschließend wirken: Kann ein Produzent die von einem Standard geforderten Leistungen nicht erbringen, hat keinen Zugang zu einem Zertifizierungssystem oder kann die mit der Einhaltung verbundenen Kosten nicht aufbringen, so wird sein Produkt faktisch vom Markt ausgeschlossen. Schließlich besteht die Gefahr, dass private Standardisierungsanbieter ein Monopol auf diese Standards entwickeln. Damit würden dann einige wenige nicht-staatliche Organisationen weltweit den Marktzugang kontrollieren, was wiederum wettbewerbsrechtlich und demokratie-theoretisch problematisch ist.

Im Agrarsektor hat sich eine Vielzahl solcher Standards entwickelt. In Bezug auf die Lebensmittelsicherheit ist der deutsche Standard GLOBALG.A.P. (früher EURO GAP) führend. Bei der Produktion nachhaltiger Güter sind vor allem niederländische Unternehmen wie UTZ und Max Havelaar tonangebend, während der Markt für Arbeitsbedingungen vor allem von FairTrade dominiert wird. Daneben besteht eine Vielzahl privater und sektorspezifischer Standards, die jeweils ein anderes Ziel verfolgen und oft von einzelnen Supermarktketten ins Leben gerufen wurden. Als Antwort auf die Durchdringung der Märkte durch solche Standards sind Produzenten in der Landwirtschaft in erster Line um die Einhaltung von deren Anforderungen und entsprechende Zertifizierung bemüht. Dadurch, dass diese Standards häufig auf (supra-)nationalem Recht aufgebaut sind, spielt das (supra-)nationale Recht in der Wahrnehmung der Produzenten kaum eine Rolle. Vielmehr setzt der private Standard den Maßstab, da dieser auch weitere Märkte über diejenigen eröffnet, den das (supra-)nationale Recht umfasst.

Diese privatrechtlichen Standards haben daher ein wesentlich größeres Regulierungspotenzial als das nationale Recht. Gelingt es dem Gesetzgeber, den privaten Standard als Regulierungsinstrument zu begreifen und somit von einer SelbstRegulierung zu einer Ko-Regulierung zu kommen, hat dies ein deutlich größeres Regulierungspotenzial. Reguliert man den Standard (idealerweise in Abstimmung mit dem Standardisierer), reguliert man zeitgleich auch den grenzüberschreitenden Warenverkehr und verhindert damit Verdrängungs- und Leakage-Effekte. Für eine an Zielen des Natur- und Umweltschutzes orientierte Agrarpolitik kann die KoRegulierung durch privatrechtliche Standards daher ein interessanter Ansatz sein.

Ansatzpunkt der Regulierung kann sowohl der Standard selbst als auch die Standardisierungsorganisation sein. Regulierungspotenzial besteht dabei sowohl in der klassischen Rechtssetzung als auch in koordinierenden Elementen der Regulierung. In der klassischen Rechtssetzung kann zum Beispiel im Recht der Allgemeinen Geschäftsbedingungen (AGB) angesetzt werden, da solche Standards häufig durch AGB in den Vertrag einbezogen werden. Werden Verträge am Ende der Kette (in Deutschland) durch AGB reguliert, so kann der Standardsetzer gezwungen sein, das 
Regelwerk vom Anfang der Kette (Herstellungsort) zu ändern. Wären beispielsweise nur nachhaltige Standards einbeziehungsfähig in den Vertrag, müssten die Herstellungsbedingungen in den Ländern, in denen das entsprechende Produkt hergestellt wird, angepasst werden. Aber auch andere Mechanismen können solche Standardsetzung beeinflussen, etwa die Aufsicht und Kontrolle von Standardisierungsorganisationen, die Haftung von Standardisierungsorganisationen oder die Beteiligung an Beschlussgremien.

\subsection{Neuere Ansätze zur Integration von Umweltanliegen in den Governance-Rahmen}

\subsubsection{Systemische Konzepte: Resilienz, Ökosystemdienstleistungen, Wasser-Energie- Nahrung-Nexus}

Die traditionelle Land- und Forstwirtschaft hatte einen ganzheitlichen Ansatz. Der moderne Begriff der ökologischen Nachhaltigkeit, eines haushälterischen Umgangs mit den Ressourcen der Natur, wurde in den Forstwissenschaften geprägt (von Carlowitz im 17. Jahrhundert). Im 20. Jahrhundert fand jedoch der Wandel hin zu einer intensiveren Landbewirtschaftung statt. Das Ziel einer Maximierung des Gewinns wurde nun zunehmend durch die Strategie einer Spezialisierung der landwirtschaftlichen Betriebe und Regionen verfolgt. In Europa wurde dieser Trend durch die europäische Agrarpolitik stark gefördert. Seit dem ausgehenden 20. Jahrhundert erhalten eher ganzheitliche Ansätze wieder mehr Beachtung. Das Konzept der Multifunktionalität ist seit 1992 fest in der GAP verankert. Die Bestandsaufnahme zum Zustand der Umweltressourcen in Kap. 3 hat jedoch gezeigt, dass von einer effektiven Integration von Belangen des Natur- und Umweltschutzes in die landwirtschaftliche Praxis im Allgemeinen noch nicht die Rede sein kann. Die im Folgenden vorgestellten innovativen Ansätze und Instrumente der Umweltpolitik setzen daher auf verschiedenen Ebenen an: systemische Leitprinzipien, neue Ansätze des skalenübergreifenden Umweltmanagements, innovative Instrumente der Verhaltenssteuerung hin zu mehr nachhaltigen landwirtschaftlichen Praktiken, eine verstärkte Einbindung von Interessengruppen in den Umsetzungsprozess und Aufklärung der Verbraucherinnen und Verbraucher als Grundlage für verantwortungsbewusste Kaufentscheidungen. Diesen Ansätzen liegt ein erweitertes Verständnis von Governance zugrunde, das berücksichtigt, dass staatliches Handeln, marktwirtschaftliche Instrumente und kooperative Ansätze gezielt verknüpft werden müssen.

Im Folgenden wird zuerst das Potenzial systemischer Konzepte diskutiert, einen Paradigmenwechsel zu unterstützen, um die Wahrnehmung von unauflöslichen Zielkonflikten zwischen ökologischen, ökonomischen und sozialen Zielen zu überwinden und um Synergien, die sich durch gleichzeitige Verfolgung von verschiedenen Zielen ergeben, aufzuzeigen. Dabei werden die folgenden Konzepte diskutiert: 
Resilienz; Wasser-Energie-Nahrung-Nexus (englisch Water-Energy-Food Nexus, kurz WEF) und Ökosystemdienstleistungen als ein Ansatz, die beiden ersten Konzepte zu operationalisieren.

Das Konzept der Resilienz hat seinen Ursprung in der Ökologie (Holling 1974; Folke 2006). Es drückt die Fähigkeit von ökologischen Systemen aus, Störungen zu absorbieren und gleichzeitig seine Funktionen zu erhalten. In der Systemdynamik spricht man davon, dass ein System nach einer Störung zum ursprünglichen Zustand zurückkehrt, dem sogenannten „Attraktor“. Das Zurückkehren zum ursprünglichen Systemzustand kann als statisches Konzept, welches keine Fähigkeiten zur Anpassung und Veränderung besitzt, wahrgenommen werden. Jedoch beinhaltet Resilienz immer auch die Fähigkeit eines Systems zur Selbstorganisation und zur Veränderung nach Schocks und Störungen. Eine oft zitierte Definition stammt von Walker et al. (2004, S. 4) : ,the capacity of a system to absorb disturbance and reorganize while undergoing change so as to still retain essentially the same function, structure, identity, and feedbacks". Der Resilienz-Ansatz wurde von Folke et al. (2010) so interpretiert, dass er sowohl die Fähigkeit eines Systems zur Regeneration wie zur Transformation umfasst. ${ }^{30}$ Dabei erhalten auch die Wechselwirkungen von Transformationsprozessen auf verschiedenen Ebenen eines Systems bzw. in verknüpften Systemen (,nested systems“) zunehmende Aufmerksamkeit. Die Resilienz-Perspektive lenkt dabei den Blick auf die Fähigkeit sozial-ökologischer Systeme, welche auf großer Skalenebene in Zeit und Raum weder nachhaltig noch resilient sind, zu transformativem Wandel. Neuere Arbeiten haben das Konzept beispielsweise auf biobasierte Produktionssysteme angewandt (Ge et al. 2016).

Der Nexus-Ansatz zielt auf eine integrative Betrachtung der systemischen $\mathrm{Zu}-$ sammenhänge zwischen der Versorgung mit Wasser, Energie und Nahrungsmitteln. Daraus ergibt sich die Anforderung einer ganzheitlichen Betrachtung der institutionell zumeist stark separierten Politikfelder Wasser, Energie und Nahrung (Benson et al. 2015). Der Nexus-Ansatz nimmt die Interaktionen zwischen den verschiedenen

\footnotetext{
${ }^{30}$ „Der Resilienz-Ansatz beachtet die Dynamiken und Entwicklungen von komplexen sozial-ökologischen Systemen. Hierbei sind drei Aspekte von zentraler Bedeutung: Resilienz, Anpassungsfähigkeit und die Fähigkeit zur Transformation. Diese Aspekte stehen über Skalen hinweg in Verbindung. In diesem Kontext ist Resilienz die Fähigkeit von sozial-ökologischen Systemen, sich kontinuierlich verändern und anpassen zu können, während sie sich innerhalb von kritischen Systemschwellen bewegen. Die Anpassungsfähigkeit ist ein Teil der Resilienz. Sie steht für die Fähigkeit, sich an verändernde äußere Einflüsse und innere Prozesse anzupassen und somit eine Entwicklung entlang des Bestehenden (Stabilitätsbereich) ermöglicht. Die Fähigkeit zur Transformation ist die Möglichkeit, Systemschwellen hin zu neuen Entwicklungspfaden zu überschreiten. Transformationen auf kleiner Skalenebene ermöglichen Resilienz auf größerer Skalenebene. Die Fähigkeit zur Transformation auf kleiner Skalenebene wiederum fußt auf der Resilienz auf mehreren Ebenen, wobei Krisen als Gelegenheitsfenster für Innovation und Erneuerung genutzt und Wissens- und Erfahrungsquellen neu kombiniert werden, um sozial-ökologische Transformationen zu navigieren. Die Gesellschaft muss ernsthaft über Wege nachdenken, die Resilienz von kleineren, besser kontrollierbaren sozial-ökologischen Systemen zu erhöhen, die zur Resilienz des Erdsystems beitragen, und Optionen für eine kontrollierte Transformation von sozial-ökologischen Systemen ausloten, welche die Resilienz des Erdsystems bedrohen“ (Folke et al. 2010, S. 1, eigene Übersetzung).
} 
Politikfeldern in den Blick, anstatt diese getrennt voneinander zu betrachten. Der Ansatz kommt aus dem Bereich der Wasserwirtschaft, wo erkannt wurde, dass Wasserprobleme nicht ohne die konzeptionelle und praktische Integration der anderen Bereichen gelöst werden können, insbesondere weil die Wasserkreisläufe auch als Verbindung zwischen den anderen Bereichen fungieren (z. B. Nitratbelastung siehe Beispiel unten).

Der Water-Energy-Food(WEF)-Nexus-Ansatz wurde im Jahr 2008 von Unternehmen beim Weltwirtschaftsforum in Davos beworben, bei dem Wasser zu einem Thema von weltweiter Relevanz wurde. Anschließende Berichte verbreiteten den WEF-Ansatz weiter (WEFWI 2011). Das Weltwirtschaftsforum hob die Gefahren, aber auch die Möglichkeiten für Unternehmen hervor. Marktmechanismen und die „grüne Ökonomie“ werden als effektive und effiziente Lösungsansätze angesehen, auf Ressourcenknappheit zu reagieren. Die deutsche Regierung hat im Vorfeld zum Nachhaltigkeitsgipfel Rio+20 im Jahr 2012 für die Einführung des Nexus-Konzepts geworben, indem sie im Jahr 2011 in Bonn eine Konferenz zum WEF-Nexus organisierte. Der Diskurs griff wiederum ein weiter gefasstes Konzept auf, welches Auswirkungen auf die Politik und die soziale, ökonomische und ökologische Nachhaltigkeit hervorhebt (BMU 2011; Hoff 2011). Allerdings wurde bezweifelt, ob der WEF-Nexus Ansatz bei der Überwindung von vorherrschenden GovernanceProblemen (etwa fehlender Koordination, ineffektiver Umsetzung) hilft. Allouche et al. (2015) bemängeln, dass dem Ansatz der Bezug zu der jeweiligen Marktlogik innerhalb der Sub-Nexus fehle. Er unterschätze die Schwierigkeit der Integration und ignoriere die tatsächlichen Prozesse der Politikgestaltung sowie die damit verbundene strategische Rolle von Wissen und Konzepten. Tatsächlich basiert der Ansatz des WEF-Nexus auf einer primär wissenschaftlich und fachspezifisch motivierten Grundidee, welche eine integrierte Herangehensweise fordert. Um sein transformatives Potenzial zu entwickeln, müsste der Nexus-Ansatz Machtkonstellationen und Aspekte der politischen Ökonomie und deren Veränderung auf verschiedenen räumlichen und zeitlichen Ebenen mehr berücksichtigen.

Eine gute Illustration von misslungener Koordination im WEF-Nexus sind die Entwicklungen in Nordwestdeutschland, wo über Jahrzehnte eine starke Intensivierung der Landwirtschaft, insbesondere der Tierproduktion, stattgefunden hat. Eine Zunahme des Nutztierbestandes, ermöglicht durch den Import von Futtermitteln (zumeist Soja aus Südamerika), und die damit verbundene erhöhte Gülleausbringung führten zu starken Nitratbelastungen der Binnengewässer. Politische Versuche, die Tierdichte und die Nitratbelastung zu reduzieren (z. B. Obergrenzen für Viehbestand pro Landeinheit oder Düngemittelmärkte) führten noch nicht zu umweltpolitisch befriedigenden Ergebnissen. Die Situation wurde seit ca. 2005 durch den Aufschwung beim Anbau von Energiepflanzen verschärft, der wesentlich durch die Förderinstrumente des Erneuerbare-Energien-Gesetzes (EEG) angetrieben wurde. Zu den Folgen gehören eine weiter erhöhte Grundwasserbelastung und stark gestiegene Pachtpreise für landwirtschaftliche Flächen. Bereits existierende Probleme wurden durch die fehlende politische Integration verschlimmert und führen sogar zum Verstoß gegen bestehende rechtliche Grenzwerte, so dass die EU im Jahr 2016 ein Vertragsverletzungsverfahren gegen Deutschland eröffnete, da die Nitratgrenzwerte der EU-Wasserrahmenrichtlinie in mehreren Regionen überschritten wurden. 
Um den Nexus-Ansatz für eine verbesserte Koordination fruchtbar zu machen, ist eine Operationalisierung der Zielgrößen notwendig. Hierbei könnte das Konzept der Ökosystemleistungen (ÖSL) eine entscheidende Rolle übernehmen, die sich definieren lassen als ,the conditions and processes through which natural ecosystems, and the species that make them up, sustain and fulfil human life" (Daily 1997). Seit der Veröffentlichung des Millennium Ecosystem Assessment im Jahr 2005 steigen die Veröffentlichungen zu ÖSL exponenziell an. Das Konzept findet auch zunehmend Resonanz auf der politischen Agenda oder in wirtschaftlichen Entscheidungsprozessen (Fisher et al. 2009) bis hin zum Finanzsektor (Gómez-Baggethun et al. 2010). Das Konzept der ÖSL kann u. a. helfen, intersektorale Trade-offs bei politischen Entscheidungen und im Ressourcenmanagement besser zu verstehen (Knüppe und Pahl-Wostl 2013).

Die neuen integrativen Ansätze problematisieren strikt sektorale Politiken und Praktiken, die durch sektorbasierte institutionelle Arrangements auf Dauer gestellt sind, welche es sektorexternen Akteuren schwer machen, ihre Sichtweisen und Interessen zur Geltung zu bringen (Primmer und Furman 2012). Die neuen integrativen Ansätze sind verbunden mit Überlegungen zur Kombination verschiedener Governance-Formen: Märkte, Regulierung, bürokratische Hierarchien und Netzwerke. Regulation etwa ist für das Setzen von Rahmenbedingungen wichtig, die etwa als Zielzustand und Verhandlungsbasis fungieren. Netzwerke unterstützen Lern- und Beratungsprozesse, welche zum Lösen von komplexen Problemen unter Ungewissheit notwendig sind. Das EU-Projekt ESAWADI hat beispielsweise das Potenzial des ÖSL-Ansatzes untersucht, komplexe Zielkonflikte bei der Umsetzung der Wasserrahmenrichtlinie zu identifizieren und zu verhandeln (ESAWADI 2013). Die gewonnenen Erkenntnisse unterstreichen, dass ein umfassender ÖSL-Ansatz das Potenzial hat, Verhandlungsformen und Kooperationen zu verbessern, welche die Komplexität von Ökosystemleistungen und deren Funktion für die Gesellschaft abbilden.

\subsubsection{Vom theoretischen Konzept zur praktischen Umsetzung?}

Das Resilienz-Konzept findet zunehmend Eingang in den politischen Diskurs, insbesondere im Zusammenhang mit der Anpassung an den Klimawandel, aber auch in der Beschreibung der Zielrichtung nationaler und internationaler Umweltpolitik. Beispiele dafür sind die Biodiversitäts-Konvention UNCBD oder die australische Strategie zum Schutz der Biodiversität (Newton 2016). Allerdings kann die noch nicht endgültig geklärte Interpretation des Resilienz-Konzeptes in der praktischen Anwendung zu Bewertungsschwierigkeiten führen (Newton 2016). Ähnlich wie das Nachhaltigkeitskonzept lässt auch das Resilienz-Konzept große Interpretationsspielräume zu, beispielsweise darüber, welcher Systemzustand als schützenswert und welche Fluktuationen im Systemzustand als tolerabel angesehen werden.

Biggs et al. (2012) identifizieren sieben politikrelevante Grundsätze, um die Resilienz von ÖSL zu verbessern: (1) Erhalt von Diversität und Redundanz, (2) Steuerung der Konnektivität, (3) Management von langsamen Variablen und Feedbacks, (4) Unterstützung des Verständnisses von sozial-ökologischen Systemen als 
komplexe, adaptive Systeme, (5) Förderung von Lernen und Experimentieren, (6) Unterstützung von Partizipation, und (7) Förderung von polyzentrischen Governance-Systemen. Obwohl diese Grundsätze einen eher allgemeinen Charakter haben, können sie eine Orientierung bieten und Spannungen zwischen vorherrschenden politischen Praktiken und Management-Ansätzen und den Anforderungen zur Verbesserung der Resilienz identifizieren.

Schipper und Langston (2015) haben ein ausführliches Review von Frameworks zur Bewertung der Resilienz von Systemen durchgeführt, welche den Schwerpunkt auf den Entwicklungskontext setzten. Demnach mangelt es nicht an Vorschlägen zu operativen Messgrößen. Von größerer Bedeutung ist jedoch, unabhängig vom Kontext, die Beantwortung der Frage, für welche betroffenen Personengruppen und für welche Zwecke das Konzept angewendet wird. Dahinter steht die Frage, welche Interessen und Werte durch die Resilienz eines Systems geschützt werden.

Im landwirtschaftlichen Kontext könnten dabei insbesondere die folgenden drei Bereiche von Interesse sein:

1. Die durch die Landwirtschaft beeinflusste Resilienz von Ökosystemen: Resilienz kann zum Leitmotiv und zum funktionalen Instrument werden, um die Widerstands- und Regenerationsfähigkeit von Ökosystemen zu erfassen. Das Konzept fördert einen integrativeren Ansatz zur Gestaltung von resilienten Landschaften mit einem Netzwerk von „grüner Infrastruktur“. So wurden beispielsweise bereits Methoden zur Identifikation und zur Kartierung von ,grünen Infrastrukturelementen" auf Landschaftsebene entwickelt. Sie basieren auf den Konzepten der ökologischen Vernetzung, der Multifunktionalität von Ökosystemen und der Nutzenmaximierung sowohl für den Menschen als auch für den Naturschutz sowie der Erhöhung der ökologischen und sozialen Resilienz (Liquete et al. 2015).

2. An den Klimawandel angepasste - also resiliente - Produktionssysteme: Der Klimawandel hat das Interesse an Resilienz und adaptivem Ressourcenmanagement gestärkt. Manche Resilienz-Strategien, wie die Anbaudiversifizierung bei Kulturpflanzen, sind zudem sowohl vorteilhaft für die Umwelt als auch für Biodiversität-Aspekte (Lin 2011). Ein gemeinsam von der FAO und der OECD organisierter Workshop über die Bildung von Resilienz im Agrarsektor und gegen den Klimawandel (Meybeck et al. 2012) kam u. a. zu dem Ergebnis, dass das Resilienz-Konzept eine systemische Perspektive unterstützt und die integrierte Betrachtung von verschiedenen Bereichen - biophysikalische, ökonomische, soziale und institutionelle - und von verschiedenen zeitlichen und räumlichen Skalen erleichtert. Große Bedeutung wurde der Identifikation und dem Monitoring von potenziellen Risiken und Schwachstellen beigemessen, vor allem im Hinblick auf die Vermeidung kumulativer und langfristiger Folgen. Es wurden verschiedene Maßnahmen als notwendig angesehen, um die Resilienz von Landwirten, aber auch von ganzen Produktionssystemen zu verbessern. Verstärkte Beachtung sollten dabei die Interaktionen zwischen Bereichen und Skalenebenen erhalten, um die Ausbreitung von Schocks zu vermeiden. 
3. Resilienz der Ernährungssicherheit: Der Großteil der Literatur beschäftigt sich mit dem Thema Ernährungssicherung in Bezug auf Entwicklungsländer. Resilienz und Ernährungssicherheit sind in der Entwicklungszusammenarbeit zu Leitprinzipien geworden. In Bezug auf den Klimawandel ist das Thema auch für Europa von Bedeutung. Die Resilienz des Nahrungsmittelsystems als Ganzes (Produktion, Transport und Konsum) hat bisher wenig Aufmerksamkeit bekommen.

Das Resilienz-Konzept kann dazu dienen, den Beitrag einzelner Politikmaßnahmen auf die Robustheit und Anpassungsfähigkeit von landwirtschaftlichen Produktionssystemen zu bewerten bzw. entsprechende Ansätze im praktischenen Kontext zu entwickeln, wie die folgenden beiden Beispiele illustrieren:

- Resilienz durch standortgerechte Fruchtfolgen: Vielfältige Fruchtfolgen und die Nutzung von regionaltypischen Kulturpflanzensorten und Nutztierrassen erhalten die Bodenfruchtbarkeit und stärken damit die Resilienz von landwirtschaftlichen Produktionssystemen. Durch angepasstes Management und vielfältige Nutzungen werden die ökosystemaren Dienstleistungen agrarisch genutzter Flächen und der umgebenden Landschaft gesichert und tragen zu einer resilienten, multifunktionalen Landnutzung bei.

- Partizipative Verfahren zur Planung und Umsetzung von Natur- und Umweltschutzmaßnahmen können etabliert werden, um alle von den ökosystemaren Leistungen betroffenen gesellschaftlichen Gruppen einzubinden (z. B. Wasserwirtschaft, Tourismus, Naturschutz- und Umweltverbände, Spezialitätenerzeuger, Forstwirtschaft). Kooperative erarbeitete Pläne zu regional angepassten Nutzungsformen sowie Natur- und Umweltschutzmaßnahmen auf Agrarflächen erhöhen die Landschaftskomplexität und die Konnektivität von wertvollen Habitaten, lassen eine differenzierte Vielfalt der Landschaften zu und minimieren die negativen Auswirkungen der Landwirtschaft. Durch eine auf regionale Bedürfnisse abgestimmte Projektierung können flächendeckend und Betriebstypen übergreifende Ansätze für eine sachgerechte (ggf. kleinräumige) Umsetzung der Maßnahmen verfolgt werden. Landwirtinnen und Landwirte können so maßgeblich zum Erhalt der biologischen Vielfalt und einem funktional orientierten Biotopverbundsystem beitragen.

Ansätze zur Operationalisierung des Wasser-Energie-Nahrung-Nexus fokussieren oft auf den Bereich der Sicherheit. Dies kann dazu führen, dass lediglich die jeweiligen sektoral üblichen Definitionen von Wasser-, Energie- und Nahrungssicherheit reproduziert werden, ohne sich um ein integriertes, sektorübergreifendes Konzept von Sicherheit im Rahmen des WEF-Nexus zu bemühen. Interessant ist daher die weit verbreitete Definition von Wassersicherheit von Grey und Sadoff (2007, S. 545): ,the availability of an acceptable quantity and quality of water for health, livelihoods, ecosystems and production, coupled with an acceptable level of water-related risks to people, environments and economies." Diese weitgefasste und auf die Reduzierung von Risiken bezogene Definition verknüpft ein Verständnis der Rolle von Wasser als Ursprung verschiedener Leistungen mit einer risikobezogenen 
Betrachtung. Welche Risiken als tolerierbar gelten, muss dabei in einem transparenten und inklusiven Prozess geklärt werden, der dem Nexus-Ansatz zufolge sektorübergreifend angelegt sein muss.

Eine Serie von internationalen Workshops des Programms Future Earth zur Thematik „Sustainability for Water, Energy, and Food through Integrated Water Information and Improved Governance" hat die Möglichkeiten von neuen Datenquellen aus Erdbeobachtungssystemen für ein besseres Verständnis der komplexen Zusammenhänge im WEF-Nexus und für eine bessere interdisziplinäre Zusammenarbeit aufgezeigt. „Good Governance“ ist dabei eine grundlegende Voraussetzung, um das Potenzial von neuen Datenquellen für ein nachhaltiges Management im Rahmen des WEF-Nexus zu ermöglichen.

Sowohl im Resilienz- wie im Nexus-Ansatz haben Investitionen in eine sektorenübergreifende grüne Infrastruktur einen hohen Stellenwert (Ozment et al. 2015). Das Konzept der ökologischen oder grünen Infrastruktur hat in den letzten Jahren sowohl in der Forschungsliteratur als auch in Politikdokumenten seit ca. 2009 großes und rapide wachsendes Interesse gefunden, wie eine Abfrage in der Literaturdatenbank Scopus ergab. Die Planung der Entwicklung einer grünen Infrastruktur auf Landschaftsebene ermöglicht sektorenübergreifende Synergien. In der Praxis stoßen eine integrierte Landschaftsplanung und Investitionen in grüne Infrastruktur auf Hemmnisse infolge einer sektoralen Fragmentierung der Entscheidungs- und Regulierungsstrukturen.

Das Konzept der Ökosystemleistungen kann systemische Ansätze der Landschaftsanalyse und -planung und damit ein stärker integriertes Management von multifunktionalen Landschaften unterstützen. Eine Vielzahl von GovernanceInstrumenten wurde unter dem konzeptionellen Dach der ,Zahlungen für Ökosystemleistungen“ („Payments for Ecosystem Services“) entwickelt. Viele dieser Ansätze sind bisher jedoch auf einzelne Arten oder Leistungen fokussiert und betrachten nicht das ganze System. Hier besteht Bedarf zur Entwicklung stärker systemischer Konzepte.

Als Fazit ist festzuhalten, dass der Resilienz- und der WEF-Nexus-Ansatz interessante Konzepte sind, um systemische Wechselwirkungen zu erkennen und $\mathrm{zu}$ bewerten und dadurch ein integriertes Management von Naturressourcen auf der Landschaftsebene zu unterstützen. Auch in der agrarpolitischen Diskussion werden diese Konzepte aufgenommen. Beispielsweise fördert die Europäische Kommission im Rahmen des Forschungsprogramms Horizon 2020 Verbundprojekte zu den Themen Resilienz und integriertes Ressourcenmanagement. Allerdings sind diese Konzepte noch nicht im Zentrum der agrarpolitischen Debatte angekommen - nicht zuletzt dadurch bedingt, dass Operationalisierung und praktische Bedeutung noch in der Diskussion sind. In dem Maße, wie mit der Aufnahme dieser Konzepte ein Paradigmenwechsel in der Agrarpolitik verbunden wäre, ist jedoch mit Widerständen zu rechnen (vgl. McMichael 2011). 


\subsubsection{Verhaltenswissenschaftliche Ansätze}

Im Zuge der Veränderungen des agrarpolitischen und europarechtlichen Rahmens (siehe Abschn. 4.1 und 4.2) wird auch die Landwirtschaft zum Adressaten neuer Regulierungsstrategien. Weltweit, in der EU und in Deutschland gewinnt die Auffassung an Boden, dass politische Ziele ,von oben herab“, also durch staatliche Ge- und Verbote für individuelles Verhalten, oftmals nicht effektiv und effizient durchgesetzt werden können. Vielmehr ist es wichtig, das Verhalten der einzelnen Akteure in einer Art und Weise zu beeinflussen, dass sich entsprechende gesamtwohlfördernde Maßnahmen durchsetzen lassen. Als vielversprechend wird hierbei der „Nudging“-Ansatz gesehen (Thaler und Sunstein 2008), der auf verhaltenswissenschaftlichen Erkenntnissen aufbaut, um eine erwünschte Verhaltensänderung zu erzielen. Versteht man, so die Annahme, wann Menschen wie auf welche Veränderungen im Entscheidungskontext reagieren, so kann man sich dies auch bei der Verhaltenslenkung durch Regulierung zunutze machen. Der Nudging-Ansatz geht davon aus, dass sich jeder Mensch in einer solchen Entscheidungsstruktur bewegt. Verhaltensbasierte Regulierung macht sich das Wissen um diese Entscheidungsstruktur zunutze und greift bewusst in diese Strukturen ein; sie schafft eine intendiert gestaltete „Architektur der Wahl“.

\subsubsection{Allgemeine verhaltenswissenschaftliche Befunde und mögliche Anwendungen zum Umweltverhalten von Landwirten}

Neuere Arbeiten aus den Bereichen der Sozialpsychologie und der Verhaltensökonomie zeigen auf, dass neben wirtschaftlichen Anreizen weitere Faktoren die Teilnahme von Landwirten an freiwilligen Umweltmaßnahmen beeinflussen (Wilson und Hart 2000; Lütz und Bastian 2002; Shogren und Taylor 2008). Bei der Gestaltung von Politik-Instrumenten zum Umweltschutz in der Landwirtschaft sollten daher auch kognitive Prozesse (z. B. Entscheidungsheuristiken), unterschiedliche Präferenzen (z. B. pro-sozial, pro-umwelt) und Annahmen der regulierten Akteure (z. B. über das Verhalten anderer Landwirte) mit einbezogen werden, die zur Entscheidungsfindung beitragen können (de Snoo et al. 2013). Im Folgenden sollen beispielhaft einige der für diese Studie relevanten Befunde skizziert und deren Implikationen für eine zukunftsfähige Agrarpolitik diskutiert werden (basierend auf Colen et al. 2015):

Als Verlustaversion (Loss Aversion) wird die Tendenz von Menschen bezeichnet, einen Verlust stärker zu gewichten als einen nominell gleich großen Gewinn (Kahneman 2003). Bei Landwirten ist beispielsweise denkbar, dass sie die möglichen Ertragsverluste infolge einer Agrarumweltmaßnahme stärker negativ empfinden als deren monetäre Kompensation und deshalb nicht an entsprechenden 
Maßnahmen teilnehmen. Auch die Greening-Auflagen der GAP können Angst vor einem Verlust auslösen, da die Landwirte fürchten müssen, einen Teil ,ihrer ' (oft als legitimer Besitzstand wahrgenommenen) Direktzahlungen einzubüßen, wenn sie - und sei es versehentlich und geringfügig - gegen die Auflagen verstoßen. In diesem Sinne liefert das Konzept Verlustaversion ein Argument dafür, dass monetäre Strafen effizienter sein könnten als monetäre Vergütungen, insbesondere, wenn letztere noch unter einem Kontrollvorbehalt stehen. Empirische Belege für diese These wurden u. a. bei Maßnahmen zur Förderung der Tiergesundheit gefunden (Valeeva et al. 2007; Hogeveen et al. 2011).

Unter Kontrollaversion (Control Aversion) wird die negative Verhaltensreaktion von Menschen auf exogene Zwänge und Einschränkungen ihrer Selbstbestimmtheit verstanden (Frey und Stutzer 2006). Besonders in Kontexten, in denen bereits eine soziale Norm etabliert ist, können Vorschriften unter Umständen pro-soziale bzw. umweltfreundliche Verhaltensweisen reduzieren (Frey und Stutzer 2006). Im landwirtschaftlichen Kontext kann also beispielsweise die Verschärfung von Auflagen (z. B. Greening) oder die Erhöhung der Frequenz von Monitoring-Programmen nachteilige, der Regelungsintention gegenläufige Effekte haben und insgesamt zu weniger umweltfreundlichen Verhaltensweisen führen. D'Adda (2011) demonstriert diesen Effekt in einer experimentellen Studie zu einem Wiederaufforstungsprojekt in Bolivien.

Verlust-und Kontrollaversion können also unterschiedliche Reaktionsrichtungen bewirken und je nach relativer Ausprägung die Performanz von Politik-Instrumenten positiv wie negativ beeinflussen. Als vermittelnder Faktor hat sich in einer Vielzahl an Studien das sogenannte Framing erwiesen (Levin et al. 1998). Der Begriff bezieht sich in diesem Zusammenhang auf die wörtliche Beschreibung und den Kontext, in dem zum Beispiel ein Politik-Instrument präsentiert wird. Menschen beurteilen Entscheidungen oder Gegebenheiten stark in Abhängigkeit von dem Frame, in dem diese präsentiert werden, zum Beispiel ob eher die positiven oder die negativen Aspekte und Konsequenzen einer Entscheidung betont werden (Levin et al. 1998). Im Kontext von Verlustaversion hat sich etwa gezeigt, dass es in Bezug auf Agrarumweltmaßnahmen vorteilhafter ist, deren gesellschaftlichen Nutzen zu betonen, als zum Beispiel lediglich darauf zu verweisen, in welcher Höhe entstehende Kosten entschädigt werden (Grolleau et al. 2015).

Als Status-Quo-Bias wird die Tendenz von menschlichen Individuen bezeichnet, lieber bei bekannten und etablierten Praktiken zu bleiben als neue Dinge auszuprobieren (Thaler und Sunstein 2008). Dieser Effekt wurde auch bei Landwirten beobachtet (Espinosa-Goded et al. 2010). Er ist relevant, wenn Agrarumweltmaßnahmen die Verwendung neuer Techniken erfordern oder sogar darauf abzielen, neue Techniken einzuführen. Freiwillige Vereinbarungen mögen hier den gesetzlichen Standards als Instrument unterlegen sein (siehe auch die Erläuterungen zur Verlustaversion). Allerdings zeigt sich, dass durch die geschickte Gestaltung der Standardoption (des „Defaults“, z. B. bei Vertragsabschluss) das Entscheidungsverhalten beeinflusst werden kann (Choi et al. 2003). Auch der Aufbau von Vertrauen, Bildungs- und Trainingsmaßnahmen sowie Gelegenheit zur Partizipation können die Akzeptanz neuer Praktiken fördern (Luz 2000). Kooperative Ansätze, bei denen 
die Landwirte und Landwirtinnen in Gruppen organisiert sind, sind in dieser Hinsicht ebenfalls förderlich, da sie durch den Kontakt mit anderen Landnutzern lernen können, dass sich die Einführung von Innovationen auch lohnen kann (Prager 2015).

Auch die verinnerlichten Werte und Normen haben einen Einfluss auf das Entscheidungsverhalten der Landwirtinnen und Landwirte. Viele von ihnen haben eine produktivistische Ethik internalisiert und besitzen ein Selbstbild, das ihre Fähigkeiten und ihr Wissen in den Vordergrund stellt (Lütz und Bastian 2002; Burton et al. 2008; Emery 2014). Maßnahmen, die diese Ethik in Frage stellen oder die nicht explizit die Fähigkeiten der Landwirtinnen und Landwirte ansprechen, können daher auf geringere Akzeptanz stoßen als Maßnahmen, die eher in Einklang mit diesen Werten präsentiert werden (Burton et al. 2008). Eine ergebnisorientierte Honorierung kann geeignet sein, Landwirtinnen oder Landwirte darin zu bestärken, dass es ihr Wissen und ihr Können sind, die benötigt werden, um die Umwelt zu schonen (Burton und Schwarz 2013). In diesem Sinne könnte etwa versucht werden, das „Produzieren“ von Trinkwasser und Biodiversität mit der produktivistischen Ethik vieler Landwirtinnen und Landwirte zu verkoppeln (Burton und Schwarz 2013).

Ein letzter Aspekt, der hier betont werden soll, ist die Tatsache, dass sich die Präferenzen von Landwirten mit der Zeit verändern können (Bocquého et al. 2013). Entscheidungen, die Landwirte zu einem gegebenen Zeitpunkt treffen, mögen daher in Konflikt zu ihren Präferenzen zu einem späteren Zeitpunkt stehen. Ein typisches Beispiel im Kontext von Agrarumweltmaßnahmen ist der Fall, in dem die unmittelbaren Kosten einer Maßnahme im Vergleich zu dem langfristigen (monetären) Nutzen zu stark bewertet werden und aus diesem Grunde die Maßnahme nicht ausgeführt wird, auch wenn es aus der langfristigen Perspektive mehr Sinn machen würde. Wenn Akteure Probleme haben, in Aktivitäten zu investieren, die erst in der mittelfristigen oder fernen Zukunft einen Nutzen abwerfen, können beispielsweise veränderte Zahlungsschemata (Vorschüsse oder häufigere Auszahlungen) Abhilfe schaffen.

\subsubsection{Faktoren und Beeinflussung umweltbezogener Entscheidungen von Landwirten}

Welches sind die wichtigsten Antriebskräfte für umweltbezogene Entscheidungen von Landwirten? Diese können sowohl psychologische Determinanten wie auch wahrgenommene praktische Hilfen und Barrieren beinhalten.

In der Literatur finden sich viele Studien, die unterschiedliche theoretische Modelle benutzen und sich auf verschiedene Zielverhaltensweisen beziehen, z. B. bezüglich Heckenmanagement, Pestizidausbringung, oder generell bei der Teilnahme an AUKM. Das am häufigsten verwendete theoretische Modell war die Theorie des geplanten Verhaltens (Theory of Planned Behaviour) von Ajzen (1991).

Gailhard und Bojnec (2015) berichten über eine Studie zur Beziehung zwischen Betriebsgröße und Teilnahme an AUKM in Slowenien. Sie finden, dass sich die Beweggründe für die Teilnahme von großen und kleinen Betrieben unterscheiden. Beispielsweise steigt bei großen Betrieben die Wahrscheinlichkeit der Teilnahme mit dem Waldanteil, bei kleinen Betrieben gibt es hingegen keine solche Beziehung. 
Mit stärkerem Fokus auf psychologische Antriebskräfte finden Beedell und Rehman (1999), dass Landwirte, die starke positive Überzeugungen über den Nutzen von Naturschutz haben und die sozialen Druck im Hinblick auf Naturschutz erfahren, mit höherer Wahrscheinlichkeit auch stärker naturschutzorientiert sind. Eine spätere Studie, in der hohes Umweltbewusstsein und ein größerer Einfluss von Naturschutzbezugsgruppen die auffälligsten Treiber für Naturschutzanstrengungen waren, bestätigte die früheren Ergebnisse (Beedell und Rehman 2000).

Wilson und Hart (2000) berichten über die Ergebnisse eines internationalen Forschungsprojekts zur Teilnahme an AUKM. Allgemeine Teilnahmemuster umfassen die Bedeutung der finanziellen Anreize und die „Passgenauigkeit“ der Maßnahme sowie den Einfluss von Faktoren wie Betriebsgröße, Pachtanteil, und Betriebstyp. Die Befunde weisen auf eine wachsende Bedeutung Naturschutz-orientierter Motivationen für die Beteiligung an AUKM in allen Teilen Europas hin. Die Autoren schlagen als ,neue Hypothese“ vor, dass die finanziellen Motive für die Teilnahmen nicht ausschließen, dass umweltbezogene Motive gleichermaßen wichtig sind.

Lokhorst et al. (2011) berichten über eine Studie zu den sozialpsychologischen Grundlagen der Naturschutzpraktiken von Landwirten in den Niederlanden. Sie unterscheiden zwischen staatlich unterstütztem und nicht unterstütztem Naturschutz und finden, dass staatlich unterstützter Naturschutz allein durch die Einstellungen der Landwirte motiviert ist, während nicht unterstützter Naturschutz auch durch sozialen Druck und die Selbst-Identität der Landwirte angetrieben ist: das Ausmaß, in dem Landwirte den Naturschutz als Teil ihrer eigenen Identität wahrnehmen.

Lokhorst et al. (2014) verfolgen diese Befunde in einer Fragebogenstudie weiter, um die psychologischen Determinanten für privaten Naturschutz zu analysieren. Die Ergebnisse bestätigen die zentrale Rolle der Selbst-Identität und zeigen, dass die Verbindung mit der Natur, die Landwirte erleben, ein wichtiger Antrieb für ihre Naturschutzanstrengungen ist.

Wie die Organisation von AUKM in den Niederlanden die Entscheidungsfindung von Landwirten beeinflusst, haben van van Dijk et al. (2015) untersucht. In den Niederlanden werden einige AUKM kollektiv von großen regionalen Gruppen von Landwirten durchgeführt, sogenannten Umweltkooperativen. Die Autoren nehmen an, dass diese Kooperativen die Absicht von Landwirten zur Teilnahme erhöhen, indem sie die Antragstellung erleichtern, aber auch, indem sie Gruppendruck aufbauen. Die Ergebnisse belegen, dass die Absicht der Landwirte, am Grabenmanagement teilzunehmen, mit der Einstellung der Landwirten und ihrer wahrgenommenen persönlichen Fähigkeit zur Teilnahme assoziiert ist. Hingegen korreliert die Bereitschaft, an Programmen zum Schutz von Weidevögeln teilzunehmen, mit sozialem Druck, Selbst-Identität und der Vermittlung durch die Umweltkooperative.

Pedersen et al. (2012) gehen von der Idee aus, dass Landwirte verschiedene Ziele verfolgen und selbst bereit sein könnten, auf Gewinn zu verzichten, um andere Ziele zu erreichen. In ihrer Studie zum Pestizideinsatz bei dänischen Landwirten ließen sie explizit ökonomische und nicht-ökonomische Rationalitäten gegeneinander antreten und fanden, dass einige Landwirte v. a. ökonomisch motiviert waren, während andere sich darauf konzentrierten, den Ertrag zu steigern. Bemerkenswerterweise 
sprachen Landwirte, die sich auf Ertragssteigerung konzentrierten, weniger auf ökonomische Politik-Instrumente an. Die Ergebnisse weisen darauf hin, dass es wichtig ist, eine große Bandbreite an Politik-Instrumenten anzubieten, um die unterschiedlichen Denkweisen verschiedener Landwirte anzusprechen.

Van Herzele et al. (2013) verfolgen einen anderen Ansatz. Basierend auf einem Fragebogen und Interviews mit belgischen Landwirten unterscheiden sie sechs Modi oder Stile der Teilnahme an AUKM: opportunistisch, kalkulatorisch, kompensatorisch, optimierend, katalytisch und engagiert. Die Autoren kommen zu dem Schluss, dass Geld zwar ein wichtiges Motiv für die Teilnahme ist, dass die monetären Aspekte aber verschiedene Rollen spielen, je nach den Abwägungen des einzelnen Landwirts und der Bedeutung, die er/sie anderen Überlegungen beimisst.

McCracken et al. (2015) fokussieren auf die Frage, wie soziale Faktoren sich zur Qualität der Naturschutzpraktiken bei einer Gruppe von britischen Farmern verhalten. Im Ergebnis fanden sie, dass insbesondere frühere Erfahrungen die Qualität der bereitgestellten Habitate beeinflussen. Sie verweisen auf die Bedeutung, landwirtschaftlichen Naturschutz als Lernprozess für Landwirte zu begreifen.

Welche Schlussfolgerungen können aus diesem kurzen Literaturbericht gezogen werden? Politische Entscheidungsträger neigen dazu, Landwirte als Gewinnmaximierer zu betrachten, die nur auf finanzielle Anreize reagieren. Dies ist aber eine vereinfachte Sichtweise. Wenn finanzieller Ausgleich notwendig ist, beruht die Motivation von Landwirten auf mehr als monetären Überlegungen. Aus der Literatur ergeben sich Belege für Antriebskräfte wie Selbst-Identität, sozialer Druck und Naturverbundenheit. Daher stellt sich die Frage, wie solche alternativen Antriebskräfte durch politische Instrumente angesprochen werden können.

Wie können die umweltbezogenen Entscheidungen von Landwirten beeinflusst werden? Hier diskutieren wir Forschungsansätze zu Interventionen, die darauf abzielen, das Umweltmanagement von Landwirten zu verändern, und geben einen Überblick über erfolgreiche Ansätze zur Verhaltensbeeinflussung.

Empirische Arbeiten zu der Frage, wie das Natur- und Umweltschutzverhalten von Landwirten verändert werden kann, sind schwer zu finden. Die Studie von Lokhorst et al. (2010) berichtet über das Design und den Test einer Intervention, welche die Mechanismen Feedback und Selbstverpflichtung kombiniert. Feedback bedeutet, den Teilnehmern Informationen über ihr gegenwärtiges Verhalten zu geben. Wird diese Information mit dem Verhalten von anderen verglichen, kann dies soziale Normen in Bezug auf das fragliche Verhalten aktivieren. Wenn Teilnehmer eine Selbstverpflichtung eingehen, dann geben sie ein Versprechen oder eine formale Verpflichtung ab, ein bestimmtes Verhalten zu verändern. Wissenschaftliche Studien zeigen, dass Teilnehmer ihre Selbstverpflichtungen eher einhalten (für eine Meta-Analyse siehe Lokhorst et al. 2013). Selbstverpflichtungen sind daher ein vielversprechendes Instrument, um Verhaltensänderungen zu bewirken. In der Studie von Lokhorst et al. (2010) war insbesondere die Kombination von Feedback und Selbstverpflichtung effektiv. Landwirte, die beiden Interventionen ausgesetzt waren, berichteten eine stärkere Naturschutzabsicht, verbrachten mehr Zeit mit Naturschutz und erhöhten die Fläche mit natürlichen Habitaten. Diese Ergebnisse waren ausgeprägter für nicht-staatlich unterstützten als für staatlich unterstützten Naturschutz. 
Nach unserem Wissen ist die Arbeit von Lokhorst et al. (2010) die einzige Studie, die den Effekt von nicht-monetären Anreizen experimentell testet. Dies ist überraschend, da wiederholt nach Interventionen gerufen wurde, die nicht allein auf monetären Zuweisungen basieren (Burton und Wilson 2006; Burton und Paragahawewa 2011; de Snoo et al. 2013). Wie könnte ein solcher alternativer Ansatz zur Verhaltensbeeinflussung aussehen?

Wie oben gezeigt, behandelt das gegenwärtige System von Zuwendungen Landwirte als Individuen, die vorwiegend von finanziellen Anreizen angetrieben sind. Ein erster Schritt wäre es demgegenüber, die nicht-finanziellen (psychologischen) Motive zu beachten. Hier wäre etwa daran zu denken, die Selbst-Identität von Landwirten als naturorientierte Landschaftsschützer zu adressieren. „Labeling“6 bezeichnet den Prozess, bei dem ein bestimmter Teil der Identität hervorgehoben wird, indem diese Identität mit einem bestimmten Verhalten einer Person in Zusammenhang gebracht wird (Cialdini et al. 1998). In ihrer Kommunikation mit Landwirten könnten staatliche Stellen versuchen, Landwirte konsistent als Naturschützer anzusprechen (zu ,labeln“) und um auf diese Weise deren Selbst-Identität zu beeinflussen.

Eine andere Erfolg versprechende Möglichkeit könnte der Versuch sein, Landwirte eher als Gruppe und nicht als Individuen zu adressieren. Regionale, sozial eingebettete Organisationen, wie die Umweltkooperativen in den Niederlanden, sind auch für andere Länder als Brückenbauer und Moderator für kollektive AUKM vorgeschlagen worden (Franks und Mc Gloin 2007; Burton und Paragahawewa 2011; Mills 2012). Solche lokalen Organisationen könnten bei ihren Mitgliedern ein Gefühl des sozialen Drucks wachrufen, etwas für den Naturschutz zu tun, ihnen aber auch das Gefühl vermitteln, dass sie bei ihren Naturschutzanstrengungen unterstützt werden (van Dijk et al. 2015).

Die Anwendung verhaltenswissenschaftlicher Einsichten hat sich in den letzten Jahren über verschiedene Politikbereiche hinweg verbreitet (Dolan et al. 2012). Zunehmend im Zusammenhang mit dem „Nudging“-Konzept diskutiert, unterstreicht die Anwendung solcher Einsichten, dass bei vielen politischen Herausforderungen unserer Zeit die Notwendigkeit der Veränderung von Verhaltensmustern zentral ist (für den deutschen Kontext siehe Purnhagen und Reisch 2016). In Großbritannien hat das beim Büro des Premierministers angesiedelte Behavioural Insights Team (BIT) einen Rahmen zur Verhaltensbeeinflussung entwickelt, der aus vier grundlegenden Prinzipien besteht, die sowohl zur Entwicklung neuer Politiken wie zur kritischen Bewertung bestehender Politiken verwendet werden können. Um ein Verhalten zu beeinflussen, muss dieses 1) einfach, 2) attraktiv, 3) sozial und 4) zeitlich angepasst sein (EAST-Formel: Easy, Attractive, Social, and Timely; Behavioural Insights Team 2014).

Es leicht zu machen, verweist auf die Einfachheit oder Schwierigkeit, mit der Bürger das gewünschte Verhalten annehmen können. Der Wechsel von Opt-in zu Opt-out, das Design von Verfahren, die den bürokratischen Aufwand begrenzen, und vereinfachte Botschaften sind Beispiele für die Anwendung des EAST-Prinzips. Im Hinblick auf Naturschutz in der Landwirtschaft kann dies bedeuten, die bestehenden 
AUKM kritisch daraufhin zu bewerten, ob sie für Landwirtinnen und Landwirte zugänglich sind, und Wege zur Teilnahme aus Sicht der Landwirtinnen und Landwirte zu untersuchen.

Verhalten attraktiv zu machen bedeutet, die Aufmerksamkeit der Zielgruppe zu erlangen und sicherzustellen, dass die Anreize, die angeboten werden, für diese einen positiven Wert haben. Für den Naturschutz kann dies etwa bedeuten, Botschaften zu personalisieren und mediale Kanäle zu nutzen, die für die landwirtschaftliche Gemeinschaft relevant sind. Man könnte auch die gegenwärtigen Anreizsysteme überdenken, um zu sehen, ob diese für Landwirtinnen und Landwirte attraktiver gestaltet werden können. Einige Autoren haben beispielsweise vorgeschlagen, dass eine ergebnisorientierte Zahlung stärkere Resonanz mit dem Gefühl der Landwirte haben könnte, den Naturschutz aktiv gestalten zu können (de Snoo et al. 2013).

Verhalten sozial zu machen, bedeutet anzuerkennen, dass menschliches Verhalten üblicherweise in gesellschaftlichen Zusammenhängen stattfindet und dass das Verhalten anderer einen großen Einfluss ausübt. Um ein erwünschtes Verhalten zu ermutigen, kann man eine positive deskriptive Norm aktivieren, die darauf verweist, dass andere sich auch auf die erwünschte Weise verhalten; man kann bestehende Netzwerke und Gemeinschaften von gleichgesinnten Bürgern nutzen; und man kann versuchen, die Leute dazu zu bringen, dass sie Selbstverpflichtungen gegenüber ihren eigenen sozialen Bezugsgruppen abgeben, ihr Verhalten zu ändern. Besonders die beiden letzteren Vorschläge stimmen mit den oben genannten Studien überein, insbesondere mit denen zu Umweltkooperativen und zur Selbstverpflichtung zum Naturschutz (Lokhorst et al. 2010).

Zeitlich angepasst schließlich bezieht sich auf die zeitliche Ausgestaltung von Politiken. Dies bedeutet anzuerkennen, dass Menschen je nach Zeitpunkt unterschiedlich auf Aufforderungen reagieren. Dem EAST-Konzept zufolge sind Politiken dann am effektivsten, wenn sie die Menschen dann zu erreichen suchen, wenn diese am empfänglichsten sind, wenn sie die unmittelbaren Kosten und Vorteile abwägen und wenn die angebotenen Maßnahmen den Menschen helfen, ihre Handlungen in Bezug auf künftige Ereignisse zu planen. Im Fall des Natur- und Umweltschutzes müssen wir wissen, wann Landwirtinnen und Landwirte ihre Schlüsselentscheidungen treffen - zum Beispiel in einer bestimmten Jahreszeit - um sie dann zu erreichen, wenn es am sinnvollsten ist. Auch könnte es lohnender sein, kurzfristige und sehr spezifische Ziele zu setzen als Generalpläne zu entwerfen, die sich über lange Zeiträume erstrecken.

Insgesamt lässt sich feststellen, dass die gegenwärtigen AUKM in dem Sinne konzeptionell begrenzt sind, als sie Landwirte tendenziell lediglich als Individuen (und nicht als Mitglieder gesellschaftlicher Netzwerke) sehen, die vor allem durch Aussicht auf finanziellen Gewinn motiviert sind und weniger durch eine Verbundenheit mit der Natur. Bestehende Politiken und Programme könnten effektiver ausgestaltet werden, wenn sie verhaltenswissenschaftliche Erkenntnisse darüber berücksichtigen, a) welche Antriebe Landwirtinnen und Landwirte bei naturschutzbezogenen Entscheidungen motivieren und b) wie verhaltensorientierte Politiken generell effektiver gestaltet werden können. 


\subsubsection{Design von Agrar- und Umweltpolitik aus der Perspektive der Zahlungen für Umweltleistungen}

\subsubsection{Das Konzept der Zahlungen für Umweltleistungen}

Der Begriff „Zahlungen für Umweltleistungen“ (Payments for Environmental Services, PES) beschreibt ein positives Anreizinstrument, welches dazu verwendet werden kann, die Attraktivität umweltfreundlicher Praktiken (bspw. in der Landoder Forstwirtschaft) zu erhöhen und somit zum Schutz von Umweltleistungen beizutragen. Im Kern geht es um die Schaffung eines Marktes oder marktähnlicher Zustände: Bereitsteller von Umweltgütern (bspw. Landnutzer) können freiwillig an PES teilnehmen und erhalten von den Käufern eine Prämie entweder (i) für die direkte Bereitstellung der Leistung oder (ii) für das Ausführen einer Aktivität, die direkt mit der Bereitstellung der Leistung verknüpft ist (Engel 2015). Das Konzept ist besonders in Situationen geeignet, in denen der generierte gesellschaftliche Nutzen die privaten Kosten der Bereitstellung übersteigt (Pannell 2008). Es sind sowohl Konstellationen mit privatwirtschaftlichen (Coasean PES) als auch staatlichen Käufern denkbar (Pigouvian PES) (Engel et al. 2008). Ein bekanntes Beispiel für Coasean PES ist der Fall des französischen Trinkwasserherstellers Vittel (Perrot-Maître 2006). Dieser konnte sich nach intensiven Verhandlungen mit ansässigen Landwirten auf einen Preis für die gewässerschonende Bewirtschaftung der Ländereien im Umfeld von Trinkwassergewinnungsgebieten einigen. Staatlich finanzierte PES finden sich in vielen Industrienationen zur Abmilderung der negativen Auswirkungen intensiver Landwirtschaft auf die Umwelt. Dabei wird versucht, neben dem Ausgleich der entgangenen Gewinne, die sich aus der Anwendung umweltschonenderer Praktiken ergeben, einen positiven Anreiz für deren Einführung zu schaffen. Für die Bestimmung der Höhe des Anreizes gibt es unterschiedliche Ansätze: Aus Sicht der Kosteneffizienz erscheint es vorteilhaft, nur die Kosten der Aktivität selbst (bzw. der Bereitstellung der Umweltleistung) zu vergüten. Diese bestehen zum einen aus den Transaktionskosten, die sich zum Beispiel aus der Informationsbeschaffung und Antragstellung ergeben, und zum anderen aus den Opportunitätskosten, die durch den Wechsel der Landnutzung entstehen. Es ist jedoch auch möglich, durch die Zahlung einer zusätzlichen Prämienkomponente den Gewinn und damit auch den Anreiz der Landnutzer zur Teilnahme an PES zu erhöhen.

Weiterhin wird als wichtiges Designmerkmal mit Bezug zum Erfolg von PESInstrumenten deren Konditionalität angesehen (Engel 2015). Dies bedeutet, dass die Zahlung nur erfolgen sollte, wenn die Umweltleistung bzw. die dafür notwendige Maßnahme auch erbracht wird. Dies erfordert eine klare Definition des angestrebten Umweltziels bzw. der durchzuführenden Maßnahme sowie die Überwachung des Ergebnisses. In diesem Zusammenhang bezeichnet man als Additionalität den generierten Mehrwert eines Programms im Gegensatz zum Zustand bei dessen Nicht-Einführung (Engel 2015). Gerade im Bereich der Landwirtschaft ist es häufig schwierig, diesen Mehrwert genau zu erfassen. Dies liegt sowohl am komplexen Gefüge der Agrarökosysteme und am Vorhandensein paralleler, teilweise gegenläufiger Anreizstrukturen als auch an der diffusen Natur vieler negativer Umwelteffekte und 
den damit verbundenen Problemen bei deren Monitoring. Auch müssen zur Bewertung der ökologischen Effizienz eines PES-Programms sogenannte Leakage-Effekte (Engel 2015) mit einbezogen werden. Hierbei tritt eine zeitlich oder räumlich verschobene Intensivierung schädlicher Aktivitäten als (Spät-)Folge auf. Beispielsweise ist es möglich, dass Landnutzer nur mit solchen Flächen an einem Umweltprogramm teilnehmen, die eine geringere Produktivität aufweisen, während sie gleichzeitig die Landbewirtschaftung an anderer Stelle intensivieren. Auch ist die zeitlich verschobene Ausnutzung eventueller positiver Folgen eines Programmes auf die Bodenfruchtbarkeit denkbar, indem nach Ablauf des Vertrages vormals eingeschriebene Landflächen wieder intensiv bewirtschaftet werden.

Ob PES in der Landwirtschaft von Erfolg gekrönt sind, hängt nicht nur von der Ausgestaltung des Instruments selbst ab. Auch das Zusammenspiel mit anderen Instrumenten muss betrachtet werden (Segerson 2013). Ist beispielsweise das Ordnungsrecht bezüglich der Umweltvorgaben bereits relativ strikt, engt dies den Spielraum zur freiwilligen Vergütung darüber hinausgehender Leistungen ein. Umgekehrt muss das Ordnungsrecht aber auch eine Ausgangsbasis etablieren, auf deren Grundlage die freiwilligen Vereinbarungen operieren. Ohne eine solche Grundlage können PES keine anspruchsvollen Umweltziele anpeilen und Vergütungen müssten bereits für sehr geringe Leistungen vergeben werden. In Deutschland deckt das Ordnungsrecht hauptsächlich den abiotischen Ressourcenschutz ab, während aktives Umweltmanagement häufig Bestandteil von freiwilligen Vereinbarungen wie PES ist.

Basierend auf dieser Einführung soll das folgende Kapitel zunächst die Rolle von PES in der Gemeinsamen Agrarpolitik (GAP) der Europäischen Union (EU) aufzeigen. Im Anschluss daran werden innovative Ansätze vorgestellt, die sich dazu eignen können, die in der SWOT-Analyse (Kap. 5) dargelegten Probleme der entsprechenden Programme zu mindern und deren Effektivität und Akzeptanz zu steigern.

\subsubsection{Zahlungen für Umweltleistungen in der GAP}

Bereits seit 1992 ist es für die Mitgliedstaaten der EU verpflichtend, im Rahmen der Zweiten Säule der GAP sogenannte Agrarumwelt- und Klimamaßnahmen (AUKM) anzubieten. Damit werden umweltschonende landwirtschaftliche Praktiken bezeichnet (wie etwa Schutz- oder Blühstreifen, pestizidfreies Wirtschaften etc.), die einzelne Landwirte auf ihren Ländereien ausführen und damit zum Schutz der Umwelt beitragen können. Diese Maßnahmen lassen sich als eine Variante von PES verstehen (Sattler und Matzdorf 2013), bei denen der Staat (in seiner Funktion als Stellvertreter für die Gesellschaft) als einziger Käufer sowie als Preisgeber auftritt (Pigouvian PES). Mit Blick auf das Gesamtbudget der EU für AUKM handelt es sich damit um eines der größten PES-Programme dieser Art weltweit. Die genaue Ausgestaltung des angebotenen Maßnahmenkataloges obliegt dabei den Mitgliedstaaten. Die EU muss jeden dieser nationalen Kataloge einzeln billigen. In Deutschland werden die entsprechenden Maßnahmen-Portfolios auf Länderebene konzipiert. Dabei überwiegen derzeit konkrete Managementanweisungen, die einen geringen bis moderaten Aufwand erfordern und flächendeckend angeboten werden 
(in der Regel 5-Jahres-Verträge). Solchen ,hellgrünen“ AUKM lassen sich „,dunkelgrüne" gegenüberstellen, bei denen ein wesentlich höherer Aufwand betrieben werden muss und die teilweise nur in abgegrenzten Zielkulissen angeboten werden. Seltener lassen sich ergebnisorientierte AUKM finden, bei denen nicht bereits bei Ausführung einer Managementanweisung, sondern erst bei Erreichen eines zuvor definierten Ergebnisses vergütet wird. Einige Bundesländer wie etwa Niedersachsen bieten beispielsweise eine Zahlung für das Vorkommen bestimmter Zielarten auf Grünlandstandorten an. Obwohl das EU-Rahmenrecht auch eine gruppenorientierte Vergütung ermöglicht (siehe unten), wird derzeit von dieser Option in Deutschland kein Gebrauch gemacht. Bezüglich der Höhe der Zahlungen sind die EU-Regularien relativ strikt. AUKM werden in ihrer jetzigen Form nach WTORecht als „Green Box“-Zahlungen eingestuft, die lediglich für die Produktion nicht-warenförmiger Gemeingüter gewährt werden dürfen (Wynne-Jones 2013). Um diesen Status zu erhalten, wurde unter anderem eine vormals bestehende Anreizkomponente abgeschafft, sodass derzeit nur die durch die Teilnahme am AUKM-Programm entstehenden Kosten ersetzt werden (Hampicke 2013). Als gesetzlicher Sockel für AUKM fungieren die Cross-Compliance- und GreeningRegelungen. Das bedeutet, dass eine Maßnahme über die in den Cross-Complianceund Greening-Richtlinien gemachten Vorgaben hinausgehen muss, um überhaupt als vergütungswürdig eingestuft zu werden (einige AUKM können hingegen zur Erfüllung der ÖVF-Vorgaben herangezogen werden, allerdings werden dann Greening-Prämie und AUKM-Vergütung miteinander verrechnet). Die Einhaltung der Vorgaben wird durch ein Monitoring-Programm durchgesetzt, in dessen Rahmen jährlich $5 \%$ aller teilnehmenden Betriebe kontrolliert werden müssen.

An dieser Stelle soll angemerkt sein, dass es neben den AUKM in der EU noch weitere Möglichkeiten für PES gibt. Innerhalb der Zweiten Säule findet sich eine Vielzahl weiterer Programme, die als PES kategorisiert werden können. Ein Beispiel stellen die freiwilligen Vereinbarungen zum Gewässerschutz dar. Diesen Maßnahmenkatalog können unter anderem Wasserverbände nutzen, um mit Landwirten Verträge über gewässerschonende Praktiken abzuschließen.

Den AUKM-Programmen in der EU werden von wissenschaftlicher Seite einige Schwächen im Design vorgehalten, welche deren ökologische Effektivität sowie ökonomische Effizienz mindern. In der Literatur findet sich eine Vielzahl von Ansätzen, die unter bestimmten Bedingungen dazu geeignet sein könnten, diese Probleme zu adressieren (Wätzold und Schwerdtner 2005; Engel 2015). Einige davon werden im nachfolgenden Abschnitt vorgestellt.

\subsubsection{Gestaltungsansätze}

Ein zentrales Problem vieler AUKM-Programme ist ein Mangel an Zielgenauigkeit (Armsworth et al. 2012). Um diese Programme leichter administrierbar und kontrollierbar zu machen, wurden die Maßnahmen vielfach standardisiert. Durch diese „Vereinfachung“ ihres Designs geht ein Großteil des möglichen positiven Nutzens für die Biodiversität verloren (Armsworth et al. 2012). Daraus lässt sich ableiten, 
dass (a) mit den verfügbaren finanziellen Mitteln mehr im Sinne der Umwelt zu erreichen wäre und (b) ein Spielraum besteht, komplexere Designs mit höheren Transaktionskosten zu implementieren und dennoch den Natur- und Umweltnutzen zu erhöhen. Der Ansatz des „Targeting“ versucht, durch eine zielgenauere Verteilung der verfügbaren finanziellen Mittel die Kosteneffizienz bzw. den ökologischen Nutzen der AUKM zu maximieren (Segerson 2013). Die Hintergrundannahme ist dabei, dass durch die heterogenen Bedingungen der Ökosysteme und ihrer Bewirtschafter bestimmte Gebiete bzw. Landwirte besser geeignet sein können, eine Leistung zu produzieren, als andere (Khanna und Ando 2009). Dabei können prinzipiell folgende Varianten zum Einsatz kommen (Hanley et al. 2012; Engel 2015):

- Nutzen-Targeting: die Konzentration der Mittel in Gebieten oder bei Landwirten, die am ehesten einen ökologischen Effekt produzieren können (hoher erwarteter Nutzen);

- Kosten-Targeting: die Konzentration der Mittel in Gebieten oder bei Landwirten, die möglichst kostengünstig eine Leistung bereitstellen können (geringe erwartete Kosten);

- Kosten-Nutzen-Targeting: die Konzentration der Mittel in Gebieten oder bei Landwirten, die ein möglichst günstiges Kosten-Nutzen-Verhältnis bei der Bereitstellung einer Leistung aufweisen (gesellschaftlich erwünschte Balance der Kosten und des Nutzens).

Im Agrarumweltprogramm in Deutschland ist bereits ein gewisser Grad an Targeting realisiert, da einige Maßnahmen ausschließlich in speziellen Zielkulissen angeboten werden. Das betrifft zum Beispiel solche zum Schutz von Gewässern oder bestimmten Zielarten (z. B. Ortolan, Feldhamster, Rotmilan). Eine konsequentere Verfolgung dieses Ansatzes könnte geeignet sein, weitere Effizienzsteigerungen zu realisieren (Schuler und Sattler 2010; Meyer et al. 2015a). Konkret könnte eine Programmierung der Maßnahmen auf regionaler oder lokaler Ebene helfen, gezielter Umweltleistungen bereitzustellen und Mitnahmeeffekte zu verringern. Auch ist es möglich, einen Kosten-Nutzen-Index als Basis für eine Programmierung aufzustellen, um eine sinnvollere Verteilung der Fördermittel zu gewährleisten (Wünscher und Engel 2012). Dieser Index könnte zum Beispiel regionsspezifisch aufzeigen, welche Ökosystemleistungen oder Ziele (z. B. Artenschutz) an welchen Orten kostengünstig realisierbar sind. Eine Studie zum PES-Programm in Costa Rica konnte den erwarteten Nutzen eines solchen Index exemplarisch aufzeigen (Wünscher et al. 2006, 2008). Weiterhin ist durch eine Kombination mit RemoteSensing-Daten und GIS-basierter Software ein weiterer Effizienzgewinn zu erwarten (van Haaren und Bathke 2008; Arponen et al. 2013), da dies sowohl die Programmierung als auch die Antragstellung erleichtern könnte. Sollen multiple Ziele verfolgt werden oder unterliegen die Kosten und der Nutzen einer Maßnahme starken zeitlichen Schwankungen, können weniger zielgerichtete Instrumente wirkungsvoller sein (Segerson 2013; Uthes und Matzdorf 2013). Targeting eignet sich besonders dann, wenn begrenzte finanzielle Mittel möglichst effizient verteilt werden sollen und es eine große Varianz in den Kosten und/oder dem Nutzen einer Maßnahme gibt (Engel 2015). 
Ein weiteres Konzept zur Steigerung der Kosteneffizienz ist die Zahlungsdifferenzierung (Engel 2015). Hier wird versucht, den Landbewirtschaftern einen Betrag auszuzahlen, der ihren tatsächlichen Bereitstellungskosten entspricht oder geringfügig darüber liegt. Durch eine Differenzierung der Zahlungen wäre der Staat in der Lage, Landbewirtschaftern mit hohen Kosten entsprechend höhere Zahlungen zu gewähren, sollte deren ökologischer Beitrag dies rechtfertigen. Damit kann unter Umständen eine höhere Akzeptanz von Maßnahmen in Intensivgebieten erreicht werden, da hier die Opportunitätskosten meist weit über den derzeitigen Zahlungen liegen. Eine aktuelle Metaanalyse zur Wirksamkeit von PES-Programmen kommt zu dem Schluss, dass eine Zahlungsdifferenzierung eng mit der ökologischen Effektivität der Programme korreliert ist (Ezzine-de-Blas et al. 2016). Ein Problem bei der Umsetzung stellt jedoch die Tatsache dar, dass der Staat meist nicht genau bestimmen kann, wie hoch die Bereitstellungskosten einzelner Landwirte sind (Hanley et al. 2012). Sind diese Kosten stark durch lokal korrelierte Faktoren wie etwa die Bodenqualität beeinflusst, kann das Problem evtl. durch eine regionale oder lokale Differenzierung umgangen werden. Besteht diese Möglichkeit nicht, muss der Staat versuchen, einen Mechanismus zu etablieren, der die Bereitstellungskosten einzelner Landwirte offenlegt.

Ein Ansatz, der diese Anforderung erfüllen kann, ist der Gebrauch von Auktionen, mit denen die AUKM ähnlich einer Ausschreibung vergeben werden. Grundsätzlich gibt es unterschiedliche Varianten des Auktionsdesigns, die im Kontext des Umweltschutzes interessant sein können (Freese et al. 2011). Der Staat kann zum Beispiel definierte Maßnahmen oder Umweltleistungen ausschreiben und die Landwirte kompetitiv um deren Ausführung bzw. Bereitstellung bieten lassen (LataczLohmann und Van der Hamsvoort 1997). Durch den Bieterprozess verringern die Landwirte ihre Gebote sukzessive auf ein Niveau, das nahe dem ihrer Bereitstellungskosten liegt (Hanley et al. 2012). Damit wird die Kosteneffizienz gegenüber einer regionalen Durchschnittszahlung deutlich erhöht. Im Falle eines limitierten Gesamtbudgets für die Erreichung spezifischer Umweltziele kann der Staat entscheiden, welche Bieter den Zuschlag bekommen, und dadurch ein Kosten-Targeting realisieren. Ein häufig als positives Beispiel angeführter Fall ist das Conservation Reserve Program in den USA, das 1985 zum Zwecke des Erosionsschutzes ins Leben gerufen wurde und einen Kosten-Nutzen-Index zur Bewertung der Gebote nutzt (Claassen et al. 2008). In Deutschland gab es bisher nur einige Pilotprojekte. In der Region Northeim in Niedersachsen wurde der Auktionsansatz mit einer quasiexperimentellen Evaluierung verbunden (Ulber et al. 2011). Zwar konnte eine hohe Additionalität nachgewiesen werden, allerdings blieben die erwarteten Kostensenkungseffekte der Maßnahmen aus. Im Gegenteil, die Landwirte boten teilweise weit über ihren Opportunitätskosten und die Partizipation blieb insgesamt gering (Ulber et al. 2011). Ähnliche Erfahrungen wurden bei einem Projekt in der Region Fuhrberg gemacht, bei dem die Landwirte ihre geringe Partizipationsbereitschaft mit der Angst begründen, das Verfahren könnte die etablierten sozialen Strukturen in ihrer Gemeinschaft zerstören (van Haaren und Bathke 2008). Eine genügend große Anzahl an Teilnehmern ist bei Auktionen allerdings zwingend notwendig, um einen ausreichenden Wettbewerb zu gewährleisten (Uthes und Matzdorf 2013; Hanley 
und White 2014). Experimentelle Studien zeigen weiterhin, dass Auktionsteilnehmer bei wiederholten Auktionen lernen und sich daher im zeitlichen Verlauf durch strategisches Bieten die Kosteneffizienz wieder verringert (Schilizzi und LataczLohmann 2007). Ebenso zeigen die Modellvorhaben, dass Auktionsschemata mit hohen Transaktionskosten für beide Seiten verbunden sind (Glebe 2008; Ulber et al. 2011). Auch ist unklar, ob und inwieweit ein Auktionsdesign derzeit mit europäischem Recht vereinbar ist. Sicher ist, dass es eines umfangreichen Anerkennungsprozesses seitens der EU bedürfte (Freese et al. 2011).

Die ökologische Effektivität der Programme kann weiterhin auch durch das einseitige Vertrauen in die maßnahmenorientierte Honorierung verringert werden. Hier besteht das Risiko, dass die Landbewirtschafter die Managementanweisungen (a) falsch oder nur oberflächlich bzw. (b) überhaupt nicht durchführen. Je nach Monitoring-Häufigkeit und Sanktionshöhe mag es für die Landbewirtschafter sogar wirtschaftlich opportun sein, sich so zu verhalten (Becker 1974). Der Staat muss durch intensives Monitoring feststellen, ob die Landnutzerinnen und Landnutzer sich an die Anweisungen halten. Beim Ansatz der ergebnisorientierten Honorierung erhalten die Landbewirtschafter ihre Prämie nur, wenn sie ein vorher festgelegtes Niveau einer bestimmten Leistung erbringen. Auf diese Weise ist es für die Bereitsteller nicht nur weniger opportun zu schummeln, auch steht es ihnen frei, die anzuwendenden Managementpraktiken selbst zu wählen. So können private Informationen der Landwirte, beispielsweise darüber, wann, wie und wo ihr Land am besten zu pflegen ist, genutzt werden. Diese Informationen sind sonst für den Staat nicht nutzbar, obwohl ihre Nutzung durch die Landbewirtschafter häufig mit einer Steigerung der ökologischen Effektivität einhergeht (Hanley et al. 2012). Ebenso könnte es sich als vorteilhaft herausstellen, dass die Landwirte stärker intrinsisch motiviert sind, wenn sie ergebnisorientiert arbeiten, da sie unter Umständen einen Imagegewinn damit verbinden, Lernprozesse und Innovationen unterstützt werden und an die Produzenten-Ethik der Landnutzer appelliert wird (van Haaren und Bathke 2008; Matzdorf und Lorenz 2010; Schleyer und Plieninger 2011). Andererseits bedeutet diese Form der Vergütung auch ein erhöhtes Risiko. Verfehlt der Landwirt oder die Landwirtin trotz Anstrengungen das gesteckte Ziel, bekommen sie im äußersten Fall keine Prämie ausgezahlt und tragen die Kosten allein (Schleyer und Plieninger 2011). Als Lösungen werden neben Risikoprämien auch kombinierte Zahlschemata diskutiert, welche das Risiko zwischen Staat und Landbewirtschaftern aufteilen (Holm-Müller et al. 2006; Loisel und Elyakime 2006; Derissen und Quaas 2013; Hanley und White 2014). Weiterhin ist es möglich, eine schrittweise oder kontinuierliche Auszahlung einzuführen, bei der beispielsweise die Anzahl an vorkommenden Zielarten aus einer Liste vergütet wird. Der Ansatz der ergebnisorientierten Honorierung eignet sich vor allem dann, wenn die Ergebnisse kostengünstiger überwacht werden können als die Ausführung einer Maßnahme selbst. Der Erfolg des Instrumentes hängt maßgeblich vom Vorhandensein eines messbaren und praktikablen Indikators ab (Zabel und Roe 2009; Freese et al. 2011). Weder darf der Indikator einen zu hohen Grad an Ambivalenz zulassen, noch sollte er zu schwierig zu erfassen sein, da häufig die Landwirte selbst an der Monitoring-Prozedur beteiligt sind (Burton und Schwarz 2013). Auch sollten weder der 
Indikator selbst (z. B. im Falle einer Ziel-Art) noch seine Messung zu sehr mit gewöhnlichen landwirtschaftlichen Praktiken und Abläufen in Konflikt stehen (Burton und Schwarz 2013). In mehreren Pilotprojekten (van Haaren und Bathke 2008) wie auch in Befragungen mit Landwirten (Matzdorf und Lorenz 2010) konnte gezeigt werden, dass diese Art der Vergütung auf wesentlich mehr Akzeptanz stößt als beispielsweise die Auktionsverfahren. In mehreren Bundesländern wird daher bereits heute eine ergebnisorientierte Honorierung eingesetzt. Es handelt sich überwiegend um Programme zur Steigerung der Biodiversität in Graslandschaften, die als Indikator eine Liste mit Ziel-Arten nutzt. Doch auch eine Nutzung im Bereich des Nährstoffmanagements ist erprobt (Runge und Osterburg 2007). Für bestimmte Leistungen bleibt es jedoch kosteneffizienter, mit Managementvorgaben zu arbeiten, vor allem dann, wenn diese sich gut überwachen lassen (Moxey und White 2014).

Ein weiteres Argument in der Debatte um AUKM ist die Beobachtung, dass die geringe ökologische Effektivität mit der Tatsache zusammenhängen könnte, dass einzelne Landwirte einzelne Maßnahmen ergreifen und somit nur fragmentierte Wirkungen erzielt werden können (Concepción et al. 2008; Smits et al. 2008). Aus dieser Sicht mangelt es an einer Steuerung und Abstimmung der Aktivitäten unter den Landwirten. Aus diesem Grunde gibt es Überlegungen, die AUKM mit kooperativen Ansätzen zu verbinden. Die Idee dahinter ist einfach: statt wie bisher mit einzelnen Landwirten Verträge über AUKM zu schließen, sollen die Vereinbarungen mit Gruppen oder Zusammenschlüssen von Landnutzern abgeschlossen werden. Die Liste der möglichen Vorteile ist groß (Hodge und Adams 2013; Prager 2015):

- Ökologische Effekte: Verringerung der Habitat-Fragmentierung, Aufrechterhaltung eines Biotopverbundes, Erhöhung der Biodiversität, bessere Handhabung invasiver Arten und insgesamt erhöhte ökologische Effektivität der Programme.

- Ökonomische Effekte: Reduktion der Transaktionskosten für den Staat, z. B. durch verringerten Aufwand für Monitoring und Sanktionen (durch SelbstMonitoring innerhalb einer Gruppe), effiziente Kosten- und Aufgabenverteilung in der Gruppe und dadurch insgesamt eine verbesserte Kosteneffizienz der Programme.

- Soziale Effekte: Schnellere Kompromissfindung bei gegensätzlichen Interessen, Informationsaustausch und gegenseitige Hilfe in der Gruppe, flexible Lösungsfindung durch Aufbau technischer Expertise und erhöhter sozialer Interaktion, erhöhte Selbstwirksamkeit.

- Möglichkeiten zur Kombination mit Auktionen und ergebnisorientierter Honorierung.

Auf der anderen Seite wird argumentiert, zu viele Variablen würden den Erfolg kooperativer Ansätze beeinflussen, so dass die Landwirtinnen und Landwirte keinen Anreiz sähen, sich diesem Risiko auszusetzen. Es mangele an Vertrauen und Zeit, die Gruppen aufzubauen, die Absprachen innerhalb der Gruppe erhöhten die Transaktionskosten einzelner Landwirtinnen und Landwirte und letztlich würden Landwirte lieber vermeiden, Teile ihres Einkommens von anderen abhängig zu machen (Emery und Franks 2012; Uthes und Matzdorf 2013; Prager 2015). Auch gibt es 
verschiedene Möglichkeiten, die Zahlungen zu arrangieren (Engel 2015): Entweder die Zahlung geht direkt an einzelne Landwirte oder an die Gruppe als Ganzes, welche die Verteilung dann selbst übernimmt. Evidenz über die Wirksamkeit konnte bisher in verschiedenen Pilotprojekten mit unterschiedlichem Erfolg gesammelt werden (Prager 2015). Eine großskalige Implementierung des Ansatzes findet derzeit in den Niederlanden statt. Als besonders förderlich erweist sich in diesem Zusammenhang, dass es hier bereits langjährige Erfahrungen mit Umweltkooperativen in der Landwirtschaft gibt (van Dijk et al. 2015). Seit 2016 werden die AUKM ausschließlich über 40 regionale Umweltkooperativen abgewickelt, welche die Funktion als Vertragsnehmer gegenüber dem Staat übernehmen. Aus Sicht des Staates wird damit die Anzahl der Anträge um ein Vielfaches reduziert. Innerhalb der jeweiligen Kooperative kann dann die Verteilung der Aufgaben und Mittel autonom abgestimmt werden.

Eine weitere und eng mit den kooperativen Ansätzen verwandte Möglichkeit zur Erzielung von Effekten auf der Landschaftsebene stellt der sogenannte Agglomerationsbonus dar (Parkhurst et al. 2002). Adressat dieses Anreizinstruments kann sowohl eine Gruppe als auch eine einzelne Person sein. Je nach Design erhalten beispielsweise Landwirtinnen oder Landwirte, die sich entschließen, mit einer Parzelle teilzunehmen, einen Bonus ausgezahlt, sollte diese an eine ebenfalls eingeschriebene Parzelle eines Nachbarn angrenzen. In Laborexperimenten konnte die Wirksamkeit dieses Anreizes zum Herstellen verschiedener Landschaftsstrukturen bereits bewiesen werden (Parkhurst et al. 2002; Parkhurst und Shogren 2007). Die Teilnehmer benötigten allerdings einige Zeit, um den Mechanismus zu erlernen und geeignete Kommunikationsstrukturen aufzubauen, so dass in jedem Falle Transaktionskosten anfallen (Banerjee et al. 2015). Durch die Unterschiede in den Bereitstellungskosten einzelner Landwirte könnten auch Ausgleichszahlungen der Landwirte untereinander die Performanz des Instrumentes verbessern (Wätzold und Drechsler 2014). Weiterhin bestätigen auch verhaltensökonomische Studien dessen Nutzen. So kann ein solcher Bonus die Erwartungen der Landwirte über die Teilnahme ihrer Nachbarn verändern und sich daher wie eine soziale Norm auswirken (Kuhfuss et al. 2015). Anwendung in der Praxis findet der Agglomerationsbonus unter anderem in den USA (Conservation Reserve Enhancement Program) und der Schweiz (Hanley et al. 2012).

Ein letzter Designaspekt, der in der Literatur diskutiert wird, sind die sogenannten gesamtbetrieblichen Verpflichtungen. Dabei wird argumentiert, dass nur dann, wenn die Managementpraktiken eines ganzen Betriebes umgestellt würden, langfristige ökologische Wirkungen zu erwarten seien und ein tatsächlicher Paradigmenwechsel in der Landwirtschaft greifbar würde (Emery und Franks 2012; Meyer et al. 2015a). Fraglich ist dabei jedoch, wie ein solcher „Ganz-odergar-nicht"-Ansatz, bei dem quasi direkt auf eine umweltschonende Bewirtschaftung auf allen Ländereien eines Betriebes umgestellt werden muss, bei einer Mehrheit der Landwirtinnen und Landwirte auf Akzeptanz stoßen kann. Derzeit gibt es in Deutschland in vielen Bundesländern die Maßnahme „Ökologischer Landbau", die allerdings immer Teil eines größeren Portfolios mit anderen Maßnahmen ist. 


\subsubsection{Partizipatorische Ansätze}

In dieser Literaturübersicht soll das Potenzial partizipatorischer Ansätze zur Mobilisierung und Vernetzung unterschiedlicher Akteure dargelegt werden. Vernetzte Akteure können helfen, agrarische Systeme durch einen stetigen Lernprozess nachhaltig und adaptiv zu managen. Eine Schlüsselrolle spielt dabei vor allem der Zugang zum vielfältigen Wissen der beteiligten Akteure. Dieses Wissen kann helfen, Probleme und Lösungen im Bereich der Governance natürlicher Ressourcen zu identifizieren (Pahl-Wostl 2015). Hinzu kommt, dass die Beteiligung von Interessenvertretern an der Entscheidungsfindung die Legitimität der getroffenen Entscheidungen erhöht (OECD 2002).

Partizipationsmöglichkeiten für die breite Öffentlichkeit oder lokale Interessenvertreter werden mittlerweile regelmäßig zur Unterstützung von (Raum-)Planung, Politikgestaltung und Entscheidungsfindung angeboten. Sie gelten als Schlüsselelement im Management natürlicher Ressourcen. Der Ansatz gibt sowohl staatlichen als auch nicht-staatlichen Akteuren die Möglichkeit, gemeinsam Lösungen zu finden, die insbesondere auch lokale und regionale Interessen wahren. Im Prozess der kooperativen Entscheidungsfindung gewinnen die Teilnehmer ein gemeinsames Verständnis der Problemlagen und möglicher Lösungen und können so die Vor- und Nachteile alternativer Politikoptionen und Maßnahmen realisieren (Basurto und Ostrom 2009). Durch die Erhöhung der Akzeptanz alternativer Politikoptionen, insbesondere solcher mit einem starken Nachhaltigkeitsbezug, können partizipative Ansätze nachweislich zu einer erfolgreicheren Politikgestaltung beitragen. Die Implementierung der Europäischen Wasserrahmenrichtlinie (WRRL) unter Beteiligung der Landwirtschaftskammern ist ein gut dokumentiertes Beispiel hierfür. Es handelt sich jedoch um eines von bisher nur sehr wenigen im Bereich der Agrarpolitik.

Die Dokumentation von Anwendung und Effektivität partizipatorischer Ansätze im Bereich der Landwirtschaft und der Agrarpolitik im deutschen und europäischen Kontext ist limitiert. Aus diesem Grunde deckt dieser Literaturüberblick auch weitere Bereiche des Managements natürlicher Ressourcen ab, in denen die Anwendung partizipatorischer Instrumente systematischer untersucht wurde. Dazu zählen beispielsweise die Sektoren des Forst- und des Wassermanagements. Mit großem Aufwand wurde hier unter anderem die Effektivität partizipatorischer Ansätze bei der Implementierung nachhaltiger Praktiken mit Bezug zur WRRL empirisch untersucht.

Die erfolgreiche Implementation von Maßnahmen zur nachhaltigen Landnutzung hängt von einer Reihe von Faktoren ab. Nach (Penker 2002) werden neben qualifiziertem Personal und dem Willen aller beteiligten Interessengruppen, auch eine angemessene Kombination von Maßnahmen sowie eine kontinuierliche und adaptive Planung benötigt. Durch die Kooperation mit Naturschutzorganisationen sind staatliche Behörden in der Lage, wertvolles lokales Wissen zu sichern und von einem positiveren Ansehen in der Region zu profitieren. Die Beteiligung von lokalen Interessengruppen und eine dezentrale Entwicklung von Strategien erhöhen die Wahrscheinlichkeit einer erfolgreichen Maßnahmenimplementation. 
Die sozialen Aspekte eines Projektes können als Bestimmungsfaktoren der lokalen Akzeptanz und Machbarkeit angesehen werden. Um die Akzeptanz innerhalb der Interessengruppen zu erhöhen, ist es insbesondere wichtig, die Sprache der Experten in die Sprache der lokalen Öffentlichkeit und der lokalen Entscheidungsträger zu „übersetzen“. Runde Tische und andere Kommunikationsformen erhöhen das gegenseitige Verständnis und damit auch die Akzeptanz (Luz 2000). Eine OECD-Studie aus dem Jahre 2001 evaluierte 4 verschiedene Initiativen im Bereich des Haushaltskonsums in den Niederlanden, Dänemark, den USA und Australien, um den Einfluss von partizipativen Entscheidungsverfahren auf Verhalten und Umweltbewusstsein zu demonstrieren (Geyer-Allély und Zacarias-Farah 2001). Die Fallstudien heben den Wert partizipativer Verfahren für eine Erhöhung des Umweltbewusstseins und des Verständnisses der Umweltpolitik, die Einflussnahme auf neue Politiken sowie eine Veränderung des Konsumentenverhaltens hervor.

Im Bereich der Landwirtschaft ist die Partizipation in der Form von Produzentengruppen vor allem im Bereich kleiner Betriebe vorzufinden, z. B., um so die Wünsche der Konsumenten besser erfüllen oder strukturelle Probleme frühzeitig erkennen und adressieren zu können (AIOTI WG06 2016). Kollaborationen zwischen den verschiedenen Akteuren der Wertschöpfungskette (beispielsweise Landwirtschaftsverbände, Konsumentenverbände oder kleine und mittlere Unternehmen) erhöhen Nahrungsmittelsicherheit und Nachvollziehbarkeit (AIOTI WG06 2016). Der Multiakteursansatz wird für ein großskaliges EU-Pilotprojekt im Bereich der intelligenten Landwirtschaft und der Nahrungsmittelsicherheit genutzt. Federführend ist hier die „Alliance for the Internet of Things Innovation“ der Europäischen Kommission. Sie testet neue Technologien im Agrar- und Nahrungsmittelsektor (AIOTI WG06 2016).

Partizipation wird weiterhin auch als essenzieller Bestandteil bei der Entwicklung von Visionen angesehen. Die Evaluierung eines partizipativen Verfahrens mit multiplen Akteuren in Flandern hebt einige essentielle Faktoren für die erfolgreiche Entwicklung gemeinsamer Visionen hervor. Das flämische Policy Research Centre for Sustainable Agriculture entwickelte einen Multiakteursdialog zur Entwicklung einer Vision für die Landwirtschaft in Flandern. Nevens et al. (2008) identifizieren mehrere Schlüsselelemente eines solches Prozesses, die dazu beitragen können, Menschen zu mobilisieren und die entwickelte Vision zu verankern: „Entdeckung von zentralen Werten, Angabe einer präzisen Mission, Aufstellung leitender Grundprinzipien und das Arbeiten mit lebendigen Beschreibungen der ins Auge gefassten zukünftigen Systeme.“

Juerges und Newig (2015) untersuchten Interessengruppen in Deutschland, die Entscheidungsprozesse im Bereich des Waldmanagements beeinflussen. Das mehrstufige Governance-System in Deutschland (im Besonderen Bund und Länder) und die europäische Ebene haben erheblichen Einfluss auf die Effektivität dieser Gruppen. Die Komplexität des Governance-Systems verlangt von den ökologischen wie ökonomischen Interessengruppen eine hohe Anpassungsfähigkeit, da das System selbst nicht statisch ist und ständigen Veränderungen unterworfen ist. Die Ergebnisse der Studie legen nahe, dass Interessengruppen diese Herausforderung wahrnehmen und lernen, ihre Strategien entsprechend anzupassen. 
Innerhalb des Wassersektors wird vor allem durch die Wasserrahmenrichtlinie (Richtlinie 2000/60/EG) deutlich, welche Vorteile, aber auch welche Herausforderungen durch partizipative Prozesse im Bereich des Ressourcenmanagements entstehen. Die Richtlinie verlangt von den Mitgliedstaaten, die interessierte Öffentlichkeit und Interessensgruppen aktiv in den Entscheidungsprozessen zu beteiligen. Artikel 14, Absatz 1 der Richtlinie besagt: „Die Mitgliedsstaaten fördern die aktive Beteiligung aller interessierten Stellen an der Umsetzung dieser Richtlinie, insbesondere an der Aufstellung, Überprüfung und Aktualisierung der Bewirtschaftungspläne [...]“. Damit konnte die Wasserrahmenrichtlinie dazu beitragen, ein partizipatives Mehrebenen-Governance-System zu schaffen und große, von den Interessengruppen getriebene EU-Forschungsprojekte umzusetzen (u. a. HarmoniCA, HarmoniCOP, NeWater, SPI-Water). Ferner führte sie zur Publikation verschiedener Leitlinien und Handbücher (Ridder et al. 2005; van Bers et al. 2011) sowie empirischer Studien, die versuchen, die Effektivität der partizipativen Prozesse in der EU zu evaluieren und zu verbessern (van Ast und Boot 2003; Newig et al. 2005; Huitema et al. 2009). Nach Newig und Koontz (2014) führt die Anordnung von partizipativen Planungsprozessen bei der Umsetzung von großen Politikinitiativen wie etwa der EU-Wasserrahmenrichtlinie und der EU-Hochwasserrisikorichtlinie zu einem zukunftsorientierten und stärker durch die Gesellschaft unterstützen Politikprozess. Die Wasserrahmenrichtlinie war dabei im Wesentlichen erfolgreich bei der Einbringung organisierter Interessen und weniger bei denen der breiten Öffentlichkeit. Im Gegensatz dazu partizipierten bei der Umsetzung der Hochwasserrisikorichtlinie wesentlich mehr Mitglieder der Öffentlichkeit, möglicherweise da hier das öffentliche Interesse stärker direkt betroffen war (Newig et al. 2014).

Die derzeitige Entwicklung deutet an, dass im Konsens verfasste Lösungen, partizipative Planungsansätze und Modelle für ein individuelles Marktmanagement immer wichtiger werden (Luz 2000; Penker 2002). Weiterhin führt die Umsetzung von Umweltschutzzielen auf der lokalen Ebene zu einer verstärkten Bedeutung lokaler Nichtregierungsorganisationen (Penker 2002).

Werden in einer Region neue Politik-Instrumente erfolgreich getestet und dabei neues Wissen gewonnen, können diese Ergebnisse unter Umständen auch auf andere Regionen übertragen werden. Beispielsweise können Politikempfehlungen formuliert werden, die darlegen, welche Aktionen in den Bereichen Umweltschutz, Raumplanung und ländliche Entwicklung auf den verschiedenen Ebenen (regional, national, supranational) umgesetzt werden sollten (GREENNET 2010).

Die Ko-Produktion von Wissen ist ein weiterer Ansatz zur Verbesserung der Einflussmöglichkeiten der Öffentlichkeit und auch der Wissenschaft an sich. Das Cornell Lab of Ornithology an der Cornell University entschied sich nach einer Durchsicht vieler ähnlicher US-basierter Programme, ein breiteres Spektrum der Öffentlichkeit einzubeziehen, da diese häufig in Programmen für den Umweltschutz unterrepräsentiert sind (Porticella et al. 2013). Die Ergebnisse sind vielversprechend und haben solche Faktoren identifiziert, die zu einer erfolgreichen öffentlichen Partizipation in Forschungsprogrammen führen können. 


\subsubsection{Instrumente auf der Konsumentenebene}

Nur eine integrierte Politik für den Nahrungssektor als Ganzes (von der Produktion bis zur Konsumption) kann einen signifikanten positiven Effekt auf den Agrarbereich haben und zu einer nachhaltigen Entwicklung führen (Reisch 2006). Konsumenten spielen eine zentrale Rolle in der Ausrichtung der Landwirtschaft (nachhaltig bzw. nicht nachhaltig) und des Naturschutzes. AIOTI WG06 (2015, S. 4) betont: „Die Nachfrage der Konsumenten ist derzeit die stärkste Triebkraft, die dazu führt, dass die Nahrungsmittelindustrie gesündere und sicherere Produkte herstellt, die den höchsten Qualitätsanforderungen genügen“. Die Wahl der Nahrung spielt ebenfalls eine zentrale Rolle für Investitionen der Landwirte in Möglichkeiten zur Herstellung hochwertiger und nachhaltig angebauter Produkte.

Es gibt zahlreiche Studien zum relativen Einfluss verschiedener Maßnahmen, die versuchen, die Wahl der Nahrungsmittel zu beeinflussen, um eine nachhaltigere Landwirtschaft anzutreiben. Dazu zählen das Produktlabeling, die Direkt- bzw. Lokalvermarktung, verschiedene Informations- und Bildungsangebote sowie die Einbeziehung von Schlüsselgruppen mit Relevanz für die menschliche Ernährung. Letzteres können sowohl Individuen oder Haushalte sein, aber auch Schulen, Restaurants und andere Institutionen, in denen Lebensmittel zubereitet und verzehrt werden. Obwohl bekannt ist, dass die Art und Weise des Nahrungsmittelkonsums signifikante Auswirkungen auf die Umwelt haben kann, wurde bis zuletzt der Bewältigung von Problemen auf der Seite der Nahrungsmittelproduzenten mehr Aufmerksamkeit zuteil. In der letzten Dekade war schließlich festzustellen, dass ein stärkerer Fokus auch auf die Einkaufsgewohnheiten und die Nahrungsmittelzubereitung sowie die Verzehrsgewohnheiten gelegt wurde. Eine Veränderung dieser Gewohnheiten kann positive Auswirkungen auf unser Nahrungsmittelsystem generieren.

Der für Nahrungsmittel ausgegebene Anteil des verfügbaren Einkommens eines typischen deutschen Haushalts hat innerhalb des 20. Jahrhunderts stetig abgenommen. Anfang der 1960er-Jahre betrug der Anteil noch ca. ein Drittel des verfügbaren Einkommens. Heute liegt unter $15 \%$ (Statista 2014). Darin spiegelt sich wider, dass die verfügbaren Einkommen stärker gestiegen sind als die Preise für Nahrungsmittel, weil die Einkommenselastizität der Nachfrage nach Lebensmitteln geringer ist als nach anderen Konsumgütern. Preisorientierte Verbraucherinnen und Verbraucher wählen bei vergleichbarer Qualität das günstigere Produkt und verlassen sich in Deutschland und Europa auf die Sicherheit und Umweltfreundlichkeit der auf den Markt kommenden Erzeugnisse. Die Nachhaltigkeitsimplikationen der Nahrungsmittelerzeugung sind - wie bei anderen Produkten in komplexen Wertschöpfungsketten auch - für die Endverbraucher häufig nicht ohne Weiteres erkennbar. Gleichzeitig werden große Mengen Nahrungsmittel weggeworfen. Aus einer rein ökonomischen Perspektive widerspricht dies der Forderung nach günstigem Essen. Der Wert des Nahrungsmittelabfalls in Deutschland im Bereich der Haushalte wird auf ca. $935 € / J a h r$ für eine vierköpfige Familie geschätzt (Kranert et al. 2012).

Aktuelle Trends im Bereich des Konsums in Deutschland wie auch in anderen Industrie- und Schwellenländern erfordern eine Steigerung der weltweiten Nahrungsmittelproduktion, die bei derzeitigen Produktionsmethoden erhebliche Auswirkungen 
auf das Klima, die Luft-, Wasser-, und Bodenverschmutzung, die Ressourcenausbeutung, den Habitatwandel und Biodiversitätsverlust haben werden. Die erhöhte Nachfrage nach Lebensmitteln (und anderen landwirtschaftlichen Erzeugnissen wie Biomasse und Bioenergie) führt zu Preiserhöhungen selbst für Grundnahrungsmittel, die arme Bevölkerungsgruppen in Entwicklungsländern einem erhöhten Risiko von Hunger und Unterernährung aussetzt. Diese Auswirkungen und Trends sind mittlerweile gut dokumentiert (Rockström et al. 2009b; Hertwich et al. 2010; FAO et al. 2014; Rockström et al. 2015).

Bestimmungsfaktoren des Nahrungsmittelkonsums: Obwohl sich zunehmend ein Bewusstsein über die Risiken und Auswirkungen des nicht-nachhaltigen Konsums herausbildet, wandelt sich die tatsächliche Nachfrage nur langsam. Dies liegt unter anderem an der geringen Aufmerksamkeit für die systemischen Ursachen und komplexen Wechselwirkungen der Nachhaltigkeitsproblematik. Ein Teil der Forschung widmet sich derzeit dem politischen und ethischen Konsum, untersucht die Beweggründe und Auswirkungen konsumorientierter Lebensstile und stellt eine Verbindung zwischen der Forschung zu sozialen Bewegungen und Studien zu Konsumverhalten her (Wahlen und Laamanen 2015). Die Analysen identifizieren die folgenden Faktoren für nicht-nachhaltigen Konsum: die Zahlungsbereitschaft der Konsumenten, die Qualität des Nahrungsmittel (Nährwert, Verfügbarkeit, Sicherheit, Geschmack, Aussehen), Werbung, Labels und Zertifikate, Bequemlichkeit und kulturelle Akzeptanz (Hughner et al. 2007; Han und Hansen 2012). Dieses Wissen kann genutzt werden, um Ansätze, die darauf abzielen, das Konsumentenverhalten zu beeinflussen, hinsichtlich ihrer voraussichtlichen Wirksamkeit zu bewerten.

Folgende Faktoren motivieren Konsumenten zu einem nachhaltigeren Konsum:

1. Verbesserte Information über die Nahrung und ihre Herstellung (Produktion, Verarbeitung und Verteilung) durch innovative Labels und Werbemaßnahmen.

2. Regulatorische Ansätze (z. B. Steuern auf nicht-nachhaltige Lebensmittel) und freiwillige bottom-up Maßnahmen (z. B. fleischfreie Tage in öffentlichen Kantinen)

3. Bildungs- und Weiterbildungsangebote (z. B. Lebensmittellehre und Kochkurse in Schulen)

4. Verbesserter Zugang zu qualitativ hochwertigen Nahrungsmitteln in Supermärkten, Restaurants und anderen Lebensmittelgeschäften

Produktinformationen und Labels: Der konventionelle Ansatz zur Information der Konsumenten über die Qualität und die Eigenschaften bezüglich Herstellung und Verteilung eines Produktes beinhaltet eine Kombination aus Werbung, Verpackung und Labels. Das digitale Zeitalter eröffnet neuartige Wege für die Konsumenten, sich über ihre Einkäufe zu informieren. Das Internet hat somit eine Optimierung der Wertschöpfungskette ermöglicht. Eine stetig wachsende Innovation der letzten zwei Jahrzehnte ist E-Commerce. Ein geläufiges Beispiel ist das Bestellen und Bezahlen von Lebensmitteln im Internet mit anschließender Lieferung an die private Adresse. Als vorteilhaft stellen sich hier insbesondere der reduzierte $\mathrm{CO}_{2}$-Ausstoß durch eine Verringerung der Nutzung privater PKW sowie die verbesserte Verfügbarkeit von Detailinformationen zu den eingekauften Produkten 
heraus (Dannenberg und Franz 2014). Seit Kurzem ermöglichen Anwendungen auf Mobilgeräten das Einscannen von Barcodes durch die Konsumenten, um dadurch mehr Informationen über die angebotenen Produkte abrufen zu können. Ein Beispiel ist das sogenannte „OpenLabel“, das den Konsumenten gezielte Informationen bezüglich der Nachhaltigkeit der gescannten Produkte darbietet (The Guardian 2015). Eine abschließende Bewertung der positiven wie negativen Effekte dieser Innovationen von wissenschaftlicher Seite steht jedoch noch aus.

Um bewusste Entscheidungen treffen zu können, benötigen Konsumenten produktbezogene Informationen bezüglich der Inhaltsstoffe sowie der Produktionsund Verarbeitungsbedingungen der Nahrungsmittel. Informationen zu Inhaltsstoffen sind oft kompliziert und schwer verständlich. Die Informationen zu den Produktions- und Verarbeitungsverfahren sind oft minimal. Verbraucherinnen und Verbraucher orientieren sich daher oft an Labels. Wissenschaftliche Studien belegen, dass sich Konsumenten ein breit gefächertes Angebot an Labels wünschen, angefangen bei ökologischen und ethischen Belangen bis hin zu solchen mit Informationen zum Nährwert (Tansey und Worsley 2014). Dabei ist jedoch zu beachten, dass die relative Wichtigkeit dieser unterschiedlichen Arten von Labels je nach Nation oder Region sehr unterschiedlich ausfallen kann. Beispielsweise legen Menschen in Europa (Spanien, Italien und Frankreich) im Vergleich zu Nordamerika größeren Wert auf produktionsbezogene Faktoren, wie etwa die Qualität der Böden, das Klima und die Berücksichtigung traditionellen Wissens, die als Bestimmungsfaktoren der Nahrungsqualität dienten (Bureau und Marette 2000). Dies spiegelt sich auch in der EU-Politik in Bezug auf freiwillige Nahrungsmittel-Labels, wie die Kennzeichnung geschützter regionaler Herkünfte und traditioneller Lebensmittel, wider (Bureau und Valceschini 2003).

Sofern die ökologische Landwirtschaft mit erhöhten Leistungen im Natur- und Umweltschutz gleichgesetzt werden kann, dürfen Öko-Labels in Europa und insbesondere in Deutschland als erfolgreich in der Ermöglichung umweltfreundlicher Konsummuster angesehen werden. Für die Kennzeichnung ökologisch hergestellter Produkte müssen gesetzlich definierte Anforderungen erfüllt werden. Bei der Zertifizierung können hohe Gebühren anfallen. Nach dem Forschungsinstitut für biologischen Landbau (FiBL) und der International Federation of Organic Agriculture Movements (IFOAM) war der größte Markt im Jahre 2012 für biologisch angebaute Produkte in Deutschland angesiedelt. Insgesamt wurden hier 7 Mrd. $€$ umgesetzt, gefolgt von Frankreich mit 4 Mrd. $€$ und dem Vereinigten Königreich mit umgerechnet 1,95 Mrd. € (Willer und Lernoud 2014). Demnach war Deutschland für ein Drittel der Nachfrage für ökologisch erzeugte Produkte in der EU verantwortlich. In Deutschland wurden im Jahr 2014 insgesamt 1.032.941 ha biologisch bewirtschaftet (Willer und Lernoud 2014). Dies war jedoch nicht ausreichend, um die Nachfrage in Deutschland zu decken. Bis heute halten finanzielle, administrative und aufwandsbezogene Hindernisse viele Landwirte davon ab, auf die biologische Landwirtschaft umzusteigen. Politikmaßnahmen sollten daher darauf abzielen, diese Hindernisse zu minimieren. Es muss jedoch auch konstatiert werden, dass aus Sicht vieler Landwirte der Wechsel zu ökologischer Produktion nicht ihre vorrangigen Probleme adressiert (Kuhnert et al. 2005). 
Labels im Bereich landwirtschaftlicher Produkte sind ein Teilbereich einer wesentlich breiteren gesellschaftlichen Diskussion. Allerdings kann ein engerer Fokus auf Labels zum Zwecke des Naturschutzes einige innovative Möglichkeiten offenlegen. Der Wert der regionalen Direktvermarktung landwirtschaftlicher Produkte zum Zwecke des Umweltschutzes wurde bereits 2006 vom BfN unterstrichen (Gehrlein und Fick 2007). Ein Treffen zu diesem Thema gipfelte in einer Reihe von Empfehlungen für Maßnahmen zur Stärkung von Netzwerken und Kooperationen zwischen Akteuren des Naturschutzes und des regionalen Marketings. Ein weiterer Ansatz ist das sogenannte, ,landscape labeling“6, das Produktzertifizierung mit Zahlungen für Umweltleistungen verknüpft (Ghazoul et al. 2009, 2011). Die Vergabe eines „Landschaftslabels“ für Produkte, die Ökosystemleistungen sowie von der Gemeinschaft definierte kulturelle und symbolische Werte bereitstellen, hat das Potenzial, besser als herkömmliche Labels wahrgenommen zu werden und Landwirten den Zugang zu Nischenmärkten zu erleichtern. Der praktischen Implementation stehen allerdings noch einige Hindernisse im Wege, wie etwa der Bedarf nach „fairen und transparenten Gemeinschaftsinstitutionen“ und das Risiko der Ausnutzung durch „Free-Rider“. Im Rahmen der Europäischen Grünen Woche 2016 gab es bereits einen Workshop zu diesem Thema. Dieser beschäftigte sich damit, wie „Landschaftslabels“ die multifunktionelle Landwirtschaft unterstützen können. ${ }^{31}$

Regulatorische und freiwillige Maßnahmen: Die am weitesten verbreitete regulative Maßnahme zur Lenkung der Entscheidungen von Konsumenten ist die Besteuerung von solchen Produkten, die besonders ressourcenintensiv sind oder die Umwelt verschmutzen. Diese Steuer fungiert dann als Anreiz für einen nachhaltigeren Konsum. Als Alternative eignet sich auch die Subventionierung nachhaltiger Produkte bzw. Nahrungsmittel. Studien in Großbritannien versuchen derzeit, die Auswirkungen einer solchen Steuer auf die Gesellschaft und die Umwelt zu evaluieren. Zum Beispiel hätte eine Besteuerung von rotem Fleisch das größte Potenzial, den Ausstoß von klimaschädlichen Gasen zu reduzieren. Kehlbacher et al. (2016) beziffern, dass eine Steuer von $2841 \mathrm{GBP} / \mathrm{t} \mathrm{CO}_{2}$ auf alle in Großbritannien verkauften Lebensmittel, die durch den Nahrungsmittelsektor verursachten Emissionen um $6,2 \%$ senken könnte. Weiterhin könnte eine Steuer auf Lebensmittel mit überdurchschnittlich hohen Emissionen zu einer Reduktion von 4,3\% führen. Allerdings hätte dies negative Umverteilungseffekte für Menschen mit geringeren Einkommen.

Regulative Maßnahmen können auch zur Beeinflussung von Werbung und Marketing eingesetzt werden, so dass nachhaltigere Produkte bevorzugt gekauft werden. Solche Praktiken sind im Bereich der Genussmittel (Alkohol, Tabak) bereits weit verbreitet, jedoch gibt es keine verlässlichen Analysen zur Performanz dieser Instrumente im Lebensmittelbereich (OECD 2016). Ein gutes Beispiel ist die EU-Verordnung, die für genetisch veränderte Nahrungsmittel eine entsprechende Kennzeichnung vorschreibt.

Im Bereich der freiwilligen Maßnahmen gibt es innerhalb Europas unzählige Beispiele für Initiativen zur Veränderung der Konsummuster und zur Erhöhung der Nachhaltigkeit des Nahrungsmittelsektors. Auf der internationalen Ebene gibt es

\footnotetext{
${ }^{31} \mathrm{http} / / / \mathrm{www}$.greenweek2016.eu/partner-event/the-potential-of-labelling-in-landscape-management-1.
} 
freiwillige Standards wie den „Marine Stewardship Council““ und die „Rainforest Alliance“. Produzenten und weiterverarbeitende Betriebe in der Nahrungsmittelbranche können sich für die angebotenen Zertifikate qualifizieren, um somit besser für Verbraucherinnen und Verbraucher sichtbar zu sein. Wenn auch etwas weniger stringent, können lokale Initiativen Leitlinien für die Verwendung nachhaltiger Produkte etwa für Restaurants herausgeben oder fleischlose Tage in Mensen und Cafeterien begleiten.

Die Beschaffung von nachhaltig produzierten Lebensmitteln im öffentlichen Sektor ist ein weiteres Feld, das in Deutschland derzeit große Aufmerksamkeit genießt und das Potenzial hat, Bewusstsein und Verhalten der Konsumenten positiv zu beeinflussen. Eine nachhaltige und sozial verantwortliche öffentliche Beschaffung erlangt in Europa stetig eine größere Bedeutung und ist häufig sogar von den Regierungen vorgeschrieben. Beispielsweise müssen in Deutschland Organisationen auf der Länderebene sowohl bei der Wahl der Energieversorgung als auch beim Kauf von holzbasierten Produkten explizit Nachhaltigkeitsaspekte mit einbeziehen (Kahlenborn et al. 2011). Ein ähnliches Modell wäre auch für die Beschaffung von Nahrung und Catering denkbar. Fünf EU-Länder haben bereits ein solches Ziel formuliert (Kahlenborn et al. 2011).

Nichtregierungsorganisationen haben ebenfalls eine große Bedeutung in der öffentlichen Information zu Themen wie nachhaltiges Essen und Landwirtschaft. Prominente Organisationen sind hier z. B. der BUND, NABU und Greenpeace. Die Rolle von lokalen Organisationen oder Einrichtungen darf nicht unterschätzt werden. Projekte wie Gemeinschaftsgärten unterstützen die Vernetzung und Mobilisierung und können dazu beitragen, das Interesse und das Bewusstsein für nachhaltige Ernährung zu verstärken.

Eine weitere freiwillige Maßnahme stellt die Gründung lokaler „Ernährungsräte“ dar (Pothukuchi und Kaufman 1999; Morgan 2015). Diese Institutionen sollen u. a. Produzenten und Konsumenten vernetzen und damit ländliche Regionen besser an urbane Zentren koppeln. Die Räte sind oft gemeinsame Initiativen von lokalen Verwaltungen und gesellschaftlichen Gruppen, um mehr Nachhaltigkeit im Nahrungsmittelsektor durch Forschung, Bildung, Lobbyarbeit und gesellschaftliche Entwicklung zu fördern. Die ersten Ernährungsräte sind in den 1980er-Jahren in den USA entstanden. Mittlerweile finden sich immer mehr dieser Räte auch in europäischen Städten. Im Jahr 2016 wurden entsprechende Institutionen z. B. in Berlin und Köln eingerichtet. Diese Räte sind oft Zusammenschlüsse von lokalen Regierungen (z. B. Stadt Köln) und lokalen Gruppen oder Organisationen, um ein nachhaltiges Ernährungssystem durch Forschung, Erziehung, Lobby-Arbeit, Serviceangebote in Bezug auf Essen und Kommunalentwicklung zu fördern. Der Rat in Berlin fungiert unter dem Motto: „Ernährungsrat in Berlin für eine zukunftsfähige Ernährungs- und Landwirtschaftspolitik in der Region“.

Aus- und Weiterbildung: Schulen und Universitäten haben ein großes Potenzial, den Nahrungsmittelkonsum der zukünftigen Generationen zu beeinflussen. Allein in Deutschland gibt es zahlreiche Initiativen an Bildungsinstitutionen. Diskutiert werden u. a. die Wiedereinführung von Hauswirtschafts- und Ernährungslehre in die Lehrpläne an Schulen sowie Angebote von Kochkursen für Erwachsene 
(Dyen und Sirieix 2016). Dazu zählt beispielsweise das Lehren von Konzept und Praxis der nachhaltigen Lebensmittelbeschaffung und die Zubereitung in Kochkursen von Berufskollegs und Berufsfachschulen (z. B. am Berufskolleg Tecklenburger Land in Ibbenbühren). Obwohl Hauswirtschaftslehre nicht verpflichtend ist, wird es wieder in als ein wichtiger Teil der Lehre in Sekundarschulen wahrgenommen. Ebenfalls in dieser Kategorie anzusiedeln sind Kurse zu Ernährungs- und Hauswirtschaftslehre an Hochschulen (z. B. das Masterprogramm „Nachhaltige Dienstleistungs- und Ernährungswirtschaft" an der FH Münster). Ein anderes Beispiel ist das Programm „CookUOS“ der Universität Osnabrück, welches unter dem Dach des UNESCO-Weltaktionsprogramms „Bildung für nachhaltige Entwicklung“ steht. Es vermittelt einer breiten Öffentlichkeit aus Lehrkräften, Eltern und Studierenden Wissen über den Beitrag des Kochens zur Nachhaltigkeit. Durch offene Kochvorführungen und Seminare bilden sich Teilnehmer zu nachhaltiger Ernährung und nachhaltigem Essen.

Zugang zu nachhaltig produzierten Nahrungsmitteln: Die große Mehrheit der Konsumenten erwirbt Nahrungsmittel in Supermärkten. In Deutschland haben insbesondere die so genannten Discounter einen großen Marktanteil. 32 \% des Umsatzes im Lebensmitteleinzelhandels fallen in ihre Kategorie, während beispielsweise in Frankreich diese Form der Supermärkte nur einen Anteil von 6,6 \% am Markt hat (Tackett 2014). Dies verstärkt die Herausforderungen in Bezug auf das Konsumverhalten der Menschen in Deutschland. Es muss jedoch konstatiert werden, dass einige Discounter mittlerweile einzelne Produktlinien eingeführt haben, die regionale, biologische oder fair gehandelte Waren enthalten. Die Marktführer der Branche nach Verkäufen in 2013 waren Edeka (50 Mrd. €), Rewe (36 Mrd. €) und Lidl (34 Mrd. €) (Tackett 2014). Die großen Einzelhandelsketten haben daher einen erheblichen Einfluss auf die ökologische Qualität der Produkte, die ihren Kundinnen und Kunden angeboten werden.

Der Gegenpol zu Supermärkten sind Wochenmärkte, lokale Hofläden oder regionale Lieferdienste für landwirtschaftliche Produkte. Diese Formen der Direktvermarktung zwischen Landwirt und Konsument haben in Deutschland eine lange Tradition. In Europa wird diese Entwicklung maßgeblich von der Nachfrage nach hochwertigen Nahrungsmitteln getrieben (Renting et al. 2003). Die Märkte eröffnen für viele Landwirte die Gelegenheit, ihr Einkommen unter Umgehung der herkömmlichen Vermarktungsketten und -wege zu erhöhen. Diese Art der Vermarktung kann auch für Landwirte attraktiv sein, die vor den hohen Kosten und dem administrativen Aufwand einer Zertifizierung als Ökobetrieb zurückschrecken. Wochenmärkte verbessern nicht nur die Verfügbarkeit lokal und regional produzierter Nahrungsmittel, sondern stärken auch die Bindung zwischen Landwirten und Konsumenten sowie zwischen ländlichen und urbanen Gebieten. Sie führen weiterhin zu einer Erhöhung des Bewusstseins in der breiten Bevölkerung, da die Konsumenten in die Lage versetzt werden, mehr über die Bedingungen zu lernen, unter denen ihre Nahrungsmittel hergestellt wurden.

Ein stärkere Verbindung und mehr Kontakt zwischen Konsumenten und Erzeugern kann ein wichtiges Element einer umfassend ansetzenden Strategie zur Verbesserung der Naturschutz- und Umweltleistungen der Landwirtschaft sein, 
denn positive Einstellungen zu Natur- und Umweltschutz sind weit verbreitet. Solche umweltfreundlichen gesellschaftlichen Normen können durch Interaktion aktiviert und verstärkt werden. Dies wiederum kann einerseits zu einer erhöhten Zahlungsbereitschaft für Produkte mit erhöhter Natur- und Umweltschutzleistung beitragen, zum anderen aber auch nicht-monetäre Motive für den Natur- und Umweltschutz stärken.

\subsection{Fazit}

Die hier vorgestellte Analyse der agrarpolitischen Rahmenbedingungen zeigt, dass sich die GAP historisch als Einkommenspolitik für den Agrarsektor etabliert hat. Auch wenn Anliegen des Natur- und Umweltschutzes in die GAP Einzug gehalten haben, besteht nach wie vor eine durch institutionelle Pfadabhängigkeit verstetigte Dominanz der Einkommenslogik gegenüber den anderen Zielen der GAP. Allerdings ist parallel zur GAP ein ordnungsrechtlicher Rahmen entstanden, der den Anliegen des Natur- und Umweltschutzes zur Geltung verhelfen soll. Bei der Durchsetzung des Natur- und Umweltschutzes bestehen jedoch Regelungs- und Implementationsdefizite, die zumindest teilweise auf eine Sonderbehandlung des Agrarsektors hindeuten. Die in der einkommenspolitischen Ausrichtung der GAP institutionalisierte Sonderbehandlung der Agrarwirtschaft im Vergleich zu anderen Wirtschaftssektoren - der landwirtschaftliche Exzeptionalismus (Skogstad 1998; Daugbjerg und Feindt 2017) - kommt damit auch im Ordnungsrecht zum Ausdruck. Die Einbeziehung der Agrarmarktordnungen in die allgemeinen Regelungen des Binnenmarkts eröffnen jedoch die Perspektive, dass im Agrarsektor in Zukunft die von den Europäischen Verträgen geforderten hohen Standards im Umwelt- und Verbraucherschutz verstärkt und konsequenter zur Geltung gebracht werden. Weiterhin könnte sich aus den möglichen haftungsrechtlichen Implikationen der zunehmend anspruchsvolleren Verbraucherschutzstandards innerhalb von Wertschöpfungsketten zunehmender Handlungsdruck ergeben. Angesichts offener Märkte für Agrarerzeugnisse kommt auch den transnationalen privatrechtlichen Standards erhöhte Bedeutung zu, weil sich deren Anforderungen auch auf die Importware erstrecken. Neben den neueren rechtlichen Entwicklungen stehen neue konzeptionelle Ansätze, aus denen sich innovative Perspektiven für eine bessere Integration des Natur- und Umweltschutzes in die Landwirtschaft ergeben. Konzepte wie Resilienz, Ökosystemleistungen und Wasser-Energie-Nahrungs-Nexus eröffnen die Perspektive eines integrierten Ressourcenmanagements in agrarischen Produktionssystemen und auf Landschaftsebene. Allerdings sind diese Ansätze noch nicht durchweg politiktauglich operationalisiert. Demgegenüber sind verhaltenswissenschaftliche Ansätze zur Ausgestaltung des Regelungsrahmens hinreichend ausgearbeitet, um Hinweise auf konkrete Verbesserungen des agrarpolitischen Instrumentariums zu ermöglichen. Dabei sollten insbesondere nicht-monetäre Verhaltensmotive verstärkt berücksichtigt werden, um zu einer effektiveren und effizienteren Politikgestaltung zu gelangen. Schließlich weisen partizipatorische Ansätze, wie 
neuere Initiativen zur besseren Integration von landwirtschaftlicher Produktion einerseits und Verbraucherverhalten und Ernährungsgewohnheiten andererseits, in Richtung einer gesellschaftlich integrierten Agrar- und Ernährungspolitik.

Im weiteren Verlauf des Projekts konnten nicht alle diese Perspektiven gleichermaßen verfolgt werden. Die Aufgabenstellung des Projekts erforderte eine Konzentration auf das agrarpolitische Instrumentarium im engeren Sinne. Bei der Entwicklung von Politik-Optionen hat das Projektteam jedoch Wert darauf gelegt, dass diese mehr Spielräume für eine Weiterentwicklung von stärker integrativen, kooperativen und lernorientierten Ansätzen bieten. Bevor wir zur Entwicklung von alternativen politischen Handlungsoptionen kommen, diskutieren wir im folgenden Kapitel zunächst die Stärken und Schwächen der derzeitigen Agrarpolitik. Diese SWOTAnalyse bildet die methodische Brücke zwischen der wissenschaftlich basierten Analyse und der Entwicklung und Bewertung politischer Gestaltungsoptionen.

Open Access Dieses Kapitel wird unter der Creative Commons Namensnennung 4.0 International Lizenz (http://creativecommons.org/licenses/by/4.0/deed.de) veröffentlicht, welche die Nutzung, Vervielfältigung, Bearbeitung, Verbreitung und Wiedergabe in jeglichem Medium und Format erlaubt, sofern Sie den/die ursprünglichen Autor(en) und die Quelle ordnungsgemäß nennen, einen Link zur Creative Commons Lizenz beifügen und angeben, ob Änderungen vorgenommen wurden.

Die in diesem Kapitel enthaltenen Bilder und sonstiges Drittmaterial unterliegen ebenfalls der genannten Creative Commons Lizenz, sofern sich aus der Abbildungslegende nichts anderes ergibt. Sofern das betreffende Material nicht unter der genannten Creative Commons Lizenz steht und die betreffende Handlung nicht nach gesetzlichen Vorschriften erlaubt ist, ist für die oben aufgeführten Weiterverwendungen des Materials die Einwilligung des jeweiligen Rechteinhabers einzuholen.

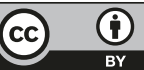




\section{Kapitel 5}

\section{SWOT-Analyse der derzeitigen Agrarpolitik aus Sicht des Natur- und Umweltschutzes}

\subsection{Vorgehen bei der SWOT-Analyse}

SWOT-Analysen sind ein etabliertes und weit verbreitetes Instrument der Strategieentwicklung. Sie ermöglichen eine systematische und verdichtete Bestandsaufnahme und Diskussion derjenigen Aspekte einer Politik, Institution oder Organisation, die besonderer Beachtung bedürfen. Stärken und Schwächen beziehen sich dabei auf interne Aspekte, Chancen und Risiken auf den Kontext der analysierten Einheit. SWOT-Analysen sollen dabei helfen, bei der Formulierung von Entwicklungsstrategien bestehende Stärken zu nutzen, Schwächen abzubauen oder zu kompensieren, Chancen zu nutzen und Risiken zu begegnen.

Im Projekt ZA-NExUS dient die SWOT-Analyse als Brücke zwischen der Bestandsaufnahme der Probleme auf Basis der bestehenden Literatur und der Entwicklung von neuen Handlungsansätzen. Zur Erstellung der SWOT-Analyse haben die Projektpartner in einem ersten Schritt auf Basis ihrer jeweiligen Literaturauswertungen die Stärken, Schwächen, Chancen und Risiken der derzeitigen Agrarpolitik aus Sicht des Natur- und Umweltschutzes zusammengefasst. Auf dieser Grundlage erstellte der Projektkoordinator eine Synthese, die auf dem Projektworkshop am 12. April 2016 diskutiert und in der Folge weiterentwickelt wurde.

Generell ist anzumerken, dass einige der Aussagen in der SWOT-Analyse aus Sicht des Natur- und Umweltschutzes ambivalent sein können. So stellen beispielsweise die generell hohe Stabilität des politischen und finanziellen Rahmens sowie die hohe Legitimationswahrnehmung unter Landwirten einerseits eine Stärke der derzeitigen Agrarpolitik dar. Aus Sicht des Natur- und Umweltschutzes ist dies jedoch nur insofern positiv, wenn angenommen wird, dass die Agrarpolitik insgesamt zu einem höheren Niveau des Natur- und Umweltschutzes beiträgt. Wenn aber die genannten Stabilitätsfaktoren zur Abwehr neuer, legitimer gesellschaftlicher Anliegen oder gar zur Ignorierung neuer Herausforderungen führen, können sie auch zur Schwäche werden, vor allem, wenn sie die Lernfähigkeit der Akteure oder die Anpassungsfähigkeit des Systems vermindern. 


\subsection{Stärken der derzeitigen europäischen Agrarpolitik}

Aus Sicht der GAP stellt die generelle Stabilität des politischen Rahmens sicherlich eine Stärke dar. Die Ziele der GAP sind in den Europäischen Verträgen verankert. Es gibt in Brüssel eine eigene Generaldirektion und in den meisten Mitgliedstaaten ein eigenes Agrarministerium.

Eine zweite wichtige Stärke ist der mittelfristig verlässliche finanzielle Rahmen. Zudem scheint bei vielen Mitgliedstaaten die Bereitschaft zu bestehen, die GAP auch über 2020 hinaus mit erheblichen Finanzmitteln auszustatten.

Drittens basiert die GAP auf einem relativ breit aufgestellten ideellen Paradigma. Die Nutzung von Ideen aus dem produktivistischen, dem neoliberalen und dem Multifunktionalitätsdiskurs erleichtert zum einen die Konsensbildung. Sie ermöglicht zum anderen aber auch einen vielfältigen und zielgruppenspezifisch differenzierten Legitimationsdiskurs durch (selektive) positive Darstellung gegenüber verschiedenen Adressatengruppen.

Viertens sind die Maßnahmen und Programme der GAP WTO-konform, das heißt, es drohen keine Handelskonflikte aufgrund der erheblichen staatlichen $\mathrm{Zu}$ wendungen an den Agrarsektor.

Aus Sicht der Anhänger des Status quo besteht eine Stärke der GAP darin, dass verschiedene Mechanismen ein positives, d. h. selbstverstärkendes PolicyFeedback sicherstellen. Dazu zählen die starke Unterstützung bei den Landwirtinnen und Landwirten, die institutionellen Eigeninteressen zahlreicher agrarpolitisch spezialisierter Administrationen sowie die Stabilisierung der GAP durch die verteilungsorientierten Verhandlungsmechanismen zwischen den Mitgliedstaaten auf EU-Ebene. Zu dieser Stabilisierung trägt auch die ideologische Verknüpfung mit dem breit akzeptierten ,europäischen Modell der multifunktionalen und bäuerlichen Landwirtschaft" bei. Aus Sicht der Kritiker des Status quo handelt es sich bei diesen Mechanismen jedoch um ausgeprägte Schwächen der GAP, welche eine Anpassung an neue Herausforderungen verzögern oder sogar unmöglich machen.

Aus Sicht des Natur- und Umweltschutzes stellt die Integration von Natur- und Umweltbelangen eine Stärke der GAP dar. Diese Feststellung schließt jedoch nicht aus, dass andere natur- und umweltpolitische Strategien u. U. erfolgreicher sein könnten. Die Cross-Compliance-Verpflichtungen, also die Verknüpfung der Direktzahlungen mit einem Minimum von Naturschutz- und Umweltanforderungen, bilden bei konsequenter Implementation eine flächendeckende Baseline. Es steht eine breite Instrumentenkiste von den flächendeckenden Instrumenten in der Ersten Säule bis zur Ermöglichung regional differenzierter Strategien in der Zweiten Säule zur Verfügung. Nach einzelnen Aussagen von Landwirtinnen und Landwirten tragen die umweltpolitischen Aspekte der GAP auch dazu bei, dass sie sich mehr mit Umweltfragen beschäftigen. Falls die GAP tatsächlich das Umweltbewusstsein unter Landwirtinnen und Landwirte verstärkt, wäre dies ebenfalls eine Stärke.

Dass die Erste Säule mit ihren Direktzahlungen einen Stabilisierungseffekt für die Einkommen landwirtschaftlicher Betriebe hat, ist aus Sicht des Einkommensziels der GAP sowie der betroffenen Empfänger sicherlich eine Stärke. Der Umfang 
dieses Effekts wird jedoch durch Überwälzungseffekte auf den Pacht- und Bodenmärkten vermindert. Angesichts der Höhe der Greening-Zahlungen besteht eine geringe „Ausstiegsgefahr“ aus dem System der Direktzahlungen und damit den Cross-Compliance-Auflagen. Über Cross-Compliance- und Greening-Auflagen sowie mögliche Kürzungen der Direktzahlungen als Sanktion bei Verstößen besteht die Möglichkeit, auf alle landwirtschaftlichen Flächen Einfluss zu nehmen - auch auf produktionsintensive Flächen, auf denen andere freiwillige Maßnahmen bei derzeitigem Kompensationsniveau kaum umgesetzt würden und welche jedoch oft unter starkem ökologischen Druck stehen. Dieser Effekt hängt ab von a) der Opt-out-Architektur des Greenings (das heißt, die landwirtschaftlichen Betriebe sind erst einmal ins Greening einbezogen), b) der verbreiteten Wahrnehmung unter Landwirten, dass man das Greening machen muss, sowie c) dem relativ hohen Zahlungsniveau.

Die Agrarumweltmaßnahmen in der Zweiten Säule enthalten eine Reihe von Maßnahmen für den Umwelt- und Naturschutz (Biodiversität, gefährdete Arten, Landschaftsheterogenität, Gewässer- und Erosionsschutz). Landwirtschaftliche Flächen auf ertragsschwachen Böden können durch Agrarumweltmaßnahmen noch in der Produktion gehalten werden (Schutz vor Brachfallen). Die AUKM leisten einen Beitrag zum Erhalt der Kulturlandschaft und von traditionellen Bewirtschaftungsweisen sowie zum Erhalt „,bäuerlicher“ Strukturen (Erfahrungswissen, Agrarkultur, soziale Funktionen im ländlichen Raum). Eine prinzipielle Stärke, die aber nicht immer eingesetzt wird, ist die Möglichkeit zur leistungsorientierten Vergütung und für kollektive Maßnahmen. Dass viele „hellgrüne“ Maßnahmen den Einstieg für Landwirte in dieses Betätigungsfeld erleichtern, kann ebenfalls als Stärke angesehen werden wie die Festschreibung eines Mindestanteils von $30 \%$ der Mittel in den ländlichen Entwicklungsprogrammen für freiwillige Maßnahmen im Bereich der Umwelt- und Klimaschutzes. Eine weitere Stärke ist auch die Einbettung in Beratungsprogramme, welche die Landwirtinnen und Landwirte bei der Umsetzung von AUKM unterstützen.

\subsection{Schwächen der derzeitigen europäischen Agrarpolitik}

Die Gemeinsame Agrarpolitik ist tief geprägt durch die Dominanz der Einkommenslogik für die landwirtschaftliche Bevölkerung. Das Einkommensziel ist der GAP als Kern eingeschrieben. Durch die Umwandlung der Marktkompensation in eine Flächenprämie ist der redistributive Charakter der GAP sichtbar geworden. Dies erhöht den Legitimationsbedarf. Es bestehen dringende Fragen hinsichtlich der Zielgenauigkeit der redistributiven Komponente und der ineffektiven Mittelverwendung bei den leistungsbezogenen Komponenten. In beiden Punkten besteht Evidenz für erhebliche Defizite, aus denen eine „Drohkulisse“ für die Fortsetzung der bisherigen Politik erwächst.

Die Naturschutz- und Umweltwirkungen der GAP sind nicht befriedigend. In ihrer derzeitigen Ausgestaltung ist die GAP nicht effizient im Erreichen von 
Naturschutz- und Umweltzielen, auch weil diese im Vergleich zu den einkommenspolitischen Zielen keine Priorität erhalten,. u. a. schreiten die Biodiversitätsverluste in der Agrarlandschaft fort. Im agrarpolitischen Diskurs werden Maßnahmen zum Erhalt der Biodiversität vorwiegend als Einschränkung bzw. Last aus Sicht der Landwirte gesehen, während positive Leistungen der Biodiversität (z. B. durch Ökosystemleistungen wie Bestäubung und natürliche Schädlingskontrolle) nur am Rande behandelt und nicht systematisch abgesichert werden. Zudem werden negative Umweltauswirkungen andernorts ausgelöst und in Kauf genommen, z. B. durch den Import von Futtermitteln aus Ländern mit niedrigeren Naturschutz-, Umwelt- und Sozialstandards.

Im Bereich der Normsetzung bestehen erhebliche Schwächen. In einigen Bereichen des Agrarumweltrechts bestehen keine Zielwerte bzw. die bestehenden Zielwerte sind unzureichend, um Verbesserungen im Natur- und Umweltschutz zu erreichen. Die ordnungsrechtlichen Mindestanforderungen sind zum Teil zu gering, um Naturressourcen und Umweltgüter überall hinreichend zu schützen.

Schwächen im Bereich der Agrarumweltpolitik sind sicherlich auch die komplexen und aufwändigen Entscheidungs- und Programmierungsstrukturen, welche diesen Politikbereich zum Monopol für ausgewiesene Spezialisten machen und jede Partizipation erschweren. Die anhaltende institutionelle Abschirmung des Kernbereichs der GAP gegen Umwelt- und Naturschutzakteure und gegen Umweltund Naturschutzpolitiken stellt angesichts der Dringlichkeit des Natur- und Umweltschutzes sicherlich eine weitere Schwäche der GAP dar. Der institutionell verankerte agrarpolitische Exzeptionalismus ist zudem schwer mit dem veränderten Verständnis des Binnenmarkts im Primärrecht zu vereinbaren.

Im Bereich von Implementation und Monitoring weist die derzeitige Politik für den Agrarsektor erhebliche Schwächen auf. Es bestehen signifikante Implementationsdefizite im Ordnungsrecht. Auflagen und Gesetze im Natur- und Umweltschutz werden vielerorts nicht eingehalten. Die Datenlage ist lückenhaft und viele Daten sind für ein Erfolgs-Monitoring schwer zugänglich. Die Implementationsdefizite werden durch die Komplexität der verwaltungstechnischen Arrangements verschlimmert. Die Kontroll- und Sanktionsmechanismen sind insofern mangelhaft, als hohem Kontrollaufwand in einigen Bereichen Kontrolldefizite in anderen Bereichen gegenüber stehen. Der Verwaltungsaufwand ist hoch, v. a. bei der Zweiten Säule, für Cross Compliance und Greening. Eine weitere Schwäche ist die ungleichmäßige Belastung der landwirtschaftlichen Betriebstypen durch die Auflagen, die indirekt zu einer Verstärkung des Strukturwandels beträgt.

Durch das System der Direktzahlungen sind die Kosten der GAP sichtbar geworden. Das trägt zwar zur verbesserten Transparenz der gesellschaftlichen Kosten wie der sektoralen Umverteilungspolitik bei. Durch die weitgehende Steuerfinanzierung wird die GAP in Zeiten knapper öffentlicher Haushalte aber anfälliger für finanzpolitische Verteilungskämpfe. Eine Schwäche der GAP besteht in diesem Umfeld darin, dass die GAP sich schwer tut, den gesellschaftlichen Nutzen der Agrarausgaben zu belegen. 
Eine wichtige Schwäche der derzeitigen Agrarpolitik ist ihre begrenzte Lernfähigkeit und Partizipation. Die agrarpolitischen Lernprozesse sind langsam und die GAP weist eine begrenzte Anpassungsfähigkeit durch die starke Orientierung an etablierten Paradigmen auf. Eine damit zusammenhängende Schwäche ist die vorrangige Orientierung an der Erhaltung bestehender Strukturen statt an einer sozial-ökologischen Transformation der landwirtschaftlichen Systeme, um mehr Ökosystemleistungen bei zumindest gleich bleibender Produktivität zu ermöglichen. Die Bürgerbeteiligung jenseits etablierter Verbände ist gering. Die vorwiegend einzelbetrieblich ausgerichteten Maßnahmen enthalten wenige Anreize zum Handeln auf kollektiver oder landschaftlicher Ebene.

Die Erste Säule der Agrarpolitik mit den Direktzahlungen weist verschiedene Schwächen auf: eine geringe Leistungsorientierung der Zahlungen; Unklarheit der Zielsetzung (die Einkommens- und Verteilungsziele werden nicht spezifiziert); keine Berücksichtigung der Motivation von Landwirten zur Erbringung von Umweltleistungen; vielerorts erhebliche Überwälzung der Zahlungen auf Pachtpreise (je nach Pachtflächenanteil und Region); und die Einjährigkeit der Maßnahmen im Greening begrenzt die möglichen ökologischen Erfolge.

Auch die Agrarumwelt- und Klimaschutzmaßnahmen in der Zweiten Säule sind nicht ohne Schwächen: Viele Maßnahmen sind nicht auf der räumlichen Skala definiert, die für Umwelt- und Biodiversitätsziele relevant ist. Der Erfolg der Agrarumweltmaßnahmen ist oft relativ gering. Die länderspezifischen Verfahren der Programmierung in der Zweiten Säule sind komplex und die Umwelt- und Naturschutzaspekte sind oft nur einer unter vielen Gesichtspunkten. Innerhalb der Zweiten Säule besteht eine Mittelkonkurrenz der AUKM mit anderen Programmen. Es fehlt vielfach an einer sektorenübergreifenden Planung. Durch die Notwendigkeit der nationalen (und sub-nationalen) Kofinanzierung sind die Programme im Vergleich zu den zu $100 \%$ von der EU finanzierten Direktzahlungen bei den agrarpolitischen Akteuren in den Mitgliedstaaten eher weniger beliebt. Der Erfolg der AUKM hängt ab von der Bereitschaft der Landwirte teilzunehmen - und damit von fluktuierenden Marktund Pachtpreisen. Aufgrund ihrer Komplexität ist der Erfolg vieler AUKM zudem abhängig von unterstützender Beratung und Information. Das Policy-Design der AUKM weist insofern Schwächen auf, als nur wenige Ansätze andere als finanzielle Motivationen zur Erbringung von Umweltleistungen ansprechen. Vielerorts werden die Anreizwirkungen der AUKM zudem durch andere Anreizinstrumente wie etwa die Biogasförderung unterlaufen. Laut WTO-Recht ist es zudem nicht möglich, bei der Vergütung von AUKM eine Anreizkomponente vorzusehen (allerdings bestehen bei der Berechnung der pauschalierten Kosten Spielräume, diese Maßnahmen so zu vergüten, dass sie für Landwirtinnen und Landwirte attraktiv sind). Weitere Schwächen sind der Umstand, dass nur relativ wenige AUKM zielgerichtete „,dunkelgrüne“ Maßnahmen enthalten, der generell hohe Bürokratieaufwand sowie das erhöhte Kontroll- und Anlastungsrisiko bei Teilnahme an AUKM im Vergleich zu einer Nicht-Teilnahme. Weitere Schwächen sind die oft zu geringe regionale Ausdifferenzierung, Mitnahmeeffekte und Überkompensation vor allem bei ,hellgrünen“ Maßnahmen sowie die geringe Reichweite der AUKM in Gebieten mit Intensivlandwirtschaft. 
Generell erscheint es als eine Schwäche der Agrarpolitik, dass sie systemischen Risiken im Vergleich zu den einkommenspolitischen Themen relativ wenig Aufmerksamkeit widmet, auch wenn es bei vielen Beteiligten durchaus ein Bewusstsein dafür gibt. Die neuen Herausforderungen (Klima, Wasser, Biodiversität) werden nur mit marginalen Mitteln angegangen und es geht dringend benötigte Zeit zur Anpassung und Umsteuerung der landwirtschaftlichen Produktionssysteme verloren. Auch könnte ein weiterer Akzeptanzverlust der Landwirtschaft in der Bevölkerung zu abrupten Änderungen der Rahmenbedingungen führen, beispielweise wenn Zoonose-Risiken aus der Intensivtierhaltung in dicht besiedelten und international stark vernetzten Regionen als nicht mehr akzeptabel angesehen werden. Beispiele für solche abrupten Änderungen aus der jüngeren Vergangenheit sind die deutsche Energiepolitik nach Fukushima oder der Zusammenbruch von Märkten und die schnelle Einführung des QS-System nach der BSE-Krise.

\subsection{Chancen aus Sicht des Natur- und Umweltschutzes}

Auch wenn die Ausgestaltung der GAP im Zuge der Cioloș-Reform 2013 aus Sicht des Natur- und Umweltschutzes enttäuschend war, ergibt sich aus der grundlegenden Verknüpfung der Legitimation der Direktzahlungen mit den vorgeblich besonders hohen Standards im Natur-, Umwelt- und Verbraucherschutz sowie im Tierwohl eine Chance. Denn die GAP wird sich an der Erreichung dieser Ziele messen lassen müssen. Die Zweifel an der Wirksamkeit und Sinnhaftigkeit der erheblichen öffentlichen Zahlungen an den Agrarbereich sind mittlerweile in der breiten Öffentlichkeit angekommen. Dies geht einher mit einem generellen Paradigmenwechsel hin zum Leitsatz, dass steuerfinanzierte Finanzleistungen an Gegenleistungen geknüpft sein sollten (nach dem Grundsatz „öffentliches Geld für öffentliche Güter") sowie der Leitlinie ,,ergebnisorientierter Politik".

Eine zweite Chance besteht darin, dass die einkommenspolitischen Wirkungen der Direktzahlungen zunehmend problematisiert werden. Die ungleiche Verteilung der Direktzahlungen könnte vor dem Hintergrund einer breiteren gesellschaftlichen Debatte über Ungleichverteilung zum politischen Problem werden. Die Beobachtung, dass die Direktzahlungen dazu beitragen, die Bodenmärkte für institutionelle Finanzinvestoren attraktiv zu machen, wirft Zweifel bei den Steuerzahlern auf, ob ihr Geld den richtigen Gruppen zugutekommt. Würden die Direktzahlungen politisch angreifbar, könnte sich eine Möglichkeit eröffnen, die erheblichen Mittel des Agrarbudgets in Maßnahmen umzuleiten, die der Erbringung öffentlicher Güter dienen.

Eine dritte Chance besteht darin, die anhebende Diskussion um die Wirksamkeit des Greenings in der Ersten Säule dazu zu nutzen, entweder auf eine 
wesentliche Anhebung der Anforderungen des Greenings zu drängen oder die zur Verfügung stehenden Mittel für gezieltere und stärker ergebnisorientierte Maßnahmen einzusetzen.

Viertens besteht ein wachsendes Verbraucherbewusstsein und in Teilsegmenten eine wachsende Zahlungsbereitschaft für besondere Leistungen im Natur-, Umwelt- und Verbraucherschutz sowie im Tierwohl. Agrarpolitische Themen führen zu einer erheblichen Mobilisierung der Öffentlichkeit (zum Beispiel Demonstrationen „Wir haben es satt" und „Wir machen Euch satt").

Fünftens ergeben sich aus der europarechtlichen Integration der GAP ins allgemeine Binnenmarkt- und Wettbewerbsrecht neue Ansätze, den Grundsatz eines hohen Schutzniveaus im Umwelt- und Verbraucherschutz auch in der Agrarpolitik voll zur Geltung zu bringen.

Sechstens eröffnen die rasanten technischen Entwicklungen im Bereich der Fernerkundung und des Geo-Monitoring neue Möglichkeiten des Monitorings von Landschaftsstrukturen und Landnutzung inklusive Fruchtfolgen, die mittelfristig für eine ergebnisorientierte Honorierung von Natur- und Umweltleistungen in diesem Bereich genutzt werden können.

Siebtens ermöglicht die digitale Revolution eine engere Vernetzung und Interaktion zwischen Produzenten und Verbrauchern sowie eine hohe Transparenz entlang der gesamten Wertschöpfungskette, die auch für natur- und umweltschutzrelevante Informationen genutzt werden kann.

Achtens können Prozesse einer ,guten“ Globalisierung zur globalen Verbreitung hoher Naturschutz-, Umwelt-, Verbraucherschutz- und Tierwohlstandards auch jenseits der EU genutzt werden. Zum einen könnten hochwertige Exportprodukte eine Vorbildwirkung haben, wenn sie denn auf transparente Weise mit besonders hohen Standards erzeugt werden. Zum anderen kann die Nachfragemacht europäischer Verbraucherinnen und Verbraucher die Etablierung von hohen Standards auch jenseits der EU ermöglichen. Eine zentrale Rolle wird dabei transnationalen Standards wie GlobalGAP zukommen.

Schließlich zeigen Pilotprojekte und Experimente, dass neue ökosystemare und verhaltenswissenschaftliche Ansätze in erfolgreichen Handlungsstrategien zur Verbesserung des Naturschutz- und Umweltverhaltens der Landwirtinnen und Landwirte sowie für ein integriertes Landschafts- und Ressourcenmanagement genutzt werden können.

\subsection{Risiken der derzeitigen Agrarpolitik aus Sicht des Natur- und Umweltschutzes}

Risiken für die Ziele des Natur- und Umweltschutz im Bereich des Agrarsektors ergeben sich zum einen aus den sich verändernden Rahmenbedingungen, zum anderen aus politischen Risiken. 
Das vermutlich unmittelbarste Risiko ergibt sich aus den finanziellen Rahmenbedingungen. Die strukturelle Krise der öffentlichen Haushalte in Europa könnte den Finanzbedarf der GAP delegitimieren, wenn hinreichende Gegenleistungen nicht erkennbar sind. Der finanzielle Druck würde sich bei einem Wiederaufleben der Finanzmarktkrise oder der Eurokrise verschärfen.

Die Niedrigzinspolitik trägt dazu bei, die Boden- und Pachtpreise nach oben zu treiben, was wiederum den Ertragsdruck auf die Fläche erhöhen und damit ertragsmindernde Maßnahmen zum Natur- und Umweltschutz weniger attraktiv machen könnte. Umgekehrt kann der Natur- und Umweltschutz kaum von den niedrigen Zinsen profitieren, da in diesem Bereich kreditfinanzierte Investitionen nicht von Bedeutung sind.

Das bevorstehende Ausscheiden Großbritanniens aus der EU („Brexit“), Rufe nach einer europäischen Flüchtlings- und Sicherheitspolitik sowie einer effektiven Wachstumspolitik mit der Schaffung von Arbeitsplätzen könnten zu einer Verschärfung der Verteilungskämpfe im EU-Haushalt führen. Es besteht die Gefahr, dass Themen des Natur- und Umweltschutzes angesichts der diversen Krisenszenarios an Priorität verlieren.

Veränderungen im Rechtsrahmen führen zu einem schrittweisen Verlust der Ausnahmeregelungen für den Agrarsektor durch die Integration der GAP ins Binnenmarktrecht, internationale Handelsabkommen (z. B. CETA) sowie die neuere Rechtsprechung (Whiskey-Urteil des EuGH). Dies könnte den Spielraum für sektorspezifische Strategien des Natur- und Umweltschutzes, wie beispielsweise die AUKM, vermindern.

Die digitale Revolution und die Entwicklung von Big-Data-Strategien der Gewinnung und Auswertung von personen- und betriebsbezogenen Informationen könnte nicht nur zu einer Konzentration von Informationsmacht führen, sondern auch zur Abwertung von kontextuellem und Erfahrungswissen (u. a. von Landwirtinnen und Landwirten) sowie Möglichkeiten des Missbrauchs eröffnen (Boyd und Crawford 2012; Cukier und Mayer-Schoenberger 2013).

Anthropogene Veränderungen der Agrarökosystemen stellen erhebliche Risikofaktoren dar. Dazu gehören

- Folgen des Klimawandels, v. a. durch Veränderung der hydrologischen Kreisläufe, Witterungsextreme und Schädlingsdruck;

- Folgen des Biodiversitätsverlusts;

- Folgen der Bodenschäden;

- invasive Schädlinge (häufig anthropogen, da durch Bewegungen im Waren- oder Personenverkehr eingeschleppt).

Aus Sicht des Natur- und Umweltschutzes bestehen außerdem erhebliche politische Risiken, vor allem im Hinblick auf die Weiterentwicklung der GAP. Die Agrarpolitik ist anfällig gegenüber einer kurzfristigen Mobilisierung für Einkommensinteressen der Produzenten, wie etwa in der Milch- und Schweinepreiskrise. In weiten Teilen der EU, insbesondere in Frankreich, Spanien und 
Teilen Osteuropas, bestehen starke Sympathien für gekoppelte Direktzahlungen und ähnliche Maßnahmen, die einen Rückfall ins produktivistische Politikparadigma bedeuten könnten, wenn sie nicht eng mit Natur- und Umweltanliegen verknüpft werden. Ein weiteres Risiko ist, dass in der agrarpolitischen Debatte zu viel Energie auf die sterile Wiederholung alter Argumentationen und Konfrontationen und zu wenig auf die Suche nach kreativen Lösungen verwendet wird. Im Kontext eines insgesamt stärker polarisierten politischen Klimas könnte es auch zu einer Radikalisierung und zu politischem Protestverhalten von Landwirtegruppen kommen, die sich existenziell bedroht sehen. Eine mögliche Zunahme von konfrontativen Aktions- und Protestformen solcher Gruppen, aber auch beispielsweise von Tierschützern, könnte die Rahmenbedingungen für einen kooperativen Politikansatz verschlechtern. Und schließlich könnte die digitale Revolution zu einer zunehmenden Orientierung der Menschen an virtuellen Realitäten mit einer einhergehenden Entfremdung von der natürlichen Umwelt führen (Zaradic und Pergams 2007).

\subsection{Zusammenfassung und Schlussfolgerungen}

\subsubsection{Kernpunkte der SWOT-Analyse}

Die SWOT-Analyse der derzeitigen europäischen Agrarpolitik ergibt ein ambivalentes Bild. Auf der einen Seite (Stärken) verfügt die GAP über einen stabilen politischen und institutionellen Rahmen mit bisher guter und verlässlicher Finanzausstattung sowie eine breite Instrumentenkiste, die ein erhebliches Potenzial für den Natur- und Umweltschutz hat. Auf der anderen Seite (Schwächen) wird das natur- und umweltpolitische Potenzial der GAP durch sektorspezifische Mechanismen unterlaufen. Die Dominanz agrarpolitischer Akteure bei der Ausgestaltung der GAP führt zur Priorität für die einkommenspolitischen Ziele und zur systematischen Schwächung der natur- und umweltpolitischen Ansätze. Dies drückt sich in Regelungs- und Implementationsdefiziten, mangelnder Datenlage und nahezu durchgängiger Überkompensation für Leistungen im Natur- und Umweltschutz aus. Die Verwaltungsstrukturen sind extrem komplex. Das Ergebnis sind mangelnde Effektivität und Effizienz der Politik. Zudem herrscht vielfach ein Status-quo-Denken vor, die Partizipationsmöglichkeiten sind gering und systemische Risiken und neue Herausforderungen erhalten zu wenig Aufmerksamkeit.

Vor diesem Hintergrund ergeben sich Chancen aus der Tatsache, dass der Natur-, Umwelt- und Verbraucherschutz sowie das Tierwohl als Legitimationsgrundlage für die Agrarzahlungen etabliert sind. Die öffentliche Kritik an der mangelnden Ergebnisorientierung der GAP sowie verstärkte juristische Anforderungen an ein hohes Schutzniveau im Binnenmarkt treffen auf ein erhebliches öffentliches Mobilisierungspotenzial für agrarpolitische Themen. Neue technische 
Entwicklungen und systemische wie verhaltenswissenschaftliche Ansätze ermöglichen die Entwicklung von neuen Policy-Designs zur systematischen und flächendeckenden Integration von Natur- und Umweltschutz in die Agrarpolitik. Zugleich besteht das Risiko, dass sich die finanzpolitischen Verteilungskämpfe verschärfen, sich das politische Klima insgesamt polarisiert und radikalisiert und vor diesem Hintergrund ein defensives Status-quo-Denken die Oberhand gewinnt. Zugleich könnte sich der rechtliche Spielraum für sektorspezifische Strategien des Natur- und Umweltschutzes verengen, während die anthropogenen Veränderungen der Agrarökosysteme die Herausforderungen für den Natur- und Umweltschutz noch verstärken. Tab. 5.1 gibt einen Überblick über die Kernpunkte der SWOT-Analyse.

Tab.5.1 SWOT-Analyse der derzeitigen GAP aus Sicht des Natur- und Umweltschutzes - Kernpunkte

\section{Stärken}

- Stabilität des politischen und institutionellen Rahmens

- Mittelfristig verlässlicher finanzieller Rahmen

- Breit aufgestelltes ideelles Paradigma

- WTO-konform

- Integration von Natur- und Umweltbelangen mit breiter Instrumentenkiste

- Cross-Compliance- und GreeningAuflagen wirken in die Fläche

- AUKM erlauben zielgerichtete, regionale, ergebnisorientierte und kollektive Maßnahmen

- Einbettung in Beratungsprogramme

\section{Schwächen}

- Dominanz der Einkommenslogik

- Hoher Legitimationsbedarf für redistributive Politik

- Institutionelle Abschirmung des Kernbereichs der GAP gegen Umwelt- und Naturschutzakteure

- Erste Säule: geringe Leistungsorientierung, unklare Zielsetzung, Ungleichverteilung, Überwälzungseffekte

- Greening: geringe Wirksamkeit, Überkompensation

- Naturschutz- und Umweltordnungsrecht: Fehlende oder unzureichende Zielwerte und Mindestanforderungen, Implementationsdefizite, teilweise ineffiziente Kontroll- und Sanktionsmechanismen

- AUKM: komplexe und aufwändige Entscheidungs- und Programmierungsstrukturen, oft fehlende Ergebnisorientierung, meist kein sektorenübergreifender Ansatz für integriertes Landschafts- und Ressourcenmanagement, Mittelkonkurrenz in Zweiter Säule, Kontroll- und Anlastungsrisiko

- Generell: lückenhafte Datenlage, hoher Verwaltungsaufwand

- langsame Lernprozesse, geringe Partizipationsmöglichkeiten

- zu wenig Aufmerksamkeit und Mittel für systemische Risiken (u. a. Zoonosis, Antibiotika) und neue Herausforderungen (Klima, Wasser, Biodiversität) 
Tab. 5.1 (Fortsetzung)

\section{Chancen}

- Natur-, Umwelt- und Verbraucherschutz sowie Tierwohl als Legitimationsgrundlage für Agrarzahlungen

- Mehr Rufe nach ,ergebnisorientierter Politik“

- Wachsende Kritik an Direktzahlungen: ungleiche Verteilung, Wirkung auf Bodenmärkte, institutionelle Finanzinvestoren - Möglichkeiten der Neustrukturierung

- Diskussion um Wirksamkeit des Greenings und damit Möglichkeit der Überarbeitung

- Wachsendes Verbraucherbewusstsein, Zahlungsbereitschaft für besondere Leistungen, Mobilisierung der Öffentlichkeit

- Europarechtliche Integration der GAP ins allgemeine Binnenmarkt- und Wettbewerbsrecht: Grundsatz eines hohen Schutzniveaus im Umwelt- und Verbraucherschutz

- Technische Entwicklungen im Bereich der Fernerkundung und des Geo-Monitoring

- Digitale Revolution: Vernetzung und Transparenz

- Globale Verbreitung hoher Naturschutz-, Umwelt-, Verbraucherschutz- und Tierwohlstandards, u. a. durch transnationale Standards

- Neue ökosystemare und verhaltenswissenschaftliche Ansätze für integriertes Landschafts- und Ressourcenmanagement

\section{Risiken}

- Krise der öffentlichen Haushalte und Verschärfung der finanzpolitischen Verteilungskämpfe (v. a. im EU-Haushalt nach dem Brexit)

- Niedrigzinspolitik: erhöhte Bodenpreise und Ertragsdruck auf die Fläche

- Veränderungen im Rechtsrahmen: Verlust des Spielraums für sektorspezifische Strategien des Natur- und Umweltschutzes

- Anthropogene Veränderungen der Agrarökosysteme: Klimawandel, Biodiversitätsverlust, Bodenschäden, invasive Schädlinge usw.

- Status-quo-Denken und politische Unterstützung für Rückfall ins produktivistische Paradigma

- Insgesamt stärker polarisiertes politisches Klima mit möglicher Radikalisierung und mehr konfrontativen Aktions- und Protestformen von allen Seiten

- Zentralisierung von Informationsmacht durch Big Data und möglicher Missbrauch

\subsubsection{Fazit: Fünf Hypothesen zur Agrarpolitik}

Die Schlussfolgerungen, die sich aus der SWOT-Analyse für die künftige Ausgestaltung einer Agrarpolitik ergeben, welche die Anliegen des Natur- und Umweltschutzes wirkungsvoll integriert, lassen sich zu fünf Hypothesen zusammenfassen.

Hypothese 1: Die Gemeinsame Agrarpolitik ist tief geprägt durch die Dominanz der Einkommenslogik für die landwirtschaftliche Bevölkerung. Durch die Umwandlung der Marktkompensation in eine Flächenprämie ist der redistributive Charakter der GAP sichtbar geworden. Dies erhöht den Legitimationsbedarf.

- Die Architektur und Legitimationslogik der derzeitigen GAP wirft dringende Fragen nach der Zielgenauigkeit der redistributiven Komponente und der effektiven Mittelverwendung bei den leistungsbezogenen Komponenten auf. In beiden Punkten besteht Evidenz für erhebliche Defizite, aus denen eine „Drohkulisse“ für die Fortsetzung der bisherigen Politik erwächst. 
Hypothese 2: Naturschutz- und Umweltaspekte (Cross Compliance, Greening, AUKM) erhöhen die Legitimation der GAP, indem diese nun nicht als bloße steuerfinanzierte Transferpolitik (staatliche Zahlungen ohne spezifische Gegenleistung) erscheint. Sie haben in der Architektur der GAP wie in der Mittelausstattung aber eine untergeordnete Rolle und dienen offenbar dazu, den einkommenspolitischen Kern der GAP zu legitimieren.

- Aus Sicht des Natur- und Umweltschutzes stellt sich die Frage, inwiefern die Strategie einer Integration dieser Belange mit der Rationalität einer sektoralen Einkommenspolitik zu vereinbaren ist. Im Kern stehen sich eine redistributive Logik und eine Logik des Entgelts öffentlicher Güter gegenüber.

Hypothese 3: Die „multifunktionale“ Begründung der Agrarpolitik, dass sie der Sicherung öffentlicher Güter und vielfältiger Funktionen der Landwirtschaft dient, wird nicht hinreichend eingelöst, weil die gesetzlichen Anforderungen oft nicht hinreichend anspruchsvoll oder vage formuliert sind und weil erhebliche Implementationsdefizite bestehen.

- Aus Sicht des Natur- und Umweltschutzes müsste die Agrarpolitik konsequent von der Aufgabe her gedacht werden, die nachhaltige Nutzung natürlicher Ressourcen zu sichern. Das wiederum erfordert, dass die landwirtschaftlichen Produktionssysteme ökologisch, ökonomisch und sozial resistent sind.

Hypothese 4: Eine zukunftsfähige Agrarpolitik muss so instrumentiert sein, dass sie sowohl die Entscheidungen der Landbewirtschafter effektiv im Sinne des Naturund Umweltschutzes beeinflusst als auch die Unterstützung der Landbewirtschafter und der Öffentlichkeit genießt.

- Der erste Aspekt zielt auf die Effektivität und Effizienz der Politik ab. Wenn man wirkliche Verbesserungen im Natur- und Umweltschutz erreichen will, müssen die Entscheidungen derjenigen, die das Land bewirtschaften, beeinflusst werden. Es geht also um effektive Verhaltensänderungen. Die Politik-Instrumente müssen daher konsequent von den Adressaten her entwickelt werden. Dabei sind zum einen Wechselwirkungen zwischen verschiedenen Instrumenten und Politikbereichen, zum anderen wahrscheinliche Ausweichoptionen und Nebenwirkungen zu beachten. Dies erfordert eine verhaltenswissenschaftlich fundierte Adressatenorientierung.

- Der zweite Aspekt betrifft die Dauerhaftigkeit der Politik. Dazu muss ein „Policy-Feedback“ erreicht werden in dem Sinne, dass die Politik hinreichend Unterstützung erfährt und nicht bei nächster Gelegenheit wieder revidiert oder abgeschafft wird. Die Politik muss also so angelegt werden, dass sie eine breite politische Unterstützerkoalition schaffen und zusammenhalten kann.

- Die derzeitige Agrarpolitik erfüllt nur einen Teil der zweiten Bedingung. Viele Vorschläge aus dem Bereich des Natur- und Umweltschutzes erfüllen nur die erste und teilweise die zweite Bedingung. 
Hypothese 5: Bei den bisher erprobten agrarpolitischen Instrumenten besteht ein Trade-Off zwischen Zielgenauigkeit und Transaktionskosten; daher besteht ein Bedarf, neue Policy-Instrumente zu entwickeln.

- Zielgenaue Maßnahmen im Bereich der Immissionen und Emissionen würden im Prinzip ein komplexes Monitoring diverser landwirtschaftlicher Praktiken wie Düngung oder Pestizideinsatz erfordern.

- Zielgenaue Maßnahmen im Bereich der Landnutzung erfordern im Prinzip ein Monitoring der tatsächlichen Flächennutzung.

- Die relative Attraktivität von freiwilligen Maßnahmen, bei denen die Landwirte für bestimmte Praktiken oder ergebnisabhängig honoriert werden, hängt von den Opportunitätskosten, im Wesentlichen also von den Marktpreisen, sowie den Transaktionskosten ab.

- Neue Instrumente müssen auf eine Verminderung der Transaktionskosten, Zuverlässigkeit des Monitorings und effektive Beeinflussung des Verhaltens der Landwirte abstellen. Zwischen diesen Zielen bestehen Trade-Offs.

Die fünf Hypothesen dienen als Leitorientierung für die Entwicklung eines zukunftsfähigen agrarpolitischen Leitbilds sowie von Handlungsansätzen und Optionen für eine Agrarpolitik, welche die Anliegen des Natur- und Umweltschutzes wirkungsvoll integriert.

Um die in der SWOT-Analyse identifizierten Chancen zu nutzen, erscheinen dabei die folgenden generellen Ansätze geeignet:

- Nutzung des erheblichen Finanzvolumens für zielgerichtete und erforderliche Maßnahmen;

- Einbindung in die mittelfristige Finanzplanung für eine glaubwürdige mittelfristige Umsteuerung nutzen (z. B. gleitender Ausstieg aus den Direktzahlungen);

- Nutzung neuer digitaler Technologien für Monitoring und Verwaltung, Vernetzung und Transparenz;

- Nutzung vorhandener Implementationsstrukturen für Greening- und ZweiteSäule-Programme können für Natur- und Umweltschutzmaßnahmen;

- Ermöglichung von Märkten für Produkte mit besonderen Prozessqualitäten und Aktivierung privater Zahlungsbereitschaft (nach dem Vorbild von Bio, geografische Herkunftsbezeichnung, traditionelles Lebensmittel etc.);

- Stärkung des Verbraucherbewusstseins (regionale, umweltfreundliche, naturschützende, ethische Lebensmittel und andere Produkte) und entsprechender Zahlungsbereitschaft;

- Weiterentwicklung des Multifunktionalitätsanspruchs ernst nehmen, z. B. in Richtung In-Wert-Setzung von Ökosystemleistungen;

- Formulierung eines Anspruchs Europas auf eine internationale Führungsrolle bei nachhaltiger Agrarpolitik, um transnationale Standardsysteme zu prägen.

Um den Risiken einer politischen Polarisierung und einer politischen Dynamik der Angst im Zeichen finanzpolitischer Verteilungskämpfe entgegenzuwirken, erscheint 
es geboten, die Agrarpolitik transparenter, ergebnisorientierter und partizipationsorientierter zu gestalten. Zugleich müssen die Globalisierungsprozesse aktiv im Sinne des Natur-, Umwelt und Verbraucherschutzes gestaltet werden, um auch die Akzeptanz der Bevölkerung für offene Märkte zu erhalten.

Insgesamt diente die SWOT-Analyse der strategisch orientierten Verdichtung der Bestandsaufnahme auf Basis der Literaturanalyse in den Kap. 3 und 4. Im Folgenden werden wir zunächst ein Leitbild für eine zukunftsorientierte Agrarpolitik formulieren (Kap. 6). Anschließend diskutieren wir alternative Handlungsoptionen zur Weiterentwicklung der verschiedenen Elemente und Instrumente der Agrarpolitik (Kap. 7), ehe wir diese zu drei strategischen Optionen für die künftige Agrarpolitik verdichten (Kap. 8).

Open Access Dieses Kapitel wird unter der Creative Commons Namensnennung 4.0 International Lizenz (http://creativecommons.org/licenses/by/4.0/deed.de) veröffentlicht, welche die Nutzung, Vervielfältigung, Bearbeitung, Verbreitung und Wiedergabe in jeglichem Medium und Format erlaubt, sofern Sie den/die ursprünglichen Autor(en) und die Quelle ordnungsgemäß nennen, einen Link zur Creative Commons Lizenz beifügen und angeben, ob Änderungen vorgenommen wurden.

Die in diesem Kapitel enthaltenen Bilder und sonstiges Drittmaterial unterliegen ebenfalls der genannten Creative Commons Lizenz, sofern sich aus der Abbildungslegende nichts anderes ergibt. Sofern das betreffende Material nicht unter der genannten Creative Commons Lizenz steht und die betreffende Handlung nicht nach gesetzlichen Vorschriften erlaubt ist, ist für die oben aufgeführten Weiterverwendungen des Materials die Einwilligung des jeweiligen Rechteinhabers einzuholen. 


\section{Kapitel 6 \\ Ein zeitgemäßes Leitbild für eine nachhaltige Agrarpolitik}

\subsection{Einleitung}

\subsubsection{Funktionen eines Leitbilds}

Die Integration von Anliegen des Umwelt- und Naturschutzes in die Agrarpolitik bewegt sich in einem komplexen institutionellen und rechtlichen Rahmen, der das Verhältnis zwischen den Rechten der Landbesitzer und Landbewirtschafter einerseits und andererseits ihren Pflichten gegenüber den Interessen der Allgemeinheit, im Zusammenhang mit dieser Studie insbesondere den Interessen des Natur- und Umweltschutzes, regelt. In der konkreten Politikgestaltung ist dieser Rahmen jedoch notwendigerweise unvollständig. Erstens ist oft unklar, welche Werte und Normen im Konfliktfall den Vorrang haben sollen. Zweitens sind viele Rechtsbegriffe, z. B. die Gemeinwohlverpflichtung des Eigentums nach Artikel 14 Absatz 2 Grundgesetz, unbestimmt und bedürfen der Konkretisierung im Lichte der jeweiligen Problemlagen. Drittens geben die bestehenden Normen keine Entwicklungsrichtung vor. Und viertens sind Rechtsnormen nicht geeignet und haben auch nicht den Zweck, gemeinsame Initiativen von staatlichen und nicht-staatlichen Akteuren zu motivieren, wo dies beispielsweise im Landschafts- oder Wassermanagement wünschenswert wäre.

Vor diesem Hintergrund haben politische Leitbilder mehrere Funktionen. Im politischen Gestaltungsprozess und gegenüber den gesellschaftlichen Gruppen dienen sie als programmatische Orientierungshilfe, die Aufschluss über die angestrebte Entwicklungsrichtung in einem Politikfeld gibt und an dem die Träger eines Leitbilds sich messen lassen müssen. Im administrativen und judikativen Prozess dienen Leitbilder als Auslegungshilfe bei der Ausgestaltung von Ermessensspielräumen und bei der Entscheidung von Normkonflikten.

Im Hinblick auf die Rolle von Leitbildern im Verwaltungshandeln erklärt Wewer (1998, S. 155): „Leitbilder beschreiben in relativ knapper Form den ,Sinn“ oder die ,Philosophie“ von Organisationen (also deren Selbstverständnis) bzw. ihre , Vision ‘ 
oder ,Mission' (nämlich die längerfristigen Ziele) ... [Sie] beschreiben strategische Ziele, also einen Soll-Zustand, nicht den Ist-Zustand“. Leitbilder geben demnach einerseits Orientierung und haben andererseits die Funktion von Selbstverpflichtungen, denn die Träger eines Leitbilds müssen sich daran messen lassen, ob sie bei dessen Verwirklichung erfolgreich sind, also dem Soll-Zustand näher kommen, oder zumindest geeignete Anstrengungen unternehmen.

\subsubsection{Gegenstand des Leitbilds: Das Was und das Wie}

Bei der Entwicklung einer zukunftsfähigen Agrarpolitik, welche die Anliegen des Natur- und Umweltschutzes integriert, muss ein Leitbild dazu dienen, die angestrebte Entwicklungsrichtung in dreierlei Hinsicht zu positionieren: erstens im Hinblick auf die Problemlagen (siehe Kap. 3), zweitens im Hinblick auf die kontroversen agrarpolitischen Paradigmen (siehe Kap. 4) und drittens im Hinblick auf die Kontroversen im Natur- und Umweltschutz selbst:

- Die Problemlagen (siehe Kap. 3) lassen sich dabei zu drei Komplexen zusammenfassen: (1) Stoffeinträge in Boden, Wasser und Luft inklusive Klimagase, (2) Wirkungen der Flächennutzung sowie (3) Implementations-, Partizipations- und umweltorientierte Innovationsdefizite. Das Leitbild sollte klare Vorstellungen darüber enthalten, wie diese Problemlagen adressiert und welche Zielwerte erreicht werden sollen.

- In der agrarpolitischen Ausrichtung muss das Leitbild klären, wie man sich positionieren will zwischen den Leitbildern einer marktorientierten, einer staatsabhängigen, einer globalisierten und einer multifunktionalen Landwirtschaft bzw. zwischen den politischen Paradigmen des einkommensorientierten agrarpolitischen Exzeptionalismus, der Marktliberalisierung, der Marktharmonisierung und Standardisierung in globalen Wertschöpfungsketten sowie der Multifunktionalität (siehe Kap. 4).

- Im Naturschutz selbst gilt es insbesondere zu klären, ob eine räumliche Integration oder Separation von Landwirtschaft und Naturschutz angestrebt wird (Sharing- vs. Sparing-Debatte), welcher relative Stellenwert dem Schutz von biotischen und abiotischen Ressourcen bzw. dem Tierwohl zukommen soll (z. B. Weidehaltung vs. Rückhalt von Emissionen in geschlossenen Stallsystemen), und ob beispielsweise das Konzept der Planetaren Leitplanken (Rockström et al. 2009a, b) in Leitplanken auf kleineren räumlichen und zeitlichen Skalen übersetzt werden soll.

Um die im ersten und dritten Punkt angesprochenen Fragen zu beantworten, ist es notwendig, ein Leitbild für die Landwirtschaft zu formulieren: Wie soll eine Landwirtschaft in Zukunft aussehen, die den Zielen des Umwelt- und Naturschutzes angemessen Rechnung trägt? Dies ist die Frage nach dem Was. Eine Antwort auf den zweiten Punkt erfordert hingegen Leitlinien für eine zukunftsfähige Agrarpolitik 
und betrifft die Frage nach dem Wie. Wir formulieren daher sowohl ein Leitbild für eine multifunktionale, natur- und umweltverträgliche Landwirtschaft sowie Leitlinien für eine zukunftsfähige Agrarpolitik. Bei der Formulierung des Leitbilds wurde eine systematische Analyse bestehender agrarpolitischer Leitbilder vorgenommen (vgl. den Überblick im Anhang).

\subsubsection{Einbettung in das agrarpolitische Leitbild der Bundesregierung}

Um Verbindlichkeit für die Träger und Adressaten zu erzeugen, werden Leitbilder üblicherweise in einem mehr oder weniger partizipativen und iterativen Prozess erarbeitet. Abgesehen von Rückkopplungen im Rahmen der Projektbegleitenden Arbeitsgruppe und seitens der Peer Reviewer war dies im Rahmen des ZA-NExUSProjekts weder möglich noch vorgesehen. Das im Folgenden formulierte Leitbild stellt also einen Diskussionsvorschlag dar, der als Anker für einen Leitbildprozess dienen kann.

Aus pragmatischen Gründen halten es die Autoren für sinnvoll, dass sich das Leitbild in das agrarpolitische Leitbild der Bundesregierung (BMEL 2015a, S. 8) einfügt. ${ }^{1}$ Dieses nimmt im ersten Absatz , attraktive, lebenswerte und vitale ländliche Räume“ als Aufhänger und strebt ,eine nachhaltige, ökologisch verantwortbare, ökonomisch leistungsfähige und multifunktional ausgerichtete Land-, Forst-, und Fischereiwirtschaft" an. Es hebt „landwirtschaftliche Familienbetriebe und Unternehmen mit bäuerlicher Wirtschaftsweise" hervor. Der zweite Absatz stellt dann die Produktions- und Versorgungsfunktion in den Mittelpunkt und bezieht die „Ernährungswirtschaft" mit ein. Das Leitbild der Bundesregierung kombiniert also einen prominenten Raumbezug (erster Halbsatz und dritter Satz) mit Elementen des Multifunktionalitätsparadigmas (zweiter Halbsatz) und des produzentenorientierten Exzeptionalismus (v. a. im zweiten und dritten Satz). Der globale Bezugsrahmen kommt durch den Bezug auf die „Welternährung“ zum Ausdruck, die eine Exportorientierung impliziert.

Das agrarpolitische Leitbild der Bundesregierung bietet durchaus gute Anknüpfungspunkte für eine Agrarpolitik, die am Schutz der Natur und der Sicherung von Umweltressourcen orientiert ist. Allerdings ist das Leitbild der Bundesregierung im

\footnotetext{
${ }^{1}$ Wörtlich heißt es: „(1) Das agrarpolitische Leitbild der Bundesregierung umfasst attraktive, lebenswerte und vitale ländliche Räume und eine nachhaltige, ökologisch verantwortbare, ökonomisch leistungsfähige und multifunktional ausgerichtete Land-, Forst- und Fischereiwirtschaft. Landwirtschaftliche Familienbetriebe und Unternehmen mit bäuerlicher Wirtschaftsweise entsprechen diesem Leitbild in besonderer Weise. Sie sind für eine Entwicklung der ländlichen Regionen und den gesellschaftlichen Zusammenhalt von großer Bedeutung. (2) Die deutsche Land- und Ernährungswirtschaft erzeugt sichere, gesunde und bezahlbare Lebensmittel und leistet ihren Beitrag zur Sicherung der Welternährung. Sie trägt daneben zur Versorgung mit erneuerbaren Energien und nachwachsenden Rohstoffen bei.“
} 
Hinblick auf die ökologischen Ziele sehr allgemein gehalten und bietet insbesondere keine Anhaltspunkte dafür, welchen Stellenwert ökologische Ziele im Konfliktfall haben sollen. Auch bietet es keine Hinweise, wie die Ziele erreicht werden sollen. Ein Leitbild für eine zukunftsfähige Agrarpolitik, die sich am Naturund Umweltschutz orientiert, hat daher insbesondere die Aufgabe, diese konzeptionellen Freiräume auszufüllen. Das folgende Leitbild einer multifunktionalen Landwirtschaft und die sich anschließenden Leitlinien einer zukunftsfähigen Agrarpolitik sind ein Vorschlag, wie die Unbestimmtheiten des allgemeinen Leitbilds der Bundesregierung aus Sicht des Natur- und Umweltschutzes so ausgefüllt werden könnten, dass die diagnostizierten ökologischen Problemlagen der derzeitigen Agrarpolitik besser adressiert werden können.

\subsection{Leitbild für eine multifunktionale, natur- und umweltverträgliche Landwirtschaft}

Das agrarpolitische Leitbild für eine multifunktionale, natur- und umweltverträgliche Landwirtschaft umfasst eine flächendeckende Landwirtschaft, welche zu einer vielfältigen Kulturlandschaft beiträgt, die Tragfähigkeit der ökologischen Systeme beachtet und die ökosystemaren Dienstleistungen erhält.

Die Landwirtinnen und Landwirte erzeugen marktfähige, qualitativ hochwertige und gesunde Lebensmittel und andere landwirtschaftliche Produkte auf nachhaltige und umweltverträgliche Weise. Sie halten die natürlichen Ressourcen in gutem $\mathrm{Zu}$ stand, bewahren die Leistungsfähigkeit der Ökosysteme und wirtschaften innerhalb der ökologischen Leitplanken. ${ }^{2}$ Sie minimieren schädliche Einträge in Boden, Wasser und Luft und tragen aktiv zur Erhaltung der genetischen Vielfalt, zum Schutz der biologischen Vielfalt und zum Klimaschutz bei.

Die Landwirtinnen und Landwirte und ihre Partner in den Wertschöpfungsketten sind umweltorientiert und innovationsfähig. Sie entwickeln besonders umweltfreundliche Methoden der Landbewirtschaftung sowie umweltorientierte Geschäftsmodelle und genießen die Wertschätzung der Verbraucherinnen und Verbraucher für ihre Leistungen im Natur- und Umweltschutz. Alle Beteiligten verstehen die Erbringung von Gemeinwohlleistungen als integrale Einkommensmöglichkeit für die deutsche Landwirtschaft.

Als Landschaftsgestalter tragen die Landwirtinnen und Landwirte in Kooperation mit anderen gesellschaftlichen Kräften dazu bei, dass vielfältige und gut strukturierte Landschaften Lebensräume für eine große Vielfalt von Tieren und Pflanzen bieten und als Teil von attraktiven, lebenswerten und vitalen ländlichen Räumen wahrgenommen werden.

\footnotetext{
${ }^{2}$ Z. B. Beachtung der Tragfähigkeit und Regenerationsraten ökologischer Systeme oder der Zeit-
} räume, die für das Nachwachsen erneuerbarer Ressourcen notwendig sind. 


\subsection{Leitlinien einer zukunftsfähigen Agrarpolitik}

Eine zukunftsfähige Agrarpolitik unterstützt die Landwirtschaft bei der Erfüllung ihrer unterschiedlichen, auf das Gemeinwohl ausgerichteten Aufgaben in effektiver und effizienter Weise. Dabei stellt die nachhaltige, umweltverträgliche Erzeugung von marktfähigen, qualitativ hochwertigen und gesunden Lebensmitteln und anderen landwirtschaftlichen Produkten die tragende Säule dar. Die Agrarpolitik stellt zugleich sicher, dass die Landwirtschaft die natürlichen Ressourcen und die Leistungsfähigkeit der Ökosysteme bewahrt und innerhalb der ökologischen Leitplanken wirtschaftet. Sie unterstützt die Vernetzung der Landwirtschaft mit anderen gesellschaftlichen Akteuren sowie die umweltorientierte Lern- und Innovationsfähigkeit des Sektors. Dazu stärkt sie die Entwicklung von besonders umweltorientierten Geschäftsmodellen, die Wertschätzung der Verbraucherinnen und Verbraucher für die Umweltleistungen der Landwirtschaft sowie die Motivation der Landwirte zu umweltorientiertem Handeln.

Eine zukunftsfähige Agrarpolitik orientiert sich insbesondere an folgenden Leitlinien:

1. Eine zukunftsfähige Agrarpolitik geht davon aus, dass die langfristige Sicherung der Produktion von Lebensmitteln und landwirtschaftlichen Rohstoffen die Sicherung der ökologischen Voraussetzungen der Produktion wie Biodiversität, Bodenfruchtbarkeit und sauberes Wasser erfordert. Sie stärkt Maßnahmen zur langfristigen Sicherung der Natur- und Umweltressourcen gegenüber Tendenzen zur kurzfristigen, aber nicht nachhaltigen Steigerung der Produktion. Dabei orientiert sie sich am Verursacher-, Kooperations-, Vorsorge- und Nachhaltigkeitsprinzip.

2. Eine zukunftsfähige Agrarpolitik unterstützt die Entwicklung von resilienten, nachhaltigen und ressourceneffizienten landwirtschaftlichen Produktionssystemen, die im Einklang mit dem Erhalt intakter und leistungsfähiger ökologischer Systeme und der natürlichen Ressourcen wirtschaften und sich im Zuge des ökonomischen, ökologischen und sozialen Wandels dynamisch und ohne abrupte Strukturbrüche entwickeln. Sie sichert und stärkt zu diesem Zweck insbesondere die ökologische und ökonomische Anpassungsfähigkeit landwirtschaftlicher Produktionssysteme sowie die Lernfähigkeit der beteiligten Personen und Organisationen.

3. Eine zukunftsfähige Agrarpolitik ist darauf ausgerichtet, dass neben der Produktion von Lebensmitteln und anderen landwirtschaftlichen Erzeugnissen die Gemeinwohlleistungen der Landwirtschaft gesichert werden. Insbesondere der Erhalt und die Pflege von ökologisch intakten Landschaften, Habitaten und Ökosystemleistungen stellen wichtige Leistungen der Landnutzung durch Landwirte dar.

4. Eine zukunftsfähige Agrarpolitik stellt einen zufriedenstellenden Zustand der Ressourcen des Natur- und Umweltschutzes sicher. Sie sorgt dafür, dass gesetzliche Mindeststandards eindeutige ökologische Leitplanken für die landwirtschaftliche Produktion definieren, die sich am Stand der Wissenschaft sowie 
am Vorsorgeprinzip orientieren und dabei systemische Zusammenhänge und schädliche kumulative Effekte berücksichtigen. Sie duldet keine Vollzugs- und Regelungsdefizite bei der Durchsetzung gesetzlicher Mindeststandards.

5. Eine zukunftsfähige Agrarpolitik ist vorrangig darauf ausgerichtet, dass die Anliegen des Natur- und Umweltschutzes in der gesamten Fläche berücksichtigt werden. Eine räumliche Separierung von Landnutzung und Naturschutz wird nur dort vorgesehen, wo dies zur Erreichung von Natur- und Umweltschutzzielen notwendig ist. Das bedeutet, dass die Landwirtschaft an allen Standorten so betrieben wird, dass den Anliegen des Natur- und Umweltschutzes in angemessener Weise Rechnung getragen wird.

6. Eine zukunftsfähige Agrarpolitik ist darauf ausgerichtet, dass einerseits alle landwirtschaftlichen Erzeugnisse, die den Markt erreichen, unter Bedingungen erzeugt werden, die mit der langfristigen Sicherung der Ressourcen des Naturund Umweltschutzes vereinbar sind, und dass andererseits eine flächendeckende landwirtschaftliche Produktion in Deutschland stattfindet, welche die ökologischen Leitplanken beachtet und möglichst viele positive Umwelt- und Naturschutzleistungen erbringt.

7. Eine zukunftsfähige Agrarpolitik berücksichtigt, dass die Leistungen des Natur- und Umweltschutzes nicht automatisch als Koppelprodukt der landwirtschaftlichen Erzeugnisse entstehen. Sie stellt geeignete Rahmenbedingungen her, welche den Landnutzern die dauerhafte Erbringung dieser Gemeinwohlleistungen ermöglicht. Dazu gehört insbesondere die Sicherung fairer Wettbewerbsbedingungen, Transparenz der Umweltleistungen von Produkten und Produzenten sowie ggf. die Honorierung derjenigen Gemeinwohlleistungen, die vom Markt nicht angemessen entlohnt werden.

8. Eine zukunftsfähige Agrarpolitik setzt finanzielle Mittel zielgerichtet und effektiv für die Sicherung der Gemeinwohlleistungen ein. Sie gewährt keinen finanziellen Ausgleich für die bloße Einhaltung gesetzlicher Mindeststandards, es sei denn zur Kompensation von daraus entstehenden Wettbewerbsnachteilen auf offenen Märkten. Sie stellt sicher, dass eventuelle Kompensationen auf wissenschaftlich nachgewiesenen Mehrkosten beruhen und die Regeln der WTO beachten.

9. Eine zukunftsfähige Agrarpolitik sorgt dafür, dass Natur- und Umweltschutz in Deutschland nicht auf Kosten Dritter geschieht. Sie vermeidet ökologische Überwälzungseffekte in Drittländer und stellt sicher, dass Nachhaltigkeitsdefizite reduziert und nicht bloß ins Ausland verlagert werden.

10. Eine zukunftsfähige Agrarpolitik bezieht die Rolle der Verbraucherinnen und Verbraucher konsequent ein. Sie trägt dazu bei, dass möglichst viele Verbraucherinnen und Verbraucher die Naturschutz- und Umweltleistungen von landwirtschaftlichen Produkten und Produktionsweisen erkennen und kompetent beurteilen können. Sie erreicht, dass die Verbraucherinnen und Verbraucher ein hohes Vertrauen in die landwirtschaftliche Erzeugung haben.

11. Eine zukunftsfähige Agrarpolitik verfolgt einen systemischen Ansatz. Sie sorgt dafür, dass ökologische Problemlagen der landwirtschaftlichen Produktion auf den entsprechenden räumlichen und zeitlichen Skalen adressiert und beispielsweise standörtliche Gegebenheiten berücksichtigt werden können. 
12. Eine zukunftsfähige Agrarpolitik sorgt für eine Vernetzung der Akteure, die zur Sicherung oder Erbringung von ökosystemaren Leistungen zusammenarbeiten müssen oder dazu beitragen können. Sie unterstützt die Beteiligung der betroffenen gesellschaftlichen Gruppen und eine wertschätzende, ergebnisorientierte Kommunikation zwischen allen Beteiligten.

13. Eine zukunftsfähige Agrarpolitik sorgt dafür, dass jeglicher administrativer Aufwand zielführend, notwendig, und verhältnismäßig ist. Sie nutzt neue Techniken der Erfassung und Verarbeitung von umweltrelevanten Daten zur Minimierung der Transaktionskosten für alle Beteiligten.

14. Eine zukunftsfähige Agrarpolitik stärkt die ökonomische, ökologische und soziale Kompetenz und Lernfähigkeit der landwirtschaftlichen Akteure. Sie stellt sicher, dass die Aus- und Fortbildung sowie die Beratung auf allen Ebenen die in der Landwirtschaft Tätigen befähigt und ermutigt, die Naturschutz- und Umweltaspekte der Landnutzung sowie die ökologischen Zusammenhänge in ihrer ganzen Bedeutung zu verstehen und angemessen zu bewerten. Sie strebt an, dass möglichst viele Landnutzer in der Lage sind, einen optimalen Beitrag zu einem zufriedenstellenden Zustand der Umweltressourcen und zum Aufbau resilienter landwirtschaftlicher Produktionssysteme zu leisten.

15. Eine zukunftsfähige Agrarpolitik unterstützt das Erfahrungswissen der Landwirte durch eine praxisnahe, gemeinwohlorientierte staatliche Forschung und entsprechende Beratungs- und Bildungsangebote. Sie setzt darauf, dass Expertisen und neueste Erkenntnisse der Wissenschaft von gesamtgesellschaftlichem Nutzen zeitnah an allen Lehr- und Weiterbildungsstätten vermittelt werden. Gleichzeitig fördert sie den Wissenstransfer zwischen der Landwirtschaft und den Forschungs- und Bildungseinrichtungen.

\subsection{Dilemmata und Zielkonflikte}

Für die Bearbeitung der Problemkomplexe des Natur- und Umweltschutzes in der Landwirtschaft wird es keine einfachen, linearen Lösungen geben, denn die Problemlagen sind systemischer Natur. Auf Basis der Bestandsaufnahmen haben wir 13 Dilemmata und Zielkonflikte identifiziert, für die auch das Leitbild einer zukunftsfähigen Agrarpolitik Lösungsansätze enthalten muss:

1. Flächenkonkurrenz zwischen Nahrungsmittelerzeugung, Biodiversität und Bioenergie

Aus Sicht des Leitbilds hat die Erreichung der Biodiversitätsziele Vorrang vor kurzfristig maximierenden Produktionszielen.

2. Zielgenauigkeit von Maßnahmen vs. Verwaltungsvereinfachung

Das Leitbild strebt durch Nutzung neuer Methoden der raumbezogenen Datenerfassung und -verarbeitung eine Reduzierung des Zielkonflikts an.

3. Hoher Naturschutz- und Umweltanspruch von Maßnahmen vs. Akzeptanz bei Landwirten 
Das Leitbild strebt an, durch Maßnahmen in der Aus- und Fortbildung sowie der besseren Vernetzung von Landwirtschaft, Wissenschaft und relevanten Akteuren die umweltorientierte Leistungs- und Innovationsfähigkeit der Landwirtschaft zu erhöhen. Zugleich soll die Landwirtschaft dabei unterstützt werden, ihre Leistungen im Umwelt- und Naturschutz durch neue Geschäftsmodelle in Wert zu setzen. Auf diese Weise soll langfristig der Zielkonflikt vermindert werden.

4. Verbraucherinteresse an hohem Umwelt- und Verbraucherschutz vs. Zahlungsbereitschaft der Verbraucherinnen und Verbraucher

Das Leitbild beinhaltet Maßnahmen zur Verbraucherbildung und zur Entwicklung von Geschäftsmodellen, welche die Zahlungsbereitschaft der Verbraucherinnen und Verbraucher für Umweltleistungen erhöhen. Dies beinhaltet, dass entsprechende Umweltleistungen sichtbar gemacht und mit Produkten oder Dienstleistungen vertrauenswürdig in Zusammenhang gebracht werden, etwa durch geeignete Kennzeichnungs- und Informationssysteme.

5. Verstärkung eines beschleunigten Strukturwandels durch hohe Anforderungen im Natur- und Umweltschutz

Das Leitbild ist neutral gegenüber Betriebsstrukturen. Maßnahmen sollten so ausgestaltet sein, dass kleine und Nebenerwerbsbetriebe nicht strukturell benachteiligt werden. Allerdings wird auch von Klein- und Nebenerwerbslandwirten die entsprechende Sachkunde für eine natur- und umweltverträgliche Landbewirtschaftung erwartet.

6. Yield gap zwischen Produktionsmethoden mit hohem Input und/oder sehr intensiver Flächennutzung, die zu Emissionsproblemen und Habitatverlusten führen, einerseits, und naturverträglicheren Produktionsmethoden andererseits

Das Leitbild stellt den langfristigen Erhalt der ökologischen Systeme und der ökosystemaren Dienstleistungen in den Mittelpunkt. Deren Bewahrung wird von allen Produktionsmethoden verlangt. Dabei gilt das Vorsorge- und Vorsichtsprinzip.

7. Konflikt zwischen Natur- und Umweltschutz in Europa und Verlagerung von Produktion an Standorte mit geringeren Natur- und Umweltschutzstandards (Leakage-Problematik, insbesondere bei Futtermittelimporten)

Das Leitbild sieht vor, dass eventuelle Leakage-Probleme systematisch erfasst werden. Dafür sind ggf. geeignete Methoden zu entwickeln und anzuwenden. Bei Evidenz entsprechender Problemlagen erfordert das Leitbild, geeignete Gegenmaßnahmen zu treffen, nach Möglichkeit in Kooperation mit den Handelspartnern. Dabei sollten Anstrengungen zur Verbesserung der Umweltsituation bei den Handelspartnern im Vordergrund stehen. Das Leitbild darf nicht dazu dienen, dass vermeintliche Leakage-Probleme zum Vorwand für protektionistische Anliegen zu nehmen.

8. Zielkonflikte zwischen Naturschutz und Artenschutz in der Feinsteuerung von Maßnahmen

Das Leitbild geht von einem systemischen Ansatz aus. Mögliche Zielkonflikte zwischen Naturschutz und Artenschutz sind aus dieser Perspektive im jeweiligen Kontext zu bewerten und zu entscheiden. 
9. Umweltschutz europäischer Prägung vs. Freihandelsregime - Importkonkurrenz

Das Leitbild beinhaltet, dass die Landwirte in Deutschland faire Wettbewerbsbedingungen erwarten können. Die Rahmenbedingungen sind dabei so auszugestalten, dass die Umweltleistungen aller Wettbewerber transparent sind und damit vorrangig als positive Komponente und nicht als Kostennachteil in den Wettbewerb eingehen. Kostennachteile durch gesetzliche Auflagen rechtfertigen aus Sicht des Leitbilds eine Kompensation, sollten aber auf nachweisbaren, wissenschaftlich fundierten Berechnungen beruhen. Dies dient langfristig auch der WTO-Kompatibilität solcher Zahlungen.

10. Anspruch und Zielgenauigkeit von Programmen vs. Zustimmungsfähigkeit in der EU

Das Leitbild räumt der Effektivität und Zielgenauigkeit politischer Maßnahmen und Programme einen hohen Stellenwert ein. Inwiefern das fachlich und gesellschaftlich Wünschenswerte am Ende in komplexen Verhandlungen durchgesetzt werden kann, ist eine politische Frage jenseits des Aussagebereichs des Leitbilds.

11. Rolle Deutschlands als Nettozahler vs. mehr Ko-Finanzierung (bei der Zweiten Säule)

Das Leitbild enthält keine Aussagen zur Finanzierung. Angesichts der Erfahrungen mit früheren GAP-Reformen wird es aus pragmatischen Gründen geboten sein, strategische Handlungsoptionen zu entwickeln, die weitgehend verteilungsneutral sind (siehe Kap. 7 und 8).

12. Wunsch nach sektoraler Koordination vs. Wunsch nach Bürokratieabbau

Das Leitbild misst der Koordination und Kooperation zwischen landwirtschaftlichen und anderen relevanten Akteuren in einer Region große Bedeutung zu. Koordination bedeutet aus Sicht des Leitbilds dabei gerade nicht Bürokratie, sondern ergebnisorientierte und innovative Zusammenarbeit. Zur Minimierung des Zeitaufwands und zur positiven und wertschätzenden Gestaltung solcher Prozesse sieht das Leitbild vor, dass Mittel zur problemorientierten Vernetzung der Akteure bereitgestellt werden.

13. Berücksichtigung systemischer Zusammenhänge und schädlicher kumulativer Effekte bei der Rechtssetzung und Implementation vs. Anforderung der eindeutigen kausalen Zurechnung auf Verursacher

Das Leitbild geht von einer systemischen Perspektive aus, räumt dem im Grundgesetz verankerten Rechtsstaatsprinzip aber unbedingten Vorrang ein. Rechtlich relevante kausale Zurechnungen müssen daher belastbar sein. Beweislastumkehr kann in gut begründeten Fällen angemessen und geeignet sein, um Implementationsdefiziten bei schädlichen kumulativen Effekten entgegenzuwirken. 


\subsection{Qualitative und quantitative Zielwerte und Soll-Ist- Vergleich mit dem derzeitigen Zustand}

Im Folgenden werden anhand des Leitbildes einer multifunktionalen, natur- und umweltverträglichen Landwirtschaft Leitindikatoren abgeleitet. Für die Leitindikatoren und ggf. Unterindikatoren werden Zielwerte beschrieben. Die Beschreibung der Zielwerte erfolgt dabei qualitativ oder quantitativ. Anschließend werden die Zielwerte soweit als möglich mit Daten der Ist-Situation verglichen, so dass sich aus diesem Vergleich der Handlungsbedarf ableiten lässt.

\subsection{Prioritäre Problemlagen}

Während ein Leitbild eine allgemeine Orientierung für die erwünschte Entwicklungsrichtung geben kann, ist es notwendig, diejenigen Problemlagen zu identifizieren, bei denen sich der Abstand zwischen dem erwünschten und dem derzeitigen Zustand besonders gravierend darstellt. In diesem Abschnitt soll daher die Analyse der Problemlagen verdichtet werden.

Die Übersicht in Tab. 6.1 sowie die Problemanalyse in Kap. 3 und 4 zeigen zwei größere Problemkomplexe. Wichtige Natur- und Umweltschutzproblemlagen ergeben sich zum einen aus den stofflichen Einträgen der Landwirtschaft in die Naturressourcen, zum anderen durch die Art und Weise der Flächennutzung. Im Folgenden werden diese beiden Bereiche als prioritäre Problemlagen bezeichnet. Des Weiteren zeigt sich, dass sich Probleme des Natur- und Umweltschutzes daraus ergeben, dass zum einen Indikatoren und Zielwerte für einige Problembereiche fehlen (Orientierungs- und Analysedefizit, Regelungsdefizit). Zum anderen werden bestehende Zielwerte nicht erreicht (Vollzugsdefizit). Zusammenfassend wird dieser Bereich als Implementationsdefizit bezeichnet. Doch es bestehen auch Defizite hinsichtlich der Beteiligung relevanter Akteursgruppen (Partizipationsdefizite) und der Entwicklung und Implementierung von Innovationen. Es ergeben sich somit drei Problemkomplexe, auf die eine zukunftsfähige Agrarpolitik vorrangig ausgerichtet sein sollte, und in denen jeweils vier Aspekte hervorzuheben sind:

Komplex 1: Stoffeinträge in Boden, Wasser und Luft

- Verlust von Biodiversität und Habitatqualität durch Stoffeinträge;

- Nitratproblematik, Arzneimitteleinträge, Pestizideinträge (Auswirkungen u. a. auf Wasserqualität);

- Antibiotikaresistenz;

- Klimaschutz: Treibhausgas-Emissionen.

Komplex 2: Flächennutzung

- Biodiversitäts- und Habitatverlust;

- Bodenschutz: Erosion, Schadverdichtung;

- Treibhausgas-Emissionen durch Landnutzungsänderungen;

- Anpassung an den Klimawandel. 


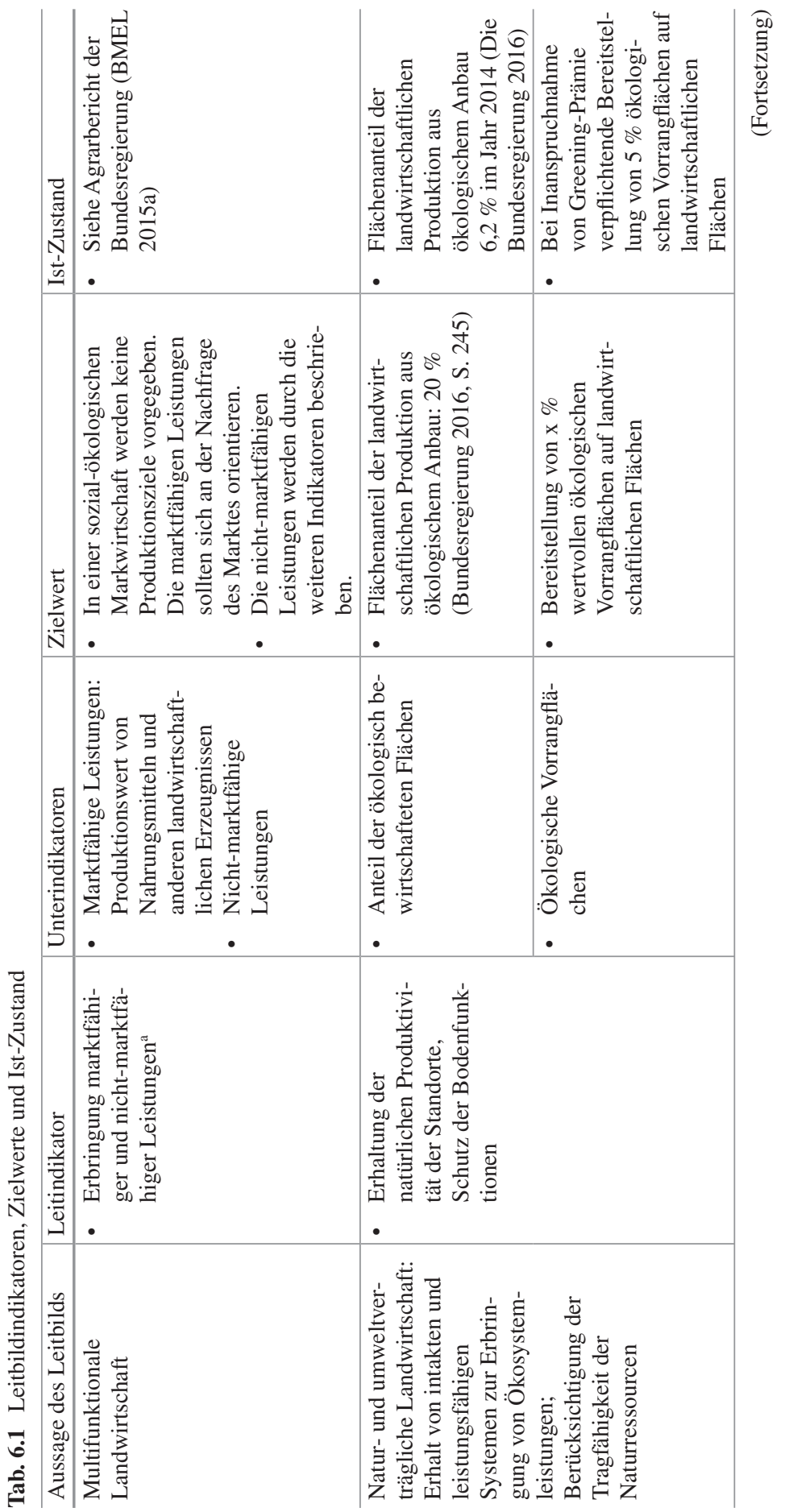




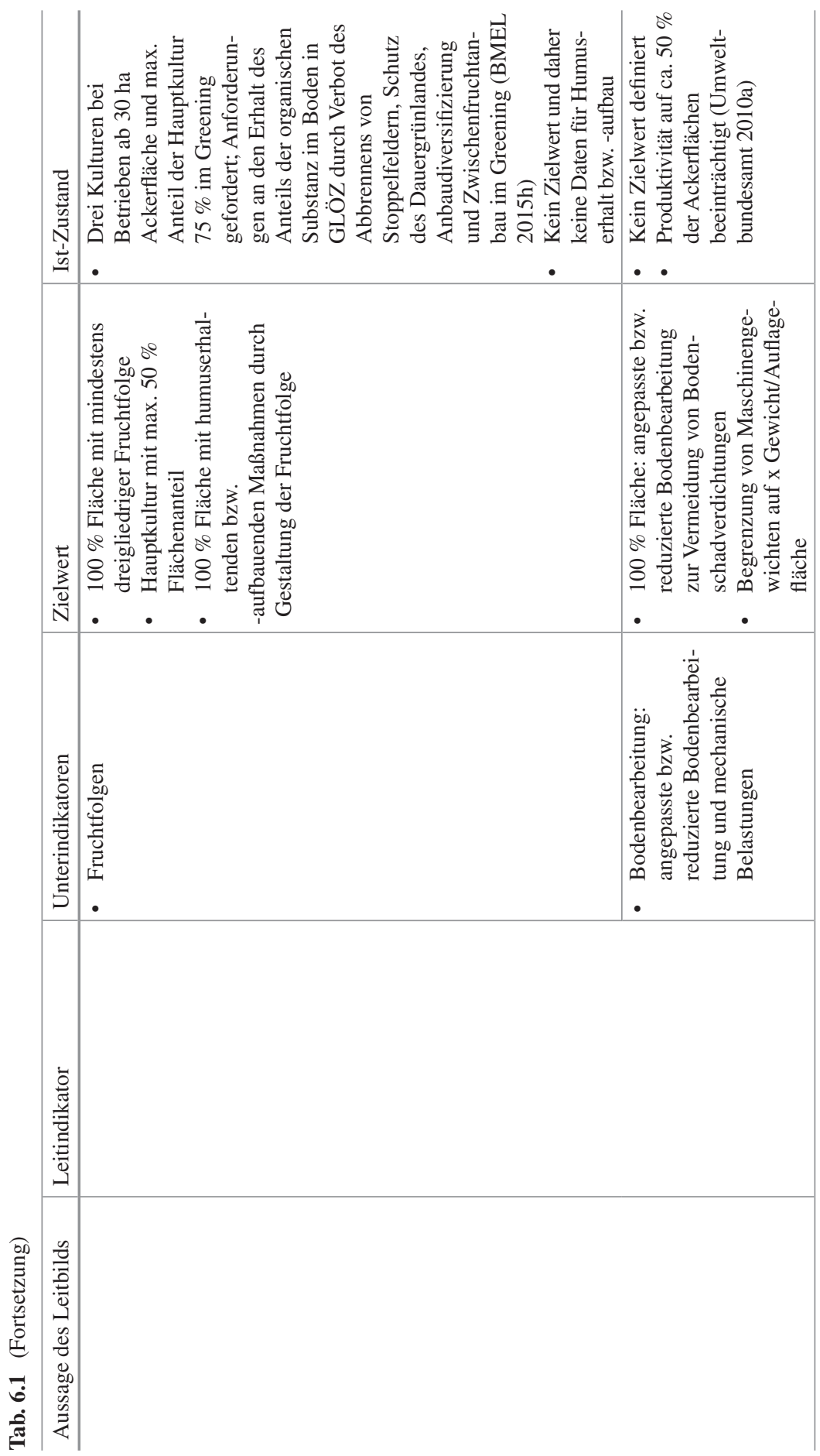




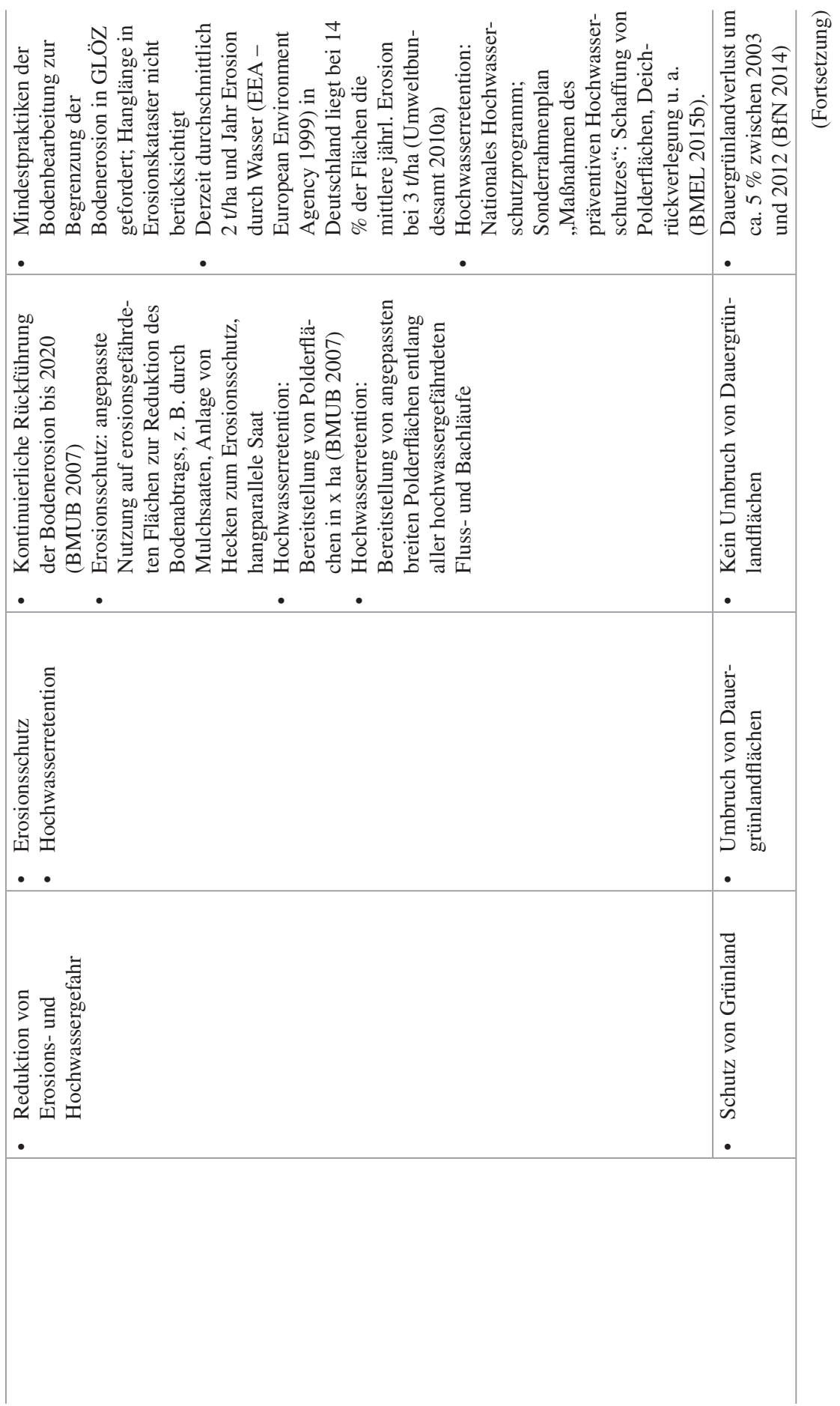




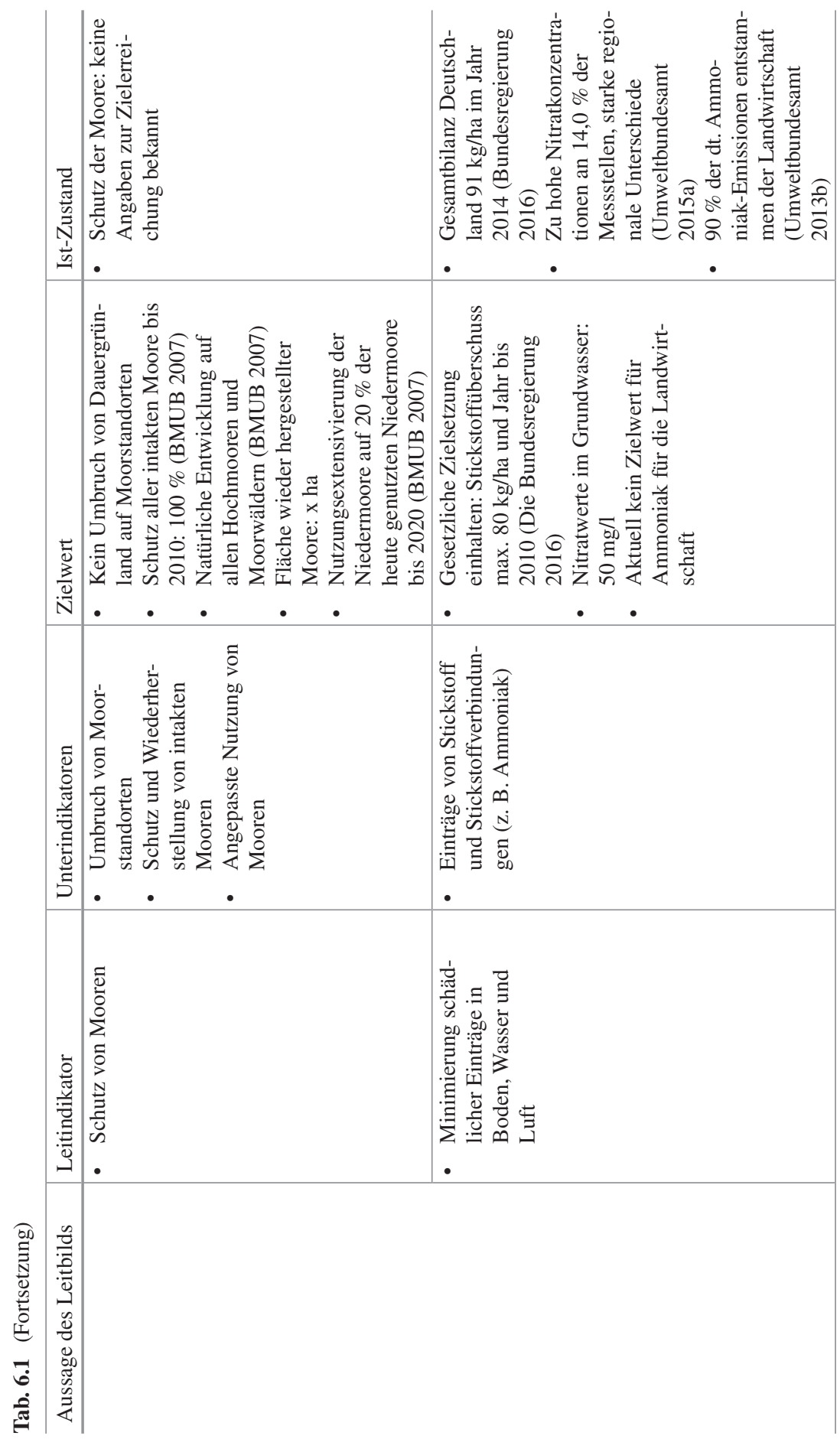




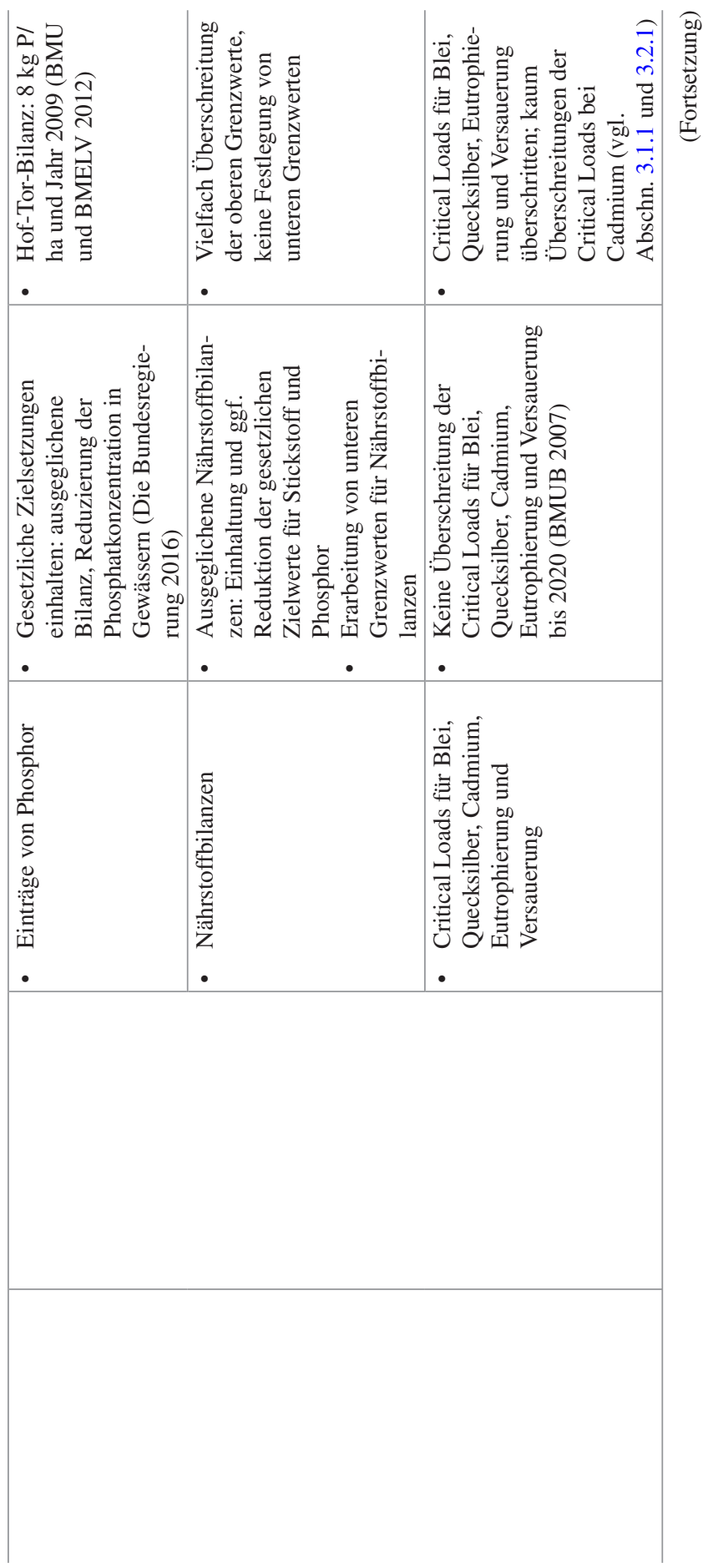




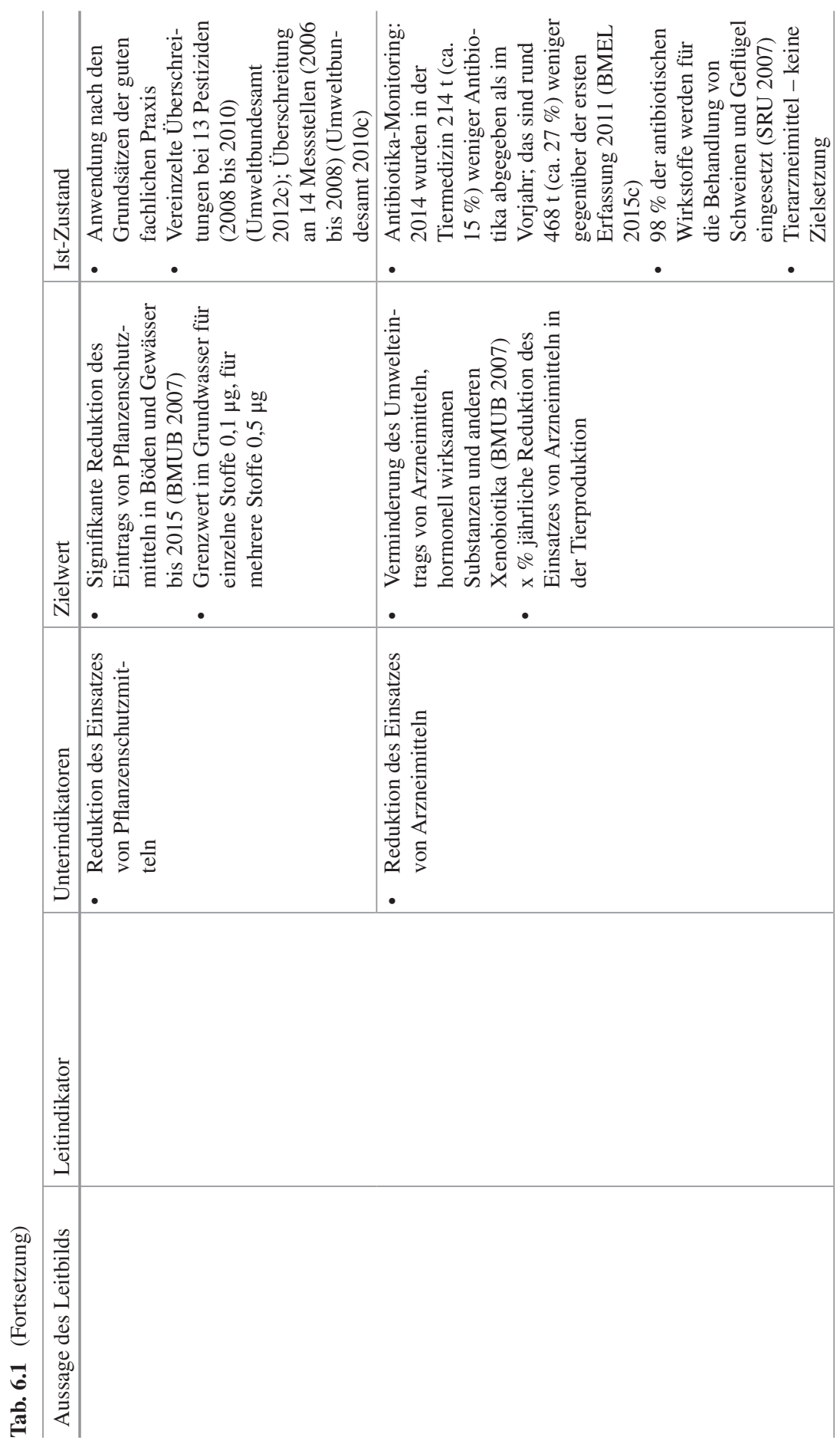




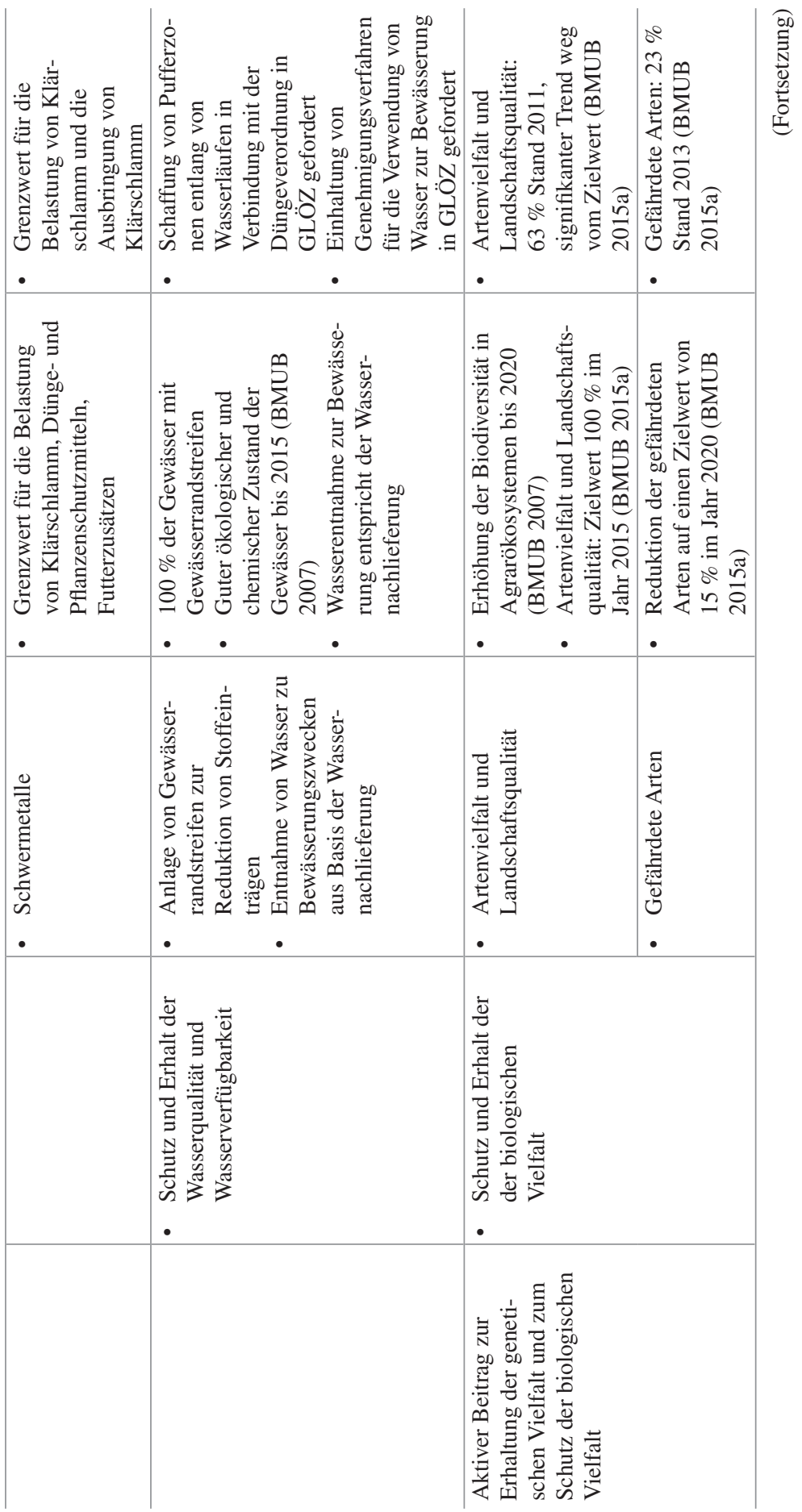




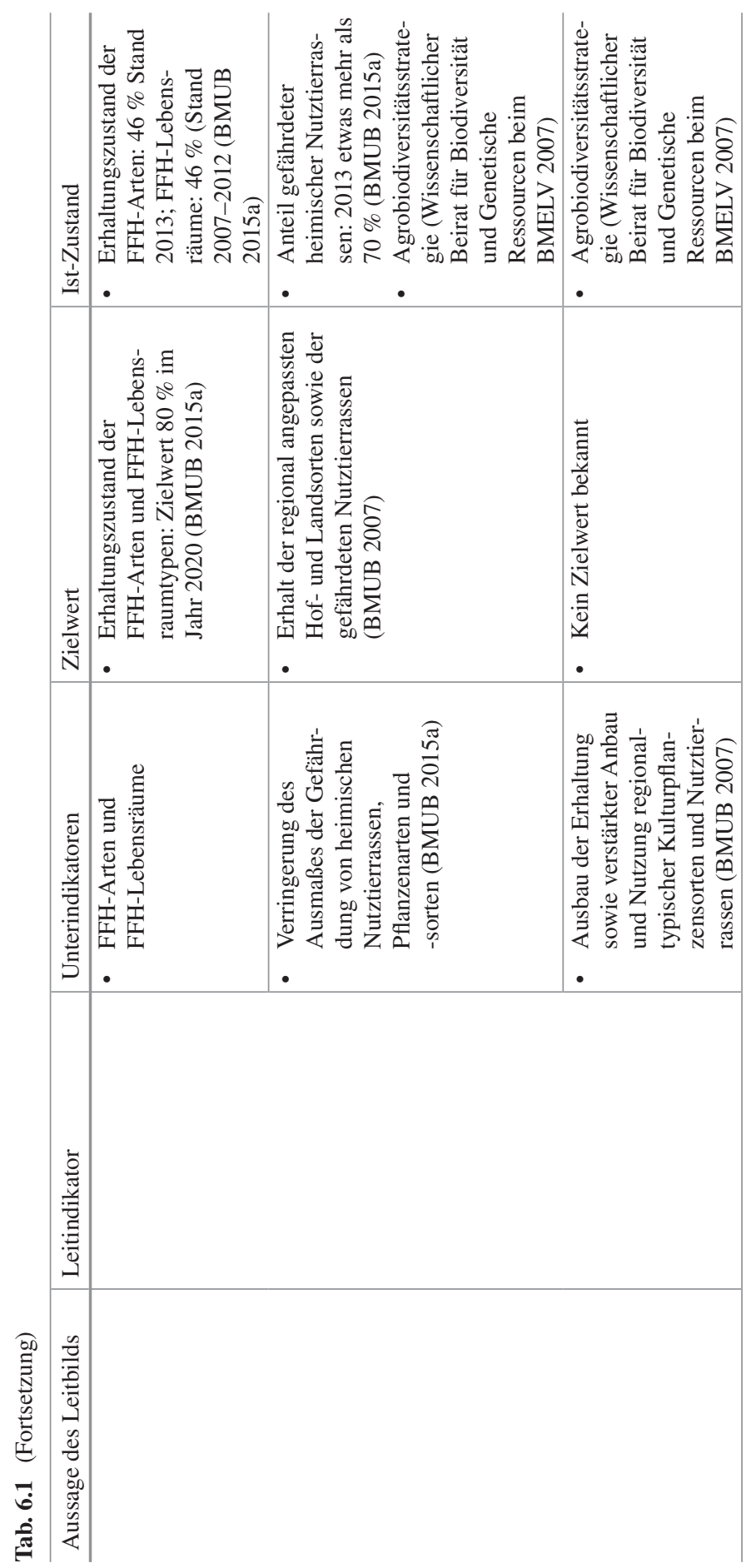




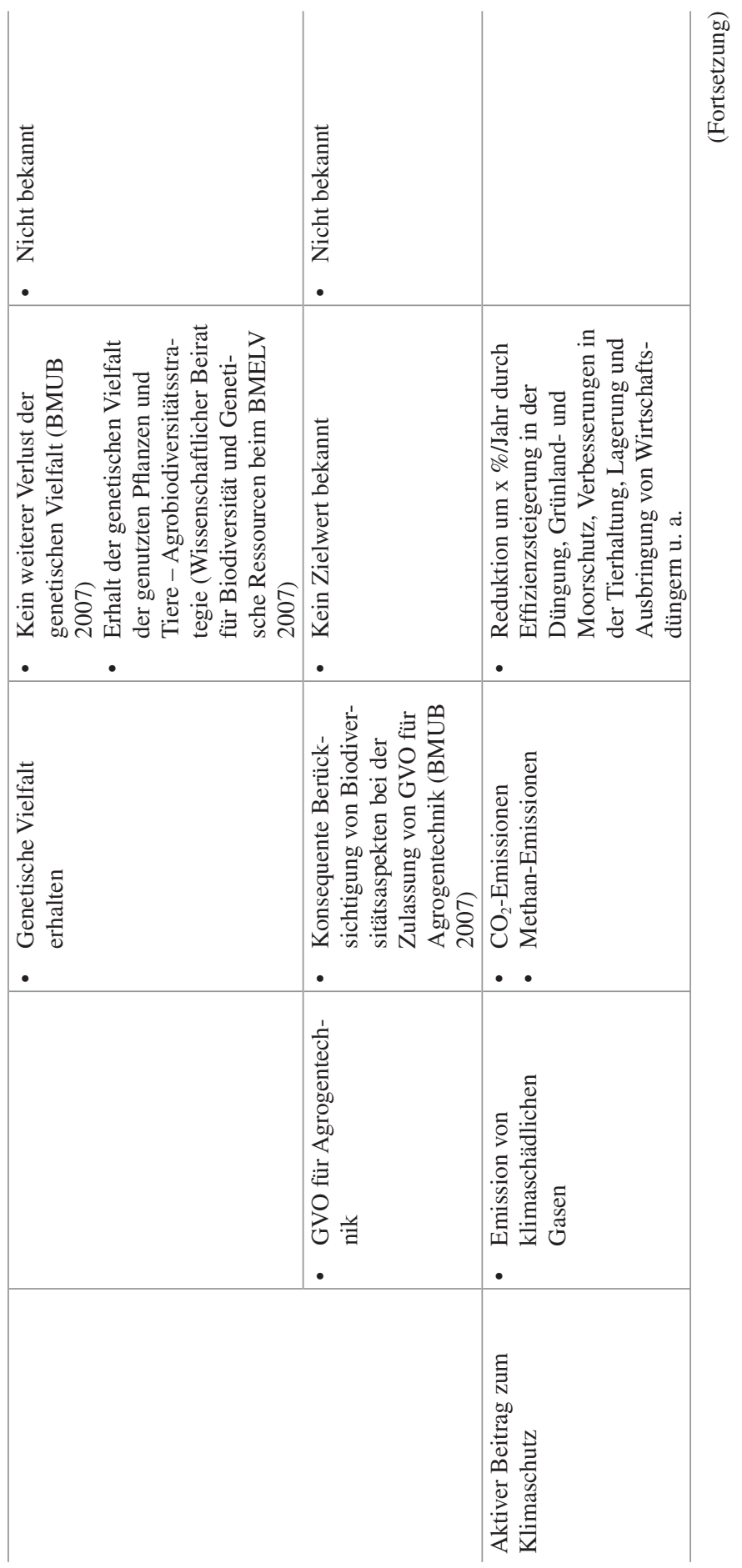




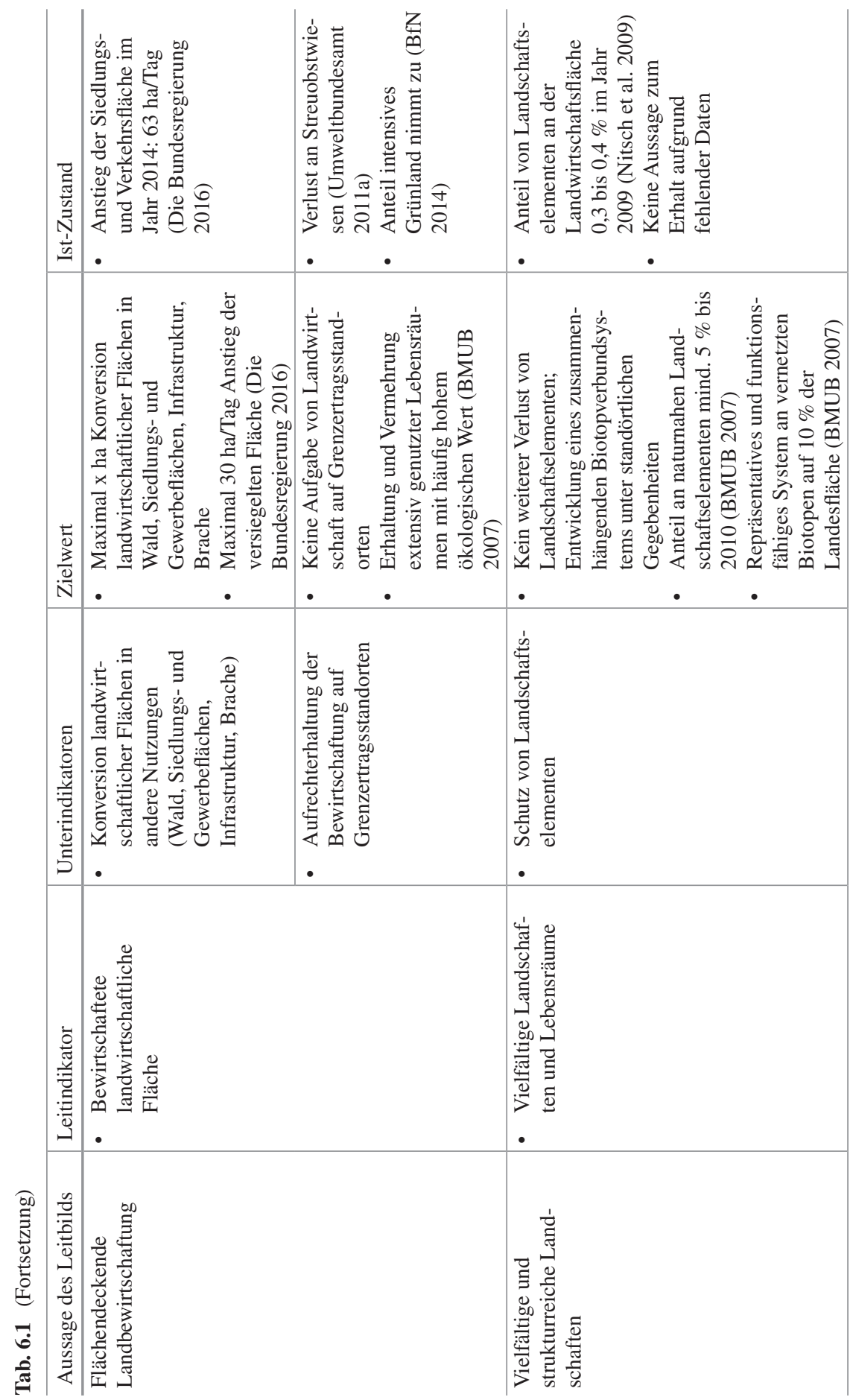




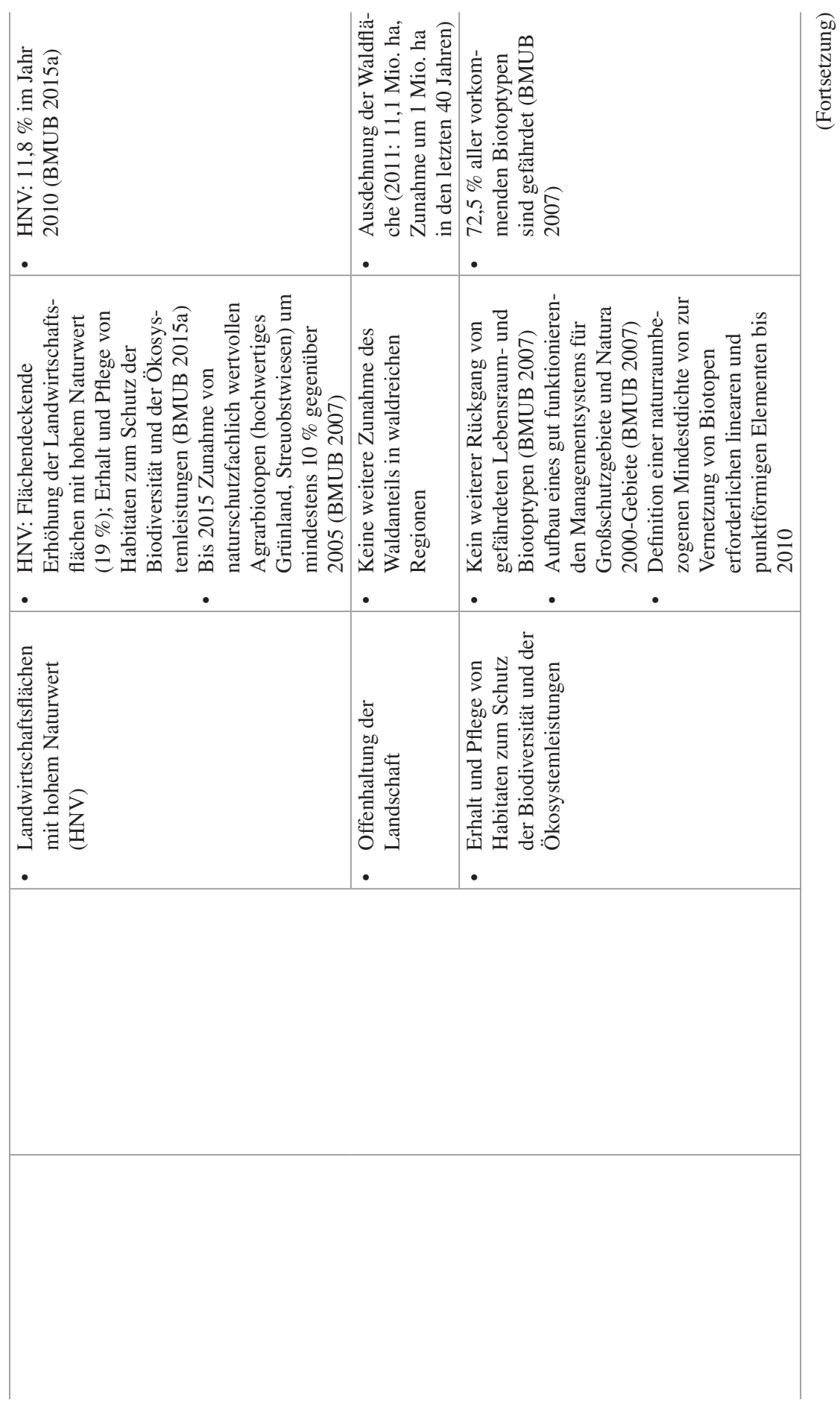




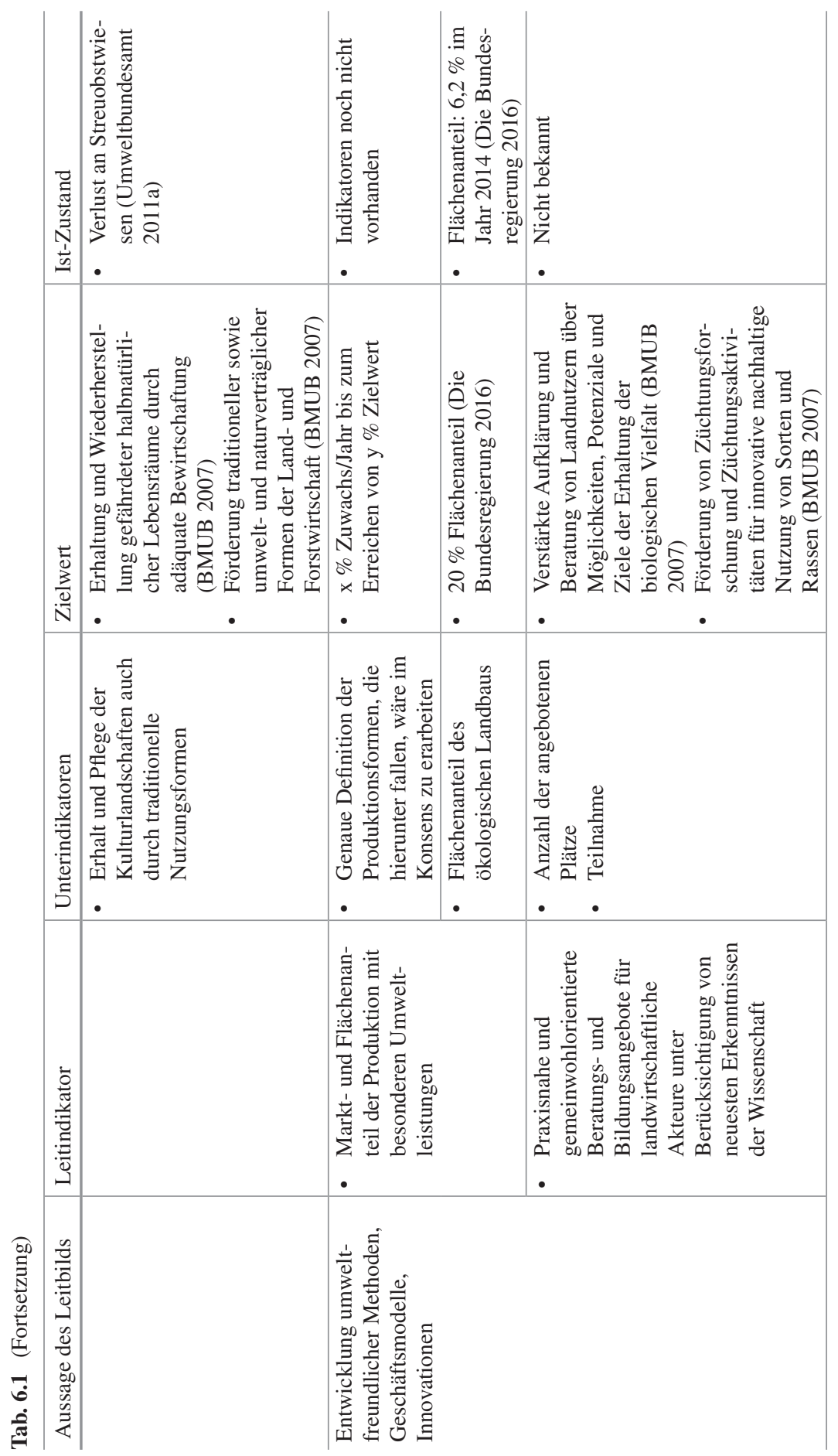




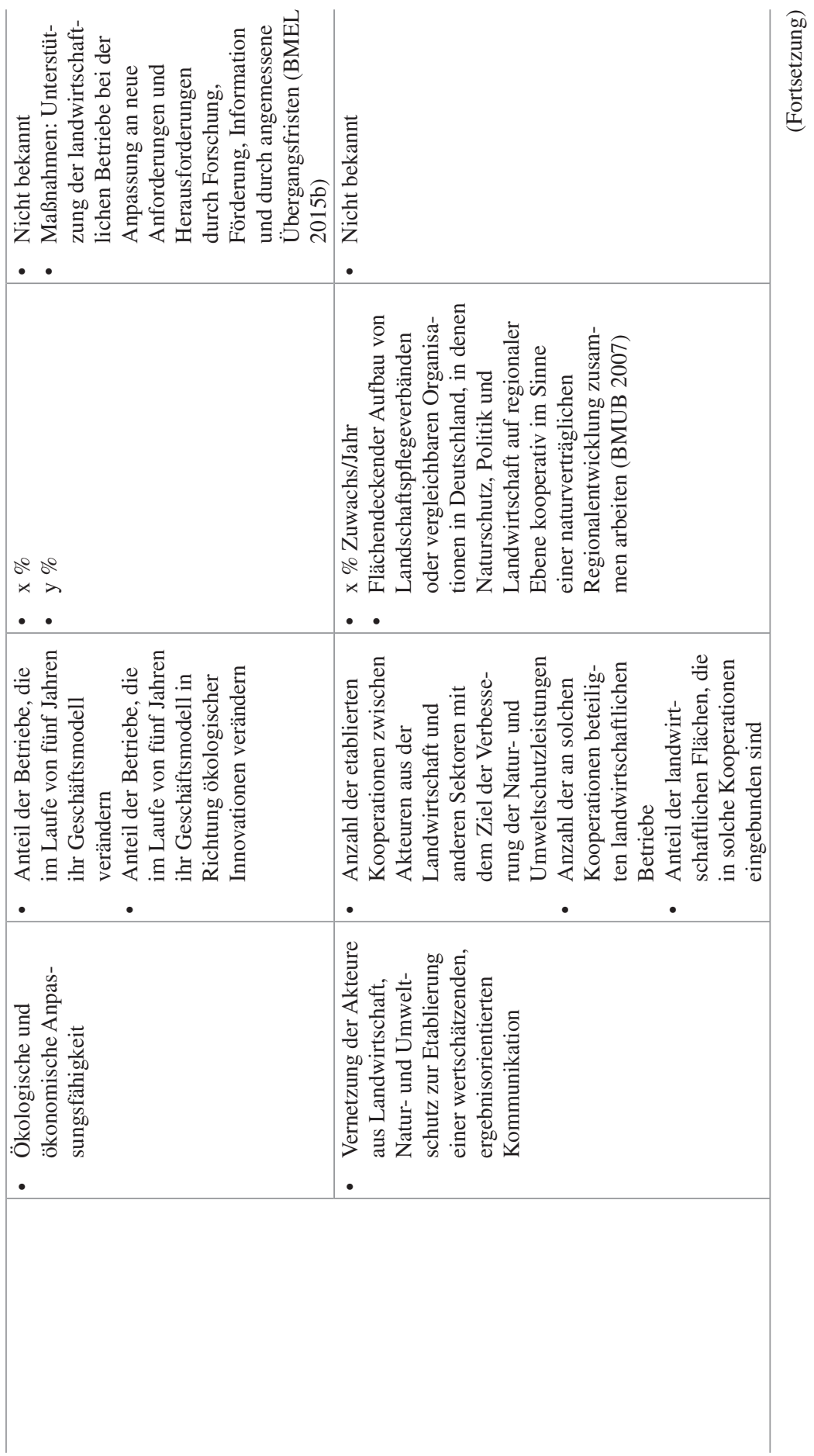




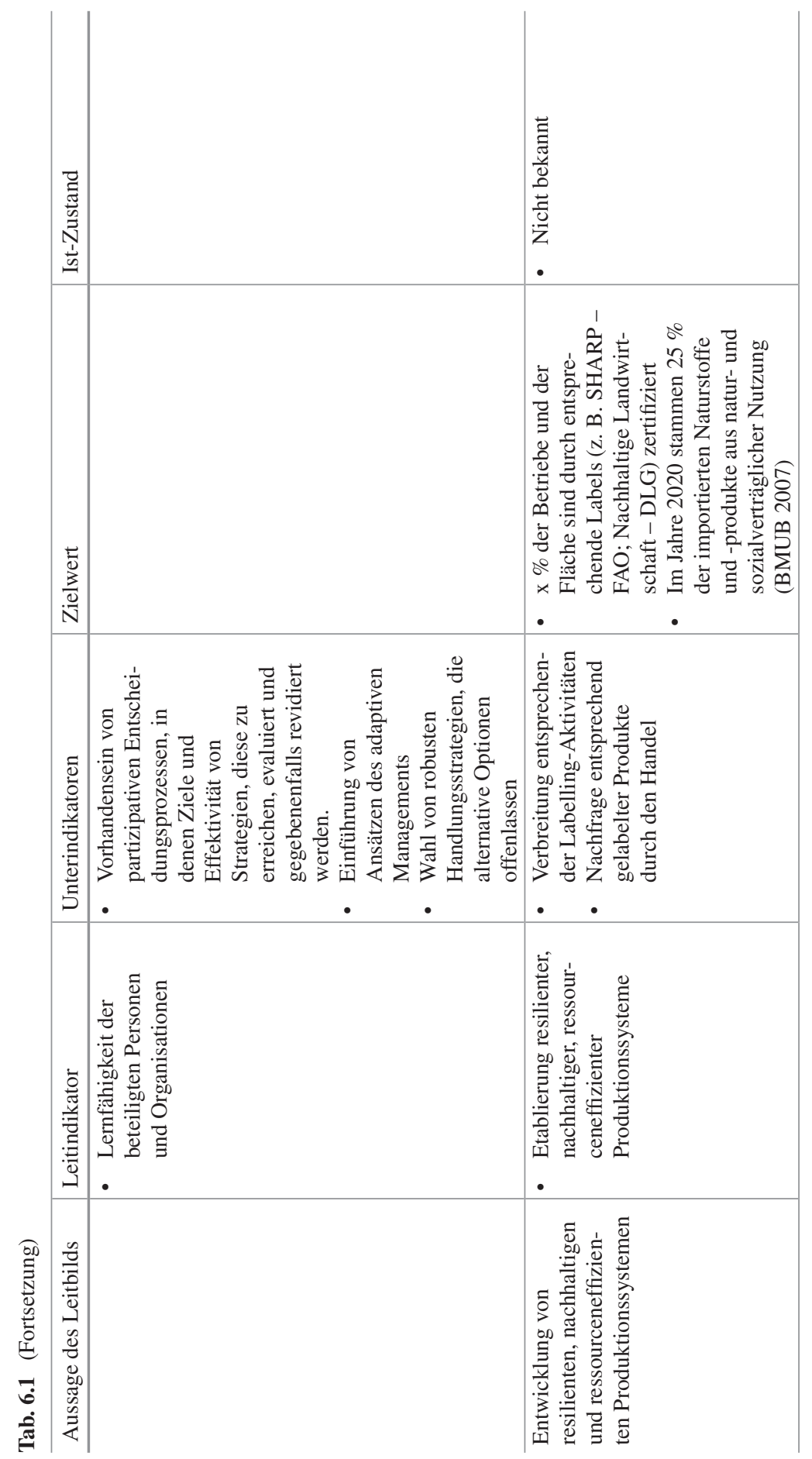




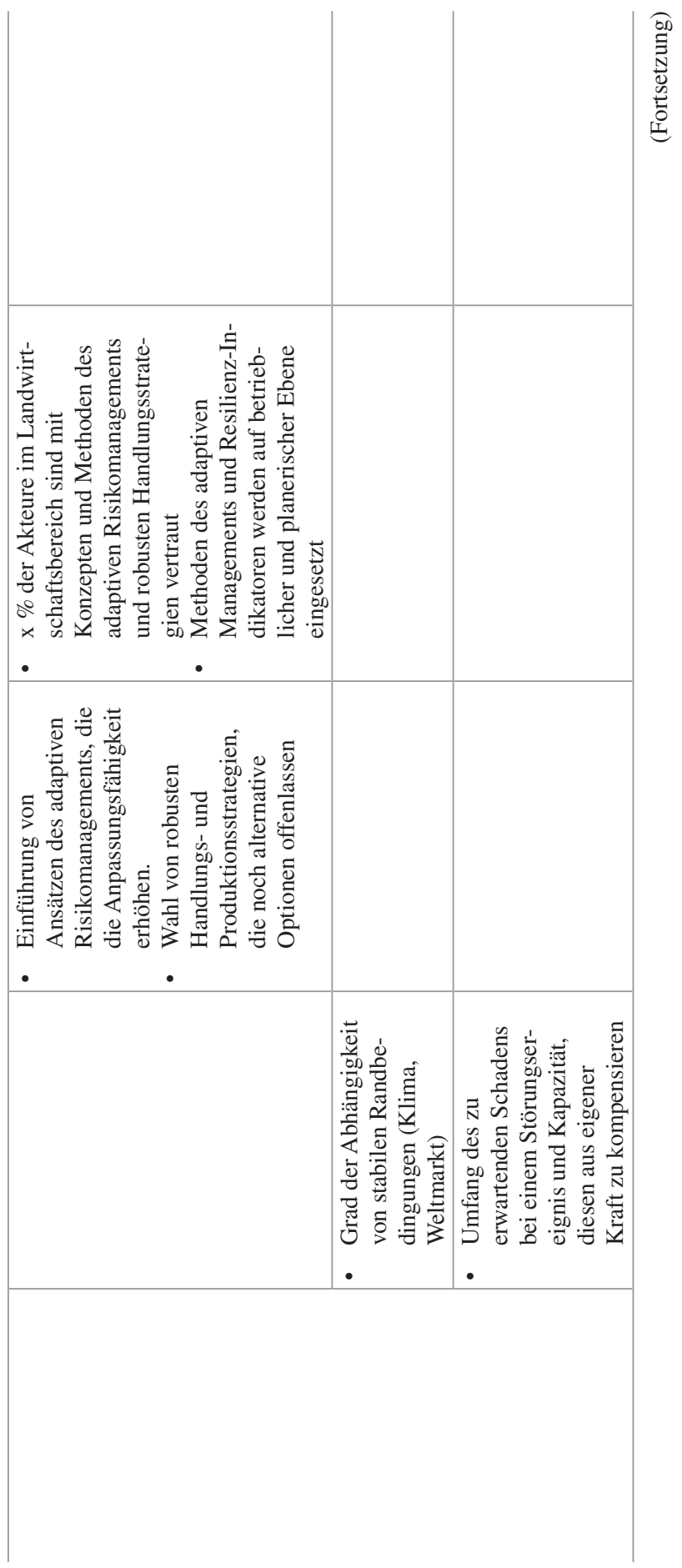




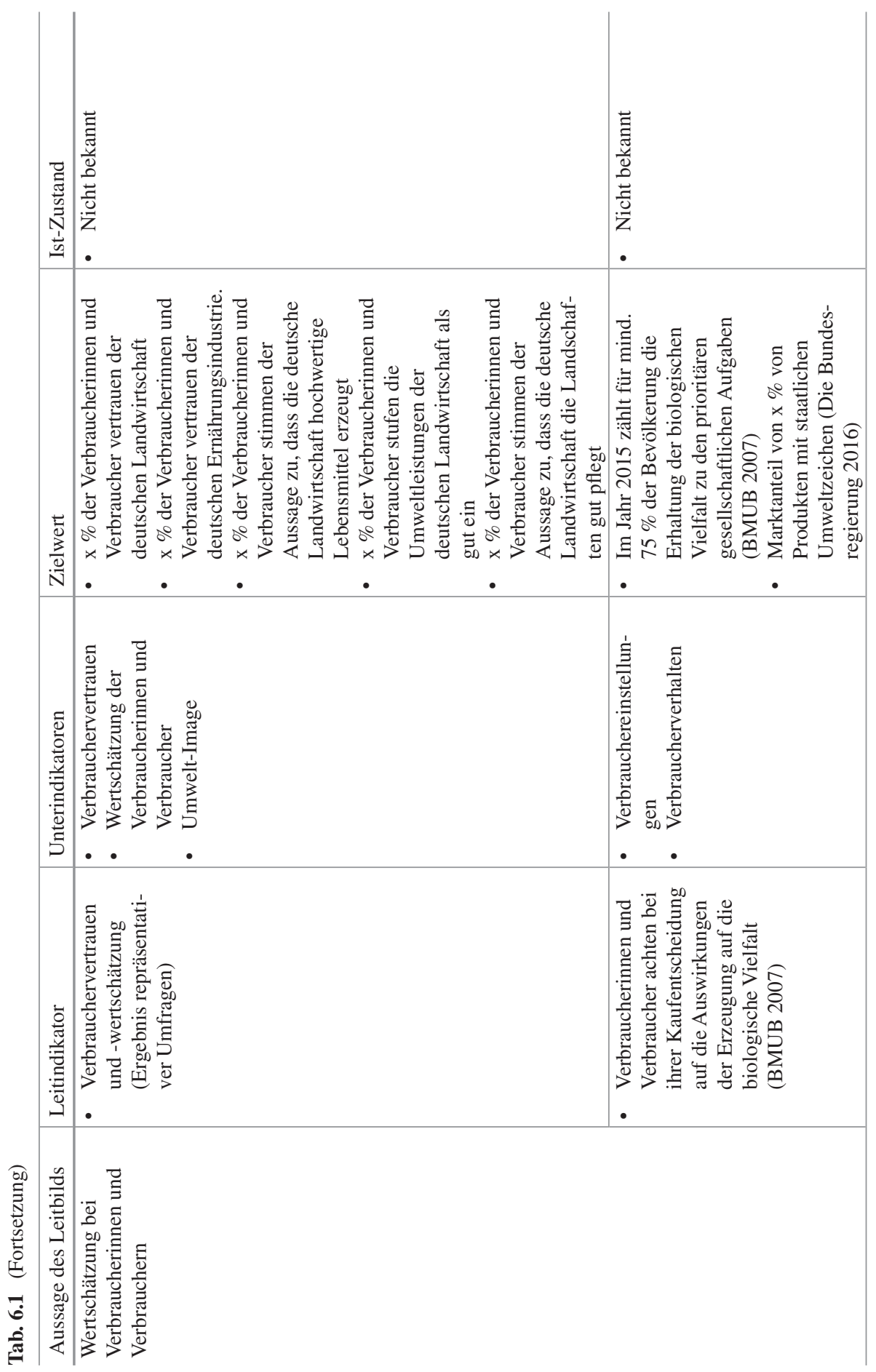




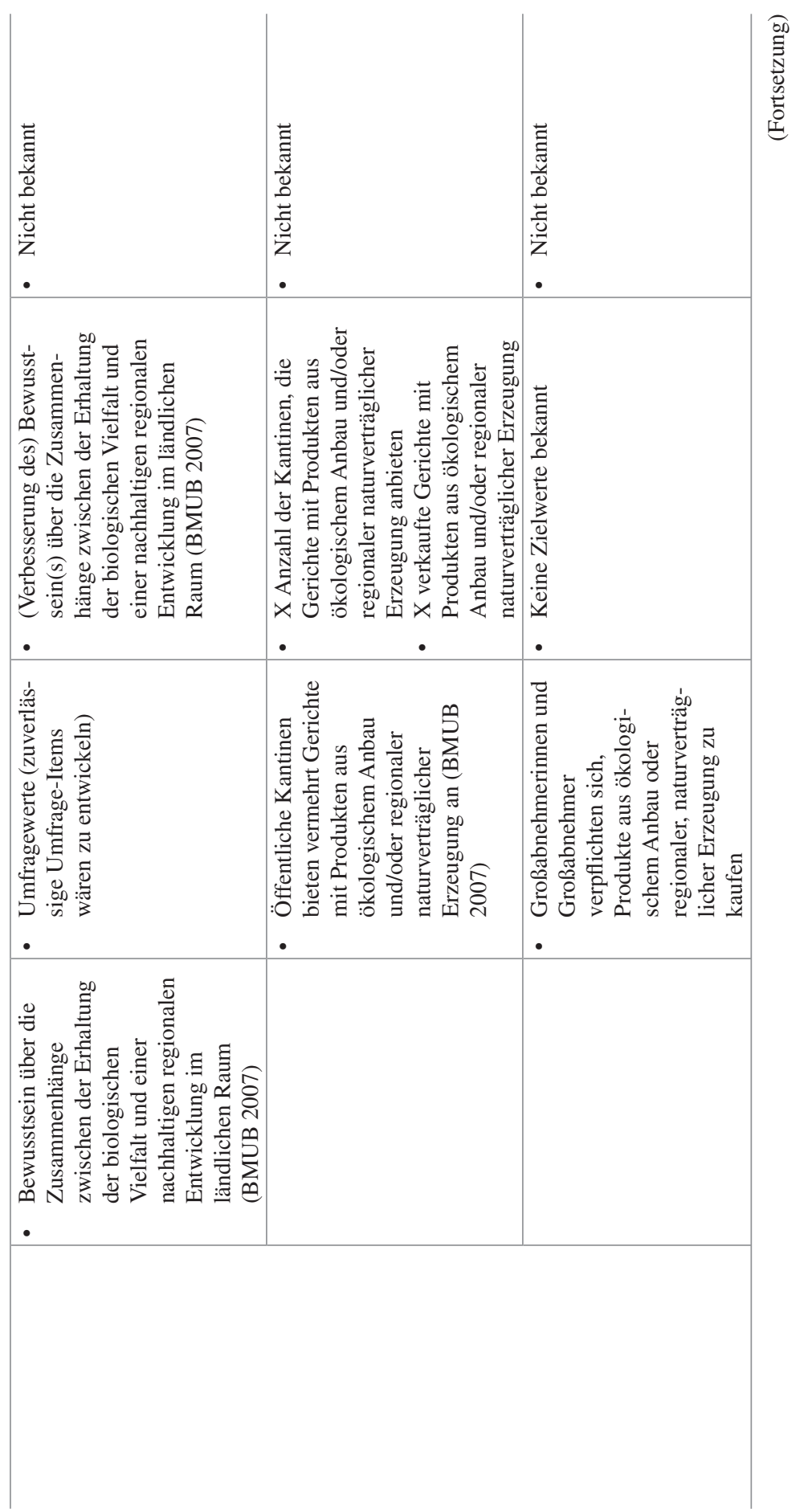




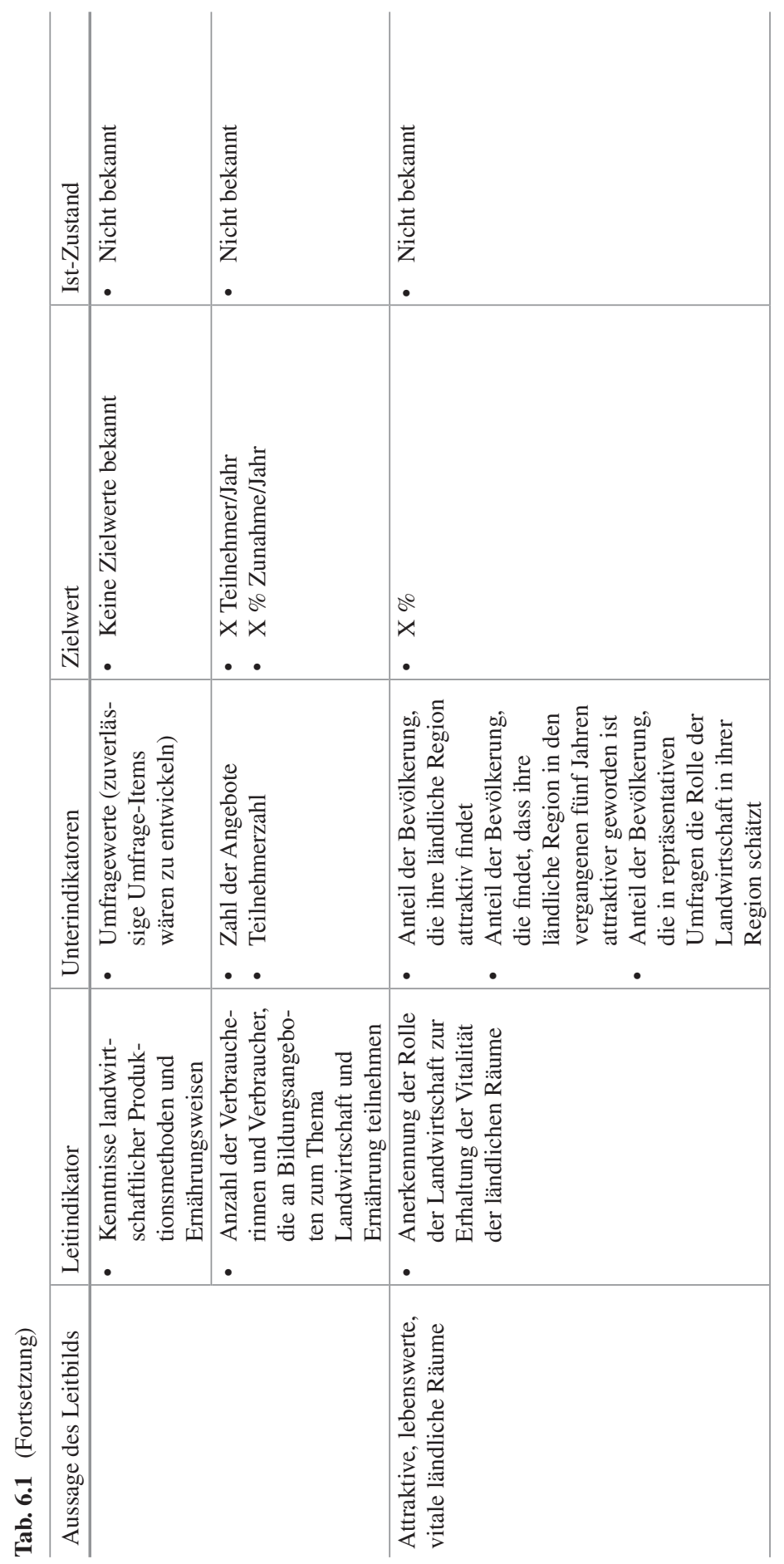




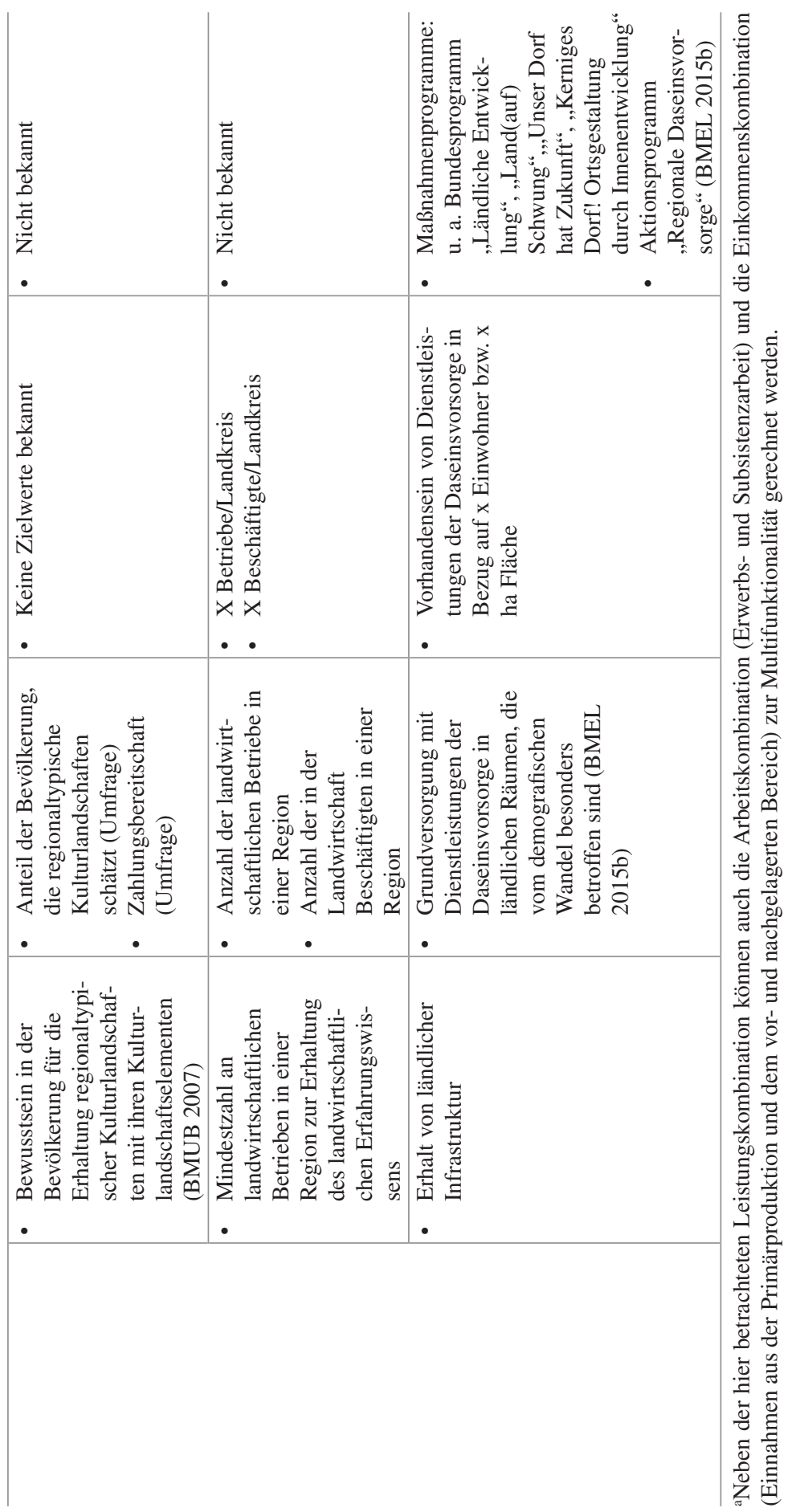


Komplex 3: Implementations-, Partizipations- und Innovationsdefizite

- Implementationsdefizite;

- Fehlende operationalisierte Ziele und unklare Rechtsbegriffe;

- Unzureichende Kontroll- und Sanktionsmechanismen;

- Zu wenige Lernprozesse und zu wenig sektorübergreifende Kooperation.

Der Problemkomplex der Stoffeinträge in Boden, Wasser und Luft legt einen im Kern regulatorischen Politikansatz auf europäischer Ebene nahe, da die Emissionen im Zusammenhang mit der Erzeugung von agrarischen Produkten entstehen, die im EU-Binnenmarkt oder auf internationalen Märkten abgesetzt werden. Die Herstellung gleicher Standards für alle Erzeuger dient daher zugleich dem fairen Wettbewerb und der Sicherung eines hohen Schutzniveaus. Ob als Ausgleich für eventuelle Kostennachteile, die durch die Verwirklichung eines gegenüber Wettbewerbern von außerhalb der EU möglicherweise höheren Schutzniveaus eintreten, Zahlungen an die landwirtschaftlichen Erzeuger gerechtfertigt sind, muss geprüft werden. Sowohl der Anspruch eines höheren Schutzniveaus als auch eventuelle Mehrkosten wären dabei auf transparente Weise und nach wissenschaftlichen Kriterien nachzuweisen.

Der Problemkomplex Flächennutzung erfordert eher einen räumlich differenzierten, flächenbezogenen Politikansatz mit einem vielfältigen Instrumentenkasten. Allerdings können flächendeckende Ge- oder Verbote angemessen und erforderlich sein, um überall den Bodenschutz und eine Mindestausstattung an Habitaten sicherzustellen oder um unerwünschten Landnutzungsänderungen vorzubeugen. Diesen Zielen entsprechende Auflagen zur Flächennutzung, wie etwa ein Mindestanteil von ökologischen Vorrangflächen, können die Wettbewerbsfähigkeit von Betrieben gegenüber Wettbewerbern ohne solche Auflagen beeinträchtigen. Daher kann eine flächenbezogene Kompensation nach Art der Direktzahlungen angemessen sein, wenn ihre Höhe nachgewiesenermaßen in Relation zu den durch die Auflagen entstehenden Kostennachteilen steht.

Der Problemkomplex der Implementations-, Partizipations- und Innovationsdefizite liegt quer zu den ersten beiden Komplexen. Er umfasst die systemischen Defizite in der Bestimmung der Rechtsbegriffe, der Implementation von natur- und umweltschutzbezogenen Rechtsnormen, der unzureichenden Kontroll- und Sanktionsmechanismen sowie die vielerorts mangelnde Offenheit für neue Ansätze und sektorübergreifende Kooperationen. Diese Defizite werden oft mit dem Paradigma des landwirtschaftlichen Exzeptionalismus in Verbindung gebracht, der eine besondere und oft isolierte Rolle der Agrarpolitik zu rechtfertigen sucht (vgl. Abschn. 4.1). Demgegenüber zielt das neue Leitbild auf eine bessere Integration landwirtschaftlicher Erzeugung in gesellschaftliche Kooperationen und eine höhere Responsivität gegenüber gesellschaftlichen Anforderungen ab.

\subsection{Abgleich der derzeitigen Agrarpolitik mit den agrarpolitischen Leitlinien}

Die folgende Tabelle gibt eine schematische Übersicht, in welchem Maße die derzeitige Agrarpolitik den vorgeschlagenen agrarpolitischen Leitlinien entspricht (Tab. 6.2). 


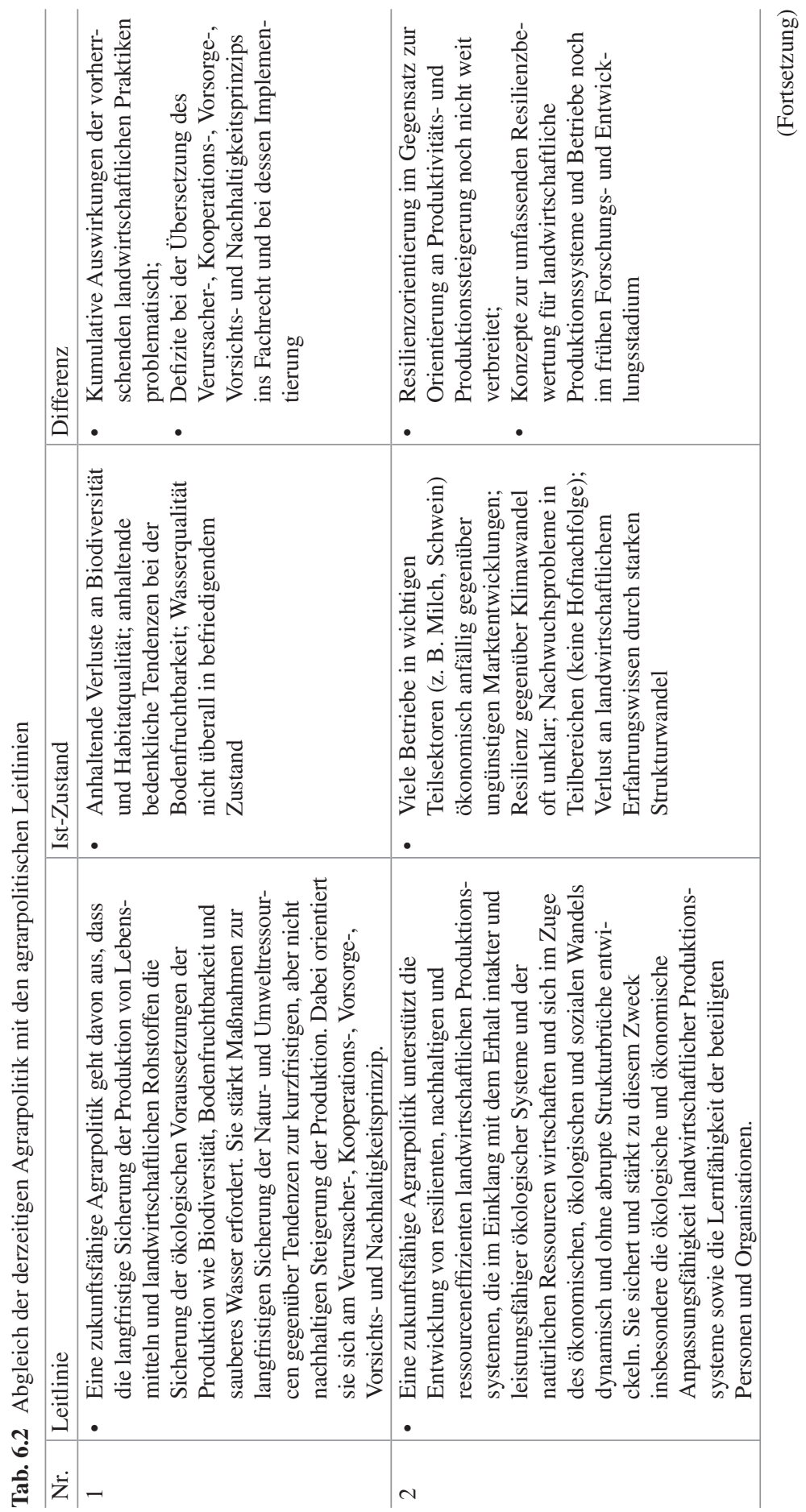




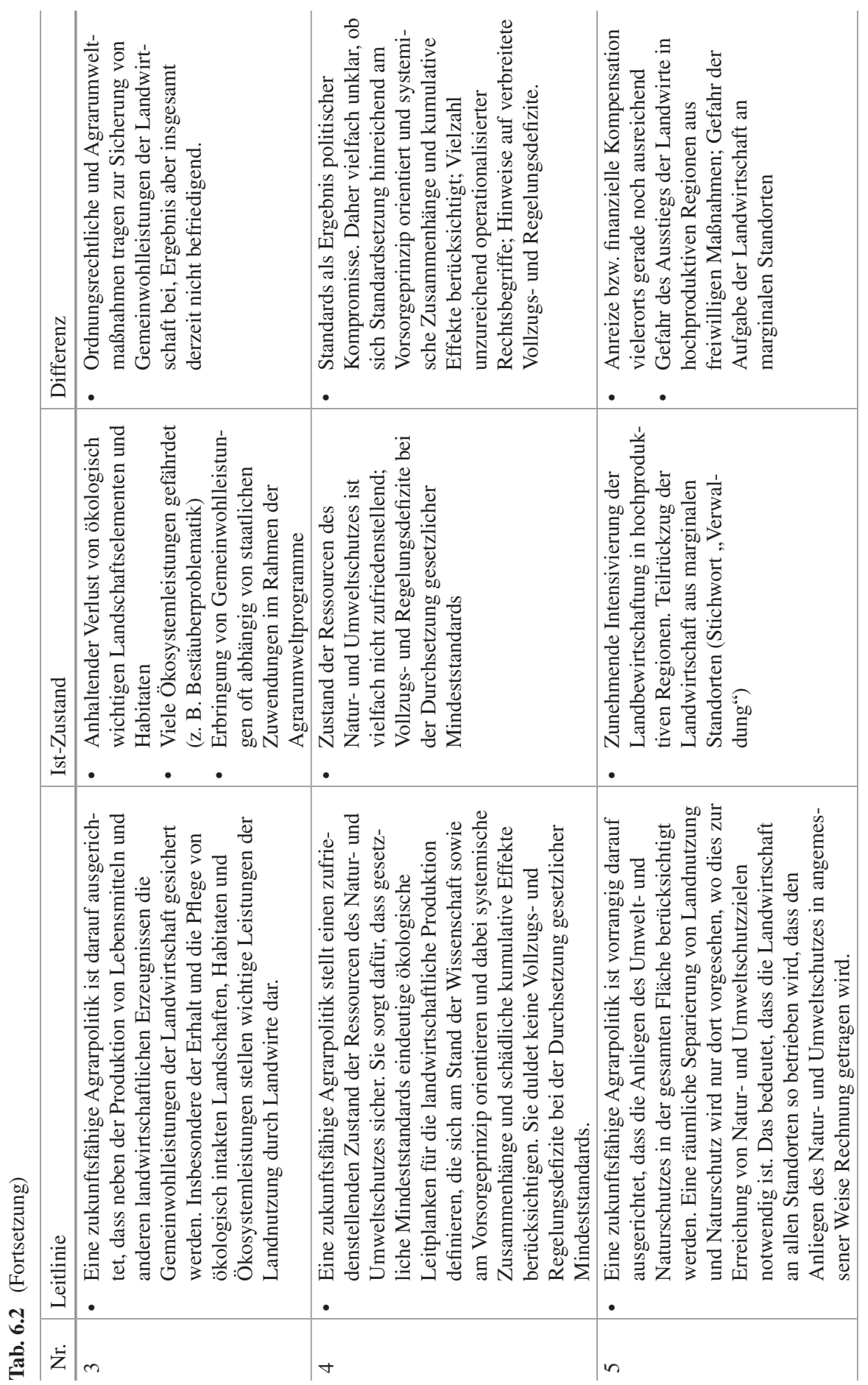




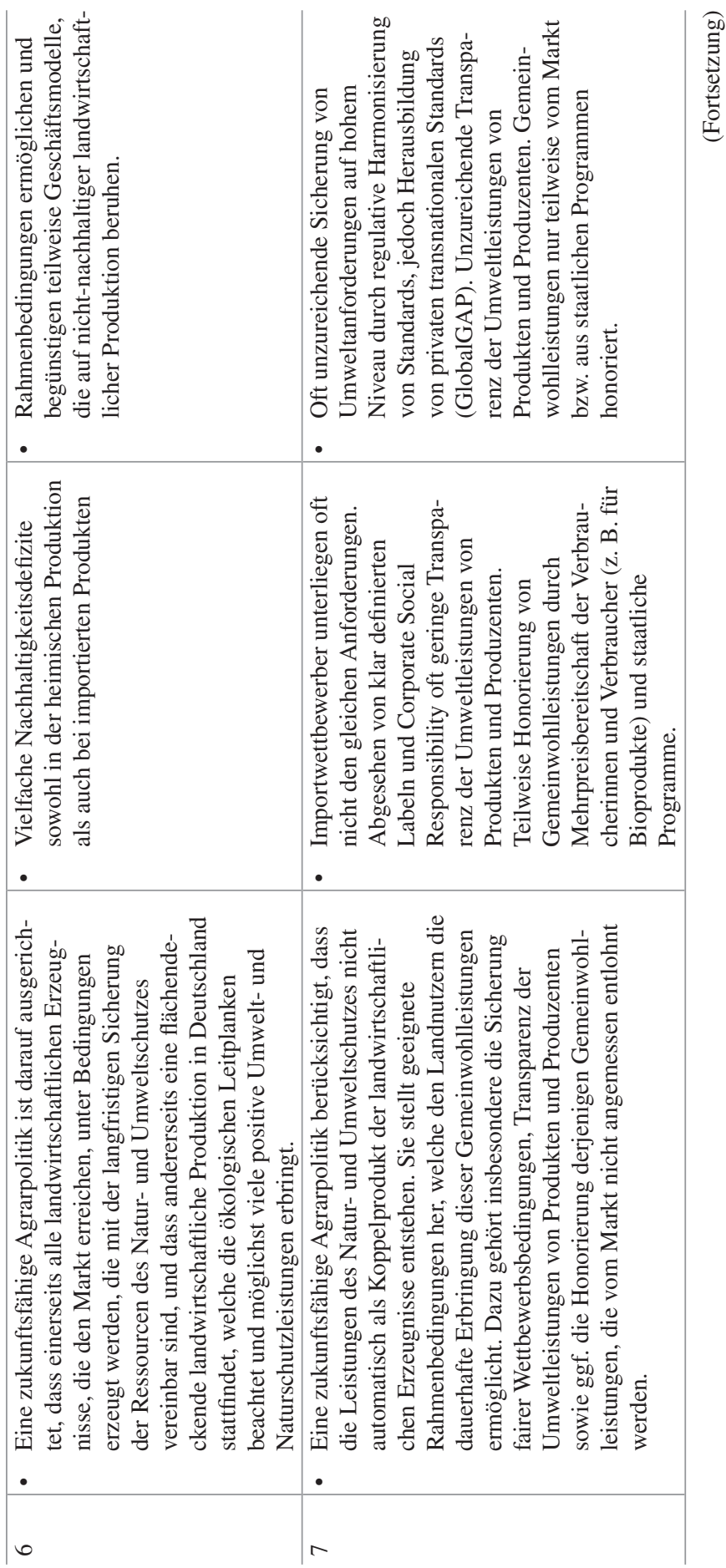




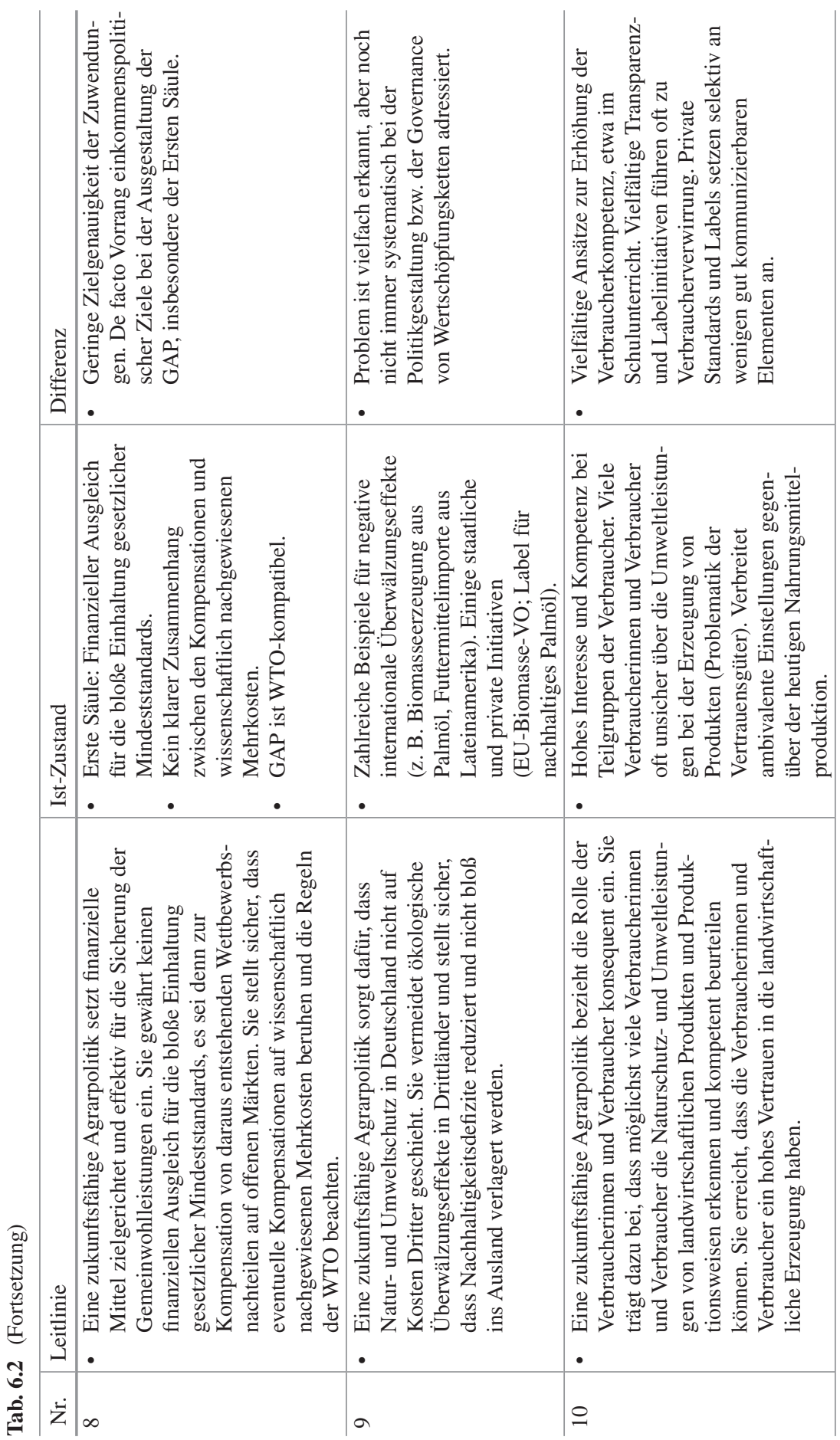




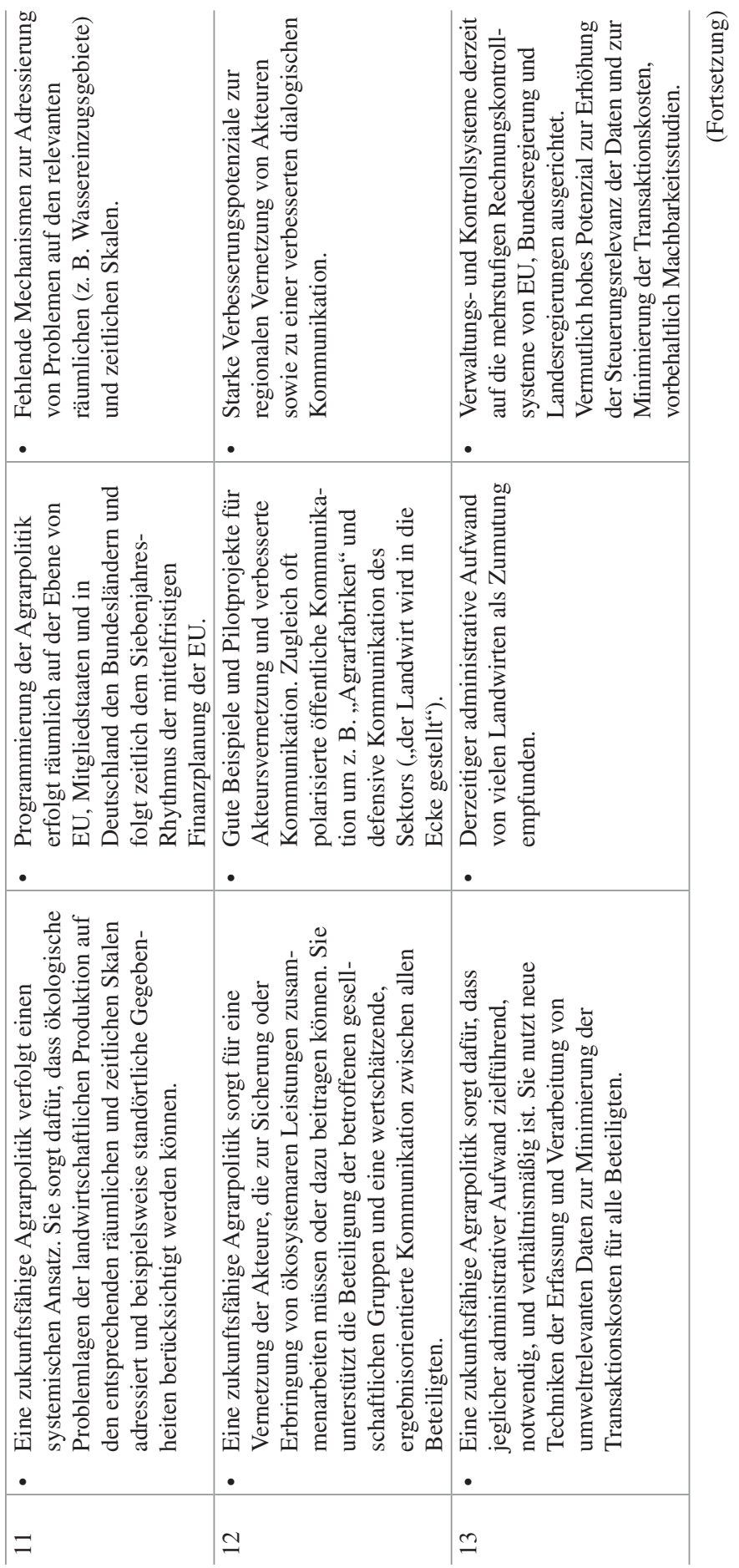




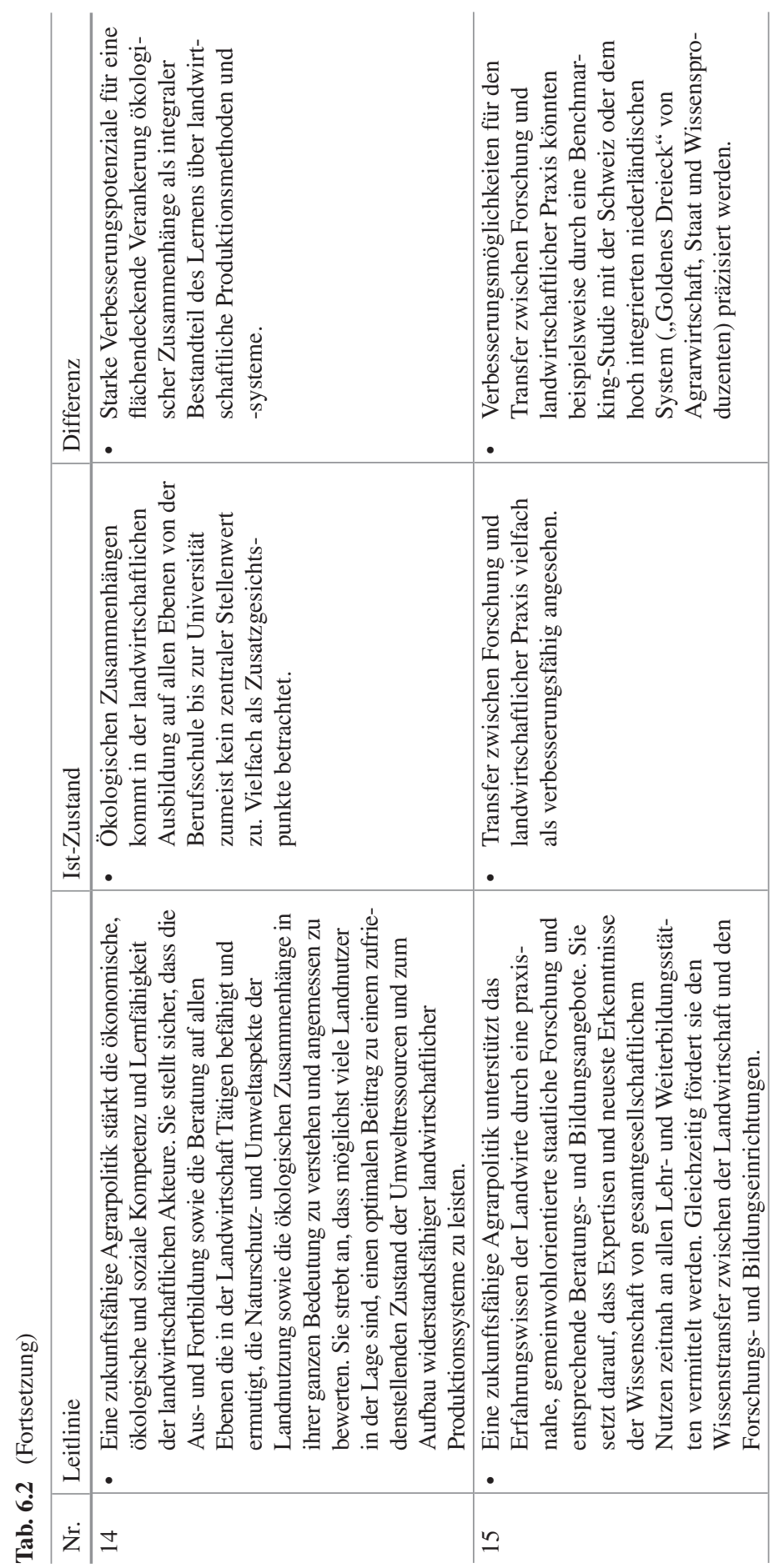




\subsection{Fazit}

Nach einem Überblick über die Natur- und Umweltschutzprobleme der Landwirtschaft, einer Analyse der Entwicklungslogik der europäischen Agrarpolitik und einer SWOT-Analyse der derzeitigen Agrarpolitik stellt das in diesem Kapitel vorgestellte Leitbild den Versuch dar, einen Gegenentwurf zu formulieren, der geeignet ist, eine Brücke zwischen der derzeit dominierenden Produktions- und Einkommensorientierung und den Anliegen des Natur- und Umweltschutzes zu schlagen. Es umfasst zum einen das relativ allgemein gehaltene Leitbild einer multifunktionalen, natur- und umweltverträglichen Landwirtschaft (das Was), zum anderen 15 Leitlinien für eine zukunftsfähige Agrarpolitik (das Wie). Das Leitbild wurde mit konkreten quantitativen und qualitativen Zielen unterfüttert, mit denen der Fortschritt beim Was gemessen werden kann. Die Diskussion von zentralen Dilemmata und Zielkonflikten der Agrarpolitik diente dazu, die von dem Leitbild vorgeschlagene Positionierung, aber auch die impliziten Opportunitätskosten seiner Verwirklichung zu verdeutlichen. Ein qualitativer Soll-Ist-Vergleich zwischen den 15 Leitlinien für eine zukunftsfähige Agrarpolitik und der derzeitigen Agrarpolitik schließlich dient dazu, die Aufgaben zu verdeutlichen. Die beiden folgenden Kapitel stellen nun die Handlungsoptionen einer zukunftsfähigen Agrarpolitik dar, die am Leitbild einer multifunktionalen, natur- und umweltverträglichen Landwirtschaft orientiert ist. Das nächste Kap. 7 diskutiert zunächst die instrumentellen „Bausteine“, bevor Kap. 8 dann alternative strategische Optionen formuliert.

Open Access Dieses Kapitel wird unter der Creative Commons Namensnennung 4.0 International Lizenz (http://creativecommons.org/licenses/by/4.0/deed.de) veröffentlicht, welche die Nutzung, Vervielfältigung, Bearbeitung, Verbreitung und Wiedergabe in jeglichem Medium und Format erlaubt, sofern Sie den/die ursprünglichen Autor(en) und die Quelle ordnungsgemäß nennen, einen Link zur Creative Commons Lizenz beifügen und angeben, ob Änderungen vorgenommen wurden.

Die in diesem Kapitel enthaltenen Bilder und sonstiges Drittmaterial unterliegen ebenfalls der genannten Creative Commons Lizenz, sofern sich aus der Abbildungslegende nichts anderes ergibt. Sofern das betreffende Material nicht unter der genannten Creative Commons Lizenz steht und die betreffende Handlung nicht nach gesetzlichen Vorschriften erlaubt ist, ist für die oben aufgeführten Weiterverwendungen des Materials die Einwilligung des jeweiligen Rechteinhabers einzuholen. 


\section{Kapitel 7 \\ Bausteine zur Integration des Natur- und Umweltschutzes in eine zukunftsfähige Agrarpolitik}

In diesem Kapitel werden zunächst Gestaltungsmöglichkeiten für die verschiedenen agrarpolitischen Instrumente diskutiert. Dabei soll vor dem Hintergrund der SWOT-Analyse diskutiert werden, welche Veränderungen im agrarpolitischen Instrumentenkasten dazu beitragen könnten, dem Leitbild einer multifunktionalen, natur- und umweltverträglichen Landwirtschaft näher zu kommen. Es soll also erörtert werden, wie die verschiedenen agrarpolitischen Komponenten besser naturund umweltschutzfachlich ausgerichtet und ausgestaltet werden können.

Wir diskutieren zunächst die Rolle und Entwicklungsmöglichkeiten des Ordnungsrechts und der gesetzlichen Mindeststandards für die landwirtschaftliche Praxis (Abschn. 7.1). Es folgt eine Erörterung möglicher Varianten zur Entwicklung des Budgets der Agrarpolitik (Abschn. 7.2) sowie der beiden Hauptelemente, mit denen Zahlungen und Anforderungen an die landwirtschaftlichen Betriebe verknüpft werden können - die Direktzahlungen (Abschn. 7.3) sowie die regional und standörtlich ausgerichteten Zahlungen, die derzeit in der Zweiten Säule verankert sind (Abschn. 7.4). Es folgen Überlegungen zu nicht-staatlichen Standards und Ko-Regulierung (Abschn. 7.5), zu den Monitoring- und Sanktionssystemen (Abschn. 7.6) sowie zu unterstützenden Elementen wie Beratung und verbraucherorientierte Maßnahmen (Abschn. 7.7). Ein kurzes Fazit schließt das Kapitel ab. Die Darstellung wird ergänzt durch einen Überblick über eine Auswahl von zielführenden, beispielhaften Management-Maßnahmen zum Schutz der Umweltressourcen, der biologischen Vielfalt und der Ökosystemleistungen, der sich im Anhang findet. 


\subsection{Baustein 1: Ordnungsrecht und gesetzliche Mindeststandards}

Der gesetzliche Mindeststandard legt fest, welche Rahmenbedingungen und Auflagen von den Landwirten einzuhalten und welche Maßnahmen ggf. ergriffen werden müssen. Diese Standards sind damit überall und jederzeit die Grundlage der Bewirtschaftung und für jeden Landwirt verbindlich (BMEL 2014b). Die gesetzlichen Mindeststandards werden durch das Ordnungs- und Fachrecht in Gesetzen und Verordnungen festgelegt, mit denen zumeist Richtlinien der EU in nationales Recht umgesetzt werden. Zum Ordnungsrecht gehören Ge- und Verbote, Anzeigepflichten, Genehmigungsvorbehalte, Kontroll-, Untersuchungs- und Anordnungsbefugnisse der Behörden (Möckel 2013). Es ,zeichnet sich durch seinen direkten Zugriff auf das zu steuernde Verhalten als ausgesprochen ökologisch treffsicher aus" (Rothstein und Schröder 2016).

Der gesetzliche Mindeststandard legt insbesondere die Abgrenzung zwischen Verursacher- und Gemeinlastprinzip fest. Die Kosten der Maßnahmen und Auflagen, die im Rahmen des gesetzlichen Mindeststandards umzusetzen sind, sind von den Verursachern, also den Landwirtinnen und Landwirten, zu tragen. Dem gegenüber steht das Gemeinlastprinzip. Die Kosten von Maßnahmen, die über den gesetzlichen Mindeststandard hinausgehen, werden demnach von der Allgemeinheit übernommen.

Dem gesetzlichen Mindeststandard liegt außerdem das Vorsorgeprinzip zugrunde. Nach dem Vorsorgeprinzip soll die Entstehung von Schäden vermieden werden. Auf Grundlage der Kenntnisse der Umweltwirkungen, der Weiterentwicklung der guten fachlichen Praxis und dem allgemeinen Stand der Technik sowie den gesellschaftlichen Erwartungen werden die gesetzlichen Mindeststandards immer wieder angepasst, wie dies auch Anfang 2017 im Zusammenhang mit der Novellierung der Düngeverordnung geschehen ist. Möckel et al. (2014, S 533) empfehlen eine „Dynamisierung der Anforderungen der guten fachlichen Praxis am besten durch eine regelmäßige (z. B. alle fünf Jahre) und wissenschaftlich fundierte Überprüfung der Standards“.

Die Definition des gesetzlichen Mindeststandards für die Landbewirtschaftung erscheint nicht immer ganz eindeutig. So können die in Tab. 7.1 aufgeführten Ansätze für die Abgrenzung des gesetzlichen Mindeststandards herangezogen werden.

Nach Maßgabe des Verursacherprinzips sind in der heutigen Ausgestaltung die Einhaltung des Stands der Technik und die gute fachliche Praxis als gesetzlicher Mindeststandard anzusehen, bei dem das Verursacherprinzip greift (SchröterSchlaack et al. 2016). Allerdings ist eine Durchsetzung eines neuen Stands der Technik teils mit langen Übergangsfristen verbunden, begründet u. a. mit der langen Abschreibungs- und Nutzungsdauer landwirtschaftlicher Maschinen und Stallanlagen. Eine rasche Anhebung des einzusetzenden Stands der Technik ist auf den Betrieben teils mit hohen Kosten verbunden und wird i. d. R. mit Investitionsförderprogrammen bzw. langen Übergangsfristen abgemildert. 


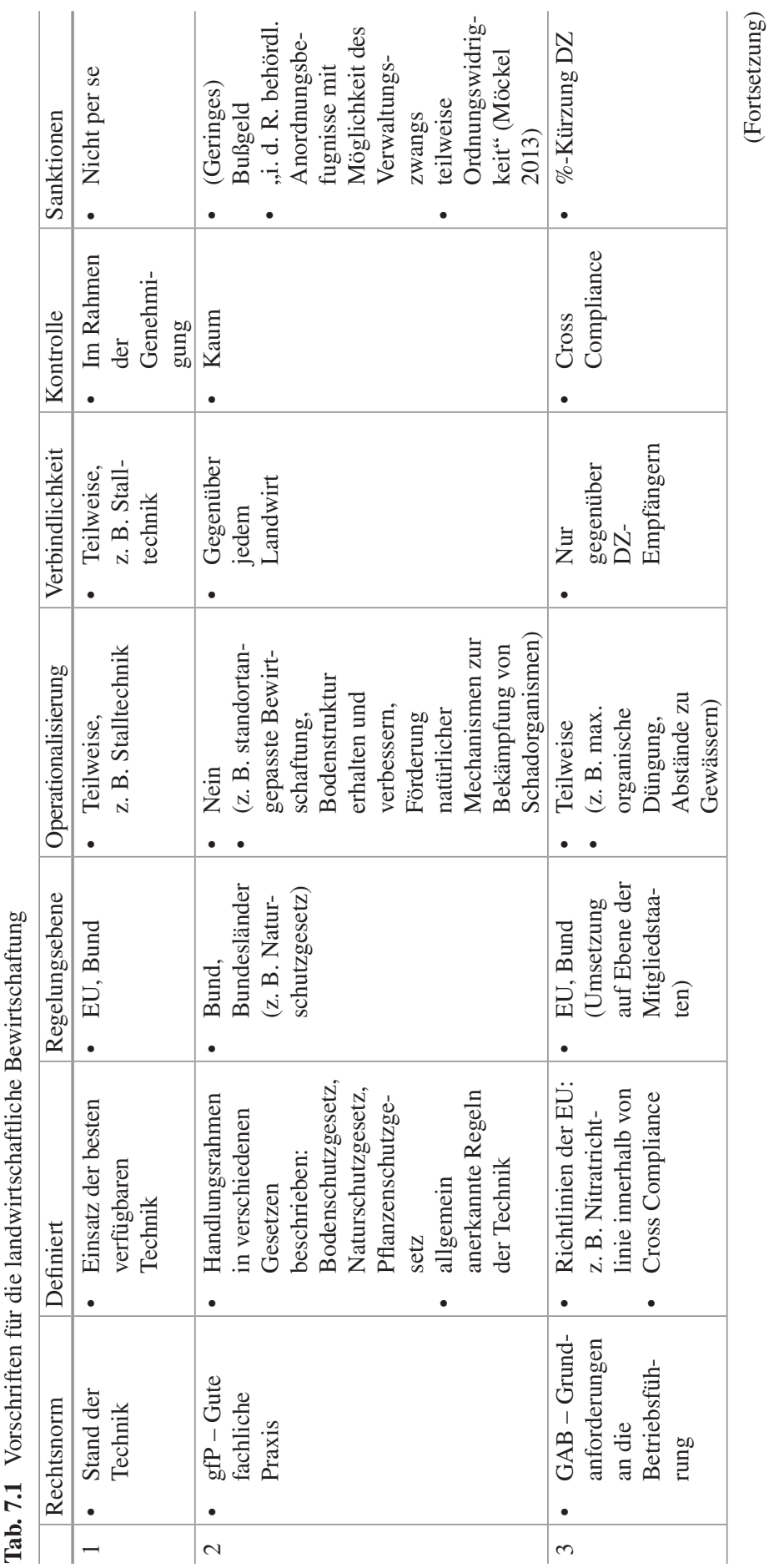


1647 Bausteine zur Integration des Natur- und Umweltschutzes in eine zukunftsfähige ...

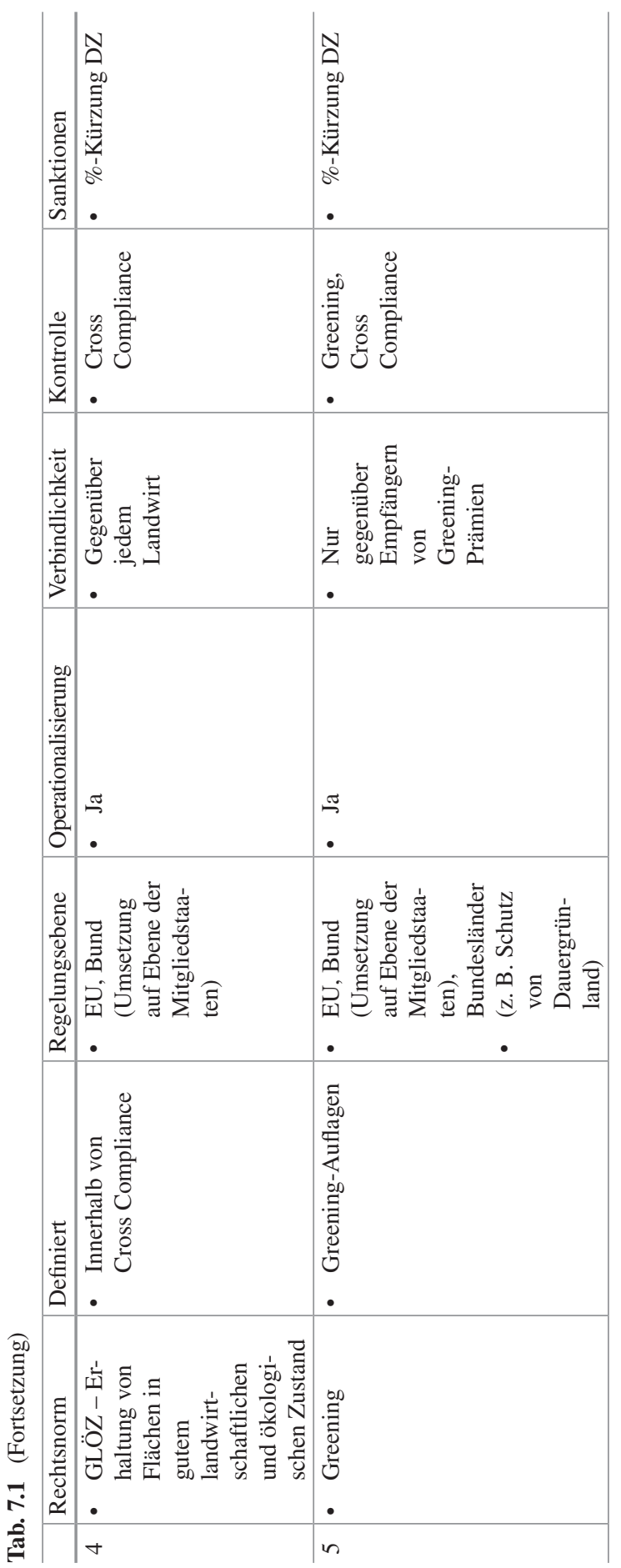




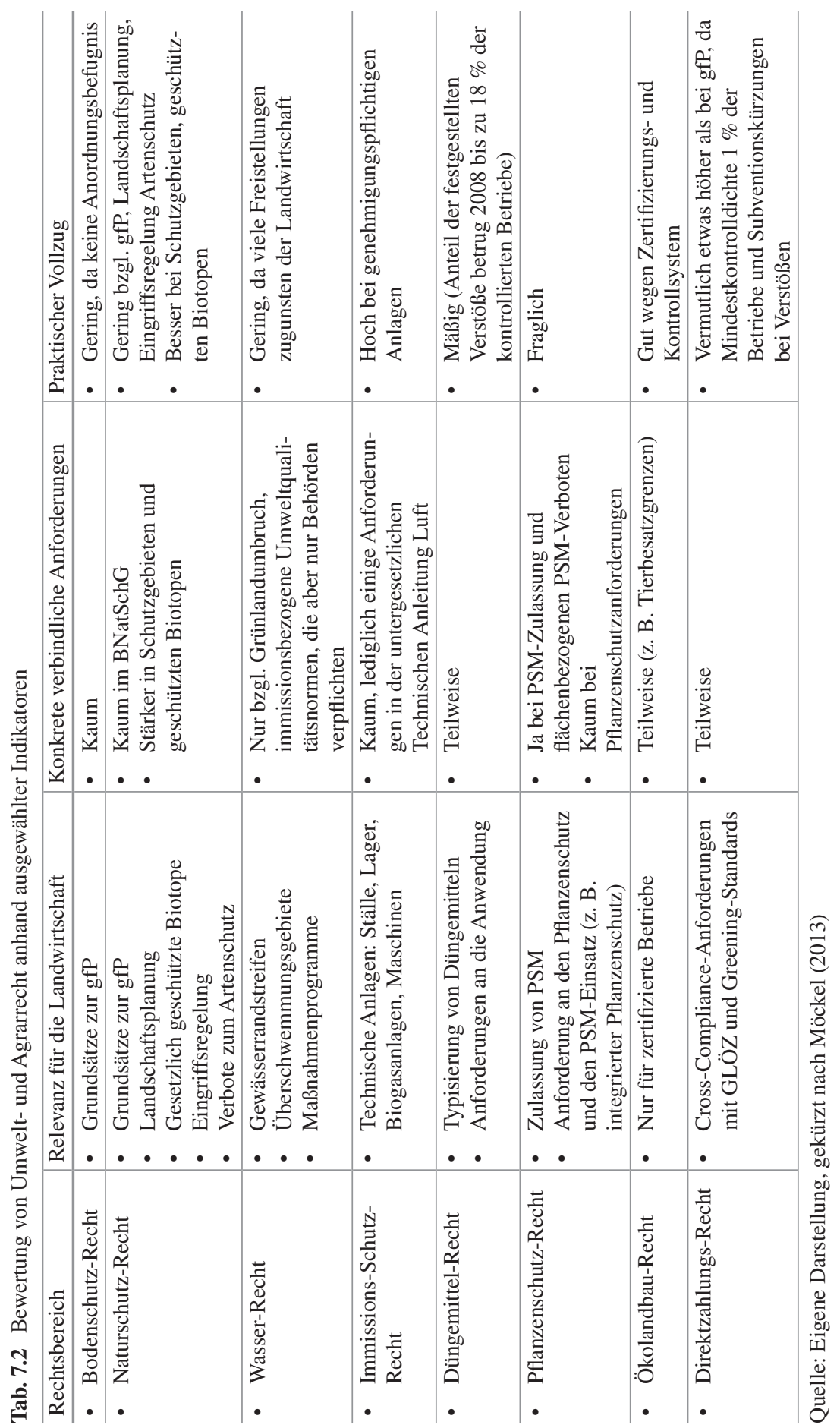


Auch die Einhaltung der GAB kann als verbindlicher Mindeststandard angesehen werden, da auch Betriebe, die keine Direktzahlungen erhalten, diese Auflagen einzuhalten haben. Es wird des Weiteren die Ansicht vertreten, dass auch die GLÖZ-Standards in das Ordnungsrecht integriert werden sollten, ,um eine Harmonisierung auf einem insgesamt höhere $(\mathrm{m})$ Umweltschutzniveau zu erreichen und die Anforderungen als verpflichtende Mindeststandards dauerhaft abzusichern, [...] da es sich bei den Cross-Compliance-Anforderungen um Mindestanforderungen im Sinne der guten fachlichen Praxis und nicht um zu honorierende darüber hinausgehende ökologische Leistungen der Landwirte handelt" (Möckel et al. 2014).

Ausgehend von den dargestellten Überlegungen wird im Folgenden der gesetzliche Mindeststandard als die Einhaltung des Stands der Technik, der guten fachlichen Praxis und der GAB angesehen. Die Durchsetzung der GLÖZ- und Greening-Auflagen ist in Verbindung mit der Gewährung von Direktzahlungen zu sehen.

\begin{tabular}{ll|l}
\hline \multicolumn{2}{l}{ Definition des gesetzlichen Mindeststandards } \\
\hline - Stand der Technik, gfP, GAB: & gesetzlicher Mindeststandard (für alle Betriebe verbindlich) \\
\hline - GLÖZ, Greening-Auflagen: & $\begin{array}{l}\text { bisher kein gesetzlicher Mindeststandard (Ausstiegsoption } \\
\text { gegen Verlust von bis zu } 125 \% \text { der Greening-Zahlungen) }\end{array}$ \\
\hline
\end{tabular}

Die folgende Tab. 7.2 zeigt, welchen Einfluss verschiedene Rechtssetzungen auf die Landwirtschaft haben, welche verbindlichen Anforderungen bestehen und wie der praktische Vollzug gestaltet ist.

Betrachtet man Tab. 7.1 und 7.2 zusammen, so zeigt sich, dass sowohl der gesetzliche Mindeststandard als auch das Agrar-Beihilferecht sowohl mit einem Regelungsdefizit wie mit einem Vollzugsdefizit zu kämpfen haben. So fehlt eine entsprechende Operationalisierung der guten fachlichen Praxis fast vollständig. Die Durchsetzung der GAB beruht in erster Linie auf Cross Compliance-Prüfungen und bei Verstößen drohen prozentuale Kürzungen der Direktzahlungen. So konnte mit der Einführung von Cross Compliance, d. h. einer Kopplung der Einhaltung der gesetzlichen Mindeststandards an den Erhalt von Direktzahlungen, die Durchsetzung des gesetzlichen Mindeststandards verbessert werden.

Die Behebung der Regelungsdefizite muss an der Operationalisierung der Begrifflichkeiten ansetzen. Hierfür ist die Festlegung geeigneter Indikatoren, Messund Grenzwerte notwendig. Grenzwerte können festgelegt werden als Ober- oder Untergrenzen, gesetzliche Unterlassungspflichten (Verbote) oder gesetzliche Handlungspflichten (Möckel 2013). Dabei ist jedoch insbesondere die Möglichkeit der praktikablen Feststellung der Mess- und Grenzwerte zu berücksichtigen. Mess- und Grenzwerte oder Verhaltensregeln, die nicht oder nur unter großem Aufwand festgestellt werden können, eignen sich nicht zur Durchsetzung der entsprechenden Rechtsnormen (z. B. längere Zeiträume für die Einarbeitung organischer Dünger). Des Weiteren sollten die Grenzwerte so festgelegt werden, dass ihre Einhaltung zu einer Verbesserung der Umwelt- und Naturschutzwirkungen der Landwirtschaft beiträgt. „Das gegenwärtige Anforderungsniveau der guten fachlichen Praxis [ist jedoch] unzureichend, um durch die Landwirtschaft verursachte Umweltschäden vollkommen abzuwenden“" (Möckel et al. 2014). Auch durch die Umsetzung der 
GAB-, GLÖZ- und Greening-Auflagen ist nur ein geringer positiver Effekt auf die Ressourcen des Naturschutzes festzustellen bzw. zu erwarten (Europäische Kommission 2016).

Zur Operationalisierung der guten fachlichen Praxis werden beispielsweise die Ausweisung von Schadschwellen für den integrierten Pflanzenschutz, Grenzwerte zur Beschränkung der Bewirtschaftungsintensität (z. B. verbindliche Obergrenzen für Stickstoffüberschüsse und den Viehbesatz pro Hektar) sowie weiterführende Untersuchungs- und Dokumentationspflichten hinsichtlich Stickstoff und Humusgehalt (Möckel et al. 2014) empfohlen. Da jedoch die Art und Weise der landwirtschaftlichen Bewirtschaftung im Zusammenhang mit standörtlichen Faktoren unterschiedliche Wirkungen in Bezug auf den Natur- und Umweltschutz entfalten kann, wäre die

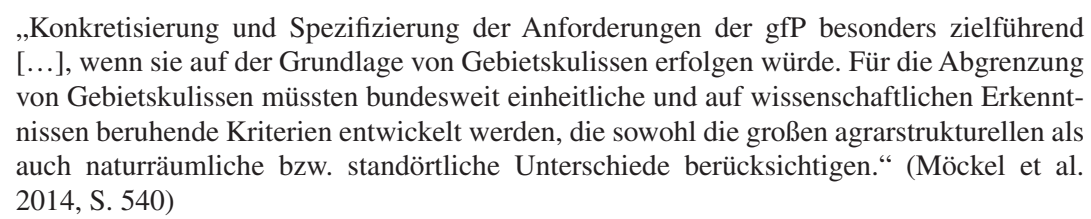

Vollzugsdefizite sind zum einen durch unbestimmte Rechtsbegriffe und einen Mangel an kontrollierbaren Indikatoren bedingt, zum anderen durch geringe Kontrollfrequenzen und Sanktionshöhen. Mit mangelnder Durchsetzung durch ungeeignete Kontroll- und Sanktionsmechanismen sinkt jedoch ceteris paribus die Bereitschaft der Landwirte, sich normgerecht zu verhalten (Rothstein und Schröder 2016). Die Durchsetzung des gesetzlichen Mindeststandards ist dabei unabhängig von der Gewährung von Direktzahlungen zu sehen. Die Kopplung der Einhaltung des gesetzlichen Mindeststandards an die Gewährung von Direktzahlungen birgt bisher den Vorteil, dass - neben der Operationalisierung von Auflagen - ein einheitliches Kontroll- und Sanktionssystem in allen Mitgliedstaaten der EU etabliert wurde. Dessen Weiterführung wäre grundsätzlich jedoch auch unabhängig von der Gewährung von Direktzahlungen möglich.

Eine Verminderung des Vollzugsdefizits könnte erreicht werden durch:

- höhere Kontrollfrequenzen und Sanktionen, wobei die Höhe der Kontrollfrequenzen und Sanktionen in einem umgekehrten Verhältnis zu einander stehen;

- Beweislastumkehr, so dass beispielsweise der Landwirt oder die Landwirtin die Einhaltung der Stickstoffbilanzgrenzwerte nachweisen muss, etwa durch Erhebung der Stickstoffgehalte im organischen Dünger oder Wiegeprotokolle der Erträge;

- Plausibilitätskontrollen, z. B. durch den zwischenbetrieblichen Vergleich von Nährstoffbilanzen in einer Region oder den Abgleich von Daten aus anderen Datenbanken;

- Einbindung externer Kontrolleinrichtungen, wie z. B. im ökologischen Landbau;

- Ziehung von Rückstellmustern, z. B. um Stickstoffgehalte in organischen Düngern zu belegen;

- Nutzung von GIS-Daten und Vernetzung digitaler Datensysteme. 
Für die Zukunft ist vorstellbar, anhand digitaler Daten und elektronischer Datenverarbeitung den bürokratischen Aufwand für landwirtschaftliche Unternehmen und Verwaltung zu reduzieren. So könnten bisher in verschiedenen Datenbanken erhobene und gespeicherte Daten zur Durchführung von Kontrollen zusammengeführt (z. B. HIT und INVEKOS) und mit Daten der Selbstkontrolle (z. B. Ackerschlagbilanzen, Daten des Precision Farming) kombiniert werden. Die gewonnen Daten könnten damit insgesamt als Kontroll-, aber auch als Monitoring- und Beratungstool für die landwirtschaftlichen Betriebe eingesetzt werden, z. B. durch Vergleich des eigenen Betriebes mit anderen Betrieben aus der Region zu Zwecken des (anonymen) Bench-Marking. Dabei sind jedoch Datenschutzerwägungen zu berücksichtigen.

Es ist auch möglich, den Erhalt von Direktzahlungen an den Nachweis der Einhaltung von Auflagen zu koppeln. Allerdings müssten die einzuhaltenden Auflagen dann über den gesetzlichen Mindeststandard hinausgehen (z. B. ökologischer Landbau), um eine Honorierung der bloßen Einhaltung der Gesetze zu vermeiden. Allerdings wird durchaus argumentiert, dass bereits die Einhaltung des gesetzlichen Mindeststandards mit einem finanziellen Ausgleich verbunden sein sollte, weil europäische Landwirtinnen und Landwirte gegenüber dem Weltmarkt höhere Natur- und Umweltschutzauflagen erfüllten, was z. B. im Ackerbau zu Kostennachteilen von ca. $50 € /$ ha führt (Wissenschaftlicher Beirat für Agrarpolitik beim BMELV 2010). Andere Quellen schätzen den Kostennachteil gegenüber der Konkurrenz außerhalb der EU eher auf ca. $20 € /$ ha (Plankl et al. 2010). Es wird jedoch auch vorgebracht, dass die Umweltleistungen der europäischen Landwirtschaft nicht notwendigerweise höher seien und Länder z. B. in Südamerika durch den Erhalt des Regenwaldes möglicherweise höhere Umweltleistungen erbringen.

$\mathrm{Zu}$ diskutieren sind des Weiteren die Wechselwirkungen, die im Zusammenhang mit der Durchsetzung eines effektiven gesetzlichen Mindeststandards entstehen könnten. Dies betrifft zum einen die oben erwähnten Wirkungen auf den Strukturwandel, die insbesondere mit der Durchsetzung von investiven Maßnahmen verbunden sein können. Hier besteht die Möglichkeit, z. B. durch Investitionsförderung gegenzusteuern (Möckel et al. 2014). Es werden jedoch auch Innovationen induziert, die dazu beitragen, die Grenzwerte möglichst effektiv und effizient einzuhalten (Rothstein und Schröder 2016; Taube 2016). Mit der Durchsetzung eines effektiven gesetzlichen Mindeststandards können jedoch auch Wirkungen auf die Verbraucherpreise verbunden sein. Durch den gesetzlichen Mindeststandard werden zumindest teilweise externe Kosten der Produktion internalisiert. Dies erhöht die Erzeugungskosten, was je nach Preiselastizität mehr oder weniger an die Abnehmer „durchgereicht“ werden kann. Sind höhere Erzeugerkosten durch EUStandards am Markt nicht durchsetzbar, könnte der Marktanteil europäischer Produkte zurückgehen. Die Größe dieses Effekts hängt u. a. von der Preiselastizität der Nachfrage ab.

In diesem Kontext können Labelling-Initiativen dazu dienen, Produkte mit höherer Umweltleistung sichtbar zu machen und den Substitutionseffekt durch Produkte, die aufgrund niedrigerer Standards preiswerter sind, zu vermindern. Eine Beschränkung des Marktzugangs von Produkten aufgrund ihrer Prozessqualität 
ist hingegen im allgemeinen nicht WTO-konform, es sei denn, dies ist zur Abwendung von Gefahren für die Gesundheit von Menschen, Tieren oder Pflanzen notwendig.

Die teilweise oder vollständige Substitution von inländischen Produkten durch Importe aus dem außereuropäischen Ausland infolge kostenintensiver Vorschriften kann mit einem Export negativer Umweltfolgen in die Erzeugerländer einhergehen. Es käme dann zu einer bloßen räumlichen Verschiebung negativer Natur- und Umweltwirkungen der Produktion (Leakage-Effekt). Angesichts der oben genannten Kosten der europäischen Standards, denen weit höhere Direktzahlungen gegenüber stehen, ist derzeit nicht davon auszugehen, dass die Mindeststandards von GAB und GLÖZ zu einer massiven Verlagerung von Produktion ins außereuropäische Ausland beitragen.

Insgesamt sind also fünf Aspekte zu beachten, um den gesetzlichen Mindeststandard effektiv und effizient durchzusetzen:

1. Praktikable Operationalisierung bisher unzureichend operationalisierter Begrifflichkeiten, wie z. B. gfP (ggf. differenziert nach Gebietskulissen);

2. Weiterführung des flächendeckenden europäischen Kontroll- und Sanktionssystems, unabhängig vom Fortbestehen einer Verknüpfung mit den Direktzahlungen (Cross Compliance);

3. Angemessene Kontrolldichte und Sanktionen mit spürbarer Anreizwirkung;

4. Adressatenorientierte Kommunikation auf Basis verhaltenswissenschaftlicher Ansätze;

5. Regelmäßige Nachjustierung der Standards nach dem Stand der Technik und neuen Erkenntnissen von Wissenschaft und Forschung.

\subsection{Baustein 2: Budget}

Das Projekt ZA-NExUS beschäftigt sich nicht vorrangig mit finanziellen Fragen der Agrarpolitik. Allerdings spielen bei der weiteren Integration des Natur- und Umweltschutzes in der Agrarpolitik die zur Verfügung stehenden finanziellen Mittel eine wesentliche Rolle. Neben dem Volumen geht es dabei auch um die Frage, welche Maßnahmen im Rahmen der Gemeinsamen Agrarpolitik vollständig von der EU finanziert werden und welche eine Ko-Finanzierung seitens der Mitgliedstaaten (aus nationalen, regionalen oder öffentlichen Haushalten oder seitens privater Geldgeber) erfordern. Die hier vorgestellten Überlegungen beschränken sich auf die Ausgabenseite des Budgets und klammern die Einnahmenseite - abgesehen von der Frage der Ko-Finanzierung - aus.

Hinsichtlich der Bedeutung des finanziellen Volumens lassen sich unter der heutigen Situation zwei Problemkreise für den Natur- und Umweltschutz unterscheiden:

- zum einen die Mittel, die für Programme und gezielte Maßnahmen des Naturund Umweltschutzes zur Verfügung stehen (inklusive der damit verbundenen Verwaltungskosten); 
- zum anderen die Mittel für flächenbezogene Direktzahlungen, deren Höhe mit darüber entscheidet, ob Betriebe in marginalen Lagen die landwirtschaftliche Produktion aufrechterhalten und ob Betriebe in hoch produktiven Lagen sich weiterhin Cross-Compliance-Auflagen unterwerfen, die über die gesetzlich verbindlichen Minimalstandards hinausgehen.

Der derzeitige Finanzrahmen für die deutsche und europäische Agrarpolitik ist erheblich. Im Jahre 2015 flossen gut 6 Mrd. € aus dem EU-Haushaltstitel 2 (Nachhaltiges Wachstum: Natürliche Ressourcen) nach Deutschland (siehe Tab. 7.3). Die Obergrenze für Direktzahlungen lag bei etwas mehr als 5,1 Mrd. €. 113 Mio. € standen für Exporterstattungen zur Verfügung. Diese beiden Posten werden zu $100 \%$ aus dem EU-Haushalt beglichen. 723 Mio. $€$ standen für Programme der ländlichen Entwicklung bereit, zu denen auch die Agrarumweltprogramme gehören. Diese Mittel konnten nur in Anspruch genommen werden, insofern es eine entsprechende Ko-Finanzierung gab. Weitere jeweils knapp 35 Mio. € flossen aus Mitteln der Gemeinsamen Fischereipolitik sowie für das Umwelt- und Klimaaktionsprogramm Life.

Der Mehrjährige Finanzrahmen 2014-2020 sieht europaweit relativ konstante Ausgaben von jährlich ca. 44,5 Mrd. € für die Direktzahlungen und marktbezogenen Ausgaben sowie ca. 13,6 Mrd. € für die ländliche Entwicklungspolitik vor (Tab. 7.4).

Tab. 7.3 EU-Ausgaben aus dem Haushaltstitel 2 (Nachhaltiges Wachstum: Natürliche Ressourcen) an deutsche Empfänger im Jahr 2015

\begin{tabular}{|c|c|c|}
\hline Titel & Bezeichnung & $\begin{array}{l}\text { Betrag in } \\
\text { Mio. } €\end{array}$ \\
\hline \multirow[t]{2}{*}{2} & SUSTAINABLE GROWTH: NATURAL RESOURCES & 6041,0 \\
\hline & Davon & \\
\hline \multirow[t]{3}{*}{2.0 .1} & $\begin{array}{l}\text { European Agricultural Guarantee Fund (EAGF) - Market related } \\
\text { expenditure and direct payments }\end{array}$ & 5247,9 \\
\hline & Direct Aid & 5135,3 \\
\hline & Export refunds & 112,7 \\
\hline 2.0 .2 & European Agricultural Fund for Rural Development (EAFRD) & 723,3 \\
\hline 2.0 .3 & $\begin{array}{l}\text { European Maritime and Fisheries Fund (EMFF), Regional Fisheries } \\
\text { Management Organisations (RFMOs) and Sustainable Fisheries } \\
\text { Agreements (SFAs) }\end{array}$ & 34,8 \\
\hline 2.0 .31 & European Maritime and Fisheries Fund (EMFF) & 34,7 \\
\hline 2.0 .32 & $\begin{array}{l}\text { Regional Fisheries Management Organisations (RFMOs) and } \\
\text { Sustainable Fisheries Agreements (SFAs) }\end{array}$ & 0,1 \\
\hline 2.0 .4 & Programme for the Environment and Climate Action (Life) & 34,5 \\
\hline 2.0.DAG & Decentralised agencies & 0,3 \\
\hline 2.0.OTH & Other actions and programmes & 0,0 \\
\hline 2.0.PPA & Pilot projects and preparatory actions & 0,1 \\
\hline 2.0.SPEC & $\begin{array}{l}\text { Actions financed under the prerogatives of the Commission and specific } \\
\text { competences conferred to the Commission }\end{array}$ & 0,0 \\
\hline
\end{tabular}

Quelle: Eigene Darstellung, nach European Commission (2016) 
Tab. 7.4 Mehrjähriger Finanzrahmen der EU 2014-2020, Haushaltstitel 2 (Nachhaltiges Wachstum: Natürliche Ressourcen)

\begin{tabular}{|c|c|c|c|c|c|c|c|c|}
\hline Titel & 2014 & 2015 & 2016 & 2017 & 2018 & 2019 & 2020 & $\begin{array}{l}2014- \\
2020 \\
\end{array}$ \\
\hline $\begin{array}{l}\text { Nachhaltiges } \\
\text { Wachstum: } \\
\text { natürliche } \\
\text { Ressourcen }\end{array}$ & 59.303 & 59.599 & 59.909 & 60.191 & 60.267 & 60.344 & 60.421 & 420.034 \\
\hline \multicolumn{9}{|l|}{ Davon } \\
\hline $\begin{array}{l}\text { Europäischer } \\
\text { Garantiefonds für } \\
\text { die Landwirt- } \\
\text { schaft (EGFL) - } \\
\text { marktbezogene } \\
\text { Ausgaben und } \\
\text { Direktzahlungen }\end{array}$ & 44.130 & 44.368 & 44.628 & 44.863 & 44.889 & 44.916 & 44.941 & 312.735 \\
\hline $\begin{array}{l}\text { Europäischer } \\
\text { Landwirtschafts- } \\
\text { fonds für die } \\
\text { Entwicklung des } \\
\text { ländlichen } \\
\text { Raums (ELER) }\end{array}$ & 13.652 & 13.652 & 13.653 & 13.653 & 13.654 & 13.654 & 13.655 & 95.577 \\
\hline $\begin{array}{l}\text { Europäischer } \\
\text { Meeres- und } \\
\text { Fischereifonds } \\
\text { (EMFF) }\end{array}$ & 1017 & 1035 & 1049 & 1058 & 1069 & 1078 & 1095 & 7404 \\
\hline $\begin{array}{l}\text { Umwelt- und } \\
\text { Klimapolitik } \\
\text { (Life) }\end{array}$ & 404 & 435 & 462 & 493 & 523 & 555 & 581 & 3456 \\
\hline Agenturen & 52 & 53 & 54 & 55 & 56 & 57 & 58 & 387 \\
\hline Spielraum & 47 & 54 & 61 & 67 & 74 & 81 & 89 & 473 \\
\hline
\end{tabular}

Mittel für Verpflichtungen in Mio. $€$ in laufenden Preisen.

Quelle: Eigene Darstellung, nach European Commission (2016)

\subsubsection{Institutioneller Rahmen}

Die Budget-Frage geht dabei weit über den Bereich der Agrarpolitik hinaus. Der Umfang des Agrarbudgets wird im Rahmen der Verhandlungen über den Mehrjährigen Finanzrahmen der EU vom Rat der EU auf Vorschlag der Kommission festgelegt. Laut Artikel 310 des Vertrags von Lissabon über die Arbeitsweise der Europäischen Union (AEUV) muss die EU einen ausgeglichenen Haushalt vorlegen. Laut Artikel 312 AEUV legt die EU einen mehrjährigen Finanzrahmen über einen Zeitraum von mindestens fünf Jahren fest. Dieser wird in einer Verordnung geregelt, deren Verabschiedung Einstimmigkeit im Rat und eine Mehrheit der Mitglieder des Europäischen Parlaments erfordert. Der Finanzrahmen legt jährliche Obergrenzen für die Haupttätigkeitsbereiche der Union fest. Daneben enthält der Finanzrahmen ,,auch alle sonstigen für den reibungslosen Ablauf des jährlichen Haushaltsverfahrens 
sachdienlichen Bestimmungen “. Im Finanzrahmen werden daher in der Regel bereits wesentliche Grundlinien der GAP mittelfristig bestimmt, deren Details dann in den agrarpolitischen Verhandlungen ausgefüllt werden, bei denen DG Agri, die Fachministerien der Mitgliedstaaten und der Agrarausschuss des Europäischen Parlaments maßgeblich sind (Matthews 2015) (siehe auch Abschn. 4.1).

Die Einflussfaktoren auf den Budgetrahmen der GAP liegen daher wesentlich jenseits der Agrarpolitik. Bei der Erstellung der strategischen Optionen haben wir daher kein bestimmtes finanzielles Szenario zugrunde gelegt. Bei den Erwartungen über das künftige finanzielle Volumen und die finanziellen Arrangements in der GAP nach 2020 sind zum einen systematische, zum anderen politische Überlegungen einzubeziehen.

\subsubsection{Systematische Aspekte}

Aus systematischer Sicht haben öffentliche Haushalte drei Funktionen: makroökonomische Stabilisierung, allokative sowie distributive Funktionen. Angesichts des Volumens von lediglich ca. $1 \%$ des BIP der Mitgliedstaaten kommt dem EU-Haushalt keine wesentliche Stabilisierungsfunktion zu. Bei den allokativen Funktionen ist der EU-Haushalt entsprechend der Lissabon-Strategie vor allem auf die Stärkung des Wachstumspotenzials in den Mitgliedstaaten und vor allem in benachteiligten Regionen ausgerichtet, etwa durch Infrastrukturausgaben. Bei den Struktur- und Kohäsionsfonds spielen verteilungspolitische Aspekte zwar eine Rolle, im Vordergrund steht aber das Ziel, Wachstumshemmnisse in benachteiligten Regionen zu vermindern sowie die grenzübergreifenden Kooperation zu fördern (Bos 2010, S. 91).

In einer Kommunikation zur Entwicklung des EU-Haushalts hat die Europäische Kommission fünf Prinzipien für den EU-Haushalt formuliert. Dieser soll der Realisierung der Schlüsselprioritäten dienen, Skaleneffekte ermöglichen, ergebnisorientiert sein, Stabilität für alle durch Solidarität mit den schwächeren Mitgliedern schaffen sowie den Prinzipien der Autonomie, Transparenz und Fairness entsprechen (European Commission 2010). Nach der Einführung der produktionsentkoppelten Direktzahlungen gab es daher Stimmen, die sich dafür aussprachen, die Politik zur Einkommensstützung zu dezentralisieren, da interpersonale Verteilungspolitik in die Kompetenz der Mitgliedstaaten fällt (Bos 2010, S. 93). Eine vorrangig einkommenspolitische Begründung der Direktzahlungen könnte daher deren Vollfinanzierung in Frage stellen. Da entkoppelte Direktzahlungen zudem weder Skaleneffekte noch Externalitäten mit sich bringen, entfallen weitere Argumente für eine Zentralisierung dieser Zahlungen (Grethe 2008).

Hinzu kommt die Überlegung, dass Maßnahmen mit Ko-Finanzierung in der Regel zu größerem Kostenbewusstsein bei den Mitgliedstaaten führen und damit zu einer stärker ergebnisorientierten und besser vorbereiteten Implementation. Beide Effekte sind geeignet, die Effektivität und Effizienz von Maßnahmen zu erhöhen. Andererseits könnte die Gefahr bestehen, dass bei knappen öffentlichen Haushalten keine hinreichenden Mittel bereitgestellt werden bzw. „Naturschutz nach Kassenlage“ betrieben wird. 
Insgesamt sind aus systematischer Sicht also sowohl die Vollfinanzierung von Maßnahmen durch die EU wie auch die zentrale Finanzierung von vorwiegend einkommenspolitischen Maßnahmen kritisch zu beurteilen.

\subsubsection{Politische Aspekte}

Im Hinblick auf die politischen Prozesse der Budgetverhandlungen ist zu berücksichtigen, dass die Mitgliedstaaten die von ihnen bereitgestellten Beiträge an die EU als ihr eigenes Geld ansehen, von dem sie einen angemessenen Rückfluss erwarten, auch wenn es sich rechtlich und technisch um Eigenmittel der EU handelt (Bos 2010, S. 89). Bei den Finanzverhandlungen spielt daher die buchhalterische Nettozahlerposition eine zentrale Rolle.

Diese Betrachtungsweise kann eine Reform der Instrumente der Agrarpolitik erschweren, wenn dadurch Unsicherheiten über die Verteilungswirkungen entstehen oder wenn einzelne Mitgliedstaaten eine Verschlechterung ihrer Nettozahlerposition hinnehmen müssten, die nicht an anderer Stelle (wenn auch nicht notwendigerweise für die gleichen Empfänger) kompensiert wird. Im Ergebnis begünstigt die Nettozahlerperspektive eine starke Status-quo-Orientierung und Pfadabhängigkeit der GAP (Ackrill 2005).

Dabei ist zu beachten, dass die GAP Umverteilungswirkungen zwischen den Mitgliedstaaten erzeugt, die zumindest in den 1990er-Jahren zwischen -20\% und +60\% des Produktionswerts des Agrarsektors streuten (Zanias 2002). Durch die teilweise Angleichung der Direktzahlungen zwischen 2014 und 2020 wurden explizit Umverteilungswirkungen im Vergleich zum Status quo zwischen den Mitgliedstaaten angestrebt, in diesem Fall im Sinne des Kohäsionsziels von den reicheren EU-15 zu den ärmeren neuen Mitgliedstaaten in Osteuropa (vgl. European Commission 2011b, S. 60). Studien mit größerer räumlicher Differenzierung finden regionale Umverteilungseffekte der GAP zugunsten ländlicher Regionen, die auch unterhalb der Ebene der Bundesländer stark variieren können (Elsholz 2009). Eine Renationalisierung der GAP könnte demnach zu einer Verminderung dieser Umverteilungseffekte führen (Elsholz 2009). Weil die Agrarzahlungen letztlich steuerfinanziert sind, ,findet eine Einkommensumverteilung von urbanen und wohlhabenden Regionen zu ländlichen und ärmeren Regionen statt" und die GAP trägt zum Kohäsionsziel der EU bei (Hansen 2009, S. 180 f.)

Aufgrund der Verteilungseffekte haben Mitgliedstaaten und Regionen, die netto von der GAP im Allgemeinen und insbesondere von den Direktzahlungen profitieren, ein Interesse an deren Fortführung zu relativ unveränderten Bedingungen. Da die Verabschiedung des Mehrjährigen Finanzrahmens Einstimmigkeit im Rat erfordert, sind die Nettoempfänger der GAP in einer relativ starken Position. Dies trägt wesentlich zur Erklärung des Entwicklungspfads der europäischen Agrarpolitik seit der MacSharry-Reform von 1992 bei: Zunächst wurde vereinbart, dass das GAP-Budget geringer steigen sollte als das BIP und damit auch als der Haushalt der EU insgesamt (Bos 2010). Für die Finanzperiode 2014-2020 bleiben die Ausgaben 
der GAP nun ungefähr konstant, was auf eine weitere Verminderung des relativen Anteils der GAP sowohl am BIP wie am Budget der EU hinausläuft und auf diese Weise gewisse Freiräume für andere Aufgaben der EU schafft.

Eine weitere Frage ist, ob in den nächsten Jahren überhaupt mit einer politischen Initiative zu rechnen ist, die auf eine grundlegende Reform der GAP abzielt. Die relativ weitgehenden GAP-Reformen von 1992 und 2003 wurden jeweils von einem Agrarkommissar mit einer konzeptionell gut unterfütterten Reform-Agenda und unter starkem äußerem Druck vorangetrieben (vgl. Abschn. 4.1). Bei unseren Hintergrundgesprächen ergab sich der Eindruck, dass derzeit eher nicht mit einer gröBeren Reforminitiative für die GAP zum Zeitpunkt 2020 gerechnet wird. Dabei spielt eine Rolle, dass die reguläre Amtszeit der derzeitigen Kommission 2019 endet und es als wenig opportun angesehen werden könnte, der Nachfolgekommission die Implementation einer radikalen Reform, die sie nicht selbst angestoßen hat, zu hinterlassen. Als wahrscheinliche Szenarien gelten daher entweder eine relativ kleine Reform für die GAP 2021-2027 oder eine Verlängerung der derzeitigen GAP um ein bis zwei Jahre mit der Gelegenheit für eine umfassendere Reform für die Zeit ab 2022.

Vor diesem Hintergrund erscheint die folgende Annahme hinsichtlich des GAP-Budgets 2021-2027 plausibel, die von einer Fortsetzung des bisherigen Politikpfads ausgeht:

- Der für die GAP zur Verfügung stehende Anteil am EU-Haushalte bleibt konstant. Unter Berücksichtigung der durch den Brexit bedingten Verminderung des Haushaltsvolumens um derzeit geschätzte $10 \mathrm{Mrd}$. €/Jahr würden die verbleibenden 27 Mitgliedstaaten ca. 4 Mrd. €/Jahr weniger als bisher für die GAP zur Verfügung stellen.

- Verschiebungen zwischen den Anteilen mit Voll- und Ko-Finanzierung sind möglich, wenn die Umverteilungseffekte relativ gering bleiben und als beherrschbar angesehen werden.

Diese Annahmen liegen der Formulierung der strategischen Optionen in Kap. 8 zugrunde.

\subsubsection{Dilemma aus Sicht der Naturschutz- und Umweltpolitik}

Aus Sicht des Naturschutz- und Umweltschutzes ergibt sich aus der finanzpolitischen Konstruktion der GAP ein Dilemma. Einerseits könnten die umweltbezogenen Maßnahmen in der Zweiten Säule bei anspruchsvoller Ausgestaltung deutlich effektiver und effizienter zur Erreichung von Umweltzielen sein als die relativ allgemeinen und wenig anspruchsvollen Instrumente in der Ersten Säule. Andererseits werden die Direktzahlungen der Ersten Säule bisher voll aus dem EU-Haushalt finanziert, während die Maßnahmen der Zweiten Säule derzeit eine nationale KoFinanzierung erfordern, die gerade in ärmeren Regionen eine Herausforderung darstellen kann. Daher besteht von Seiten der Mitgliedstaaten und Regionen, die 
Nettoempfänger der Ersten Säule sind, Widerstand gegen eine Umschichtung von Mitteln der Ersten in die Zweite Säule. Dieser Widerstand war in der letzten Reformdiskussion wirkmächtig. Nachdem der Anteil der Zweiten Säule zuvor in mehreren Reformschritten jeweils erhöht wurde, stagnierte er in der Reform von 2013 nicht nur, sondern war sogar leicht rückläufig (Anania und Pupo D'Andrea 2015). Ein mögliches Argument könnte dabei gewesen sein, dass durch die Einführung des Greenings in der Ersten Säule die Zweite Säule entlastet wurde und damit für weitergehende Maßnahmen genutzt werden sollte.

Die Teilnehmer in den Hintergrundgesprächen waren eher skeptisch, ob es zu größeren Veränderungen der Aufteilung der Mittel zwischen Vollfinanzierung und Ko-Finanzierung kommen wird. Die Erhöhung des Anteils mit erforderlicher KoFinanzierung würde - bei insgesamt gleichbleibendem GAP-Budget - erfordern, dass die Mitgliedstaaten insgesamt noch mehr Geld für die Agrarpolitik aufwenden würden, da mehr Finanzmittel zur Ko-Finanzierung aufgebracht werden müssten. Dies ist angesichts der Fülle anderer Aufgaben sowie der schrumpfenden ländlichen Bevölkerung aus polit-ökonomischer Perspektive eher unwahrscheinlich.

Mehr Erfolg verspricht daher der im Rahmen der 2013-Reform verfolgte Ansatz, den Mitgliedstaaten die Option einer freiwilligen Teilmodulation von der Ersten in die Zweite Säule bei Beibehaltung der Vollfinanzierung zu eröffnen. Die Mitgliedstaaten gewinnen dadurch mehr Spielraum für eine von finanziellen Erwägungen unabhängige Auswahl der geeignetsten Instrumente. Bis August 2017 haben die Mitgliedstaaten die Möglichkeit, die Modulation weiter auf bis zu 15 \% zu erhöhen.

Eine andere Möglichkeit wäre es, die bisherige Verknüpfung zwischen Vollfinanzierung mit Direktzahlungen/Cross Compliance einerseits und Ko-Finanzierung mit freiwilligen/regionsspezifischen Maßnahmen andererseits zu lockern oder sogar ganz aufzuheben.

\subsubsection{Handlungsalternativen}

Vor dem Hintergrund der Überlegungen ergeben sich fünf alternative Ansätze, die sich nicht gegenseitig ausschließen, sondern auch kombiniert werden können:

(1) Verlagerung von Mitteln aus der GAP in z. B. einen ,Naturschutzfonds“6: Diesem Ansatz liegt die Erfahrung zugrunde, dass nach Festlegung des Mehrjährigen Finanzrahmens 2014-2020 die Ausgestaltung der darin vorgesehenen sachlichen Rahmenrichtlinien von agrarischen Interessen dominiert wurde. Eine teilweise Verlagerung von Mitteln aus der GAP in einen „Naturschutzfonds“ würde sicherstellen, dass bei deren Vergabe die Anliegen des Natur- und Umweltschutzes Priorität erhalten. Der Nachteil dieser Strategie ist ihr redistributiver Charakter, der regelmäßig zu einem hohen Konfliktniveau führt. Dies kann einerseits Mobilisierungseffekte für den Natur- und Umweltschutz haben, andererseits wäre aber auch mit einer Mobilisierung derjenigen Kräfte zu rechnen, die befürchten, dass man ihnen ,ihr“ Budget ,wegnehmen“ will. Angesichts der 
Interessenlagen und Kräfteverhältnisse wäre hier vermutlich ein hoher politischer Krafteinsatz bei ungewissen Erfolgsaussichten erforderlich. Daneben ist zu berücksichtigen, dass auch ein bei der DG Umwelt angesiedeltes Budget für Programme des Agrarumweltschutzes den Regeln des EU-Rechts unterliegt. Zudem könnte die Koordination mit anderen Maßnahmen der Agrarpolitik schwieriger sein, und es könnten parallele Verwaltungsabläufe entstehen.

(2) Verschiebung von Mitteln von der Ersten in die Zweite Säule: Dies erschien über 20 Jahre als der evolutionäre Entwicklungspfad der GAP. Seit 2013 stagniert dieser Reformpfad jedoch. Ein erstes Hindernis dieser Strategie sind die Verteilungseffekte zuungunsten von Vetospielern, denen jedoch grundsätzlich durch Kompensations- und Paketlösungen begegnet werden kann. Ein zweites wichtiges Problem ist, dass bei Beibehaltung der (sub-)nationalen Ko-Finanzierung zu derzeitigen Sätzen insgesamt noch mehr Mittel für die Agrarpolitik aufgewendet werden müssten. Dem könnte dadurch begegnet werden, dass die nationalen Ko-Finanzierungssätze so vermindert werden, dass das finanzielle Gesamtvolumen (Aufwendungen der EU plus Mitgliedstaaten) ungefähr konstant bleibt. Bei diesem Ansatz ist weiterhin zu beachten, dass es nicht nur eine Kritik an der oberflächlichen Legitimierung der Ersten Säule durch wenig wirksame Cross-Compliance-Regelungen gibt, sondern auch eine Kritik an mangelnder Wirksamkeit der Maßnahmen der Zweiten Säule (siehe Abschn. 7.4.2).

(3) Stärkung der Agrarumweltprogramme innerhalb der ländlichen Entwicklungspolitik: Weiterhin ist zu berücksichtigen, dass innerhalb der Zweiten Säule eine Mittelkonkurrenz zwischen Agrarumweltprogrammen, ländlicher Entwicklung und betrieblichen Investitionsprogrammen besteht (Hart et al. 2016). Die Naturschutz- und Umweltkomponente könnte dadurch gestärkt werden, dass hier höhere finanzielle Mindest- und Höchstanteile innerhalb der ländlichen Entwicklungsprogramme verankert werden. Auf der nationalen und regionalen Ebene wäre dann sicherzustellen, dass die vorhandenen finanziellen Räume für Naturschutz- und Umweltmaßnahmen möglichst weitgehend ausgeschöpft werden.

(4) Dominante Direktzahlungen akzeptieren und umwelteffektiver machen: Geht man davon aus, dass die Nutznießer starker direktfinanzierter Direktzahlungen eine nicht zu überwindende Vetoposition im Rat einnehmen, dann bestehen folgende Möglichkeiten:

- stärkere Verknüpfung der Direktzahlungen mit Naturschutz- und Umweltleistungen durch Weiterentwicklung des Greenings und Neugestaltung der Instrumente (siehe Abschn. 7.3);

- fortlaufende Erhöhung des Anteils, den die Mitgliedstaaten von der Ersten in die Zweite Säule modulieren können, ggf. unter Beibehaltung der Vollfinanzierung; dadurch Erhöhung der nationalen Spielräume für zielgenauere und anspruchsvollere Maßnahmen nach Art der Zweiten Säule.

(5) Erschließen und Aktivieren neuer Finanzquellen: Eine andere Variante ist die Erschließung neuer Finanzierungsquellen für Maßnahmen des Natur- und 
Umweltschutzes. Hier könnte etwa ein Agrarumwelt-Investitionsfonds von Interesse sein, der Umweltinvestitionen ko-finanziert oder - nach dem Vorbild der Unterstützung von Energiesparmaßnahmen durch die Kreditanstalt für Wiederaufbau - mit vorteilhaften Krediten ermöglicht. Hier wäre auch darüber nachzudenken, ob aus Marktaktivitäten weitere Zahlungen aktiviert werden können, etwa durch die Einführung eines Umweltcents auf bestimmte Güter durch Einzelhändler mit Marktmacht, aus dem dann Pilotprojekte oder andere Maßnahmen gefördert oder ko-finanziert werden können.

\subsection{Baustein 3: Erste Säule der GAP - Flächenbezogene Direktzahlungen}

\subsubsection{Bewertung des derzeitigen Systems der Direktzahlungen}

Die Erste Säule der GAP beinhaltet die Gewährung von Direktzahlungen, die seit der Reform 2003 unabhängig von der Art und Höhe der Produktion (etwa Anzahl gehaltener Tiere, angebaute Kulturen) als Flächenprämie gezahlt werden. In Deutschland wurden die Direktzahlungen vollständig von der Produktion entkoppelt und spätestens ab 2019 werden in ganz Deutschland für Acker- und Grünland einheitliche Flächenprämien ausbezahlt. Seit 2015 gliedern sich die Direktzahlungen in die Basisprämie, die Greening-Prämie, die Umverteilungsprämie und die Junglandwirteprämie. Dabei sind die Basis- und die Greening-Prämie an die Einhaltung der Cross-Compliance-Verpflichtungen (GAB, GLÖZ) und der Greening-Auflagen (Anbaudiversifizierung, Erhalt von Dauergrünland, Bereitstellung ökologischer Vorrangflächen) gebunden. Mit der Umverteilungsprämie werden die ersten Hektare eines Betriebes zusätzlich gefördert, hierdurch sollen kleinere Betriebe verstärkt begünstigt werden. Ebenso werden mit der Junglandwirteprämie Berufseinsteiger stärker gefördert. Das BMEL definiert als Ziele der Gewährung der Direktzahlungen (BMEL 2015e):

- Erstens sollten damit die vielfältigen gesellschaftlichen Leistungen der Landwirtschaft honoriert und gesichert werden.

- Zweitens seien die staatlichen Förderungen ein Ausgleich dafür, dass Landwirte in Europa gerade in den Bereichen Umwelt-, Tier- und Verbraucherschutz deutlich höhere Standards einhalten müssten als viele ihrer Kollegen in anderen Teilen der Welt.

- Drittens trügen die Direktzahlungen zur Einkommenssicherung und Einkommensstabilisierung der Landwirte bei, indem sie die Auswirkungen der zum Teil extremen Preisschwankungen bei Agrarprodukten abfedern.

Die Direktzahlungen der Ersten Säule sind seit der Reform von 2003 an die Einhaltung von Natur- und Umweltschutzauflagen gebunden (Cross Compliance). Damit werden die Aspekte der Einkommenssicherung und Versorgungssicherheit als 
Ziele der GAP systematisch mit der Kontrolle der Einhaltung von Mindeststandards verknüpft. Die Zahlungen erscheinen daher als Honorierung landwirtschaftlicher Leistungen. An Grenzertragsstandorten unterstützen die Direktzahlungen zudem die Aufrechterhaltung einer flächendeckenden Bewirtschaftung, denn sie werden schon bei Offenhaltung der Flächen durch einmaliges Mähen oder Mulchen pro Jahr gewährt. Auf diese Weise wurde mit der Reform von 2003 eine argumentative Engführung von Erster und Zweiter Säule eingeleitet, denn bis dahin wurden Naturund Umweltleistungen ausschließlich in der Zweiten Säule honoriert.

Allerdings wird vielfach Kritik an diesem System geäußert. So gehen die Cross-Compliance-Auflagen nur geringfügig über den gesetzlichen Mindeststandard hinaus - nämlich in Form der GLÖZ- und seit 2015 der Greening-Auflagen. Die positiven Effekte für den Natur- und Umweltschutz sind dabei vermutlich gering (Hart et al. 2016).

Da die Landwirte nicht dazu verpflichtet sind, Cross Compliance und Greening einzuhalten (mit Ausnahme der GAB), steht ihnen die Möglichkeit offen, auf die Greening- und die Basisprämie zu verzichten, um damit die Einhaltung der GLÖZund Greening-Auflagen zu umgehen. Zwar ist die Einhaltung der GreeningAuflagen Voraussetzung für den Erhalt der Basisprämie, bei Verstößen werden jedoch max. $125 \%$ der Greening-Prämie gekürzt. In Deutschland entspricht das ca. $100 € /$ ha. Bisher erhalten die meisten Betriebe in Deutschland jedoch Direktzahlungen (siehe Tab. 7.5) und müssen damit die Cross-Compliance-Auflagen einhalten. Aussagen zur Einhaltung der Greening-Auflagen bzw. zum Umfang von Verstößen gegen die Greening-Auflagen sind noch nicht bekannt.

Wie Tab. 7.5 zeigt, nehmen konstant mehr als $90 \%$ der Betriebe in Deutschland die Direktzahlungen in Anspruch. Insgesamt wurden in Deutschland nur für ca. 49.000 ha landwirtschaftlicher Fläche keine Direktzahlungen bezogen, wobei es sich vorwiegend um Sonderkulturflächen handelte (Krämer 2017, S. 40). Diese hohe Teilnahmebereitschaft lässt sich damit erklären, dass die Höhe der Direktzahlungen die Kosten, die durch die Einhaltung der Bedingungen für die Gewährung von Direktzahlungen anfallen, typischerweise bei weitem übersteigt. Berechnungen zeigen, dass die durch die Auflagen verursachten Mehrkosten im Ackerbau gegenüber dem Weltmarkt bei nur ca. $20 € /$ ha (Plankl et al. 2010) bis $50 € /$ ha liegen (Wissenschaftlicher Beirat für Agrarpolitik beim BMELV 2010).

Auch bezüglich der Einhaltung der Greening-Auflagen ist davon auszugehen, dass die durch die Einhaltung der Auflagen verursachten Kosten durch die Greening-Prämie mehr als kompensiert werden. Im Vorfeld wurden die Kosten der Ein-

Tab. 7.5 Anteil

landwirtschaftlicher Betriebe, die keine Direktzahlungen beziehen. (Quelle: Eigene Darstellnug, nach BMELV (2013a); BMEL (2014a, 2015d))

\begin{tabular}{l|l}
\hline & $\begin{array}{l}\text { Anteil der Betriebe } \\
\text { ohne } \\
\text { Direktzahlungen (\%) }\end{array}$ \\
\hline $2014 / 15$ & 6,8 \\
\hline $2013 / 14$ & 6,4 \\
\hline $2012 / 13$ & 7,2 \\
\hline $2011 / 12$ & 6,7 \\
\hline
\end{tabular}


haltung der Greening-Auflagen im EU-Durchschnitt auf ca. $35 € /$ ha geschätzt (European Commission 2011a). Seitens der Landwirtschaftsverbände stand dabei vor allem die Auflage, 5 \% ökologische Vorrangflächen bereit zu stellen, stark in der Kritik. Nimmt man nun an, dass die Direktzahlung ab 2019 in Deutschland $281 €$ / ha betragen, dann beträgt die Höhe der Greening-Prämie 84,30 €/ha (30 \% der Direktzahlungen). Nimmt man weiterhin an, dass die beiden anderen GreeningAuflagen - die Verpflichtung zum Grünlanderhalt und die Fruchtfolgegestaltung ohne Verursachung wesentlicher Kosten eingehalten werden können, dann ergibt sich so für die Bereitstellung von 1 ha ökologischer Vorrangfläche ein „Pachtpreis“ von $1686 € /$ ha. ${ }^{1}$ Selbst in aktuellen Gunstlagen ist ein Pachtpreis in dieser Höhe „,konkurrenzfähig“. Dabei ist zusätzlich zu berücksichtigen, dass die Bereitstellung ökologischer Vorrangflächen einen vollkommenen Nutzungsverzicht gar nicht vorsieht. Es können ökologische Vorrangflächen gemeldet werden, welche die Bereitstellungskosten der ökologischen Vorrangfläche weiter senken. So ist z. B. der Anbau von Eiweißpflanzen möglich. Auch wenn durch die Auflagen des Grünlanderhalts und der Einhaltung der Fruchtfolgeauflagen in einigen Betrieben Kosten entstehen können, ist davon auszugehen, dass diese durch die Greening-Prämie kompensiert werden. So wird festgestellt, dass auch unter Berücksichtigung der Kosten des Greenings, die mit 10 bis $100 € /$ ha berechnet werden, die Einhaltung der Greening-Auflagen aus betrieblicher Sicht langfristig meist sinnvoll ist (de Witte und Latacz-Lohmann 2014). Die Folgenabschätzung der Europäischen Kommission bezifferte die zu erwartenden Kosten aller Greening-Auflagen auf etwas mehr als $30 € /$ ha im EU-Durchschnitt und für Deutschland auf durchschnittlich ca. $50 € /$ ha (European Commission 2011a).

Es lässt sich somit festhalten, dass die Cross-Compliance- und GreeningAuflagen zwar nicht garantieren, dass von allen Betrieben in allen Regionen die entsprechenden Auflagen eingehalten werden, da ein „Ausstieg“ aus dem System der Direktzahlungen möglich ist. Aktuell findet ein solcher Ausstieg aber nur in sehr geringem Umfang statt, da die gewährten Prämien die entstehenden Kosten bei Weitem übersteigen. Darüber hinaus liegen die Direktzahlungen deutlich über dem Wert, der für einen Ausgleich für höhere Auflagen gegenüber dem Weltmarkt gerechtfertigt wäre.

Wenn die Direktzahlungen tatsächlich vorrangig dem Zweck dienen sollen, einen Ausgleich für die aus den Cross-Compliance- und Greening-Verpflichtungen entstehenden Mehrkosten zu gewähren, dann müssten die erheblichen Unterschiede zwischen den verschiedenen Betriebstypen deutlich stärker berücksichtigt werden (siehe Tab. 7.6). Dies betrifft z. B. tierhaltende Betriebe, die im Vergleich zu Ackerbaubetrieben mehr Auflagen einhalten müssen und dadurch zum einen höhere Kosten haben (Bürokratie, Produktion, Investitionen), aber auch ein höheres Anlastungsrisiko aufweisen (gegen die Tierkennzeichnungsverordnung wird am häufigsten

\footnotetext{
${ }^{1}$ Die Summe von $1686 € /$ ha ergibt sich, wenn man die Greening-Prämie von 84,30 €/ha mit der Anzahl der Hektare multipliziert, für welche die Greening-Prämie gezahlt wird. Diese ist 20 mal so groß wir die ökologischen Vorrangflächen, die bereitgestellt werden müssen, um die Greening-Auflagen zu erfüllen.
} 
Tab. 7.6 Steigerung der betrieblichen Kosten für die Einhaltung der EU-Auflagen im Bereich Umwelt, Tierwohl und Verbraucherschutz $(\%)$ nach Betriebstypen

\begin{tabular}{l|l|l}
\hline & Deutschland & EU \\
\hline Milchbetriebe & $1,1-1,5$ & $0,7-2,9$ \\
\hline Rindermastbetriebe & & $0,5-3,0$ \\
\hline Schafmastbetriebe & & $0,4-3,2$ \\
\hline Schweinemastbetriebe & 8,8 & $2,9-8,8$ \\
\hline Geflügelmastbetriebe & $1,4-2,2$ & $1,4-5,6$ \\
\hline $\begin{array}{l}\text { Ackerbaubetriebe } \\
\text { (Weizen) }\end{array}$ & $1,4-2,2$ & $2,1-3,4$ \\
\hline Apfelproduktion & $0,9-3,0$ & $0,9-3,0$ \\
\hline Weinbaubetriebe & & $0,1-4,1$ \\
\hline
\end{tabular}

Quelle: Eigene Darstellung, nach Menghi et al. (2014)

verstoßen). In der EU kommt es aufgrund der Auflagen im Bereich des Umwelt-, Tier- und Verbraucherschutzes zu Steigerungen der Produktionskosten bei Schweine und Geflügel haltenden Betrieben von rund 5-10\%, bei Milchvieh, Rinder und Schafe haltenden Betrieben um 2-3\% und bei viehlosen Betrieben um 1-3,5\% (Menghi et al. 2014).

Des Weiteren ist anzuführen, dass Betriebe, die z. B. in der Vergangenheit Landschaftselemente erhalten und/oder Grünland nicht umgebrochen haben, nun von den entsprechenden Auflagen und Anlastungsrisiken betroffen sind. Dies steht im Gegensatz zur Situation von Betrieben, die sich in der Vergangenheit weniger naturund umweltschutzorientiert verhalten haben. Umgekehrt erfordert die Bereitstellung ökologischer Vorrangflächen oder die Einhaltung der Fruchtfolgeauflagen von Betrieben, die dies bereits in der Vergangenheit geleistet haben, wenige Veränderungen, während diese Auflagen für andere Betriebe mit Kosten verbunden sein könnten.

Durch die Modulation bis 2014 und die Umverteilungsprämie, welche kleinere Betriebe pro Hektar besser stellen, erhalten die Direktzahlungen eine strukturpolitische Komponente. Allerdings ist diese kaum geeignet, den Strukturwandel aufzuhalten, der hin zu weniger Betrieben führt, die sich aber stärker spezialisieren. Weiterhin kommt es zu einer räumlichen Konzentration ähnlich spezialisierter Betriebe in einzelnen Regionen (z. B. die Veredelungsbetriebe im Raum Vechta-Cloppenburg). Die regionale Spezialisierung kann wiederum Umweltprobleme mit sich bringen (z. B. hoher Anfall organischer Dünger). Mit der abnehmenden Zahl an Landwirten könnte darüber hinaus Erfahrungswissen verloren gehen. In Deutschland führt die stärkere Förderung der ersten Hektare zu einer Umverteilung der Zahlungen zwischen den Bundesländern, wobei die neuen Bundesländer 85 Mio. € verlieren, während Baden-Württemberg und Bayern 48 Mio. $€$ gewinnen (Balmann und Sahrbacher 2014). Es wird erwartet, dass die Prämie geringe Effekte hinsichtlich der Entlastung kleinerer Betriebe bewirkt, aber einen hohen administrativen Aufwand verursacht (Forstner et al. 2012).

Das Hauptziel der Direktzahlungen ist die Einkommenssicherung für Landwirtinnen und Landwirte. Die Direktzahlungen machen zwar ca. die Hälfte des betrieblichen Einkommens aus, jedoch nur ca. $10 \%$ der betrieblichen Erträge (siehe Tab. 7.7). Die Abhängigkeit der Betriebe von den Direktzahlungen wird häufig mit 
Tab. 7.7 Einkommensstabilisierende Wirkung der Direktzahlungen in Deutschland

\begin{tabular}{l|l|l}
\hline & $\begin{array}{l}\text { Anteil der Direktzahlungen am } \\
\text { landwirtschaftlichen Einkommen }(\%)\end{array}$ & $\begin{array}{l}\text { Anteil der Direktzahlungen an den } \\
\text { betrieblichen Erträgen (\%) }\end{array}$ \\
\hline $2014 / 15$ & 55,8 & 11,2 \\
\hline $2013 / 14$ & 44,4 & 10,4 \\
\hline $2012 / 13$ & 45,3 & 10,8 \\
\hline $2011 / 12$ & 54,0 & 12,3 \\
\hline
\end{tabular}

Quelle: Eigene Darstellung, nach BMELV (2013a), BMEL (2014a, 2015d)

dem hohen Anteil der Direktzahlungen am landwirtschaftlichen Einkommen begründet. Die absolute Höhe der gewährten Direktzahlungen als Anteil des landwirtschaftlichen Einkommens auszuweisen und damit die Abhängigkeit der Landwirtschaft von den Direktzahlungen zu begründen, erscheint allerdings nur gerechtfertigt, wenn die Gewährung der Direktzahlungen keine Kosten sowie „Überwälzungseffekte“ beim landwirtschaftlichen Betrieb hervorruft. Dies ist jedoch nicht der Fall. Zum einen entstehen Kosten der Bürokratie und Umsetzung der Cross-Compliance- und Greening-Auflagen, zum anderen werden die Direktzahlungen zumindest zum Teil und in Abhängigkeit von der regionalen Bodennachfrage auf den Pachtpreis überwälzt (Wissenschaftlicher Beirat für Agrarpolitik beim BMELV 2010). Eine Ausweisung der Anteile der Direktzahlungen an den betrieblichen Erträgen könnte somit besser geeignet sein, die Abhängigkeit landwirtschaftlicher Betriebe von den Direktzahlungen darzustellen. Ein Anteil der Direktzahlungen an den betrieblichen Erträgen von ca. $10 \%$ scheint mit einer Übergangsfrist durchaus kompensierbar zu sein.

Des Weiteren wird häufig angemahnt, dass einkommens- und verteilungspolitische Fragestellungen nach dem Subsidiaritätsprinzip eher auf der Ebene der Mitgliedstaaten zu regeln sind (Wissenschaftlicher Beirat für Agrarpolitik beim BMELV 2010; Forstner et al. 2012). Auch ist der sektorbezogene Zuschnitt der Direktzahlungen nicht geeignet, verteilungspolitische Ziele zu verfolgen, denn ein niedriges landwirtschaftliches Einkommen kann beispielsweise einem Haushalt mit hohem Einkommen aus anderen Quellen zufließen, so dass keine verteilungspolitisch relevante Bedürftigkeit gegeben ist.

Schließlich wird argumentiert, dass die Fortführung der Direktzahlungen notwendig ist, in Grenzertragsregionen, wie etwa den Mittelgebirgen, die Aufrechterhaltung der Landwirtschaft zu ermöglichen. Tatsächlich wären zu diesem Zweck stärker räumlich fokussierte und zielgerichtete Maßnahmen effizienter, wie sie heute z. B. durch die Ausgleichszulage in der Zweiten Säule angeboten werden (Wissenschaftlicher Beirat für Agrarpolitik beim BMELV 2010).

Auch werden die Direktzahlungen zum Teil an den vor- und nachgelagerten Bereich „,durchgereicht“. Im Ergebnis ist ,,die Einkommenstransfereffizienz der meisten Unterstützungsmaßnahmen [...]<1“ (Moreddu 2011). Eine Studie aus dem Jahr 2008 zeigt, dass in Deutschland 30-60 \% der Direktzahlungen an die Landeigentümer weitergegeben werden (Swinnen et al. 2008). Bei hohen Pachtanteilen - in Deutschland im Durchschnitt ca. $60 \%$ - verbleiben damit große Teile der Direktzahlungen nicht bei den aktiven Landwirten, sondern subventionieren den Grundbesitz (Forstner et al. 
2012). Es wird davon ausgegangen, dass bei sinkenden Direktzahlungen unter ansonsten unveränderten Rahmenbedingungen auch die Pachtpreise sinken würden (Wissenschaftlicher Beirat für Agrarpolitik beim BMELV 2010).

Zusammenfassend lässt sich demnach festhalten, dass die Direktzahlungen die mit ihnen verbundenen Zielsetzungen nicht oder nur sehr ineffizient erfüllen. Durch die Cross-Compliance- und Greening-Auflagen werden nur geringe positive Effekte auf den Natur- und Umweltschutz erzielt, während eine Einhaltung der Auflagen in allen Regionen und Betrieben aufgrund der Möglichkeit zum „Ausstieg“ nicht garantiert werden kann. Die Greening-Prämie übersteigt die mit den Greening-Auflagen verbundenen Kosten für die allermeisten Betriebe bei Weitem. Auch die Basisprämie wäre als Ausgleich für die betrieblichen Kosten höherer Auflagen gegenüber dem Weltmarkt viel zu hoch angesetzt. Damit wird deutlich, dass die Direktzahlungen vor allem einkommenspolitischen Zielen dienen. Aufgrund des sektoralen Zuschnitts und der fehlenden Bindung an Bedürftigkeitskriterien stellen die Direktzahlungen aber kein effektives und effizientes Instrument der Einkommenspolitik dar. Hinzu kommt, dass - je nach den Verhältnissen auf den regionalen Bodenmärkten - ein erheblicher Teil der Direktzahlungen auf Pacht- und Landpreise überwälzt wird, so dass die Einkommenseffekte zum Teil bei Grundbesitzern anfallen, die weder landwirtschaftlich tätig noch im ländlichen Raum ansässig sind.

\subsubsection{Vorschläge zur Neustrukturierung der Ersten Säule}

Insbesondere in Hinblick auf die mangelnde Erreichung der Ziele des Natur- und Umweltschutzes werden aktuell folgende Vorschläge zur weiteren Entwicklung der Ersten Säule der GAP diskutiert (vgl. dazu das Projekt des Instituts für Agrarökologie (Oppermann et al. 2016)und Biodiversität (ifab) sowie die Vorschläge des Institute for European Environmental (Hart et al. 2016):

- Weitere Begrünung der Ersten Säule;

- Ausbau der Zweiten Säule;

- Umbau des Systems der GAP in ein integriertes System zur Honorierung öffentlicher Leistungen.

Eine Weiterentwicklung des Greenings der Ersten Säule wird in Abschn. 8.2 eingehender dargestellt, eine Stärkung der Zweiten Säule durch Umschichtung von Geldern aus der Ersten Säule in Abschn. 8.3. An dieser Stelle wird im Folgenden auf einen Umbau des Systems der GAP eingegangen. Dabei entwickeln die folgenden Überlegungen zum Umbau der Ersten Säule in ein Instrument zur Honorierung öffentlicher Leistungen der Landwirtschaft den eingeschlagenen Weg der Gewährung von Zahlungen für die Einhaltung von Umwelt- und Naturschutzauflagen weiter und orientieren sich strikt am Prinzip ,öffentliche Gelder für öffentliche Leistungen“. Als öffentliche Leistungen wird dabei im Rahmen der neuen Ersten Säule der Ausgleich von wirtschaftlichen Nachteilen und Maßnahmen verstanden, die auf Betriebsebene im Rahmen eines „Baukastensystems“ umgesetzt werden. 
Es werden verschiedene Politik-Elemente formuliert, welche die adressierten Problemlagen jeweils gezielt ansprechen. Damit sollen die Umweltwirkungen der Landwirtschaft verbessert werden, indem gezielt die Einhaltung anspruchsvollerer Auflagen honoriert wird. Die Höhe der betrieblichen Prämien würde sich dann nach den tatsächlich erbrachten öffentlichen Leistungen richten. Dies würde auch die Motivation der Landwirte erhöhen, öffentliche Leistungen zu erbringen. Da eine einheitliche Honorierung in Form einer Flächenzahlung nicht mehr gewährt würde, ist davon auszugehen, dass Prämien nur noch in geringerem Maße auf die Pacht- und Bodenpreise überwälzt würden. Eine Einkommensverbesserung der Landwirtschaft würde dadurch erreicht, dass neben der Produktion landwirtschaftlicher Erzeugnisse die Erbringung von öffentlichen Leistungen als Einkommensquelle an Bedeutung gewinnt.

Die nachfolgenden vorgestellten Elemente des Systems grenzen sich gegenüber einer neu strukturierten Zweiten Säule (siehe Abschn. 7.4) dadurch ab, dass

- sie auf EU-Ebene oder Ebene der Mitgliedstaaten programmiert und finanziert werden;

- die Maßnahmen weitgehend unabhängig von regionalen und standörtlichen Bedingungen einen positiven Mehrwert für den Natur- und Umweltschutz erwarten lassen.

Nachfolgend wird die den Elementen zugrundeliegende Idee skizziert, Ausführungen zur detaillierten Ausgestaltung der Elemente werden an dieser Stelle nicht gemacht.

\subsubsection{Element 1: Gesetzlicher Mindeststandard zur Aufrechterhaltung der natürlichen Produktivität der Standorte}

Bisher sind in Cross Compliance verschiedene Auflagen zusammengefasst. Die GAB sind dabei als Ordnungsrecht anzusehen, dessen Einhaltung prinzipiell nicht entlohnt werden sollte. Die GLÖZ- und Greening-Auflagen können aufgrund der „Ausstiegsmöglichkeit“ als freiwillige Maßnahmen angesehen werden. Dabei sind Teile der GLÖZ- und Greening-Auflagen jedoch landbautechnisch zur dauerhaften Aufrechterhaltung der Produktivität der Standorte notwendig und sollten damit einerseits im langfristigen Eigeninteresse der Bewirtschafter liegen, andererseits aber nicht der Freiwilligkeit überlassen bleiben. Daher könnte es geboten sein, weitere verpflichtende Auflagen zu definieren, die weitgehend den heutigen GLÖZ- und Greening-Auflagen entsprechen, um auch langfristig und für zukünftige Generationen die Produktivität der Standorte zu sichern. Diese Auflagen wären von allen Landwirten einzuhalten. Zu diesen Auflagen zur Aufrechterhaltung der natürlichen Produktivität könnten insbesondere zählen:

- Erhalt von Dauergrünland;

- Maßnahmen des Erosionsschutzes entsprechend dem Erosionskataster (zur Einordnung im Erosionskataster ist zusätzlich die Hanglänge zur berücksichtigen), z. B. Erosionsschutzstreifen, Winterbegrünung;

- Maßnahmen des Humuserhalts;

- Bereitstellung wertvoller ökologischer Vorrangflächen; 
- Anspruchsvollere mehrgliedrige Fruchtfolge auf der Fläche (Flächenanteil der Hauptfrucht max. $50 \%) ;^{2}$

- Erhalt von Landschaftselementen.

\subsubsection{Element 2: Prämien zur Aufrechterhaltung der Landbewirtschaftung in Gebietskulissen zum Erhalt der Landschaftsvielfalt}

Direktzahlungen können aus Sicht des Umwelt- und Naturschutzes wichtig sein, um die Landbewirtschaftung an Standorten aufrecht zu erhalten, wo dies für eine vielfältige Landschaft und die damit verbundenen Vorteile für den Erhalt der Biodiversität und der Ökosystemleistungen notwendig ist. Um diese Ziele zu erreichen, ist es jedoch effektiver und effizienter, die entsprechenden Zahlungen auf die betroffenen Standorte zu konzentrieren.

Um die landwirtschaftliche Erzeugung auch auf Grenzertragsstandorten aufrecht zu erhalten, wo dies zur Erhaltung einer Landschaftsvielfalt wünschenswert ist, könnte daher - in Weiterentwicklung der heutigen Ausgleichszulage - eine Prämie gewährt werden, deren Höhe sich daran orientiert, welche Standortnachteile ausgeglichen werden müssen, um die Bewirtschaftung attraktiv zu machen. $\mathrm{Zu}$ den möglichen Kriterien für die Bestimmung der Prämienhöhe gehören die Vegetationsdauer (ausgedrückt in Höhe über NN oder langjährige Mittel der Länge der Vegetationsperiode), die Hangneigung, die Bodenpunkte u. a. Grundsätzlich sollten Prämien für Grenzertragsstandorte so bemessen werden, dass sie die tatsächlichen Opportunitätskosten der Bewirtschaftung widerspiegeln (Hart et al. 2016). Da die Benachteiligung anhand relativ einfacher Indikatoren gut abgebildet werden kann, könnten zukünftig die Kriterien für die Höhe einer solchen Prämie auf Ebene der EU einheitlich festgelegt werden. Allerdings sollte die Zahlung einer solchen Prämie auf zielorientierte Gebietskulissen beschränkt sein. Zudem sollte die Prämie auf der Ebene von Standorten (Schlagebene) und nicht von relativ großflächigen Regionen definiert werden, um Mitnahmeeffekte zu minimieren.

Eine Finanzierung im Rahmen der Ersten Säule wäre anzuraten. Schon heute besteht die Möglichkeit für die Mitgliedstaaten, benachteiligte Standorte durch die Erste Säule zu fördern, ${ }^{3}$ die jedoch von Deutschland nicht genutzt wird (Isermeyer et al. 2014).

Ergebnisse aus der Schweiz zeigen, dass Betriebe mit einem hohen Anteil von „Erschwernisflächen" aufgrund des hohen Arbeitsaufwandes häufig in ihrem betrieblichen

\footnotetext{
${ }^{2}$ Ein minimaler Flächenanteil von $10 \%$ und ein maximaler Flächenanteil von $50 \%$ sind für jede der drei Feldfrüchte aus Sicht der Agrobiodiversität angemessen. Die Früchte müssen sich auf jeder Fläche mit den mindestens bzw. höchsten vorgegebenen Anteilen abwechseln (Wissenschaftlicher Beirat für Biodiversität und Genetische Ressourcen beim BMELV 2011).

${ }^{3}$ Deutschland macht hiervon keinen Gebrauch. Möglich wären max. $5 \%$ der nationalen Obergrenze für die Direktzahlungen. Jedoch ist die diesbezügliche Förderung über die Erste Säule bei der Bemessung der Ausgleichszulage in der Zweiten Säule zu berücksichtigen (Isermeyer et al. 2014).
} 
Wachstum beschränkt sind (Bosshard und Häusler 2012), so dass diese Prämienkomponente auch der Förderung kleinerer (Familien-)Betriebe dient. Gleiches kann auch für die folgende Prämienkomponente gelten.

\subsubsection{Element 3: Honorierung der landschaftlichen Vielfalt}

Zu einer reformierten Ersten Säule könnte auch eine Honorierung der landschaftlichen Vielfalt gehören. Da das Vorhandensein von Strukturelementen in der Landschaft sowie kleine Schlag- bzw. Gewanngrößen im Allgemeinen mit einem erhöhten Wert für die Biodiversität einher gehen, aber häufig mit einem höheren Arbeitsaufwand und Ertragsverlusten auf den angrenzenden Flächen verbunden sind, könnte eine Prämie eingeführt werden, welche landschaftliche Vielfalt belohnt. Hierbei könnten an Landschaftselemente angrenzende Flächen, die Gewanngröße unter Berücksichtigung der integrierten Schläge, lineare Landschaftselemente, ${ }^{4}$ Puffer-, Rand- und Blühstreifen (z. B. entlang von Bächen, Wassergräben und Feldwegen), Steine und Felsen (die z. B. in Steilwiesen vorkommen) in oder angrenzend an die Fläche honoriert werden, aber auch Flächen, die durch extensive, häufig traditionelle Nutzungsverfahren (z. B. Streuobstwiesen, Terrassen) gekennzeichnet sind. Neben dem Vorhandensein landschaftlicher Strukturen könnte auch die beeinflusste landwirtschaftliche Nutzfläche, z. B. Randstreifen entlang von Hecken, in die Prämienberechnung einbezogen werden. Dabei ist es unabhängig, in wessen Besitz das beeinflussende Landschaftselement ist. Im Rahmen einer solchen Prämie würde das Vorhandensein von Strukturen der landschaftlichen Vielfalt gefördert. Deren Anlage, Pflege und ggf. Bewirtschaftung würde weiterhin im Rahmen der Zweiten Säule gefördert (ähnlich dem heutigen Verfahren zum Erhalt und zur Pflege von Landschaftselementen). Da Strukturen der landschaftlichen Vielfalt als überwiegend ungenutzte Elemente unabhängig von sonstigen standörtlichen, lokalen und regionalen Bedingungen meist einen positiven Einfluss auf die Schutzgüter, insbesondere Biodiversität und Landschaftsbild, haben, aber beispielsweise im Allgemeinen auch dem Erosions- und Hochwasserschutz dienen (Hampicke 2009; Batáry et al. 2011; Kremen und Miles 2012; Scheper et al. 2013; Vogel und Inauen 2013), könnte eine Förderung im Rahmen der Ersten Säule systematisch gerechtfertigt sein.

\subsubsection{Exkurs: Ansätze zur Honorierung der landschaftlichen Vielfalt}

Landwirte pflegen und erhalten die landschaftliche Vielfalt der Kulturlandschaft. Damit erbringen sie eine gesellschaftlich gewünschte, nicht marktgängige Leistung sowohl aus Sicht des Naturschutzes als auch durch die Schaffung von Erholungsräumen

${ }^{4}$ ÖVF - lineare und/oder dauerhafte Landschaftselemente sollten anrechenbar sein (Wissenschaftlicher Beirat für Biodiversität und Genetische Ressourcen beim BMELV 2011). 
(Plankl et al. 2010). Eine gezielte Honorierung dieser Leistung ist sinnvoll und soll im Weiteren kurz skizziert werden.

\section{Der Wert landschaftlicher Vielfalt}

Der Erhalt der biologischen Vielfalt ist im Sinne des Naturschutzes wie auch des Schutzes der Stabilität von Agrarökosystemen und den mit ihnen verbundenen Ökosystemleistungen (z. B. Bestäubung, biologische Schädlingsbekämpfung) notwendig.

Landschaftliche Vielfalt fördert den Artenreichtum in der Agrarlandschaft (Duelli und Obrist 2003; Tscharntke et al. 2005). Dies trifft vor allem für generalistische (euryöke) Arten zu (Jedicke 1994).

Die hohe Diversität in komplexen Kulturlandschaften resultiert dabei in einer großen Anzahl an Arten, die Saumbiotope an den zahlreichen Grenzlinien besiedeln, und Arten die im Laufe ihrer Entwicklung zwischen verschiedenen Habitaten wechseln (Jedicke 1994). Zum Schutz spezialisierter (stenöker) Arten werden zusätzlich zu diesem Netz kleinflächiger Strukturelemente in der Agrarlandschaft großflächige hochwertige natürliche und semi-natürliche Landschaftsstrukturen benötigt (Jedicke 1994).

\section{Herausforderungen für die Landwirtschaft}

Die Bewirtschaftung von Agrarflächen in vielfältigen Landschaften mit einer hohen Anzahl an Grenzlinien (aufgrund kleiner Schlaggrößen und/oder einer hohen Anzahl angrenzender wie auch in den Schlägen enthaltener Landschaftselemente) bedeutet für die Landwirte einen erhöhten Bewirtschaftungsaufwand. Diese für den Landwirt nachteilige, aber aus gesellschaftlicher Sicht positive Mehrleistung sollte für die Landwirte honoriert werden (Abb. 7.1).

\section{Indikatoren für landwirtschaftlich erbrachte Leistungen zur Förderung landschaftlicher Vielfalt}

Folgende Parameter sind für eine Bewertung der von den Landwirten im Rahmen ihrer landwirtschaftlichen Tätigkeit erbrachten Mehrleistung sinnvoll und sollten bei einer Honorierung berücksichtigt werden:

- Schlaggröße

- Einschränkungen durch die Schlagform;

- Einschränkungen durch identische Kulturen auf benachbarten Schlägen.

Abb. 7.1 Artenreichtum von Ackerwildkräutern in Abhängigkeit von der Landschaftsstruktur. (Quelle: Eigene Darstellung nach Tscharntke et al. 2005)

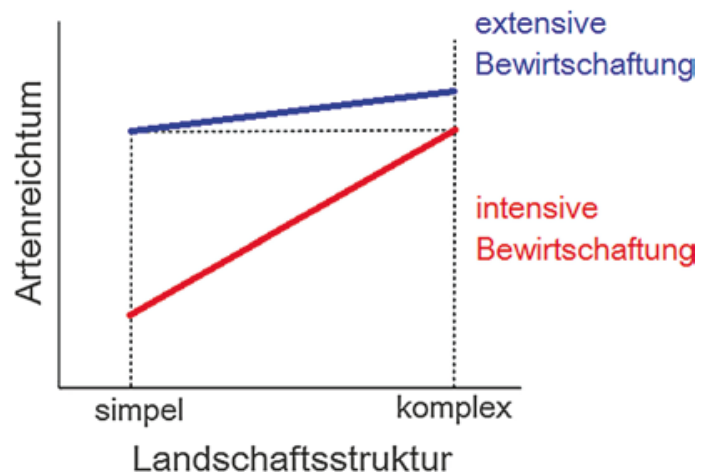


- Grenzlinien zu Landschaftselementen (innerhalb des Schlags und angrenzend an den Schlag)

- Größe und Wertigkeit der Landschaftselemente.

Schlaggröße: Ein Mosaik kleiner Schläge weist deutlich mehr Grenzlinien mit Saumbiotopen auf als wenige große Schläge. Als ökologisch sinnvoll werden von den meisten Autoren maximale Schlaggrößen im Bereich von 5 bis 12 ha, in Regionen mit großflächigen naturbetonten Zwischenflächen von 20 bis 25 ha genannt (Haber 1998; Bosch und Partner GmbH 2000; Christen et al. 2009; Ring 2013). Aus landwirtschaftlicher Sicht steigt der relative Arbeitsaufwand pro ha mit abnehmender Schlaggröße im Bereich von ca. 5 bis 1 ha besonders stark an (Engelhardt 2004).

Abb. 7.2 skizziert eine mögliche Honorierung in Abhängigkeit von der Schlaggröße.

Einschränkung durch die Schlagform: Die Schlagform in Abhängigkeit von der Schlaggröße definiert die Abstände von Saumbiotopen bzw. von Landschaftselementen (soweit diese vorhanden sind). Geringe Abstände von Saumbiotop zu Saumbiotop bzw. zu Landschaftselement sind aus ökologischer Sicht wünschenswert. Randstrukturen haben auf einer Distanz von 20 m einen deutlichen Effekt in benachbarte Agrarflächen hinein und die Angaben zu Maximalabständen zwischen zwei Saumbiotopen bzw. Landschaftselementen liegen zwischen 50 und $250 \mathrm{~m}$ (Kaule 1991; Bosch und Partner GmbH 2000; Nentwig 2000). Diese Forderung beeinflusst die Honorierungswürdigkeit vor allem von größeren Schlägen. Ein 20 ha großer Schlag hat in Form eines Quadrates mit einem Rand-zu-Rand-Abstand von $447 \mathrm{~m}$ und trägt damit über seine Schlagform nicht positiv zum ökologischen Wert der Landschaft bei. Geht man von einem wünschenswerten Mindestabstand von z. B. $100 \mathrm{~m}$ zwischen 2 gegenüberliegenden Schlaggrenzen aus, so kann für die verschiedenen Schlaggrößen (unter der vereinfachenden Annahme, dass jeder Schlag

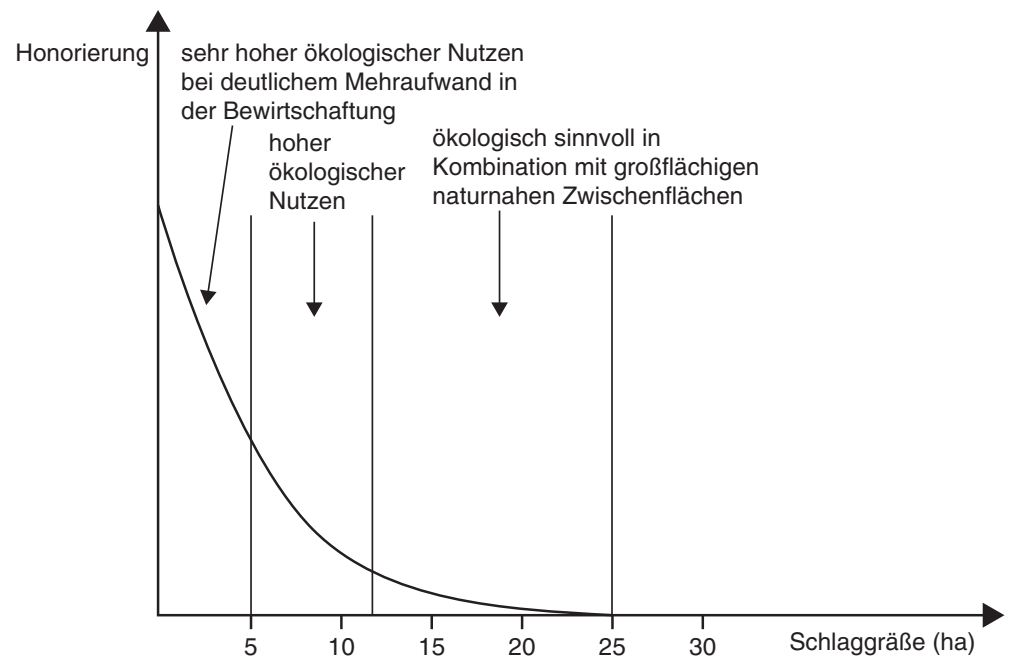

Abb. 7.2 Honorierung von Landschaftsvielfalt in Abhängigkeit von der Schlaggröße. (Quelle: Eigene Darstellung nach Engelhardt 2004) 
als Rechteck betrachtet wird) der minimale Umfang berechnet werden, bei dem der wünschenswerte Mindestabstand zwischen 2 gegenüberliegenden Schlaggrenzen noch eingehalten wird. Nur Schläge mit diesem oder einem höheren Schlagumfang sind demnach für ihre geringe Schlaggröße honorierungswürdig (Abb. 7.3). Bei Schlägen unter 1 ha ist diese Bedingung für alle Schlagformen erfüllt. Schläge mit einer Dreiecksform oder andere Vielecke werden bei dieser Methode geringfügig benachteiligt, da bei diesen Schlagformen in Teilen der Schläge die Bedingungen des Mindestabstandes erfüllt sind, ohne dass dafür automatisch eine Honorierung erfolgt.

Einschränkung durch gleiche Kulturen auf angrenzenden Schlägen: Bei angrenzenden Schlägen mit gleicher Kultur kann eine geringe Schlaggröße keine positive Wertung erhalten, da keine Grenzlinie mit Saumbiotopen zwischen den beiden Schlägen zu erwarten ist. In einem kooperativen Ansatz können Landwirte mit ihren Bewirtschaftungsnachbarn Absprachen in der Anbauplanung treffen. Dies ist eine wichtige Voraussetzung, um langfristig eine vielfältige Fruchtfolge in der Agrarlandschaft zu sichern. Sind keine Absprachen möglich, bietet sich hier die Möglichkeit zur gezielten Anlage von Randstreifen. Unabhängig von der Schlaggröße sind daher nur Schläge honorierungswürdig, die keine (nur in geringem Umfang) angrenzenden Schläge mit identischer Ackerkultur aufweisen.

Grenzlinien zu Landschaftselementen: Landschaftselemente spielen als potenziell hochwertige Habitatinseln bzw. als Trittsteinhabitate eine entscheidende Rolle beim Artenschutz in der Agrarlandschaft (Jedicke 1994; Haber 1998). Die Bewirtschaftung entlang der Grenzlinien zu Landschaftselementen sind aus landwirtschaftlicher Sicht teilweise mit einem deutlichen Mehraufwand verbunden (u. a. kein Befahren möglich, schlechte Wendemöglichkeiten). Neben den an die Schläge angrenzenden Landschaftselementen fallen dabei auch die in den Schlägen

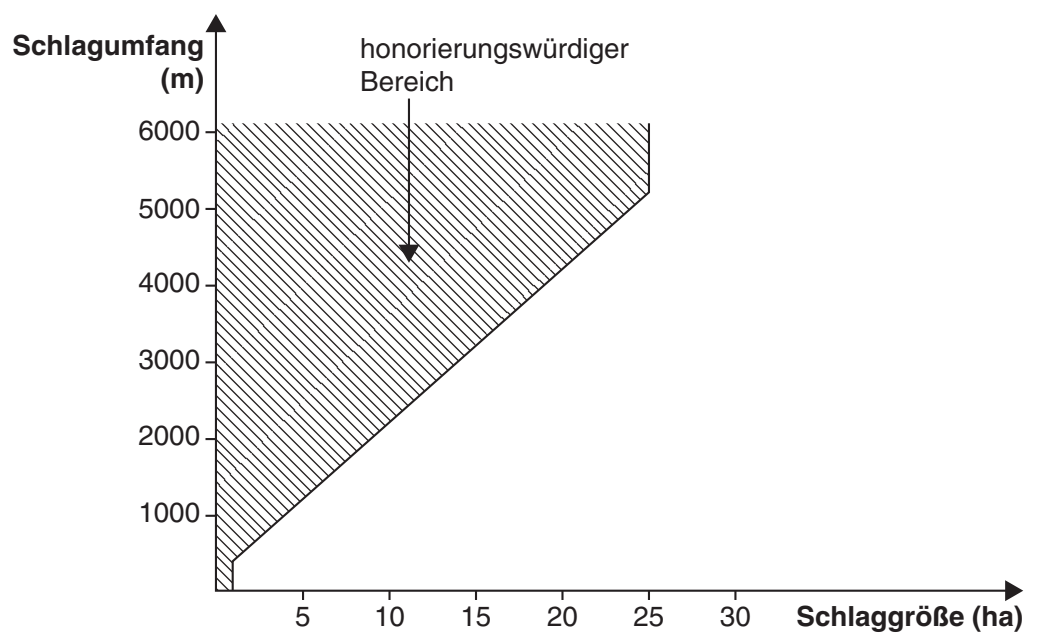

Abb. 7.3 Beispiel für honorierungswürdige Schlagformen, dargestellt am Schlagumfang in Abhängigkeit von der Schlaggröße. (Quelle: Eigene Darstellung) 
enthaltenen Landschaftselemente ins Gewicht, denen eine besondere Bedeutung als Trittsteinbiotope, besonders in größeren Schlägen zukommt (Bosch und Partner GmbH 2000). Abb. 7.4 skizziert eine mögliche Honorierung der Bewirtschaftung entlang von Grenzlinien zu Landschaftselementen.

Größe und Wertigkeit der Landschaftselemente: Je nach Größe und Wertigkeit der Landschaftselemente steigt auch ihr ökologischer Nutzen. Der Anreiz zum Erhalt besonders hochwertiger Landschaftselemente sollte daher auch höher angesetzt sein als bei weniger wertigen Landschaftselementen. Der High-Nature-Value (HNV) Indikator zur Bewertung der Umweltsituation in der Agrarlandschaft bietet ein System, Landschaftselemente in drei Wertigkeitsstufen einzuordnen (Bundesamt für Naturschutz 2016a). Diese Wertigkeit kann in die Honorierung der Bewirtschaftung entlang von Grenzlinien zu Landschaftselementen über die Einführung eines Faktors $\mathrm{X}$ berücksichtigt werden, z. B.:

- Honorierung $=$ Grenzlinienbezogene Honorierung $* \mathrm{X}$

\subsubsection{Exkurs: Praktikabilität einer GIS-gestïtzten Honorierung von Landschaftsvielfalt}

Die Vielgestaltigkeit der Landschaft und damit die entsprechende Prämie könnten anhand von GIS-Daten berechnet werden. Eine solche Berechnung wurde im Rahmen eines Werkvertrags von Herrn Dr. Machl, Weihenstephan, beispielhaft für drei bayerische Regionen durchgeführt. Die Regionen sind folgendermaßen charakterisiert:

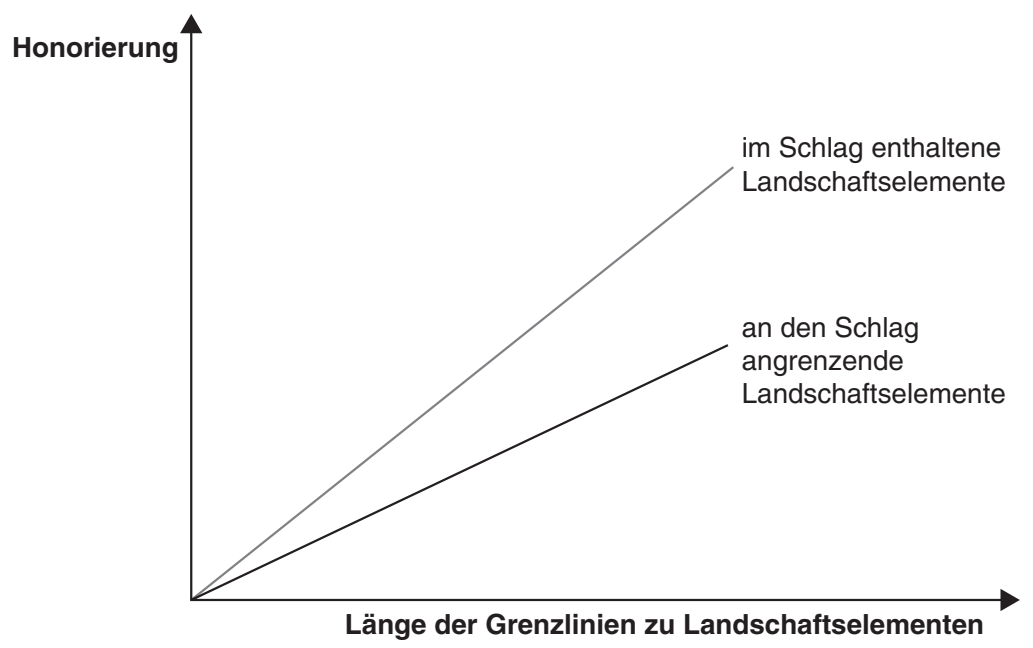

Abb. 7.4 Honorierung der Landschaftsvielfalt in Abhängigkeit von der Länge der Grenzlinien eines Schlages zu Landschaftselementen. (Quelle: Eigene Darstellung) 
- Testregion 1: Kleinstrukturierte Region mit zahlreichen Landschaftselementen und umgebenden Wäldern;

- Testregion 2: Region mit großen landwirtschaftlichen Parzellen und wenigen Landschaftselementen und Wäldern;

- Testregion 3: Strukturreiche Grünlandregion mit eher unförmigen Parzellen.

Für die Analyse wurde der Datensatz „Tatsächliche Nutzung“verwendet, der detaillierte Angaben zu verschiedenen Nutzungen der Erdoberfläche (Hecken, Gewässer, Wälder, Landwirtschaft etc.) enthält. Für die Berechnung der Kennzahlen wurde folgendermaßen vorgegangen: Zunächst wurden den Flächen der Klasse „Landwirtschaft" die angrenzenden Nachbarn zugeordnet. Als angrenzend galten dabei sowohl die unmittelbar benachbarten Flächen als auch Flächen in einer Distanz von bis zu $3 \mathrm{~m}$. Ausgehend von der Grenzlinie des betreffenden Polygons wurde dann ein $20 \mathrm{~m}$ Puffer nach innen erzeugt und die dadurch entstandenen Polygone nach Typ der angrenzenden Fläche aggregiert. Als mögliche Säume für Saumflächen kamen dabei folgende Typen in Frage: „Stehendes Gewässer“, „Fließgewässer“, „Wald“, „Gehölz“, „Moor“ und „Unland bzw. vegetationslose Fläche“. „Unland bzw. vegetationslose Fläche“" gilt dabei erst ab einer Mindestbreite von $3 \mathrm{~m}$ als „wirklicher“ Nachbar bzw. als „Kandidat“ für ein Saumbiotop. Für jede einzelne Parzelle wurde die Summe der potenziellen Saumbiotopfläche sowohl in absoluter wie auch in relativer Fläche (bezogen auf die Gesamtfläche) angegeben. Die Summe über die verschiedenen Kategorien konnte dabei die Gesamtfläche einer Parzelle übersteigen. Das konnte z. B. dann der Fall sein, wenn eine sehr kleine landwirtschaftliche Parzelle von einem Entwässerungsgraben umgeben ist und sich zwischen der Parzelle und dem Graben ein Gehölzstreifen befindet. In diesem Fall ist die betreffende Parzelle einerseits sowohl vollständig ein Saumbiotop zu Gewässer als auch ein Saumbiotop zu Gehölz. Die folgenden Abbildungen zeigen den Anteil der Saumbiotope in den Testregionen (Abb. 7.5, 7.6 und 7.7).

Die drei Abbildungen zeigen jeweils einen gleich großen Ausschnitt der Agrarlandschaft. Die strukturellen Unterschiede lassen sich in den Abbildungen deutlich erkennen.

Der genutzte Datensatz „Tatsächliche Nutzung“ erlaubt jedoch nicht (immer), einzelne Feldstücke oder Schläge zu identifizieren, da angrenzende Flächen gleicher Nutzung zu einem Polygon zusammengefasst werden. Es liegen allerdings bereits seitens der Mitgliedstaaten der EU bzw. auf Ebene der Bundesländer schärfere Flächeninformationen vor. Auch sind im genutzten Datensatz die Strukturelemente nicht vollständig erfasst. Dies ist besonders deutlich in der Abb. 8.1 zu Testgebiet 3 ersichtlich. Auch hier liegen den Behörden jedoch wahrscheinlich deutlich bessere Daten bereits vor. Nicht berücksichtigt wurden in der beispielhaften Berechnung der Daten für die drei Testgebiete jene Landschaftselemente, die innerhalb der bewirtschafteten Flächen liegen.

Anhand der dargestellten Beispiele der Testregionen zeigt sich, dass bereits Daten vorliegen, mit denen der Strukturreichtum von Regionen und die Zuordnung zu einzelnen landwirtschaftlichen Parzellen erfolgen kann. Mit einer zu entwickelnden Formel könnten die genannten Strukturen und beeinflussten landwirtschaftlich 


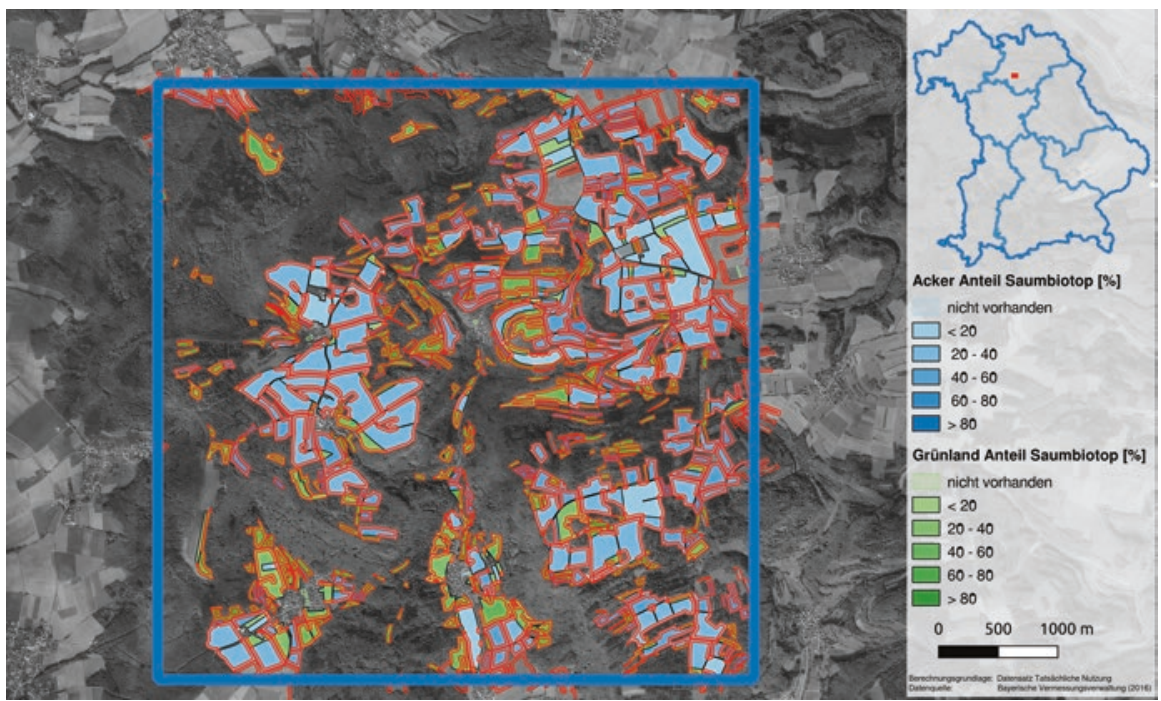

Abb. 7.5 Anteil Saumbiotope, Testregion 1. (Quelle: Orthophoto: geoservices.bayern.de; Lizenz: creativecommons.org/licenses/by/3.0/deed.de), (Analyse und Visualisierung: Thomas Machl (vgl. Machl 2016))

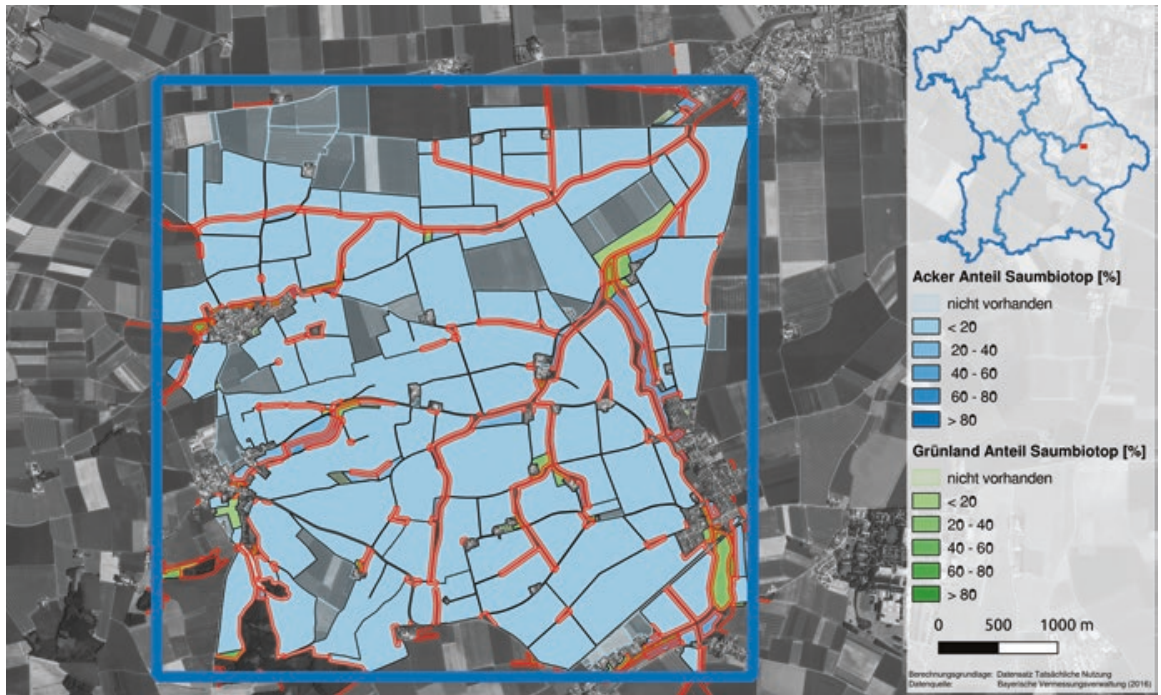

Abb. 7.6 Anteil Saumbiotope, Testregion 2. (Quelle: Orthophoto: geoservices.bayern.de; Lizenz: creativecommons.org/licenses/by/3.0/deed.de), (Analyse und Visualisierung: Thomas Machl (vgl. Machl 2016)) 


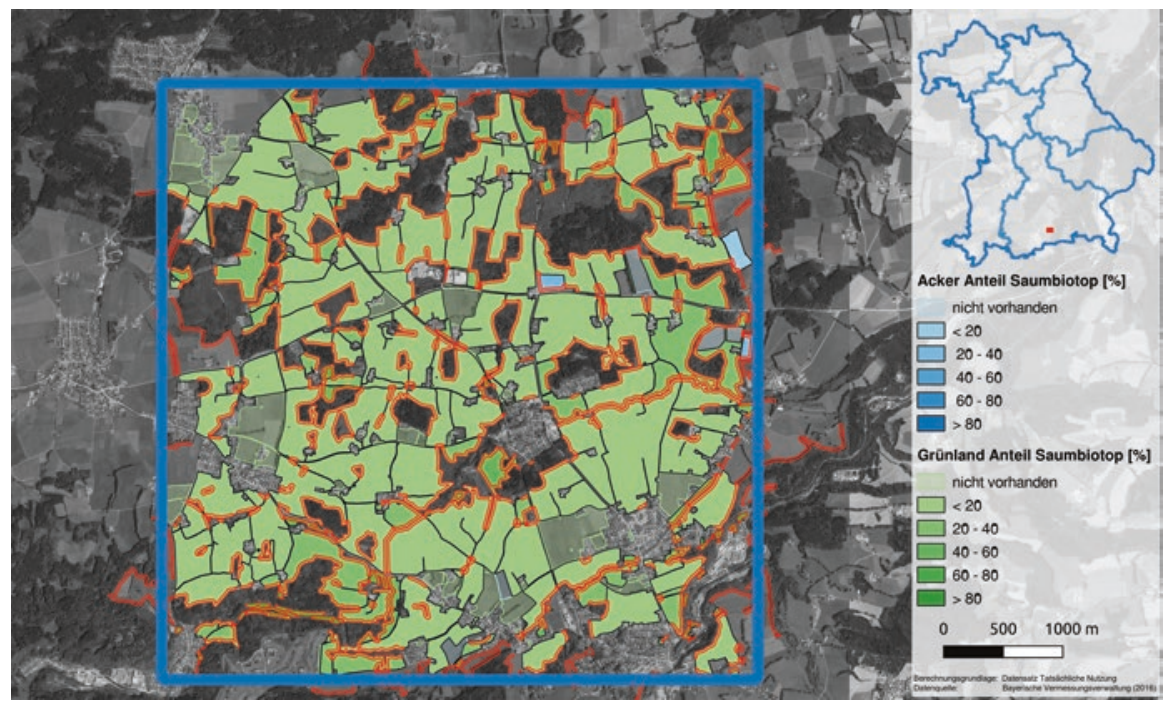

Abb. 7.7 Anteil Saumbiotope, Testregion 3. (Quelle: Orthophoto: geoservices.bayern.de; Lizenz: creativecommons.org/licenses/by/3.0/deed.de), (Analyse und Visualisierung: Thomas Machl (vgl. Machl 2016))

genutzten Flächen berücksichtigt werden und eine individuelle flächenbezogene Berechnung der Prämienhöhe erfolgen. Die Zuweisung der Prämien erfolgt automatisch mit Antragsstellung für die entsprechenden Flächen. Für Landschaftselemente in der Agrarlandschaft besteht ein Beseitigungsverbot (siehe Element 1). Mit zunehmend präziseren Datensätzen werden zukünftig weitere Aspekte sowie auch die ökologische Wertigkeit der Landschaftsstrukturen honoriert werden können. Mit präziseren Datensätzen könnten zukünftig weitere Aspekte sowie auch die ökologische Wertigkeit von Landschaftsstrukturen bei der Berechnung der Prämienhöhe berücksichtigt werden. ${ }^{5}$

\subsubsection{Element 4: Honorierung besonderer Leistungen im landwirtschaftlichen Natur- und Umweltmanagement}

Als viertes Element einer neuen Prämienstruktur könnte ein Punkte- bzw. „Baukastensystem" zur ökologischen Leistungserbringung auf Ebene der landwirtschaftlichen Betriebe (oder Kooperationen) eingeführt werden. Dort könnten Maßnahmen

\footnotetext{
${ }^{5}$ „Nachvollziehbare und mit quantitativen Daten hinterlegte Erfassung von Grünlandlebensräumen als Grundlage für effektives Monitoring und angepasstes Management“, nicht nur zur Kontrolle, sondern auch für die Beratung und ergebnisorientierte Anpassung bisheriger Managementpraktiken (NABU 2012).
} 
honoriert werden, die in allen Regionen, auf jedem Standort und in jedem Betrieb positive Umwelt- und Naturschutzeffekte erwarten lassen. Die in Tab. 7.8 aufgeführten Maßnahmen lassen positive Effekte auf die Bereitstellung von Ökosystemleistungen und die Umweltressourcen unabhängig von der räumlichen Allokation erwarten und könnten daher flächendeckend gefördert werden. Ziele der aufgelisteten Maßnahmen sind dabei die Verminderung negativer Einträge aus der Landwirtschaft, eine erhöhte Diversität der Feldfrüchte, die Gewährleistung einer natürlichen Nährstoffversorgung, der Schutz natürlicher Prozesse des Bodens sowie die Förderung und Extensivierung der Grünlandnutzung. Ansätze in dieser Richtung wurden auch vom DVL (Neumann 2016) und der Land Use Policy Group (Hart et al. 2016) vorgeschlagen.

Die Liste in Tab. 7.8 stellt eine Auswahl an möglichen Maßnahmen dar, erhebt jedoch keinen Anspruch auf Vollständigkeit. Ebenso können an dieser Stelle nicht die Zielwerte für die einzelnen Maßnahmen diskutiert werden. Innerhalb des Ansatzes besteht die Möglichkeit, verschiedene Untergruppen an Maßnahmen zu bilden, z. B. entsprechend der verschiedenen Schutzgüter.

Basierend auf einer solchen Liste könnten die Landwirtinnen und Landwirte selbst aus dem „Baukasten“ auswählen, welche Maßnahmen sie erbringen wollen. Es werden demnach nicht alle Maßnahmen von allen Landwirten in allen Regionen erwartet, sondern eine sinnvolle Auswahl. Die Leistungen (Maßnahmen auf Betriebsebene) werden in eine Punktzahl umgerechnet, die Honorierung ergibt sich aus der Formel Punkte*Punktwert (€)*ha. Ähnliche Ansätze werden beispielsweise bereits in der Schweiz (Birrer et al. 2014, 2015) und in Niederösterreich (Ökopunktemodell: Umsetzung der Zweiten Säule in Niederösterreich) umgesetzt und innerhalb von Pilotprojekten in Nord- und Ost-Deutschland erprobt (Neumann und Dierking 2014; Gottwald und Stein-Bachinger 2015). Während im Ökopunktemodell Niederösterreichs eine Mindestpunktzahl zu erreichen ist, sind im Schweizer Ansatz Maßnahmen aus verschiedenen Untergruppen zu wählen, so dass ein Nutzen für verschiedene Naturgüter erbracht wird. Um eine Entlastung beim Verwaltungsaufwand zu erreichen, könnte das System anstelle einer jährlichen eine mehrjährige Antragstellung vorsehen. Des Weiteren sollten die Maßnahmen in der Liste eine relativ einfache Dokumentation und Kontrolle ermöglichen. Das bedeutet, dass sie im Allgemeinen anhand von GIS-gestützten Fernerkundungsdaten, der Nutzung der InVeKoS- und HIT-Datenbank sowie amtlichen Dokumente und Zertifikaten nachgewiesen werden können.

\subsubsection{Diskussion}

Die folgende Tab. 7.9 gibt einen Überblick über das hier skizzierte mögliche neue Prämiensystem.

Systematik und Verhältnis zur Zwei-Säulen-Struktur: Das hier dargestellte System folgt dem Grundsatz „Öffentliches Geld für öffentliche Güter“. Alle Zahlungen 
Tab. 7.8 „Baukasten“ zur Honorierung von Basisleistungen im landwirtschaftlichen Natur- und Umweltmanagement

\section{Maßnahmen auf Schlagebene:}

- Zwischenfruchtanbau;

- Gemenge/Polykulturen/Mischkulturen;

- Verzicht auf chemisch-synthetischen Dünger, Pflanzenschutzmittel bzw. Wachstumsregulatoren;

- Verzicht oder Einschränkung von Managementmaßnahmen (Striegeln, Pflügen, Walzen, Schleppen etc.);

- Anbau alter Nutzpflanzenarten;

- Brache (auf Acker mit Selbstbegrünung).

Maßnahmen auf Betriebsebene:

- Fruchtfolge ab 4 Fruchtfolgeglieder;

- Bereitstellung wertvoller ökologischer Vorrangfläche;

- GV-Besatz (<2,0 GV/ha) (Kooperationen auf Regionsebene evtl. ermöglichen)a , bei einem Mindestviehbesatz von $0,3 \mathrm{GV} / \mathrm{ha}$;

- Einsatz von Festmist, Kompost und Gründüngung Dauergrünland/Ackergras/Kleegras pro Raufutter-GV (Ziel: hoher Grundfutteranteil) ${ }^{\mathrm{b}}$;

- Umsetzung von Tierschutzmaßnahmen (z. B. Tierbesatz/Stallfläche);

- Erhaltung der genetischen Vielfalt (z. B. seltene Nutztierrassen);

- Amtl. Nachweise der Teilnahme an hochwertigen Fortbildungsmaßnahmen (z. B. Tierschutz, Biodiversitätsschutz);

- Amtl. Nachweis erhöhter Stickstoffnutzungseffizienz (Ertrag/Düngung);

- Amtl. Nachweis eines geringen Arzneimitteleinsatz;

- Amtl. Nachweis reduzierter Ammoniak-Emissionen (z. B. entsprechende Stall- und Ausbringtechnik);

- Amtl. Nachweis entsprechender Betreuungs-Indices pro Tier (und Fläche);

- Whole-Farm-Ansätze (z. B. ökologischer Landbau), Nachweis von Zertifikaten (z. B. nachhaltige Landwirtschaft (Bereich Ökologie) der DLG usw.).

${ }^{a}$ Die Flächenintensität der Tierhaltung sollte bereits innerhalb des Greenings berücksichtigt werden (Wissenschaftlicher Beirat für Biodiversität und Genetische Ressourcen beim BMELV 2011) ${ }^{\mathrm{b}}$ Graslandbasierte Milch- und Fleischproduktion ist in der Schweiz als Maßnahme definiert

sind nachweisbar an die Erbringung von öffentlichen Leistungen geknüpft. Ein solches System kombiniert Elemente aus dem Ordnungsrecht (Element 1a), der Ersten Säule (Element 1b) sowie Instrumente, die dem ähneln, was derzeit in der Zweiten Säule angeboten wird (Elemente 2, 3 und 4). Der Ansatz löst sich daher von der derzeitigen Zwei-Säulen-Struktur. Die Verwirklichung eines solches Ansatzes wäre wahrscheinlich auch im Rahmen der Zwei-Säulen-Struktur möglich, würde dann aber vermutlich eine bessere Ausstattung der Zweiten Säule erfordern (siehe dazu auch Abschn. 7.2 und 7.4).

Programmierung: Bei der Frage einer EU-weiten oder nationalen Programmierung wäre eine durchgehende EU-weite Programmierung aller vier Elemente vorstellbar. Dies ist jedoch nicht zwingend und unterscheidet sich zwischen den Elementen:

- Element 1 betrifft den Rechtsrahmen des Binnenmarkts sowie eine Basiskompensation. Die Ausgestaltung sollte daher EU-einheitlich geregelt sein.

- Bei Element 2 ist es sinnvoll, die Bestimmung der Gebietskulissen, der prämienberechtigten Schläge und der notwendigen Prämienhöhe den Mitgliedstaaten 


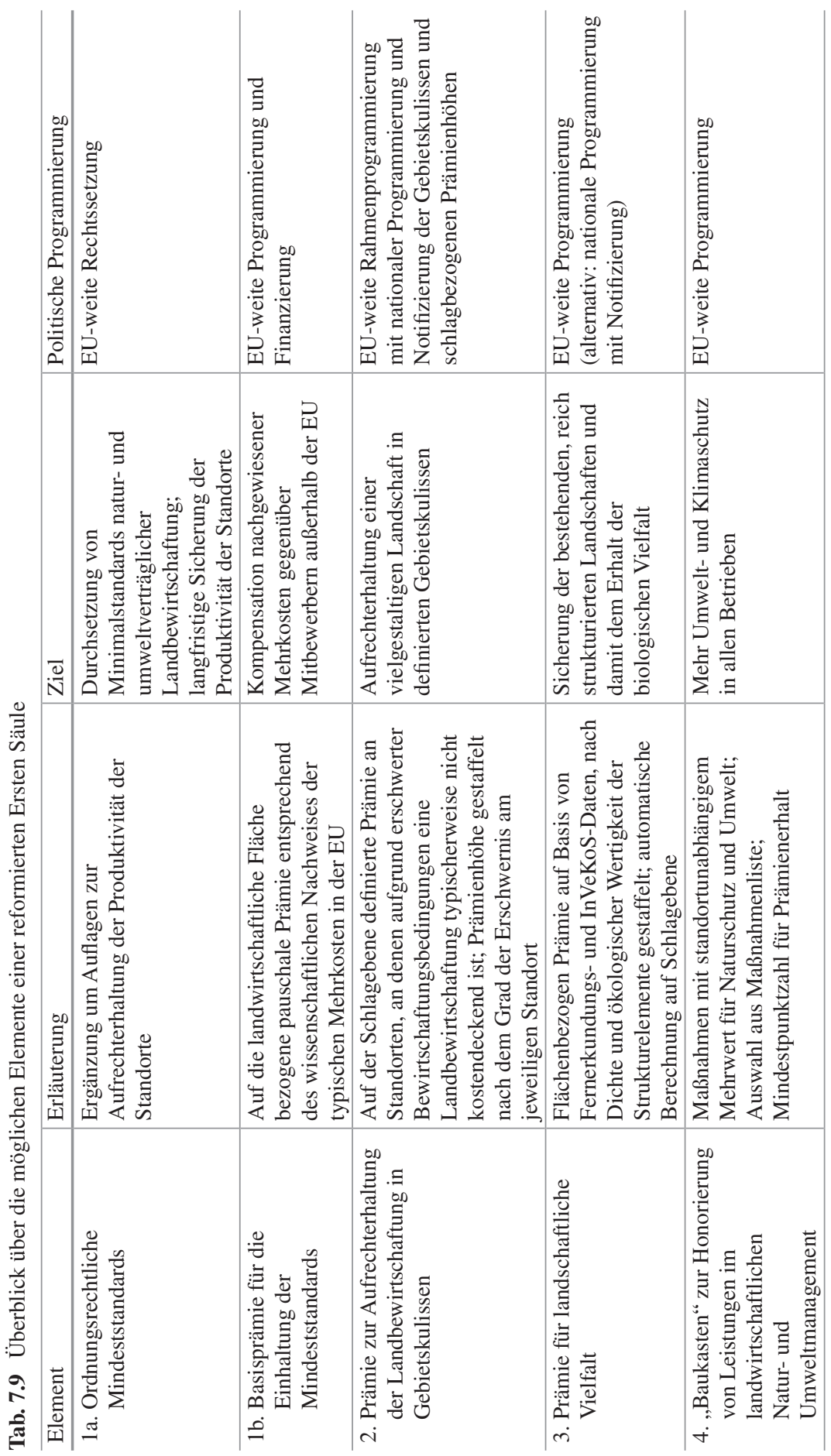


(bzw. in Deutschland den Bundesländern) im Rahmen klarer Rahmenvorgaben zu überlassen. Die Programmierung sollte dann bei der EU-Kommission notifiziert und von dieser genehmigt werden.

- Bei Element 3 könnte die Programmierung sowohl auf der EU- wie der nationalen Ebene erfolgen. Die wesentlichen Variablen sind dabei die Bestimmung des Bewertungsalgorithmus und des monetären Multiplikators. Letzterer könnte variabel gestaltet werden, so dass ein vorgegebenes Budget eingehalten wird. Bei Programmierung auf nationaler Ebene wäre wiederum ein Notifizierungsverfahren bei der EU-Kommission vorzusehen. Eine nationale Programmierung wäre möglicherweise weniger komplex, würde aber nicht die Notwendigkeit einer wissenschaftlich basierten Bestimmung der EU-weiten Rahmenregelung aufheben.

- Bei Element 4 sieht das Modell eine EU-weite Programmierung vor. Es handelt sich hier in gewisser Weise um ein weiterentwickeltes Greening.

Finanzierung: Die Finanzierung der Elemente 1 und 4 wäre im Anschluss an die bisherige Finanzierungssystematik in der GAP zu $100 \%$ bei der EU anzusiedeln (Kompensation für EU-weite gehobene Standards und weiterentwickeltes Greening). Bei den Elementen 2 und 3 wäre zu argumentieren, in welchem Verhältnis hier der europäische und der nationale Nutzen stehen. In der verteilungspolitischen Diskussion zwischen den Mitgliedstaaten würde sicherlich eine Rolle spielen, dass der Anteil der Flächen, die unter die Anforderungen des Elements 2 (Prämie zur Aufrechterhaltung der Landbewirtschaftung in Gebietskulissen) fallen, zwischen den Mitgliedstaaten stark variieren dürfte. In ähnlicher Weise werden sich die Anteile der landschaftlichen Flächen, die eher hohe oder eher niedrige Zahlungen aus einer Prämie für landschaftliche Vielfalt (Element 3) erwarten könnten, zwischen den Mitgliedstaaten unterscheiden. Ob die mit den Elementen 2 und 3 gewollte Umverteilung hin zu Flächen mit relativ hohem Wert für die landschaftliche Vielfalt eher auf europäischer oder nationaler Ebene organisiert werden sollte, würde einer weiteren Diskussion bedürfen. Generell hätte eine Vollfinanzierung aus EU-Mitteln den Vorteil, dass Natur- und Umweltschutz nicht „,nach Kassenlage“ der einzelnen Mitgliedstaaten und Regionen erfolgen würde.

Mögliche Ergänzungen: Das in diesem Abschnitt vorgestellte System würde eine betriebsgrößenabhängige Förderungskomponente zunächst nicht vorsehen. Eine höhere Förderung kleiner Betriebe könnte jedoch aufgrund der geringeren Möglichkeit, Skaleneffekte zu realisieren, gerechtfertigt sein. So wird in ,der EU-VO 1307/2013 [...] die größenabhängige Ausgestaltung der Direktzahlungen mit Effektivitäts- und Verteilungsaspekten vor dem Hintergrund des Ziels der Einkommensstützung begründet" (Isermeyer et al. 2014). In dem hier vorgeschlagenen System könnte eine strukturpolitische Komponente - ähnlich wie in der derzeitigen Basisprämie - mit dem Element 1 verknüpft werden. Allerdings ist dabei zu bedenken, dass ein kleiner Betrieb nicht notwendig mit einer einkommenspolitischen Bedürftigkeit der Betriebsinhaber einhergeht. Auf die begrenzte Eignung sektorspezifischer Instrumente, wie den Direktzahlungen als einkommenspolitisches Instrument, ist an anderer Stelle hingewiesen worden (Wissenschaftlicher 
Beirat für Agrarpolitik beim BMELV 2010) mit der Schlussfolgerung, dass, um abrupte Übergänge zu vermeiden, Maßnahmen zur Einkommenssicherung ,nur noch in Form zeitlich begrenzter, sozial begründeter Maßnahmen Bestandteil einer zukünftigen Agrarpolitik sein“"sollten (Bosshard et al. 2010). Alternative Ansätze zur Verfolgung einkommenspolitischer Ziele im Agrarbereich werden diskutiert. Anders als die Direktzahlungen setzen etwa Überlegungen zu einem „Sicherheitsnetz“ am Problem der Preisschwankungen an. Dieses Konzept findet in naturschutz- und umweltpolitischen Konzeptionen durchaus Widerhall (Hart et al. 2016). Ähnliche Überlegungen zu Preis-Ausgleichszahlungen wurden vom Kasseler Institut für ländliche Entwicklung e.V. vorgelegt (Thomas et al. 2016). Eine offene Frage ist dabei, ob nicht im Endeffekt wieder ein Mindestpreis für Erzeuger eingeführt würde, der produktionsstimulierend wirken und damit auch negative Auswirkungen auf die Ziele des Natur- und Umweltschutzes haben könnte.

Das in diesem Abschnitt vorgestellte System dient dem Zweck, ein EU-weites System von Mindeststandards und Mindestleistungen für den Natur-, Umwelt- und Verbraucherschutz sowie das Tierwohl zu etablieren und den landwirtschaftlichen Betrieben die daraus entstehenden Mehrkosten zu kompensieren. Die Verwirklichung eines solchen Ansatzes würde dem Prinzip einer ergebnisorientierten Politik entsprechen. De facto würden durch die Einführung eines solchen Systems die Gemeinwohlleistungen der Landwirtschaft zu einer neuen Einkommensquelle neben der Produktion führen. Die Höhe der entsprechenden Zahlungen sollte dabei auf transparenten Berechnungen beruhen und einen effizienten Mitteleinsatz unter Vermeidung von systematischen Überkompensationen und Mitnahmeeffekten ermöglichen.

Ein solches EU-weites System würde weiterhin der Flankierung durch regionale und standörtliche Programme zum Agrarumwelt- und Klimaschutz bedürfen. Dieses diskutieren wir im nächsten Abschnitt.

\subsection{Baustein 4: Regional und standörtlich ausgerichtete AUKM}

\subsubsection{Ziele von Agrarumwelt- und Klimaschutzmaßnahmen}

Dieser Abschnitt befasst sich mit den leistungsbezogenen Zahlungen der GAP im Agrarumwelt- und Klimaschutz, die derzeit im Bereich der Zweiten Säule angesiedelt sind. Gemäß dem Leitsatz ,öffentliches Geld für öffentliche Güter“ sind solche Zahlungen spätestens seit 1992 Teil der GAP und richten sich vor allem an einzelne Landwirte, die auf vertraglicher Basis und gegen Entgelt ökologische Leistungen auf ihren bewirtschafteten Flächen erbringen.

Die Agrarumwelt- und Klimaschutzmaßnahmen (AUKM) der GAP sind derzeit in die Programme zur Entwicklung des ländlichen Raums integriert, in denen mindestens 
$30 \%$ der Finanzmittel für umwelt- und klimabezogene Maßnahmen ${ }^{6}$ angesetzt werden müssen. Derzeit sehen die auf der Ebene der Mitgliedstaaten oder auf regionaler Ebene (in Deutschland: Bundesland) aufgestellten Maßnahmenkataloge sehr divers aus, sodass eine große Anzahl verschiedener Ziele verfolgt wird:?

- Erhaltung oder Verbesserung der Umweltsituation;

- Klimaschutz durch Reduzierung der Treibhausgas-Emissionen;

- Erhalt und Steigerung der biologischen Vielfalt;

- Pflege und Erhalt der Kulturlandschaft;

- Schutz der natürlichen Ressourcen, der Gewässer und des Bodens;

- Erhalt und Steigerung der genetischen Diversität.

\subsubsection{Bewertung der gegenwärtigen Ausgestaltung der Agrarumwelt- und Klimaschutzmaßnahmen}

Empirische Studien zur Wirksamkeit der AUKM in der EU können trotz des relativ hohen Finanzeinsatzes für diesen Politikbereich kein eindeutiges Bild liefern. Bezüglich der ökologischen Effektivität wurde eine Debatte im Jahre 2001 mit einem Artikel in Nature ausgelöste (Kleijn et al. 2001). Dieser bescheinigte durch eine quasi-experimentelle Analyse, dass die untersuchten AUKM nicht in der Lage waren, die Biodiversität in der beabsichtigten Art und Weise zu schützen. Seither hat sich eine Vielzahl weiterer Studien mit diesem Sachverhalt beschäftigt und kommt zu gemischten Ergebnissen: Einige Studien können einen positiven Effekt feststellen (Kleijn und Sutherland 2003; Knop et al. 2006; Batáry et al. 2011, 2015), andere nicht unmittelbar (Berendse et al. 2004; Kleijn et al. 2006; Wilson et al. 2007). Uthes und Matzdorf (2013) listen mehrere Beispiele von Programmen auf, die entgegengesetzte Effekte produziert haben. Es zeichnet sich ab, dass die Ergebnisse stark variieren, je nachdem welche und wie viele Arten über welchen Zeitraum und in welcher geografischen Ausdehnung bzw. in welchem Landschaftskontext untersucht werden (Whittingham 2007; Armsworth et al. 2012). Auch die Frage, ob AUKM in intensiven oder extensiven Regionen einen größeren Erfolg versprechen, konnte noch nicht abschließend geklärt werden (Duelli und Obrist 2003; Tscharntke et al. 2005; Batáry et al. 2011). Eine Evaluierung der ökonomischen Effizienz gestaltet sich noch schwieriger, da die tatsächlichen Kosten der Landwirtschaft für den Staat

\footnotetext{
${ }^{6}$ Umwelt- und klimabezogene Maßnahmen umfassen die folgenden Artikel der ELER-Verordnung: Art. 17 Umwelt- und klimaschutzbezogene Investitionen; Art. 21 Aufforstung und Agroforstsysteme; Art. 28 Agrarumwelt- und Klimamaßnahmen; Art. 29 Ökologischer Landbau; Art. 30 Zahlungen für Natura 2000 und WRRL; Art. 31/32 Zahlungen für benachteiligte Gebiete; Art. 34 Waldumwelt- und -klimamaßnahmen.

${ }^{7}$ Verordnung (EU) Nr. 1305/2013 des Europäischen Parlaments und des Rates vom 17. Dezember 2013 über die Förderung der ländlichen Entwicklung durch den Europäischen Landwirtschaftsfonds für die Entwicklung des ländlichen Raums (ELER) und zur Aufhebung der Verordnung (EG) Nr. 1698/2005, AB1. L 347 vom 20.12.2013, S. 487-548.
} 
nicht direkt messbar sind. Umfassende empirische Studien lassen sich daher nicht finden. Marggraf (2003) attestiert den deutschen Agrarumweltprogrammen in einer Expertenanalyse große Unterschiede in ihrer Kosteneffizienz im Vergleich zwischen den Ländern. Andere, vor allem modellgestützte Evaluationen finden hohe Mitnahmeeffekte und eine geringe Kosteneffizienz (Drechsler et al. 2007; Chabé-Ferret und Subervie 2010).

Als Ursache werden in der Literatur vier Problemkomplexe ausgemacht, die im Folgenden kurz skizziert werden:

Problemkomplex 1: Vielen Maßnahmen fehlt ein Bezug zu den eigentlichen Umweltzielen, so dass die angestrebten Ziele nicht oder nur unzureichend erreicht werden. Es können hier verschiedene Ursachen ausgemacht werden:

- Maßnahmenportfolios werden nicht evidenzbasiert programmiert und es bestehen kaum Möglichkeiten zur institutionalisierten Evaluierung der Programme (Primdahl et al. 2003; Whitfield 2006);

- Mangelnde Berücksichtigung der regionalen Charakteristika und von Effekten der Intensivierung und Extensivierung der Landnutzung;

- Wenig gezielte Wahl und Allokation von Einzelmaßnahmen zur Nutzung von Synergieeffekten (z. B. Schaffung von Nahrungs- und Nisthabitaten mit gleichzeitiger Vernetzungs- und Erosionsschutzfunktion);

- Mangel an räumlicher und zeitlicher Passgenauigkeit;

- Vage Zielsetzungen der maßnahmenorientierten AUKM (Uthes und Matzdorf 2013) und wenig Anreiz, landwirtschaftliche Praktiken langfristig zu ändern (Matzdorf und Lorenz 2010).

- Die angebotenen Maßnahmen sind starr und offerieren den Landwirten eine zu geringe Flexibilität (Mettepenningen et al. 2009). Es wird kein Anreiz zur Entfaltung von Innovationspotenzial gegeben (Hodge 2001).

Problemkomplex 2: Nahezu allen AUKM-Programmen fehlt ein Bezug zu unterschiedlichen räumlichen Skalen (sieht auch Abschn. 4.3). Es wird überwiegend die Feldebene angesprochen, selten die Betriebsebene und niemals die Landschaftsebene (Concepción et al. 2008).

Problemkomplex 3: Das angewandte Zahlungsschema wird weder der Heterogenität der Landnutzer noch den Eigenarten der jeweiligen Landschaften gerecht (Hanley et al. 2012). Der Kostenersatz beruht auf regionalen Durchschnittswerten und führt zu Unter- und Überkompensation sowie Mitnahmeeffekten (Armsworth et al. 2012). Dies hat zur Folge, dass Intensivregionen nicht hinreichend erreicht werden (Pretty et al. 2001; Uthes und Matzdorf 2013). Ferner werden Transaktionskosten bei der Berechnung der Zahlungshöhe nur unzureichend mit einbezogen (Mettepenningen et al. 2009). Auch werden Verträge teilweise nach dem Prinzip ,wer zuerst kommt, mahlt zuerst“" vergeben (Smits et al. 2008). Bei knappen Budgets führt dies zu Einbußen bei der Kosteneffizienz, da die Verträge nicht auf Basis einer Kosten-Nutzen-Berechnung vergeben werden (Armsworth et al. 2012).

Problemkomplex 4: Maßnahmenorientierte Honorierung und geringe MonitoringHäufigkeiten kreieren einen Anreiz zur Nicht-Befolgung von Managementvorgaben (Hodge 2001; Hanley et al. 2012). Häufig werden nur sehr geringe Strafen bei Missachtung der Vorgaben angesetzt. 


\subsubsection{Ziele und Struktur zukünftiger regionaler und standörtlicher AUKM}

Der im vorigen Abschnitt vorgestellte Vorschlag eines EU-weiten Systems von Zahlungen für erhöhte Mindeststandards, vielfältige Landschaften und einem „Baukasten“ von Basismaßnahmen für den Umweltschutz würde EU-weit eine Grundlage für die Sicherung der von der Landwirtschaft betroffenen Natur- und Umweltgüter etablieren. Darauf aufbauend werden Programme für gezielte Maßnahmen des regionsund standortspezifischen Natur- und Umweltschutzes benötigt. Um die Effektivität der Maßnahmen bezüglich ihrer Wirkungen auf die Umweltressourcen zu steigern, wird in der Literatur ein Management auf unterschiedlichen räumlichen und zeitlichen Skalen gefordert (van Dijk et al. 2015; Tscharntke et al. 2012; Prager 2015). Entscheidend dabei ist die Unterscheidung von Maßnahmen, welche die Bereitstellung von Ökosystemleistungen fördern und meist auf lokaler Ebene anzusetzen sind, und Maßnahmen des Arten- und Biotopschutzes, die oft eher auf Landschaftsebene ansetzen sollten (Scheper et al. 2013; Ekroos et al. 2014). Darüber hinaus sollte gezielt versucht werden, die überbetriebliche Ebene und die Landschaftsebene zu adressieren. Zu diesem Zweck werden im Folgenden drei Elemente vorgestellt.

\subsubsection{Element 1: Betriebsübergreifende, langfristige Ansätze auf der Landschaftsebene zur Steigerung der Landschaftsvielfalt und zum Schutz der natürlichen Ressourcen}

Das erste Element dient dem Schutz und der Förderung der landschaftlichen Vielfalt und dem Schutz der natürlichen Ressourcen. Im Mittelpunkt steht die gezielte Ausdehnung und Zustandsverbesserung von Strukturen in der Landschaft mit hohem Naturwert und der damit verbundenen Reduktion der negativen Auswirkungen der Landwirtschaft auf die Umwelt. In Abgrenzung von der im Abschn. 7.3.5 vorgestellten Prämie für Landschaftsvielfalt geht es hier weniger um die Erhaltung und Belohnung als vielmehr um eine Erhöhung der strukturellen Landschafts- und Habitatvielfalt.

Im Fokus stehen dabei Flächen, die halbnatürliche Vegetation tragen oder extensiv genutzt werden und auf Landschaftsebene ein zusammenhängendes Mosaik von halbnatürlichen oder angepassten Flächen bzw. Kleinstrukturen bilden. Solche Strukturen dienen Tier- und Pflanzenarten als Lebensraum und Trittsteinbiotop und lassen einen positiven Einfluss auf die Schutzgüter erwarten (Batáry et al. 2011; Kremen und Miles 2012; Scheper et al. 2013). Zur gezielten Steigerung von Landschaftsheterogenität, -komplexität und -konnektivität unter Berücksichtigung der regionalen Besonderheiten und entsprechender Maßnahmenimplementierung ist ein betriebsübergreifendes Management auf der Landschaftsebene notwendig. Mögliche Maßnahmen sind hierbei die Förderung der Anlage von linearen Landschaftselementen in und angrenzend an landwirtschaftliche Flächen (Blüh-, Randund Pufferstreifen, z. B. entlang von Bächen, Hecken und Baumreihen). 
In Kopplung mit einer Prämie für Landschaftsvielfalt, wie sie im Abschn. 7.3.5 vorgeschlagen wurde, könnte die Sicherung von Strukturelementen in der Landwirtschaft durch eine langfristige Honorierung unterstützt werden. Darüber hinaus würde ein solcher Ansatz auch zur Erreichung des im Bundesnaturschutzgesetz festgeschriebenen Ziels eines Biotopverbundes von $10 \%$ der Fläche beitragen.

Die angebotenen Maßnahmen könnten von relativ einfachen, „hellgrünen“ Maßnahmen bis hin zu komplexeren und wesentlich gezielter programmierten „dunkelgrünen“ Maßnahmen reichen. Dabei wäre abzuwägen, ob eine regionale Zahlungsdifferenzierung angebracht ist, so dass auch Landwirte in Intensivgebieten einen Anreiz erhalten, in eine vielfältige Landschaftsstruktur zu investieren. Unter dem Gesichtspunkt der Anreizwirkung könnten sowohl maßnahmen- als auch ergebnisorientierte Honorierungsansätze geeignet sein. Zusätzlich könnte ein Agglomerationsbonus eingesetzt werden, um bei Abwesenheit lokaler Koordinationsstrukturen (siehe dazu Element 3 in diesem Abschnitt) einen Anreiz zur Koordination zu geben.

\subsubsection{Element 2: Zielgerichtete Maßnahmen des Arten- und Biotopschutzes}

Eine zweite Komponente könnte dazu dienen, zielgerichtete Maßnahmen des Artenund Biotopschutzes zu honorieren, die insbesondere der Umsetzung der europäischen und nationalen Ziele der Biodiversitätserhaltung dienen. Es handelt sich hier also hauptsächlich um ,dunkelgrüne“ AUKM (Maßnahmen zur gezielten Erhaltung und Förderung der biologischen Vielfalt), die lokal oder regional programmiert werden, da ihre Effektivität je nach Landschaftskontext räumlich und zeitlich sehr stark variieren kann. Mögliche Maßnahmen können sowohl auf der Feldebene als auch auf Landschaftsebene angesiedelt sein:

- Feldebene: Förderung von Nist-, Ruhe- und Nahrungshabitaten (z. B. Blühstreifen), gezielte Erhaltungsmaßnahmen wertvoller Flächen (z. B. extensiver Ackerbau, artenreiches Grünland, Streuobst);

- Landschaftsebene: Maßnahmen zur Förderung des Biotopverbunds für Zielarten auf Landschaftsebene entsprechend Biotopvernetzungsplan (z. B. angepasste Nutzung für Wiesenbrüter, Feldhamster etc.).

Dieses Element beinhaltet ein explizites Nutzen-Targeting, da die Maßnahmen nur dort angeboten werden, wo ein positiver Nutzen sehr wahrscheinlich ist. Da ,dunkelgrüne“ Maßnahmen häufig sehr arbeitsintensiv sind und die damit verbundenen Kosten je nach Standort stark variieren können, kann eine Zahlungsdifferenzierung angezeigt sein, um eine effektive Allokation der finanziellen Mittel zu gewährleisten. Dazu können entweder regional angepasste Zahlungshöhen oder Auktionen dienen. Weiterhin kann bei Vorhandensein geeigneter und praktikabler Indikatoren eine erfolgsorientierte Honorierung die ökologische Effektivität der Programme steigern. Um Risiken auf der Seite der Landwirte zu minimieren, kann 
eine maßnahmenbasierte Grundprämie mit einer mittelfristigen Erfolgshonorierung kombiniert werden. Wie auch beim vorherigen Element könnte auch hier eine regionale Kooperation durch einen Agglomerationsbonus stimuliert oder durch kooperative Ansätze organisiert werden.

\subsubsection{Element 3: Förderung von lokalen oder regionalen Organisationsstrukturen zur gezielten Programmierung und Koordination von AUKM}

Im Zuge der verstärkten Aufmerksamkeit für Ökosystemleistungen, für die Bedeutung der Landschaftsstrukturen für die biologische Vielfalt und für die im NexusAnsatz beschriebenen Wechselwirkungen zwischen den Sektoren Land- und Forstwirtschaft, Energie und Wasser kommt einem integrierten Ressourcen- und Landschaftsmanagement zunehmende Bedeutung zu. Daher sind Mittel erforderlich, um Kooperationen zwischen der Landwirtschaft einerseits und den Akteuren des Natur- und Umweltschutzes andererseits zu stimulieren.

Kooperative Ansätze für ein integriertes regionales Ressourcen- und Landschaftsmanagement könnten nicht nur beratend die Programmierung von AUKM unterstützen, sondern auch eine verbesserte Koordination von Maßnahmen zum Gewässerschutz, Biotopverbund, ,grüne“ und „,blaue“ Infrastruktur (also ein Netzwerk natürlicher und naturnaher Flächen) usw. auf der Landschaftsebene ermöglichen. In solche Kooperationen wären landwirtschaftliche und naturschutzfachliche Sichtweisen und Interessen gleichermaßen einzubinden.

Lokale oder regionale Kooperationen könnten einen integrierten Plan zur Verbesserung beim Landschafts- und Ressourcenmanagement entwickeln und implementieren, indem sie die Expertise (z. B. lokale Artenkenntnis) und das soziale Kapital (z. B. Netzwerk der Landwirte) unterschiedlicher Akteursgruppen (z. B. Landwirtinnen und Landwirte, Agrar- und Umweltämter, Landschaftspflegeverbände, Umweltverbände, Forstwirtschaft, Jagdverbände, Wasserverbände) bündeln. Neben der Vernetzung könnten auch die Bereitstellung und Fortschreibung der notwendigen ökologischen Daten gefördert werden.

Ein wichtiges Ziel solcher lokalen oder regionalen Kooperationen sollte es sein, eine übergreifende und längerfristige Wirkungsabschätzung der Maßnahmen für die Ressourcen Boden, Wasser, Klima, Biodiversität und Landschaftsbild zu möglichen, so dass auch Trade-Offs und Synergien frühzeitig identifiziert werden.

In Zusammenarbeit mit solchen lokalen oder regionalen Kooperationen könnten unter direktem Bezug zu lokalen Umweltzielen auch Maßnahmenprioritäten in den AUKM entwickelt und lokale Zielregionen und Standorte (z. B. durch die Erstellung eines Biotopvernetzungsplans) priorisiert werden.

Ein weiterer Schritt wäre es, lokale oder regionale Kooperationen mit der Implementierung der vorgeschlagenen Maßnahmen in den jeweiligen Regionen zu betrauen. Ein solches Vorgehen könnte sich an den Erfahrungen mit den Agrarumweltkooperativen in den Niederlanden orientieren (Renting und Ploeg 2001; van Dijk et al. 2015). Die lokalen oder regionalen Kooperationen müssten dazu mit einem 
geeigneten Organisationsstatus und einem Budget ausgestattet werden. Die Mittelvergabe an die Kooperationen könnte in einem Wettbewerbsverfahren erfolgen.

Zur Umsetzung der von ihnen erarbeiteten Pläne könnten die Kooperationen dann sowohl mit Einzellandwirten als auch mit Gruppen von Landwirten oder mit anderen Akteuren Maßnahmenverträge schließen, die entweder ein maßnahmenoder ein ergebnisorientiertes Entgelt vorsehen könnten. Vertragsdetails, wie etwa die Laufzeit oder der Zahlungsmodus, können direkt innerhalb der lokalen Organisationsstruktur abgestimmt werden. Auch ist es möglich, die Gruppen von Landwirten im Rahmen eines Budgets und einer Zielvorgabe selbst über die Verteilung der Pflichten und Prämien entscheiden zu lassen, was zu einem effizienteren Einsatz der Ressourcen beitragen könnte. Allerdings sind die Traditionen der Zusammenarbeit in Gruppen unter Landwirten in Deutschland weniger ausgeprägt als in den Niederlanden, so dass möglicherweise erst Zeit und Moderationskapazität in die Etablierung einer kooperativen Kultur investiert werden müsste.

Ein solcher kooperativer Ansatz könnte eine Reihe von positiven Effekten ermöglichen (siehe auch Abschn. 4.3). Neben den naturschutzfachlichen Vorteilen einer stärker integrierten, systemischen Planung könnten die Interaktionsformen mit den Landwirten verbessert werden, indem diese nicht einer Bürokratie, sondern einem interaktiven Planungsprozess begegnen. Ein partizipatorischer Prozess der Maßnahmenprogrammierung und Implementierung schafft Gelegenheit für Lernprozesse, Vernetzung und Vertrauensbildung. Eine etablierte regionale Organisationsstruktur könnte eine Schlüsselrolle in der vertrauensvolleren Zusammenarbeit zwischen Landwirtschaft und Bevölkerung einnehmen (z. B. gegenseitige Wissensvermittlung, Abbau von Stigmatisierung, regionales Bewusstsein, Vermarktungsplattform).

\subsubsection{Diskussion}

Abschließend soll noch dargestellt werden, wie die drei hier vorgestellten Elemente die in Abschn. 7.4.2 identifizierten Problemkomplexe adressieren.

Für Problemkomplex 1, den fehlenden Bezug zu den eigentlichen Umweltzielen, wurde eine größere Anzahl an Ursachen ausgemacht. Generell gilt, dass die hier vorgestellten Handlungsansätze ein starkes Nutzen-Targeting aufweisen. Die Maßnahmen würden kleinräumig programmiert und nach Möglichkeit in kooperativer Weise geplant und ausgeführt werden. Dadurch könnten lokale und regionale Charakteristika besser adressiert und eine höhere räumliche und zeitliche Passgenauigkeit erreicht werden. Durch die kooperativen Ansätze könnte gezielt fachliche Expertise aus dem Umweltbereich einbezogen werden, so dass eine evidenzbasierte Programmierung und die gezielte Nutzung von Synergien ermöglicht werden.

Ein multiskalares Landschaftsmanagement (Problemkomplex 2) wird explizit durch alle drei beschriebenen Elemente angestrebt. Die Elemente 1 und 2 stellen Maßnahmen bereit, die über die Feldebene hinausgehen, während Element 3 versucht, eine entsprechende Kooperationsstruktur zu etablieren. Ein Agglomerationsbonus kann bei den Elementen 1 und 2 weitere Anreize für betriebsübergreifende Kooperationen setzen. 
Probleme mit Über- und Unterkompensation sowie den Transaktionskosten (Problemkomplex 3) werden zunächst einmal durch eine regionale Zahlungsdifferenzierung adressiert. Bei einigen der Maßnahmen können auch Auktionen geeignet sein, die finanziellen Mittel effizienter zu verteilen. Die Transaktionskosten verringern sich beispielsweise, wenn Landwirte in der Gruppe Verträge abschließen, die über einen längeren Zeitraum laufen.

Das Problem der bislang oft unzureichenden Anreize zur Erfüllung der Managementvorgaben (Problemkomplex 4) würde auf drei Wegen angegangen: durch eine verstärkte Nutzung von ergebnisorientierter Honorierung; durch die partizipative Einbindung der Landwirtinnen und Landwirte in die Erarbeitung von lokalen und regionalen Maßnahmenplänen, welche Selbstbindung und einen „,sense of ownership“ generieren; sowie bei Honorierung auf Gruppenebene durch Prozesse der sozialen Kontrolle, weil Verfehlungen einzelner dann auch die anderen betreffen. Fragen von Monitoring und Sanktionierung werden vertieft in Abschn. 7.6 diskutiert.

Kooperative Ansätze wurden in der deutschen Agrarpolitik bisher wenig verfolgt. Ein Problem kann daher die Etablierung der entsprechenden Organisationsstrukturen darstellen (Prager 2015). Vertrauensbildung zwischen den Landwirtinnen und Landwirten sowie anderen gesellschaftlichen Gruppen ist dabei wichtig. Der Aufwand zur Etablierung kooperativer Strukturen (Sitzungen, Anfahrt, Abstimmungen, Meinungsverschiedenheiten etc.) erfordert Kompensation der Transaktionskosten und Unterstützung durch professionelle Moderation. Dabei handelt es sich jedoch um Investitionen in die Zukunft des ländlichen Raums, da die sektorübergreifende Vernetzung und Vertrauensbildung positive Wirkungen weit über die unmittelbaren Fragen des Landschafts- und Ressourcenmanagements hinaus erwarten lässt.

\subsection{Baustein 5: Nicht-staatliche Standards und Ko-Regulierung}

Regulierung bezeichnet staatliche Interventionen zur Beeinflussung des Verhaltens von Individuen und Organisationen (Levi-Faur 2009). Eigenregulierung bezeichnet demgegenüber ein Governance-Arrangement, bei dem diejenigen, von denen die Befolgung bestimmter Regeln, Normen und Verfahren erwartet wird, diese auch selbst formulieren und vereinbaren (Führ 2003; Porter und Ronit 2006). In der internationalen Diskussion sind dafür auch die Begriffe „private Regulierung“ (Vogel 2010) und ,private governance“ (Busch 2011) gebräuchlich. Private Regulierung fungiert dabei oft komplementär zu staatlicher Regulierung und ihre Durchsetzung vollzieht sich vor dem Hintergrund des staatlich konstituierten regulatorischen Rahmens, insbesondere Ordnungs-, Privat- und Haftungsrechts (Ménard und Valceschini 2005; Porter und Ronit 2006). Mitunter beziehen sich private auch auf staatliche Regelungen. Daher ist der Begriff der Ko-Governance (Tosun et al. 2016) bzw. der Ko-Regulierung geeignet, das Zusammenspiel von staatlicher und nichtstaatlicher Regulierung zu bezeichnen. 
Die Diskussion um Eigenregulierung setzt sich im Kern auseinander mit nicht-staatlichen und in diesem Sinne privaten Regeln in Bezug auf:

1. Standards für die Charakteristika und Inhaltsstoffe von Produkten (Produktstandards);

2. Standards für die Verfahren zur Erzeugung oder Verarbeitung von Produkten (Prozessstandards);

3. die Nachvollziehbarkeit der Herkunft von Produkten bzw. von deren Bestandteilen;

4. die Kennzeichnung von Produkten aufgrund von einem oder mehreren der Aspekte 1-3.

In der wissenschaftlichen Literatur diskutierte Beispiele sind etwa das Label Filière Qualité Carrefour der französischen Supermarktkette für Qualitätsfleisch (Ménard und Valceschini 2005) oder das britische Kennzeichen Red Tractor. Im internationalen Raum haben sich neben den zwischenstaatlichen Regeln der WTO transnationale Handelsstandard wie GlobalG.A.P. (Botterill und Daugbjerg 2015) oder auch ethisch ausgerichtete Lebensmittelstandards - z. B. Fairtrade, Marine Stewardship Council, ECOFAM - etabliert (Daugbjerg und Botterill 2012). Im Sinne des Ansatzes der Corporate Social Responsibility versuchen nicht zuletzt Handelsketten zunehmend, sich von ethisch problematischen Praktiken zu distanzieren. Dies kann entweder dazu führen, dass als problematisch empfundene Produkte ausgelistet werden, wie zum Beispiel Eier aus Käfighaltung. Zunehmend versuchen Handelsketten aber auch, aktiv eigene Nachhaltigkeitsstandards für ihre Lieferanten zu setzen. Ein Beispiel ist die Sieben-Siegel-Kampagne von ALDI, in welcher der Discounter die Bekanntheit ausgewählter Nachhaltigkeitslabel zu erhöhen und sich kommunikativ mit diesen zu assoziieren sucht (ALDI SÜD 2016).

Bei privater oder Eigenregulierung stellt sich grundsätzlich die Frage der Verbindlichkeit und Durchsetzbarkeit. Hinsichtlich der Verbindlichkeit reicht das Spektrum von freiwilliger Teilnahme bis zu staatlich unterstützter privater Regulierung.

Die Durchsetzbarkeit privater Standards ist von der Frage der Marktmacht nicht zu trennen. In diesem Zusammenhang ist der Unterschied zwischen privater Regulierung von Marktnischen einerseits und flächendeckender privater Regulierung wie GlobalGAP oder dem QS-System zu unterscheiden.

- Standards oberhalb der gesetzlich vorgesehenen Mindeststandards können dabei helfen, Marktnischen im Premiumsektor zu schaffen. Beispiele sind Öko-Labels oder die Kennzeichnung regionaler Herkunft. Dabei liegt insofern viel Marktmacht beim Handel, als dieser über die Listung entscheidet und erfolgreiche Konzepte oft durch Eigen-Labels kopiert, etwa biologische Eigenmarken wie das ,biobio“-Label von Plus.

- Die Durchsetzung privater Standards auf breiter Ebene geht in der Regel vom Handel aus, also der Stufe mit der höchsten Marktkonzentration. Große Supermarktketten sind damit de facto zu privaten Regulatoren geworden, die einen starken Einfluss auf die Erzeugung, Verarbeitung und Distribution von Nahrungsmitteln nehmen (Burch et al. 2013). 
Der Markterfolg von privaten Standards hängt wesentlich vom Vertrauen der potenziellen Käufer ab. Handel und Erzeuger kooperieren daher oft mit Nichtregierungsorganisationen, um die Glaubwürdigkeit ihrer Standards und Labels zu erhöhen (Busch 2011; Fuchs et al. 2011; Ponte und Cheyns 2013; Botterill und Daugbjerg 2015). Aus Sicht der Verbraucherinnen und Verbraucher besteht oft eine verwirrende Label-Vielfalt, welche die Mehrpreisbereitschaft für Produkte mit besonderen ethischen Merkmalen unterlaufen kann (Zander und Hamm 2010; Janssen und Hamm 2012). Bei vielen Nachhaltigkeits-Labels ist der tatsächliche Mehrwert unter Nachhaltigkeitsgesichtspunkten oft fraglich, weshalb „Standards für Standards“ gefordert werden und der Transparenz der Informationen hinter einem Label große Bedeutung zukommt (Adger und Jordan 2009).

In vielen Bereichen bestehen zunehmend staatliche und private Standards nebeneinander. Im Bereich biologischer Lebensmittel gibt es beispielsweise einerseits die zwischenstaatlichen Richtlinien des Codex Alimentarius und andererseits die privaten Richtlinien der International Federation of Organic Agriculture Movements (FOAM). Oft erteilen auch private Organisationen Zertifikate auf der Basis von staatlichen Standards. In der WTO wird daher diskutiert, ob eine staatliche Verantwortung für die Qualität solcher Labels besteht und diese ggf. als technisches Handelshemmnis gegen WTO-Recht verstoßen (Daugbjerg 2012).

Staatliche Regulierungsstrategien sind wesentlich für die Entwicklung privater Standards. Zum einen definieren die staatlichen Mindeststandards den Raum, der zur Profilierung mit höherwertigen Standards zur Verfügung steht (Vogel 2010). Zum anderen dient Eigenregulierung oft dazu, drohender staatlicher Regulierung zuvorzukommen, wie etwa bei Selbstverpflichtungen zu zucker- und fetthaltigen Lebensmitteln (Jensen und Ronit 2015).

Während eine optimistische Perspektive erwarten lässt, dass der Profilierungswettbewerb am Markt ein „race to the top“ auslöst, entsteht aus einer pessimistischen Perspektive Skepsis gegenüber dem Anspruchsniveau privater Standards sowie den Problemen bei der privaten Durchsetzung und Kontrolle (Vogel 2010; Jensen und Ronit 2015).

Eine wesentliche Diskussion betrifft auch die Frage, ob Eigenregulierung den Landwirten und Umweltorganisationen mehr Einfluss verschaffen kann oder ob private Standards zur Individualisierung und Privatisierung der Verantwortung für öffentliche Aufgaben und öffentliche Güter führen (Ménard und Valceschini 2005; Fuchs et al. 2011; Botterill und Daugbjerg 2015).

Auf globalisierten Märkten müssen Standards zudem in globalen Normsetzungsgremien abgestimmt werden. Der europäische Einfluss gilt hier als begrenzt (Young 2014).

Insgesamt ist davon auszugehen, dass auf internationalisierten Märkten transnationale private Standards wie GlobalGAP erheblich an Bedeutung gewinnen, um die Qualität von Produkten zu harmonisieren und den Akteuren entlang der Wertschöpfungskette einklagbare Qualitätserwartungen über gehandelte Ware zu ermöglichen. Dabei spielen auch Umwelt- und Nachhaltigkeitsstandards eine zunehmende Rolle (Havinga et al. 2015). Ob sich daraus eine Triebkraft für eine multifunktionale, natur- und umweltgerecht Landwirtschaft ergibt, ist offen. Allerdings haben 
transnationale Standards den Vorteil, dass sie auch die Importkonkurrenz betreffen und damit das Problem umgehen, dass staatliche Erzeugerstandards immer nur für diejenigen Produzenten verbindlich sind, die im territorialen Geltungsbereich der betreffenden Regeln operieren.

Fazit: Die Entwicklung von Vorschlägen für spezifische Labels würde über den Rahmen dieses Projekts hinausgehen. Im Zuge der Entwicklung von strategischen Handlungsoptionen ist jedoch festzuhalten, dass Kennzeichnungen ein wichtiger Baustein für die bessere Verankerung von Natur- und Umweltschutz in den landwirtschaftlichen Wertschöpfungsketten sind. Denn sie ermöglichen es nicht nur, die Naturschutz- und Umweltleistungen besser in Wertschöpfung am Markt umzusetzen, sondern sie unterstützen auch die Herausbildung sozialer Normen und erstrecken sich potenziell auf importierte Erzeugnisse.

\subsection{Baustein 6: Monitoring- und Sanktionssysteme}

\subsubsection{Aufgaben, Zielkonflikte und Problemlagen von Monitoring- und Sanktionssystemen}

Mehrere Elemente des agrarpolitischen Rahmens erfordern ein flächendeckendes Monitoring-System, um sicherzustellen, dass gesetzliche Regelungen befolgt und ggf. Sanktionen verhängt werden können:

- die Durchsetzung der ordnungsrechtlichen Mindestanforderungen an die landwirtschaftliche Betriebsführung;

- die Einhaltung der Auflagen, an welche die betrieblichen Direktzahlungen geknüpft sind (derzeit in der Ersten Säule der GAP);

- die tatsächliche Durchführung vereinbarter freiwilliger Maßnahmen (derzeit in der Zweiten Säule der GAP).

Bei der Einrichtung von Monitoring- und Sanktionssystemen besteht grundsätzlich ein Zielkonflikt zwischen

- dem Interesse an einer flächendeckenden und zuverlässigen Erfassung derjenigen Tatbestände, die für die angestrebte Verhaltensbeeinflussung (politische Steuerung) im weiteren Sinne sowie die Implementation der Rechtsnormen im engeren Sinne relevant sind;

- den direkten und indirekten Kosten von Monitoring- und Sanktionssystemen, die im Allgemeinen mit dem Detaillierungsgrad, der Neuheit des Systems sowie der Qualität der Daten (Validität der Indikatoren, Reliabilität der Datenerhebung) steigen. Dies beinhaltet zum einen die Kosten der Systemadministration, die bei den Kontrollinstanzen anfallen, andererseits die Transaktions- und ComplianceKosten für die Normadressaten und ggf. andere Akteure.

Monitoring- und Sanktionssysteme sind zudem nicht neutral, sondern haben eine „Performanz“, wie Politik-Instrumente generell (Lascoumes und Le Gales 2007; 
Turnhout et al. 2010). Die verschiedenen Akteure interagieren mit diesen Systemen, z. B. als Antragsteller oder als Administrator, und erleben sich dabei nicht nur in den durch das Recht zugewiesenen Rollen, sondern auch in den Rollen, die ihnen durch die Interaktionsformen zugewiesen werden. So berichten Landwirte, dass sie sich etwa als Bittsteller oder als permanent latent Verdächtiger erleben. Diese PerformanzFunktion der Monitoring- und Sanktionssysteme kann die Wirksamkeit anderer Politik-Instrumente unterstützen oder behindern. Beispielsweise können Monitoring- und Sanktionssysteme einerseits die Durchsetzung ordnungsrechtlicher Normen unterstützen. Andererseits können sie der Herausbildung von Normen der Eigenverantwortung oder dem Leitbild des kreativen Unternehmers widersprechen, indem sie den Normadressaten Formen der Interaktion aufzwingen, in denen sie sich als passiv und unterworfen erleben. Wünschenswert wäre, dass bei der Setzung von Standards und dem flankierenden Monitoring- und Sanktionssystem soziale Normen der Einhaltung der Standards entstehen und so Verstöße gegen die Standards von der Gesellschaft nicht akzeptiert würden (im Gegensatz zum heutigen Umgang etwa mit Steuerhinterziehung).

Monitoring-Systeme können auch mit Formen der Selbst- oder Ko-Regulierung verbunden sein, wie beispielsweise die Kontrollen im Rahmen privater Standardund Zertifizierungssysteme (z. B. QS oder GlobalGAP). Sind solche Systeme hinreichend weit verbreitet, können sie für die Zwecke staatlicher Kontrolle anerkannt werden.

Im Zusammenhang mit Monitoring- und Sanktionssystemen ergeben sich folgende relevante Problemlagen:

- in Teilbereichen fehlende Datengrundlage (siehe Kap. 3);

- ungenaue Indikatoren (zum Beispiel der Vogelindikator als Leitindikator für Biodiversität);

- Zuverlässigkeit der Datenerhebung, insbesondere bei Angewiesenheit auf Aktivitäten Dritter;

- mangelnde Durchsetzung der Auflagen - Vollzugsdefizit;

- hohe Kosten der Datenerfassung;

- hierarchische Interaktionsformen (Instrumentenperformanz).

Im Folgenden diskutieren wir Ansatzpunkte für eine Verbesserung des Monitoringund Sanktionssystems für natur- und umweltbezogene Problemlagen im Bereich der Agrarpolitik.

\subsubsection{Baustein 6.1: Investition in das Indikatorensystem}

Abschn. 6.5 enthält eine umfassende Liste möglicher Indikatoren für die Verwirklichung des Leitbilds einer natur- und umweltverträglichen Landwirtschaft. Die Auflistung zeigt, dass für wichtige Aspekte einer solchen Politik keine oder keine hinreichend operationalisierten Indikatoren zur Verfügung stehen. 
Wie in Kap. 3 und 4 dargestellt, sind auch viele gesetzliche Vorgaben des Naturund Umweltschutzes nicht hinreichend operationalisiert, um ihre Durchsetzung zu ermöglichen.

Vor diesem Hintergrund erscheint es geboten, ein Review der Indikatorensysteme durchzuführen und Mängel bei der Operationalisierung, vor allem bei rechtlich kodifizierten Zielen, abzustellen. Dazu sollte in einen entsprechenden, wissenschaftlich basierten Konsultationsprozess, bei Bedarf unterstützt durch Forschungs- und Entscheidungsvorhaben, investiert werden.

\subsubsection{Baustein 6.2: Digitale Informationssysteme und Fernerkundung zur automatischen Erhebung}

Für das Monitoring der Flächennutzung werden heute in erster Linie InVeKoSDaten genutzt. Zukünftig werden daneben andere GIS-Daten verwendet, um die angrenzende Flächennutzung zu dokumentieren, zu beobachten und zu überwachen. So kann beispielsweise mit Hilfe von GIS-Daten die landschaftliche Vielfalt erfasst werden. Auch können Informationen zur Flächennutzung - z. B. Fruchtfolgegestaltung - aus den InVeKoS-Daten oder anhand von anderen GIS-Daten überprüft werden.

Die Zunahme der Rechenleistung und das Vorhandensein mehrjähriger Daten ermöglichen ein automatisiertes Monitoring der Flächennutzung. Auf dieser Basis könnten in Zukunft die Einhaltung von Auflagen etwa für Fruchtfolgen (und nicht nur Diversität der Anbaufrüchte), aber auch Kennzahlen für die Vielfalt der Landschaftsnutzung berechnet werden, die als Grundlage für entsprechende Prämien dienen könnten.

In dem Maße, wie GIS-gestützte digitale Informationssysteme zur automatisierten Erfassung von Landnutzung und Landschaftsstrukturen beitragen, können sie geeignet sein, den bürokratischen Aufwand bei der Ausgestaltung von flächenbezogenen Monitoring- und Informationssystemen und der Berechnung von landschaftsbezogen Prämien zu verringern. Fachgespräche mit Experten der Fernerkundung haben Aufschluss über derzeitige Entwicklungen bei der Nutzung von digitalen Technologien gegeben. Neue Erdbeobachtungssatelliten stellen räumlich und zeitlich hoch aufgelöste Datensätze zur Verfügung und ermöglichen so die flächendeckende Aufnahme von Landnutzungsstrukturen.

Das europäische Erdbeobachtungsprogramm Copernicus (Programm der Europäischen Kommission und der Europäischen Weltraumorganisation) stellt eine leistungsfähige Infrastruktur für Erdbeobachtung und Dienstleistungen der Geoinformation zur Verfügung. Die Copernicus-Satelliten Sentinel-2A und -2B überfliegen die globale Landoberfläche alle 5 Tage, was zur Verfügbarkeit einer zeitlich hoch aufgelösten Zeitreihe von Daten führt. Sie liefern mit einer räumlichen Auflösung von 10 m seit Kurzem Fernerkundungsdaten, die aufgrund ihrer hohen Qualität (räumliche und zeitliche Auflösung) grundsätzlich für das Monitoring von 
landwirtschaftlichen Flächen geeignet sind. Die Daten des europäischen Copernicus-Erdbeobachtungsprogramms stehen offen zur Verfügung und sind gezielt für die Entscheidungsfindung bei Politikprozessen und für das Monitoring und Management von Umweltbelangen eingeführt worden.

Fernerkundungstechniken werden auf nationaler Ebene bereits zur Unterstützung des Integrated Administrative Control System (IACS) der EU eingesetzt. Hierbei werden u. a. Flächengrößen, Nutzungsarten und Landschaftselemente identifiziert sowie Angaben von Landwirten überprüft. Zum Einsatz kommt hier beispielsweise die GIS-Umgebung des Land Parcel Identification Systems (LPIS) innerhalb des Integrierten Verwaltungs- und Kontrollsystems (InVeKoS). Die genutzten Analysesysteme beruhen aktuell allerdings noch nicht in vollem Maße auf den neuen Sentinel-Satelliten und sind nicht voll automatisiert. Genutzt werden in der Regel etablierte Sensoren wie Landsat, RapidEye und WorldView, welche nicht die Vorzüge der sowohl räumlich wie zeitlich hohen Auflösung bei flächendeckender und kostenfreier Verfügbarkeit bieten.

Für die voll automatisierte Extraktion der genannten Informationen besteht aktuell jedoch noch Forschungsbedarf. Projekte zur Entwicklung entsprechender Copernicus-Services für die Landwirtschaft, an denen auch das Deutsche Zentrum für Luft- und Raumfahrt (DLR) beteiligt ist, werden u. a. von der EU gefördert. Hierbei spielt jedoch der Aspekt von Landschafts- und Strukturelementen bisher noch keine große Rolle. Diese Daten könnten eine deutlich verbesserte Grundlage zur Erfassung von Schlaggrößen, Fruchtfolgen, Länge der Vegetationsperiode und Strukturelementen, die bei einer Pixelgröße von $10 \mathrm{~m}$ x $10 \mathrm{~m}$ erkannt werden, darstellen. Landschaftselemente könnten zukünftig über Fernerkundung automatisch erfasst werden. Forschungsbedarf besteht bei der automatischen Erfassung von kleinen Strukturen, wie z. B. Einzelbäumen, die bei einer Pixelgröße von $10 \mathrm{~m}$ x $10 \mathrm{~m}$ nicht ohne Weiteres erfasst werden.

Derzeit laufen mehrere Forschungsprojekte, die sich mit der Klassifizierung von Agrarräumen mit Hilfe von Fernerkundungsdaten beschäftigen, u. a. an den Universitäten in Würzburg, Halle und Hannover. Feldfrüchte können z. B. über die spektrale und phänologische Signatur mittlerweile recht eindeutig bestimmt werden. Für eine europäische automatisierte Erfassung der Landnutzungsstrukturen erscheint derzeit jedoch die Erstellung der Trainingsdaten über Beobachtung oder Kalibrierung noch als relativ aufwendig. Hier könnte die partielle Verschneidung mit aktuellen Vektordatensätzen (InVeKoS, Amtliches Liegenschaftskatasterinformationssystem ALKIS) Abhilfe schaffen. Ein vielversprechender Datensatz zur Erfassung der Hangneigung ist das neue Höhenmodell TandDEM-X DEM. Je nach Nutzungszweck und gewünschter Flächengröße ist der Zugang zu den Daten unter verschiedenen Voraussetzungen möglich.

Die Überwachung einer auf die Landschaftsstruktur bezogenen Prämie mittels GIS-gestützter Systeme mit Hilfe von Fernerkundungsdaten erscheint daher mittelfristig als eine realistische Option. Voraussetzung dafür wäre es, dass die Fehlerhaftigkeit unter den von EU-Kontrollsystemen geforderten Wert von 2 \% fällt. Dies erfordert weitere Investitionen in ein solches System. Im Ergebnis könnte dadurch jedoch ein Kontrollsystem ermöglicht werden, das die laufenden Administrations- 
kosten und die bürokratische Last für Landbewirtschafter vermutlich deutlich verringern könnte. Voraussetzung für die Zahlung einer auf Daten zur Landschaftsstruktur basierenden Prämie wäre eine Einigung auf einen fachlich belastbaren Satz von nachvollziehbaren, gewichteten Faktoren für die Zuteilung der Prämienansprüche und die Berechnung der Prämienhöhe.

\subsubsection{Baustein 6.3: Monitoring stofflicher Einträge - Plausibilitätsprüfungen}

Stoffliche Einträge sind auf absehbare Zeit kaum durch Fernerkundung zu erfassen, so dass in diesem Bereich weiterhin Dokumentation und Bilanzerstellung auf betrieblicher Ebene notwendig sein werden. Um sicherzustellen, dass die ausgewiesenen Bilanzwerte den tatsächlichen stofflichen Einträgen entsprechen, sind jedoch zum einen die Vorschriften der Bilanzierung in einigen Bereichen zu überarbeiten (z. B. anrechenbare Verluste in der Düngeverordnung), zum anderen sind auf Seiten der Überwachung entsprechende Plausibilitätsprüfungen einzuführen. Am Beispiel der Düngeverordnung hieße dies, dass z. B. die angegebene Ertragsmenge bzw. Bilanzwerte mit regions- und betriebsformtypischen Werten auf Plausibilität verglichen werden sollten. Entsprechende Prüfmechanismen können ebenfalls automatisiert in den Systemen der Datenverarbeitung hinterlegt werden. Werden Werte angeben, die von den definierten Grenzwerten - z. B. für den Stickstoffgehalt im organischen Dünger - abweichen, kann hierfür die Vorlage entsprechender amtlicher Belege oder Rückstellmuster gefordert werden. Für den Einsatz zugekaufter Düngeund Pflanzenschutzmittel ${ }^{8}$ sind des Weiteren die Belege auf Verlangen der Kontrollbehörden vorzulegen.

\subsubsection{Weitere Bausteine}

\section{Baustein 6.4: Amtliche Belege bei komplexen Problemlagen}

Des Weiteren kann für Aspekte, wie etwa die Erstellung von Humusbilanzen oder den Einsatz von Tierarzneimitteln, die Vorlage amtlicher Belege eingeführt werden. Diese Praxis wird heute schon teils angewendet, sollte zukünftig aber ausgebaut werden, so dass eine Einbindung externer (Kontroll-) Einrichtungen, wie z. B. im ökologischen Landbau, erfolgt. Dieses Vorgehen bietet sich vor allem für Maßnahmen an, deren Messwerte nur mit hohem betrieblichen und/oder verwaltungstechnischen Aufwand zu erheben sind und/oder deren Messung spezifisches Fachwissen und entsprechende technische Ausrüstung erfordert.

\footnotetext{
${ }^{8}$ Da z. B. auch bei hohem Anfall organischer Dünger zusätzlich hohe Mengen an mineralischem Dünger eingesetzt werden (Taube 2016).
} 


\section{Baustein 6.5: Mehrjährige Antragstellung und Gruppenanträge}

Eine bürokratische Entlastung kann des Weiteren durch mehrjährige Antragsstellung auch in der Ersten Säule erreicht werden. Weiterhin würde eine Reduzierung der Ausnahmetatbestände den Verwaltungsaufwand vermindern (Hart et al. 2016). Daneben könnte die Verlagerung der Antragstellung auf die Ebene von lokalen oder regionalen Kooperativen wie in den Niederlanden zu einer Reduzierung des bürokratischen Aufwandes führen, da nicht nur Verantwortlichkeiten weitergegeben werden, sondern auch Mechanismen sozialer Kontrolle verstärkt greifen. Der anfangs eventuell erhöhte Zeitaufwand für Planung, Ausführung und Erfassung der Auflageneinhaltung würde im Zeitverlauf durch Routinebildung typischerweise abnehmen.

\section{Baustein 6.6: Abstimmung von Kontrollfrequenzen und Sanktionshöhe}

Die Durchsetzung der gesetzlichen Mindestanforderungen und weiteren Auflagen hat häufig mit einem Vollzugsdefizit zu kämpfen. Die Durchsetzung der GAB beruht bspw. in erster Linie auf Cross Compliance-Prüfungen mit prozentualen Kürzungen der Direktzahlungen bei Verstößen. Mit der Einführung von Cross Compliance konnte ein flächendeckendes Kontrollsystem mit der Verteilung der Direktzahlungen verknüpft werden, was die systematische Durchsetzung der gesetzlichen Mindeststandards erleichtert. Um die Durchsetzung des gesetzlichen Mindeststandards und der genannten Auflagen zu sichern, könnte die Anhebung der Kontrollquote und -frequenz in Kombination mit der Sanktionshöhe angebracht sein. Dabei sollte die Höhe der Kontrollquoten und -frequenzen unter Berücksichtigung der möglichen Schäden bei Verstoß in einem umgekehrten Verhältnis zur Sanktionshöhe stehen. Die Einführung eines effektiven Kontroll- und Sanktionsmechanismus bestärkt auch die Einsicht der Landwirte in die Bedeutung der Maßnahmen und den Wunsch der Gesellschaft, dass diese eingehalten werden. Zugleich sollte der Kontrollaufwand in einem angemessenen Verhältnis zur erwarteten Häufigkeit und Schwere der Verstöße stehen. Hier kann ein risiko-basierter Ansatz zu einer an den Prinzipien der Effektivität, Effizienz und Angemessenheit orientierten Kontrollpraxis beitragen.

\section{Baustein 6.7: Beweislastumkehr}

Eine Umkehr der Beweislast kann ebenso wie ein starker Kontroll- und Sanktionsmechanismus dazu beitragen, die Bedeutung der Grenzwerte und Maßnahmen und deren Einhaltung zu bestärken. Bei Anlastung der Beweislast müssten die Landwirtinnen und Landwirte aber auch in die Lage versetzt werden, die Einhaltung der Auflagen selbst nachweisen zu können. So könnten bspw. die landwirtschaftlichen Betriebe durch Erhebung der Stickstoffgehalte im organischen Dünger und Wiegeprotokolle der Erträge die Einhaltung der Stickstoffbilanzgrenzwerte nachweisen, falls hieran Zweifel bestehen.

\subsubsection{Fazit}

Zusammenfassend ist festzuhalten, dass es für die Zukunft vorstellbar ist, anhand digitaler Daten und elektronischer Datenverarbeitung den bürokratischen Aufwand für die landwirtschaftliche Betriebe und die Verwaltung zu reduzieren. So können bisher in verschiedenen Datenbanken erhobene und gespeicherte Daten 
zur Durchführung von Kontrollen zusammengeführt werden (z. B. HIT und InVE$\mathrm{KoS}$ ) und mit Daten der Selbstkontrolle (z. B. Ackerschlagbilanzen, Daten des Precision Farming) kombiniert werden. Die gewonnenen Daten können damit insgesamt als Kontroll- aber auch als Monitoring- und Beratungstool für die landwirtschaftlichen Betriebe eingesetzt werden, z. B. durch Vergleiche des eigenen Betriebes mit anderen Betrieben aus der Region (Tab. 7.10).

\subsection{Baustein 7: Unterstützende Elemente}

Die Verwirklichung des Leitbilds einer multifunktionalen, natur- und umweltverträglichen Landwirtschaft erfordert von den Beteiligten viel neues Wissen und zum Teil auch eine Umorientierung. Dazu könnten unterstützende Maßnahmen angeboten werden. Dies betrifft zum einen Ausbildung und Beratung für die in der Landwirtschaft tätigen Personen, zum anderen verbraucherorientierte Maßnahmen.

\subsubsection{Ausbildung und Beratung}

Die Entwicklung einer multifunktionalen, natur- und umweltverträglichen Landwirtschaft erfordert von den Landwirten erhebliches Wissen. Die Verwirklichung des Leitbildes könnte dadurch unterstützt werden, dass Workshops, Kurse oder Informationsbroschüren neuere Ansätze vermitteln und dadurch sowohl das Wissen wie die Akzeptanz erhöhen (Frondel et al. 2012). Weitere sinnvolle Ansätze sind Demonstrationsprojekte (Schleyer und Plieninger 2011), Beratung durch Fachleute (Meyer et al. 2015a) oder gegenseitige Hilfe der Landwirte untereinander (Prager 2015). Für die Einführung neuer, zum Teil komplizierter Verfahren, wie etwa Auktionen, ist eine intensive Begleitung, vor allem in den Anfangsstadien, unerlässlich (Freese et al. 2011; Ulber et al. 2011). Ebenso benötigen viele ergebnisorientierte Maßnahmen eine fachliche Begleitung (Schroeder et al. 2013). Auch kann fachliche Expertise helfen, Transaktionskosten zu senken, die als wesentliche Teilnahmehürde vieler AUKM angesehen werden (Meyer et al. 2015a).

Einführung eines Curriculums an den landwirtschaftlichen Fachschulen: $\mathrm{Zu}$ nächst ist es wichtig, die neuesten wissenschaftlichen Erkenntnisse zu den Umweltleistungen der Landwirtschaft überall im Curriculum zu verankern.

Angeboten und gefördert werden könnten laufende Seminarangebote für Landwirte zu Themen wie:

- Auswirkungen landwirtschaftlicher Aktivitäten auf die Bodenqualität;

- nachhaltige Landnutzungssysteme;

- Wasserhaushalt, Artenvielfalt;

- ökologischer Pflanzenbau;

- EU-/Landes-Verordnung;

- Verwaltungsverfahren. 


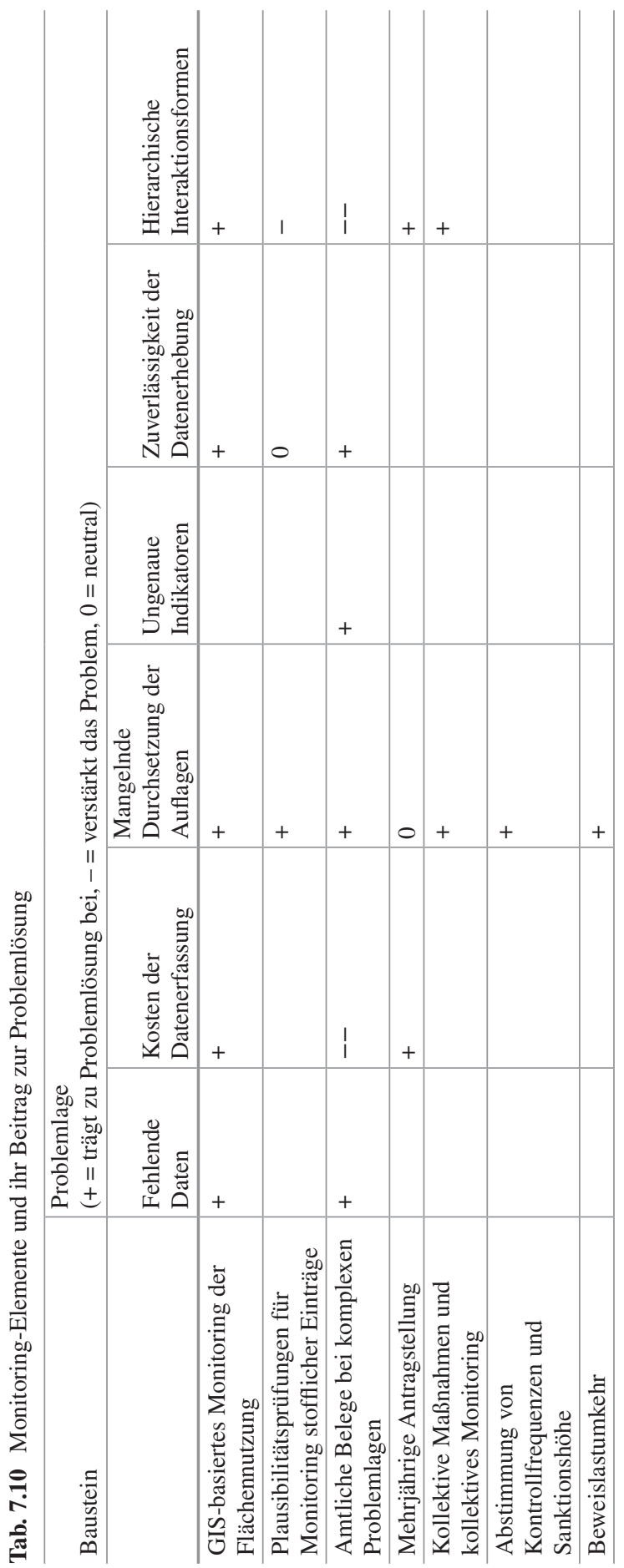


Weiterhin könnte durch Leistungsvereinbarungen zwischen Landesregierungen und Hochschulen/Universitäten ein Curriculum zum Thema ,Nachhaltige Landwirtschaft" etabliert werden mit Inhalten wie:

- systemisches Denken;

- Flächennutzungskonzepte;

- Wasserkreislauf;

- umweltorientiertes Marketing und regionale Märkte;

- Energiefragen;

- Artenvielfalt;

- Konsequenzen landwirtschaftlicher Aktivitäten auf lokalem, regionalem und globalem Niveau;

- Tierschutz, Agrarpolitik;

- Plurale Ökonomik ${ }^{9}$

- EU-/Landes-Verordnung und Verwaltungsverfahren.

Beratungsdienste können mit Hilfe von Training und Vorführungen im Feld (Demonstrationsprojekte), in Zusammenarbeit mit Hochschulen, gerade bei fehlenden Kapazitäten viel erreichen (Klair et al. 1998).

Weiterhin könnte ein anerkannter, fachlich hochwertiger Sachkundenachweis für nachhaltige Landtechnik, Tierhaltung, und Pflanzenbau eingeführt werden. Eine Auffrischung dieser Kenntnisse alle drei bis fünf Jahre wäre wünschenswert, um auf dem neuesten Stand zu bleiben. Damit würden Kapazitäten der Landwirte aufgebaut und gefestigt.

Die Erstellung oder Unterstützung der Entwicklung von umweltorientierten Betriebsentwicklungsplänen für Pflanzen- und Tierproduktionsbetriebe könnte durch Beratungsstellen gefördert und angeboten werden. ${ }^{10}$

\subsubsection{Verbraucherorientierte Maßnahmen}

Das Engagement von Individuen, Haushalten, Schulen und anderen Einrichtungen, in denen Lebensmittel zubereitet und serviert werden, kann auch einen Multiplikatoreffekt haben. Hier spielen Regeln, Konsumentensteuerung, Produktkennzeichnung, Direktmarketing, Bewusstseinsbildung und Bildung von Konsumenten wichtige Rollen.

\footnotetext{
${ }^{9}$ Plurale Ökonomik, auch als Real World Economics oder Post-autistische Ökonomie bekannt, fordert die neoklassischen Standardannahmen heraus und integriert Ideen aus der Soziologie und Psychologie in die wirtschaftliche Analyse (Fullbrook 2007).

${ }^{10} \mathrm{Vgl}$. dazu die Einrichtung eines „,Kompetenzzentrums für ländliche Entwicklung“ bei der Bundesanstalt für Landwirtschaft und Ernährung (BLE) mit Informationsplattform zur Intensivierung der landwirtschaftlichen Beratung und Innovationsförderung in gezielten Bundesprogrammen (BMEL 2015a, S. 29).
} 


\subsubsection{Steuern und Regulation}

Konsumenten spielen eine zentrale Rolle für die Chancen einer nachhaltigen (oder nicht nachhaltigen) Landwirtschaft. Fördernde Maßnahmen, darunter regulierende, steuerliche und freiwillige Maßnahmen, Produktlabels, direktes/lokales Marketing, Bewusstseinsbildung über Nahrungsmittelentscheidungen und Ernährung, sowie individuelles Engagement als auch Engagement von Haushalten, Schulen und sonstigen Organisationen in denen Nahrung vorbereitet und konsumiert wird, können effektiv sein.

Grundsätzlich kann das Konsumentenverhalten durch die erhöhte Besteuerung von Produkten beeinflusst werden, welche nicht nachhaltig produziert wurden oder als gesundheitsschädigend (bspw. Tabak oder Alkohol) oder umweltschädigend (bspw. Dünger, Plastikverpackungen, ölbasierte Kraftstoffe) eingestuft werden. Der Vorschlag, den reduzierten Mehrwertsteuersatz für tierische Erzeugnisse in Frage zu stellen, hat jedoch zu einer kontroversen öffentlichen Diskussion geführt. Andere Ansätze wären die Besteuerung von Inputs in die Produktion, wie Mineraldünger oder Pestizide (Pedersen et al. 2012). Die ökonomische Begründung für eine solche Steuer wäre immer die Beeinflussung der relativen Faktorpreise. Der Umfang, in dem eine Besteuerung zur Reduzierung des Einsatzes der besteuerten Faktoren führt, hängt von den Faktorpreiselastizitäten ab. Beim Pestizideinsatz ist weiterhin zu berücksichtigen, dass die Umweltverträglichkeit durch die Genehmigungsverfahren und die Auflagen beim Gebrauch sichergestellt werden sollen. Eine gesonderte Produktsteuer bedürfte daher in diesem Fall einer besonderen Begründung.

Regulative Ansätze können beispielsweise die Werbung und Produktinformation betreffen. Während dies eine übliche Methode ist, den Alkohol- und Tabakkonsum zu regulieren, gibt es nur unzureichende Analysen der Wirksamkeit regulativer Ansätze im Nahrungsmittelbereich (OECD 2016).

\subsubsection{Produktkennzeichnung}

Ein effektives Marketing von nachhaltig produzierten landwirtschaftlichen Produkten von Landwirten und Nahrungsmittelindustrie braucht gut informierte Verbraucherinnen und Verbraucher. Insbesondere müssen Unterschiede zwischen konventionellen und nachhaltig hergestellten Produkten verstanden werden. Zudem sollten die Gründe für die höheren Preise klar kommuniziert werden (Klair et al. 1998; von Meyer-Höfer und Spiller 2014). Auch wenn Verbraucherinnen und Verbraucher eine positive Einstellung gegenüber nachhaltig erzeugten Lebensmitteln besitzen, gibt es immer noch Hindernisse, welche die Beachtung und Auswahl dieser Produkte hemmen, wie etwa Wahrnehmung, Wissen über die Produkte, Konflikte mit anderen Prioritäten (z. B. Geschmack, Preis), mangelndes Bewusstsein oder mangelnde Motivation (Kuhnert et al. 2005; Grunert 2011).

Um die Nachhaltigkeit von Produkten zu kommunizieren, wurde ein , übergreifendes Nachhaltigkeitslabel“" als effektivste regulierende Maßnahme empfohlen 
(von Meyer-Höfer und Spiller 2014). Als allgemeine Anforderungen an ein solches Label gelten (Grunert 2011):

- Es muss die Aufmerksamkeit des Käufers auf sich ziehen;

- den Käufer an seine ,guten Absichten“ erinnern;

- einfach zu verstehen und zu lesen sein und

- glaubwürdig und vertrauenswürdig sein.

Die Einführung eines allgemein anerkannten Nachhaltigkeitslabels ist jedoch bisher nicht gelungen, u. a. weil dadurch der Spielraum für einzelne Anbieter, sich durch Eigen-Labels zu profilieren, vermindert würde.

Eine direkt mit dem Naturschutz verbundene Kennzeichnung ist das ,landscape label", welches die Produktzertifikation mit der Entrichtung von Zahlungen für Ökosystemdienstleistungen kombiniert (Ghazoul et al. 2009). Zu den vielen Herausforderungen bei der Einführung eines solchen Labels gehören der Aufbau von „fairen und transparenten community-basierten Organisationen“ und die Gefahren vor „Trittbrettfahrern“ (Ghazoul et al. 2009). Verbraucherbefragungen haben gezeigt, dass die beiden genannten Punkte wichtig für die Glaubwürdigkeit und das Vertrauen für Label sind (McEachern et al. 2005; Eden et al. 2008; Golan et al. 2001). Obwohl dies für die meisten Nahrungsmittel der Fall ist, ist der Marktanteil von Bio-Produkten in Deutschland mit 7 \% (2011) sehr gering und stieg in den letzten Jahren nur minimal an (BMUB und Umweltbundesamt 2013). Höhere Verkaufspreise, Zweifel an Umweltnutzen und korrekter Deklaration sowie die verwirrende Label-Vielfalt ${ }^{11}$ vermindern das Vertrauen und damit die Wirksamkeit von Umwelt- und Nachhaltigkeitslabels (van der Zee 2016). Die Einführung eines staatlich garantierten Labels, welches auf transparente und nachvollziehbare Weise die mit einem Produkt verbundenen Leistungen im Natur- und Umweltschutz zertifiziert, wäre daher ein wichtiger Schritt zur Aktivierung der Verbrauchernachfrage nach nachhaltigen Produkten.

\subsubsection{Bewusstseinsbildung und Verbindung zwischen Konsumenten und Erzeugern}

Eine Vielzahl von freiwilligen „Bottom-up“-Initiativen zielt darauf ab, das Bewusstsein bei Verbrauchern für Ernährungsfragen und für den Einfluss ihrer eigenen Kaufentscheidungen zu stärken sowie stärkere Verbindungen zwischen Konsumenten und Erzeugern herzustellen (siehe dazu auch Abschn. 4.3.6). Beispiele reichen von einfachen Maßnahmen, wie fleischfreien Tagen in Kantinen, bis hin zur Förderung von nachhaltigem Nahrungsmittelkonsum im öffentlichen Sektor, wie zum Beispiel Gerichte aus saisonaler, regionaler und biologischer Produktion. Durch

\footnotetext{
${ }^{11}$ So gab es laut der Verbraucherinitiative Label-online.de allein aus Deutschland 34 Labels, welche „Essen und Trinken“ als „Bio“ deklarieren. Zugleich wurden 21 Nachhaltigkeits-Labels aufgeführt (www.label-online.de. Zugegriffen am 28.06.2016).
} 
systematische Planung, niedrige Darlehenszinsen und auch durch Wissensaustausch können solche freiwilligen Initiativen unterstützt werden.

Direktvermarktung ist nicht nur eine Möglichkeit für Landwirtinnen und Landwirte, ihr Einkommen zu steigern, indem sie kommerzielle Weiterverarbeitung und Zwischenhändler in der Lebensmittelkette umgehen. Es stellt auch sozial eine direkte Verbindung zwischen Verbrauchern und Erzeugern her. Die sichtbarste und aktuell vorherrschende Form von Direktvermarktung in Deutschland sind regionale Wochenmärkte. Wochenmärkte stellen nicht nur eine Quelle lokaler und regionaler Produkte dar, sondern stärken die Verbindung sowohl zwischen Landwirten und Endverbrauchern als auch zwischen ländlichen und städtischen Regionen. Sie tragen zur Bewusstseinsbildung bei und können Konsumenten dazu motivieren, mehr über das Essen, welches sie kaufen, und die dafür benötigten Ressourcen für landwirtschaftliche Betriebe, zu lernen. Andere Formen des Direktmarketings von Landwirten an Konsumenten sind der Hofverkauf via Hofläden und Hofautomaten. Allerdings sind diese Initiativen nicht immer eindeutig mit besonderen Leistungen im Natur- und Umweltschutz verknüpft.

Auch könnte die Arbeit von Ernährungsräten wie den 2016 gegründeten Initiativen in Berlin und Köln dabei unterstützt werden, eine Verbindung zwischen städtischen Verbraucherinnen und Verbrauchern und besonders natur- und umweltfreundlichen Formen der Landwirtschaft herzustellen.

\subsubsection{Investitionshilfen}

Betriebliche und ländliche Infrastrukturinvestitionen werden im Rahmen der Zweiten Säule der GAP gefördert. Oft dienen diese Maßnahmen bereits jetzt der Verbesserung des Umweltschutzes. Zur Verwirklichung des Leitbilds einer multifunktionalen, natur- und umweltverträglichen Landwirtschaft könnten die Investitionshilfen konsequent an dem neuen Leitbild ausgerichtet werden. Im Bereich der Tierhaltung sollen im Prinzip bereits jetzt ,geförderte Investitionen besondere Anforderungen an den Umwelt-, Klima- und Ressourcenschutz erfüllen, die über die geltenden Standards hinausgehen" (BMEL 2015i).

Eine neuere Untersuchung aus dem Thünen-Institut (2016) zu den Agrarinvestitionsförderungsprogrammen findet dabei zwar positive Effekte, aber wenig Belege dafür, dass die Förderung tatsächlich zu zusätzlichen Leistungen im Natur-, Umwelt- und Tierschutz (Additivität) führt. Auch wird die Frage aufgeworfen, welche Funktion angesichts niedriger Zinsen und hoher Kreditvergabewilligkeit der Banken eine zusätzliche staatliche Investitionsförderung haben kann.

Neben staatlichen wäre dabei auch an private Kreditprogramme zu denken. Oft werden dabei die Aspekte der Kostensenkung und der Ökologisierung verknüpft, um ,die Anpassung der Betriebe an z. B. neue oder wechselnde Rahmenbedingungen [zu ermöglichen]. Die Produktionskosten sollen gesenkt, die Ökologisierung gefördert und damit die Wettbewerbsfähigkeit einer nachhaltig produzierenden Landwirtschaft gestärkt werden“ (Weber 2005, S. 400). Beispielsweise finanziert 
die GLS-Bank spezifisch die Umstellung auf Ökolandbau, ökologische Betriebserweiterungen und Modernisierungen bis hin zu Hofgemeinschaften, die neben der Landwirtschaft auch einen Hofladen, Kulturprogramme oder Übernachtungen anbieten. Das Förderprogramm „Nachhaltigkeit“ der Landwirtschaftlichen Rentenbank dient der Verbesserung der Lebens-, Produktions- und Arbeitsbedingungen unter Berücksichtigung der Verbraucherinteressen und dem Erhalt der biologischen Vielfalt, aber auch Investitionen zur Erfüllung besonderer Anforderungen im Bereich Tierschutz.

\subsection{Fazit}

In diesem Kapitel wurden die verschiedenen Instrumente der Agrarpolitik im Hinblick auf ihren Beitrag zur Realisierung des Leitbilds einer multifunktionalen, natur- und umweltverträglichen Landwirtschaft diskutiert. Die Übersicht in Tab. 7.11 zeigt, dass eine Kombination der Bausteine notwendig ist, um die verschiedenen prioritären Problemkomplexe (vgl. Abschn. 6.6) zu adressieren. Daher ist ein koordinierter Gesamtansatz, eine Policy-Architektur, notwendig, die ein abgestimmtes Design der verschiedenen Politik-Instrumente ermöglicht. Dafür werden im folgenden Kapitel mehrere strategische Entwicklungsoptionen aufgezeigt. Natürlich folgt auch die derzeitige Agrarpolitik einem Gesamtansatz. Dieser ist aber historisch entstanden und reformbedürftig.

Zugleich zeigt die Diskussion in diesem Kapitel, dass in jedem der Elemente der Agrarpolitik verschiedene Instrumenten-Varianten bestehen, die mehr oder weniger geeignet sind, zur Verwirklichung des in Kap. 6 entwickelten Leitbilds beizutragen. Bei der Verwirklichung des Leitbilds kommt es also sowohl auf eine überzeugende Gesamtkonzeption wie auch auf die Ausgestaltung der verschiedenen Instrumente im Detail an.

Die Übersicht in Tab. 7.11 bestätigt den Befund aus der SWOT-Analyse, dass die Instrumentenvielfalt eine Stärke der gegenwärtigen Agrarpolitik darstellt, die genutzt werden sollte, um die vielfältigen Probleme des Natur- und Umweltschutzes im Zusammenhang mit der Landwirtschaft zu adressieren. Zugleich hat die Diskussion in diesem Kapitel gezeigt, dass eine effizientere und effektivere Ausgestaltung der meisten Elemente der Agrarpolitik möglich wäre. Dies wird zum Teil zusätzliche Ressourcen erfordern. Da die Diskussion in Abschn. 7.2 zu dem Ergebnis geführt hat, dass bestenfalls mit einer Aufrechterhaltung des Anteils der GAP am EU-Budget zu rechnen ist, aber nicht mit einer Steigerung des Agrarbudgets, müssten die Mittel innerhalb der bisherigen Agrarpolitik anders eingesetzt werden. Die Diskussion des Instruments der Direktzahlungen hat dabei ergeben, dass diese in ihrer derzeitigen Form insgesamt wenig effektiv und effizient sind. Ein stärker am Prinzip der Ergebnisorientierung ausgerichteter Einsatz der erheblichen Mittel, die derzeit für Direktzahlungen verwendet werden, erscheint daher geboten. Dafür bestehen grundsätzliche drei Optionen: (1) eine stärkere Verknüpfung der Direktzahlungen mit der Erbringung von öffentlichen Leistungen, etwa 
Tab. 7.11 Zuordnung der Instrumenten-Bausteine zu den prioritären Problembereichen

\begin{tabular}{|c|c|}
\hline Zielgröße/Problembereich & $\begin{array}{l}\text { Baustein/Variante, welche das Ziel bzw. den } \\
\text { Problembereich prioritär adressiert }\end{array}$ \\
\hline \multicolumn{2}{|c|}{ Komplex 1: Stoffeinträge in Boden, Wasser und Luft } \\
\hline $\begin{array}{l}\text { Verlust von Biodiversität und } \\
\text { Habitatqualität durch Stoffeinträge } \\
\text { Nitratproblematik, } \\
\text { Arzneimitteleinträge, } \\
\text { Pestizideinträge (Auswirkungen } \\
\text { u. a. auf Wasserqualität) } \\
\text { - Antibiotikaresistenz } \\
\text { - Klimaschutz: Treibhausgas- } \\
\text { Emissionen }\end{array}$ & $\begin{array}{l}\text { Ordnungsrecht und Mindeststandards, ggf. } \\
\text { Ausgleich erhöhter regulationsbedingter Kosten } \\
\text { im Vergleich zu Nicht-EU-Erzeugern auf } \\
\text { wissenschaftlicher Basis } \\
\text { - Investitionshilfen bei Anhebung der Standards im } \\
\text { Ordnungsrecht }\end{array}$ \\
\hline
\end{tabular}

Komplex 2: Flächennutzung

- Biodiversitäts- und Habitatverlust

- Bodenschutz: Erosion, Schadverdichtung

- Treibhausgas-Emissionen durch Landnutzungsänderungen

- Anpassung an den Klimawandel

- GLÖZ, Greening-Auflagen (ggf. teilweise Überführung ins Ordnungsrecht)

- Mindeststandards zur Aufrechterhaltung der natürlichen Produktivität der Standorte

- Prämien zur Aufrechterhaltung der Bewirtschaftung in Gebietskulissen mit erschwerten Bewirtschaftungsbedingungen

- Prämien zur Honorierung der landschaftlichen Vielfalt

- Flächendeckendes Minimum von Natur- und Umweltschutzmaßnahmen gegen Honorierung

- Honorierung besonderer Leistungen im landwirtschaftlichen Natur- und Umweltmanagement

- Regional und standörtlich ausgerichtete AUKM

- Koordinationsansätze zum integrierten Landschafts- und Ressourcenmanagement

Komplex 3: Implementations-, Partizipations- und Innovationsdefizite

- Implementationsdefizite

- Unzureichende Kontroll- und Sanktionsmechanismen

- Ko-Regulierung und nicht-staatliche Standards

- Verbesserte Monitoring- und Sanktionssysteme inkl. GIS-gestützter Fernerkundungssysteme

- Monitoring stofflicher Einträge Plausibilitätsprüfungen

- Fehlende operationalisierte Ziele und unklare Rechtsbegriffe

- Priorisierung und Operationalisierung der naturschutz- und umweltpolitischen Zielgrößen

- Schließung von Operationalisierungsdefiziten bei Ordnungsrecht und Mindeststandards

- Zu wenige Lernprozesse und zu wenig sektorübergreifende Kooperation
- Ausbildung und Beratung

- Verbraucherorientierte Ansätze

- Partizipative und kooperative Instrumente bei der Planung und Implementation regionaler und standörtlicher Programme und Maßnahmen

- Integriertes Ressourcen- und Landschaftsmanagement 
durch eine anspruchsvollere Ausgestaltung der Cross-Compliance- und Greening-Anforderungen; (2) eine Umschichtung von Mitteln von den Direktzahlungen in der Ersten Säule hin zu den stärker ziel- und ergebnisorientierten Maßnahmen der Zweiten Säule; und/oder (3) der Übergang zu einem integrierten System aufeinander aufbauender Prämien und Programme, welche zusammen mit einem verbesserten Ordnungsrecht systematisch die verschiedenen Problemlagen des Naturund Umweltschutzes im Agrarsektor adressieren.

Im folgenden Kapitel werden diese drei strategischen Optionen weiter ausformuliert und diskutiert.

Open Access Dieses Kapitel wird unter der Creative Commons Namensnennung 4.0 International Lizenz (http://creativecommons.org/licenses/by/4.0/deed.de) veröffentlicht, welche die Nutzung, Vervielfältigung, Bearbeitung, Verbreitung und Wiedergabe in jeglichem Medium und Format erlaubt, sofern Sie den/die ursprünglichen Autor(en) und die Quelle ordnungsgemäß nennen, einen Link zur Creative Commons Lizenz beifügen und angeben, ob Änderungen vorgenommen wurden.

Die in diesem Kapitel enthaltenen Bilder und sonstiges Drittmaterial unterliegen ebenfalls der genannten Creative Commons Lizenz, sofern sich aus der Abbildungslegende nichts anderes ergibt. Sofern das betreffende Material nicht unter der genannten Creative Commons Lizenz steht und die betreffende Handlung nicht nach gesetzlichen Vorschriften erlaubt ist, ist für die oben aufgeführten Weiterverwendungen des Materials die Einwilligung des jeweiligen Rechteinhabers einzuholen. 


\section{Kapitel 8 \\ Skizzierung und Bewertung \\ umweltpolitischer Strategien für eine zukünftige Agrar- und Umweltpolitik}

\subsection{Einführung und Überblick}

\subsubsection{Einführung}

Nach der Formulierung eines Leitbilds (Kap. 6) und der Darstellung verschiedener Gestaltungsmöglichkeiten auf der Ebene der einzelnen Bausteine der Agrarpolitik (Kap. 7) diskutiert dieses Kapitel die Optionen für eine zukunftsfähige Agrarpolitik, welche die Anliegen des Natur- und Umweltschutzes wirksam integriert, aus einer stärker strategischen Perspektive. Angestrebt wird, verschiedene mögliche Entwicklungsrichtungen darzustellen, die von der derzeitigen Architektur der Gemeinsamen Agrarpolitik der Europäischen Union ausgehen und diese jeweils so verändern, dass die in Kap. 3 dargestellten negativen Auswirkungen der Landwirtschaft auf die Natur- und Umweltgüter vermindert und die positiven Natur- und Umweltleistungen der Landwirtschaft gestärkt werden.

Wir geben zunächst einen Überblick über die strategischen Alternativen, klären die gemeinsame Minimalbasis und heben diejenigen „Bausteine“ hervor, die alle Optionen flankieren müssen. Anschließend stellen wir die drei strategischen Optionen vor. Es folgt eine Abschätzung der Stärken und Schwächen der Optionen in Bezug auf die Verwirklichung des Leitbilds einer multifunktionalen, natur- und umweltverträglichen Landwirtschaft.

\subsection{2 Überblick: Strategische Entwicklungsrichtungen für eine zukünftige Agrar- und Umweltpolitik}

Für die zukünftige Gestaltung der Agrar- und Umweltpolitik sind verschiedene Optionen vorstellbar. Im Folgenden werden drei Optionen dargestellt, die als mögliche strategische Entwicklungsrichtungen einer Agrarpolitik zu verstehen sind, die 
sich am Leitbild einer multifunktionalen, natur- und umweltverträglichen Landwirtschaft orientiert (vgl. Kap. 6). Die ersten zwei Optionen knüpfen an in der Vergangenheit begonnenen Entwicklungspfaden der Agrarpolitik an und setzen jeweils an einem der Elemente der derzeitigen agrarpolitischen Architektur an: der Ersten Säule mit den Direktzahlungen beziehungsweise der Zweiten Säule mit der Honorierung freiwilliger Agrarumweltleistungen. Die Optionen 1 und 2 stellen damit unterschiedliche Gewichtungen der eingesetzten politischen Instrumente dar (Anreize, Verordnungen und Gebote). Die dritte Option löst sich von der bisherigen Zwei-Säulen-Struktur der GAP und schlägt stattdessen ein Modell mit mehreren Ebenen vor, in denen zunehmend anspruchsvollere Umweltleistungen gestaffelt honoriert werden. Daraus ergeben sich die folgenden drei strategischen Entwicklungsrichtungen:

- Weiterentwicklung des Greenings der Ersten Säule;

- Stärkung der Zweiten Säule;

- Integriertes Modell jenseits der Zwei-Säulen-Struktur.

Eine vierte strategische Entwicklungsrichtung wäre die Umstellung der Regulierungslogik auf prinzipienbasierte Regulation. Dieser Ansatz kann grundsätzlich mit den vorigen drei Strategien kombiniert werden. Er wird als Exkurs in Abschn. 8.5 dargestellt.

Die Grundlogik der ausgewählten Optionen ist nachfolgend anhand wesentlicher Charakteristika, insbesondere der Instrumentierung, skizziert. Diese Grundlogik wird durch die Möglichkeiten der Einbindung der verschiedenen Bausteine flankiert (siehe Kap. 7). So sind beispielsweise Maßnahmen zur Verbesserung von Aus- und Fortbildung sowie Beratung (siehe Abschn. 7.7.1) in alle Optionen zu integrieren.

\subsubsection{Gemeinsamkeiten aller Optionen}

Mehrere wichtige Elemente sollten in allen Optionen enthalten sein.

1. Mindeststandards: Alle Optionen basieren auf den Grundanforderungen der Betriebsführung (GAB), deren Wirkungen auf den Natur- und Umweltschutz sich im Vergleich zur Ist-Situation dadurch verbessern, dass die Einhaltung gesetzlicher Normen konsequenter kontrolliert und sanktioniert wird (Verminderung des Vollzugsdefizites). Der gesetzliche Mindeststandard sollte überprüft und gegebenenfalls nachjustiert (wie z. B. in der aktuellen Novellierung der Düngeverordnung) und erweitert werden (z. B. Grünlandumbruchverbot, Schutz von Landschaftselementen). Über die gesetzlichen Mindeststandards können die Natur- und Umwelteffekte auch derjenigen landwirtschaftlichen Betriebe, die auf Direktzahlungen und die dafür notwendige ökologische Leistungserbringung verzichten, eingegrenzt werden. 
2. Eine Reduzierung der bürokratischen Belastungen durch die Nutzung neuer digitaler Informationssysteme (z. B. Nutzung von Remote Sensing- und GIS-Daten) sollte angestrebt werden.

Bei allen Optionen sollten zudem die folgenden Elemente gestärkt werden:

1. Beratung und Unterstützung der Landwirte/Öffentlichkeitsarbeit:

- Um die Akzeptanz für Natur- und Umweltschutzbelange bei Landwirtinnen und Landwirten zu erhöhen und Programme zielorientierter und flexibler einzusetzen, könnten einzelbetriebliche Umweltberatungen und gesamtbetriebliche Beratungen angeboten werden. Diese Beratungsansätze würden darüber hinaus der Rückkopplung mit den Landwirtinnen und Landwirten über die Beratungsstellen dienen, um eventuelle Missstände des Bewertungssystems (siehe folgender Punkt 2) zu erkennen und gegebenenfalls Anpassungen vorzunehmen.

- Die regelmäßige Teilnahme von Landwirtinnen und Landwirten an Fortbildungskursen und Tagungen sollte unterstützt werden, um aktuelle Leistungsbewertungen und Agrarumwelt- und Klimaschutzmaßnahmen zu vermitteln. Die Teilnahme an solchen Veranstaltungen könnte beispielsweise über ein Top-up zu anderen Prämienelementen honoriert werden.

- Den Landwirtinnen und Landwirten sollten gut verständliche Informationsmedien, wie z. B. Filme, Handbücher und andere Publikationen, zur Verfügung gestellt werden.

- Den Landwirtinnen und Landwirten sollten regelmäßig Benchmarks zur Verfügung gestellt werden, um ihre einzelbetriebliche Situation im regionalen und überregionalen Kontext hinsichtlich verschiedener Kriterien zu vergleichen.

2. Monitoring und Evaluierung/Adaptives Management

- Das Monitoring der freiwilligen AUKM sollte nach Möglichkeit mit dem Monitoring der Maßnahmen, die an die Direktzahlungen geknüpft sind, gekoppelt werden, um den Kontrollaufwand für alle Beteiligten zu minimieren.

- Das Monitoring sollte so gestaltet werden, dass es ein adaptives Ressourcenmanagement auf Landschaftsebene ermöglicht, um gegebenenfalls Maßnahmen anzupassen, falls Ziele nicht erreicht werden, und um auf neue Erkenntnisse und unerwartete Entwicklungen reagieren zu können.

3. Zahlung zur Aufrechterhaltung der Landbewirtschaftung in Gebietskulissen

- Darüber hinaus könnten Landwirte Zahlungen für die Bewirtschaftung von Standorten erhalten, an denen andernfalls eine großflächige Aufgabe der Landbewirtschaftung eintreten könnte und dies nicht erwünscht ist. Die Höhe einer solche Zahlung sollte sich vorrangig am Ziel orientieren, die flächendeckende Landbewirtschaftung in Zielgebieten aufrecht zu erhalten. Angesichts der mittlerweile vorhandenen feinskaligen GIS-Systeme sollte angestrebt werden, im Sinne einer höheren Zielgenauigkeit diese Zahlungen von einer regionsbezogenen auf eine standörtliche Förderung umzustellen. 
- Bei einer solchen Zahlung sollten ausschließlich standörtliche Indikatoren zur Ermittlung der Höhe der zur Fortsetzung der Bewirtschaftung notwendigen Unterstützung herangezogen werden. Regionale und soziale Indikatoren, wie bisher z. B. die Bevölkerungsentwicklung, würden nicht in eine solche Systematik passen.

- Die Höhe der Zahlungen sollte dabei gestaffelt werden und sich an den Opportunitätskosten der Bewirtschaftung des jeweiligen Standorts (im Vergleich zur nicht-landwirtschaftlichen Nutzung oder der Nicht-Bewirtschaftung) orientieren.

Alle Optionen können eine zeitliche Entwicklung aufweisen, in deren Verlauf das Anforderungsniveau ansteigen kann. In dem Maße, wie das Anforderungsniveau der Mindeststandards steigt, würden auch die Ansprüche an Maßnahmen oder Ergebnisse steigen, die darüber hinaus, etwa in AUKM, honoriert werden können (Additionalität, Vermeidung der Mehrfachentlohnung einer Leistung). Die Begründung für eine Erhöhung der Ansprüche im Zeitablauf ergibt sich zum einen aus dem agronomischen Fortschritt, zum anderen aus einer zu erwartenden zunehmenden Knappheit an Umweltgütern, die höhere Standards erfordern.

Wir kommen nun zur Darstellung und Diskussion der drei strategischen Entwicklungsoptionen der GAP.

\subsection{Option 1 - Weiterentwicklung des Greenings der Ersten Säule}

\subsubsection{Zugrundeliegende Idee}

Die Grundidee dieser Option ist, die flächendeckende Einbeziehung eines Großteils der landwirtschaftlichen Flächen und Betriebe in der derzeitigen Ersten Säule der GAP dazu zu nutzen, die Naturschutz- und Umweltleistungen in der EU flächendeckend zu verbessern. Dabei wird davon ausgegangen, dass die erheblichen Finanzmittel für die Erste Säule weiterhin in ähnlichem Maße zur Verfügung stehen. Der wichtigste Mechanismus zur Realisierung einer multifunktionalen, natur- und umweltverträglichen Landwirtschaft ist die Erhöhung der Anforderungen zunächst im Greening und perspektivisch eventuell auch im GLÖZ. Abb. 8.1 stellt den Ansatz der Option dar.

Option 1 führt damit die grundlegende Architektur der momentanen Agrarpolitik weiter. Jedoch wird der Zahlungsanspruch verstärkt an eine tatsächliche Leistungserbringung gekoppelt und erhält zunehmend den Charakter einer Honorierung von Gemeinwohlleistungen. Im Zeitablauf könnten ggf. die Anforderungen, welche Voraussetzung für den Bezug von Basiszahlungen aus der Ersten Säule sind, schrittweise erhöht werden (z. B. aufgrund der Etablierung des technischen Fortschritts für umweltfreundliche Verfahren). 


\section{Auflagen / zu erreichender \\ Umweltzustand}

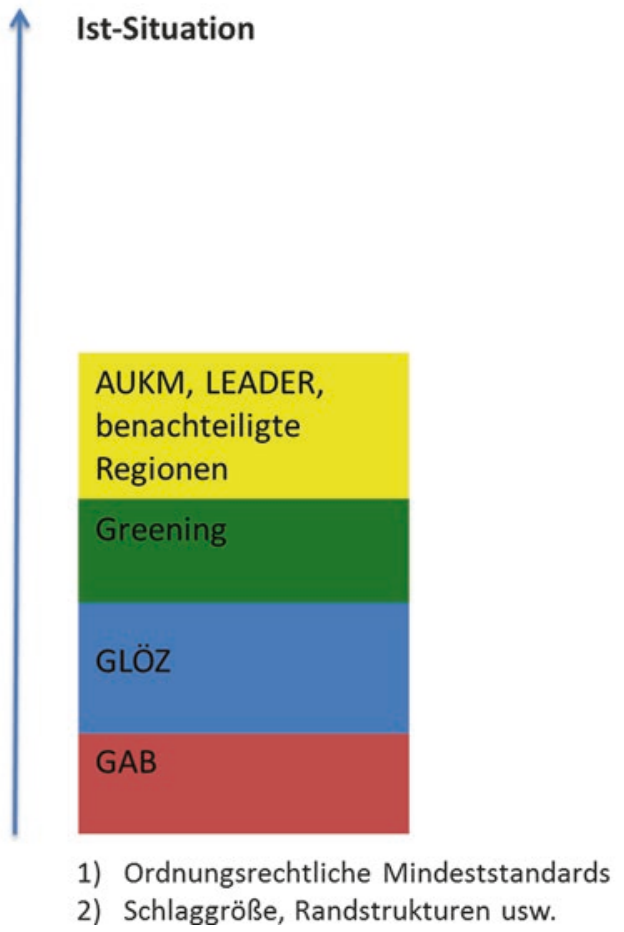

Option 1:

Weitere Begrünung der Ersten Säule

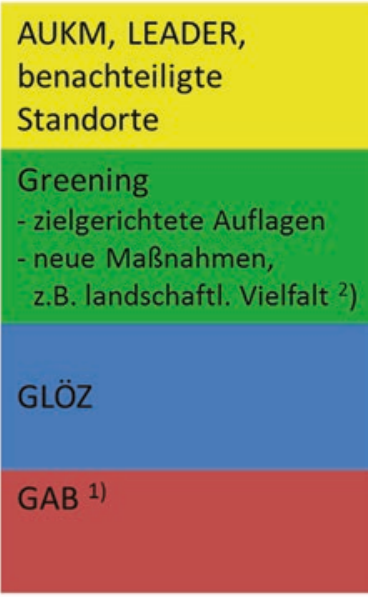

Abb. 8.1 Option 1 - Weiterentwicklung des Greenings der Ersten Säule (Quelle: Eigene Darstellung)

In diesem Modell setzen sich die Direktzahlungen aus zwei Komponenten zusammen:

- Eine Basisprämie zur Abgeltung regulationsbedingt höherer Kosten in der EU im Vergleich zu den internationalen Wettbewerbern auf wissenschaftlich gesicherter Basis. Hier ist zu diskutieren, ob dafür weiterhin ein Cross-Compliance-Mechanismus notwendig ist. Wenn ein Zusammenhang zwischen den Mehrkosten der höheren EU-Standards und der Höhe der Basisprämie bestehen soll, würde letztere deutlich unterhalb der ab 2019 gewährten Flächenprämie von 196/€ ha liegen. ${ }^{1}$

- Eine pauschalierte Prämie für flächenbezogene betriebliche Leistungen im Natur- und Umweltschutz (oberhalb von GLÖZ). Als anspruchsvollere Form des Greenings würde der Bezug eines Teils der Direktzahlungen an einige relativ allgemein gehaltene Leistungen gekoppelt.

${ }^{1}$ Direktzahlung von $281 € /$ ha abzüglich der Greening-Prämie von $85 € /$ ha $=196 € /$ ha. 
Vorstellbar für die Umsetzung eines reformierten Greenings sind zwei Varianten:

Variante 1: Es werden Maßnahmen formuliert, die von jedem landwirtschaftlichen Betrieb zu erbringen sind, der an diesem System teilnehmen will. Dies entspricht dem heutigen Ansatz des Greenings. Dabei würden solche Maßnahmen definiert, die einen Mehrwert für den Natur- und Umweltschutz erbringen. In Bezug auf das heutige Greening könnte dies beispielsweise bedeuten:

- Reduzierung der Gestaltungsmöglichkeiten der Ökologischen Vorrangflächen mit geringem ökologischem Mehrwert (z. B. Anbau von Eiweißpflanzen, Erlaubnis der Anwendung von Pflanzenschutzmitteln);

- Beschränkung der Hauptfrucht auf $50 \%$.

Auch können weitere sinnvolle Maßnahmen aufgenommen werden (siehe Abschn. 7.3.8).

Variante 2: Es wird ein „Baukasten“ an Maßnahmen angeboten, aus denen die Landwirte diejenigen wählen, die sie auf ihrem Betrieb umsetzen (siehe Abschn. 7.3.8). Mit der Maßnahmenumsetzung wäre eine Mindestanzahl an Punkten zu erreichen, um diese Prämienkomponente zu erhalten.

Die Finanzierung der Direktzahlungen könnte in dieser Option weiterhin zu $100 \%$ aus dem EU-Haushalt der Gemeinsamen Agrarpolitik erfolgen. Dies sollte auch dann gelten, falls die Variante gewählt wird, die Zuständigkeit für die Programmierung der Maßnahmen-Module für die Greening-Komponente der Direktzahlungen bei den Mitgliedstaaten (nach Vorgaben der EU und unter Kontrolle der EU-Kommission) anzusiedeln.

Gegebenenfalls könnte eine Prämie für die Aufrechterhaltung der Landbewirtschaftung in ausgewählten Gebietskulissen mit erschwerten Bewirtschaftungsbedingungen hinzukommen (mit standörtlicher Abgrenzung im Gegensatz zu der heute angewendeten regionalen Abgrenzung, siehe oben).

Weiterhin könnten im Rahmen der Zweiten Säule weiterhin freiwillige, gezielte Maßnahmen auf spezifischen Flächen (Flächenbezug) gefördert werden, etwa bestimmte Bewirtschaftungsverfahren zur Sicherung der Biodiversität (z. B. Spätmahd von Orchideenwiesen, Bewirtschaftung von Natura 2000-Flächen).

Tab. 8.1 gibt einen Überblick über die Architektur der strategischen Option 1.

\subsubsection{Einbettung in die agrarpolitische Diskussion}

Mit dieser Option würde der in den beiden GAP-Reformen seit 2003 eingeschlagene Weg fortgesetzt, den Erhalt von Direktzahlungen mit der Einhaltung von Natur- und Umweltschutzauflagen zu verknüpfen. Die Option geht mit einer Verschiebung der Begründung für die Gewährung von Direktzahlungen an die Landwirtschaft einher. Sie geht davon aus, dass diese immer weniger ,mit den ursprünglichen Argumenten ,Einkommensausgleich“ und ,Vertrauensschutz" zu begründen“" sind (Wissenschaftlicher Beirat für Agrarpolitik beim BMELV 2010). Stattdessen beruht Option 1 auf dem Argument, dass die Zahlungen an die Landwirtschaft der Bereitstellung öffentlicher 
Tab. 8.1 Überblick über die Elemente der strategischen Option 1 (Weiterentwicklung des Greenings der Ersten Säule)

\begin{tabular}{|c|c|c|}
\hline Element & Anforderung & Varianten \\
\hline $\begin{array}{l}\text { Ordnungsrechtli- } \\
\text { che Mindeststan- } \\
\text { dards }\end{array}$ & - $\mathrm{GAB}$ & - Keine \\
\hline - Basisprämie & - GLÖZ & $\begin{array}{l}\text { Mit oder ohne Cross Compli- } \\
\text { ance-Mechanismus } \\
\text { - Modulation (degressive } \\
\text { Ausgestaltung) } \\
\text { - Obergrenze (Capping) }\end{array}$ \\
\hline - Greening-Prämie & $\begin{array}{l}\text { Flächenbezogene, relativ } \\
\text { allgemein gehaltene, aber } \\
\text { höhere Leistungen } \\
\text { - Eventuell höhere Anforderun- } \\
\text { gen im Zeitablauf }\end{array}$ & $\begin{array}{l}\text { - Opt-In oder Opt-Out der } \\
\text { Landwirte } \\
\text { - EU-weite, nationale oder } \\
\text { regionale Spezifizierung der } \\
\text { Anforderungen } \\
\text { - Art der Anforderungen } \\
\text { - Ausnahmen von Modulation } \\
\text { und Capping }\end{array}$ \\
\hline $\begin{array}{l}\text { Zahlung für } \\
\text { benachteiligte } \\
\text { Standorte }\end{array}$ & $\begin{array}{l}\text { Bewirtschaftung eines } \\
\text { benachteiligten Standorts. } \\
\text { - Gestaffelte Höhe der } \\
\text { Zahlungen }\end{array}$ & $\begin{array}{l}\text { - Räumlicher Zuschnitt (Standort } \\
\text { statt Region) und je nach } \\
\text { Standortnachteil (Opportuni- } \\
\text { tätskosten) gestaffelte Höhe der } \\
\text { Zahlungen }\end{array}$ \\
\hline - $\mathrm{AUKM}$ & $\begin{array}{l}\text { Freiwillige vertragliche } \\
\text { Opt-in-Maßnahmen im } \\
\text { Rahmen der ländlichen } \\
\text { Entwicklungsprogramme }\end{array}$ & $\begin{array}{l}\text { - Ausgestaltung der AUKM- } \\
\text { Programme }\end{array}$ \\
\hline
\end{tabular}

Güter dienen sollen (SRU 2009, S. 5; Kommission Landwirtschaft am Umweltbundesamt 2011, S. 4), darunter insbesondere Natur- und Umweltschutzleistungen. Daher wird in dieser Option eine vermehrte Erbringung solcher Leistungen von zumindest denjenigen landwirtschaftlichen Betrieben eingefordert, die weiterhin Direktzahlungen erhalten wollen. Die im Zusammenhang mit der Ausgestaltung des Greenings bestehende Kritik der unzureichenden Zielerreichung führt dazu, dass über eine „Verschärfung“ der Greening-Auflagen nachgedacht wird. Aktuell ist ein Großteil der landwirtschaftlichen Flächen und Betriebe in die derzeitige Erste Säule der GAP eingebunden. Dies legt die Überlegung nahe, dass mit den Direktzahlungen verknüpfte Natur- und Umweltschutzauflagen flächendeckend zu Verbesserungen des Zustandes der Ressourcen des Naturschutzes in der EU führen könnten.

\subsubsection{Instrumentelle Ausgestaltung}

Basisprämie: Die Landwirte könnten weiterhin eine Basiszahlung pro Hektar bei Einhaltung der GLÖZ-Auflagen erhalten. Um dem Vorwurf zu begegnen, dass landwirtschaftliche Betriebe Zahlungen für die bloße Einhaltung von Gesetzen 
und Anforderungen, die der guten fachlichen Praxis entsprechen, erhalten, könnten die Direktzahlungen an den wissenschaftlichen Nachweis geknüpft werden, dass regulationsbedingte Mehrkosten gegenüber Mitbewerbern von außerhalb der EU entstehen.

Anforderungen in der Greening-Komponente: Um zukünftig vermehrt Natur- und Umweltleistungen der Landwirtschaft zu realisieren, wäre eine effektivere Ausgestaltung der Greening-Auflagen denkbar. Hierfür wird z. B. vorgeschlagen, die Möglichkeiten der Ausgestaltung der ökologischen Vorrangflächen vermehrt auf den Biodiversitätsschutz auszurichten, d. h. aktuelle Möglichkeiten der ökologisch geringwertigen Nutzung von ÖVF einzuschränken (Hart et al. 2016) oder die Beschränkung des Anbaus einer Fruchtart auf maximal $50 \%$ der Ackerfläche (Expertengespräche). Des Weiteren könnte über weitere Auflagen, wie eine Beschränkung des Stickstoffsaldos auf $50 \mathrm{~kg}$ N/ha LF und der Tierbesatzdichte auf max. 2,0 GV/ha LF (Kommission Landwirtschaft am Umweltbundesamt 2011, S. 7 ff.), die Begrenzung des Einsatzes von Dünge- und Pflanzenschutzmitteln (Hart et al. 2016) bzw. eine Fokussierung der Maßnahmen auf Kohlenstoff-, Nährstoff- und Biodiversitätsmanagement (Hart et al. 2016), nachgedacht werden. Auch könnte ein Ansatz zur Honorierung kleiner Schlagbzw. Gewanngrößen und Randstrukturen integriert werden (Ansatz zur Honorierung von landschaftlicher Vielfalt). Durch die Reduktion von Ausnahmeregelungen könnte auch der bürokratische Aufwand reduziert werden (Hart et al. 2016).

Höhe der Zahlungen: Allerdings ist zu berücksichtigen, dass die Teilnahme der Landwirte an dem System der Direktzahlungen (und des Greenings) freiwillig ist und die hohe Teilnahmebereitschaft darauf beruht, dass die Zahlungen je Hektar so hoch sind, dass eine Teilnahme selbst auf sehr guten Standorten meist ökonomisch vorteilhaft ist (de Witte und Latacz-Lohmann 2014). Bisher verzichten ca. 7 \% der Betriebe in Deutschland auf Direktzahlungen (BMELV 2013a; BMEL 2015d). Eine weiterhin hohe Teilnahme wird nur durch relativ hohe Direktzahlungen pro Hektar erreicht werden können. An weniger produktiven Standorten, wo die Opportunitätskosten des Greenings niedriger sind, ist jedoch mit hohen Mitnahmeeffekten zu rechnen, so dass von einem wenig effizienten Einsatz von Steuergeldern auszugehen ist. Es ist jedoch $\mathrm{zu}$ fordern, dass die Instrumente der GAP ,die angestrebten Ziele zu den geringsten oder doch jedenfalls vertretbaren volkswirtschaftlichen Kosten erreich(en)" (Wissenschaftlicher Beirat für Agrarpolitik beim BMELV 2010). Bei sinkenden Hektarprämien im Verhältnis zu den Erzeugerpreisen könnten vor allem in intensiv genutzten Regionen, in denen die Betriebe durch die Auflagen wesentlich beeinflusst werden, viele Landwirtinnen und Landwirte aus dem System ,aussteigen“.

Ausgestaltung als Opt-in oder Opt-out: Grundsätzlich ist bereits heute der Bezug von Direktzahlungen freiwillig. Die Teilnahme an den Direktzahlungsprogrammen insgesamt, wie insbesondere die Teilnahme am Greening, kann daher im Prinzip entweder als Opt-in- oder als Opt-out-Variante angeboten werden. Beide Varianten eröffnen den Landwirten die Möglichkeit, sich zwischen der Einhaltung der höheren Auflagen und dem Erhalt der Zahlungen einerseits bzw. dem Verzicht auf die Zahlungen bei Verpflichtung auf die niedrigeren Anforderungen der GAB zu entscheiden. Eine Ausgestaltung als Opt-out unterstützt jedoch die Kommunikation einer sozialen Norm, dass Landwirte normalerweise die höheren Natur- und Umweltleistungen 
erbringen. Andererseits besteht dann bei Landwirten möglicherweise das Gefühl, das man ihnen ,ihr' Geld wegnimmt, wenn es zu Sanktionen kommt. Daher kann eine Ausgestaltung von Direktzahlungen und Greening als Opt-in eine positive Einstellung der Landwirte gegenüber den zu erbringenden Leistungen fördern. In diesem Fall sollte der Akt des Opt-in aber sehr einfach ausgestaltet sein, um unnötige Barrieren zu vermeiden.

Natur- und Umweltschutzeffekt: Da die Auflagen, die mit dem Erhalt von Direktzahlungen verbunden sind, weitgehend undifferenziert für alle Betriebstypen und unabhängig von standörtlichen Gegebenheiten gelten, ist bisher auch ein geringer positiver Effekt für den Natur- und Umweltschutz festzustellen. Der WBA merkte 2010 daher an, dass eine flächendeckende Gewährung einzelbetrieblicher Zahlungen an alle Landwirte wenig erfolgsversprechend erscheint (Wissenschaftlicher Beirat für Agrarpolitik beim BMELV 2010, S. 29). Allerdings kann hierauf durch die Gestaltung der Auflagen wesentlich Einfluss genommen werden.

Prämien zur Aufrechterhaltung der Bewirtschaftung in Gebietskulissen mit erschwerten Bewirtschaftungsbedingungen: Die Förderung benachteiligter Standorte könnte weiterhin in der Zweiten Säule und im Rahmen der ländlichen Entwicklungsprogramme erfolgen. Aufgrund der starken Streueffekte wäre aber eine Ausgestaltung als standortspezifische Prämie zur Aufrechterhaltung der Landbewirtschaftung in Gebietskulissen, wo dies gesellschaftlich erwünscht ist, zu bevorzugen.

AUKM und LEADER: Neben der Förderung der Landwirte über die Direktzahlungen aus der Ersten Säule würde bei dieser Politikoption die bestehende Förderung von freiwilligen, gezielten Agrarumwelt- und Klimaschutzmaßnahmen (AUKM) und LEADER-Projekten durch die Zweite Säule der GAP fortgeführt. Empfangsberechtigt wären jedoch nur solche Betriebe, die einen ökologischen Leistungsnachweis in der Ersten Säule erbringen. Die Maßnahmen der Zweiten Säule würden sich weiterhin auf spezifische Problemlagen und Flächen beziehen, etwa zur Förderung von gezielten Bewirtschaftungsverfahren (z. B. Spätmahd von Orchideenwiesen, Bewirtschaftung von Natura 2000-Flächen). Durch Erhöhung der Anforderungen im Greening könnte sich u. U. das Anforderungsniveau der Maßnahmen in der Zweiten Säule erhöhen. Hierdurch würden jedoch auch finanzielle Mittel für weitergehende Maßnahmen in der Zweiten Säule freigesetzt. Ansätze zur Optimierung der Zweiten Säule wie in Option 2 und Kap. 7 beschrieben, könnten auch in dieser Option umgesetzt werden.

Betriebsgrößenbezogene Elemente: Diese Option könnte weiterhin eine Kleinbetriebsregelung, eine Degression oder Kappung der Basiszahlungen oder eine Zusatzförderung für Junglandwirte vorsehen.

\subsubsection{Diskussion und Bewertung}

Insgesamt würde es sich bei Option 1 um eine inkrementelle Weiterentwicklung des bisherigen Entwicklungspfads der GAP handeln. Die politischen Instrumente sind im Wesentlichen etabliert, würden in dieser Option aber anspruchsvoller ausgestaltet 
werden. Das setzt einen entsprechenden politischen Willen und Mehrheiten voraus. Dem Einwand, dass es Direktzahlungen allein für die Einhaltung der Gesetze gibt, würde dadurch begegnet, dass es diese erst für die Einhaltung der GLÖZ-Anforderungen und darüber hinaus gehende Leistungen gibt.

Entwicklung im Zeitablauf: Auch im Zeitablauf würde diese Option eine inkrementelle Weiterentwicklung des derzeitigen Pfads der GAP darstellen. Konsequent fortgeführt, eröffnet diese Option die Perspektive eines kumulativen Paradigmenwechsels zu einer nachhaltigen, multifunktionalen Landwirtschaft. Ein Wechsel zu den Optionen 2 und 3 (siehe unten) wäre grundsätzlich möglich. So wäre als Variante eine Nationalisierung oder Regionalisierung der Anforderungen im Rahmen eines allgemeinen Systems denkbar, die notifiziert und von der EU-Kommission genehmigt werden müssten. Dies würde die Option 1 in Richtung Option 2 entwickeln.

Machbarkeit: Wegen der relativ geringen Neuheit der Instrumenten-Architektur ist dies vermutlich die am einfachsten zu implementierende der drei Optionen. Da die Förderungsstruktur an das bestehende System der Agrarverwaltung anknüpfen würde, erscheint ein derartiges Fördersystem praktisch möglich und würde durch die vermehrte Nutzung von Fernerkundung und GIS-Technologien perspektivisch Vereinfachungen in den Verwaltungsabläufen beinhalten.

Effektivität und Effizienz: Es ist plausibel, dass diese Option zu deutlichen Verbesserungen im Natur- und Umweltschutz beitragen könnte, wenn das Greening anspruchsvoller würde. Allerdings verspricht diese Option eine lediglich inkrementelle Verbesserung bei den Problemen der mangelnden Zielgenauigkeit und der Überkompensierung der derzeitigen Direktzahlungen.

Variante: Das Greening könnte auch als „Baukasten“ angeboten werden, wie er in Baustein 3 (Abschn. 7.3) skizziert wurde und dem Vorschlag des DVL entspricht. Dabei könnten die Betriebe Maßnahmen wählen, die sie umsetzten möchten. Um die Greening-Prämie zu erhalten, wäre dann eine Mindestpunktzahl durch die gewählten Maßnahmen zu erreichen. Bei dieser Variante würden nicht in allen Regionen die gleichen Maßnahmen umgesetzt werden. Allerdings wäre durch diese Variante eine höhere Akzeptanz bei den Landwirten zu erwarten.

Implikationen und mögliche Nebenwirkungen:

- Auch wenn bereits die Basisprämie in dieser Option an die Einhaltung der gegenüber den GAB höheren Anforderungen der GLÖZ geknüpft bliebe, würde sie auch Einkommensschwankungen an den Produktmärkten ausgleichen und damit der Einkommensverstetigung der landwirtschaftlichen Betriebe dienen, sofern nicht die Prämie durch Erhöhung der Pachtpreise an die Grundbesitzer „,durchgereicht" wird. Gleiches gilt für die Greening-Prämie, und zwar auch dann, wenn das Anforderungsniveau steigt. Sowohl die Basis- als auch die GreeningPrämie erhöhen damit die ökonomische Resilienz der Betriebe.

- Es besteht die Möglichkeit, dass Landwirte aus den Direktzahlungen aussteigen, wenn die Anforderungen höher werden. Dies könnte zu Verschlechterungen der Umweltsituation führen, v. a. wenn dieser Effekt in einzelnen Regionen flächendeckend auftritt. 
- Durch den inkrementellen Ansatz verbleibt diese Option im Rahmen des derzeitigen agrarpolitischen Pfads. Das könnte einerseits dazu führen, dass die Impulse für technische und agronomische Innovationen im Natur- und Umweltschutz relativ begrenzt bleiben. Andererseits würden die politischen und ökonomischen Ungewissheiten für die Landwirte begrenzt.

- Akteurskoalitionen: Es ist plausibel, dass sich hinter einer solchen Option eine moderate Reformkoalition bilden könnte. Das würde allerdings von den perzipierten Alternativen abhängen. Der Umstand, dass die Standards und Anforderungen sichtbar und für alle landwirtschaftlichen Betriebe, die weiterhin Direktzahlungen erhalten wollen, erhöht werden, ohne dass es zu höheren Zahlungen kommt, könnte zu Widerständen aus der Landwirtschaft führen.

\subsection{Option 2 - Stärkung der Zweiten Säule}

\subsubsection{Zugrundeliegende Idee}

Während Option 1 Verbesserungen im Natur- und Umweltschutz vorwiegend dadurch zu erreichen sucht, dass die bestehenden Direktzahlungen an zunehmend höhere Anforderungen in der Ersten Säule geknüpft werden, setzt Option 2 auf eine Verlagerung der finanziellen Mittel in die Zweite Säule.

Option 2 sieht ein Abschmelzen der Direktzahlungen in der Ersten Säule und eine Umschichtung der dadurch frei werdenden Mittel in Haushaltstitel vor, aus denen gezielte AUK-Maßnahmen oder Zahlungen für benachteiligte Standorte finanziert werden. Auf diese Weise wird angestrebt, zum einen die flächenbezogenen Zahlungen zielgenauer auf diejenigen Gebiete zu konzentrieren, in denen diese zur Aufrechterhaltung einer natur- und umweltgerechten Landwirtschaft notwendig sind. Vor allem aber würden mehr Mittel für standortrelevante Natur-, Umwelt- und Klimaschutzmaßnahmen bereitstehen, die dadurch anspruchsvoller ausgestaltet werden können. Die folgende Abb. 8.2 stellt Option 2 im Vergleich zur Ist-Situation dar.

\subsubsection{Einbettung in die agrarpolitische Diskussion}

Option 2 knüpft an der erheblichen Kritik an, die seit langem an den Direktzahlungen der Ersten Säule geübt wird. Die Option nimmt den Einwand ernst, dass die relativ pauschalen Auflagen, wie sie derzeit im Allgemeinen in der Ersten Säule zur Voraussetzung zum Erhalt von Direktzahlungen gemacht werden, nur sehr basale Naturund Umweltschutzeffekte erzielen können. Des Weiteren wird kritisiert, dass die Direktzahlungen in der Ersten Säule der GAP für einen Großteil der landwirtschaftlichen Betriebe und Flächen eine Überkompensation der Opportunitätskosten darstellen, welche sich aus den Grundanforderungen der Betriebsführung und darüber hinausgehenden GLÖZ- und Greening-Anforderungen ergeben. Sie verstoßen daher 


\section{Auflagen / zu erreichender \\ Umweltzustand}

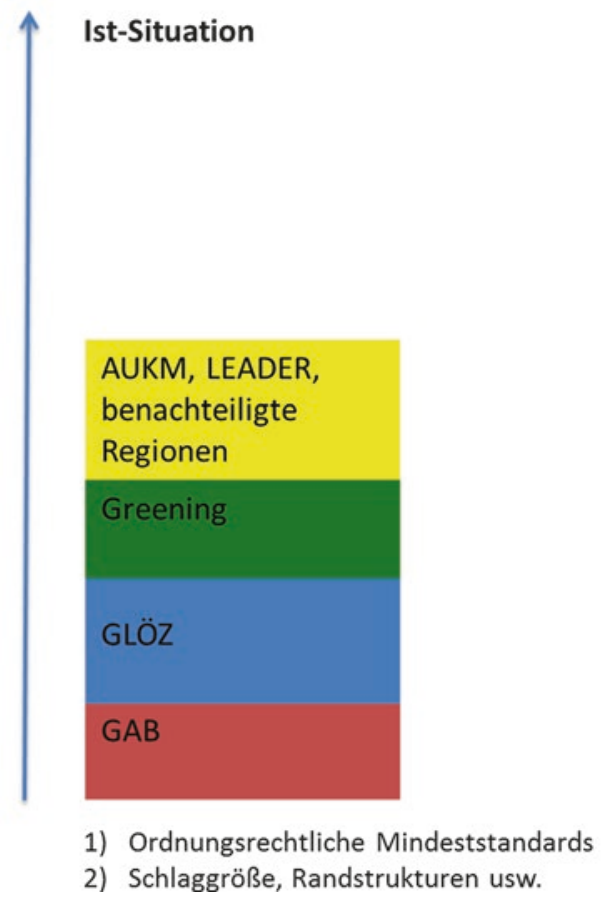

Option 2:

Ausbau der Zweiten Säule

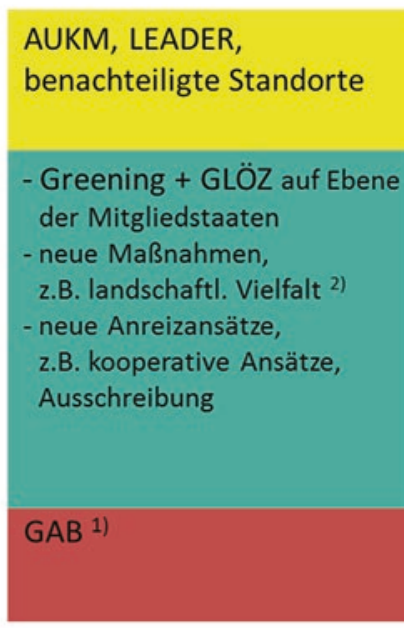

Abb. 8.2 Option 2 - Eine starke Zweite Säule. (Quelle: Eigene Darstellung)

nach verbreiteter wissenschaftlicher und politischer Kritik in ihrer heutigen Ausgestaltung gegen das Prinzip der effizienten Mittelverwendung. Auch als Instrument der Einkommensstützung für landwirtschaftliche Erzeuger gelten die Direktzahlungen in ihrer heutigen Ausgestaltung im Allgemeinen als nicht effizient, da es keine systematische Verknüpfung mit der Bedürftigkeit der Empfänger gibt und es offenbar in Regionen mit hohem Pachtanteil zu erheblichen Überwälzungseffekten kommt. Direktzahlungen, ,die nicht auf die ländlichen Problemregionen fokussiert sind“ und zudem häufig an die Grundeigentümer überwälzt werden (Wissenschaftlicher Beirat für Agrarpolitik beim BMELV 2010, S. 27), sind daher als Instrument zur Förderung einer multifunktionalen, natur- und umweltverträglichen Landwirtschaft weder effektiv noch effizient. Eine Kürzung des finanziellen Volumens der Ersten Säule wird etwa vom Wissenschaftlichen Beirat für Agrarpolitik beim BMELV (2010) schon lange gefordert.

Durch die Einführung der Greening-Auflagen in der Ersten Säule war ursprünglich angestrebt worden, das Ausgangsniveau für AUK-Maßnahmen in der Zweiten Säule zu erhöhen, so dass - selbst bei stagnierendem Finanzvolumen der Zweiten Säule, mit den AUK-Maßnahmen vermehrt ambitioniertere Ziele verfolgt werden können und - wegen des Doppelförderungsverbots - müssen (Hart et al. 2016). 
Diese Hoffnung des Natur- und Umweltschutzes erfüllte sich angesichts einer relativ anspruchslosen Ausgestaltung der Greening-Anforderungen in vielen Mitgliedstaaten nicht; zudem wurden die für AUK-Maßnahmen zur Verfügung stehenden Mittel vielerorts reduziert (Hart et al. 2016). Im Rahmen der freiwilligen Modulation haben die Mitgliedstaaten bereits heute die Möglichkeit, Finanzmittel von der Ersten in die Zweite Säule zu verlagern. In Deutschland wurden im Jahr 2015 4,5 \% der Mittel umgeschichtet, bis 2018 könnte sich dieser Anteil bis auf $15 \%$ erhöhen (BMEL 2015h).

\subsubsection{Grundlegende Struktur}

In der Grundstruktur entsprechen die Elemente der Option 2 (siehe Tab. 8.2) zunächst denen der Option 1. Durch die Verlagerung der finanziellen Mittel würden jedoch die Basisprämie und das Greening an Bedeutung verlieren. Angesichts des Abschmelzens der Basisprämie wäre es schwer zu vermitteln, die GLÖZ-Standards zu erhöhen. Das Greening (und GLÖZ) könnte in dieser Option nationalisiert oder regionalisiert werden (was nicht zwangsläufig mit einer Umstellung von Voll- auf Ko-Finanzierung einhergehen müsste, siehe unten). Da für die Ausgestaltung der Zahlungen für benachteiligte Standorte und vor allem für AUK-Maßnahmen mehr Geld bereit stehen würde, wäre hier eine differenziertere und anspruchsvollere Ausgestaltung, auch mit neuen Instrumenten, möglich (siehe Abschn. 8.4 zu Option 3).

Tab. 8.2 Abgrenzung der strategischen Option 2 (Starke Zweite Säule) von Option 1

\begin{tabular}{l|l|l}
\hline Element & Anforderung & Unterschied zu Option 1 \\
\hline $\begin{array}{l}\text { Ordnungsrechtli- } \\
\text { che Mindeststan- } \\
\text { dards }\end{array}$ & $\begin{array}{l}\text { GAB } \\
\text { - Basisprämie }\end{array}$ & $\begin{array}{l}\text { Verstärkte Bedeutung für die } \\
\text { flächendeckende Durchsetzung } \\
\text { der Mindeststandards, falls } \\
\text { Direktzahlungen langfristig } \\
\text { ganz abgeschmolzen werden }\end{array}$ \\
\hline - Greening-Prämie & $\begin{array}{l}\text { Flächenbezogene, relativ } \\
\text { allgemein gehaltene } \\
\text { Leistungen; } \\
\text { höhere Anforderungen im } \\
\text { Zeitablauf }\end{array}$ & $\begin{array}{l}\text { Eventuell Nationalisierung oder } \\
\text { Regionalisierung im Zeitablauf }\end{array}$ \\
\hline $\begin{array}{l}\text { Zahlung für } \\
\text { benachteiligte } \\
\text { Standorte }\end{array}$ & $\begin{array}{l}\text { Nationalisierung oder } \\
\text { benachteiligten Standorts; } \\
\text { gestaffelte Höhe der } \\
\text { Zahlungen }\end{array}$ & $\begin{array}{l}\text { Eventuell mehr Finanzmittel, } \\
\text { Priorität liegt aber bei AUKM }\end{array}$ \\
\hline AUKM & $\begin{array}{l}\text { Freiwillige Opt-in-Maßnah- } \\
\text { men im Rahmen der } \\
\text { ländlichen Entwicklungspro- } \\
\text { gramme }\end{array}$ & $\begin{array}{l}\text { Mehr Finanzmittel erlauben } \\
\text { anspruchsvollere Ausgestaltung } \\
\text { der AUKM-Programme }\end{array}$ \\
\hline
\end{tabular}




\subsubsection{Instrumentelle Ausgestaltung}

In Option 2 würde das finanzielle Volumen der Ersten Säule stark gekürzt. Aus Sicht des Natur- und Umweltschutzes sollte dies sowohl die Basisprämie als auch die Greening-Prämie betreffen (Hart et al. 2016). In der Diskussion ist eine Basisprämie in Höhe von ca. 20 bis 50 €/ha (Plankl et al. 2010; Wissenschaftlicher Beirat für Agrarpolitik beim BMELV 2010; Wissenschaftlicher Beirat für Agrarpolitik beim BMEL 2015) bei Einhaltung von GAB und GLÖZ, die mit den Kostennachteilen durch höhere gesetzlichen Mindeststandards gegenüber den Wettbewerbern auf dem Weltmarkt gerechtfertigt werden könnte. Die freiwerdenden Mittel würden in die Zweite Säule umgeschichtet und stünden für Zahlungen für benachteiligte Standorte und AUKM zur Verfügung.

Die Ausgestaltung der Greening-Auflagen könnte nationalisiert oder regionalisiert werden. Die Mitgliedstaaten hätten dann einen flächendeckenden GreeningAnsatz umzusetzen. Von der Systemlogik könnte ein solches nationalisiertes oder regionalisiertes Greening sowohl als nationale Differenzierung innerhalb der Ersten Säule wie auch als verpflichtende Komponente der ländlichen Entwicklungsprogramme in der Zweiten Säule verankert werden. Das Finanzvolumen dieser Komponente könnte sich an der Fläche der jeweiligen Region orientieren. Die Mittel der derzeitigen Greening-Komponente der betrieblichen Direktzahlungen würden dann beispielsweise in einen „Regionstopf“ fließen. Den Mitgliedstaaten stünde es dann frei, ob und in welchem Maße sie bei der Ausgestaltung der nationalen oder regionalen Greening-Programme Umverteilungen in Kauf nehmen wollen. Aus Sicht des Natur- und Umweltschutzes wäre jedoch anzustreben, dass die Greening-Maßnahmen anspruchsvoller werden, was zur Folge haben würde, dass einige Betriebe ,ausstiegen“ und damit mehr Geld für anspruchsvollere Maßnahmen zur Verfügung stünde - entweder bei den im Greening verbleibenden Betrieben oder in den AUK-Maßnahmen. Die erste Variante würde eine entsprechende Ausgestaltung und Programmierung des Greenings erfordern, die letztere Variante würde einen entsprechenden Modulationsmechanismus voraussetzen.

Je nach Instrumentierung (Opt-in- oder Opt-out-Architektur) könnte der freiwillige Charakter des Greenings mehr oder weniger hervorgehoben werden.

Durch eine Verlagerung in den Bereich der Integrierten Ländlichen Entwicklungsprogramme würden die Greening-Maßnahmen nicht nur deutlicher als freiwillige Opt-in-Maßnahmen konstituiert, es könnten gegebenenfalls auch mehrjährige Maßnahmen einbezogen werden. Dies könnte aus Sicht des Natur- und Umweltschutzes anspruchsvollere Maßnahmen und unter dem Gesichtspunkt des Bürokratieabbaus eine geringere Frequenz von Antragstellung und Kontrollen ermöglichen.

Eine regionale Zahlungsdifferenzierung für die Teilnahme an Greening-Maßnahmen könnte durch Einbeziehung z. B. der Bodengüte (und den sich damit ergebenden Preisen, Pachten etc.) verwirklicht werden. Dies könnte es ermöglichen, die Teilnahmebereitschaft auch bei intensiv wirtschaftenden Betrieben zu erhöhen. 
Eine Überarbeitung der Berechnungsbasis der Maßnahmen in der Zweiten Säule könnte geboten sein. Vor allem die Transaktionskosten der Landwirte wären stärker zu berücksichtigen bzw. könnte die Koordinierung von überbetrieblichen Maßnahmen gefördert werden. Auch die Wiedereinführung einer Anreizkomponente erscheint sinnvoll (Wissenschaftlicher Beirat für Agrarpolitik beim BMELV 2010, S. 23; Kommission Landwirtschaft am Umweltbundesamt 2011, S. 12), um die Teilnahmebereitschaft zu erhöhen. Die Ausgestaltung müsste aber dabei die Grenzen des WTO-Rechts beachten, Überkompensierung vermeiden und Mitnahmeeffekte minimieren.

Bei der Ausgestaltung der nun besser ausgestatteten AUKM könnte die Anwendung weiterer Mechanismen, wie z. B. Ausschreibungen, in Erwägung gezogen werden (siehe Abschn. 4.3.4). Eine Verringerung der Transaktionskosten der Landwirte könnte auch durch die Nutzung neuer Technologien (z. B. Internet-Applikationen, Kartierungstools, Remote Sensing) unterstützt werden. Bei Einbeziehung von mehrjährigen Maßnahmen sowie durch verstärkten Einsatz GIS-gestützter Datenerfassung und digitaler Datenverarbeitung könnte der bürokratische Aufwand reduziert werden. Zudem würden nur jeweils diejenigen Maßnahmen kontrolliert, zu denen ein Betrieb sich verpflichtet hat.

Zur Finanzierung der Zweiten Säule würden weiterhin EU-Mittel dienen, die - je nach Art der Maßnahmen - zumindest teils durch Bund und Länder ko-finanziert würden. Umgeschichtete Mittel müssten nicht unbedingt ko-finanziert werden. Je nach Regionalisierungsgrad der Maßnahmen könnte auch eine Beteiligung unterer Verwaltungsebenen (z. B. Bezirk, Landkreis) ermöglicht werden, die besonders anspruchsvolle Maßnahmen ermöglichen wollen (vgl. Wissenschaftlicher Beirat für Agrarpolitik beim BMELV 2010, S. 26). Die Finanzierung aus Mitteln der EU, des Bundes und der Länder sollte im Zusammenspiel mit den Mindeststandards geeignet sein, überall einen befriedigenden bis guten Zustand der Umweltressourcen sicherzustellen.

Im weiteren Kontext könnten die sechs Prioritäten der ländlichen Entwicklungsprogramme beibehalten werden (Dies sind: 1. Wissenstransfer und Innovation, 2. Förderung der Wettbewerbsfähigkeit, 3. Förderung einer Organisation der Wertschöpfungskette und eines Risikomanagements, 4. Wiederherstellung, Erhaltung und Verbesserung der mit der Landwirtschaft verbundenen Ökosysteme, 5. Förderung der Ressourceneffizienz, 6. Förderung der sozialen Inklusion, der Armutsbekämpfung und der wirtschaftlichen Entwicklung). Dabei sind die Maßnahmen aus den Prioritäten 2 und 6 auf ihre Umweltverträglichkeit zu überprüfen oder in eine Innovationsförderung zu überführen (Wissenschaftlicher Beirat für Agrarpolitik beim BMELV 2010). Auch könnte eine Anhebung der Mindest- und der Obergrenze für das Budget der umwelt- und klimabezogenen Maßnahmen (derzeit ca. $30 \%$ ) diskutiert werden. Aus Sicht des Natur- und Umweltschutzes ist anzustreben, dass ein möglichst großer Teil der aus der Ersten Säule umgeschichteten Mittel dem Agrar-Umwelt-Bereich zur Verfügung steht.

Im Bereich der AUKM sollte ein begrenztes Portfolio an einfachen, relativ unspezifischen und aktionsorientierten Maßnahmen weiterhin angeboten werden. Dieses 
könnte regional differenziert werden und zur Senkung der Einstiegshürden in die komplexeren Maßnahmen dienen (Mante 2010). Der Einsatz eines Agglomerationsbonus wäre zu erwägen. Die Förderung gesamtbetrieblicher Ansätze (,,whole-farmapproaches“) wie in Form des Ökolandbaus sollte flächendeckend erhalten bleiben. ${ }^{2}$ Eine ergebnisorientierte Honorierung könnte eingesetzt werden, wenn ausreichend aussagekräftige, einfach zu erhebende Indikatoren zur Verfügung stehen, beispielsweise eine Verbesserung der Fruchtartenvielfalt und -folge in Intensivgebieten. Insgesamt wären die AUKM effektiver zu gestalten, z. B. durch eine räumliche Allokation auf Landschaftsebene (z. B. Prioritätsregionen in Biotopvernetzungsplan integrieren) und - soweit möglich - eine evidenzbasierte Programmierung der Maßnahmen. Auch könnten die AUKM so gestaltet werden, dass sie Möglichkeiten der Nutzung von Synergieeffekten bieten (z. B. Schaffung von Nahrungs- und Nisthabitaten mit gleichzeitiger Vernetzungs- und Erosionsschutzfunktion).

Die Einführung kooperativer Ansätze wäre zu prüfen. Dabei wäre zwischen zwei Varianten abzuwägen. Entweder könnten die Agrarumweltmaßnahmen nach dem Vorbild der Niederlande konsequent in regionalen Umweltkooperativen organisiert werden, oder es wird die Bildung von freiwilligen Kooperativen unterstützt, etwa durch Anschubhilfen oder Unterstützung für die Moderation und Koordination. Es kann dann gemeinsam mit der Kooperative ein regionales Programm ausgearbeitet werden, für dessen Umsetzung die Kooperative als Ganzes verantwortlich ist. Die Bildung und Förderung von Umweltkooperativen könnte langfristig ebenfalls zu einer Minderung des bürokratischen Aufwandes und zur Steigerung von Effizienz und Effektivität beitragen, jedenfalls wenn die AUKM vollständig auf der Ebene solcher Kooperativen organisiert würden und damit die einzelbetriebliche Kontrolle dieser Maßnahmen weitgehend durch geeignete Mechanismen innerhalb der Kooperativen ersetzt würde.

Eine periodische Evaluierung und Neuprogrammierung der AUKM im Sinne eines adaptiven Managements etwa nach dem Vorbild der Wasserrahmenrichtlinie kann sinnvoll sein. Durch partizipative und kooperative Prozesse können die beteiligten Akteure ein gemeinsames Verständnis der verschiedenen lokalen und regionalen Prioritäten und Probleme gewinnen (sowohl der Umweltbelange als auch der sozio-ökonomischen Gegebenheiten). Auf dieser Basis können gemeinsam Maßnahmen erarbeitet werden, die einen effektiven Natur- und Umweltschutz für alle akzeptabel gestalten und die sozio-ökonomischen Hintergründe der Landwirte und Regionen berücksichtigen.

\subsubsection{Diskussion und Bewertung}

Die Verbesserungen der Naturschutz- und Umweltqualität werden in Option 2 im Wesentlichen durch zusätzliche und anspruchsvollere freiwillige Leistungen im Rahmen von AUK-Maßnahmen erbracht, für die mehr Mittel zur Verfügung stehen

${ }^{2}$ Hier sind auch andere Ansätze denkbar, siehe z. B. Hope Farm in Großbritannien. 
würden. Weiterhin würden mehr Mittel für die Aufrechterhaltung der Bewirtschaftung von benachteiligten Standorten aufgewendet. Hinzu käme ein möglicher Naturschutz- und Umwelt-Mehrwert der Greening-Maßnahmen, wenn diese national oder regional und damit angepasster programmiert würden.

Wie stark diese strategische Option zur Verwirklichung des Leitbilds einer multifunktionalen, natur- und umweltverträglichen Landwirtschaft beiträgt, hängt wesentlich von der instrumentellen Ausgestaltung und finanziellen Ausstattung der verschiedenen Komponenten ab.

Eine erste Frage ist, ob eine Nationalisierung und Regionalisierung der Greening-Maßnahmen tatsächlich den Effekt hätte, dass diese mit erhöhten Umwelt-Anforderungen verknüpft würden. Die bisherigen Erfahrungen mit der Ausgestaltung der nationalen Spielräume im Greening zeigen eine Neigung der Akteure in vielen Mitgliedstaaten, das Greening zu ,verwässern“, also weniger anspruchsvoll zu gestalten. Um einen solchen Effekt zu verhindern, wäre zum einen sicherzustellen, dass fachliche Belange des Natur- und Umweltschutzes eine starke Rolle bei der Ausgestaltung erhalten. Zum anderen müssten die Rahmenvorgaben der EU eine anspruchsvolle Ausgestaltung abfordern, die dann im Notifizierungsverfahren zu überprüfen wären. Auch hier wäre den fachlichen Gesichtspunkten des Natur- und Umweltschutzes eine wichtige Rolle einzuräumen.

Eine zweite Frage ist, in welchem Umfang die Mittel, die durch eine eventuelle Reduktion der Direktzahlungen frei würden, auch tatsächlich in erheblichem Umfang in AUKM fließen. Eine pauschale Überführung solcher Mittel in die Zweite Säule könnte zur Folge haben, dass eine Aufteilung auf die verschiedenen Programmlinien erfolgt. Dies kann auch sinnvoll sein, wenn etwa die Investitionsförderung systematisch mit einer Nachhaltigkeitsausrichtung verknüpft wird. Hier würden bei dieser strategischen Option aber erhebliche Spielräume und Ungewissheiten im Programmierungsprozess bestehen.

Vor diesem Hintergrund lässt sich drittens fragen, ob es zweckmäßiger wäre, die finanziell stärker ausgestatteten AUKM außerhalb oder weiterhin im Rahmen der ländlichen Entwicklungspolitik, also der Zweiten Säule der GAP, anzusiedeln. Eine Ansiedlung innerhalb der bestehenden Strukturen der ländlichen Entwicklung hätte den Vorteil, dass die bestehenden Verwaltungsstrukturen und -erfahrungen genutzt werden können. Aus Sicht des Natur- und Umweltschutzes könnte sich jedoch ein Einwand daraus ergeben, dass diese Strukturen historisch aus einer vorrangig produktionsorientierten Landwirtschaftspolitik erwachsen sind, die aus ökologischer Sicht eher Teil des Problems als Teil der Lösung war. Es gibt daher im Natur- und Umweltschutzbereich die Überlegung, einen aus der GAP ausgelagerten eigenen Fonds zur Finanzierung von Naturschutzmaßnahmen auf europäischer Ebene zu fordern.

Ein vierte Frage bei dieser Option ergibt sich daraus, dass die Umsetzung von Natur- und Umweltschutzmaßnahmen vom politischen Willen und von den finanziellen Möglichkeiten der Mitgliedstaaten und der Regionen abhängig wäre (Schulz 2010), wenn diese AUK-Maßnahmen kofinanziert werden müssen. Eine mögliche Lösung könnte darin liegen, Maßnahmen, die zur Erfüllung von 
EU-Anforderungen dienen, vollständig durch die EU zu finanzieren (SRU 2009; Schulz 2010). Grundsätzlich ist die Frage einer Mittelverlagerung - von den Direktzahlungen der Ersten Säule hin zu natur- und umweltbezogenen Maßnahmen im Rahmen der ländlichen Entwicklungspolitik - logisch zu trennen von der Frage der Ko-Finanzierung.

Fünftens könnten die regionale und lokale Programmierung zusätzlichen bürokratischen Aufwand bedeuten. Eine Abhilfe könnte hier darin bestehen, nur Maßnahmen, die nationale und regionale Anforderungen erfüllen, auf den entsprechenden Ebenen zu programmieren. Grundsätzlich kann die Verteilung der Kompetenzen auf verschiedene Ebenen es erschweren, zentrale Lösungsstrategien umzusetzen, die für einige Herausforderungen notwendig wären (Wissenschaftlicher Beirat für Agrarpolitik beim BMELV 2010, S. 29).

Eine sechste Frage hängt mit der Wahl der Instrumente im dezentralen Programmierungsprozess zusammen. Die Erfahrung zeigt, dass häufig einfache, bekannte Maßnahmen programmiert werden, die einen planmäßigen Mittelabfluss und ein geringes Anlastungsrisiko gewährleisten (Wissenschaftlicher Beirat für Agrarpolitik beim BMELV 2010, S. 29), jedoch nur geringe zusätzliche positive Effekte für den Natur- und Umweltschutz realisieren (,hellgrüne Maßnahmen“). Bei einem verstärkten Mittelzufluss in die AUKM könnte es daher geboten sein, die Instrumente und die Programmierung darauf hin zu überprüfen, ob sie den Anforderungen des Natur- und Umweltschutzes gerecht werden. In diesem Zusammenhang könnte es auch angezeigt sein, die anderen Förderbereiche der Zweiten Säule zu überarbeiten (vgl. Abschn. 7.4 und 7.7).

Ein grundsätzlicher Einwand könnte sein, dass bei einer erhöhten Sichtbarkeit der Freiwilligkeit der Greening-Maßnahmen und bei stärkerer instrumenteller Entkopplung von der Basisprämie die Erbringung der entsprechenden Leistungen weniger gesichert sein könnte, als dies bei der aktuellen Überkompensation der Auflagen in der Ersten Säule der Fall ist. Hier wäre sicherzustellen, dass durch Gewährung entsprechender Prämien auch bei einer Verankerung des Greenings in der Zweiten Säule die Teilnahmebereitschaft gesichert wird.

Insgesamt besteht bei der strategischen Option 2 eine hohe Wahrscheinlichkeit, dass Betriebe mit hohen Opportunitätskosten für Agrar- und Umweltleistungen aus allen freiwilligen Elementen des agrarpolitischen Systems aussteigen und sich daher auch von GLÖZ und Greening ,,verabschieden“. Daher kommt in dieser Option einer wirksamen Ausgestaltung und Implementation der Mindeststandards des Natur- und Umweltschutzes im ordnungsrechtlichen Rahmen eine besondere Bedeutung zu.

Insgesamt handelt es sich bei dieser Option zunächst zwar um eine Weiterentwicklung der bisherigen GAP, die Rückführung von den Direktzahlungen und das hohe Gewicht national und regional programmierter Maßnahmen stellen jedoch erhebliche Veränderungen gegenüber dem Status quo dar.

Machbarkeit: Die Option knüpft an bestehende Instrumente und Mechanismen der GAP an, entwickelt diese aber weiter. Aufgrund der erhöhten dezentralen 
Diskretion bei der Ausgestaltung der Maßnahmen wird den Implementationsprozessen große Bedeutung zukommen. Hier besteht die Gefahr einer „Verwässerung“ der Ansätze aus Sicht des Natur- und Umweltschutzes.

Effektivität und Effizienz: Grundsätzlich hat die Option das Potenzial, die Wirksamkeit und Effizienz der Politik im Vergleich zum derzeitigen System zu erhöhen. Insbesondere könnten bessere Koordinierungsmöglichkeiten geschaffen und der Aspekt der Leistungserbringung in Intensivregionen besser adressiert werden. $\mathrm{Ob}$ dieses Potenzial realisiert wird, würde aber von einer Vielzahl dezentraler Entscheidungen im Implementationsprozess abhängen.

Implikationen und mögliche Nebenwirkungen:

- Wegen der Verminderung der Direktzahlungen besteht die Möglichkeit, dass viele Landwirte keine Maßnahmen mehr umsetzen, die durch das heutige GLÖZ und Greening gefordert werden, wenn sie keine Möglichkeit erhalten, an attraktiven natur- und umweltbezogenen Maßnahmen teilzunehmen.

- Die Nationalisierung und Regionalisierung der Greening-Maßnahmen könnte zu Wettbewerbsverzerrungen führen, wenn es keinen wirksamen europäischen Koordinations- und Notifizierungsmechanismus gibt.

- Es könnte zu einer bloßen Verschiebung von Geldern aus der Ersten Säule in Zahlungen für weniger ertragsstarke Standorte kommen, wenn nicht auch für intensiv genutzte Regionen attraktive Maßnahmen angeboten werden.

- Die zunehmende Verknüpfung der Direktzahlungen an nachweisbare Leistungen der Landbewirtschaftung könnte auf Pachtflächen zu einer geringeren Überwälzung der Zahlungen an die Landbesitzer führen.

- Eine Umschichtung der finanziellen Mittel der Greening-Maßnahmen von der Ersten in die Zweite Säule wäre gleichbedeutend mit einer Nationalisierung oder Regionalisierung der Ausgestaltung der Greening-Anforderungen. Dann könnte sich die Frage stellen, ob nicht auch die Ausgestaltung der GLÖZ-Anforderungen nationalisiert oder regionalisiert werden könnte. In letzter Konsequenz könnte eine solche Entwicklung auf ein weitgehendes oder gar vollständiges PhasingOut der EU-weiten Direktzahlungen der Ersten Säule hinauslaufen. Denn wenn, wie in der vorigen Option diskutiert, der Erhalt der Direktzahlungen letztlich an die Einhaltung der GLÖZ-Auflagen geknüpft würde, die Ausgestaltung der GLÖZ-Auflagen aber nationalisiert oder regionalisiert würde, dann wäre das Ergebnis eine an bestimmte Natur- und Umweltschutzauflagen geknüpfte, flächenbezogene Zahlung, wie sie im Rahmen der AUKM in der Zweiten Säule bereits heute für Flächen bezahlt werden, auf denen bestimmte Bewirtschaftungsformen angewandt werden.

- Nach Nationalisierung der Greening-Komponente wäre ein Überstieg in Option 3 schwierig.

- Akteurskoalitionen: Die erhöhten nationalen und regionalen Gestaltungsspielräume könnten für viele Akteure attraktiv sein. Sie werden auch von vielen Umweltverbänden sowie Wissenschaftlerinnen und Wissenschaftlern gefordert. 


\subsection{Option 3 - Integriertes Modell}

\subsubsection{Zugrundeliegende Idee}

Option 3 geht von der Annahme aus, dass der mit Etablierung von Cross Compliance begonnene Weg der instrumentellen und argumentativen Verknüpfung von Erster und Zweiter Säule sowie Ordnungsrecht nun zu einem integrierten strategischen Aufbau der verschiedenen Elemente führen könnte. Die dritte Option löst sich daher konzeptionell von der derzeitigen vertikalen Zwei-Säulen-Architektur und entwickelt ein aufeinander aufbauendes System der Honorierung von Natur- und Umweltschutzleistungen.

Hinsichtlich der Finanzierungsmodi können dabei weiterhin Maßnahmen mit einem vorrangig EU-weiten Bezug vollfinanziert und Maßnahmen mit vorrangig nationalem oder regionalem Bezug kofinanziert werden. Die konzeptionelle, programmatische und administrative Trennung der zwei Säulen wird aufgegeben und ein integriertes System der Honorierung von Natur- und Umweltleistungen aufgebaut (vgl. Abb. 8.3). Damit wird angestrebt, mehr Raum für Instrumente zu schaffen, die dem systemischen Umweltzusammenhang der landwirtschaftlichen Produktionssysteme besser gerecht werden.

\section{Auflagen / zu erreichender Umweltzustand}

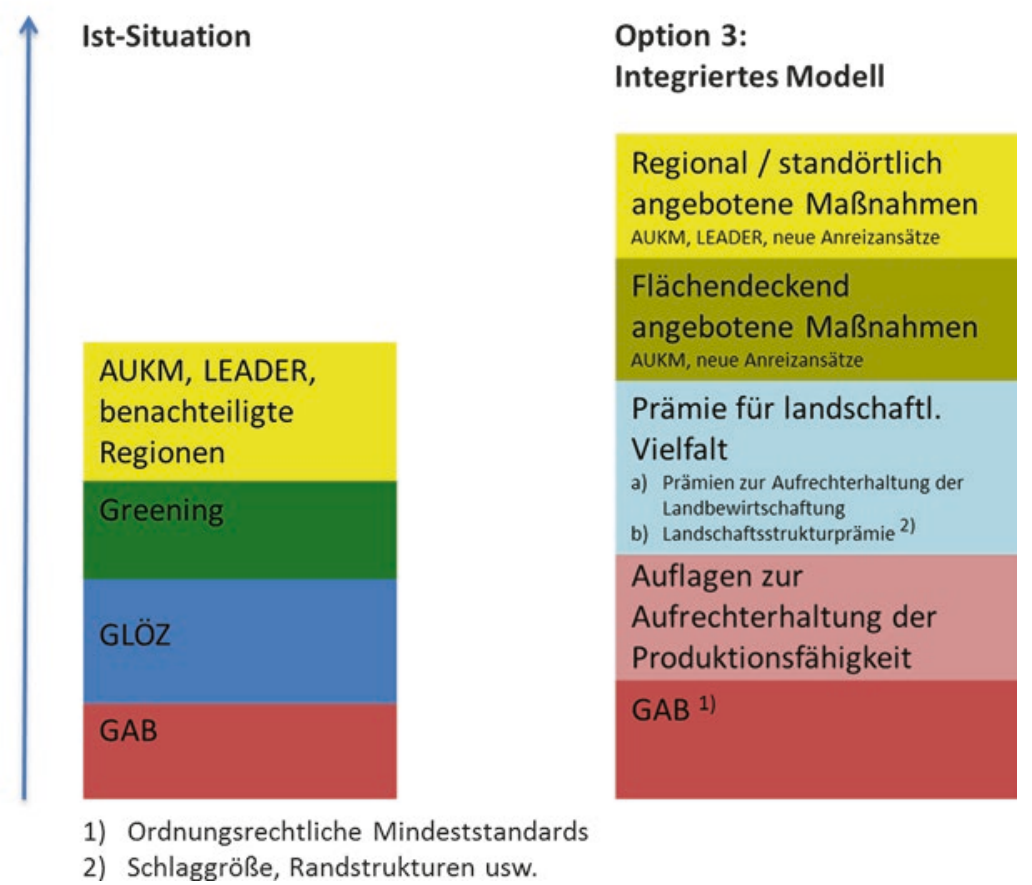

Abb. 8.3 Option 3 - Integriertes Modell (Quelle: Eigene Darstellung) 


\subsubsection{Einbettung in die agrarpolitische Diskussion}

Der mögliche Übergang von der historisch gewachsenen Zwei-Säulen-Architektur der GAP zu einem stärker konzeptionell integrierten System der Prämienzahlungen an den Agrarsektor wird vielfach diskutiert. Ein integriertes System der Honorierung von Natur- und Umweltleistungen wird beispielsweise Hart et al. (2016) vorgeschlagen.

Der Übergang von der Zwei-Säulen-Struktur zu einem integrierten Ansatz würde auch die relativ starre Kopplung von Finanzierungsmodus und Instrumentierung lösen. Es würde ermöglichen, ,die Frage der Ko-Finanzierung von der Frage der optimalen Ausgestaltung der Maßnahmen" getrennt zu behandeln (Wissenschaftlicher Beirat für Agrarpolitik beim BMELV 2010, S. 6).

Auch die EU-Strategie „Grüne Infrastruktur“ (European Commission 2013) enthält das Programm eines stärker integrierten Ansatzes zur Sicherstellung der ökosystemischen Dienstleistungen. Die integrierte Option 3 könnte hier die Anknüpfungsmöglichkeiten für eine integrierte Berücksichtigung dieser Anliegen verbessern.

\subsubsection{Grundlegende Struktur}

Das Integrierte Modell besteht aus drei aufeinander aufbauenden Schichten:

1. Basisanforderungen: Diese sollten aus den bisherigen Grundanforderungen an die Betriebsführung (GAB) sowie Auflagen zur Aufrechterhaltung der Produktionsfähigkeit der Standorte bestehen. Letztere würden einige Maßnahmen übernehmen, die bisher in GLÖZ und Greening enthalten waren. Die Auflagen sollten dann von allen Betrieben auf allen (bzw. ggf. den betroffenen) Standorten eingehalten werden. Ein „Ausstieg“ wie aus den heutigen GLÖZ- und Greening-Anforderungen wäre jedoch nicht möglich. Damit wären diese Basisanforderungen langfristig als gesetzlicher Mindeststandard anzusehen.

\section{Prämien für landschaftliche Vielfalt:}

a. Prämie zur Aufrechterhaltung der Landbewirtschaftung in Gebietskulissen zum Erhalt der Landschaftsvielfalt (vgl. Abschn. 7.3.4): Für die Bewirtschaftung benachteiligter Standorte sowie von strukturreichen Flächen (z. B. kleine Schläge, Randstreifen entlang von Waldrändern, Streuobstflächen, vgl. Baustein 3) könnte ein entsprechender standortbezogener Ausgleich gewährt werden. Hierbei würde der Mehraufwand honoriert, der durch die Bewirtschaftung von Flächen entsteht, deren fortgesetzte landwirtschaftliche Nutzung aus Sicht des Natur- und Umweltschutzes besonders (z. B. Biodiversität, Landschaftsbild) wertvoll wäre, deren Bewirtschaftung aber unter den gegebenen Rahmenbedingungen häufig nicht rentabel ist. Die Prämie könnte EUweit programmiert und gewährt werden. Alternativ wäre eine nationale Programmierung mit Notifikation bei der EU-Kommission denkbar. 
b. Eine Landschaftsstrukturprämie für relativ strukturreiche Standorte (vgl. Abschn. 7.3.5) könnte unabhängig von Gebietskulissen und Betriebsgrößen gewährt werden. Sie könnte z. B. kleinstrukturierte Landschaften, Randstreifen entlang von Strukturelementen oder Flächen mit Landschaftselementen wie z. B. Streuobstwiesen belohnen. Die Prämienhöhe könnte flächenbezogen auf Basis von Fernerkundungsdaten berechnet werden und nach Dichte und ökologischer Wertigkeit der Strukturelemente gestaffelt werden.

\section{Freiwillige Maßnahmen:}

a. Flächendeckend angebotene freiwillige Maßnahmen (vgl. Abschn. 7.3.4): Dieses Element könnte Maßnahmen enthalten, deren Umsetzung unabhängig von regionalen und standörtlichen Bedingungen positive Wirkungen auf Natur und Umwelt erwarten lassen. Diese Maßnahmen werden auf EU- oder Mitgliedstaaten-Ebene programmiert und finanziert. Die Landwirte können sich freiwillig für die Teilnahme an den angebotenen Maßnahmen entscheiden (Opt-in). Die mehrjährige Konstruktion vermindert den Bürokratieaufwand.

b. Regional/standörtliche freiwillige Maßnahmen (vgl. Abschn. 7.4): Diese Maßnahmen könnten nach dem Prinzip der heutigen AUKM der Zweiten Säule auf subnationaler Ebene programmiert und ko-finanziert werden. Es könnten vermehrt Freiräume für kooperative und partizipative Ansätze ermöglicht werden. Auch für die Teilnahme an diesen Maßnahmen können die Landwirtinnen und Landwirte sich freiwillig entscheiden (Opt-in). Eine mehrjährige Konstruktion könnte den Bürokratieaufwand vermindern.

Diese aufeinander aufbauenden Elemente könnten flankiert werden durch Maßnahmen der Aus- und Weiterbildung, Beratung und Wissenstransfer, die Förderung von Innovationen und ländlicher Infrastruktur, Maßnahmen im LEADER-Programm, Europäische Innovationspartnerschaften (EIP-AGRI) o. Ä. (vgl. Abschn. 7.7). Die anderen Elemente der ländlichen Entwicklungspolitik könnten ebenfalls weiterhin bestehen.

Darüber hinaus bestünde in dieser Option weiterhin Raum für höhere private Standards des nachgelagerten Bereichs, die durch entsprechende staatliche KoRegulierung anspruchsvoller gestaltet werden könnten (siehe Abschn. 7.5).

\subsubsection{Instrumentelle Ausgestaltung}

Basisanforderungen: Die Basisanforderungen könnten aus den heutigen Grundanforderungen für die Betriebsführung und weiteren Auflagen zur Aufrechterhaltung der Produktionsfähigkeit bestehen. Dabei wäre hinsichtlich der Grundanforderungen an die Betriebsführung zu prüfen, ob die derzeitigen Anforderungen hinreichend sind oder der Anpassung bedürfen. Die verpflichtenden Anforderungen zur Aufrechterhaltung der Produktivität könnten den Humuserhalt, den Erosionsschutz, den Dauergrünlanderhalt, die Bereitstellung von ökologischer Vorrangfläche beinhalten. 
Die Auflagen zur Aufrechterhaltung der Produktivität können als gute fachliche Praxis angesehen werden, die dazu dient, die Produktionsfähigkeit landwirtschaftlicher Standorte langfristig zu sichern. Diese Anforderungen könnten als gesetzlicher Mindeststandard festgelegt werden. Es wird angestrebt, das Vollzugsdefizit zu minimieren (siehe hierzu auch die Abschn. 7.1 und 7.6).

Anders als in Option 2 ist in Option 3 grundsätzlich keine Basisprämie vorgesehen. Sollte sich jedoch wissenschaftlich belegen lassen, dass tatsächlich in der EU Mehrkosten durch höhere Mindeststandards im Vergleich zu den Wettbewerbern entstehen, könnte eine flächendeckende Prämie zu deren Abgeltung legitim sein. Wie oben diskutiert, reichen Schätzungen der derzeitigen regulatorischen Mehrkosten der landwirtschaftlichen Betriebe in der EU im Vergleich zur Importkonkurrenz von ca. $20 € /$ ha (Plankl et al. 2010) bis über $50 € /$ ha (Wissenschaftlicher Beirat für Agrarpolitik beim BMELV 2010).

Die Prämien für Landschaftsvielfalt würden zwei Aspekte berücksichtigen. Zum einen würde auf Flächen eine Prämie gezahlt werden, die aufgrund ihrer natürlichen Eigenschaften (z. B. Höhenlage, Hangneigung, Klima) Grenzertragsstandorte darstellen, deren Bewirtschaftung am Markt nicht mehr wirtschaftlich ist, auf denen die Aufgabe der landwirtschaftlichen Nutzung aber nicht wünschenswert wäre (siehe Abschn. 7.3.4). Zum anderen würde die strukturelle Vielfalt in der Landschaft honoriert (siehe Abschn. 7.3.5 und 7.6.3). Letztere Prämie könnte nach einem EU-weit einheitlichen Schlüssel gewährt und mithilfe von GIS-gestützten Fernerkundungsdaten überwacht und administriert werden. Voraussetzung wäre eine Einigung auf einen fachlich belastbaren Satz mit nachvollziehbaren, gewichteten Faktoren, die in die Berechnung der Höhe der Prämie eingehen. Bei EU-weiter Gewährung wäre eine Vollfinanzierung aus EU-Mitteln systematisch konsequent, aber möglicherweise mit Umverteilungseffekten zwischen den Mitgliedstaaten verbunden.

Im Bereich der „hellgrünen“ Maßnahmen würde das derzeitige Greening durch einen „Baukasten“ flächendeckend angebotener Maßnahmen ersetzt, die freiwillig sind und differenziert honoriert werden (siehe Abschn. 7.3.8). Eine Honorierung in Abhängigkeit vom ökologischen Wert der Maßnahme und der Bodenpunkte am jeweiligen Standort könnte dabei auch in Intensivregionen die Bereitschaft zur Teilnahme erhöhen.

Darauf aufbauend würden regional und standörtlich programmierte freiwillige Maßnahmen die heutigen AUKM weiterentwickeln. Diese würden sich an den heutigen „dunkelgrünen“ Maßnahmen und den Vertragsnaturschutzprogrammen der heutigen Zweiten Säule orientieren. Beim Design dieser Maßnahmen könnten neue verhaltenswissenschaftliche Erkenntnisse berücksichtigt werden, z. B. die Nutzung von Selbstverpflichtungs- und Feedback-Mechanismen. Weiterhin würde in Option 3 mehr Raum für systemische, kooperative und partizipative Ansätze eines integrierten Ressourcen- und Landschaftsmanagements geschaffen werden.

Flankiert würde dieses Stufensystem durch weitere Maßnahmen. Zum einen würden unterstützende Beratungs-, Aus- und Fortbildungsangebote intensiviert werden (vgl. Abschn. 7.7.1). Des Weiteren könnte ein „Sicherheitsnetz“ für die Landwirtschaft entwickelt werden, um extreme Preisschwankungen abzufedern. Dabei wäre zu überprüfen, ob aus Sicht des Natur- und Umweltschutzes Versicherungslösungen 
(Meuwissen et al. 2003) oder Preis-Ausgleichzahlungen (Thomas et al. 2016) sinnvoller wären. Bei letzteren wäre zu verhindern, dass letztlich ein produktionsstimulierender Mindestpreis für Erzeuger mit negativen Auswirkungen auf die Ziele des Natur- und Umweltschutzes eingeführt würde.

In Option 3 würde schließlich ein konsequentes System von Kennzeichnungen für die Natur- und Umweltleistungen der Landwirtschaft etabliert werden. Durch die glaubwürdige Ankündigung staatlicher Label-Aktivitäten im Falle mangelnder privater Initiativen sowie durch staatliche Moderations- und Koordinierungsleistungen könnten Aktivitäten der Verarbeiter und des Handels stimuliert und flankiert werden.

\subsubsection{Diskussion und Bewertung}

Insgesamt enthält Option 3 eine strategische Entwicklungsrichtung, die eine Überwindung der historisch gewachsenen Zwei-Säulen-Architektur der GAP ermöglichen könnte. Andererseits ließe sich diese Option auch innerhalb des Zwei-SäulenModells etablieren.

Die einzelnen Elemente des integrierten Modells können im Zeitablauf im Lichte neuer Problemlagen und Erkenntnisse weiterentwickelt werden.

Die Option zielt auf eine Abkehr vom „Gießkannensystem“, dass durch den Ansatz einer leistungsbezogenen Honorierung der einzelbetrieblich erbrachten ökologischen Leistungen ersetzt würde (,Mehr Leistung, mehr Zahlung“). Damit würde ein Anreiz geschaffen, zusätzliche Gemeinwohlleistungen zu erbringen. Allerdings würden mit diesem Ansatz nicht mehr von allen Betrieben in allen Regionen die gleichen Leistungen erbracht und in intensiv genutzten Regionen würden Betriebe eventuell nicht an den freiwilligen Maßnahmen teilnehmen. Zwar ist das derzeitige Greening de facto auch freiwillig, aber unter Landwirten gilt die Teilnahme als die ,normale“ Option. Für die Sicherung eines Mindeststandards an Natur- und Umweltschutz auch in intensiv bewirtschafteten Regionen käme in Option 3 daher dem Ordnungsrecht und effektiven Kontroll- und Sanktionsmechanismen verstärkte Bedeutung zu. Innerhalb der Agrarpolitik würde sich der Kontrollaufwand hingegen auf die freiwillig eingegangenen Verpflichtungen sowie die fernerkundungsgestützte Administration der Prämien für landschaftliche Vielfalt beschränken. Bei vielen dieser Maßnahmen könnte ein Antragszeitraum von fünf Jahren geeignet sein, den bürokratischen Aufwand auf Betriebs- und Verwaltungsseite zu reduzieren.

Machbarkeit: Da die Förderungsstruktur an das bestehende System der Agrarverwaltung anknüpft, erscheint ein derartiges Fördersystem praktikabel. Durch die vermehrte Nutzung von Fernerkundung und GIS-Technologien könnten möglicherweise erhebliche Vereinfachungen in den Verwaltungsabläufen sowie eine Verminderung der Transaktionskosten auf Seiten der Verwaltung und der Landwirte erreicht werden. Eine wesentliche Voraussetzung für die Realisierung der Option wäre eine wissenschaftliche Begründung der Algorithmen für die EU-weite Kompensierung von Standortnachteilen. Angesichts der umfangreichen Daten wären eventuelle Datenschutz-Probleme proaktiv zu identifizieren und zu regeln. 
Effektivität und Effizienz: Grundsätzlich könnte diese Option die Zielgenauigkeit der GAP und ihre Wirksamkeit im Hinblick auf die Ziele des Natur- und Umweltschutzes erhöhen. Allerdings ist noch durch Machbarkeitsstudien zu zeigen, dass eine digitale Bewertung von Standortnachteilen und ggf. auch von flächenbezogenen Maßnahmen, wie der Erhalt von Landschaftselementen, tatsächlich weniger Bürokratie bedeutet. Weiterhin ist noch zu zeigen dass die notwendigen Daten tatsächlich vorhanden sind bzw. mit vertretbarem Aufwand gewonnen werden können. Beispielsweise sind die Landschaftselemente bereits erhoben. Aufbauend auf diesen Daten können Updates aufgrund von Luftbilddaten vorgenommen werden.

Implikationen und mögliche Nebenwirkungen:

- Die Finanzierung der Zahlungen für flächendeckende Maßnahmen könnte weiterhin zu $100 \%$ aus dem EU-Haushalt der Gemeinsamen Agrarpolitik erfolgen. Dies könnte damit begründet werden, dass die umgesetzten Maßnahmen unabhängig von standörtlichen Gegebenheiten in allen Regionen und Betrieben positive Effekte auf den Natur- und Umweltschutz erwarten lassen.

- Durch Integration einiger Maßnahmen in flächendeckende, freiwillige Programme, die bisher in der Zweiten Säule umgesetzt werden (z. B. Verzicht auf mineralische Dünger und Pflanzenschutzmittel), erhöht sich indirekt auch das Anforderungsniveau in den freiwilligen regionalen/standörtlichen Maßnahmen, die kofinanziert werden. Hierdurch könnten diese finanziellen Mittel für anspruchsvollere Maßnahmen eingesetzt werden.

- Im Vergleich zum gegenwärtigen Cross-Compliance-System könnte diese Option einen verminderten Bürokratieaufwand ermöglichen (siehe dazu die in Abschn. 7.6 dargestellten Monitoring-, Kontroll- und Sanktionsmechanismen).

- Indem diese Option in Bezug auf einige Elemente (z. B. Prämie zur Aufrechterhaltung der Landbewirtschaftung in Gebietskulissen zum Erhalt der Landschaftsvielfalt und Prämie zur Landschaftsvielfalt) relativ stark auf die Verarbeitung GIS-gestützter Daten setzt, könnte sie einen Trend zur Digitalisierung der Agrarwirtschaft verstärken, der in seinen strukturpolitischen, organisationssoziologischen und arbeitstechnischen Implikationen nicht neutral wäre. Da die digitale Technik aber nicht von den Landwirten vorgehalten werden muss, verstärkt dieser Ansatz nicht notwendigerweise den Strukturwandel. Hier besteht allerdings Forschungsbedarf.

- Durch das Abschmelzen der bestehenden Direktzahlungen könnte die Aufrechterhaltung der Landwirtschaft in Regionen mit erhöhten Produktionskosten in Frage gestellt sein. Daher müssten die Zahlungen zur Aufrechterhaltung der Landbewirtschaftung in Gebietskulissen hinreichend finanziell ausgestattet sein, um einen solchen unerwünschten Effekt zu vermeiden. Dies wiederum könnte regionale Umverteilungseffekte mit sich bringen, die weitere finanzielle Ausgleichsmaßnahmen erfordern könnten, um politisch akzeptabel zu sein. Allerdings deuten die Expertengespräche an, dass eine stärkere Förderung von Standorten, an denen eine großflächige Aufgabe der Landbewirtschaftung droht, durchaus Zustimmung finden könnte.

- Im Vergleich zum Status quo könnten die Elemente und Maßnahmen dieser Option deutlicher zeigen, was die gesellschaftlichen Erwartungen und Leistungen sind. 
- Verteilungswirkungen zwischen Mitgliedstaaten: Aus politischer Sicht könnten die Verteilungsimplikationen der Umstellung vom bestehenden auf das neue Prämiensystem kritisch sein. Um die Unsicherheit über die Umverteilungswirkungen zwischen den Mitgliedstaaten zu begrenzen, könnte beispielsweise eine Beibehaltung der nationalen finanziellen Envelopes vereinbart werden. Aus einem solchen Arrangement könnten sich aber Spannungen mit der angestrebten klaren Systematik dieser Option ergeben.

- Verteilungswirkungen zwischen den Regionen: Durch die Prämien für Landschaftsvielfalt würden Regionen, in denen viele öffentliche Leistungen erbracht werden, gegenüber Regionen, die vorwiegend der intensiven landwirtschaftlichen Produktion dienen, begünstigt. Die nach Regionen differenzierte Prämienhöhe für die „Basismaßnahmen“ würde den gegenteiligen Effekt haben. Der Gesamteffekt auf die regionale Verteilung würde von der finanziellen Ausstattung der einzelnen Elemente sowie der Ausgestaltung der verteilungsrelevanten Komponenten abhängen.

- Akteurskoalition: Diese Option könnte eine breite Koalition für die Förderung der benachteiligten Standorte und Regionen hinter sich versammeln. Für landwirtschaftliche Betriebe könnte die geringere Bürokratielast attraktiv sein. Viele landwirtschaftliche Betriebe könnten sich aber durch den Verlust des jetzigen Systems der Direktzahlungen in einer ungewissen Situation sehen. In Regionen mit starker Hypothekenbelastung der landwirtschaftlichen Betriebe könnte es bei negativen Umverteilungswirkungen zu Folgeprobleme bei einigen Finanzinstituten kommen, die stark in kreditfinanzierte Investitionen im Sektor engagiert sind.

Insgesamt könnte ein gleitender Übergang vom derzeitigen System der GAP zur Option 3 bis beispielsweise 2027 geeignet sein, ohne größere Strukturbrüche einen Übergang in eine Agrarpolitik zu gestalten, die systematisch an der Erbringung von Gemeinwohlleistungen und an der Sicherung der öffentlichen Güter des Natur- und Ressourcenschutzes ausgerichtet ist.

\subsection{Exkurs: Umstellung der Regulierungslogik auf ,prinzipienbasierte Regulation“6}

\subsubsection{Einleitung - Ein prinzipienbasierter „Neuer Ansatz“ für die GAP}

Die Anpassung an die fortschreitende Integration des Binnenmarktes und damit an den Erfolg der EU sind keine Herausforderungen, denen sich nur die GAP zu stellen hat. Dichte Regulierung, hohe bürokratische Anforderungen, das Primat nationalstaatlicher Begehrlichkeiten sowie vertikale Regelungen einzelner Produkte anstatt horizontaler Regulierung des Marktes sind Problemlagen, die in vielen Wirtschaftssektoren bekannt sind. Andere Bereiche des Binnenmarktes, insbesondere das EUProduktsicherheitsrecht, haben sich bereits erfolgreich diesen Herausforderungen 
gestellt. Insbesondere diejenigen Produkte, die heute im „Neuen Ansatz“3 geregelt sind, sowie das Lebensmittelsicherheitsrecht und das Chemikalienrecht standen vor der Neuorientierung ihrer Regulierung vor ähnlichen Problemen (Purnhagen 2013a). Es bietet sich daher an, die dort gefundenen regulativen Lösungen auf ihre Übertragbarkeit auch für die GAP und die sich hier stellenden Problemlagen zu untersuchen. Eine solche Übertragung kann selbstverständlich nicht pars pro toto erfolgen. Eine mögliche Übertragung der in anderen Bereichen gefundenen Lösungen müsste die GAP-spezifischen Besonderheiten der Regulierung und Finanzierung (beispielsweise die Unterteilung in Erste und Zweite Säule und deren unterschiedliche Finanzierung) sowie die Besonderheiten landwirtschaftlicher Märkte (wie endogene Preisschwankungen, extreme exogene Schocks durch wetter- und klimabedingte Risiken, Ernährungssicherheit) einbeziehen.

Die im EU-Produktsicherheitsrecht durchgeführten Reformen können als ein Mix der Regulierungsstrategien umschrieben werden, die allesamt auf der sogenannten prinzipienbasierten Regulierung aufbauen. Prinzipienbasierte Regulierung folgt drei grundsätzlichen Zielen (Black et al. 2007, S. 191):

1. Weit formulierte Ziele als rechtlich verbindliche Standards anstatt verbindlicher detaillierter Regeln;

2. Fokussierung auf das Ergebnis (Output) des regulativen Eingriffs;

3. Stärkung der individuellen Verantwortlichkeit der Adressaten bei der Zielerreichung.

Der letzte Punkt (Eigen-Verantwortlichkeit der Adressaten) kann, wie im „Neuen Ansatz" geschehen, als Anreizmechanismus ausgestaltet werden, sich bestimmten freiwilligen, aber im Gegensatz zu den Zielen unter Punkt 1 detaillierten Regeln zu unterwerfen. Tun die Adressaten dies, so können sie beispielsweise in den Genuss einer Beweislastumkehr kommen, welche die Verantwortlichkeit für die tatsächliche Erreichung der Ziele ein Stück weit wieder von ihnen nimmt. Im Bereich der GAP wäre eine solche Option ein großer Gewinn für Landwirtinnen und Landwirte, da hierdurch insbesondere die Haftungsrisiken, die derzeit beispielsweise mit der verschuldensunabhängigen Haftung nach der Umwelthaftungsrichtlinie 2004/35/ $\mathrm{EG}^{4}$ verbunden sind, deutlich vermindert würden. Grundsätzlich haben es allerdings weiterhin die Regelungsadressaten in der Hand, wie sie ihre Verantwortlichkeit ausgestalten und damit am Markt agieren wollen.

Ausgehend von den im EU-Produktsicherheitsrecht gemachten positiven Erfahrungen und der damit verbundenen relativen Rechtssicherheit wäre eine Option denkbar, die auf diesen Regulierungsstrategien fußt, aber an die Besonderheiten der GAP und der Agrarmärkte anknüpft. Ein solches Regelungsregime würde existierende rechtliche Mindestvorgaben, die in der Form von europäischen Prinzipien

\footnotetext{
${ }^{3}$ Entschließung des Rates vom 7. Mai 1985 über eine neue Konzeption auf dem Gebiet der technischen Harmonisierung und der Normung, ABl C 136, 04.06.1985, S. 1-9.

${ }^{4}$ Richtlinie 2004/35/EG des Europäischen Parlaments und des Rates vom 21. April 2004 über Umwelthaftung zur Vermeidung und Sanierung von Umweltschäden, AB1 L 143, 30.04.2004, S. 56-75.
} 
ausgestaltet sind, mit einem am Ergebnis orientierten freiwilligen „Baukastensystem“ für die Auswahl der Instrumente zur Zielerreichung kombinieren. Die Anwendung dieses „Baukastensystems“ könnte an ein Anreizsystem nach dem Vorbild des „Neuen Ansatzes“ geknüpft werden. Ein solches System hätte mehrere Vorteile:

1. Es gleicht die GAP an die Veränderungen des Binnenmarktrechtes an, welche die GAP mehr und mehr als integralen Bestandteil denn als Sonderrecht behandelt.

2. Es sorgt durch Ergebnisorientierung für einen effizienteren Einsatz der finanziellen Mittel der GAP.

3. Es entlastet Landwirte von dem hohen bürokratischen Aufwand.

4. Es gibt Landwirten mehr Flexibilität.

\subsubsection{Ausgangspunkt: Der „Neue Ansatz“ im EU- Produktsicherheitsrecht}

Die Regulierung technischer Produktsicherheitsstandards gehört seit dem Beginn des EU-Produktsicherheitsrechts zum Kern des Rechts des Binnenmarkts in der EU. Mehr als 30 Jahre lang erfolgte dies in der EU durch klassische Regulierung, mit genauen Vorgaben für bestimmte Produkte (sogenannte vertikale Regulierung) und genauer Adressierung an bestimmte Industriezweige (Tricker 2000; Hey et al. 2007). Für viele Produkte gab es verbindliche, detaillierte, produkt-spezifische und europaweit gültige Vorgaben für den gesamten Produktlebenszyklus (Joerges et al. 1988, S. 252ff.). Ob diese Vorgaben tatsächlich das Ziel erreichten und ob sie auch den technischen Entwicklungen standhalten konnten, war dabei durchaus fraglich. Diese Regulierungstechnik war zwar durch das Europarecht, damals Art. 100 des Vertrages über die Europäische Wirtschaftsunion (heute Art. 114 des Vertrags über die Arbeitsweise der Europäischen Union) vorgesehen, jedoch führte es zu etlichen Problemen. Die Produktstandards regelten punktuell immer nur einige wenige Aspekte der entsprechenden Produktsektoren (Joerges et al. 1988, S. 273 ff.). Sie waren zu statisch, ${ }^{5}$ konnten nicht mit der technischen Entwicklung mithalten, berücksichtigten auch nicht die unterschiedlichen Anforderungen der Verbraucherinnen und Verbraucher und Unternehmer in den Mitgliedstaaten im Binnenmarkt und waren mit der Zeit zu einem Berg an Vorschriften angewachsen, der nur noch schwer zu bewältigen war (McGee und Weatherill 1990, S. 582). Insbesondere war diese Art der Regulierung nicht geeignet, die angestrebten Ziele (Sicherheit der Produkte, Binnenmarktverwirklichung) angesichts der mit der fortlaufenden Technisierung einhergehenden Zunahme von Risiken dauerhaft zu gewährleisten (Hanson 2005, S. 37; Trubek und Trubek 2006, S. 539 ff.).

\footnotetext{
${ }^{5}$ Dynamisierende Formeln wie „Stand der Technik“ wurden erst im Zuge der Einführung des „Neuen Ansatzes“" wirksam mit europäischen Institutionen unterfüttert.
} 


\subsubsection{Der ,Neue Ansatz“6 als Alternative}

Auf der Suche nach einer Alternative für ein regulatorisches Design wurde man in der Niederspannungsrichtlinie 73/23/EWG fündig, ${ }^{6}$ deren Regulierungsdesign zu jener Zeit eine große und hoch umstrittene Ausnahme bildete. In der Niederspannungsrichtlinie wurde das generelle und abstrakte Ziel der Verbrauchersicherheit formuliert, deren konkrete Ausgestaltung in Form von Produktsicherheitsstandards beließ sie jedoch bei privaten Standardisierungsorganisationen. Wenn sich Produzenten an diese Standards hielten, so wurde die Sicherheit des Produktes vermutet (Art. 5 (1) NiederspannungsRL). Rechtlich war nur das Ziel der Verbrauchersicherheit bindend. Wenn Produzenten diese Sicherheit auf eine andere Weise als durch die Einhaltung der Standards gewährleisten konnten, so stand ihnen dieser Weg offen. Heute würden wir dies als prinzipienbasierten Ansatz beschreiben, da hauptsächlich auf die Erreichung der vorgenannten drei Ziele der prinzipienbasierten Regulierung abgestellt wird.

Der wesentliche Grund dafür, dass dieser Ansatz ausschließlich in der Niederspannungsrichtlinie Verwendung fand, waren Unsicherheiten über ihre Vereinbarkeit mit dem Europarecht. Die Kommission und Repräsentanten der Mitgliedstaaten sahen die prinzipienbasierte Regulierung in der Niederspannungsrichtlinie ohnehin lediglich als eine nicht-repräsentative Ausnahme, die nicht zur Regulierung anderer Bereiche des Binnenmarktes wiederholt werden sollte (Joerges et al. 1988, S. 326). In der Tat war die Regulierung in der Niederspannungsrichtlinie so verschieden von dem generellen Harmonisierungskonzept der Art. 30, 36 und 100 EWG (heute Art. 30, 36, 114 AEUV), dass eine rechtliche Neukonzeption der Harmonisierung notwendig geworden war, um eine solche Neuausrichtung zu unterstützen. Gleichzeitig bot der Europäische Gerichtshof mit seinen bahnbrechenden Urteilen „Dassonville“" und „Cassis de Dijon“8 eine solche Neukonzeption an. Die weite Auslegung des Begriffs „Maßnahmen gleicher Wirkung“ als jede auch nur potenziell den Binnenmarkt beeinträchtigende Maßnahme und die korrespondierende weite Auslegung der Rechtfertigungsgründe bildeten die Basis für eine Neukonzeption der Binnenmarktharmonisierung (Maduro 1998, S. 61 ff.). Produzenten konnten nach dieser neuen Sichtweise nur dann in den Genuss des Rechts auf freie Zirkulation von Produkten in der EU kommen, wenn diese nachweisbar keine Gefahr für die Sicherheit und die Gesundheit von Verbrauchern darstellten (Joerges et al. 1988, S. 294). Harmonisierungsmaßnahmen auf europäischer Ebene mussten diese Neuausrichtung berücksichtigen, und zwar durch die Breitstellung entsprechender Sicherungsmaßnahmen, um auf diese Art den Verbraucherschutz gewährleisten zu können.

\footnotetext{
${ }^{6}$ Richtlinie 73/23/EWG des Rates vom 19. Februar 1973 zur Angleichung der Rechtsvorschriften der Mitgliedstaaten betreffend elektrische Betriebsmittel zur Verwendung innerhalb bestimmter Spannungsgrenzen, AB1. L 77 vom 26.03.1973, S. 29.

${ }^{7}$ Rs. 8/74, Urteil des Gerichtshofes vom 11. Juli 1974, Procureur du Roi v Dassonville, [1974] ECR, 837.

${ }^{8}$ Rs. 120/78 Urteil des Gerichtshofes vom 20. Februar 1979, Rewe-Zentral AG v Bundesmonopolverwaltung für Branntwein (Cassis de Dijon) [1979] ECR 649.
} 
Im Produktsicherheitsrecht führte diese Notwendigkeit zu einer Ausweitung des Regulierungsansatzes der Niederspannungsrichtlinie auf andere Produktgruppen und machte damit die Ausnahme zur Regel. Dieser horizontale Ansatz zur Regulierung von Produkten löste fortan als „Neuer Ansatz“9 den alten vertikalen Ansatz in der EU ab (Burrows 1990; McGee und Weatherill 1990; Egan 2001, S. 118 ff.): Produkte im „Neuen Ansatz“ müssen lediglich ein bestimmtes, abstrakt definiertes Ziel erreichen. Private Standards, welche durch europäische Normungsorganisationen entwickelt werden, helfen Produzenten dabei, die Schutzziele der Regulierung zu erreichen. Die Einhaltung der Standards ist meistens freiwillig. Werden diese Standards eingehalten, so können Produkte die „CE“ (= Communauté Européenne, Comunidad Europea, Comunidade Europeia und Comunità Europea)-Signatur erhalten, welche sodann eine Eintrittskarte für den gesamten Binnenmarkt darstellt.

Sollte ein Mitgliedstaat Bedenken hinsichtlich der Sicherheit eines Produkts haben, so kann er innerhalb einer bestimmten Frist temporäre Maßnahmen zur Aufrechterhaltung der Sicherheit einleiten, wenn er die Kommission darüber informiert.

Zusammenfassend besteht der „Neue Ansatz“ aus sieben Schritten:

- Die EU entwirft Rahmenrichtlinien für bestimmte Produktkategorien, welche nur ein abstraktes Ziel setzen, das von den Produzenten erreicht werden muss.

- Europäische Standardisierungsorganisationen (z. B. European Committee for Standardization, European Committee for Electrotechnical Standardization, European Telecommunications Standards Institute) bekommen in dieser Richtlinie das Recht, das abstrakte Regulierungsziel im Rahmen der Entwicklung von Standards weiter zu konkretisieren. Ihre Vorschläge sind von den nationalen Standardisierungsorganisationen zu akzeptieren und werden in den Mitgliedstaaten in den entsprechenden Journalen publiziert.

- Die Einhaltung dieser Standards ist freiwillig.

- Die Einhaltung der allgemeinen Prinzipien der Rahmenrichtlinie wird vermutet, wenn der Produzent die von den Standardisierungsorganisationen entwickelten Standards einhält.

- Eine Sicherungsklausel erlaubt Mitgliedstaaten und der Kommission, auch in Fällen einzuschreiten, in denen trotz Konformität mit dem Standard das Produkt nicht sicher ist.

- Der Produzent darf die Konformität seines Produkts mit dem Standard durch ein CE-Kennzeichen deutlich machen. Üblicherweise muss dann das Produkt allerdings einen Konformitätstest der nationalen Behörde bestehen.

Vorangegangen waren nicht nur eine Unzufriedenheit mit der Effektivität der alten, punktuellen Regulierung, sondern auch ein geändertes Verständnis im Recht des Binnenmarktes. Die EuGH-Urteile „Dassonville“"10 und „Cassis de Dijon“"11 führten

\footnotetext{
${ }^{9}$ Entschließung des Rates vom 7. Mai 1985 über eine neue Konzeption auf dem Gebiet der technischen Harmonisierung und der Normung, Abl. C 136, 04.06.1985, S. 1-9.

${ }^{10}$ Rs. 8/74, Urteil des Gerichtshofes vom 11. Juli 1974, Procureur du Roi v Dassonville, [1974] ECR, 837.

${ }^{11}$ Rs. 120/78 Urteil des Gerichtshofes vom 20. Februar 1979, Rewe-Zentral AG v Bundesmonopolverwaltung für Branntwein (Cassis de Dijon) [1979] ECR 649.
} 
eine Logik ins Binnenmarktrecht ein, die eine freie Zirkulation von Produkten in der EU nur dann zuließ, wenn diese nachweisbar keine Gefahr für die Sicherheit und die Gesundheit von Verbrauchern darstellten (Joerges et al. 1988, S. 294). Dadurch wurde ein Umdenken eingeleitet: Während die Marktfreiheiten bislang nur eine de-regulierende Funktion hatten, ermöglichten die beiden EuGH-Urteile ein re-regulierendes Vorgehen auf EU-Ebene. Darüber hinaus machten sie auch den Weg für die EU frei, ganze Marktbereiche horizontal zu regulieren, anstatt nur punktuell bestimmte Produkte oder Produktgruppen. Die EU konnte nunmehr wählen, welche Regulierungsart sie für geeignet hielt, um die entsprechenden Ziele des Binnenmarktes zu verwirklichen.

Zusammenfassend wurde der „Neue Ansatz“ entwickelt, um auf die entsprechenden Bedürfnisse der damaligen Zeit zu reagieren:

- Die alte Regulierung hatte zu überbordender Bürokratie geführt, war zu kostspielig und wenig zielführend.

- Das rechtliche Verständnis des Binnenmarktes hatte sich von einer DeRegulierungs- zu einer Re-Regulierungsaufgabe geändert.

- Die Vielfalt des Marktes in der EU und die unterschiedlichen Bedürfnisse in den Mitgliedstaaten wurden nicht hinreichend berücksichtigt.

- Die bestehenden Regulierungen waren zu unflexibel, um auf technologischen Fortschritt oder unterschiedliche Bedürfnisse der Marktteilnehmer reagieren zu können.

\subsubsection{Haftungsregeln als Sicherheitsnetz}

Der „Neue Ansatz“ wird flankiert von Haftungsregeln, die der Konformitätsvermutung zur vollen Wirksamkeit verholfen haben. Neben dem „Neuen Ansatz“ wurde die Produkthaftungsrichtlinie 85/374/EEG ${ }^{12}$ eingeführt, welche eine verschuldensunabhängige Produzentenhaftung für den Hersteller defekter Produkte einführte. Dies ging Hand in Hand mit dem „Neuen Ansatz“. Hielt sich ein Produzent nämlich an die entsprechenden Standards, so wurde vermutet, dass das Produkt ,sicher“ ist. Damit wurde den Produzenten auch die Beweisführung im Rahmen der Produkthaftungsrichtlinie erleichtert und ihnen damit ein erhebliches Haftungsrisiko abgenommen. Zusätzlich wurde im Jahr 1992 durch die Richtlinie 92/59/EWG über die allgemeine Produktsicherheit $^{13}$ (Art. 3) die Verpflichtung der Hersteller, nur sichere Produkte herzustellen, horizontal, also für alle Produkte, verbindlich festgeschrieben. Damit wurde die

\footnotetext{
${ }^{12}$ Richtlinie 85/374/EWG des Rates vom 25. Juli 1985 zur Angleichung der Rechts- und Verwaltungsvorschriften der Mitgliedstaaten über die Haftung für fehlerhafte Produkte, Abl. L 210 vom 07.08.1985, S. 29-33.

${ }^{13}$ Richtlinie 92/59/EWG des Rates vom 29. Juni 1992 über die allgemeine Produktsicherheit, Abl L 228, 11.8.1992, S. 24-32. Aktuell gültige Richtlinie: Richtlinie 2001/95/EG des Europäischen Parlaments und des Rates vom 3. Dezember 2001 über die allgemeine Produktsicherheit, ABl. L 11 vom 15.01.2002, S. 4-17.
} 
Einhaltung der im „Neuen Ansatz“ geregelten Standards noch einmal attraktiver, da die damit verbundene Beweislastumkehr die einzige Möglichkeit darstellte, um die mit der Verpflichtung zur Produktsicherheit verbundenen Haftungsrisiken zu minimieren.

\subsection{3 Übertragbarkeit aufgrund ähnlicher Voraussetzungen}

Die Parallelen zur Bestandsaufnahme im Rahmen der GAP sind deutlich. Versteht man die GAP als eine im Wesentlichen landwirtschaftliche Erzeugung flankierende Politik, so geht es auch hier, wie im „Neuen Ansatz“, darum, unionsrechtliche Rahmenbedingungen für qualitativ hochwertige und europäischen Standards entsprechende landwirtschaftliche Erzeugnisse zu schaffen. Diese Erzeugnisse müssen, vergleichbar mit den Zielvorgaben im „Neuen Ansatz“, nicht nur dem maßgeblich im europäischen Lebensmittelrecht geregelten Verbraucherschutz entsprechen, sondern auch den den Landwirt verpflichtenden und freiwilligen europäischen Umwelt-, Verbraucher- und sonstigen Standards. Das bisherige System der GAP, die dies den Landwirten eigentlich ermöglichen soll, ist allerdings gekennzeichnet durch überbordende Bürokratie, komplexe und kostenträchtige Einzelfallregulierung und zu geringe Zielerreichung. Gleichzeitig verändern sich die Bedingungen im Binnenmarkt: Der Agrarsektor wird zunehmend vom Recht wie ein ,normaler“ Bereich des Binnenmarkts behandelt. Das Verbraucher- und Umweltschutzrecht reguliert mehr und mehr auch die Erzeugung landwirtschaftlicher Produkte und zwingt dabei Landwirte, sich auch an der Logik dieser Regulierungsbereiche zu orientieren. Landwirtschaft wird in diesem Sinne regulierungsstrategisch ,produktiviert“.

Der Vorteil dieser Entwicklung liegt darin, dass auch Landwirte ein Interesse daran haben, qualitativ hochwertige, marktfähige Produkte zu erzeugen, die nach außen hin, ähnlich dem CE-Siegel, für hohe Qualität bürgen. Dies hat der Landwirtschaftssektor mit den Produzenten im „Neuen Ansatz“ gemein. Unterschiedlich sind jedoch die Verknüpfung des ordnungsrechtlichen Kontrollsystems mit den einkommenspolitisch begründeten Direktzahlungen, die national und regional programmierten Möglichkeiten zur Finanzierung betrieblicher Maßnahmen zum Natur- und Umweltschutz, die nach wie vor wichtige Sonderstellung der Landwirtschaft als Primärversorger sowie die Bedeutung der Landwirtschaft für die Landschaftspflege und die ländliche Entwicklung. Eine mögliche Übertragung der Logik des „Neuen Ansatzes“ muss daher diese Aspekte mit in Betracht ziehen.

\subsubsection{Vorschlag der Übertragung des prinzipienbasierten „Neuen Ansatzes" auf die GAP}

Eine mögliche Übertragung der prinzipienbasierten Regulierung im „Neuen Ansatz", modifiziert für die Bedürfnisse des Landwirtschaftssektors, auf die GAP könnte folgendermaßen aussehen: 


\subsubsection{Rechtlich verbindliche Rahmenverordnung}

Ein rechtlich verbindlicher Rahmen in Form einer Verordnung (Rahmenverordnung oder Basisverordnung ${ }^{14}$ regelt die allgemein gültigen Prinzipien, die für jede Tätigkeit als Landwirtin oder Landwirt in der EU sowie für jedes Inverkehrbringen von landwirtschaftlichen Produkten in der EU gelten. Diese Prinzipien gelten sodann auch für Importeure. Daneben regelt die Rahmenverordnung auch den institutionellen Rahmen, der die Einhaltung der in dieser Rahmenverordnung festgelegten Prinzipien überwacht. Jeder in der EU gewerblich als Landwirtin oder Landwirt tätige Person muss diese Prinzipien erfüllen. Tun sie dies nicht, so verlieren sie als Ultima Ratio ihre Marktfähigkeit in der EU und dürfen nicht mehr am Markt tätig sein. ${ }^{15}$

\subsubsection{Gesetzliche Minimalvorgaben und freiwilliges Baukastensystem unterschiedlicher Standards}

Für alle landwirtschaftlichen Betriebe gelten rechtliche Mindeststandards, die einzuhalten sind. Dazu gehören mindestens die Anforderungen, die derzeit unter Cross Compliance zusammengefasst werden.

Den Betrieben wird ein „Baukastensystem“ von Standards zur Verfügung gestellt. Diese werden in zwei Gruppen differenziert. Die Standards der ersten Gruppe helfen den Betrieben bei der Erreichung der in der Verordnung festgelegten Minimalziele. Ihre Einhaltung begründet die Vermutung, dass die Minimalvorgaben erreicht werden. Die Standards der zweiten Gruppe gehen über die Minimalanforderungen hinaus. Ihre Einhaltung ist freiwillig, wie etwa derzeit bei den Standards für ökologischen Landbau.

Jeder Betrieb muss so ausgerichtet werden, dass die in der Rahmenverordnung festgelegten abstrakten Minimalziele erreicht werden. Dafür gibt es im System der prinzipienbasierten Regulierung zwei Möglichkeiten. Entweder die Landwirtin oder der Landwirt entwickeln eigene innovative Methoden, um diese Ziele zu erreichen. Dabei wird rechtlich vermutet, dass sie sich den in der Rahmenverordnung festgelegten Zielen entsprechend verhalten. Allerdings haften sie bei eventueller Nicht-Erreichung der Ziele (also z. B. mangelnde Sicherheit der Erzeugnisse, Verletzungen des Tierwohls oder Umweltschäden). Die andere Möglichkeit ist, dass die Betriebe nachweislich die Standards erfüllen, die typischerweise zur Einhaltung der Minimalziele hinreichen. Dann kommen sie in den Genuss der Beweislastumkehr.

\footnotetext{
${ }^{14}$ „Rahmen-“ oder „Basisverordnung“ beschreibt Gesetze auch auf europäischer Ebene, die die rechtlichen Grundlagen für einen bestimmten Marktbereich schaffen, die sodann nach und nach produkt- oder problemspezifisch mit einzelnen anderen Verordnungen verdichtet werden. Ein Beispiel ist die Lebensmittelbasisverordnung (EG) Nr. 178/2002, die beispielsweise in Art. 8 festschreibt, dass es ein Ziel des Lebensmittelrechts ist, Verbrauchern „eine sachkundige Wahl“ zu ermöglichen. Die Verordnung (EG) Nr. 1924/2006 (Health Claims) bestimmt dann für den Bereich der Gesundheitsinformationen genauer, wie dies im Detail umgesetzt werden soll.

${ }^{15}$ Das System basiert auf einer Registrierungspflicht. Deren formale Ausgestaltung ist Sache der Mitgliedstaaten. In Deutschland dient dem ein Gewerbeschein.
} 
Erfüllt ein Betrieb nachweislich darüber hinaus gehende Standards aus dem „Baukastensystem“, kann er eine entsprechende Qualitätskennzeichnung führen. Ein Modell dafür wäre wiederum das EU-Bio-Siegel: Orientiert sich ein Betrieb an den Standards für ökologischen Landbau, so kann er ein entsprechendes EU-Siegel für ökologische Produktion verwenden. Gehobene Standards könnten entweder für einzelne Ziele des Natur-, Umwelt- und Verbraucherschutzes sowie des Tierwohls entwickelt werden oder für Zielbündel (z. B. „biodiversitätsfreundlich“) oder Betriebskonzepte (z. B. ,nachhaltig“, ,ökologisch“). ${ }^{16}$ In jedem Fall müssen die Standards operationalisiert und nachprüfbar sein.

Um zu gewährleisten, dass das „Baukastensystem“ sowohl den fachlichen Standards wie den Bedürfnissen des Marktes entspricht, soll es nach dem Vorbild von Standardisierungsorganisationen mit fachkundigen Vertretern der einschlägigen europäischen Gruppen (Bauernverbände, Umweltschutzorganisationen etc.) sowie Wissenschaftlerinnen und Wissenschaftlern in einem paritätisch besetzten Gremium unter der Leitung und auf Vorschlag der Kommission entworfen werden. Bei der Ausgestaltung der Standards sollte auch auf bereits existierende Marktstandards (z. B. GlobalG.A.P. oder UTZ) zurückgegriffen werden. So kann gewährleistet werden, dass die Erzeugnisse der europäischen Landwirtschaft international marktfähig sind, die EU-Standards anschlussfähig bleiben, das Wissen der Standardisierungsorganisationen in den Prozess mit eingebunden wird und auch Abwanderungs- und Leakage-Effekte durch „Outsourcing“ ins außereuropäische Ausland minimiert werden.

\subsubsection{Mögliche Verknüpfung mit dem System der Agrarzahlungen}

Die über die ordnungsrechtlichen Mindeststandards hinausgehenden, freiwilligen Standards des „Baukastensystems“ könnten mit dem landwirtschaftlichen Fördersystem verknüpft werden, so wie dies bereits bei den vielerorts gewährten Prämien für Betriebe des ökologischen Landbaus der Fall ist. Die Knüpfung der Zahlungen an die Erfüllung von Standards, die von fachlich kompetenten Standardisierungsausschüssen erarbeitet wurden, könnte dazu beitragen, die Effektivität des Mitteleinsatzes zu erhöhen.

Im Bereich der Mindeststandards könnten die nachgewiesenen Mehrkosten für Anforderungen oberhalb der Weltmarktstandards durch finanzielle Zuwendungen ausgeglichen werden. Dafür könnten die Mittel, die derzeit in der Ersten Säule der GAP verausgabt werden, eingesetzt werden. Diese Überlegung knüpft an die Beobachtung an, dass die derzeitigen Direktzahlungen an landwirtschaftliche Betriebe u. a. als Ausgleich für die höheren Standards gegenüber der Konkurrenz von außerhalb der EU begründet werden.

Graphisch lässt sich das hier vorgeschlagene neue System wie in Abb. 8.4 darstellen.

\footnotetext{
${ }^{16}$ Ein Beispiel im Bereich Tierwohl wäre das Beter Leven Kenmerk, das von der niederländischen Tierschutzorganisation Dierenbescherming entwickelt wurde.
} 


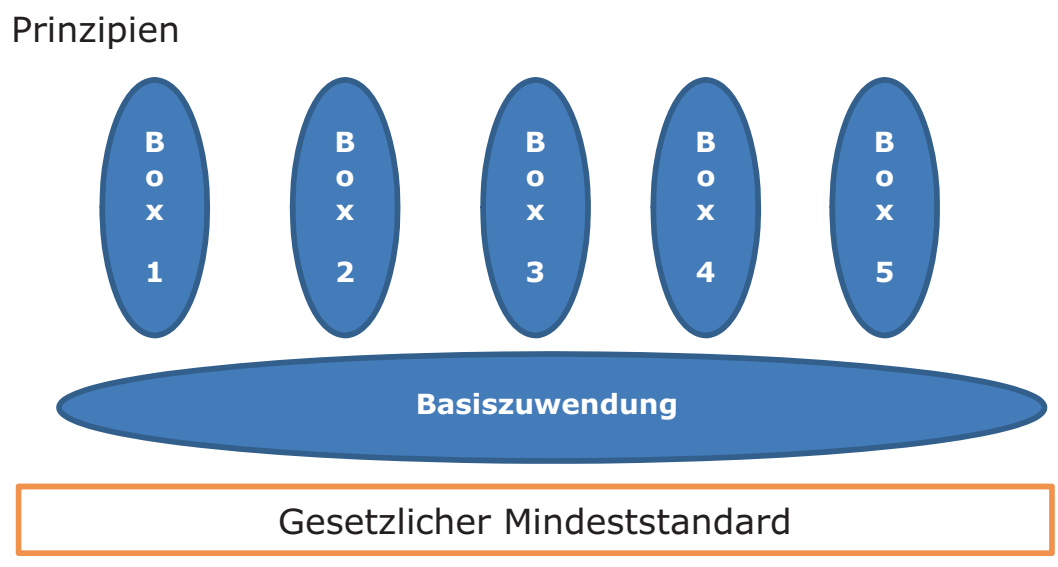

Abb. 8.4 Mögliche Struktur einer prinzipienbasierten Regulierung für den Agrarsektor. (Quelle: Eigene Darstellung)

In der rechtlichen Umsetzung könnte dieses Prinzip wie folgt ausgestaltet sein:

\section{1) Rahmenverordnung:}

Die Rahmenverordnung stellt den Grundsatz der Gebundenheit der Landwirte an die in dieser Verordnung dargelegten Pflichten in Form von Prinzipien auf. Daneben enthält sie die Vermutung der Einhaltung der Prinzipien, wenn Landwirte ihren Betrieb an den Standards des Baukastensystems ausrichten. Darüber hinaus enthält die Rahmenverordnung eine institutionelle Einbettung, die die Einhaltung der in der Rahmenverordnung festgelegten Prinzipien überwacht.

\section{a. Grundsatz und Prinzipien}

Grundsatz: Landwirte dürfen nur in der EU tätig werden, wenn sie die folgenden Prinzipien bei all ihren Tätigkeiten nachweislich erfüllen. In Anlehnung an die Regulierung von gefährlichen Stoffen auf EU-Ebene (REACH) kann eine solche Einhaltung von Prinzipien an eine Registrierungspflicht geknüpft sein. Damit ist sogleich ein Durchsetzungsmechanismus integriert, da die Registrierung bei Nicht-Einhaltung der Prinzipien entzogen werden kann.

Eine Beispielformulierung wäre:

\section{Art. 1 Registrierungspflicht}

Jeder landwirtschaftliche Betrieb in der EU muss registriert sein. Die Registrierung ermächtigt den Betrieb zu gewerblichen landwirtschaftlichen Tätigkeiten in der EU.

\section{Art. 2 Inhalt der Registrierungspflicht}

Die Registrierung wird geknüpft an die Erfüllung der nachfolgenden Prinzipien. Erfüllt der landwirtschaftliche Betrieb diese Prinzipien nicht, erlischt seine Registrierung. 
Prinzipien: Die Prinzipien werden dem Vertrag über die Europäische Union (EUV), dem Vertrag über die Arbeitsweise der Europäischen Union (AEUV), der Charta der Grundrechte der Europäischen Union, der europarechtlichen Rechtsprechung, den einschlägigen Sekundärrechtsakten und dem WTO-Recht entnommen. Die Prinzipien im AEUV und EUV sind nur an Mitgliedstaaten und Organe der EU gerichtet. Die Rahmenverordnung soll diese Ziele als Prinzipien für die Tätigkeit der Landwirte anwendbar fortschreiben. Im Einzelnen sind dies insbesondere folgende Prinzipien:

- die in Art. 39 AEUV genannten;

- hohes Umwelt-, Verbraucherschutz- und Gesundheitsniveau;

- freier Warenverkehr;

- Vorsorgeprinzip;

- Verursacherprinzip;

- Wissenschaftlichkeitsprinzip;

- Tierwohl;

- Nachhaltigkeit.

Eine Beispielformulierung für ein Prinzip ist:

\section{Art. 4 Hohes Umweltschutzniveau}

Jeder in der EU tätige landwirtschaftliche Betrieb muss sicherstellen, dass er bei all seinen Tätigkeiten ein hohes Umweltschutzniveau erreicht.

\section{b. Konformitätsvermutung}

Grundsätzlich steht es landwirtschaftlichen Betrieben frei, auf welche Weise sie diese Ziele erreichen. Die relative Unbestimmtheit der Prinzipien und deren Zielkonflikte untereinander lassen dem Landwirt zwar einen erheblichen schöpferischen Spielraum, der von besonders unternehmerfreudigen Landwirten und oder Landwirten, deren Geschäft nur wenig risikobehaftet ist, genutzt werden kann. Allerdings belässt dieses System Landwirte auch mit einer erheblichen Rechtsunsicherheit. Um einerseits den Raum für unternehmerische Freiheit (und Verantwortung) nicht zu beschneiden und um andererseits Rechtssicherheit zu bieten, enthält die Verordnung eine Klausel, die unter bestimmten Bedingungen die Vereinbarkeit der Tätigkeit des Landwirts mit diesen Prinzipien vermutet.

Eine Beispielformulierung wäre:

\section{Art. 10 Konformitätsvermutung}

Das Sicherstellen mit den oben genannten Prinzipien wird vermutet, wenn der Landwirt seine Tätigkeit an dem in Verordnung (EU) XXX/XXX festgelegten System ausrichtet. 


\section{c. Verordnung über das „Baukastensystem““}

Eine Verordnung legt das „Baukastensystem“, die entsprechenden Standards und wie sie entwickelt werden sowie die Bedingungen für die Vergabe von Kennzeichnungen fest. Das „Baukastensystem“ orientiert sich an den Berufsbildern am Markt und entwirft entsprechende Standards für diese Berufsbilder.

Eine Beispielformulierung wäre:

\section{Art. 7 Berufsbilder}

\section{(1) Nachhaltig wirtschaftender Betrieb}

Als „Nachhaltig wirtschaftender Betrieb“ darf sich bezeichnen, wer die Standards EN346, EN568 und EN789 aus dem „Baukastensystem“ erfüllt.

\section{(2) Ökologisch wirtschaftender Betrieb}

Als „Ökologisch wirtschaftender Betrieb“ darf sich bezeichnen, wer zusätzlich die Standards EN347, EN569 und EN790 aus dem „Baukastensystem“ erfüllt.

Die Standards selbst werden durch zu bildende Standardisierungsausschüsse ${ }^{17}$ entwickelt, deren Zusammensetzung sich nach EU-Recht richtet und Vertreter der betroffenen Marktbereiche umfasst. Die von diesen Ausschüssen entwickelten Standards werden im Anhang zur Verordnung veröffentlicht.

Die Qualitätssiegel sollen von der EU unter Zuhilfenahme verhaltenswissenschaftlicher Erkenntnisse entworfen und in der Verordnung festgelegt werden.

\section{d. Einbettung in ein Haftungssystem}

Der hier vorgeschlagene „Neue Ansatz“ der GAP kann, ähnlich den Regelungen des "Neuen Ansatzes“ im Produktsicherheitsrecht, in ein Haftungssystem eingebettet werden. Im Unterschied zum „Neuen Ansatz“ enthält das hier vorgeschlagene System jedoch eine Registrierungspflicht. Dadurch ist bereits ein Durchsetzungselement mit eingebaut. Darüber hinaus besteht auf EU-Ebene schon heute ein verschuldensunabhängiges Haftungssystem für Agrarprodukte, welches zum einen durch die Umwelthaftungsrichtlinie und zum anderen durch die Produktsicherheitsrichtlinie ${ }^{18}$ gewährleistet ist, ohne jedoch, wie im Produktsicherheitsrecht, Landwirten eine Beweislastumkehr durch einen „Neuen Ansatz“ zu ermöglichen. Der hier vorgeschlagene Weg würde dieses Versäumnis wett machen und somit das

\footnotetext{
${ }^{17}$ Diese könnten beispielsweise nach dem Vorbild des TÜV organisiert werden. Die Zusammensetzung sollte staatlich geregelt sein, um eine breite Einbindung der relevanten Expertise sicherzustellen. Eine staatliche Finanzierung wäre gerechtfertigt, da die Standardisierungsausschüsse die effektive Wahrnehmung hoheitlicher Aufgaben unterstützen sollen.

${ }^{18}$ Richtlinie 1999/34/EG des Europäischen Parlaments und des Rates vom 10. Mai 1999 zur Änderung der Richtlinie 85/374/EWG des Rates zur Angleichung der Rechts- und Verwaltungsvorschriften der Mitgliedstaaten über die Haftung für fehlerhafte Produkte, ABl L 141 vom 04.06.1999, S. 20-21, hat die Haftung der ProdukthaftungsRL ausdrücklich auch auf landwirtschaftliche Erzeugnisse erweitert.
} 
Haftungsrisiko von Landwirten, welche die geprüften Standards erfüllen, deutlich verringern. Die entsprechenden Richtlinien müssten sodann an den „Neuen Ansatz" angepasst werden.

\section{e. Institutionelle Einbettung}

Eine europäische Behörde wird mit der Verwaltung der Registrierung der Landwirte sowie der Administration und Weiterentwicklung des „Baukastensystems“ betraut. Diese Verwaltung kann entweder an eine existierende Behörde angegliedert (bspw. EFSA in Parma) oder durch eine neu zu gründende Agentur übernommen werden. Die Kontrolle und Implementation erfolgt weiterhin durch nationale Behörden. Zusätzlich sind privilegierte Klagebefugnisse für Mitbewerber oder Verbände denkbar, um die Einhaltung der Mindeststandards durch gerichtliche Kontrolle zu flankieren.

\subsubsection{Kompetenzgrundlage}

Rechtlich kann ein solcher Ansatz auf der Kompetenznorm des Art. 114 AEUV gestützt werden. Die aufgrund von Art. 114 AEUV erlassenen Akte müssen unter anderem der Verwirklichung der Ziele des Art. 26 AEUV dienen. Demnach müssen die Maßnahmen gem. Art. 26 Abs. 2 AEUV vor allem die Grundfreiheiten verwirklichen. Im Vergleich zum „Neuen Ansatz“ besteht bei der hier vorgeschlagenen Lösung die Besonderheit, dass nicht nur Produkte und damit „Waren“ im Sinne der Warenverkehrsfreiheit, sondern auch Dienstleistungen der Landwirte, also beispielsweise Umweltschutzdienstleistungen, im Sinne der Dienstleistungsfreiheit geregelt werden. Ist der Anwendungsbereich mehrerer Grundfreiheiten berührt, so sind diese Grundfreiheiten grundsätzlich nebeneinander anwendbar. Lässt sich indes aus der Verletzungshandlung der Grundfreiheit und der Zielrichtung der Maßnahme des Verpflichteten im Schwerpunkt die Verletzung einer Grundfreiheit zuordnen, so ist der Anwendungsbereich der anderen Grundfreiheit erst gar nicht eröffnet (Purnhagen 2011, S. 225 f.). Wo der Schwerpunkt der Regelung bei den hier vorgeschlagenen Verordnungen liegt, ist nicht ohne Weiteres zu bestimmen. Sollte der Schwerpunkt auf der Regelung der Produkte liegen, so kann ohne Weiteres die Rechtsprechung zum „Neuen Ansatz“ zu Art. 114 AEUV auf die hier vorliegenden Rechtsakte übertragen werden. Sollte der Schwerpunkt auf Dienstleistungen liegen, ist fraglich, ob die Rechtsprechung zum „Neuen Ansatz“ auch hierauf bezogen werden kann. Grundsätzlich ist hier wohl eher davon auszugehen, dass die Anwendungsbereiche mehrerer Grundfreiheiten berührt sind und daher beide nebeneinander anzuwenden sind.

\subsubsection{Schlussfolgerung und Ausblick}

Ein „Neuer Ansatz“ zur Regulierung in der GAP könnte geeignet sein, die Haftungsrisiken für Landwirte und die mit dem derzeitigen System einhergehende Bürokratie zu verringern und die GAP an die veränderten Gegebenheiten im Binnenmarktrecht 
anzugleichen. Die parallele Entwicklung der privaten Standardisierung könnte in das System integriert werden, indem beispielsweise private Standards durch die Standardisierungsausschüsse anerkannt werden. Das „Baukastensystem“ könnte es den Landwirtinnen und Landwirten erleichtern, sich durch klar definierte Qualitätssiegel auf dem Markt als Qualitätsbetrieb international zu positionieren. Sie bekämen mehr Freiraum in der Gestaltung ihrer betrieblichen Abläufe bei gleichzeitiger Möglichkeit zur Spezialisierung und Haftungsbeschränkung. Das System von Standardisierungsausschüssen, Registrierungspflicht und Zertifizierungsmöglichkeit für landwirtschaftliche Tätigkeiten würde eine angemessene Partizipation der Marktteilnehmer bei der Standardisierung erlauben. Für die genauere Ausgestaltung des „Baukastensystems“ könnte die EU auf die Erfahrung der Mitglieder der neu zu schaffenden Standardisierungsorganisationen zurückgreifen, die mit Vertretern der jeweiligen fachkundigen Kreise besetzt sein sollten.

Eine Umstellung der Regulierungsregimes des Agrarsektors auf prinzipienbasierte Regulierung könnte auch das Versprechen einlösen, dass die Direktzahlungen der Ersten Säule der GAP dabei helfen, in der EU ein höheres Niveau des Umweltund Verbraucherschutzes im Vergleich zu den Wettbewerbern von außerhalb der EU zu ermöglichen. Die von den Standardisierungsausschüssen erarbeiteten Standards lassen sich direkter mit Schätzungen des typischen betrieblichen Aufwands verknüpfen als etwa ein Umweltzustandsindikator. Insofern ließen sich auch die eventuellen Mehrkosten der ordnungsrechtlich festgeschriebenen Minimalstandards im Vergleich zur Importkonkurrenz von außerhalb der EU, die gegebenenfalls eine pauschale Basisprämie für alle landwirtschaftlichen Betriebe rechtfertigen könnten, vergleichsweise systematisch und transparent ableiten. In jedem Fall wäre eine solche pauschale Basisprämie bei prinzipienbasierter Regulierung mindestens so systematisch mit den regulatorischen Anforderungen verknüpft wie im derzeitigen Cross-Compliance-System. Dessen maßnahmenorientierter Ansatz und hoher administrativer Aufwand würden jedoch durch ein erfolgs- und haftungsorientiertes System ersetzt.

Insgesamt könnte die Umstellung auf das System einer prinzipienbasierten Regulierung für den Agrarsektor also zu einer erheblichen Verbesserung der Effektivität und Effizienz des Natur-, Umwelt-, Verbraucher- und Tierschutzes bei gleichzeitiger Verminderung der administrativen Komplexität führen. Eine weitere Ausarbeitung des Ansatzes erscheint vielversprechend.

\subsection{Bewertung der Optionen}

Die Optionen 1 bis 3 zeigen alternative strategische Entwicklungsrichtungen der Agrarpolitik auf, die dazu beitragen könnten, dass sich die deutsche Agrarwirtschaft dem Leitbild einer multifunktionalen, natur- und umweltverträglichen Landwirtschaft annähert. In Option 1 dienen die betrieblichen Direktzahlungen als Hebel, die Naturschutz- und Umweltleistungen in der EU flächendeckend zu verbessern. Der wichtigste Mechanismus ist hier die Erhöhung der Anforderungen zunächst im Greening 
und perspektivisch eventuell auch im GLÖZ. In Option 2 werden Verbesserungen der Naturschutz- und Umweltqualität vor allem durch zusätzliche und anspruchsvollere freiwillige Leistungen im Rahmen von AUK-Maßnahmen erbracht, für die durch Reduktion der Direktzahlungen mehr Mittel zur Verfügung stehen. Hinzu kommt ein möglicher Naturschutz- und Umwelt-Mehrwert der Greening-Maßnahmen, wenn diese national oder regional und damit angepasster programmiert würden. Option 3 integriert das System von Direktzahlungen, freiwilligen Maßnahmen und Ordnungsrecht zu einem aufeinander aufbauenden Stufensystem. Verbesserte Naturschutz- und Umweltleistungen werden hier angestrebt durch die Kombination verpflichtender Auflagen, flächendeckender Zahlungen zur Honorierung von landschaftlicher Vielfalt, standortbezogener Zahlungen zur Aufrechterhaltung der Landwirtschaft in von großflächiger Aufgabe bedrohten Gebietskulissen, flächendeckender freiwilliger „,hellgrüner“ Maßnahmen sowie regional/standörtlich angebotener freiwilliger „,dunkelgrüner" AUK-Maßnahmen.

Eine vergleichende Bewertung kann an dieser Stelle lediglich abschätzenden Charakter haben. Denn sowohl die politische Zustimmungsfähigkeit wie die tatsächlichen Effekte der strategischen Optionen hängen von ihrer Ausgestaltung im Detail ab. An dieser Stelle werden die oben dargestellten Befunde im Hinblick auf die Machbarkeit, die Effektivität hinsichtlich der Erreichung von Natur- und Umweltschutzzielen sowie die Implikationen und Akteurskoalitionen in vergleichender Absicht verdichtet.

\subsubsection{Machbarkeit}

Option 1 stellt eine inkrementelle Weiterentwicklung des bisherigen Entwicklungspfads der GAP dar, bei dem die etablierten politischen Instrumente im Zeitablauf anspruchsvoller ausgestaltet werden. Konsequent fortgeführt, eröffnet sie die Perspektive eines kumulativen Paradigmenwechsels zu einer nachhaltigen, multifunktionalen Landwirtschaft.

Bei Option 2 handelt es sich zunächst zwar ebenfalls um eine Weiterentwicklung der bisherigen GAP, die Rückführung der Direktzahlungen und das hohe Gewicht national und regional programmierter Maßnahmen stellen jedoch erhebliche Veränderungen dar. Die starke Dezentralisierung der Ausgestaltung der Maßnahmen erfordert, dass in den nationalen und regionalen Implementationsprozessen die Anliegen des Natur- und Umweltschutzes in eine starke Position gebracht werden. Die Erfahrungen mit der Umsetzung der Greening-Maßnahmen zeigen, dass hier Vorkehrungen zu treffen wären, um einer möglichen „Verwässerung“ entgegenzuwirken sowie der Präferenz der Verwaltungen für einfache, bekannte Maßnahmen mit planmäßigem Mittelabfluss und geringem Anlastungsrisiko Rechnung zu tragen. Dabei käme den Notifizierungsverfahren eine wesentliche Bedeutung zu. Ein Erfolg dieser Strategie setzt außerdem voraus, dass die durch den Abbau der Direktzahlungen frei werdenden Mittel in erheblichem Umfang in natur- und umweltorientierte Maßnahmen und Programme fließen. Der Sicherung der dafür 
zur Verfügung stehenden Mittel kommt bei dieser Option erhebliche Bedeutung $\mathrm{zu}$, insbesondere auch dann, wenn die AUK-Maßnahmen kofinanziert werden müssten. Die regionale und ggf. lokale Programmierung kann zusätzlichen bürokratischen Aufwand bedeuten.

Option 3 läuft auf eine Überwindung der historisch kontingenten Zwei-SäulenArchitektur der GAP hinaus. Sie ersetzt das „Gießkannensystem“ durch ein mehrstufiges System leistungsbezogener Honorierung der einzelbetrieblich erbrachten ökologischen Leistungen. Die Förderungsstruktur knüpft am bestehenden System der Agrarverwaltung an, nutzt aber konsequent Fernerkundung und GIS-Technologien zur Verwaltungsvereinfachung. Dies setzt aber eine wissenschaftliche Begründung der Algorithmen für die EU-weite Kompensierung von Standortnachteilen und Honorierung landschaftlicher Vielfalt sowie eine proaktive Regelung eventueller Datenschutz-Probleme voraus. Die Option erfordert also eine intensive konzeptionelle Vorbereitung.

\subsubsection{Effektivität und Effizienz}

Es ist plausibel, dass Option $1 \mathrm{zu}$ deutlichen Verbesserungen im Natur- und Umweltschutz beiträgt, wenn das Greening anspruchsvoller wird. Allerdings verspricht diese Option eine lediglich inkrementelle Verbesserung der Probleme der mangelnden Zielgenauigkeit und der Überkompensierung der derzeitigen Direktzahlungen.

Option 2 hat ein großes Potenzial, durch Verschiebung der Finanzmittel von den Direktzahlungen zu stärker zielorientierten Maßnahmen die Effektivität und Effizienz des Mitteleinsatzes zu erhöhen. Allerdings besteht die Gefahr, dass Betriebe mit hohen Opportunitätskosten für Agrar- und Umweltleistungen aus allen freiwilligen Programmen, inklusive GLÖZ und Greening, aussteigen. Daher kommt in dieser Option einer wirksamen Ausgestaltung und Implementation der Mindeststandards des Natur- und Umweltschutzes im ordnungsrechtlichen Rahmen eine besondere Bedeutung zu. Dennoch könnte es in dieser Option zu Verschlechterungen des Natur- und Umweltzustands vor allem in hoch produktiven Regionen kommen, wenn hier nicht attraktive Alternativen angeboten werden. Bei entsprechender Mittelausstattung kann in dieser Option aber der Aspekt der Leistungserbringung in Intensivregionen besser adressiert werden als bisher. Außerdem könnten die Koordinierungsmöglichkeiten verbessert werden. Letztlich hängt die Realisierung des Effektivitäts- und Effizienzpotenzials der Option 2 von einer Vielzahl dezentraler Entscheidungen im Implementationsprozess ab.

Option 3 könnte bei guter Vorbereitung und systematischer Umsetzung das höchste Potenzial zur Verbesserung der Effektivität und Effizienz im Sinne der Ziele des Natur- und Umweltschutzes haben. Das aufeinander aufbauende System von verpflichtenden Anforderungen sowie allgemeinen und regionsspezifischen freiwilligen Maßnahmen (mit gestaffelter finanzieller Kompensation) ermöglicht eine zielgenaue Programmierung. Die neuartigen Prämien zum Erhalt der Landschaftsvielfalt würden direkt an einem wesentlichen Ziel der Politik für den ländlichen Raum 
ansetzen und zugleich die Voraussetzungen für den Schutz der biologischen Vielfalt flächendeckend verbessern.

Im Ergebnis sind Differenzierungen der von den Betrieben erbrachten Leistungen innerhalb einer Region sowie die Nicht-Teilnahme von Betrieben in intensiv genutzten Regionen an freiwilligen Maßnahmen wahrscheinlich. Daher kommt auch hier den gesetzlichen Mindeststandards und ihrer Durchsetzung große Bedeutung zu. Vorbehaltlich der Ergebnisse von Machbarkeitsstudien ist anzunehmen, dass der Kontroll- und Verwaltungsaufwand deutlich geringer sein könnte als in Option 2, vor allem wenn freiwillige Maßnahmen grundsätzlich über einen mehrjährigen Zeitraum vereinbart würden.

\subsubsection{Akteurskoalitionen}

Option 1 verbleibt durch den inkrementellen Ansatz im Rahmen des derzeitigen agrarpolitischen Pfads. Dies begrenzt die Ungewissheit für alle Beteiligten, insbesondere die landwirtschaftlichen Betriebe und die mit der Implementierung betrauten Verwaltungsapparate. Hinter dieser Option könnte sich daher eine moderate Reformkoalition bilden, wenn die Nutznießer der Direktzahlungen den Eindruck gewinnen, dass erhöhte Gegenleistungen notwendig sind, um deren Fortführung gesellschaftlich zu legitimieren.

Option 2 folgt mit dem Ansatz, die Zweite Säule der Agrarpolitik auf Kosten der Ersten Säule zu stärken, einer langjährigen Forderung vieler Vertreter des Natur- und Umweltschutzes. Von dieser Seite wird allerdings auch die Bedeutung der Direktzahlungen betont, um eine Aufrechterhaltung der Landwirtschaft an Marginalstandorten und - über Cross Compliance und Greening - Verbesserungen in den Intensivregionen zu erreichen. Option 2 sieht für beide Ziele Ansätze vor, die zielgenauer und effizienter sein könnten. Die erhöhten nationalen und regionalen Gestaltungsspielräume könnten für viele Akteure in den Mitgliedstaaten attraktiv sein. Sie werden auch von vielen Umweltverbänden sowie Wissenschaftlerinnen und Wissenschaftlern gefordert. Die Unsicherheiten über die Instrumentierung und die Verteilungswirkungen sowie die konsequentere Kopplung von Zahlungen an nachweisbare Gegenleistungen könnten zu Widerständen aus den Reihen der Agrarwirtschaft führen.

Option 3 würde die deutlichste Transformation der bisherigen Agrarpolitik darstellen. Sie könnte für eine Koalition aus Agrarwirtschaft einerseits und Natur- und Umweltschutz andererseits attraktiv sein, wenn es gelingt, überzeugend glaubhaft zu machen, dass die angestrebten Vereinfachungen der Verwaltungsabläufe tatsächlich erreicht und die Bewertung einer Reihe von Umweltleistungen über GIS-gestützte Daten fachlich begründet werden können. Solange die Verteilungswirkungen dieser Option nicht klar sind, ist jedoch mit erheblichen politischen Widerständen zu rechnen. Eine detaillierte Operationalisierung der konzeptionellen Grundlagen, Studien zur Machbarkeit und den Effekten sowie ggf. begleitende Instrumente zur Kanalisierung der Verteilungseffekte zwischen Mitgliedstaaten und Regionen dürften daher wesentlich sein, um die notwendige politische Unterstützung zu gewinnen, diese Option auf die Reformagenda zu setzen. 


\subsection{Fazit}

Die Formulierung von drei strategischen Entwicklungsoptionen für die Agrarpolitik diente dazu, den möglichen Handlungsraum für die weitere Entwicklung der Agrarpolitik in verdichteter Weise zu beschreiben. Eine frühere Fassung der drei Optionen wurde der Projektbegleitenden Arbeitsgruppe Ende Juli 2016 vorgelegt. Die Priorisierung einer dieser Optionen stellte eine politische Aufgabe dar. Die wissenschaftliche Analyse der Voraussetzungen und Implikationen kann dabei zwar die Wissensgrundlagen einer solchen Entscheidung verbessern, diese aber nicht in die Sphäre einer wertfreien Expertenhandlung transferieren. Daher war es im Ablauf des Projekts von großer Bedeutung, dass die Auswahl derjenigen Option, die der Formulierung der Politikpapiere zugrunde gelegt werden sollte, durch die Auftraggeber vorgenommen wird.

Nach ausgiebiger Diskussion votierte die Projektbegleitende Arbeitsgruppe im September 2016 dafür, die Option 3 zur Grundlage des Policy-Papers (Kap. 2) zu machen. Im Verlauf der Erarbeitung dieser Papiere wurde die ausgewählte Option weiter kondensiert und verdichtet. Die „,neue Architektur“ für die Agrarpolitik, die im Policy-Paper vorgeschlagen wird, basiert daher auf Option 3, ist aber zugleich eine Weiterentwicklung und stärker politikorientierte Profilierung.

Mit der Formulierung der strategischen Handlungsoptionen und der Politikpapiere waren die Aufgaben des Projekts abgeschlossen. Die folgende Schlussbetrachtung reflektiert den Verlauf und die Ergebnisse des Projekts und formuliert Desiderata für die weitere Forschung.

Open Access Dieses Kapitel wird unter der Creative Commons Namensnennung 4.0 International Lizenz (http://creativecommons.org/licenses/by/4.0/deed.de) veröffentlicht, welche die Nutzung, Vervielfältigung, Bearbeitung, Verbreitung und Wiedergabe in jeglichem Medium und Format erlaubt, sofern Sie den/die ursprünglichen Autor(en) und die Quelle ordnungsgemäß nennen, einen Link zur Creative Commons Lizenz beifügen und angeben, ob Änderungen vorgenommen wurden.

Die in diesem Kapitel enthaltenen Bilder und sonstiges Drittmaterial unterliegen ebenfalls der genannten Creative Commons Lizenz, sofern sich aus der Abbildungslegende nichts anderes ergibt. Sofern das betreffende Material nicht unter der genannten Creative Commons Lizenz steht und die betreffende Handlung nicht nach gesetzlichen Vorschriften erlaubt ist, ist für die oben aufgeführten Weiterverwendungen des Materials die Einwilligung des jeweiligen Rechteinhabers einzuholen.

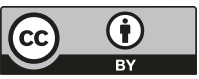




\section{Kapitel 9 \\ Schlussbetrachtung}

\subsection{Verwirklichung des inter- und transdisziplinären Anspruchs}

Das Projekt ZA-NExUS war ein dezidiert inter- und transdisziplinäres Projekt an der Schnittstelle von Wissenschaft und Politik. Weder die spezifische interdisziplinäre Zusammensetzung des Forschungskonsortiums noch der enge, an der politischen Dynamik einer langfristigen, großskaligen politischen Reformdiskussion orientierte Zeitplan entsprachen den etablierten Routinen wissenschaftlichen Arbeitens. Das Hauptziel des Projekts war es, wissenschaftlich basierte Optionen für die künftige Ausgestaltung der Agrarpolitik aus der Perspektive des Natur- und Umweltschutzes $\mathrm{zu}$ formulieren und in die öffentliche und politische Diskussion einzubringen. Für die wissenschaftliche Basierung was es notwendig, einen ausgeprägt interdisziplinären Ansatz zu wählen. Es wurde daher ein Konsortium gebildet, das Sachverstand aus den Bereichen Ökologie und Umweltwissenschaften, Agrar- und Ressourcenökonomie, Systemanalyse, Rechtswissenschaften sowie Politik- und Kommunikationswissenschaften in einer seltenen Konstellation kombinierte. Die Disziplinen übergreifende Verständigung wurde durch eine Reihe von Brückenkonzepten sowie einen geteilten systemischen Ansatz ermöglicht. Sie erforderte Dialogfähigkeit, ermöglichte aber auch ein ,Thinking out of the box“. Wichtig war der Anspruch, neue und innovative Konzepte, die noch nicht im Mainstream der politischen Debatte angekommen sind, aufzunehmen und weiterzuentwickeln. Der laufenden Qualitätssicherung dienten dabei die fünf Peer Reviewer, die langjährige Erfahrung in der wissenschaftlichen Politikberatung einbrachten und an mehreren Punkten intensive kritische Rückmeldungen zu Zwischen- und vorläufigen Endergebnissen gaben.

Transdisziplinarität ist ein Prinzip integrativer Forschung, das auf die methodische Verbindung von wissenschaftlichem und praktischem Wissen abzielt (Gibbons et al. 1994). Transdisziplinäre Forschung geht von komplexen gesellschaftlichen Problemen aus, beinhaltet sowohl interdisziplinäre Zusammenarbeit wie Kooperation zwischen Wissenschaft und Gesellschaft und ermöglicht wechselseitige Lernprozesse 
(Jahn et al. 2012). Der transdisziplinäre Charakter des ZA-NExUS-Projekts ergibt sich daraus, dass in verschiedenen Formaten - Experten- und Hintergrundgespräche, Experten-Workshops sowie die regelmäßigen Treffen mit einer Projektbegleitenden Arbeitsgruppe (PAG) - ein laufender, auf Lernen abzielender Dialog mit Personen geführt wurde, die auf langjähriger Erfahrung beruhendes Wissen über das hier relevante Praxisfeld, die Evaluierung und Formulierung von Policy-Designs, einbrachten. Auf diese Weise konnte das wissenschaftliche Team, das aufbauend auf der interdisziplinären Problemanalyse Lösungsansätze formulierte, die Problemlagen und Handlungsbedingungen aus Sicht der praktischen Politikgestaltung systematisch in die Projektarbeit einbeziehen. Dies trug wesentlich dazu bei, die Anschlussfähigkeit der Überlegungen aus dem wissenschaftlichen Ideenraum an die politische Diskussion sicherzustellen. Vor allem die regelmäßigen intensiven Diskussionen in der PAG ermöglichten wechselseitige Lernprozesse. Die Synthese der Diskussionsergebnisse und die Formulierung der Schlussfolgerungen war dabei strikt die Aufgabe des Projektteams. Die Verbindung von wissenschaftlichem und praktischem Wissen wurde insofern aus der Perspektive des wissenschaftlichen Teams vorgenommen.

Die konzeptionelle Brücke zwischen der interdisziplinären Wissenssynthese (Kap. 3 und 4) und der transdisziplinären Entwicklung von Handlungsansätzen (Kap. 7 und 8) bildete die SWOT-Analyse (Kap. 5) und die darauf aufbauende Formulierung eines Leitbilds für eine zukunftsfähige Agrarpolitik (Kap. 6).

Der transdisziplinäre Charakter des Projekts verstärkte sich in der letzten Projektphase, nachdem Ende Juli 2016 ein erster Entwurf des Projektberichts mit drei alternativen strategischen Handlungsoptionen (Kap. 8) vorgelegt worden war. Zunächst war es Aufgabe der PAG, die politische Entscheidung zu treffen, welche der drei Optionen in die öffentliche Diskussion eingebracht werden sollte. Im Anschluss waren die Mitglieder der PAG durch mehrere Rückkopplungsschleifen intensiv in die Erarbeitung des Policy-Papers (Kap. 2) eingebunden. Dies war insbesondere für die Zuspitzung und Pointierung des Papiers von großer Bedeutung. Der wissenschaftlichen Qualitätssicherung diente in dieser Phase eine fortgesetzte Einbindung der Peer Reviewer.

\subsection{Zusammenfassung der Aufgaben und Ergebnisse des ZA-NExUS-Projekts}

\section{Das Projekt ZA-NExUS erfüllte sieben Aufgaben:}

1. eine Synthese vorhandenen Wissens zu einer konzisen Problembeschreibung;

2. eine Analyse der politischen Mechanismen, welche einer besseren Berücksichtigung der Anliegen des Natur- und Umweltschutzes in der Agrarpolitik entgegenstehen;

3. die Identifizierung von Entwicklungen und Konzepten, welche eine bessere Berücksichtigung des Natur- und Umweltschutzes in der Agrarpolitik ermöglichen könnten; 
4. eine verdichtete Bewertung der Stärken und Schwächen der gegenwärtigen Agrarpolitik aus Sicht des Natur- und Umweltschutzes;

5. die Formulierung und Operationalisierung eines Leitbilds;

6. die Formulierung und Bewertung von Handlungsempfehlungen für die Politik;

7. die Vermittlung der Ergebnisse in die öffentliche und politische Diskussion.

Die erste Aufgabe war eine Synthese vorhandenen Wissens. Zu diesem Zweck wurde ein systematischer Überblick über die für den Natur- und Umweltschutz relevanten systemischen Entwicklungen im Zusammenhang mit der landwirtschaftlichen Entwicklung erstellt (Kap. 3). Das zwar nicht überraschende, in der Zusammenschau aber doch nachdrückliche Ergebnis war, dass

- der Zustand vieler schützenswerter Naturressourcen nicht gut ist;

- die Landwirtschaft in Deutschland durch stoffliche Einträge und Flächennutzung erheblich zur negativen Beeinflussung der Ressourcen beiträgt;

- die in verschiedenen Gesetzen, Richtlinien und Strategien definierten Zielwerte häufig verfehlt werden;

- in vielen Bereichen Aussagen zur Zielerreichung aufgrund der mangelnden Datenlage oder unzureichender Operationalisierung nicht zuverlässig getroffen werden können;

- zu wichtigen Teilbereichen bislang gar keine Zielwerte bestehen und insofern

- ein erhebliches Regelungsdefizit, auch durch oft wenig ambitionierte Vorgaben, sowie ein Vollzugsdefizit vorliegen.

Die zweite Aufgabe war eine Analyse der politischen Mechanismen, welche einer besseren Berücksichtigung der Anliegen des Natur- und Umweltschutzes in der Agrarpolitik entgegenstehen. Die Problembeschreibung legt die Schlussfolgerung nahe, dass ein Politikversagen beim Schutz der Natur- und Umweltressourcen vorliegt. Um dieses zu erklären, wurde die politische Logik der GAP und des ordnungsrechtlichen Rahmens analysiert (Abschn. 4.1). Hinsichtlich der GAP wurden dabei trotz erheblicher Veränderungen des institutionellen Rahmens (Einbettung in GATT/WTO, Mitentscheidung des Europäisches Parlaments bei der GAP, Ordnungsrecht), der Einführung neuer Politik-Instrumente, einer teilweisen Öffnung der agrarpolitischen Netzwerke und einer Pluralisierung der agrarpolitischen Ideen und Diskurse weiterhin erhebliche Barrieren für eine bessere Berücksichtigung des Natur- und Umweltschutzes festgestellt:

- die sektorale institutionelle Verankerung der Agrarpolitik bei der DG Agri und den Agrarministerien, wo Verbraucherschutz, Naturschutz- und Umweltanliegen eher als Zusatzaspekte denn als Kern der Agrarpolitik verstanden werden;

- die Institutionalisierung und Instrumentierung der GAP vorrangig als Einkommenspolitik für den Sektor;

- die Priorität für Verteilungsfragen (Nettozahlerposition) bei Verhandlungen zwischen den Mitgliedstaaten der EU;

- mangelnder externer Handlungsdruck bzw. institutionelle Abpufferung der Agrarpolitik gegenüber nicht-agrarischen gesellschaftlichen Problembeschreibungen;

- der mit zielgenauen Maßnahmen oft verbundene Bürokratieaufwand, der solche Maßnahmen unpopulär macht. 
Diese Barrieren müssen jedoch nicht unüberwindbar sein, und sie können auch veränderlich sein. Im Projekt wurden wichtige neue Entwicklungen und Konzepte identifiziert, welche eine bessere Berücksichtigung des Natur- und Umweltschutzes in der Agrarpolitik ermöglichen könnten (dritte Aufgabe):

- Institutioneller Rahmen: Erstens wandelt sich durch die europarechtliche Einbeziehung der GAP in das allgemeine Binnenmarktrecht die Position des landwirtschaftlichen Binnenmarkts von einer sektorspezifischen Markteinheit mit eigenen ausschließenden Regeln zu einem regulären Teil des Binnenmarktes. Dadurch wird u. a. das ,soziale” Binnenmarktrecht (insbesondere Umwelt- und Verbraucherschutz) mit der Anforderung hoher Schutzstandards auch in die GAP eingeführt. Zweitens hat der „From farm to table“-Ansatz im Verbraucherschutz die Folge, dass sich landwirtschaftliche Produktionszweige immer mehr an der Vermeidung von Haftungsrisiken orientieren müssen, die aus verbraucherschützenden Normen resultieren und sich auf die gesamte Wertschöpfungskette erstrecken. Und drittens entstehen zunehmend privatrechtliche Normen und Standards, die im Zusammenspiel mit (supra-)nationalem Recht ein größeres Regulierungspotenzial entfalten können als das nationale Recht (Abschn. 4.2).

- Einkommensorientierung der GAP: Die einkommenspolitischen Instrumente der GAP könnten in Zukunft verstärkt auf den Prüfstand gestellt werden. Durch den bevorstehenden Austritt des Nettozahlers Großbritannien aus der EU könnte das Prinzip einer ergebnisorientierten Verwendung der Haushaltsmittel größere Beachtung finden. Damit könnte dann die geringe einkommenspolitische Zielgenauigkeit der Direktzahlungen und möglicherweise sogar der Ansatz einer sektoralen Einkommenspolitik auf EU-Ebene verstärkt problematisiert werden.

- Zwischenstaatliche Verteilungslogik: Durch den Austritt Großbritanniens wird ein Nettozahler am Verhandlungstisch für die künftige GAP fehlen, der in der Vergangenheit stark auf eine deutlichere Ergebnisorientierung der GAP gedrängt hatte. Dies könnte die verteilungspolitische Dimension der Verhandlungen über die künftige GAP verschärfen.

- Handlungsdruck: Neue systemische Konzepte lenken den Blick auf die u. a. durch den Klimawandel gefährdete Resilienz vieler landwirtschaftlicher Produktionssysteme, die Resilienz von Ökosystemen, die durch die Landwirtschaft beeinflusst werden, und die Resilienz der Ernährungssicherheit. Der Handlungsbedarf erscheint zudem bei intersektoraler Betrachtung, etwa mittels des Konzepts des Wasser-Energie-Nahrung-Nexus, dringender als bei bloß sektoraler Sichtweise (Abschn. 4.3.1 und 4.3.2). Diese Betrachtung zeigt auch die Notwendigkeit einer intersektoralen Kooperation und Koordination auf.

- Ineffiziente und bürokratische Instrumente: Neuere verhaltenswissenschaftliche Ansätze ermöglichen die bessere Berücksichtigung psychologischer Mechanismen, wie beispielsweise Verlust- und Kontrollaversion, FramingMechanismen, Status-quo-Bias, veränderliche Präferenzen oder verinnerlichte Werte und Normen, bei der Ausgestaltung politischer Instrumente. Dies erleichtert die Einbeziehung und Aktivierung nicht-monetärer Motivationen zum Naturund Umweltschutz, die durch Normbildungsprozesse, sozialen Druck und die 
Stärkung von umweltbezogenen Aspekten der Selbst-Identität von Landwirtinnen und Landwirten gestärkt werden können. In der Folge könnte landwirtschaftlicher Naturschutz stärker als Lernprozess gestaltet werden, in dem Mechanismen wie Feedback und Selbstverpflichtung, ,Labeling“ oder kooperative Ansätze eine wichtige Rolle spielen. Die Effizienz von bestehenden AUKM könnte durch Orientierung am Konzept der Ökosystemleistungen verbessert werden, etwa durch Targeting, Zahlungsdifferenzierung, Auktionen, ergebnisorientierte Honorierung, kooperative Ansätze, Agglomerationsboni oder gesamtbetriebliche Verpflichtungen (Abschn. 4.3.3 und 4.3.4).

- Die häufig nicht problemadäquate sektorale Engführung der Agrarpolitik könnte zudem durch eine integrierte Agrar- und Ernährungspolitik überwunden werden, bei der u. a. partizipatorische Ansätze und die systematische Einbeziehung von Verbrauchern eine wichtige Rolle spielen würden (Abschn. 4.3.5 und 4.3.6).

Darauf aufbauend wurde als vierte Aufgabe eine verdichtete Bewertung der Stärken und Schwächen der gegenwärtigen Agrarpolitik aus Sicht des Natur- und Umweltschutzes in Form einer SWOT-Analyse vorgenommen. Die Stärken der GAP haben aus Sicht des Natur- und Umweltschutzes dabei eher den Charakter von Potenzialen: eine breite Instrumentenkiste mit einer bislang guten und verlässlichen Finanzausstattung. Dieses Potenzial wird jedoch vor allem für eine sektororientierte Einkommenspolitik genutzt, weil agrarpolitische Akteure und Interessen den politischen Gestaltungs-, Programmierungs- und Implementationsprozess dominieren (Schwächen). Bei einer möglichen Verschärfung der finanzpolitischen Verteilungskämpfe und einer Polarisierung des politischen Klimas besteht das Risiko, dass sich der politische Handlungsraum für den Natur- und Umweltschutz verengt. Dies könnte die notwendigen transformativen Strategien zur Erhöhung der Resilienz der Agrarökosysteme unmöglich machen - mit erheblichen Risiken für die Ernährungssicherheit, die Landwirtschaft und die Natur- und Umweltgüter. Demgegenüber könnte die Verankerung des Natur-, Umwelt- und Verbraucherschutzes sowie des Tierwohls als Legitimationsgrundlage für die Agrarzahlungen die Chance eröffnen, die mangelnde Ergebnisorientierung der tatsächlich implementierten Politik aufzuzeigen und den rechtlichen Anforderungen an ein hohes Schutzniveau im Binnenmarkt besser zur Geltung zu verhelfen. Neue systemische, partizipatorische und verhaltenswissenschaftliche Ansätze könnten in Kombination mit Innovationen im Bereich der Fernerkundung und der IT wesentlich effektivere und effizientere Policy-Designs ermöglichen.

Ausgehend von der SWOT-Analyse war die fünfte Aufgabe, ein Leitbild zu formulieren und zu operationalisieren. Diese wurde in die Frage nach dem Was und dem Wie aufgeteilt. Die Frage nach dem Was adressiert das agrarpolitische Leitbild für eine multifunktionale, natur- und umweltverträgliche Landwirtschaft, die eine flächendeckende Landwirtschaft umfasst, welche zu einer vielfältigen Kulturlandschaft beiträgt, die Tragfähigkeit der ökologischen Systeme beachtet und die ökosystemaren Dienstleistungen erhält. Auf die Frage nach dem Wie antworten die 15 Leitlinien einer zukunftsfähigen Agrarpolitik, welche die Landwirtschaft bei der Erfüllung ihrer unterschiedlichen, auf die Produktion und 
das Gemeinwohl ausgerichteten Aufgaben in effektiver und effizienter Weise unterstützt. Die Leitlinien wurden im weiteren Verlauf des Projekts bei der Formulierung des Policy-Papers weiter verdichtet. Ausgehend von dem Leitbild und den Leitlinien wurden 13 Dilemmata und Zielkonflikte identifiziert, die aufzeigen, dass eine zukunftsfähige Agrarpolitik Spannungen und Widersprüche aushalten muss und es keine einfachen, linearen Lösungen geben kann. Im nächsten Schritt wurden die verschiedenen Aussagen des Leitbilds mit qualitativen und quantitativen Zielwerten unterlegt und ein Soll-Ist-Vergleich mit der derzeitigen Situation vorgenommen. Auf dieser Basis wurden drei prioritäre Problemkomplexe identifiziert: Stoffeinträge in Boden, Wasser und Luft inklusive Klimagase und Arzneimitteleinträge; Wirkungen der Flächennutzung; Implementations-, Partizipations- und umweltorientierte Innovationsdefizite. Abschließend wurde die derzeitige Agrarpolitik mit den agrarpolitischen Leitlinien abgeglichen. Dabei zeigte sich, dass sich für alle Leitlinien zwar bereits Ansätze finden lassen, dass diese aber nicht hinreichend entwickelt oder noch nicht hinreichend wirksam sind. Neben den bereits oben genannten Problemen - geringe Zielgenauigkeit der Zuwendungen, Regelungs- und Vollzugsdefizite, Bürokratielasten und Defizite bei der Einbindung der Verbraucherinnen und Verbraucher - soll an dieser Stelle die mangelnde systemische Ausrichtung hervorgehoben werden (vgl. Tab. 6.2):

- zu geringe Berücksichtigung kumulativer Auswirkungen der vorherrschenden landwirtschaftlichen Praktiken;

- Defizite bei der Übersetzung des Verursacher-, Kooperations-, Vorsorge-, Vorsichts- und Nachhaltigkeitsprinzips ins Fachrecht und dessen Implementierung;

- geringe Verbreitung einer Resilienzorientierung im Gegensatz zur Orientierung an Produktivitäts- und Produktionssteigerung;

- Konzepte zur umfassenden Resilienzbewertung für landwirtschaftliche Produktionssysteme und Betriebe sind noch im frühen Forschungs- und Entwicklungsstadium;

- unzureichende Transparenz der Umweltmerkmale von Produkten und der Umweltleistungen von Produzenten;

- private Standards und Labels setzen selektiv an wenigen gut kommunizierbaren Elementen an;

- fehlende Mechanismen zur Adressierung von Problemen auf den relevanten räumlichen (z. B. Wassereinzugsgebiete) und zeitlichen Skalen;

- starke Verbesserungspotenziale zur regionalen Vernetzung von Akteuren sowie zu einer verbesserten dialogischen Kommunikation;

- starke Verbesserungspotenziale für eine flächendeckende Verankerung ökologischer Zusammenhänge als integraler Bestandteil des Lernens über landwirtschaftliche Produktionsmethoden und -systeme;

- Verbesserungsmöglichkeiten für den Transfer zwischen Forschung und landwirtschaftlicher Praxis.

Auf Basis der Problembeschreibung und -bewertung, des Leitbilds und der Defizitanalyse wurden Handlungsempfehlungen für die Politik formuliert und bewertet (sechste Aufgabe). Dies geschah auf den zwei Ebenen der agrarpolitischen 
Instrumente (,Bausteine“, Kap. 7) einerseits sowie alternativer strategischer Optionen (Kap. 8) andererseits. Auf der Ebene der „Bausteine“ wurden Entwicklungsmöglichkeiten beim Ordnungsrecht und den gesetzlichen Mindeststandards für die landwirtschaftliche Praxis (Abschn. 7.1), mögliche Varianten zur Entwicklung des Budgets der Agrarpolitik (Abschn. 7.2), der Direktzahlungen (Abschn. 7.3) sowie regional und standörtlich ausgerichteter Zahlungen (Abschn. 7.4), nichtstaatliche Standards und Ko-Regulierung (Abschn. 7.5), Monitoring- und Sanktionssysteme (Abschn. 7.6) sowie unterstützende Elemente wie Beratung und verbraucherorientierte Maßnahmen (Abschn. 7.7) diskutiert.

Anschließend wurden drei alternative strategische Optionen formuliert, die verschiedene mögliche Entwicklungsrichtungen der GAP zu mehr Natur- und Umweltschutz skizzieren. Die Optionen wurden im Hinblick auf ihre Anschlussfähigkeit, Machbarkeit, Implikationen und mögliche Akteurskoalitionen bewertet. Alle drei Optionen beinhalten im Vergleich zur gegenwärtigen Situation ergänzte Mindeststandards, Maßnahmen zur Verbesserung der Kontrollen und zur Reduzierung der Bürokratie durch Nutzung neuer digitaler und GIS-gestützter Informationssysteme, verbesserte Beratung und Unterstützung der Landwirte und Landwirtinnen sowie ein besser für laufende Lernprozesse geeignetes Monitoring- und Evaluationssystem. Alle Optionen können eine zeitliche Entwicklung aufweisen, in deren Verlauf das Anforderungsniveau ansteigen kann, um die Möglichkeiten des wissenschaftlichen und technologischen Fortschritts sowie die zu erwartende zunehmende Knappheit an Umweltgütern zu reflektieren.

Auf dieser Basis setzt die erste Option auf eine effektivere Verknüpfung der Direktzahlungen in der Ersten Säule mit Anforderungen und Leistungen des Natur- und Umweltschutzes, so dass auch die Programme in der Zweiten Säule anspruchsvoller gestaltet werden können. Dies stellt eine moderate Reformoption dar, die technisch machbar und politisch möglich erscheint, aber aus Sicht des Natur- und Umweltschutzes nicht hinreichend geeignet ist, die systemischen Probleme effektiv anzugehen. Die zweite Option setzt auf eine zunehmende Stärkung der Zweiten Säule durch Verlagerung von Mitteln aus der Ersten Säule. Bei dieser Option gibt es Skepsis bezüglich der Effektivität und der begrenzten politischen Unterstützung. Die dritte Option schließlich löst sich von der Zwei-Säulen-Struktur der GAP und sieht stattdessen ein integriertes Modell mit aufeinander aufbauenden Instrumenten vor, die zum Teil flächendeckend, zum Teil regionsspezifisch zunehmend anspruchsvollere Umweltleistungen gestaffelt honorieren. Sie enthält insbesondere zwei innovative Policy-Instrumente: eine flächendeckende Prämie für Landschaftsvielfalt, welche Strukturvielfalt in der Landschaft honoriert und damit besser in Wert setzt, sowie eine standortgenaue Prämie zur Aufrechterhaltung der Landbewirtschaftung in Gebietskulissen, in denen eine unerwünschte großflächige Aufgabe der Landwirtschaft anders nicht abzuwenden ist. Diese Option erfordert weitere Investitionen in die Machbarkeit und die Mobilisierung politischer Unterstützung, könnte bei weiterer Ausarbeitung aber das Potenzial haben, Unterstützung bei Teilen der Agrarwirtschaft, bei Fachleuten, in den politischen Parteien sowie in der breiten Bevölkerung zu finden. Als vierte mögliche strategische Entwicklungsrichtung wurde die Umstellung der Regulierungslogik auf prinzipienbasierte 
Regulation vorgestellt, die mit den drei vorigen Strategien kombiniert werden könnte (Abschn. 8.5).

Die Projektbegleitende Arbeitsgruppe sprach sich im September 2016 einhellig dafür aus, bei der Vermittlung der Ergebnisse in die öffentliche und politische Diskussion (siebte Aufgabe) die dritte Option in den Mittelpunkt zu stellen. Parallel wurden zahlreiche Expertengespräche und vier Workshops durchgeführt. Dadurch konnte die Bewertung der drei Optionen auf eine breitere Basis gestellt und die Konsistenz und Verständlichkeit der Option 3 verbessert werden. Ein Werkauftrag diente dazu, das Konzept der auf GIS-Daten basierenden Prämien für Landschaftsvielfalt auf Machbarkeit zu überprüfen (siehe Abschn. 7.3.7).

Die Vorstellung des Policy-Papers auf einer Pressekonferenz mit Ministerin Dr. Barbara Hendricks am 17. Januar 2017 erhielt ein breites nationales Medienecho. Die Grundzüge der vorgeschlagenen neuen Architektur der Agrarpolitik wurden dabei zutreffend dargestellt. Die Diskussionen des Papiers und des Vortrags auf dem Agrarkongress des BMUB „Landwirtschaft mit Zukunft“ am selben Tag in Berlin zeigten, dass die erarbeiteten Vorschläge ernsthaft diskutiert wurden und in verschiedene Richtungen anschlussfähig sind.

Das BMUB veranstaltete ein Jahr später, am 16. Januar 2018, in Berlin einen weiteren Agrarkongress unter dem Titel „Gemeinsam Zukunft wachsen lassen. Ein Gesellschaftsvertrag für Landwirtschaft, Umwelt und Verbraucher". Nach einem Einführungsvortrag der Bundesumweltministerin Dr. Barbara Hendricks und einem Grußwort des Bundesministers für Ernährung und Landwirtschaft, Christian Schmidt, wurde in mehreren Vorträgen das Konzept des Gesellschaftsvertrags exploriert. Sprecher und Sprecherinnen aus den Bereichen der Agrar- und Ernährungswirtschaft, der Umwelt- und Entwicklungsverbände, der Wasserwirtschaft und des Gesundheitswesens formulierten die verschiedenen Anforderungen und Erwartungen an einen solchen Gesellschaftsvertrag. Anschließend widmeten sich zwei Diskussionsrunden mit Wissenschaftlerinnen und Wissenschaftlern sowie mit Politikerinnen und Politikern aus der Bundes- und Europapolitik den Chancen, Risiken und Perspektiven des Konzepts eines Gesellschaftsvertrags für die Landwirtschaft.

Zur Weiterentwicklung der Konzepte aus dem ZA-NExUS-Projekt vergab das Bundesamt für Naturschutz Ende Dezember 2017 eine Vertiefungsstudie (Förderkennzeichen 3517840900), deren Ergebnisse im Jahr 2020 vorgelegt werden sollen.

\subsection{Reflexion und Ausblick}

Das Projekt ZA-NExUS hat die gesetzten Aufgaben und Ziele erfüllt, wie im vorigen Abschnitt dargestellt wurde. Abschließend sollen kurz die Grenzen des Projekts sowie die Implikationen des methodischen Vorgehens reflektiert und der weitere Forschungsbedarf formuliert werden.

Zunächst ergeben sich generell Einschränkungen aus dem zeitlichen und budgetären Rahmen des Projekts. Das Forschungskonsortium aus vier Partnern konnte nicht vollständig alle relevanten Aspekte der Natur- und Umweltauswirkungen der 
Landwirtschaft abdecken. So war beispielsweise kein Mitglied des Projektteams durch Publikationen spezifisch zum Themenkreis Landwirtschaft und Klima ausgewiesen. Allerdings waren mehrere Mitglieder des Projektteams aus der wissenschaftliche Politikberatung und der Betreuung von Studienarbeiten mit diesem Themenkreis vertraut. Die iterative Begutachtung durch fünf Peer Reviewer war ein Versuch, eventuelle Lücken zu schließen.

Aufgrund des engen zeitlichen Rahmens war es nicht möglich, eine komplette systematische Studie der gesamten relevanten wissenschaftlichen Literatur vorzunehmen. Daher wurde so weit wie möglich auf vorhandene wissenschaftliche Überblicksstudien zurückgegriffen. Nicht möglich war dadurch beispielsweise ein detailliertes Review der unterschiedlichen Methoden der berücksichtigten Studien oder die Durchführung von Meta-Studien, wo die Datenlage dieses eventuell ermöglicht hätte. Es gibt jedoch keine Hinweise, dass ein solches Vorgehen die Grundaussagen zu den Natur- und Umweltauswirkungen der Landwirtschaft wesentlich verändert hätte. Es ist daher davon auszugehen, dass die Bestandsaufnahme der Problemlagen hinreichend zuverlässig und robust ist, um die darauf aufbauende Problembewertung mit wissenschaftlichem Anspruch zu begründen.

SWOT-Analysen und Leitbildentwicklungen sollten normalerweise unter direkter Einbeziehung der Beteiligten vorgenommen werden. Beides sind in erster Linie Instrumente der reflexiven Strategieentwicklung. Ein partizipatives Vorgehen unter Einbeziehung der relevanten gesellschaftlichen Gruppen hätte jedoch ein eigenes Projekt erfordert. Die Ergebnisse der SWOT-Analyse und der Leitbildentwicklung stehen insofern unter dem Vorbehalt einer Validierung durch die gesellschaftlichen Akteure. Sie können jedoch als Grundlage einer Reflexion und Diskussion über die Stärken und Schwächen der derzeitigen Agrarpolitik sowie über erwünschte Entwicklungsrichtungen und deren Operationalisierung dienen.

Bei der Entwicklung von Handlungsansätzen war es in der relativ kurzen Projektlaufzeit nicht möglich, alle interessanten und vielversprechenden Ansätze detailliert auszuarbeiten. Die drei strategischen Politikoptionen verbleiben auf einem relativ hohen Abstraktionsgrad. Sie erfüllen das Ziel, alternative strategische Entwicklungsrichtungen zu formulieren, beantworten aber nicht alle Fragen eines vollständigen Policy-Designs. Weiterhin sind die Handlungsempfehlungen bislang nicht mit Angaben zum jeweiligen Budget und der Höhe der verschiedenen Prämien verknüpft. Selbst wenn hier Szenarien formuliert worden wären, war eine Modellierung und Abschätzung der Politikwirkungen der verschiedenen Handlungsvorschläge nicht Teil des Projekts.

In der zweiten Hälfte des Projekts gewannen Fragen der Anschlussfähigkeit der aus dem Projekt hervorgehenden Empfehlungen an Gewicht. Dies hatte den Effekt, dass einige stärker visionäre Ansätze - etwa ein skalenübergreifendes partizipatives Ressourcenmanagement oder die Umstellung auf prinzipienbasierte Regulierung nicht weiter verfolgt wurden. Im Ergebnis verblieb die Instrumentendebatte daher weitgehend innerhalb des etablierten agrarpolitischen Kosmos und das Policy-Paper stellt einen relativ moderaten Reformvorschlag dar. Allerdings würde eine Umsetzung der Empfehlungen aus dem Projekt durchaus erhebliche Politik-Innovationen beinhalten, wie etwa die Einführung einer flächendeckenden, auf GIS-Daten basierenden 
Prämie für Landschaftsvielfalt oder ein Kooperationsprogramm Natur und Landwirtschaft zur Erarbeitung von regionalen Programmen des integrierten Landschafts- und Ressourcenmanagements.

Vor diesem Hintergrund ergibt sich insbesondere folgender weiterer Forschungsund Entwicklungsbedarf:

- Für die Weiterverfolgung des im ZA-NExUS-Projekt entwickelten Ansatzes ist es zunächst notwendig, die vorgeschlagenen Instrumente im Detail auszuarbeiten. Insbesondere wäre zu klären, wie ein Algorithmus für die Zahlung einer Prämie für die Honorierung landschaftlicher Vielfalt aussehen könnte, die auf GIS-gestützten Fernerkundungsdaten und einem fachlich belastbaren Satz mit nachvollziehbaren, gewichteten Faktoren basiert, die in die Berechnung der Höhe der Prämie eingehen. Weiterhin wäre beispielsweise zu klären, mit welchen Verfahren die Gebietskulissen bestimmt werden könnten, in denen Zahlungen zur Aufrechterhaltung der Landbewirtschaftung gestattet werden. Auch hier wären die Faktoren und Gewichtungen für eine differenzierte standortspezifische Kompensierung von Standortnachteilen zu begründen. Bei der Entwicklung der leistungsbezogenen Policy-Instrumente, die sowohl attraktiv für die Landwirtinnen und Landwirte als auch effektiv für die Ziele des Natur- und Umweltschutzes sein sollten, könnten verhaltenswissenschaftliche Experimente durchgeführt werden, von denen es im Hinblick auf die GAP bisher nur wenige gibt (Colen et al. 2015).

- Auf dieser Basis müssten die Empfehlungen dann mit monetären Szenarien unterfüttert werden, um die Auswirkungen auf verschiedene Betriebstypen und Regionen abzuschätzen. Beispielsweise könnte für unterschiedliche Verteilungen eines bestimmten Budgets auf die verschiedenen Politik-Instrumente die Auswirkung auf Modellbetriebe abgeschätzt werden. Teil einer solchen Wirkungsanalyse sollten auch Interviews mit Landwirtinnen und Landwirten verschiedener Betriebstypen sein, um die Implikationen des vorgeschlagenen Modells aus Sicht der Betroffenen und mögliche Reaktionen abzuschätzen. Eine solche Wirkungsanalyse könnte auch verschiedene Budget-Szenarien berücksichtigen.

- Aufbauend auf der Analyse der Reaktionen der landwirtschaftlichen Betriebe sollte eine Abschätzung der Wirkungen des hier vorgeschlagenen Ansatzes auf die Landnutzung und den Zustand der Güter des Natur- und Umweltschutzes vorgenommen werden. Dabei sollten nach Möglichkeit verschiedene räumliche und zeitliche Skalen in ihrer Wechselwirkung berücksichtigt werden.

- Bei einer Abschätzung der Wirkungen auf die Managementpraktiken, die Betriebe sowie Natur- und Umweltgüter könnte auch ein Vergleich mit der derzeitigen GAP sowie alternativen Entwürfen für die künftige GAP vorgenommen werden, um die relativen Vor- und Nachteile der verschiedenen Politikvorschläge besser abschätzen zu können.

- Hinsichtlich der Verteilungswirkungen könnte die hier vorgeschlagene neue Förderarchitektur insgesamt dazu führen, dass es zu einer stärkeren Differenzierung von Betrieben, die ihre Produktion eher auf den Weltmarkt ausrichten, und Betrieben, die vermehrt in die „Produktion“ von Natur- und Umweltschutzleistungen einsteigen, kommen wird. Welche Konsequenzen für die landwirtschaftlichen Einkommen damit verbunden wären, hängt vor allem auch davon ab, wie 
hoch die Prämien wären und welche Anpassungsmaßnahmen die Betriebe vornähmen. Eine Untersuchung in der Schweiz zeigt, dass ,viele (von den Betriebsleitern vorgeschlagene Anpassungsmaßnahmen [...] zu geringeren Produktionskosten - beispielsweise als Folge eines verminderten Kraftfuttereinsatzes oder reduzierter Nutzungsfrequenz steiler, wenig ertragreicher Wiesen“ führen und damit zu einem vergleichbaren landwirtschaftlichen Einkommen (Bosshard und Meierhofer 2014, S. 6). Des Weiteren wäre anhand von Modellrechnungen zu klären, welche Veränderungen in der Produktion unterschiedlicher Erzeugnisse zu erwarten wären.

- In konsequenter Fortsetzung eines systemischen Ansatzes sollte die Betrachtung über die landwirtschaftlichen Flächen hinaus auf die gesamte Fläche ausgeweitet werden. So wäre beispielsweise zu überlegen, welche Rolle der GAP im Rahmen eines integrierten Landschafts- und Ressourcenmanagements zukommen könnte, das auch Waldflächen, Wassermanagement und Klimaschutz umfasst. Wünschenswert wären wissenschaftlich begleitete Pilotstudien, in denen intersektorale Kooperationen gefördert werden, die innovative Kooperationsansätze entwickeln und testen. Dabei würde es sich um transdisziplinäre Projekte im Sinne der Aktionsforschung handeln.

- Der hier vorgeschlagene Ansatz setzt relativ stark auf einen Trend zur Digitalisierung von Information und deren Verknüpfung mit GIS-gestützten Daten (z. B. bei den vorgeschlagenen Prämien zur Honorierung von Landschaftsvielfalt sowie zur Aufrechterhaltung der Landbewirtschaftung in Gebietskulissen zum Erhalt der Landschaftsvielfalt). Damit könnte ein bestehender Trend zur Digitalisierung der Agrarwirtschaft weiter verstärkt werden. Über die strukturpolitischen, organisationssoziologischen und arbeitstechnischen Implikationen besteht Forschungsbedarf.

- Weiterhin wäre der Ansatz einer integrierten Agrar- und Ernährungspolitik weiter zu entwickeln, der die gesellschaftlichen und volkswirtschaftlichen Kosten des gesamten Ernährungssystems in den Blick nimmt. Dies würde den Blick verstärkt auf die Wertschöpfungskette von der landwirtschaftlichen Urproduktion über die Verarbeitung und den Handel bis zu den Verbraucherinnen und Verbrauchern lenken.

- Daneben sollten die Potenziale einer Ko-Regulierung von öffentlichen und privaten Standards zur Verbesserung des Natur- und Umweltschutzes systematisch erforscht werden, da hier ein Ansatzpunkt bestehen könnte, das Problem einer Importkonkurrenz mit geringeren Standards und damit der bloßen geographischen Verschiebung von Naturschutz- und Umweltproblemen effektiver anzugehen.

- Nicht zuletzt sollte der Ansatz weiter ausgearbeitet werden, das Steuerungsmodell der Gemeinsamen Agrarpolitik auf prinzipienbasierte Regulierung umzustellen. Dabei sollten die Voraussetzungen und Implikationen geklärt und mit relevanten gesellschaftlichen Gruppen diskutiert werden. Weiterhin wären die Potenziale dieses Ansatzes für die Durchsetzung eines im Vergleich zu heute höheren Niveaus im Natur-, Umwelt- Tier- und Verbraucherschutz abzuschätzen. 
Im Hinblick auf die Anschlussfähigkeit des im Projekt ZA-NExUS entwickelten Ansatzes an die laufende agrarpolitische Diskussion wären die folgenden Fragen zu diskutieren:

- Kann das Konzept in der bestehenden Zwei-Säulen-Struktur verankert werden, falls sich diese als politisch gesetzt erweisen sollte?

- Welche Grade der vollständigen oder teilweisen Finanzierung aus EU-Mitteln wären für die verschiedenen Elemente des hier vorgeschlagenen Modells systematisch zu rechtfertigen?

- Welche Investitionen in Fernerkundung, GIS-Technologien und andere ICTVerfahren sind notwendig, um in einem Zeitraum bis spätestens 2027 eine Verwaltungsvereinfachung durch semi-automatische Prämienzuteilung zu ermöglichen? Welche Implikationen ergeben sich für den Datenschutz? Welche Potenziale der Verwaltungsvereinfachung und Entbürokratisierung ergeben sich daraus?

- Wie würde eine institutionelle Auslagerung der Finanzierung des Natur- und Umweltschutzes in einen separaten Naturschutzfonds außerhalb der GAP die Programmierung und Implementation der Agrarumweltpolitik verändern?

Die Etablierung einer Agrarpolitik, welche die Anliegen des Natur- und Umweltschutzes besser berücksichtigt, ohne die Produktionsfunktion der Landwirtschaft zu vernachlässigen, erfordert anspruchsvolle Mindeststandards und deren konsequente Durchsetzung, leistungs- und ergebnisorientierte politische Programme, kooperative Lernprozesse und die konsequente Beachtung systemischer Zusammenhänge auf verschiedenen räumlichen und zeitlichen Skalen. Sie erfordert eine gute Datengrundlage, Transparenz, ein lernorientiertes Monitoring und einen Dialog zwischen wissenschaftlichem und nicht-wissenschaftlichem Wissen. Dabei müssen die Landwirtinnen und Landwirte nicht nur „,mitgenommen“ werden, sondern aktiv beitragen können.

Die bisherige Entwicklung kann nicht dauerhaft fortgeführt werden. Der Status quo ist keine nachhaltige Option. Notwendig sind eine ehrliche Bestandsaufnahme, Kreativität und innovative Ansätze. Die Autorinnen und Autoren dieses Berichts wären zufrieden, wenn die hier vorgelegten Ergebnisse und Überlegungen dazu einen Beitrag leisten können.

Open Access Dieses Kapitel wird unter der Creative Commons Namensnennung 4.0 International Lizenz (http://creativecommons.org/licenses/by/4.0/deed.de) veröffentlicht, welche die Nutzung, Vervielfältigung, Bearbeitung, Verbreitung und Wiedergabe in jeglichem Medium und Format erlaubt, sofern Sie den/die ursprünglichen Autor(en) und die Quelle ordnungsgemäß nennen, einen Link zur Creative Commons Lizenz beifügen und angeben, ob Änderungen vorgenommen wurden.

Die in diesem Kapitel enthaltenen Bilder und sonstiges Drittmaterial unterliegen ebenfalls der genannten Creative Commons Lizenz, sofern sich aus der Abbildungslegende nichts anderes ergibt. Sofern das betreffende Material nicht unter der genannten Creative Commons Lizenz steht und die betreffende Handlung nicht nach gesetzlichen Vorschriften erlaubt ist, ist für die oben aufgeführten Weiterverwendungen des Materials die Einwilligung des jeweiligen Rechteinhabers einzuholen. 


\section{Anhänge}

\section{Anhang 1: Indikatoren zur biologischen Vielfalt}

Artenvielfalt und Landschaftsqualität (Vogelindikator): ${ }^{1}$ Zwischen 2001 und 2011 hat sich der Wert dieses Indikators deutlich verschlechtert. Dabei liegt der Teilindikator Agrarland mit $56 \%$ besonders weit vom Zielwert (100\%) entfernt. Viele wissenschaftliche Untersuchungen dokumentieren einen Bestandsrückgang von Vögeln in der Agrarlandschaft Deutschlands und Europas (Donald et al. 2001; Hötker 2004; Hoffmann et al. 2012; Jahn et al. 2014). Die Gefährdung der Feldvogelarten scheint dabei mit der Stärke der Bindung an Agrarlebensräume zuzunehmen (Hötker 2004).

Gefährdete Arten: ${ }^{2}$ Bei diesem Indikator wird der Gefährdungszustand der Arten auf Basis der bundesweiten Roten Listen ausgewählter Artengruppen durch den Indikator „Gefährdete Arten“ bilanziert. Im Jahr 2013 lag der Wert mit 23 \% noch weit oberhalb des Zielwertes (15\% bis 2020). Dies stellt im Vergleich zu den entsprechenden Roten Listen von 1996/1998 tendenziell eine Verschlechterung dar

\footnotetext{
${ }^{1}$,Für die Zielwertbildung hat ein Expertengremium für jede einzelne Vogelart einen Bestandswert für das Jahr 2015 festgelegt, der erreicht werden kann, wenn europäische und nationale rechtliche Regelungen mit Bezug zum Naturschutz und die Leitlinien einer nachhaltigen Entwicklung zügig umgesetzt werden. Die Zielwerte der Indikatorarten für das Jahr 2015 wurden zunächst als Vielfaches der damals bekannten Bestandsgrößen des Jahres 2002 bestimmt. Die resultierenden Indexwerte wurden nachfolgend einheitlich auf $100 \%$ normiert. Daher ergeben sich für die Teilindikatoren und den Gesamtindikator jeweils Zielwerte von $100 \%$ “(Bundesamt für Naturschutz 2015b). Diese Zielwerte für die Teilindikatoren und den Gesamtindikator wurden vorläufig unverändert auf das Zieljahr 2030 übertragen (Statistisches Bundesamt 2017, S. 101).

${ }^{2}$ „In die Berechnung des Indikators fließen die Arten in Abhängigkeit von ihrer Gefährdung mit unterschiedlichen Gewichtungsfaktoren ein. Dabei gilt: Je stärker eine Art gefährdet ist, desto stärker beeinflusst sie den Indikatorwert. Aus der Bildung des Indexes resultiert eine Skala, auf der null Prozent erreicht würden, wenn keine der Arten bestandsgefährdet, ausgestorben oder verschollen wäre. Bei 100 Prozent wären sämtliche betrachteten Arten ausgestorben oder verschollen“ (BMUB 2015a, S. 21).
} 
(BMUB 2015a). Von den rund 11.000 in den aktuellen Bänden der Roten Liste Deutschland (Haupt et al. 2009; Binot-Hafke et al. 2011; Ludwig und MatzkeHajek 2011; Becker et al. 2013) genannten heimischen Taxa (Arten und Unterarten) sind rund $30 \%$ bestandsgefährdet und weitere $6 \%$ ausgestorben.

Erhaltungszustand der FFH-Arten: ${ }^{3}$ Auch dieser Indikator weist auf den andauernden Verlust der Arten hin. So lag der Indikatorwert für FFH-Arten im Jahr 2013 (2007-2012) bei $46 \%$ und damit um gut zwei Prozentpunkte niedriger als im Berichtsjahr 2007 (2001-2006). Er ist somit nach wie vor weit vom Zielwert entfernt (BMUB 2015a).

Erhaltungszustand der FFH-Lebensräume: ${ }^{95}$ Der Wert dieses Indikators lag im Jahr 2013 (2007-2012) mit 46 \% um rund acht Prozentpunkte niedriger als im Berichtsjahr 2007 (2001-2006). Er ist also ebenfalls weit vom Zielwert entfernt (BMUB 2015a). Im Vergleich zur letzten Berichtsperiode (2007) konnte 2013 in keinem Lebensraum eine Verbesserung des Erhaltungszustands festgestellt werden - bei 13 Lebensräumen wurden sogar Verschlechterungen festgestellt. Besonders der Erhaltungszustand nutzungsabhängiger bzw. durch landwirtschaftliche Nutzung stark geprägter Schutzgüter wird als ungünstig bewertet.

Genetische Vielfalt in der Landwirtschaft: ${ }^{4}$ Dieser Indikator gibt am Beispiel der fünf wichtigsten Nutztierarten (Pferd, Rind, Schwein, Schaf und Ziege) Auskunft über das Ausmaß und die Gefährdung genetischer Ressourcen in der Landwirtschaft. Der Anteil an gefährdeten Nutztierrassen war im Jahr 2013 mit $70 \%$ sehr hoch (BMUB 2015a). Von den 74 einheimischen Tierrassen sind derzeit 52 Rassen gefährdet (Bundesanstalt für Landwirtschaft und Ernährung 2016). Als Hauptursache für die genetische Verarmung wird die langjährige, strenge züchterische Selektion aus einem begrenzten Genpool genannt. Dadurch konnten zwar hohe Leistungen erzielt werden, während jedoch gleichzeitig eine genetische Verarmung stattfand (BMELV 2007). Auch das Spektrum der genutzten Kulturpflanzensorten hat in den letzten Jahrzehnten stark abgenommen (BMUB 2007, 2015a). Seit Beginn des letzten Jahrhunderts gingen rund $75 \%$ der weltweiten (FAO, IFAD und WFP 2014) und sogar $90 \%$ der deutschen (Bundesamt für Naturschutz 2016d) genetischen Vielfalt der Nutzpflanzenvarietäten verloren.

\footnotetext{
${ }^{3}$ „Der Wert des Indikators beträgt null Prozent, wenn der Erhaltungszustand aller einfließenden Schutzgüter als ungünstig-schlecht bewertet wird, und 100 Prozent, wenn der Erhaltungszustand aller Schutzgüter als günstig bewertet wird“" (BMUB 2015a, S. 24).

${ }^{4}$ „Als Datengrundlage dienen die von den Züchtervereinigungen und herdbuchführenden Stellen zur Verfügung gestellten Bestandszahlen für die einzelnen Nutztierrassen.“ ... „Für die Berechnung des Indikators wird die Einstufung der Rassen in die Rote Liste anhand der im Rahmen des Nationalen Fachprogramms Tiergenetische Ressourcen erstellten Gefährdungskategorien ausgewertet“ (BMUB 2015a, S. 68).
} 


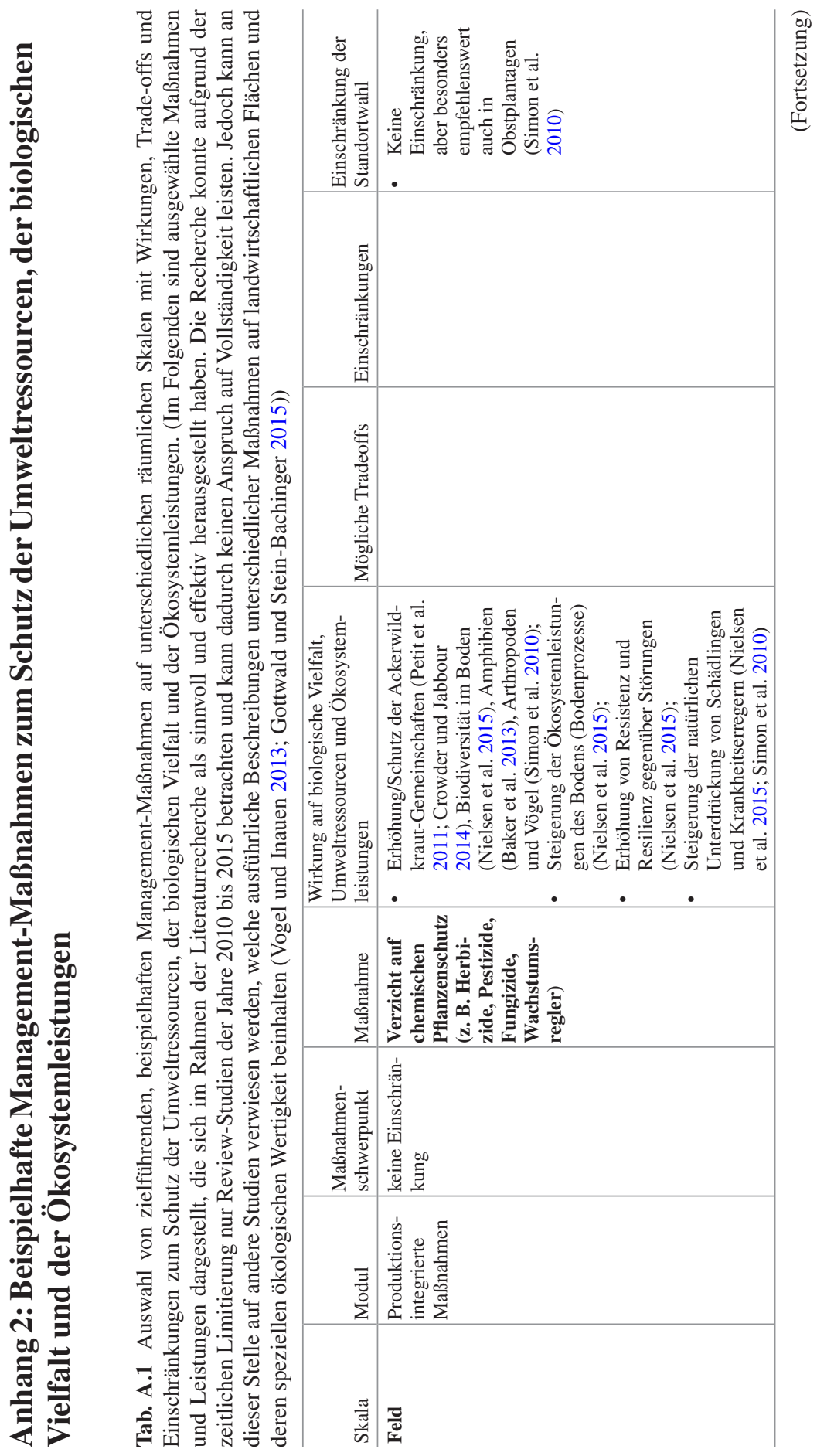




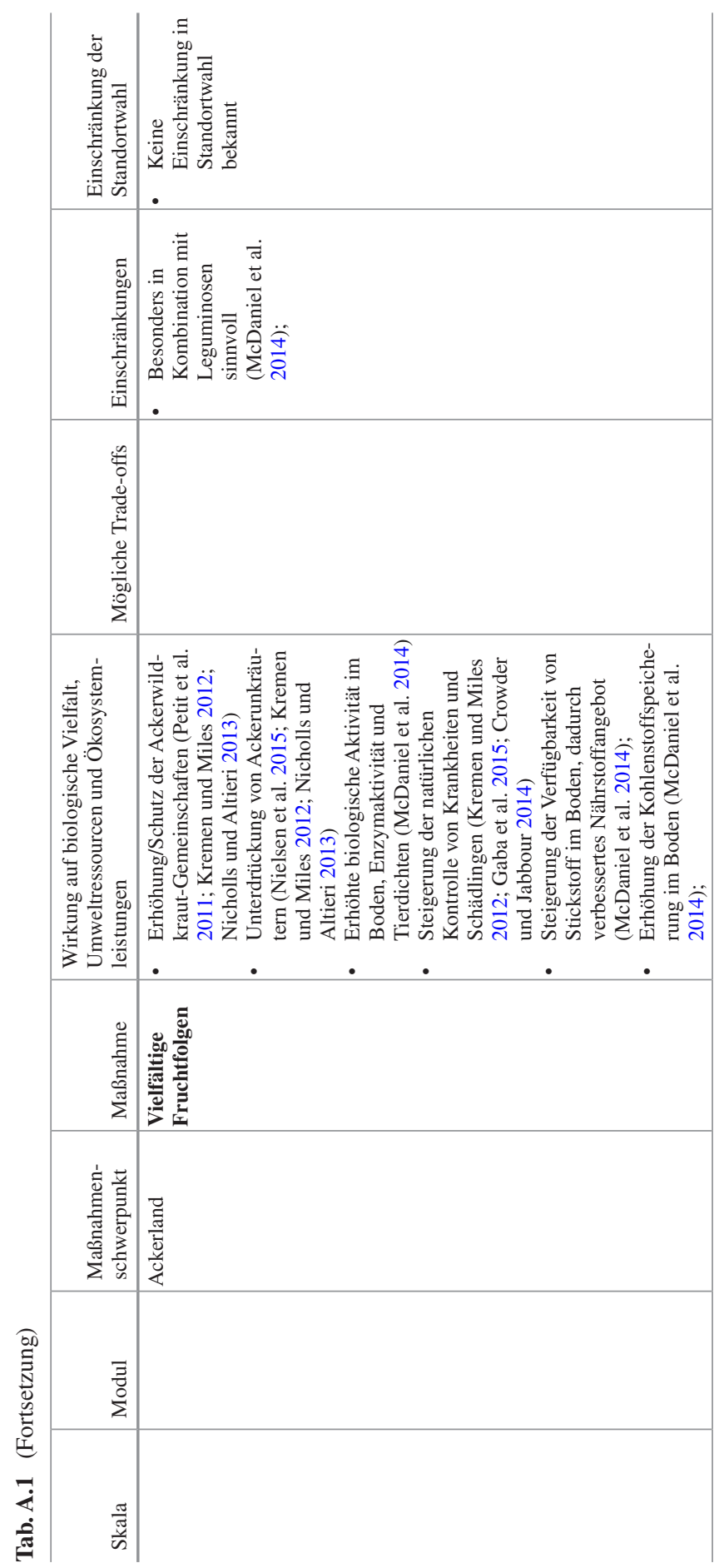




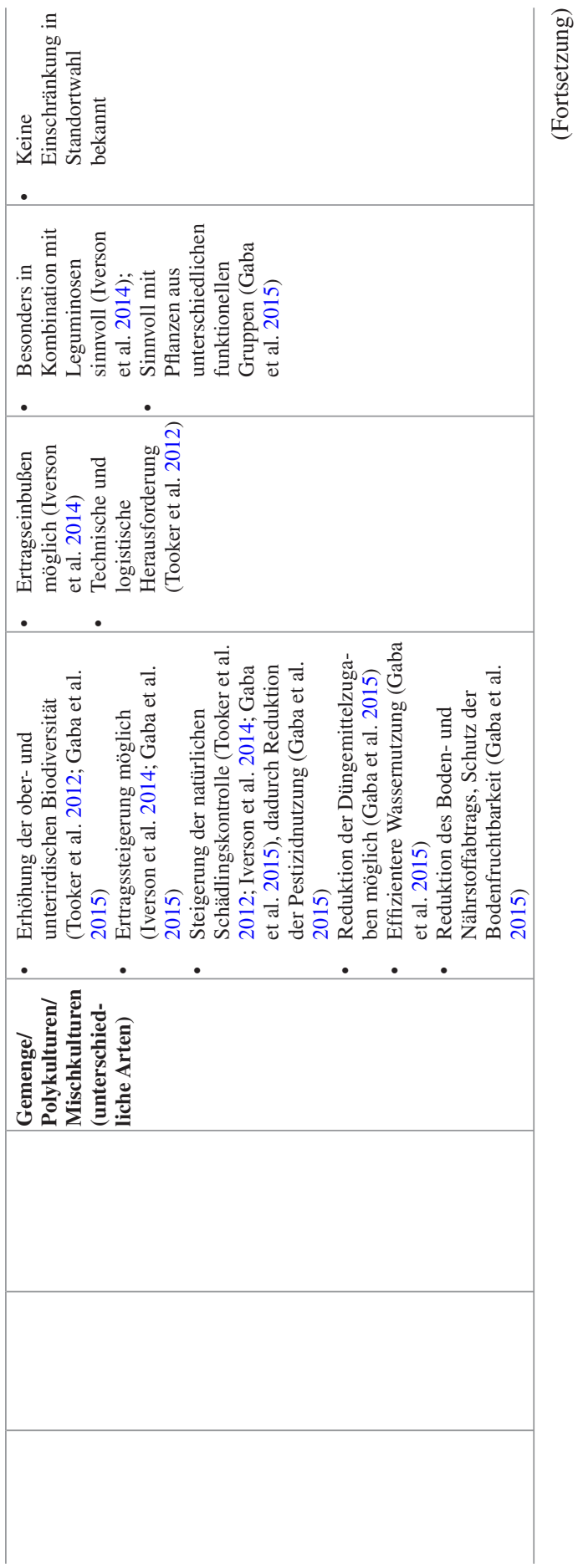




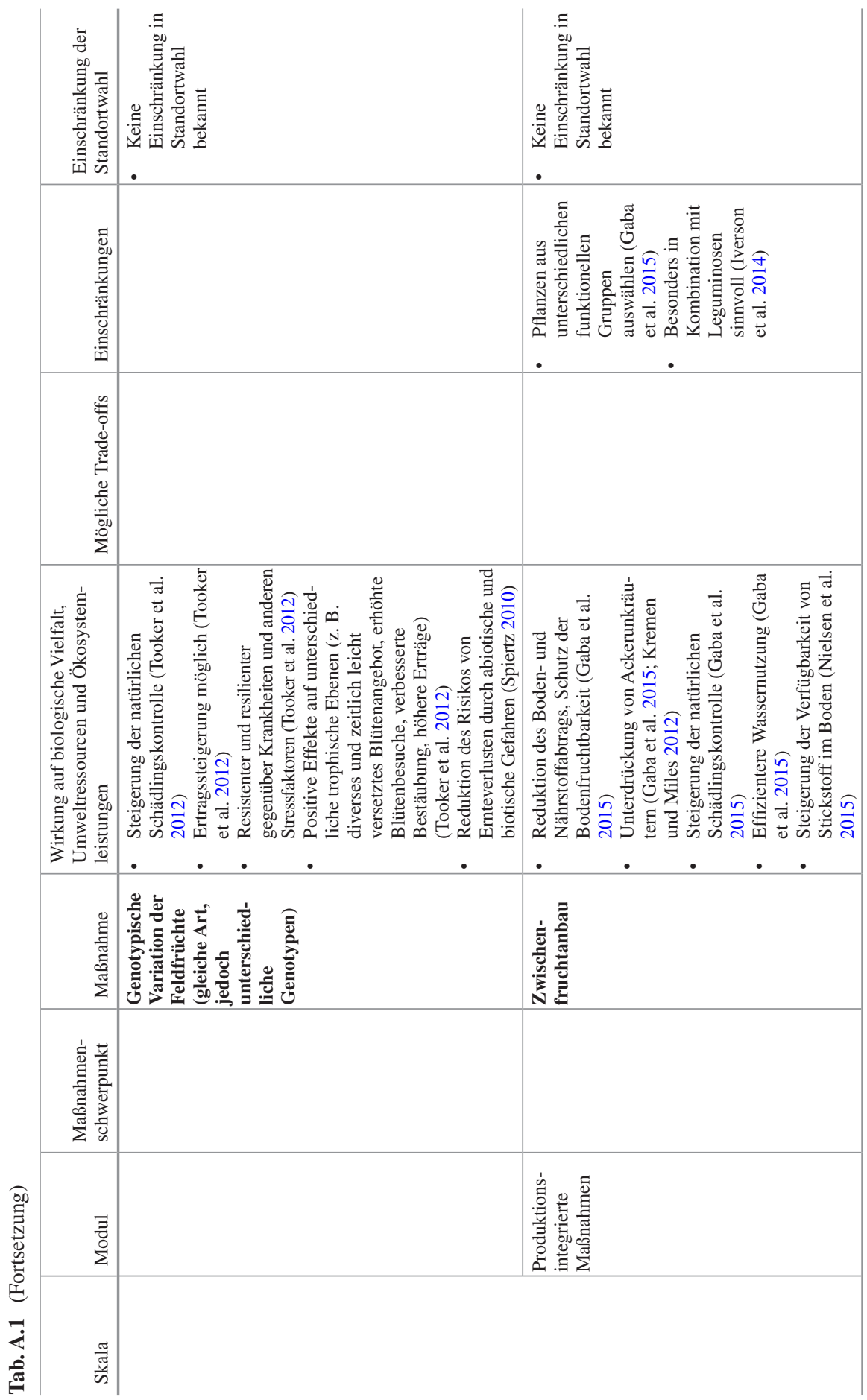




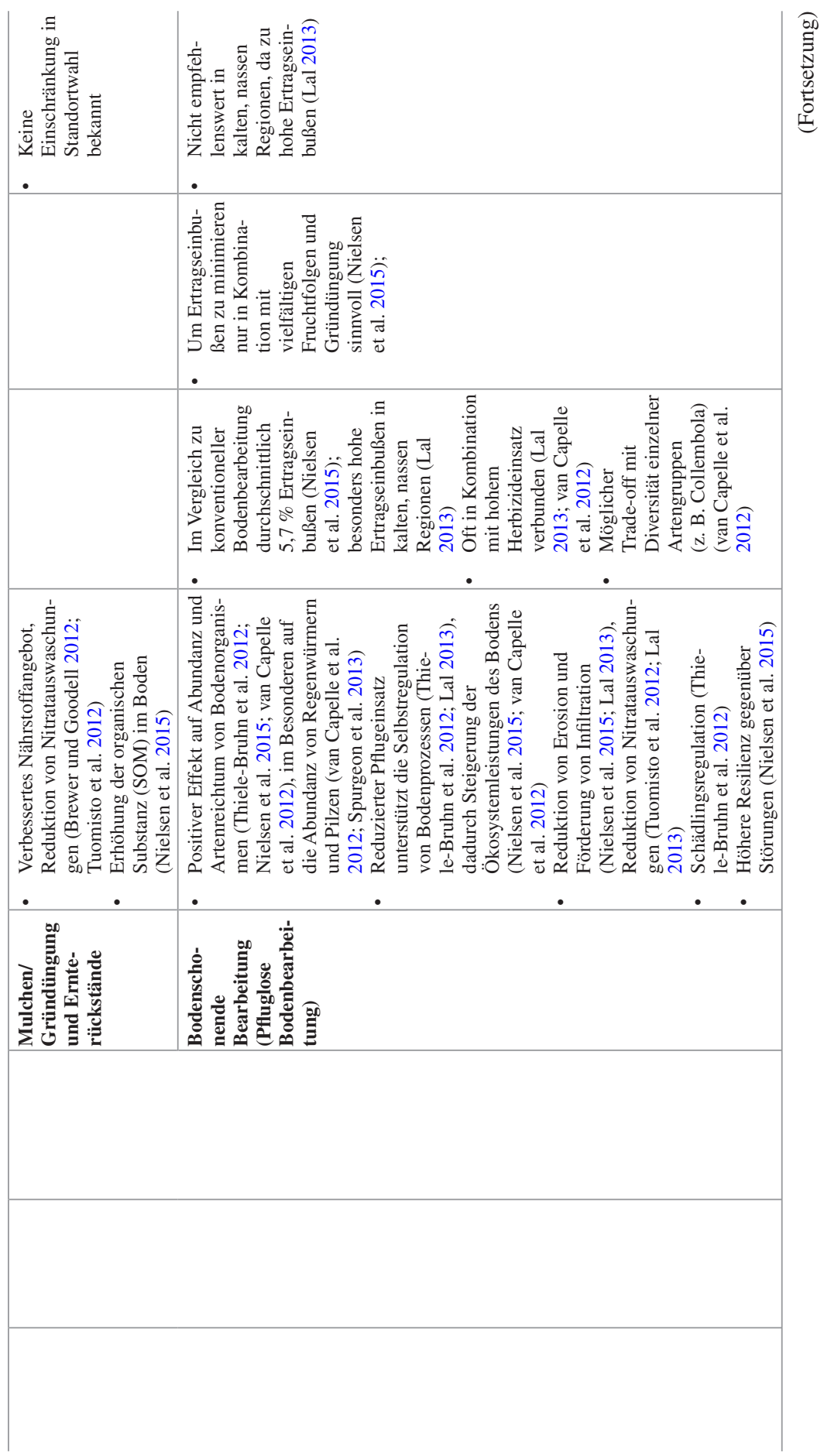




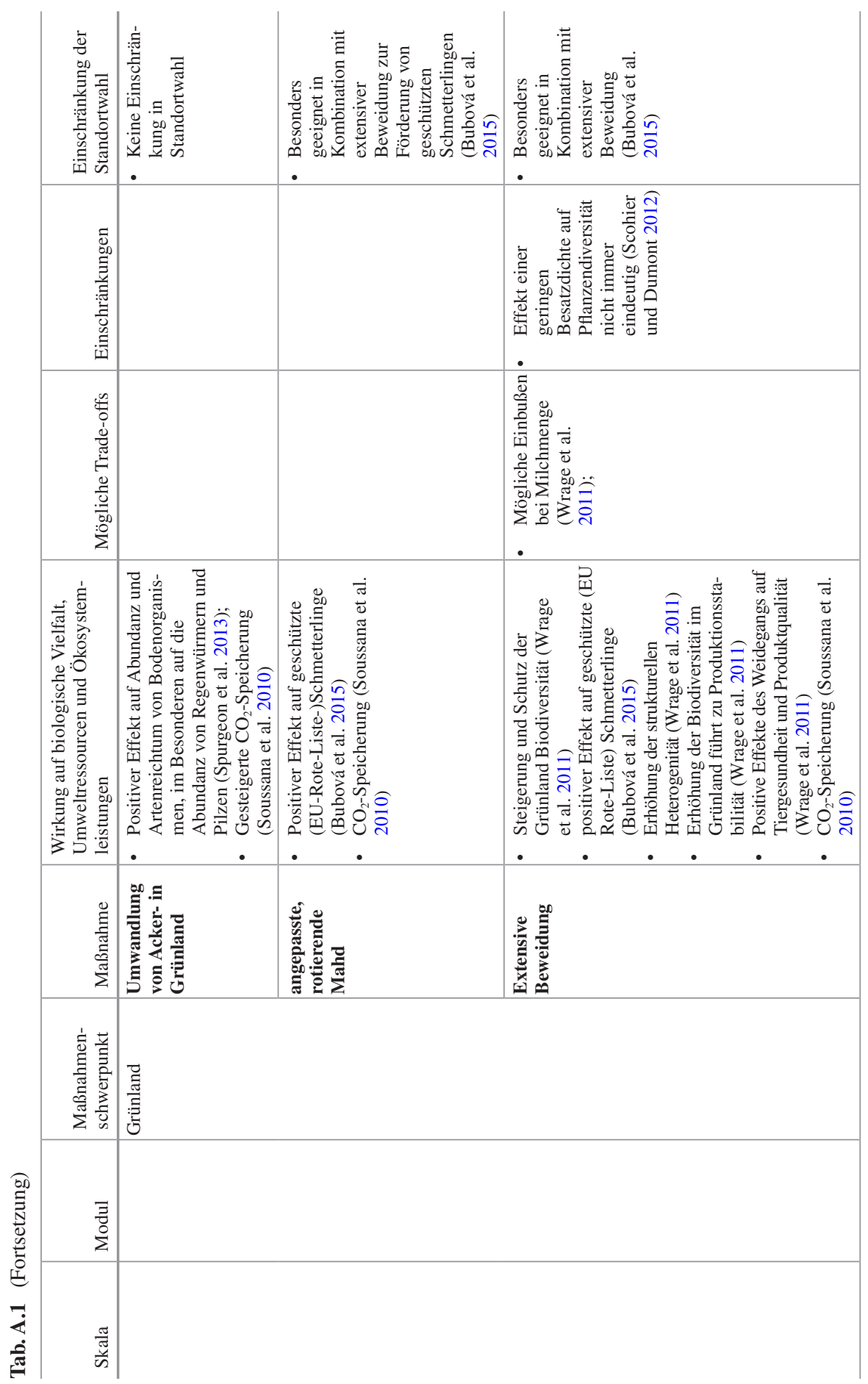



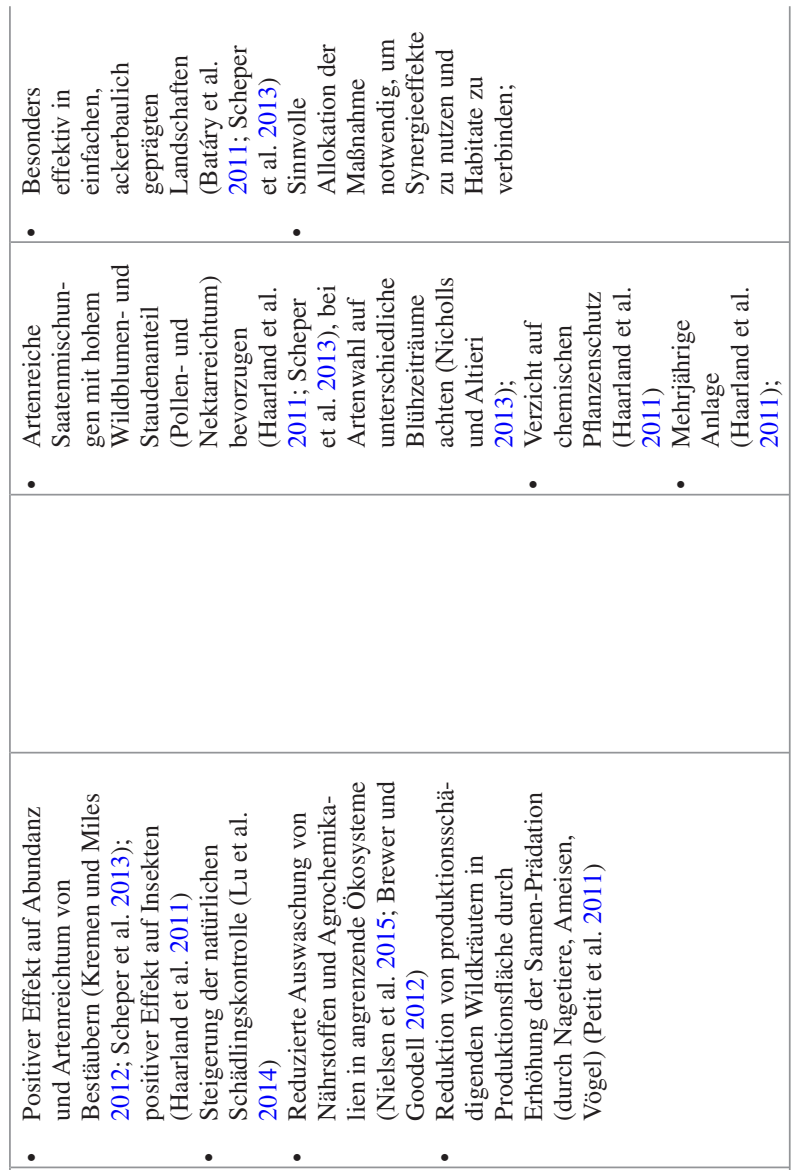

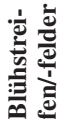

00
0
0
0
0
0

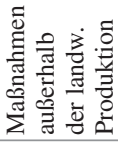




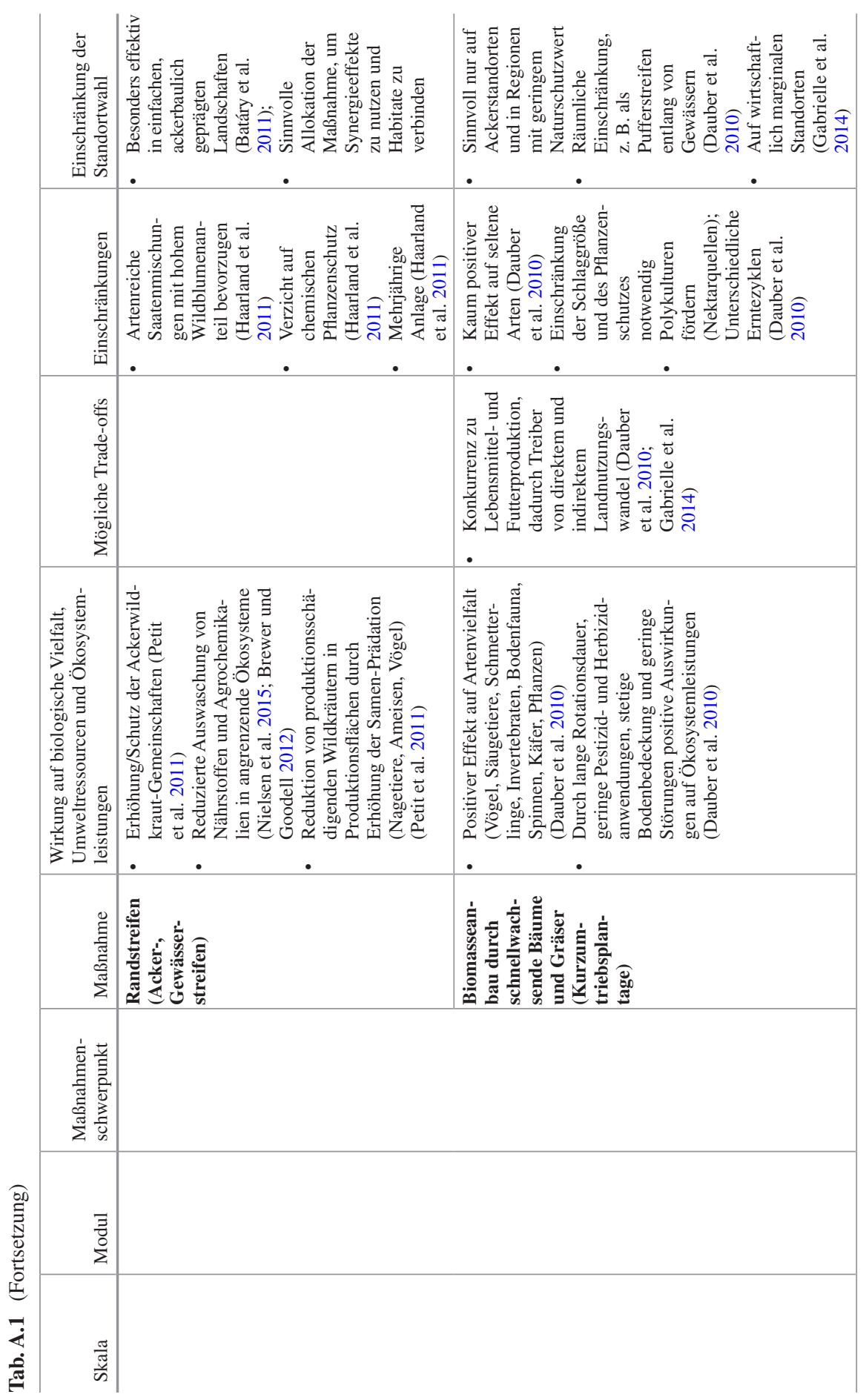




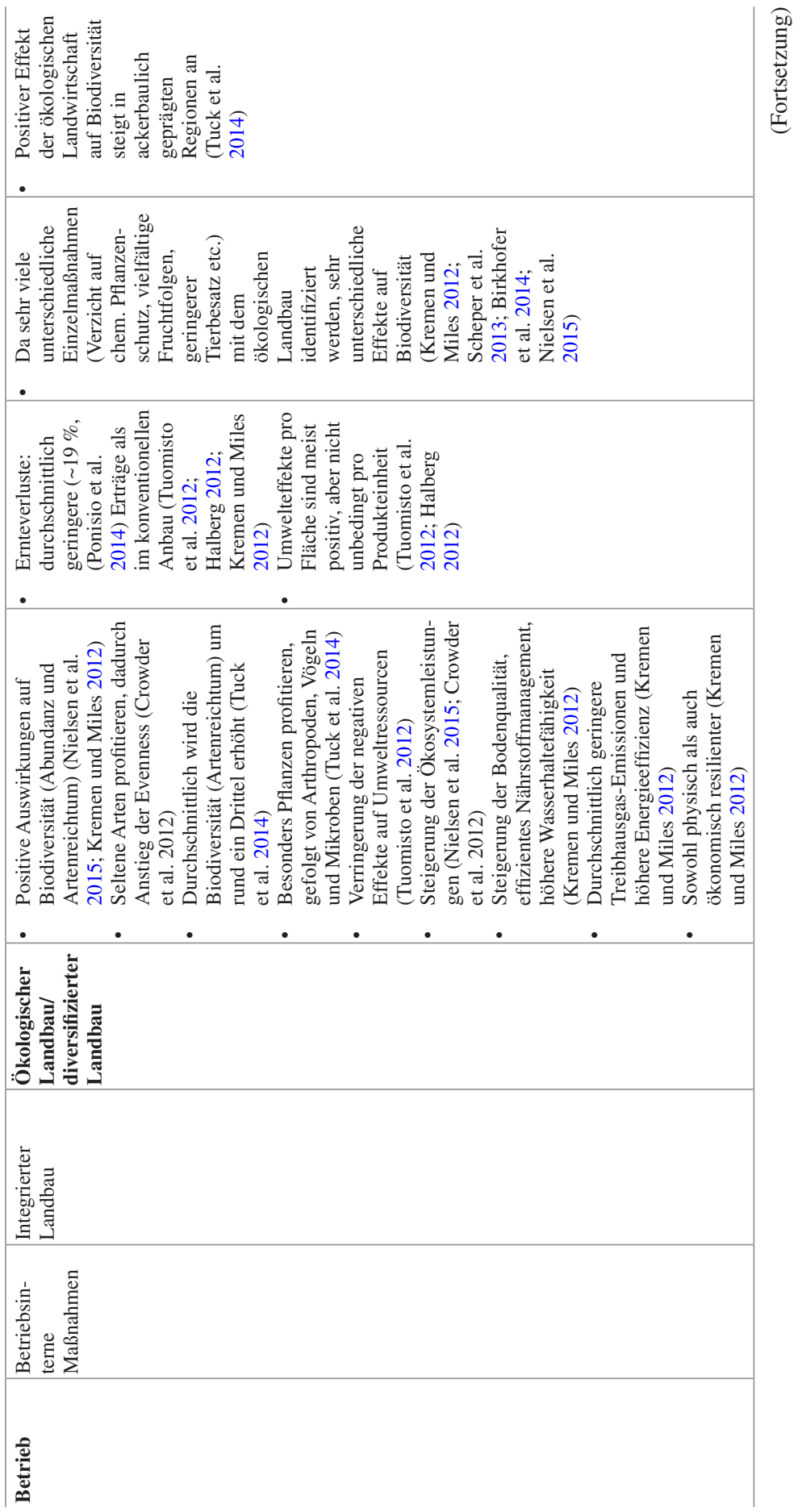




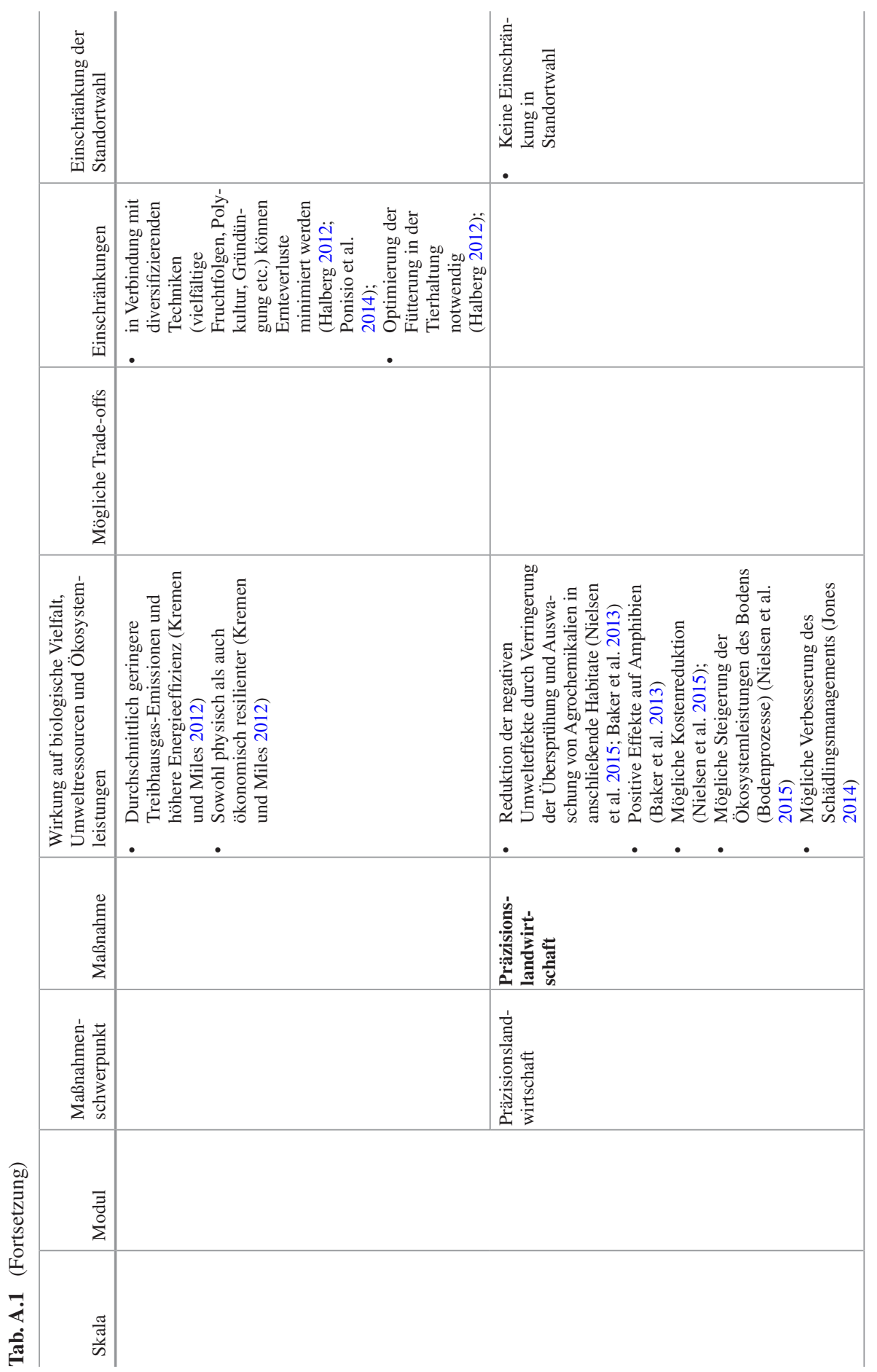




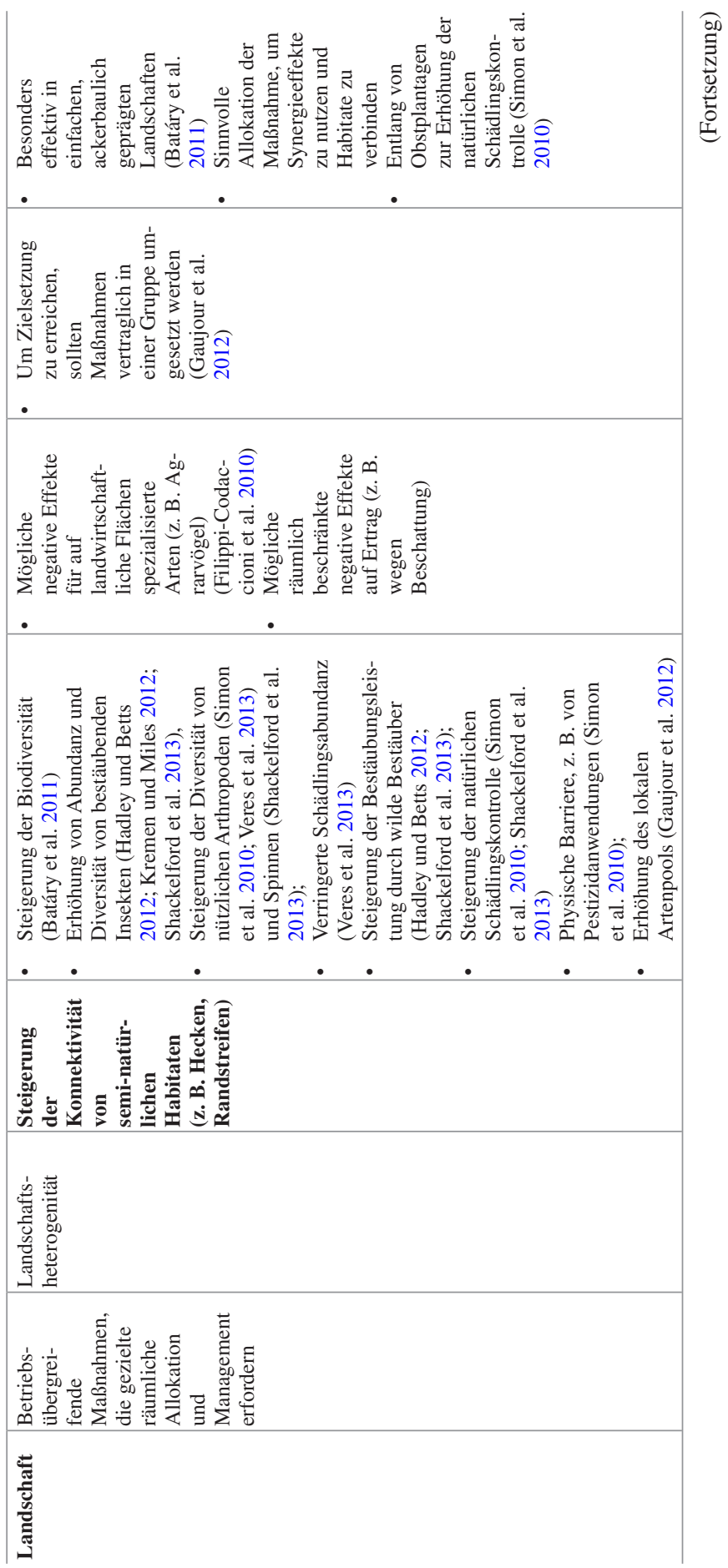




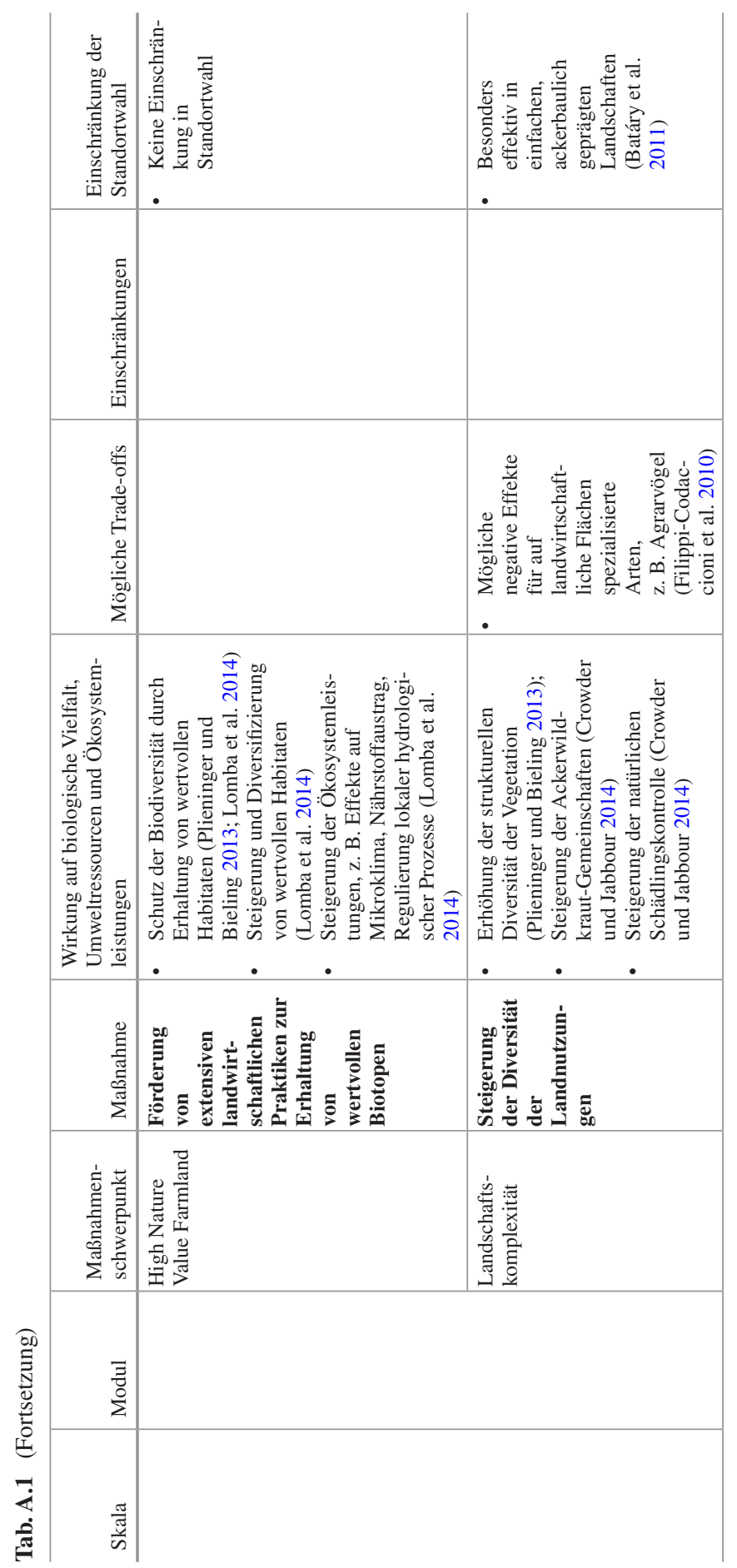




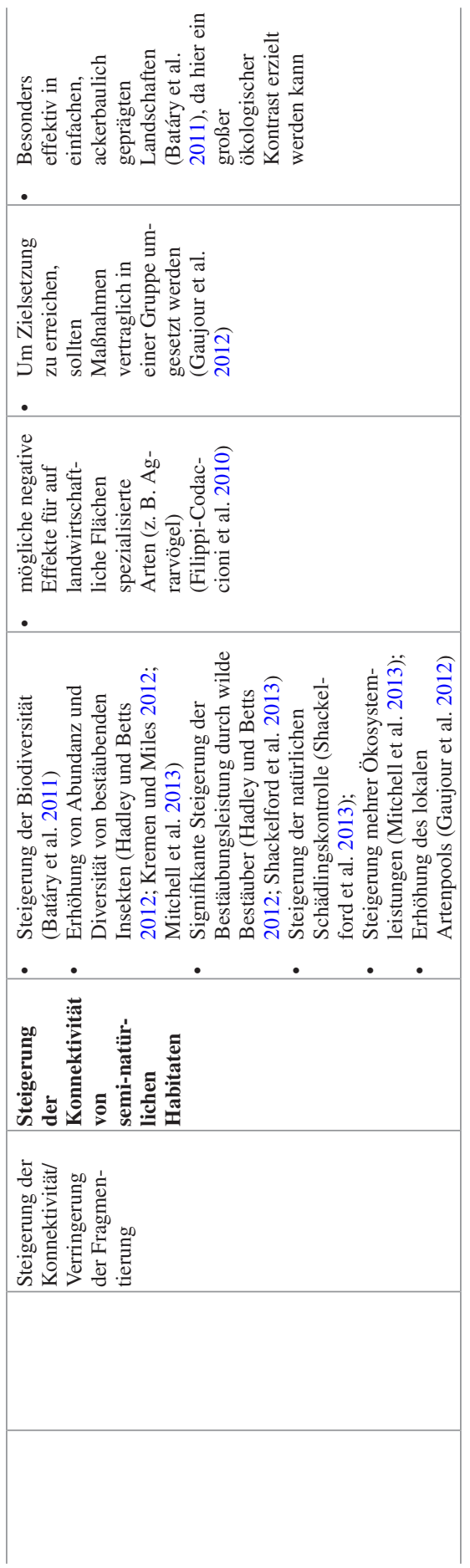




\section{Literatur}

Ackrill, R. (2005): The Common Agricultural Policy. In: M.P. van der Hoek (Hrsg.): Handbook of Public Administration and Policy in the European Union. Boca Raton: Taylor \& Francis, S. 435-487.

Ackrill, R. und Kay, A. (2006): Historical-institutionalist perspectives on the development of the EU budget system. Journal of European Public Policy, 13, S. 113-133.

Ackrill, R., Kay, A. und Morgan, W. (2008): The common agricultural policy and its reform: the problem of reconciling budget and trade concerns. Canadian Journal of Agricultural Economics/Revue canadienne d'agroeconomie, 56, S. 393-411.

Adger, W.N. und Jordan, A. (2009): Governing sustainability, Cambridge: Cambridge University Press.

AIOTI WG06 (2015): Smart Farming and Food Safety Internet of Things Applications - Challenges for Large Scale Implementations 2015, Brussels: Alliance for the Internet of Things Innovation (AIOTI).

Ajzen, I. (1991): The theory of planned behavior. Organizational Behaviour and Human Decision Making Processes, 50, S. 179-211.

ALDI SÜD (2016): ALDI SÜD bringt Licht in den Siegel-Dschungel, Pressemitteilung vom 02.05.2016. Abrufbar unter: https://unternehmen.aldi-sued.de/de/presse/pressemitteilungen/ verantwortung/2016/pressemitteilung-7-siegel-kampagne/, zuletzt abgefragt: 26.07.2016.

Alexandrovskiy, A.L. (2007): Rates of soil-forming processes in three main models of pedogenesis. Revista Mexicana da Ciencias Geologicas, 24, S. 283-292.

Allouche, J., Middleton, C. und Gyawali, D. (2015): Technical veil, hidden politics: Interrogating the power linkages behind the nexu. Water Alternatives, 8, S. 610-626.

Alons, G. und Zwaan, P. (2015): New Wine in Different Bottles: Negotiating and Selling the CAP post-2013 Reform. Sociologia Ruralis, 56, S. 349-370.

Anania, G. und Pupo D'Andrea, M.R. (2015): The 2013 Reform of the Common Agricultural Policy. In: J. Swinnen (Hrsg.): The Political Economy of the 2014-2020 Common Agricultural Policy An Imperfect Storm. London: Rowman \& Littlefield, S. 33-84.

Armsworth, P.R., Acs, S., Dallimer, M., Gaston, K.J., Hanley, N. und Wilson, P. (2012): The cost of policy simplification in conservation incentive programs. Ecology Letters, 15, S. 406-414.

Arponen, A., Heikkinen, R.K., Paloniemi, R., Pöyry, J., Similä, J. und Kuussaari, M. (2013): Improving conservation planning for semi-natural grasslands: Integrating connectivity into agrienvironment schemes. Biological Conservation, 160, S. 234-241.

Baker, N.J., Bancroft, B.A. und Garcia, T.S. (2013): A meta-analysis of the effects of pesticides and fertilizers on survival and growth of amphibians. The Science of the total environment, 449, S. $150-156$. 
Balmann, A. und Sahrbacher, C. (2014): Mehr als „Museumsprämien“? Zur Förderung der ersten Hektare und Junglandwirte im Rahmen der EU-Agrarpolitik. IAMO Policy Brief, Halle: Leibniz-Institut für Agrarentwicklung in Transformationsökonomien. Abrufbar unter: http:// hdl.handle.net/10419/92495, zuletzt abgefragt: 09.10.2018.

Banerjee, S., Cason, T.N., De Vries, F.P. und Hanley, N. (2015): Spatial Coordination in Agglomeration Bonus Schemes with Transaction Costs and Communication: An Experimental Study. University of St. Andrews Discussion Papers in Environmental Economics Paper 10, St. Andrews.

Basurto, X. und Ostrom, E. (2009): The core challenges of moving beyond Garrett Hardin. Journal of Natural Resources Policy Research, 1, S. 255-259.

Batáry, P., Baldi, A., Kleijn, D. und Tscharntke, T. (2011): Landscape-moderated biodiversity effects of agri-environmental management: a meta-analysis. Proceedings. Biological sciences/ The Royal Society, 278, S. 1894-1902.

Batáry, P., Dicks, L.V., Kleijn, D. und Sutherland, W.J. (2015): The role of agri-environment schemes in conservation and environmental management. Conservation Biology, 29, S. 1006-1016.

Baumgarten, M. (2006): Belastung der Wälder mit gasförmigen Luftschadstoffen Studie zur Beurteilung der Luftqualität an Waldstandorten des forstlichen Umweltmonitoring in den Jahren 2002 und 2003 Bericht im Auftrag des Bundesministeriums für Ernährung, Landwirtschaft und Verbraucherschutz (BMELV). Abrufbar unter: http://www.bmel.de/SharedDocs/Downloads/ Landwirtschaft/Wald-Jagd/WaldGasSchadstoffbelastung.pdf?_blob=publicationFile, zuletzt abgefragt: 13.05.2017.

BBodschG (2017): Bundes-Bodenschutzgesetz vom 17. März 1998 (BGB1. I S. 502), das zuletzt durch Artikel 3 Absatz 3 der Verordnung vom 27. September 2017 (BGBl. I S. 3465) geändert worden ist. Bundesamt für Justiz. Abrufbar unter: https://www.gesetze-im-internet.de/ bbodschg/BJNR050210998.html, zuletzt abgefragt: 09.05.2019.

Becker, G.S. (1974): Crime and Punishment: An Economic Approach. Journal of Political Economy, 76, S. 169-217.

Becker, N., Haupt, H., Hofbauer, N., Ludwig, G. und Nehring, S. (Hrsg.) (2013): Rote Liste gefährdeter Tiere, Pflanzen und Pilze Deutschlands: Band 2: Meeresorganismen, Münster: Landwirtschaftsverlag.

Beedell, J.D.C. und Rehman, T. (1999): Explaining farmers' conservation behaviour: Why do farmers behave the way they do. Journal of Environmental Management, 57, S. 165-176.

Beedell, J.D.C. und Rehman, T. (2000): Using social-psychology models to understand farmers' conservation behaviour. Journal of Rural Studies, 16, S. 117-127.

Behavioural Insights Team (2014): EAST: Four Simple Ways to Apply Behavioural Insights, London: Cabinet Office.

Benson, D., Gain, A.K. und Rouillard, J.J. (2015): Water Governance in a Comparative Perspective: From IWRM to a ,Nexus ' Approach? Water Alternatives, 8, S. 756-773.

Benton, T.G., Vickery, J.A. und Wilson, J.D. (2003): Farmland biodiversity: is habitat heterogeneity the key? Trends in Ecology \& Evolution, 18, S. 182-188.

Berendse, F., Chamberlain, D., Kleijn, D. und Schekkerman, H. (2004): Declining biodiversity in agricultural landscapes and the effectiveness of agri-environment schemes. Ambio, 33, S. 499-502.

Biesmeijer, J.C., Roberts, S.P.M., Reemer, M., Ohlemüller, R., Edwards, M., Peeters, T., Schaffers, A.P., Potts, S.G., Kleukers, R., Thomas, C.D., Settele, J. und Kunin, W.E. (2006): Parallel declines in pollinators and insect-pollinated plants in Britain and the Netherlands. Science (New York, N.Y.), 313, S. 351-354.

Biggs, R., Schlüter, M., Biggs, D., Bohensky, E.L., BurnSilver, S., Cundill, G., Dakos, V., Daw, T.M., Evans, L.S., Kotschy, K., Leitch, A.M., Meek, C., Quinlan, A., Raudsepp-Hearne, C., Robards, M.D., Schoon, M.L., Schultz, L. und West, P.C. (2012): Toward Principles for Enhancing the Resilience of Ecosystem Services. Annual Review of Environment and Resources, 37, S. 421-448.

Binot-Hafke, M., Balzer, S., Becker, N., Gruttke, H., Haupt, H., Hofbauer, N., Ludwig, G., Matzke-Hajek, G. und Strauch, M. (2011): Rote Liste gefährdeter Tiere, Pflanzen und Pilze Deutschlands: Band 3: Wirbellose Tiere (Teil 1), Bonn: Bundesamt für Naturschutz. 
Birkhofer, K., Ekroos, J., Corlett, E.B. und Smith, H.G. (2014): Winners and losers of organic cereal farming in animal communities across Central and Northern Europe. Biological Conservation, 175 , S. $25-33$.

Birrer, S., Balmer, O., Graf, R. und Jenny, M. (2010): Biodiversität im Kulturland: vom Nebenprodukt zum Marktvorteil. Julius-Kühn-Archiv, 421, S. 21-29.

Birrer, S., Chevillat, V., Graf, R., Hagist, D., Home, R., Jenny, M., Pfiffner, L., Stöckli, S. und Zellweger-Fischer, J. (2015): Mit Vielfalt punkten. Bauern beleben die Natur. Jahresbericht 2014, Sempach/Frick: Schweizerische Vogelwarte Sempach/Forschungsinstitut für biologischen Landbau (FiBL). Abrufbar unter: http://orgprints.org/28205/1/birrer-etal-2014-MVP-jahresbericht2014.pdf, zuletzt abgefragt: 17.12.2016.

Birrer, S., Zellweger-Fischer, J., Stoeckli, S., Korner-Nievergelt, F., Balmer, O., Jenny, M. und Pfiffner, L. (2014): Biodiversity at the farm scale: A novel credit point system. Agriculture, Ecosystems \& Environment, 197, S. 195-203.

Bittner, C. (2012): Art. 39 AEUV. In: J. Schwarze (Hrsg.): EU Kommentar, 3. Auflage. Baden-Baden: Nomos.

Black, J., Hopper, M. und Band, C. (2007): Making a success of principles-based regulation. Law and financial markets review, 1, S. 191-206.

BLAG (2012): Evaluierung der Düngeverordnung - Ergebnisse und Optionen zur Weiterentwicklung. Bericht im Auftrag des Bundesministeriums für Ernährung, Landwirtschaft und Verbraucherschutz: Bund-Länder-Arbeitsgruppe zur Evaluierung der Düngeverordnung.

BMEL (2012): Statistisches Jahrbuch für Ernährung, Landwirtschaft und Forsten 2012, Münster-Hiltrup: Landwirtschaftsverlag.

BMEL (2014a): Die wirtschaftliche Lage der landwirtschaftlichen Betriebe im Wirtschaftsjahr 2013/14, Berlin.

BMEL (2014b): Landwirtschaft verstehen - Fakten und Hintergründe, Berlin: Bundesministerium für Ernährung und Landwirtschaft. Abrufbar unter: https://www.bmel.de/SharedDocs/Downloads/Broschueren/Landwirtschaft-verstehen.pdf, zuletzt abgefragt: 15.05.2016.

BMEL (2015a): Agrarpolitischer Bericht der Bundesregierung 2015, Berlin.

BMEL (2015b): Agrarpolitischer Bericht der Bundesregierung 2015, Berlin: Bundesministerium für Ernährung und Landwirtschaft. Abrufbar unter: https://www.bmel.de/SharedDocs/Downloads/Broschueren/Agrarbericht2015.pdf, zuletzt abgefragt: 15.04.2017.

BMEL (2015c): Antibiotika in der Landwirtschaft, Bonn; Berlin: Bundesministerium für Ernährung und Landwirtschaft,. Abrufbar unter: http://www.bmel.de/DE/Tier/Tiergesundheit/Tierarzneimittel/_texte/Antibiotika-Dossier.html?docId=6975508, zuletzt abgefragt: 09.11.2015.

BMEL (2015d): Die wirtschaftliche Lage der landwirtschaftlichen Betriebe im Wirtschaftsjahr 2014/15, Berlin.

BMEL (2015e): Grundzüge der Gemeinsamen Agrarpolitik (GAP) und ihrer Umsetzung in Deutschland, Stand 05.01.2015. Abrufbar unter: https://www.bmel.de/DE/Landwirtschaft/Agrarpolitik/_Texte/GAP-NationaleUmsetzung.html, zuletzt abgefragt: 23.07.2016.

BMEL (2015f): Schmidt: Bauern erbringen zusätzliche Umweltleistungen durch das Greening. Pressemitteilung Nr. 191 vom 08.10.2015. Abrufbar unter: https://www.bmel.de/SharedDocs/ Pressemitteilungen/2015/191-SC-OekologischeVorrangflaechen.html, zuletzt abgefragt: 20.12.2016.

BMEL (2015g): Statistisches Jahrbuch für Ernährung, Landwirtschaft und Forsten 2014, Münster-Hiltrup: Landwirtschaftsverlag.

BMEL (2015h): Umsetzung der EU-Agrarreform in Deutschland, Berlin: Bundesministerium für Ernährung und Landwirtschaft. Abrufbar unter: https://www.bmel.de/SharedDocs/Downloads/ Broschueren/UmsetzungGAPinD.pdf, zuletzt abgefragt: 15.04.2017.

BMEL (2015i): Umsetzung der EU-Agrarreform in Deutschland. Ausgabe 2015. Abrufbar unter: http://www.bmel.de/SharedDocs/Downloads/Broschueren/UmsetzungGAPinD.pdf? blob=publicationFile, zuletzt abgefragt: 23.04.2017.

BMELV (2007): Agrobiodiversität erhalten, Potenziale der Land-, Forst- und Fischereiwirtschaft erschließen und nachhaltig nutzen. Eine Strategie des BMELV für die Erhaltung und nachhaltige Nutzung der biologischen Vielfalt für die Ernährung, Land-, Forst- und Fischereiwirt- 
schaft, Berlin. Abrufbar unter: https://www.bmel.de/SharedDocs/Downloads/Broschueren/ Agrobiodiversitaet.pdf?_blob=publicationFile, zuletzt abgefragt: 17.11.2016.

BMELV (2013a): Die wirtschaftliche Lage der landwirtschaftlichen Betriebe im Wirtschaftsjahr 2012/13, Berlin

BMELV (2013b): Ländliche Wege, Gewässer und Bodenschutz nach dem Flurbereinigungsgesetz (FlurbG) und Landwirtschaftsanpassungsgesetz (LwAnpG), Berlin.

BMELV (2015): Verordnungsentwurf: Verordnung zur Neuordnung der guten fachlichen Praxis beim Düngen, Berlin.

BMU (2011): Messages from the Bonn 2011 Conference: The Water, Energy and Food Security Nexus - Solutions for a Green Economy, Bonn. Abrufbar unter: https://www.water-energy-food.org/uploads/media/bonn2011_nexusmessages.pdf, zuletzt abgefragt: 23.04.2017.

BMU (2012): Klärschlamm-Statistik, Bonn: Bundesministerium für Umwelt, Naturschutz und Reaktorsicherheit.

BMU und BMELV (2012): Nitratbericht 2012. Abrufbar unter: http://www.bmel.de/SharedDocs/ Downloads/Landwirtschaft/Klima-und-Umwelt/Nitratbericht-2012.pdf, zuletzt abgefragt: 15.05 .2016

BMUB (2007): Nationale Strategie zur biologischen Vielfalt: Kabinettsbeschluss vom 7. November 2007. Abrufbar unter: http://www.biologischevielfalt.de/fileadmin/NBS/documents/broschuere_biolog_vielfalt_strategie_bf.pdf, zuletzt abgefragt: 14.04.2017.

BMUB (2015a): Indikatorenbericht 2014 zur Nationalen Strategie zur biologischen Vielfalt. Abrufbar unter: http://www.bmub.bund.de/fileadmin/Daten_BMU/Pools/Broschueren/indikatorenbericht_biologische_vielfalt_2014_bf.pdf, zuletzt abgefragt: 23.04.2017.

BMUB (2015b): Naturschutz-Offensive 2020: Für biologische Vielfalt: Bundesministerium für Umwelt, Naturschutz und Reaktorsicherheit. Abrufbar unter: http://www.bmub.bund.de/fileadmin/Daten_BMU/Pools/Broschueren/naturschutz-offensive_2020_broschuere_bf.pdf，zuletzt abgefragt: 23.04.2017.

BMUB (2016): Klimaschutzplan 2050. Klimaschutzpolitische Grundsätze und Ziele der Bundesregierung, Berlin.

BMUB und Umweltbundesamt (2013): Umweltbewusstsein in Deutschland 2012: Ergebnisse einer repräsentativen Bevölkerungsumfrage, Berlin/Dessau: Bundesministerium für Umwelt Naturschutz und Reaktorsicherheit/Umweltbundesamt. Abrufbar unter: https://www. umweltbundesamt.de/sites/default/files/medien/publikation/long/4396.pdf, zuletzt abgefragt: 12.03.2016.

BNatschG (2009): Bundesnaturschutzgesetz vom 29. Juli 2009 (BGBl. I S. 2542), das zuletzt durch Artikel 1 des Gesetzes vom 15.September 2017 (BGB1. I S. 3434) geändert worden ist. Bundesamt für Justiz. Abrufbar unter: http://www.gesetze-im-internet.de/bnatschg_2009/ BNatSchG.pdf, zuletzt abgefragt: 09.05.2019.

Bocquého, G., Jacquet, F. und Reynaud, A. (2013): Reversal and Magnitude Effects in Long-Term Time Preferences: Results from a Field Experiment. Economic Letters, 120, S. 108-111.

Bos, M. (2010): The EU budget. In: A. Oskam, G. Meester und H. Silvis (Hrsg.): EU policy for agriculture, food and rural areas. Wageningen: Wageningen Academic Publishers, S. 79-96.

Bosch \& Partner GmbH (2000): Kriterienkatalog zur Gestaltung von Ackerschlägen im Agrarraum - Landschaftsökologische Aspekte. Endbericht des Forschungs- und Entwicklungsvorhabens im Auftrag des Sächsischen Landesamtes für Umwelt und Geologie, Dresden: SMUL. Abrufbar unter: https://www.umwelt.sachsen.de/umwelt/download/endbericht_acker. pdf, zuletzt abgefragt: 17.12.2016.

Bosshard, A. und Häusler, L. (2012): Gezieltere Erschwernisbeiträge zugunsten einer flächendeckenden Bewirtschaftung. Faktenblatt Nr. 3, Litzibuch: Vision Landwirtschaft. Abrufbar unter: https://www.visionlandwirtschaft.ch/_visionlandwirtschaft_prod/uploads/ themen/faktenbl\%C3\%A4tter/FB\%203/Faktenblatt_3_2012_D_aV.pdf, zuletzt abgefragt: 17.12.2016.

Bosshard, A. und Meierhofer, U. (2014): Entwicklungsmöglichkeiten von Landwirtschaftsbetrieben unter der neuen Schweizer Agrarpolitik AP 2014-17. Faktenblatt Nr. 4, Litzibuch: Vision Landwirtschaft. Abrufbar unter: https://www.visionlandwirtschaft.ch/_visionlandwirtschaft_ 
prod/uploads/themen/faktenb1\%C3\%A4tter/FB4/Faktenblatt_4_VL.pdf, zuletzt abgefragt: 17.12.2016.

Bosshard, A., Schläpfer, F. und Jenny, M. (2010): Analysen und Vorschläge zur Reform der Schweizer Agrarpolitik, Litzibuch: Vision Landwirtschaft. Abrufbar unter: https://www.visionagriculture.ch/_visionlandwirtschaft_dev/uploads/themen/Faktenb1\%C3\%A4tter/Weissbuch_Faktenblatt_2010_D.pdf, zuletzt abgefragt: 17.12.2016.

Boström, M. und Klintman, M. (2006): State-centered versus nonstate-driven organic food standardization: A comparison of the US and Sweden. Agriculture and Human Values, 23, S. 163-180.

Boström, M. und Klintman, M. (2009): Eco-Standards, Product Labelling and Green Consumerism, Basingstoke: Macmillan.

Botterill, L.C. und Daugbjerg, C. (2015): Commensalistic institutions and value conflicts: the World Trade Organization and global private food standards. European Political Science Review, 7, S. 23-42.

Boyd, D. und Crawford, K. (2012): Critical questions for big data: Provocations for a cultural, technological, and scholarly phenomenon. Information, communication \& society, 15, S. 662-679.

Brewer, M.J. und Goodell, P.B. (2012): Approaches and incentives to implement integrated pest management that addresses regional and environmental issues. Annual review of entomology, 57, S. 41-59.

Bubová, T., Vrabec, V., Kulma, M. und Nowicki, P. (2015): Land management impacts on European butterflies of conservation concern: a review. Journal of Insect Conservation, 19, S. 805-821.

Buller, H., Wilson, G.A. und Höll, A. (Hrsg.) (2000): Agri-environmental Policy in the European Union, Aldershot et al: Ashgate.

BUND (ohne Datum): Streuobstprojekte - ein wichtiger Beitrag zum Artenschutz. Abrufbar unter: http://www.bund-kreis-konstanz.de/themen_projekte/naturschutz/streuobstwiesen/, zuletzt abgefragt: 23.04.2017.

Bundesamt für Naturschutz (Hrsg.) (2014): Grünland-Report: Alles im Grünen Bereich?, Bonn.

Bundesamt für Naturschutz (2015a): Artenschutz-Report 2015: Tiere und Pflanzen in Deutschland, Bonn. Abrufbar unter: https://www.bfn.de/fileadmin/BfN/presse/2015/Dokumente/Artenschutzreport_Download.pdf, zuletzt abgefragt: 31. August 2018.

Bundesamt für Naturschutz (2015b): Indikator „Artenvielfalt und Landschaftsqualität“. Abrufbar unter: https://www.bfn.de/themen/monitoring/indikatoren/indikator-artenvielfalt-und-landschaftsqualitaet.html, zuletzt abgefragt: 06.04.2017.

Bundesamt für Naturschutz (2016a): Erfassungsanleitung für den HNV-Farmland-Indikator, Version 7, Stand 2016, Bonn. Abrufbar unter: https://www.bfn.de/fileadmin/BfN/monitoring/Dokumente/Erfassungsanleitung_HNV_2016_barrfrei_.pdf, zuletzt abgefragt: 25.11.2016.

Bundesamt für Naturschutz (2016b): Landesweite Moorentwicklungskonzepte, Bonn. Abrufbar unter: https://www.bfn.de/0311_moore-entwicklungskonzepte.html, zuletzt abgefragt: 17.12.2016.

Bundesamt für Naturschutz (2016c): Moore - Entstehung, Zustand, Biodiversität: Gefährdung der Moorlebensräume in Deutschland, Bonn: Bundesamt für Naturschutz. Abrufbar unter: https:// www.bfn.de/0311_moore-entstehung-zustand.html, zuletzt abgefragt: 27.06.2016.

Bundesamt für Naturschutz (2016d): Was ist Agrarbiodiversität?, Bonn: Bundesamt für Naturschutz. Abrufbar unter: https://www.bfn.de/0313_agrobiodiv.html, zuletzt abgefragt: 23.04.2017.

Bundesanstalt für Landwirtschaft und Ernährung (2016): Gefährdung tiergenetischer Ressourcen, Bonn: GENRES Informationssystem Genetische Ressourcen. Abrufbar unter: http://www. genres.de/haus-und-nutztiere/gefaehrdung/, zuletzt abgefragt: 31.05.2016.

Bundesregierung (2016): Deutsche Nachhaltigkeitsstrategie - Neuauflage 2016, Berlin.

Burch, D., Lawrence, G. und Hattersley, L. (2013): Watchdogs and ombudsmen: monitoring the abuse of supermarket power. Agriculture and Human Values, 30, S. 259-270.

Bureau, J.-C. und Marette, S. (2000): Accounting for consumers preferences in international trade rules. Incorporating science economics and sociology in developing sanitary and phytosanitary standards in international trade, S. 170-198. 
Bureau, J.-C. und Valceschini, E. (2003): European food-labeling policy: successes and limitations. Journal of Food Distribution Research, 34, S. 70-76.

Burrows, N. (1990): Harmonisation of technical standards: reculer pour mieux sauter? The Modern Law Review, 53, S. 597-603.

Burton, R.J.F., Kuczera, C. und Schwarz, G. (2008): Exploring farmers' cultural resistance to voluntary agri-environmental schemes. Sociologia Ruralis, 48, S. 16-37.

Burton, R.J.F. und Paragahawewa, U.H. (2011): Creating culturally sustainable agri-environmental schemes. Journal of Rural Studies, 27, S. 95-104.

Burton, R.J.F. und Schwarz, G. (2013): Result-oriented agri-environmental schemes in Europe and their potential for promoting behavioural change. Land Use Policy, 30, S. 628-641.

Burton, R.J.F. und Wilson, G.A. (2006): Injecting social psychology theory into conceptual-isations of agricultural agency: towards a post-productivist farmer self-identity? Journal of Rural Studies, 22, S. 95-115.

Busch, L. (2011): The private governance of food: equitable exchange or bizarre bazaar? Agriculture and Human Values, 28, S. 345-252.

BVL (2015): Absatz an Pflanzenschutzmitteln in der Bundesrepublik Deutschland: Ergebnisse der Meldungen gemäß $§ 64$ Pflanzenschutzgesetz für das Jahr 2014, Braunschweig: Bundesamt für Verbraucherschutz unf Lebensmittelsicherheit. Abrufbar unter: http://www.bvl.bund.de/ SharedDocs/Downloads/04_Pflanzenschutzmittel/meld_par_19_2014.pdf?_blob=publicationFile \&v=5, zuletzt abgefragt: 23.04.2017.

Candel, J.J., Breeman, G.E., Stiller, S.J. und Termeer, C.J. (2014): Disentangling the consensus frame of food security: the case of the EU Common Agricultural Policy reform debate. Food Policy, 44, S. 47-58.

Chabé-Ferret, S. und Subervie, J. (2010): Evaluating Agro-Environmental Schemes by DID Matching : Theoretical Justification, Robustness Tests and Application to a French Program. Fourth World Congress of Environmental and Resource Economists, Montreal, Canada. S. 1-32.

Choi, J.J., Laibson, D., Madrian, B.C. und Metrick, A. (2003): Optimal Defaults. The American Economic Review, 93, S. 180-185.

Christen, O., Hövelmann, L., Hülsbergen, K.J., Packeiser, M., Rimpau, J. und Wagner, B. (2009): Nachhaltige landwirtschaftliche Produktion in der Wertschöpfungskette Lebensmittel. Initiativen zum Umweltschutz, Band 78, Berlin: Erich Schmidt Verlag.

Cialdini, R.B., Eisenberg, N., Green, B.L., Rhoads, K. und Bator, R. (1998): Undermining the undermining effect of reward on sustained interest. Journal of Applied Social Psychology, 28, S. 249-263.

Claassen, R., Cattaneo, A. und Johansson, R. (2008): Cost-effective design of agri-environmental payment programs: U.S. experience in theory and practice. Ecological Economics, 65, S. 737-752.

Clay, S.A., DeSutter, T.M. und Clay, D.E. (2001): Herbicide Concentration and Dissipation from Surface Wind-Erodible Soil. Weed Science, 49, S. 431-436.

Coleman, W.D., Skogstad, G.D. und Atkinson, M.M. (1997): Paradigm shifts and policy networks: cumulative change in agriculture. Journal of Public Policy, 16, S. 273-301.

Colen, L., Paloma, S.G.Y., Latacz-Lohmann, U., Lefebvre, M. und Thoyer, S. (2015): (How) can economic experiments inform EU agricultural policy?, Seville: JRC. Abrufbar unter: http:// publications.jrc.ec.europa.eu/repository/bitstream/JRC97340/jrc_report_final.pdf, zuletzt abgefragt: 05.05.2016.

Concepción, E.D., Díaz, M. und Baquero, R.A. (2008): Effects of landscape complexity on the ecological effectiveness of agri-environment schemes. Landscape Ecology, 23, S. 135-148.

Crowder, D.W. und Jabbour, R. (2014): Relationships between biodiversity and biological control in agroecosystems: Current status and future challenges. Biological Control, 75, S. 8-17.

Cukier, K. und Mayer-Schoenberger, V. (2013): The rise of Big Data: How it's changing the way we think about the world. Foreign Affairs, 92, S. 28-40.

D'Adda, G. (2011): Motivation crowding in environmental protection: Evidence from an artefactual field experiment. Ecological Economics, 70, S. 2083-2097.

DAFA (2015): Fachforum Grünland: Forschungsstrategie der DAFA. Abrufbar unter: http:// www.dafa.de/fileadmin/dam_uploads/images/Fachforen/FF_Gruenland/ff_gruenland-strategie-de_2015.pdf, zuletzt abgefragt: 23.04.2017. 
Daily, G.C. (1997): Nature's services - societal dependence on natural ecosystems, Washington: Island Press.

Dannenberg, P. und Franz, M. (2014): Essen aus dem Internet - Online-Supermärkte auf dem Weg aus der Experimentierphase? Standort - Zeitschrift für angewandte Geographie, 38, S. 237-243.

Dauber, J., Jones, M.B. und Stout, J.C. (2010): The impact of biomass crop cultivation on temperate biodiversity. GCB Bioenergy, 2, S. 289-309.

Daugbjerg, C. (1997): Policy networks and agricultural policy reforms: Explaining deregulation in Sweden and re-regulation in the European Community. Governance, 10, S. 123-141.

Daugbjerg, C. (1999): Reforming the CAP: Policy Networks and Broader Institutional Structures. Journal of Common Market Studies, 37, S. 407-428.

Daugbjerg, C. (2003): Policy feedback and paradigm shift in EU agricultural policy: the effects of the MacSharry reform on future reform. Journal of European Public Policy, 10, S. 421-437.

Daugbjerg, C. (2009): Sequencing in public policy: the evolution of the CAP over a decade. Journal of European Public Policy, 16, S. 395-411.

Daugbjerg, C. (2012): The World Trade Organization and organic food trade: potential for restricting protectionism? Organic Agriculture, 2, S. 55-66.

Daugbjerg, C. und Botterill, L.C. (2012): Ethical food standard schemes and global trade: Paralleling the WTO? Policy and Society, 31, S. 307-317.

Daugbjerg, C. und Feindt, P.H. (2017): Post-exceptionalism in Food and Agricultural Policy: Transforming Public Policies. Journal of European Public Policy, 24, S. 1565-1584.

Daugbjerg, C. und Swinbank, A. (2007): The politics of CAP reform: trade negotiations, institutional settings and blame avoidance. Journal of Common Market Studies, 45, S. 1-22.

Daugbjerg, C. und Swinbank, A. (2016): Three Decades of Policy Layering and Politically Sustainable Reform in the European Union's Agricultural Policy. Governance, 29, S. 265-280.

de Snoo, G.R., Herzon, I., Staats, H., Burton, R.J., Schindler, S., van Dijk, J., Lokhorst, A.M., Bullock, J.M., Lobley, M., Wrbka, T., Schwarz, G. und Musters, C.J.M. (2013): Toward effective nature conservation on farmland: making farmers matter. Conservation Letters, 6, S. 66-72.

de Witte, T. und Latacz-Lohmann, U. (2014): Was kostet das Greening? top agrar, 4/2014, S. 36-41.

Deimer, C. (2005): Honorierungsansätze für Umweltleistungen in der Landwirtschaft: Genese, Trends und Bewertung. Dissertation Martin-Luther-Universität Halle-Wittenberg. Abrufbar unter: https://sundoc.bibliothek.uni-halle.de/diss-online/05/05H168/prom.pdf, zuletzt abgefragt: 21.05 .2016 .

Delzeit, R., Holm-Müller, K. und Britz, W. (2012): Ökonomische Bewertung des Erneuerbare Energien Gesetzes zur Förderung von Biogas. Perspektiven der Wirtschaftspolitik, 13, S. 251-265.

Derissen, S. und Quaas, M.F. (2013): Combining performance-based and action-based payments to provide environmental goods under uncertainty. Ecological Economics, 85, S. 77-84.

Deutsches Maiskomitee e.V. (2016): Anbaufläche Silomais. Abrufbar unter: http://www.maiskomitee.de/web/public/Fakten.aspx/Statistik/Deutschland/Gesamtfl\%C3\%A4chen-_entwicklung, zuletzt abgefragt: 28.10.2016.

Dienemann, C. und Utermann, J. (2012): Uran in Boden und Wasser, Dessau-Roßlau: Umweltbundesamt. Abrufbar unter: https://www.umweltbundesamt.de/sites/default/files/medien/461/ publikationen/4336.pdf, zuletzt abgefragt: 23.04.2017.

Dierschke, H., Briemle, G. und Kratochwil, A. (2002): Kulturgrasland: Wiesen, Weiden und verwandte Staudenfluren, Stuttgart: Ulmer.

Dolan, P., Hallsworth, M., Halpern, D., King, D., Metcalfe, R. und Vlaev, I. (2012): Influencing behaviour: The mindspace way. Journal of Economic Psychology, 33, S. 264-277.

Donald, P.F., Green, R.E. und Heath, M.F. (2001): Agricultural intensification and the collapse of Europe's farmland bird populations. Proceedings. Biological sciences/The Royal Society, 268, S. 25-29.

Drechsler, M., Johst, K., Ohl, C. und Wätzold, F. (2007): Designing cost-effective payments for conservation measures to generate spatiotemporal habitat heterogeneity. Conservation Biology, 21, S. 1475-1486.

Drösler, M., Freibauer, A., Adelmann, W., Augustin, J., Bergman, L., Beyer, C., Chojnicki, B., Förster, C., Giebels, M., Görlitz, S., Höper, H., Kantelhardt, J., Liebersbach, H., Hahn-Schöfl, M., Minke, M., Petschow, U., Pfadenhauer, J., Schaller, L., Schägner, P., Sommer, M., Thuille, A. 
und Wehrhan, M. (2011): Klimaschutz durch Moorschutz in der Praxis. Ergebnisse aus dem BMBF-Verbundprojekt „Klimaschutz - Moornutzungsstrategien“ 2006-2010, Braunschweig: vTI-Institut für Agrarrelevante Klimaforschung. Abrufbar unter: http://literatur.vti.bund.de/digbib_extern/bitv/dn049337.pdf, zuletzt abgefragt: 23.04.2017.

Duelli, P. und Obrist, M.K. (2003): Regional biodiversity in an agricultural landscape: the contribution of seminatural habitat islands. Basic and Applied Ecology, 4, S. 129-138.

Düwel, O., Siebner, C., Utermann, J. und Krone, F. (2007): Gehalte an organischer Substanz in Oberböden Deutschlands: Bericht über länderübergreifende Auswertungen von Punktinformationen im FISBo BGR, Hannover: Bundesanstalt für Geowissenschaften und Rohstoffe.

Dyen, M. und Sirieix, L. (2016): How does a local initiative contribute to social inclusion and promote sustainable food practices? Focus on the example of social cooking workshops. International Journal of Consumer Studies, 40, S. 685-694.

Eden, S., Bear, C. und Walker, G. (2008): Understanding and (dis) trusting food assurance schemes: consumer confidence and the 'knowledge fix'. Journal of Rural Studies, 24, S. 1-14.

EEA - European Environment Agency (1999): Environment in the European Union at the turn of the century, Copenhagen: EEA. Abrufbar unter: https://www.eea.europa.eu/publications/92-9157-202-0, zuletzt abgefragt: 23.04.2017.

EEA - European Environment Agency (2010): 10 messages for 2010 - Agricultural ecosystems, Copenhagen: EEA. Abrufbar unter: https://www.eea.europa.eu/publications/10-messages-for-2010-agricultural-ecosystems, zuletzt abgefragt: 25.05.2018.

Egan, M. (2001): Constructing a European market: standards, regulation, and governance, Oxford: Oxford University Press.

Ekroos, J., Olsson, O., Rundlöf, M., Wätzold, F. und Smith, H.G. (2014): Optimizing agri-environment schemes for biodiversity, ecosystem services or both? Biological Conservation, 172, S. 65-71.

Ellenberg, H. und Leuschner, C. (2009): Vegetation Mitteleuropas mit den Alpen in ökologischer, dynamischer und historischer Sicht, Stuttgart: Ulmer.

Elsholz, R. (2009): Regionale Wohlfahrtseffekte der Gemeinsamen Europäischen Agrarpolitik. Vortrag anlässlich der 49. Jahrestagung der GEWISOLA in Kiel. Abrufbar unter: http://ageconsearch.umn.edu/bitstream/53257/2/v31_53257.pdf, zuletzt abgefragt: 25.07.2016.

Emery, S.B. (2014): Hard work, productivity and the management of the farmed environment in anthropological perspective. In: L. Hamilton, L. Mitchell und A. Mangan (Hrsg.): Contemporary Issues in Management. Cheltenham: Edward Elgar, S. 90-104.

Emery, S.B. und Franks, J.R. (2012): The potential for collaborative agri-environment schemes in England: Can a well-designed collaborative approach address farmers' concerns with current schemes? Journal of Rural Studies, 28, S. 218-231.

Engel, S. (2015): The devil in the detail: A practical guide on designing payments for environmental services. Abrufbar unter: http://papers.ssrn.com/sol3/papers.cfm?abstract_id=2712376, zuletzt abgefragt: 23.04.2017.

Engel, S., Pagiola, S. und Wunder, S. (2008): Designing payments for environmental services in theory and practice: An overview of the issues. Ecological Economics, 65, S. 663-674.

Engelhardt, H. (2004): Auswirkungen von Flächengröße und Flächenform auf Wendezeiten, Arbeitserledigung und verfahrenstechnische Maßnahmen im Ackerbau. Inauguraldissertation zur Erlangung des Doktorgrades beim Fachbereich Agrarwissenschaften, Ökotrophologie und Umweltmanagement der Justus-Liebig-Universität Gießen.

ESAWADI (2013): Utilizing the Ecosystem Services Approach for Water Framework Directive Implementation. Synthesis Report: iwrm-net. Abrufbar unter: http://www.iwrm-net.eu/sites/ default/files/ESAWADI\%20SCP\%20final\%20synthesis\%20july2013.pdf, zuletzt abgefragt: 17.12.2016.

Espinosa-Goded, M., Barreiro-Hurlé, J. und Ruto, E. (2010): What do farmers want from agri-environmental scheme design? A choice experiment approach. Journal of Agricultural Economics, 61, S. 259-273.

Europäische Kommission (2016): Entwicklung des ländlichen Raums 2014-2020, Brussels. Abrufbar unter: http://ec.europa.eu/agriculture/rural-development-2014-2020/index_de.htm, zuletzt abgefragt: 07.04.2016. 
European Commission (2010): The EU Budget Review (Communication). COM (2010)700, Brussels.

European Commission (2011a): Greening - Results of partial analysis on impact on farm income using FADN, Brussels.

European Commission (2011b): Impact Assessment: Common Agricultural Policy towards 2020. Commission Staff Working Paper. SEC(2011) 1153 final/2. Abrufbar unter: http://ec.europa.eu/ agriculture/policy-perspectives/impact-assessment/cap-towards-2020/report/full-text_en.pdf, zuletzt abgefragt: 25.07.2016.

European Commission (2013): Green Infrastructure (GI) - Enhancing Europe's Natural Capital. Communication from the Commission to the European Parliament, the Council, the European Economic and Social Committee and the Committee of the Regions (COM/2013/0249 final): Brussels.

European Commission (2016): Interactive: EU expenditure and revenue 2014-2020. Abrufbar unter: http://ec.europa.eu/budget/figures/interactive/index_en.cfm, zuletzt abgefragt: 25.07.2016.

Ezzine-de-Blas, D., Wunder, S., Ruiz-Pérez, M. und del Pilar Moreno-Sanchez, R. (2016): Global Patterns in the Implementation of Payments for Environmental Services. Plos One, 11, S. p. e0149847.

FAO (2008): How to Feed the World in 2050, Rome: FAO.

FAO, IFAD und WFP (2014): The state of food insecurity in the world 2014: strengthening the enabling environment for food security and nutrition, Rome: FAO.

Feindt, P.H. (2008): Interessenvermittlung in der deutschen und europäischen Agrarpolitik im Wandel. In: B. Rehder, U. Wilms und T. von Winter (Hrsg.): Interessenvermittlung in Politikfeldern im Wandel. Befunde aus Verbände- und Policyforschung zur Bundesrepublik Deutschland und in vergleichender Perspektive. Wiesbaden: VS Verlag, S. 68-89.

Feindt, P.H. (2010): Policy-learning and environmental policy integration in the Common Agricultural Policy, 1973-2003. Public Administration, 88, S. 296-314.

Feindt, P.H. und Flynn, A. (2009): Policy stretching and institutional layering: British food policy between security, safety, quality, health and climate change. British Politics, 4, S. 386-414.

Feindt, P.H., Krämer, C., Früh-Müller, A., Wolters, V., Pahl-Wostl, C., Heißenhuber, A., Bers, C.v., Thomas, F. und Purnhagen, K. (2017): A future-oriented social contract with the agricultural sector: Plea for a new agricultural policy, Policy paper from the ZANEXUS project, presented to the Federal Agency for Nature Conservation (BfN), the German Federal Environmental Protection Agency and the German Federal Ministry for the Environment, Nature Conservation and Nuclear Safety, FKZ 3515880 400, January 2017, Bonn/Berlin. Abrufbar unter: https:// www.researchgate.net/publication/313562796_A_future-oriented_social_contract_with_the_ agricultural_sector_Plea_for_a_new_agricultural_policy.

Fietz, R. (2012): Die GAP-Reform 2014 aus dem Blickwinkel der betroffenen Landwirte in Deutschland. In: J. Martinez (Hrsg.): Die Gemeinsame Agrarpolitik vor neuen Herausforderungen. Baden-Baden: Nomos, S. 19 ff.

Filippi-Codaccioni, O., Devictor, V., Bas, Y. und Julliard, R. (2010): Toward more concern for specialisation and less for species diversity in conserving farmland biodiversity. Biological Conservation, 143, S. 1493-1500.

Fisher, B., Turner, R.K. und Morling, P. (2009): Defining and classifying ecosystem services for decision making. Ecological Economics, 68, S. 643-653.

Flachowsky, G. und Lebzien, P. (2005): Weniger Spurengase durch gezielte Ernährung der Nutztiere: Potentiale und Einflussmöglichkeiten bei Wiederkäuern und Nichtwiederkäuern. Forschungsreport Verbraucherschutz, Ernährung, Landwirtschaft, 2005, S. 7-9.

Flessa, H., Müller, D., Plassmann, K., Osterburg, B., Techen, A.-K., Nitsch, H., Nieberg, H., Sanders, J., Meyer zu Hartlage, O., Beckmann, E. und Anspach, V. (2012): Studie zur Vorbereitung einer effizienten und gut abgestimmten Klimaschutzpolitik für den Agrarsektor, Braunschweig: Johann Heinrich von Thünen-Institut. Abrufbar unter: http://literatur.thuenen.de/ digbib_extern/dn050716.pdf, zuletzt abgefragt: 23.04.2017.

Folke, C. (2006): Resilience: The emergence of a perspective for social-ecological systems analyses. Global Environmental Change, 16, S. 253-267. 
Folke, C., Carpenter, S.R., Walker, B., Scheffer, M., Chaplin, T. und Rockström, J. (2010): Resilience Thinking: Integrating Resilience, Adaptability and Transformability. Ecology and Society, 15, S. 20.

Forstner, B., Deblitz, C., Kleinhanss, W., Nieberg, H., Offermann, F., Röder, N., Salamon, P., Sanders, J. und Weingarten, P. (2012): Analyse der Vorschläge der EU-Kommission vom 12. Oktober 2011 zur künftigen Gestaltung der Direktzahlungen im Rahmen der GAP nach 2013: Arbeitsberichte aus der vTI-Agrarökonomie. Abrufbar unter: http://literatur.thuenen.de/digbib_extern/bitv/dn050475.pdf, zuletzt abgefragt: 23.04.2017.

Franks, J.R. und Mc Gloin, A. (2007): Environmental co-operatives as instruments for delivering across-farm environmental and rural policy objectives: lessons for the UK. Journal of Rural Studies, 23, S. 472-489.

Freese, J., Klimek, S. und Marggraf, R. (2011): Auktionen und ergebnisorientierte Honorierung bei Agrarumweltmaßnahmen. Natur und Landschaft, 86, S. 156-159.

Frey, B.S. und Stutzer, A. (2006): Environmental morale and motivation. IEW - Working Papers 288, Zurich: Institute for Empirical Research in Economics - University of Zurich.

Frische, T., Egerer, S., Matezki, S., Pickl, C. und Wogram, J. (2016): 5-Punkte-Programm für einen nachhaltigen Pflanzenschutz, Dessau-Roßlau: Umweltbundesamt. Abrufbar unter: https:// www.umweltbundesamt.de/themen/5-punkte-programm-fuer-einen-nachhaltigen, zuletzt abgefragt: 23.04.2017.

Frondel, M., Lehmann, P. und Wätzold, F. (2012): The impact of information on landowners' participation in voluntary conservation programs-Theoretical considerations and empirical evidence from an agri-environment program in Saxony, Germany. Land Use Policy, 29, S. 388-394.

Fuchs, D.A., Kalfagianni, A. und Havinga, T. (2011): Actors in private food governance: the legitimacy of retail standards and multistakeholder initiatives with civil society participation. Agriculture and Human Values, 28, S. 353-367.

Fuchs, S., Scherer, U., Wander, R., Behrendt, H., Venohr, M., Opitz, D., Hillenbrand, T., Marscheider-Weidemann, F. und Götz, T. (2010): Berechnung von Stoffeinträgen in die Fließgewässer Deutschlands mit dem Modell MONERIS: Nährstoffe, Schwermetalle und Polyzyklische aromatische Kohlenwasserstoffe, Dessau-Roßlau: Umweltbundesamt. Abrufbar unter: https:// www.umweltbundesamt.de/publikationen/berechnung-von-stoffeintraegen-in-fliessgewaesser, zuletzt abgefragt: 12.02.2017.

Führ, M. (2003): Eigen-Verantwortung im Rechtsstaat, Berlin: Duncker \& Humblot.

Fullbrook, E. (2007): Real world economics: a post-autistic economics reader, London: Anthem Press.

Funk, R., Deumlich, D. und Steidl, J. (2013): GIS Application to Estimate the Wind Erosion Risk in the Federal State of Brandenburg. In: J.C. Ascough II und D.C. Flanagan (Hrsg.): Soil Erosion Research for the 21st Century. St. Joseph, MI: ASAE, S. 400-403.

Gaba, S., Lescourret, F., Boudsocq, S., Enjalbert, J., Hinsinger, P., Journet, E.-P., Navas, M.-L., Wery, J., Louarn, G., Malézieux, E., Pelzer, E., Prudent, M. und Ozier-Lafontaine, H. (2015): Multiple cropping systems as drivers for providing multiple ecosystem services: from concepts to design. Agronomy for Sustainable Development, 35, S. 607-623.

Gabrielle, B., Bamière, L., Caldes, N., Cara, S.d., Decocq, G., Ferchaud, F., Loyce, C., Pelzer, E., Perez, Y., Wohlfahrt, J. und Richard, G. (2014): Paving the way for sustainable bioenergy in Europe: Technological options and research avenues for large-scale biomass feedstock supply. Renewable and Sustainable Energy Reviews, 33, S. 11-25.

Gailhard, I.U. und Bojnec, S. (2015): Farm size and participation in agri-environmental measures: Farm-level evidence from Slovenia. Land Use Policy, 46, S. 273-282.

Garzon, I. (2006): Reforming the Common Agricultural Policy. History of a Paradigm Change, Basingstoke: Palgrave Macmillan.

Gaujour, E., Amiaud, B., Mignolet, C. und Plantureux, S. (2012): Factors and processes affecting plant biodiversity in permanent grasslands. A review. Agronomy for Sustainable Development, 32, S. $133-160$.

Gay, H., Osterburg, B. und Schmidt, T. (2004): Szenarien der Agrarpolitik: Untersuchung möglicher agrarstruktureller und ökonomischer Effekte unter Berücksichtigung umweltpolitischer 
Zielsetzungen, Berlin: Rat von Sachverständigen für Umweltfragen. Abrufbar unter: http:// www.umweltrat.de/SharedDocs/Downloads/DE/03_Materialien/2004_MAT37_Szenarien_ der_Agrarpolitik.pdf?_blob=publicationFile, zuletzt abgefragt: 23.04.2017.

Ge, L., Anten, N.P.R., van Dixhoorn, I., Feindt, P.H., Kramer, K., Leemans, R., Meuwissen, M.P.M., Spoolder, H. und Sukkel, W. (2016): Why we need resilience thinking to meet societal challenges in bio-based production systems. Current Opinion in Environmental Sustainability, 23, S. 17-27.

Gehrlein, U. und Fick, J. (2007): Bedeutung der Regionalvermarktung für Kulturlandschaftserhalt und Naturschutz. Dokumentation zum Fachgespräch am 13. Februar 2006, Bonn: Bundesamt für Naturschutz. Abrufbar unter: https://www.bfn.de/fileadmin/MDB/documents/service/ skript221.pdf, zuletzt abgefragt: 23.04.2017.

Geyer-Allély, E. und Zacarias-Farah, A. (2001): Background Paper on Information and Consumer Decision-Making for Sustainable Consumption, Paris: OECD Environment Directorate.

Ghazoul, J., Garcia, C. und Kushalappa, C.G. (2009): Landscape labelling: a concept for nextgeneration payment for ecosystem service schemes. Forest ecology and management, 258, S. $1889-1895$.

Ghazoul, J., Garcia, C.A. und Kushalappa, C.G. (2011): Landscape labeling approaches to PES: Bundling services, products and stewards. In: D. Ottaviani und N.E. Hage Scialabba (Hrsg.): Payments for ecosystem services and food security. Rome: FAO, S. 171-189.

Gibbons, M., Limoges, C., Nowotny, H., Schwartzman, S., Scott, P. und Trow, M. (1994): The new production of knowledge: The dynamics of science and research in contemporary societies, London, Thousand Oaks, New Delhi: Sage.

Glebe, T.W. (2008): Scoring two-dimensional bids: How cost-effective are agri-environmental auctions? European Review of Agricultural Economics, 35, S. 143-165.

Golan, E., Kuchler, F., Mitchell, L., Greene, C. und Jessup, A. (2001): Economics of food labeling. Journal of Consumer Policy, 24, S. 117-184.

Gómez-Baggethun, E., de Groot, R., Lomas, P.L. und Montes, C. (2010): The history of ecosystem services in economic theory and practice: From early notions to markets and payment schemes. Ecological Economics, 69, S. 1209-1218.

Gottwald, F. und Stein-Bachinger, K. (2015): Landwirtschaft für Artenvielfalt. Ein Naturschutzstandard für ökologisch bewirtschaftete Betriebe. Stand: Mai 2015, Berlin: WWF Deutschland.

Grant, W. (1997): The Common Agricultural Policy, New York: St. Martins.

GREENNET (2010): Förderung eines ökologischen Netzwerkes im Grünen Band Zentraleuropas. Projektvorschlag INTERREG IV B Central Europe Program. Abrufbar unter: https:// silopdf.com/download-pdf-greennet-foerderung-eines-oekologischen-netzwerkes-im-gruenen-band-zentraleuropas.html, zuletzt abgefragt: 18.04.2017.

Greer, A. (2005): Agricultural Policy in Europe, Manchester: Manchester University Press.

Greer, A. und Hind, T. (2012): Inter-institutional decision-making: The case of the Common Agricultural Policy. Policy and Society, 31, S. 331-341.

Grethe, H. (2008): Agricultural Policy: What Roles for the EU and the Member States? In: G. Gelauff, I. Grilo und A. Lejour (Hrsg.): Subsidiarity and Economic Reform in Europe. Berlin: Springer, S. 191-218.

Grey, D. und Sadoff, C.W. (2007): Sink or Swim? Water security for growth and development. Water Policy 9, S. 545-571.

Grolleau, G., Mzoughi, N. und Thoyer, S. (2015): Les Incitations Monétaires Dans La Politique Agro-Environmentale: Peut-on Faire Mieux Avec Moins? Revue d'Études en Agriculture et Environnement, 96, S. 241-257.

Grunert, K.G. (2011): Sustainability in the food sector: A consumer behaviour perspective. International Journal on Food System Dynamics, 2, S. 207-218.

Haarland, C., Naisbit, R.E. und Bersier, L.-F. (2011): Sown wildflower strips for insect conservation: a review. Insect Conservation and Diversity, 4, S. 60-80.

Haber, W. (1998): Nutzungsdiversität als Mittel zur Erhaltung von Biodiversität. Berichte der Bayerischen Akademie für Naturschutz und Landschaftspflege (ANL), 22, S. 71-76.

Haber, W. (2014): Landwirtschaft und Naturschutz, Weinheim: Wiley. 
Hadley, A.S. und Betts, M.G. (2012): The effects of landscape fragmentation on pollination dynamics: absence of evidence not evidence of absence. Biological reviews of the Cambridge Philosophical Society, 87, S. 526-544.

Halberg, N. (2012): Assessment of the environmental sustainability of organic farming: Definitions, indicators and the major challenges. Canadian Journal of Plant Science, 92, S. 981-996.

Hampicke, U. (2009): Fachgutachten über die Höhe von Ausgleichszahlungen für die naturnahe Bewirtschaftung landwirtschaftlicher Nutzflächen in Deutschland, Hamburg: Michael Otto Stiftung für Umweltschutz. Abrufbar unter: http://www.umweltstiftungmichaelotto.de/uploads/ downloads/Initiativen/Fachgutachten2014_final_LowRes.pdf, zuletzt abgefragt: 23.04.2017.

Hampicke, U. (2013): Kulturlandschaft und Naturschutz, Heidelberg: Springer.

Han, Y. und Hansen, H. (2012): Determinants of Sustainable Food Consumption: A Meta-Analysis Using a Traditional and a Structura Equation Modelling Approach. International Journal of Psychological Studies, 4, S. 22-45.

Hanley, N., Banerjee, S., Lennox, G.D. und Armsworth, P.R. (2012): How should we incentivize private landowners to "produce" more biodiversity? Oxford Review of Economic Policy, 28, S. 93-113.

Hanley, N. und White, B. (2014): Incentivizing the Provision of Ecosystem Services. International Review of Environmental and Resource Economics, 7, S. 299-331.

Hansen, H. (2009): Regionale Verteilungswirkungen der Europäischen Agrarpolitik: Theoretische Überlegungen und empirische Evidenz. Dissertation Justus-Liebig-Universität Gießen, Göttingen: Cuvillier-Verlag. Abrufbar unter: http://geb.uni-giessen.de/geb/volltexte/2009/7182/, zuletzt abgefragt: 25.07.2016.

Hanson, D. (2005): CE marking, product standards and world trade, Cheltenham: Edward Elgar.

Hart, J.D., Milsom, T.P., Fisher, G., Wilkins, V., Moreby, S.J., Murray, A.W.A. und Robertson, P.A. (2006): The relationship between yellowhammer breeding performance, arthropod abundance and insecticide applications on arable farmland. Journal of Applied Ecology, 43, S. 81-91.

Hart, K., Buckwell, A. und Baldock, D. (2016): Learning the lessons of the Greening of the CAP, London: Institute for European Environmental Policy. Abrufbar unter: http://www.ieep.eu/ assets/2028/Learning_the_lessons_from_CAP_greening_-_April_2016_-_final.pdf, zuletzt abgefragt: 22.04.2016.

Haupt, H., Ludwig, G., Gruttke, H., Binot-Hafke, M., Otto, C. und Pauly, A. (2009): Rote Liste gefährdeter Tiere, Pflanzen und Pilze Deutschlands: Band 1: Wirbeltiere, Bonn: Bundesamt für Naturschutz.

Havinga, T., van Waarden, F. und Casey, D. (Hrsg.) (2015): The Changing Landscape of Food Governance: Public and Private Encounter, Cheltenham: Edward Elgar.

Heinrich, B., Holst, C. und Lakner, S. (2013): Die Reform der Gemeinsamen Agrarpolitik: Wird alles grüner und gerechter. GAIA, 22, S. 20-24.

Heißenhuber, A., Haber, W. und Krämer, C. (2015): 30 Jahre SRU-Sondergutachten „Umweltprobleme der Landwirtschaft“ - eine Bilanz, Dessau: Umweltbundesamt. Abrufbar unter: http:// www.umweltbundesamt.de/publikationen/umweltprobleme-der-landwirtschaft, zuletzt abgefragt: 23.04.2017.

Heißenhuber, A., Kantelhardt, J., Schaller, J. und Magel, H. (2004): Visualisierung und Bewertung ausgewählter Landnutzungsentwicklungen. Natur und Landschaft, 79, S. 159-166.

Heißenhuber, A. und Krämer, C. (2013): Wasser und Landwirtschaft. In: M. Grambow (Hrsg.): Nachhaltige Wasserbewirtschaftung - Konzept und Umsetzung eines vernünftigen Umgangs mit dem Gemeingut Wasser. Wiesbaden: Springer Vieweg, S. 281-298.

Herbes, C., Jirka, E., Braun, J.P. und Pukall, K. (2014): Der gesellschaftliche Diskurs um den „Maisdeckel“ vor und nach der Novelle des Erneuerbare-Energien-Gesetzes (EEG) 2012. GAIA, 23, S. 100-108.

Hertwich, E., van der Voet, E., Suh, S., Tukker, A., Kazmierczyk, P., Lenzen, M., Mc-Neely, J. und Moriguchi, Y. (2010): UNEP (2010) Assessing the Environmental Impacts of Consumption and Production: Priority Products and Materials, Paris: United Nations Environment Programme. 
Hey, C., Jacob, K. und Volkery, A. (2007): Better regulation by new governance hybrids? Governance models and the reform of European chemicals policy. Journal of Cleaner Production, 15, S. $1859-1874$.

Higgins, V. und Lawrence, G. (Hrsg.) (2005): Agricultural Governance: Globalization and the New Politics of Regulation, London: Routledge.

Hodge, I. (2001): Beyond agri-environmental policy: Towards an alternative model of rural environmental governance. Land Use Policy, 18, S. 99-111.

Hodge, I. und Adams, W.M. (2013): The role of agri-environment measures in promoting co-ordinated land management in large conservation areas. $14^{\text {th }}$ Global Conference of the International Association for the Study of the Commons. Mt Fuji, S. 1-17.

Hoff, H. (2011): Understanding the Nexus. Background paper for the Bonn 2011 Conference: The Water, Energy and Food Security Nexus, Stockholm: Stockholm Environment Institute. Abrufbar unter: http://wef-conference.gwsp.org/fileadmin/documents_news/understanding_the_nexus.pdf, zuletzt abgefragt: 23.04.2017.

Hoffmann, J., Berger, G., Wiegand, I., Wittchen, U., Pfeffer, H., Kiesel, J. und Ehlert, F. (2012): Bewertung und Verbesserung der Biodiversität leistungsfähiger Nutzungssysteme in Ackerbaugebieten unter Nutzung von Indikatorvogelarten: Biodiversität in Ackerbaugebieten. Berichte aus dem Julius Kühn-Institut, 163, S. 215 ff.

Hoffmann, J. und Kretschmer, H. (2001): Zum Biotop- und Artenschutzwert großer Ackerschläge in Nordostdeutschland. Peckiana, S. 17-31.

Hogeveen, H., Huijps, K. und Lam, T.J.G.M. (2011): Economic aspects of mastitis: New developments. New Zealand Veterinary Journal, 59, S. 16-23.

Holling, C.S. (1974): Resilience and stability of ecological systems. Ann. Rev. of Ecol. And Syst., 4, S. 1-23.

Holm-Müller, K., Henseleit, M., Möseler, B.M. und Vollmer, I. (2006): Honorierung ökologischer Leistungen der Landwirtschaft: Einführung ergebnisorientierter Komponenten in das Kulturlandschaftsprogramm NRW, Bonn: Rheinische Friedrich-Wilhelms-Universität Bonn. Abrufbar unter: https://www.usl.uni-bonn.de/pdf/Forschungsbericht\%20141_1.pdf, zuletzt abgefragt: 16.03.2017.

Hötker, H. (2004): Vögel in der Agrarlandschaft. Bestand, Gefährdung, Schutz, Meckenheim: NABU.

Hughner, R.S., McDonagh, P., Prothero, A., Shultz II, C.J. und Stanton, J. (2007): Who are organic food consumers? A compilation and review of why people purchase organic food. Journal of Consumer Behavior, 6, S. 94-110.

Huitema, D., Mostert, E., Egas, W., Moellenkamp, S., Pahl-Wostl, C. und Yalcin, R. (2009): Adaptive water governance: assessing the institutional prescriptions of adaptive (co-) management from a governance perspective and defining a research agenda. Ecology and Society, 14, S. 26. IPCC (1996): Climate Change 1995: The Science of Climate Change, Geneva.

IPCC (2014): Climate Change 2014: Synthesis Report. Contribution of Working Groups I, II and III to the Fifth Assessment Report of the Intergovernmental Panel on Climate Change: Summary for Policymakers, Geneva.

Isermeyer, F., Forstner, B., Nieberg, H., Offermann, F., Osterburg, B., Schmidt, T., Röder, N. und Weingarten, P. (2014): Gesetzentwurf zur Durchführung der Direktzahlungen an Inhaber landwirtschaftlicher Betriebe im Rahmen von Stützungsregelungen der Gemeinsamen Agrarpolitik. Stellungnahme im Rahmen einer öffentlichen Anhörung des Ausschusses für Ernährung und Landwirtschaft des Deutschen Bundestages am 7. April 2014, Braunschweig: Thünen-Institut. Abrufbar unter: https://literatur.thuenen.de/digbib_extern/dn053441.pdf, zuletzt abgefragt: 17.12 .2016 .

Iverson, A.L., Marín, L.E., Ennis, K.K., Gonthier, D.J., Connor-Barrie, B.T., Remfert, J.L., Cardinale, B.J., Perfecto, I. und Wilson, J. (2014): REVIEW: Do polycultures promote win-wins or trade-offs in agricultural ecosystem services? A meta-analysis. Journal of Applied Ecology, 51, S. 1593-1602.

Jahn, T., Bergmann, M. und Keil, F. (2012): Transdisciplinarity: Between mainstreaming and marginalization. Ecological Economics, 79, S. 1-10. 
Jahn, T., Hötker, H., Oppermann, R., Bleil, R. und Vele, L. (2014): Protection of biodiversity of free living birds and mammals in respect of the effects of pesticides, Berlin: Umweltbundesamt.

Janssen, M. und Hamm, U. (2012): Product labelling in the market for organic food: Consumer preferences and willingness-to-pay for different organic certification logos. Food Quality and Preference, 25, S. 9-22.

Jedicke, E. (1994): Biotopverbund - Grundlagen und Maßnahmen einer neuen Naturschutzstrategie, Stuttgart: Ulmer.

Jensen, J.D. und Ronit, K. (2015): Obesity, International Food and Beverage Industries, and Self-Regulation: The Fragmentation of Information Strategies. World Medical \& Health Policy, 7, S. 278-297.

Jering, A., Klatt, A., Seven, J., Ehlers, K., Günther, J., Ostermeier, A. und Mönch, L. (2013): Globale Landflächen und Biomasse nachhaltig und ressourcenschonend nutzen, Berlin: Umweltbundesamt.

JKI (2015): Herbizidresistenz, Braunschweig: Julius Kühn-Institut. Abrufbar unter: http://www. julius-kuehn.de/no_cache/de/startseite/institute/pflanzenschutz-ackerbau-und-gruenland/arbeitsgruppen/herbologie/herbizidresistenz.html, zuletzt abgefragt: 30.06.2016.

Joerges, C., Falke, J., Micklitz, H.-W. und Brüggemeier, G. (1988): Die Sicherheit von Konsumgütern und die Entwicklung der Europäischen Gemeinschaft, Baden-Baden: Nomos.

Jones, R.A.C. (2014): Trends in plant virus epidemiology: opportunities from new or improved technologies. Virus research, 186, S. 3-19.

Joosten, H. (2012): Zustand und Perspektiven der Moore weltweit. Natur und Landschaft, 87, S. 50-55.

Joosten, H., Brust, K., Couwenberg, J., Gerner, A., Holsten, B., Permien, T., Schäfer, A., Tanneberger, F., Trepel, M. und Wahren, A. (2016): MoorFutures: Integration of additional ecosystem services (including biodiversity) into carbon credits: standard, methodology and transferability to other regions, Bonn: Bundesamt für Naturschutz. Abrufbar unter: https://www.bfn.de/filead$\mathrm{min} / \mathrm{BfN} / \mathrm{service} /$ Dokumente/skripten/skript407.pdf, zuletzt abgefragt: 17.12.2016.

Jordan, G., Maloney, W.A. und McLaughlin, A.M. (1994): Characterizing agricultural policy making. Public Administration, 72, S. 505-526.

Josling, T.E. (2002): Competing Paradigms in the OECD and Their Impacts on the WTO Agricultural Talks. In: L. Tweeten und S.R. Thompson (Hrsg.): Agricultural Policy for the 21st Century. Ames, IA: Iowa State University Press, S. 245-264.

Juerges, N. und Newig, J. (2015): How interest groups adapt to the changing forest governance landscape in the EU: A case study from Germany. Forest Policy and Economics, 50, S. 228-235.

Kahlenborn, W., Moser, C., Frijdal, J. und Essig, M. (2011): Strategic Use of Public Procurement in Europe. Final Report to the European Commission MARKT/2010/02/C, Berlin: adelphi.

Kahneman, D. (2003): Maps of Bounded Rationality: Psychology for Behavioral Economics. The American Economic Review, 93, S. 1449-1475.

Kaule, G. (1991): Arten- und Biotopschutz, Stuttgart: Ulmer.

Kay, A. (2003): Path dependency and the CAP. Journal of European Public Policy, 10, S. 405-420.

KBU (2008): Bodenschutz beim Anbau nachwachsender Rohstoffe, Dessau-Roßlau: Umweltbundesamt.

KBU (2012): Uran-Einträge in landwirtschaftliche Böden durch Düngemittel. Positionspapier der Kommission Bodenschutz am Umweltbundesamt, Dessau-Roßlau: Umweltbundesamt.

Kehlbacher, A., Tiffin, R., Briggs, A., Berners-Lee, M. und Scarborough, P. (2016): The distributional and nutritional impacts and mitigation potential of emission-based food taxes in the UK. Climatic Change, S. 1-21.

Khanna, M. und Ando, A.W. (2009): Science, economics and the design of agricultural conservation programmes in the US. Journal of Environmental Planning and Management, 52, S. 575-592.

Klair, K., Boggia, A. und Richardson, D.W. (1998): The changing information needs of farmers in the US and Europe, Minneapolis, Minnesota: Center for International Food and 
Agricultural Policy. Abrufbar unter: http://ageconsearch.umn.edu/record/14496, zuletzt abgefragt: 25.04.2017.

Kleijn, D., Baquero, R.A., Clough, Y., Diaz, M., De Esteban, J., Fernandez, F., Gabriel, D., Herzog, F., Holzschuh, A., Johl, R., Knop, E., Kruess, A., Marshall, E.J., Steffan-Dewenter, I., Tscharntke, T., Verhulst, J., West, T.M. und Yela, J.L. (2006): Mixed biodiversity benefits of agri-environment schemes in five European countries. Ecology Letters, 9, S. 243-254.

Kleijn, D., Berendse, F., Smit, R. und Gilissen, N. (2001): Agri-environment schemes do not effectively protect biodiversity in Dutch agricultural landscapes. Nature, 413, S. 723-725.

Kleijn, D., Kohler, F., Baldi, A., Batary, P., Concepcion, E.D., Clough, Y., Diaz, M., Gabriel, D., Holzschuh, A., Knop, E., Kovacs, A., Marshall, E.J.P., Tscharntke, T. und Verhulst, J. (2009): On the relationship between farmland biodiversity and land-use intensity in Europe. Proceedings. Biological sciences, 276, S. 903-909.

Kleijn, D. und Sutherland, W.J. (2003): How effective are European agri-environment schemes in conserving and promoting biodiversity? . Journal of Applied Ecology, 40, S. 947-969.

Kluser, S. und Peduzzi, P. (2007): Global Pollinator Decline: A literature Review: UNEP/GRIDEurope. Abrufbar unter: http://archive-ouverte.unige.ch/unige:32258, zuletzt abgefragt: 23.04.2017.

Knop, E.V.A., Kleijn, D., Herzog, F. und Schmid, B. (2006): Effectiveness of the Swiss agri-environment scheme in promoting biodiversity. Journal of Applied Ecology, 43, S. 120-127.

Knudsen, A.-C.L. (2009): Farmers on Welfare. The Making of Europe's Common Agricultural Policy, Ithaca and London: Cornell University Press.

Knüppe, K. und Pahl-Wostl, C. (2013): Requirements for adaptive governance of groundwater ecosystem services: insights from Sandveld (South Africa), Upper Guadiana (Spain) and Spree (Germany). Regional Environmental Change, 13, S. 53-66.

Kommission Landwirtschaft am Umweltbundesamt (2011): Für eine ökologisierte erste und eine effiziente zweite Säule, Dessau-Roßlau: Umweltbundesamt.

Krämer, C. (2017): Umsetzung der EU-GAP-Reform (2014-2020) in den EU-Nachbarstaaten, Dessau-Roßlau: Umweltbundesamt. Abrufbar unter: https://www.umweltbundesamt.de/sites/ default/files/medien/1410/publikationen/2017-06-08_texte_48-2017_gap-reform.pdf, zuletzt abgefragt: 23.03.2017.

Kranert, M., Hafner, G., Barabosz, J., Schuller, H., Leverenz, D., Kölbig, A., Schneider, F., Lebersorger, S. und Scherhaufer, S. (2012): Ermittlung der weggeworfenen Lebensmittelmengen und Vorschläge zur Verminderung der Wegwerfrate bei Lebensmitteln in Deutschland, Stuttgart: iswa. Abrufbar unter: https://www.bmel.de/SharedDocs/Downloads/Ernaehrung/WvL/ Studie_Lebensmittelabfaelle_Langfassung.pdf?_blob=publicationFile, zuletzt abgefragt: 12.01.2017.

Krause, B., Wesche, K., Culmsee, H. und Leuschner, C. (2014): Diversitätsverluste und floristischer Wandel im Grünland seit 1950. Natur und Landschaft, 89, S. 399-404.

Kremen, C. und Miles, A. (2012): Ecosystem services in biologically diversified versus conventional farming systems: benefits, externalities, and trade-offs. Ecology and Society, 17, S. 40.

Kuhfuss, L., Préget, R., Thoyer, S. und Hanley, N. (2015): Nudging farmers to enrol land into agri-environmental schemes: the role of a collective bonus. European Review of Agricultural Economics, S. 1-28.

Kuhnert, H., Feindt, P.H. und Beusmann, V. (2005): Ausweitung des ökologischen Landbaus in Deutschland. Voraussetzungen, Strategien, Implikationen, politische Optionen, Münster-Hiltrup: Landwirtschaftsverlag.

Lal, R. (2013): Enhancing ecosystem services with no-till. Renewable Agriculture and Food Systems, 28, S. 102-114.

Landwirtschaftskammer Niedersachsen (2016): Nährstoffbericht in Bezug auf Wirtschaftsdünger für Niedersachsen 2014/2015, Hannover.

Landwirtschaftskammer Nordrhein-Westfalen (2014): Nährstoffbericht: über Wirtschaftsdünger und andere organische Düngemittel für Nordrhein-Westfalen, Münster. Abrufbar unter: https:// www.landwirtschaftskammer.de/landwirtschaft/ackerbau/pdf/naehrstoffbericht-nrw-2014.pdf, zuletzt abgefragt: 23.04.2017. 
Landwirtschaftskammern (2010): Biologische Vielfalt in Agrarlandschaften bewahren und weiterentwickeln: Positionspapier des Fachausschusses Ländliche Entwicklung, Raumordnung und Ressourcenschutz, Berlin: Verband der Landwirtschaftskammern.

Lascoumes, P. und Le Gales, P. (2007): Introduction: understanding public policy through its instruments - from the nature of instruments to the sociology of public policy instrumentation. Governance, 20, S. 1-21.

Latacz-Lohmann, U. und Van der Hamsvoort, C. (1997): Auctioning Conservation Contracts: A Theoretical Analysis and an Application. American Journal of Agricultural Economics, 79, S. 407-418.

Lebert, M. (2004): Entwicklung eines Prüfkonzeptes zur Erfassung der tatsächlichen Verdichtungsgefährdung landwirtschaftlich genutzter Böden, Dessau-Roßlau: Umweltbundesamt.

Lefebvre, M., Espinosa, M. und Gomez, S. (2012): The influence of Common Agricultural Policy on agricultural landscapes, Seville: JRC. Abrufbar unter: http://publications.jrc.ec.europa.eu/ repository/bitstream/JRC73276/cap_and_landscape_final_bar_code_end.pdf, zuletzt abgefragt: 18.12.2016.

Leuschner, C., Krause, B., Meyer, S. und Bartels, M. (2014): Strukturwandel im Acker- und Grünland Niedersachsens und Schleswig-Holsteins seit 1950. Natur und Landschaft, 89, S. 386-391.

Levi-Faur, D. (2009): Regulatory capitalism and the reassertion of the public interest. Policy and Society, 27, S. 181-191.

Levin, I.P., Schneider, S.L. und Gaeth, G.J. (1998): All Frames Are Not Created Equal: A Typology and Critical Analysis of Framing Effects. Organizational Behavior and Human Decision Processes, 76, S. 149-188.

LfL - Bayerische Landesanstalt für Landwirtschaft (2010): Standorttypische Humusgehalte von Ackerböden in Bayern: Bayerische Landesanstalt für Landwirtschaft.

LfU - Bayrisches Landesamt für Umwelt (2004): Ammoniak und Ammonium, München: Bayrisches Landesamt für Umwelt.

LfU - Bayrisches Landesamt für Umwelt (2008): Pflanzenschutzmittel in der Umwelt, München: Bayrisches Landesamt für Umwelt.

Lin, B.B. (2011): Resilience in Agriculture through Crop Diversification: Adaptive Management for Environmental Change. BioScience, 61, S. 183-193.

Liquete, C., Kleeschulte, S., Dige, G., Maes, J., Grizzetti, B., Olah, B. und Zulian, G. (2015): Mapping green infrastructure based on ecosystem services and ecological networks: A Pan-European case study. Environmental Science \& Policy, 54, S. 268-280.

Loisel, P. und Elyakime, B. (2006): Incentive contract and weather risk. Environmental and Resource Economics, 35, S. 99-108.

Lokhorst, A.M., Hoon, C., le Rutte, R. und de Snoo, G.R. (2014): There is an I in nature: The crucial role of the self in nature conservation. Land Use Policy, 39, S. 121-126.

Lokhorst, A.M., Staats, H., Van Dijk, J., Van Dijk, E. und de Snoo, G.R. (2011): What's in it for me? Motivational differences between farmers' voluntary and subsidized nature conservation practices. Applied psychology - an International Review, 60, S. 337-353.

Lokhorst, A.M., Van Dijk, J., Staats, H., Van Dijk, E. und de Snoo, G.R. (2010): Using tailored information and public commitment to improve the environmental quality of farm lands: An example from the Netherlands. Human Ecology, 38, S. 113-122.

Lokhorst, A.M., Werner, C.J.M., Staats, H., van Dijk, E. und Gale, J. (2013): Commitment and behavior change: A meta-analysis and critical review of commitment-making strategies in environmental research. Environment and Behavior, 45, S. 3-34.

Lomba, A., Guerra, C., Alonso, J., Honrado, J.P., Jongman, R. und McCracken, D. (2014): Mapping and monitoring High Nature Value farmlands: challenges in European landscapes. Journal of Environmental Management, 143, S. 140-150.

Lowe, P. und Baldock, D. (2000): Integration of Environmental Objectives into Agricultural Policy Making. In: F. Brower und P. Lowe (Hrsg.): CAP Regimes and the European Countryside. Wallingford: CAB International, S. 31-52.

Lowe, P., Feindt, P.H., Laschewski, L. und Vihinen, H. (2010): Greening Agriculture and the Countryside? Changing Frameworks of Agricultural Policy. Public Administration, 88, S. 287-295. 
Lu, Z.-X., Zhu, P.-Y., Gurr, G.M., Zheng, X.-S., Read, D.M.Y., Heong, K.-L., Yang, Y.-J. und Xu, H.-X. (2014): Mechanisms for flowering plants to benefit arthropod natural enemies of insect pests: prospects for enhanced use in agriculture. Insect science, 21, S. 1-12.

Ludwig, G. und Matzke-Hajek, G. (2011): Rote Liste gefährdeter Tiere, Pflanzen und Pilze Deutschlands: Band 6: Pilze (Teil 2) - Flechten und Myxomyzeten, Bonn: Bundesamt für Naturschutz.

Lütke-Entrup, N. und Schneider, M. (2003): Nachhaltigkeit und Umweltverträglichkeit landwirtschaftlicher Systeme der Bodennutzung durch Fruchtfolgengestaltung und konservierende Bodenbearbeitung/Direktsaat. In: J. Epperlein (Hrsg.): Bodenschutz und landwirtschaftliche Bodennutzung - Umweltwirkungen am Beispiel der konservierenden Bodenbearbeitung. Braunschweig und Dessau-Roßlau: FAL und Umweltbundesamt, S. 7-35.

Lütz, M. und Bastian, O. (2002): Implementation of landscape planning and nature conservation in the agricultural landscape - a case study from Saxony. Agriculture. Ecosystems \& Environment, 92, S. 159-170.

Luz, F. (2000): Participatory landscape ecology - A basis for acceptance and implementation. Landscape and Urban Planning, 50, S. 157-166.

Machl, T. (2016): Beispielhafte Darstellung ausgewählter Landschaftsstrukturen in drei Testregionen. Unveröffentlichter Bericht für das ZA-NExUS-Projekt, Freising.

Maduro, M. (1998): We the Court: The European Court of Justice and the European Economic Constitution, London: Bloomsbury.

Mahoney, J. (2000): Path dependence in historical sociology. Theory and Society, 29, S. 507-548.

Majone, G. (1989): Evidence, Argument and Persuasion in the Policy Process, New Haven: Yale University Press.

Mante, J. (2010): Erfolgs- und Hemmfaktoren für die Umsetzung von Naturschutzmaßnahmen in intensiv genutzten Agrarregionen. Promotion an der Universität Rostock.

Marggraf, R. (2003): Comparative assessment of agri-environment programmes in federal states of Germany. Agriculture, Ecosystems and Environment, 98, S. 507-516.

Matthews, A. (2015): The Multi-Annual Financial Framework and the 2013 CAP Reform. In: J. Swinnen (Hrsg.): The Political Economy of the 2014-2020 Common Agricultural Policy. An Imperfect Storm. London: Rowman \& Littlefield, S. 169-191.

Matzdorf, B. (2011): Potentiale der ersten und zweiten Säule der GAP für die Umsetzung von Umweltmaßnahmen: Vortrag gehalten auf dem Internationalen Expertenworkshop Perspektiven für die Biodiversität in der europäischen Agrarlandschaft ab 2014 in Ladenburg. Abrufbar unter: https://www.bfn.de/fileadmin/MDB/documents/themen/landwirtschaft/vortrag_matzdorf.pdf, zuletzt abgefragt: 23.04.2017.

Matzdorf, B. und Lorenz, J. (2010): How cost-effective are result-oriented agri-environmental measures? - An empirical analysis in Germany. Land Use Policy, 27, S. 535-544.

McCracken, M.E., Woodcock, B.A., Lobley, M., Pywell, R.F., Saratsi, E., Swetnam, R.D., Mortimer, S.R. und Harris, S.J. (2015): Social and ecological drivers of success in agri-environment schemes: the roles of farmers and environmental context. Journal of Applied Ecology, 52, S. 696-705.

McDaniel, M.D., Tiemann, L.K. und Grandy, A.S. (2014): Does agricultural crop diversity enhance soil microbial biomass and organic matter dynamics? A meta-analysis. Ecological Applications, 24, S. 560-570.

McEachern, M., Seaman, C., Padel, S. und Foster, C. (2005): Exploring the gap between attitudes and behaviour: Understanding why consumers buy or do not buy organic food. British Food Journal, 107, S. 606-625.

McGee, A. und Weatherill, S. (1990): The Evolution of the Single Market - harmonisation or liberalisation. The Modern Law Review, 53, S. 578-596.

McMichael, P. (2011): Food system sustainability: Questions of environmental governance in the new world (dis)order. Global Environmental Change, 21, S. 804-812.

Ménard, C. und Valceschini, E. (2005): New institutions for governing the agri-food industry. European Review of Agricultural Economics, 32, S. 421-440. 
Menghi, A., de Roest, K., Porcelluzzi, A., Deblitz, C., von Davier, Z., Wildegger, B., de Witte, T., Strohm, K., Garming, H. und Dirksmeyer, W. (2014): Assessing farmers' cost of compliance with EU legislation in the fields of environment, animal welfare and food safety. Commissioned by the European Commission Directorate-General for Agriculture and Rural Development. AGRI-2011-EVAL-08. Brussels: European Commission Directorate-General for Agriculture and Rural Development.

Mettepenningen, E., Verspecht, A. und Van Huylenbroeck, G. (2009): Measuring private transaction costs of European agri-environmental schemes. Journal of Environmental Planning and Management, 52, S. 649-667.

Meuwissen, M.P., Huirne, R. und Skees, J.R. (2003): Income insurance in European agriculture. EuroChoices, 2, S. 12-17.

Meybeck, A., Lankoski, J., Redfern, R., Azzu, N. und Gitz, V. (Hrsg.) (2012): Building resilience for adaptation to climate change in the agriculture sector. Proceedings of a Joint FAO/OECD Workshop Rome: FAO/OECD.

Meyer, C., Reutter, M., Matzdorf, B., Sattler, C. und Schomers, S. (2015a): Design rules for successful governmental payments for ecosystem services: Taking agri-environmental measures in Germany as an example. Journal of Environmental Management, 157, S. 146-159.

Meyer, S., Bergmeier, E., Becker, T., Wesche, K., Krause, B., Leuschner, C. und Schwabe-Kratochwil, A. (2015b): Detecting long-term losses at the plant community level - arable fields in Germany revisited. Applied Vegetation Science, 18, S. 432-442.

Meyer, S., Wesche, K., Krause, B., Brütting, C., Hensen, I. und Leuschner, C. (2014): Diversitätsverluste und floristischer Wandel im Ackerland seit 1950. Natur und Landschaft, 89, S. 392-398.

Mills, J. (2012): Exploring the social benefits of agri-environment schemes in England. Journal of Rural Studies, 28, S. 612-621.

Mitchell, M.G.E., Bennett, E.M. und Gonzalez, A. (2013): Linking Landscape Connectivity and Ecosystem Service Provision: Current Knowledge and Research Gaps. Ecosystems, 16, S. 894-908.

Möckel, S. (2013): Gute fachliche Praxis, Eingriffsregelung und weitere Steuerungsinstrumente im Kontext Landnutzung. Vortrag gehalten in Kassel am 26.09.2013. Abrufbar unter: https:// www.bbn-online.de/fileadmin/Service/8_2\%20Veroeffentlichungen/Bundesfachtagung_Naturschutzrecht/Moeckel_-_Rechtliche_Instrumente_der_Landnutzungssteuerung_Kassel_26-9-2013__geschuetzt.pdf, zuletzt abgefragt: 23.04.2017.

Möckel, S. (2016): Verhältnis ordnungs- und beihilferechtlicher Mindestanforderungen im Agrarumweltrecht. Zeitschrift für Umweltrecht, S. 655-665.

Möckel, S., Köck, W., Rutz, C. und Schramek, J. (2014): Rechtliche und andere Instrumente für vermehrten Umweltschutz in der Landwirtschaft, Dessau-Roßlau: Umweltsbundesamt. Abrufbar unter: http://www.umweltbundesamt.de/sites/default/files/medien/378/publikationen/ texte_42_2014_rechtliche_und_andere_instrumente.pdf, zuletzt abgefragt: 23.04.2017.

Mohn, R., Hauffe, H.-K., Reich, R., Bortt, W. und Arheidt, M. (2003): Identifizierung von Problemen und Lösungsansätzen einer Gewannebewirtschaftung insbesondere aus landschaftsökologischer Sicht unter Berücksichtigung organisatorischer sowie technischer und arbeitswirtschaftlicher Aspekte. Schriften der Rentenbank, Sammelbände zum Symposium der Edmund-Rehwinkel-Stiftung, 18, S. 139-178.

Montgomery, D.R. (2007): Soil erosion and agricultural sustainability. Proceedings of the National Academy of Sciences of the United States of America, 104, S. 13268-13272.

Moreddu, C. (2011): Distribution of support and income in agriculture, Geneva: OECD.

Morgan, K. (2015): Nourishing the city: The rise of the urban food question in the Global North. Urban Studies, 52, S. 1379-1394.

Moxey, A. und White, B. (2014): Result-oriented agri-environmental schemes in Europe: A comment. Land Use Policy, 39, S. 397-399.

Moyer, W. und Josling, T.E. (2002): Agricultural Policy Reform: Politics and Process in the EU and US in the 1990s, Aldershot, UK/Burlington, VT: Ashgate. 
NABU (2012): Defizitanalyse Natura 2000 - Situation von artenreichem Grünland im Süddeutschen Raum, Berlin.

NABU (2013): Naturverträgliche Nutzung ökologischer Vorrangflächen - ein Mehrwert für Biodiversität und Landwirtschaft?, Berlin.

Nationale Akademie der Wissenschaften Leopoldina, acatech - Deutsche Akademie der Technikwissenschaften und Union der deutschen Akademien der Wissenschaften (2018): Artenrückgang in der Agrarlandschaft: Was wissen wir und was können wir tun?, Halle (Saale): Leopoldina/acatech/UAW. Abrufbar unter: www.leopoldina.org/biodiversitaet, zuletzt abgefragt: 28.10.2018.

Nentwig, W. (2000): Streifenförmige ökologische Ausgleichsflächen in der Kulturlandschaft: Ackerkrautstreifen, Buntbrache, Feldränder, Bern: Vaö - Verlag Agrarökologie.

Neumann, H. (2016): Öffentliches Geld für öffentliche Leistungen. Biodiversitätszahlung statt Direktzahlung, Berlin: Deutscher Verband für Landschaftspflege. Abrufbar unter: http://www. lpv.de/uploads/media/Biodiversitätsbewertung_und_Agrarzahlungen_17_03_2016.pdf, zuletzt abgefragt: 06.04.2016.

Neumann, H. und Dierking, U. (2014): Ermittlung des „Biodiversitätswerts“ landwirtschaftlicher Betriebe in Schleswig-Holstein. Ein Schnellverfahren für die Praxis. Natur und Landschaft, 46, S. $145-152$.

Nevens, F., Dessein, J., Meul, M., Rogge, E., Verbruggen, I., Mulier, A., Van Passel, S., Lepoutre, J. und Hongenaert, M. (2008): 'On tomorrow's grounds', Flemish agriculture in 2030: a case of participatory translation of sustainability principles into a vision for the future. Journal of Cleaner Production, 16, S. 1062-1070.

Newig, J., Challies, E., Jager, N. und Kochskämper, E. (2014): What Role for Public Participation in Implementing the EU Floods Directive? A Comparison with the Water Framework Directive, Early Evidence from Germany and a Research Agenda. Environmental Policy and Governance, 24, S. 275-288.

Newig, J. und Koontz, T.M. (2014): Multi-level governance, policy implementation and participation: the EU's mandated participatory planning approach to implementing environmental policy. Journal of European Public Policy, 21, S. 248-267.

Newig, J., Pahl-Wostl, C. und Sigel, K. (2005): The role of public participation in managing uncertainty in the implementation of the Water Framework Directive. Environmental Policy and Governance, 15, S. 333-343.

Newton, A.C. (2016): Biodiversity Risks of Adopting Resilience as a Policy Goal. Conservation Letters, 9, S. 369-376.

Nicholls, C.I. und Altieri, M.A. (2013): Plant biodiversity enhances bees and other insect pollinators in agroecosystems. A review. Agronomy for Sustainable Development, 33, S. 257-274.

Nielsen, U.N., Wall, D.H. und Six, J. (2015): Soil Biodiversity and the Environment. Annual Review of Environment and Resources, 40, S. 63-90.

Nitsch, H., Osterburg, B., Laggner, B. und Roggendorf, W. (2010): Wer schützt das Grünland? Analysen zur Dynamik des Dauergrünlands und entsprechender Schutzmechanismen. Vortrag anlässlich der 50. Jahrestagung der GEWISOLA „Möglichkeiten und Grenzen der wissenschaftlichen Politikanalyse“, Braunschweig, 29.09. - 01.10.2010. Abrufbar unter: https://ageconsearch.umn.edu/bitstream/93940/2/A3_3.pdf, zuletzt abgefragt: 17.12.2016.

Nitsch, H., Osterburg, B. und Roggendorf, W. (2009): Landwirtschaftliche Flächennutzung im Wandel: Folgen für Natur und Landschaft. Eine Analyse agrarstatistischer Daten, Berlin und Ansbach: NABU-Bundesverband \& Deutscher Verband für Landschaftspflege (DVL).

Nitsch, H., Osterburg, B., von Buttlar, C. und von Buttlar, H.-B. (2008): Aspekte des Gewässerschutzes und der Gewässernutzung beim Anbau von Energiepflanzen: Ergebnisse eines Forschungsvorhabens im Auftrag des Umweltbundesamtes, Braunschweig: von Thünen-Institut für Agrarökonomie.

Nohl, W. (1993): Beeinträchtigung des Landschaftsbildes durch mastenartige Eingriffe, München, Kirchheim.

OECD (2002): Participatory Decision Making for Sustainable Consumption, Paris: OECD.

OECD (2016): Alternative Futures for Global Food and Agriculture, Paris: OECD. 
Oppermann, R., Fried, A., Lepp, N., Lepp, T. und Lakner, S. (2016): Fit, fair und nachhaltig: Vorschläge für eine neue EU-Agrarpolitik, Mannheim/Göttingen: IFAB/INA. Abrufbar unter: https://www.nabu.de/imperia/md/content/nabude/landwirtschaft/agrarreform/161104-studie-neueeuagrarpolitik-langfassung.pdf, zuletzt abgefragt: 13.04.2017.

Oppermann, R., Gelhausen, J., Matzdorf, B., Reutter, M., Luick, R. und Stein, S. (2012): Gemeinsame Agrarpolitik ab 2014: Perspektiven für mehr Biodiversitäts- und Umweltleistungen der Landwirtschaft?: Empfehlungen für die Politik aus dem F\&E Vorhaben: Reform der Gemeinsamen Agrarpolitik (GAP) 2013 und Erreichung der Biodiversitäts- und Umweltziele, Bonn: Bundesamt für Naturschutz.

Oppermann, R., Kasperczyk, N., Matzdorf, B., Reutter, M., Meyer, C., Luick, R., Stein, S., Ameskamp, K., Gelhausen, J. und Bleil, R. (2013): Reform der Gemeinsamen Agrarolitik (GAP 2013) und Erreichung der Biodiversitäts- und Umweltziele, Bonn: Bundesamt für Naturschutz.

Oppermann, T., Classen, C.D. und Nettesheim, M. (2011): Europarecht, 5. Auflage, München: C.H. Beck.

Osterburg, B., Nitsch, H., Laggner, B. und Roggendorf, W. (2009): Auswertung von Daten des Integrierten Verwaltungs- und Kontrollsystems zur Abschätzung von Wirkungen der EU-Agrarreform auf Umwelt und Landschaft, Braunschweig: von Thünen Institut für Agrarökonomie.

Ozment, S., DiFrancesco, K. und Gartner, T. (2015): Natural infrastructure in the Nexus, Gland, Switzerland: IUCN. Abrufbar unter: http://www.iwa-network.org/wp-content/uploads/2016/06/Natural-Infrastructure-in-the-Nexus_Final-Dialogue-Synthesis-Paper-2015. pdf, zuletzt abgefragt: 23.04.2017.

Pahl-Wostl, C. (2015): Water governance in the face of global change: from understanding to transformation, Berlin et al.: Springer.

Pannell, D.J. (2008): Public Benefits, Private Benefits, and Policy Mechanism Choice for LandUse Change for Environmental Benefits. Land Economics, 84, S. 225-240.

Parkhurst, G.M. und Shogren, J.F. (2007): Spatial incentives to coordinate contiguous habitat. Ecological Economics, 64, S. 344-355.

Parkhurst, G.M., Shogren, J.F., Bastian, C., Kivia, P., Donner, J. und Smith, R.B.W. (2002): Agglomeration bonus: An incentive mechanism to reunite fragmented habitat for biodiversity conservation. Ecological Economics, 41, S. 305-328.

Pedersen, A.B., Nielsen, H.Ø., Christensen, T. und Hasler, B. (2012): Optimising the effect of policy instruments: a study of farmers' decision rationales and how they match the incentives in Danish pesticide policy. Journal of Environmental Planning and Management, 55, S. 1094-1110.

Penker, M. (2002): Naturschutz auf landwirtschaftlichen Flächen - eine institutionenökonomische Betrachtung. Die Bodenkultur, 53, S. 217-226.

Perrot-Maître, D. (2006): The Vittel Payments for Ecosystem Services: A "Perfect" PES Case?, London: International Institute for Environment and Development. Abrufbar unter: http://pubs. iied.org/pdfs/G00388.pdf, zuletzt abgefragt: 23.04.2017.

Petit, S., Boursault, A., Guilloux, M., Munier-Jolain, N. und Reboud, X. (2011): Weeds in agricultural landscapes. A review. Agronomy for Sustainable Development, 31, S. 309-317.

Pierson, P. (1993): When Effect Becomes Cause. Policy Feedback and Political Change. World Politics, 45, S. 595-628.

Plankl, R., Weingarten, P., Nieberg, H., Zimmer, Y., Isermeyer, F., Krug, J. und Haxsen, G. (2010): Quantifizierung „gesellschaftlich gewünschter, nicht marktgängiger Leistungen“ der Landwirtschaft, Braunschweig. Abrufbar unter: https://iteratur.thuenen.de/digbib_extern/bitv/ dk043125.pdf, zuletzt abgefragt: 17.04.2017.

Plieninger, T. und Bieling, C. (2013): Resilience-Based Perspectives to Guiding High-Nature-Value Farmland through Socioeconomic Change. Ecology and Society, 18, S. 20.

Plieninger, T., Schleyer, C., Schaich, H., Ohnesorge, B., Gerdes, H., Hernández-Morcillo, M. und Bieling, C. (2012): Mainstreaming ecosystem services through reformed European agricultural policies. Conservation Letters, 5, S. 281-288.

Ponisio, L.C., M'Gonigle, L.K., Mace, K.C., Palomino, J., Valpine, P.d. und Kremen, C. (2014): Diversification practices reduce organic to conventional yield gap. Proceedings of the Royal Society B: Biological Sciences, 282, S. 20141396. 
Ponte, S. und Cheyns, E. (2013): Voluntary standards, expert knowledge and the governance of sustainability networks. Global Networks, 13, S. 459-477.

Porter, T. und Ronit, K. (2006): Self-regulation as policy process: The multiple and criss-crossing stages of private rule-making. Policy Sciences, 39, S. 41-72.

Porticella, N., Bonfield, S., DeFalco, T., Fumarolo, A., Garibay, C., Jolly, E., Huerta Migus, L., Pandya, R., Purcell, K., Rowden, J., Stevenson, F. und Switzer, A. (2013): Promising Practices for Community Partnerships: A Call to Support More Inclusive Approaches to Public Participation in Scientific Research. A Report Commissioned by the Association of Science-Technology Centers, Washington, DC.

Pothukuchi, K. und Kaufman, J.L. (1999): Placing the food system on the urban agenda: The role of municipal institutions in food systems planning. Agriculture and Human Values, 16, S. 213-224.

Potts, S., Roberts, S., Dean, R., Marris, G., Brown, M., Jones, R., Neumann, P. und Settele, J. (2010): Declines of managed honey bees and beekeepers in Europe. Journal of Apicultural Research, 49, S. 15.

Prager, K. (2015): Agri-environmental collaboratives for landscape management in Europe. Current Opinion in Environmental Sustainability, 12, S. 59-66.

Pretty, J., Brett, C., Gee, D., Hine, R., Mason, C., Morison, J., Rayment, M., Bijl, G.V.D. und Dobbs, T. (2001): Policy Challenges and Priorities for Internalizing the Externalities of Modern Agriculture. Journal of Environmental Planning and Management, 44, S. 263-283.

Primdahl, J., Peco, B., Schramek, J., Andersen, E. und Onate, J.J. (2003): Environmental effects of agri-environmental schemes in Western Europe. Journal of Environmental Management, 67, S. 129-138.

Primmer, E. und Furman, E. (2012): Operationalising ecosystem service approaches for governance: Do measuring, mapping and valuing integrate sector-specific knowledge systems? Ecosystem Services, 1, S. 85-92.

Purnhagen, K. (2011): Anmerkung. Europäische Zeitschrift für Wirtschaftsrecht, S. 224 ff.

Purnhagen, K. (2013a): The Politics of Systematization in EU Product Safety Regulation: Market, State, Collectivity, and Integration, Berlin et al.: Springer.

Purnhagen, K. (2013b): The Virtue of Cassis de Dijon 25 years later. In: K. Purnhagen und Rott (Hrsg.): Varieties of European Economic Law and Regulation. S. $315 \mathrm{ff}$.

Purnhagen, K. (2015): Mapping Private Regulation - Classification, Market Access and Market Closure Policy and Law's Response. Journal of World Trade, 49, S. 309-323.

Purnhagen, K. und Feindt, P.H. (2017): Principles-based Regulation: Blueprint for a "New Approach" for the Internal Agricultural Market. European Law Review, 42, S. 722-736.

Purnhagen, K. und Reisch, L.A. (2016): ,Nudging Germany'? Herausforderungen für eine verhaltensbasierte Regulierung in Deutschland. Zeitschrift für Europäisches Privatrecht, S. $629 \mathrm{ff}$.

Purnhagen, K. und Schebesta, H. (2017): A Case moving at the Frontiers of Market Access, Freedom of Goods, the Common Agricultural Policy and Science in Court - Reflections on Scotch Whisky Association. European Law Review, S. forthcoming.

Purnhagen, K. und Wesseler, J. (2016): The Principle(s) of Co-existence in the Market for GMOs in Europe: Social, Economic and Legal Avenues. In: N. Kalaitzandonakes, P.W.B. Phillips, J. Wesseler und S.J. Smyth (Hrsg.): The Coexistence of Genetically Modified, Organic and Conventional Foods. Berlin et al.: Springer Science, S. 71-85.

Ratzbor, G. (2011): Windenergieanlagen und Landschaftsbild: Zur Auswirkung von Windrädern auf das Landschaftsbild. Abrufbar unter: http://fwg-weiskirchen.bitwnd.de/cms/files/thesenpapier_landschaftsbild.pdf, zuletzt abgefragt: 23.04.2017.

Reisch, L. (2006): Food-Chain-Politik: Neuorientierung der Agrarpolitik. German Journal of Agricultural Economics, 55, S. 310-322.

Renting, H., Marsden, T.K. und Banks, J. (2003): Understanding alternative food networks: exploring the role of short food supply chains in rural development. Environment and planning A, 35, S. 393-411. 
Renting, H. und Ploeg, J.D.V.D. (2001): Reconnecting nature, farming and society: environmental cooperatives in the Netherlands as institutional arrangements for creating coherence. Journal of Environmental Policy \& Planning, 3, S. 85-101.

Richner, N., Holderegger, R., Linder, H.P., Walter, T. und Westerman, P. (2015): Reviewing change in the arable flora of Europe: a meta-analysis. Weed Research, 55, S. 1-13.

Ridder, D., Mostert, E. und Wolters, H.A. (2005): Learning Together to Manage Together - Improving participation in water management, Osnabrück: University of Osnabrück, Institute of Environmental Systems Research.

Ring, I. (2013): Nachhaltige Entwicklung in Industrie- und Bergbauregionen - Eine Chance für den Südraum Leipzig?, Berlin/New York/Tokyo: Springer.

Rockström, J., Klum, M. und Miller, P. (2015): Big world, small planet: abundance within planetary boundaries: Yale University Press.

Rockström, J., Steffen, W., Noone, K., Persson, A., Chapin, F.S., Lambin, E.F., Lenton, T.M., Scheffer, M., Folke, C., Schellnhuber, H.J., Nykvist, B., de Wit, C.A., Hughes, T., van der Leeuw, S., Rodhe, H., Sorlin, S., Snyder, P.K., Costanza, R., Svedin, U., Falkenmark, M., Karlberg, L., Corell, R.W., Fabry, V.J., Hansen, J., Walker, B., Liverman, D., Richardson, K., Crutzen, P. und Foley, J.A. (2009a): A safe operating space for humanity. Nature, 461, S. 472-475.

Rockström, J., Steffen, W.L., Noone, K., Persson, A., Chapin III, F.S., Lambin, E., Lenton, T.M., Scheffer, M., Folke, C. und Schellnhuber, H.J. (2009b): Planetary boundaries: exploring the safe operating space for humanity. Ecology and Society, 14, S. 32.

Röder, N., Schmidt, T. und Osterburg, B. (2015): Grünland: Mehr als nur Viehfutter, Braunschweig: Thünen-Institut. Abrufbar unter: https://literatur.thuenen.de/digbib_extern/dn055216.pdf, zuletzt abgefragt: 23.11.2016.

Rodríguez, C. und Wiegand, K. (2009): Evaluating the trade-off between machinery efficiency and loss of biodiversity-friendly habitats in arable landscapes: The role of field size. Agriculture, Ecosystems \& Environment, 129, S. 361-366.

Rothstein, B. und Schröder, D. (2016): Ordnungsrecht versus ökonomische Instrumente im landwirtschaftlichen Bodenschutz. Zeitschrift für Umweltpolitik und Umweltrecht, 26, S. 371-391.

Runge, T. und Osterburg, B. (2007): A result-oriented approach to reward N-efficiency improvements. Paper presented at the $15^{\text {th }}$ Nitrogen Workshop, Lleida (Spain), May 28-30, 2007. Abrufbar unter: http://www.life-kuestenheiden.niedersachsen.de/download/23080/A_result-oriented_approach_to_reward_N-efficiency_improvements.pdf\&hl=de\&sa=X\&scisig=AAGBfm0UC9jc7svlG4ZNE6zuNBke57X68w\&nossl=1\&oi=scholarr, zuletzt abgefragt: 23.11.2016.

Sattler, C. und Matzdorf, B. (2013): PES in a nutshell: From definitions and origins to PES in practice - Approaches, design process and innovative aspects. Ecosystem Services, 6, S. 2-11.

Sauer, D., Schülli-Maurer, I., Sperstad, R., Sorensen, R. und Stahr, K. (2008): Podzol development with time in sandy beach deposits in southern Norway. Journal of Plant Nutrition and Soil Science, 171, S. 483-497.

Schäfer, A. (2009): Moore und Euros - die vergessenen Millionen. Archiv für Forstwesen und Landschaftsökologie, 43, S. 156-160.

Scheper, J., Holzschuh, A., Kuussaari, M., Potts, S.G., Rundlöf, M., Smith, H.G. und Kleijn, D. (2013): Environmental factors driving the effectiveness of European agri-environmental measures in mitigating pollinator loss - a meta-analysis. Ecology letters, 16, S. 912-920.

Schieß1, P., Krämer, C. und Heißenhuber, A. (2015): Transport und Aufbereitung von Wirtschaftsdünger. UBA-Texte 80/2015, Dessau-Roßlau: Umweltbundesamt.

Schilizzi, S. und Latacz-Lohmann, U. (2007): Assessing the Performance of Conservation Auctions: An Experimental Study. Land Economics, 83, S. 497-515.

Schipper, E.L.F. und Langston, L. (2015): A comparative overview of resilience measurement frameworks. Analysing indicators and approaches. ODI Working paper 422, London: Overseas Development Institute. Abrufbar unter: https://www.odi.org/sites/odi.org.uk/files/odi-assets/ publications-opinion-files/9754.pdf, zuletzt abgefragt: 25.02.2017. 
Schleyer, C. und Plieninger, T. (2011): Obstacles and options for the design and implementation of payment schemes for ecosystem services provided through farm trees in Saxony, Germany. Environmental Conservation, 38, S. 454-463.

Schmidt, B.R. (2007): Prädatoren, Parasiten und Geduld: Neue Erkenntnisse zur Wirkung von Pestiziden auf Amphibien. Zeitschrift für Feldherpetologie, 14, S. 1-8.

Schmidt, C., Hofmann, M. und Dunkel, A. (2014): Den Landschaftswandel gestalten! Potentiale der Landschafts- und Raumplanung zur modellhaften Entwicklung und Gestaltung von Kulturlandschaften vor dem Hintergrund aktueller Transformationsprozesse, Bonn: Bundesamt für Naturschutz.

Schmidt, M.H., Lauer, A., Purtauf, T., Thies, C., Schaefer, M. und Tscharntke, T. (2003): Relative importance of predators and parasitoids for cereal aphid control. Proc Biol Sci., 270, S. 1905-1909.

Schroeder, L.A., Isselstein, J., Chaplin, S. und Peel, S. (2013): Agri-environment schemes: Farmers' acceptance and perception of potential 'Payment by Results' in grassland - A case study in England. Land Use Policy, 32, S. 134-144.

Schröter-Schlaack, C., Albert, C., von Haaren, C., Hansjürgens, B., Krätzig, S. und Albert, I. (2016): Ökosystemleistungen in ländlichen Räumen - Grundlage für menschliches Wohlergehen und nachhaltige wirtschaftliche Entwicklung. Schlussfolgerungen für Entscheidungsträger. Abrufbar unter: http://www.naturkapital-teeb.de/fileadmin/Downloads/Projekteigene_Publikationen/TEEB_Broschueren/TEEB_DE_Landbericht_Kurzfassung_2_.pdf, zuletzt abgefragt: 15.12.2016.

Schuler, J., Bues, A., Henseler, M., Krämer, C., Krampe, L., Kreins, P., Liebersbach, H., Osterburg, B., Röder, N. und Uckert, G. (2015): Instrumente zur Stärkung von Synergien zwischen Natur- und Klimaschutz im Bereich Landbewirtschaftung, Bonn: Bundesamt für Naturschutz.

Schuler, J. und Sattler, C. (2010): The estimation of agricultural policy effects on soil erosion-An application for the bio-economic model MODAM. Land Use Policy, 27, S. 61-69.

Schulz, D. (2010): Agrarpolitik der EU - Umweltschutz-Anforderungen für die Jahre 2014 bis 2020 (CAP 2020), Dessau-Roßlau: Umweltbundesamt.

Scohier, A. und Dumont, B. (2012): How do sheep affect plant communities and arthropod populations in temperate grasslands? Animal: an international journal of animal bioscience, 6 , S. 1129-1138.

Segerson, K. (2013): When is reliance on voluntary approaches in agriculture likely to be effective? Applied Economic Perspectives and Policy, 35, S. 565-592.

Shackelford, G., Steward, P.R., Benton, T.G., Kunin, W.E., Potts, S.G., Biesmeijer, J.C. und Sait, S.M. (2013): Comparison of pollinators and natural enemies: a meta-analysis of landscape and local effects on abundance and richness in crops. Biological reviews of the Cambridge Philosophical Society, 88, S. 1002-1021.

Sheingate, A.D. (2003): The rise of the agricultural welfare state: institutions and interest group power in the United States, France, and Japan, Princeton and Oxford: Princeton University Press.

Shogren, J.F. und Taylor, L.O. (2008): On Behavioral-Environmental Economics. Review of Environmental Economics and Policy, 2, S. 26-44.

Simon, S., Bouvier, J.-C., Debras, J.-F. und Sauphanor, B. (2010): Biodiversity and pest management in orchard systems. A review. Agronomy for Sustainable Development, S. 139-152.

Skogstad, G. (1998): Ideas, Paradigms and Institutions: Agricultural Exceptionalism in the European Union and the United States. Governance, 11, S. 463-490.

Smits, M.J., Driessen, P. und Glasbergen, P. (2008): Governing agri-environmental schemes: lessons to be learned from the new institutional-economics approach. Environment and Planning C: Government and Policy, 26, S. 627-643.

Soliveres, S., van der Plas, F., Manning, P., Prati, D., Gossner, M.M., Renner, S.C., Alt, F., Arndt, H., Baumgartner, V., Binkenstein, J., Birkhofer, K., Blaser, S., Bluthgen, N., Boch, S., Bohm, S., Borschig, C., Buscot, F., Diekötter, T., Heinze, J., Hölzel, N., Jung, K., Klaus, V.H., Kleinebecker, T., Klemmer, S., Krauss, J., Lange, M., Morris, E.K., Müller, J., Oelmann, Y., Overmann, J., 
Pasalic, E., Rillig, M.C., Schaefer, H.M., Schloter, M., Schmitt, B., Schöning, I., Schrumpf, M., Sikorski, J., Socher, S.A., Solly, E.F., Sonnemann, I., Sorkau, E., Steckel, J., Steffan-Dewenter, I., Stempfhuber, B., Tschapka, M., Türke, M., Venter, P.C., Weiner, C.N., Weisser, W.W., Werner, M., Westphal, C., Wilcke, W., Wolters, V., Wubet, T., Wurst, S., Fischer, M. und Allan, E. (2016): Biodiversity at multiple trophic levels is needed for ecosystem multifunctionality. Nature, 536, S. 456-459.

Sommer, C.G. und Hutchings, N.J. (2001): Ammonia emission from field applied manure and it's reduction. European Journal of Agronomy, 15, S. 1071-1079.

Soussana, J.F., Tallec, T. und Blanfort, V. (2010): Mitigating the greenhouse gas balance of ruminant production systems through carbon sequestration in grasslands. Animal: an international journal of animal bioscience, 4, S. 334-350.

Spiertz, J. (2010): Nitrogen, sustainable agriculture and food security. A review. Agronomy for Sustainable Development, 30, S. 43-55.

Spurgeon, D.J., Keith, A.M., Schmidt, O., Lammertsma, D.R. und Faber, J.H. (2013): Land-use and land-management change: relationships with earthworm and fungi communities and soil structural properties. BMC ecology, 13, S. 46.

SRU (1985): Umweltprobleme der Landwirtschaft. Sondergutachten, Stuttgart: Kohlhammer.

SRU (2007): Arzneimittel in der Umwelt. Stellungnahme, Berlin: Sachverständigenrat für Umweltfragen.

SRU (2009): Für eine zeitgemäße Gemeinsame Agrarpolitik (GAP). Stellungnahme, Berlin: Sachverständigenrat für Umweltfragen.

Statista (2014): Anteil der Ausgaben der privaten Haushalte in Deutschland für Nahrungsmittel, Getränke und Tabakwaren an den Konsumausgaben in den Jahren in den Jahren 1850 bis 2013. Abrufbar unter: https://de.statista.com/statistik/daten/studie/75719/umfrage/ausgaben-fuer-nahrungsmittel-in-deutschland-seit-1900/, zuletzt abgefragt: 05.05.2016.

Statistisches Bundesamt (2011): Wer produziert unsere Nahrungsmittel? Aktuelle Ergebnisse der Landwirtschaftszählung 2010, Wiesbaden: Destatis.

Statistisches Bundesamt (2014): Nachhaltige Entwicklung in Deutschland: Indikatorenbericht 2014, Wiesbaden: Destatis.

Statistisches Bundesamt (2016): Feldfrüchte und Grünland: Dauergrünland nach Art der Nutzung im Zeitvergleich, Wiesbaden: Destatis. Abrufbar unter: https://www.destatis.de/DE/ZahlenFakten/Wirtschaftsbereiche/LandForstwirtschaftFischerei/FeldfruechteGruenland/Tabellen/ ZeitreiheDauergruenlandNachNutzung.html, zuletzt abgefragt: 23.04.2017.

Statistisches Bundesamt (2017): Nachhaltige Entwicklung in Deutschland: Indikatorenbericht 2016, Wiesbaden: Destatis. Abrufbar unter: https://www.destatis.de/DE/Publikationen/Thematisch/UmweltoekonomischeGesamtrechnungen/Umweltindikatoren/IndikatorenPDF_0230001.pdf?_blob=publicationFile, zuletzt abgefragt: 06.05.2017.

Sutton, M.A., Oenema, O., Erisman, J.W., Leip, A., van Grinsven, H. und Winiwarter, W. (2011): Too much of a good thing. Nature, 472, S. 159-161.

Swinbank, A. (1993): CAP Reform, 1992. Journal of Common Market Studies, 31, S. 359-372.

Swinbank, A. (1999): EU Agriculture, Agenda 2000 and the WTO Commitments. World Economy, 22, S. 41-54.

Swinbank, A. und Tanner, C. (1996): Farm Policy and Trade Conflict: The Uruguay Round and Common Agricultural Policy Reform, Ann Arbor: University of Michigan Press.

Swinnen, J. (2015): The Political Economy of the 2014-2020 Common Agricultural Policy: Introduction and Key Conclusions. In: J. Swinnen (Hrsg.): The Political Economy of the 2014-2020 Common Agricultural Policy: An Imperfect Storm. London: Rowman and Littlefield International, S. 1-30.

Swinnen, J., Ciaian, P. und Kancs, d.A. (2008): Study on the Functioning of Land Markets in the EU Member States under the Influence of Measures applied under the Common Agricultural Policy. Brussels: EERI.

Tackett, K. (2014): European Grocery Retailing: Change is the only constant. Planet Retail Presentation. Abrufbar unter: http://www.planetretail.net/presentations/ApexBrasilPresentation. pdf, zuletzt abgefragt: 05.05.2016. 
Tansey, G. und Worsley, A. (2014): The Food System, London: Routledge.

Taube, F. (2016): Umwelt- und Klimawirkungen der Landwirtschaft. Eine kritische Einordnung - Statusbericht, Herausforderung und Ausblick, Kiel. Abrufbar unter: https://www.grassland-organicfarming.uni-kiel.de/de/aktuelles/Taube_Umweltwirkungen_Statusbericht_final_ WiTa16_03.pdf, zuletzt abgefragt: 23.04.2017.

Taube, F., Hennig, C., Albrecht, E., Reinsch, T. und Kluß, C. (2015): Nährstoffbericht des Landes Schleswig-Holstein, Kiel: Ministeriums für Energiewende, Landwirtschaft, Umwelt und ländliche Räume des Landes Schleswig-Holstein. Abrufbar unter: https:/www.grassland-organicfarming.uni-kiel.de/de/aktuelles/naehrstoffbericht_sh_taube.pdf, zuletzt abgefragt: 23.04.2017.

TEEB DE (2012): Der Wert der Natur für Wirtschaft und Gesellschaft: Eine Einführung, München: ifuplan.

Thaler, R.H. und Sunstein, C.B. (2008): Nudge: Improving Decisions about Health, Wealth, and Happiness, New Heaven: Yale University Press.

The Guardian (2015): New OpenLabel app offers product information for eco-minded consumers. 10. Feb. 2015. Abrufbar unter: http://www.theguardian.com/sustainable-business/2015/feb/10/ openlabel-app-consumer-information-google-facebook, zuletzt abgefragt: 17.10.2015.

Thiele-Bruhn, S., Bloem, J., de Vries, F.T., Kalbitz, K. und Wagg, C. (2012): Linking soil biodiversity and agricultural soil management. Current Opinion in Environmental Sustainability, 4, S. 523-528.

Thomas, F., Poppinga, O. und Jürgens, K. (2016): Plädoyer für eine aktive Agrarstrukturpolitik durch Preis-Ausgleichszahlungen, Kassel-Witzenhausen: Kasseler Institut für ländliche Entwicklung e.V.

Thünen-Institut (2016): Projektinformation: Was bewirken Investitionshilfen in der Landwirtschaft? Abrufbar unter: https://www.thuenen.de/de/bw/projekte/was-bewirken-investitionshilfen-in-der-landwirtschaft/, zuletzt abgefragt: 27.7. 2016.

Tooker, J.F., Frank, S.D. und Steffan-Dewenter, I. (2012): Genotypically diverse cultivar mixtures for insect pest management and increased crop yields. Journal of Applied Ecology, 49, S. 974-985.

Tosun, J., Koos, S. und Shore, J. (2016): Co-governing common goods: Interaction patterns of private and public actors. Policy and Society, 35, S. 1-12.

Tracy, M. (1989): Government and Agriculture in Western Europe 1880-1988, New York/London: Harvester Wheatsheaf.

Trebilcock, M.J. (2015): International Trade Law, Cheltenham: Elgar.

Trepl, L. (2012): Die Idee der Landschaft: Eine Kulturgeschichte von der Aufklärung bis zur Ökologiebewegung, Bielefeld: transcript.

Tricker, R. (2000): CE conformity marking and new approach directives, Oxford (MA) et al.: Butterworth-Heinemann.

Trubek, D.M. und Trubek, L.G. (2006): New Governance \& (and) Legal Regulation: Complementarity, Rivalry, and Transformation. Colum. J. Eur. L., 13, S. 539.

Tscharntke, T., Klein, A.M., Kruess, A., Steffan-Dewenter, I. und Thies, C. (2005): Landscape perspectives on agricultural intensification and biodiversity - ecosystem service management. Ecology Letters 8, S. 857-874.

Tscharntke, T., Tylianakis, J.M., Rand, T.A., Didham, R.K., Fahrig, L., Batáry, P., Bengtsson, J., Clough, Y., Crist, T.O., Dormann, C.F., Ewers, R.M., Fründ, J., Holt, R.D., Holzschuh, A., Klein, A.M., Kleijn, D., Kremen, C., Landis, D.A., Laurance, W., Lindenmayer, D., Scherber, C., Sodhi, N., Steffan-Dewenter, I., Thies, C., van der Putten, W.H. und Westphal, C. (2012): Landscape moderation of biodiversity patterns and processes - eight hypotheses. Biological reviews of the Cambridge Philosophical Society, 87, S. 661-685.

Tsiafouli, M.A., Thébault, E., Sgardelis, S.P., de Ruiter, P.C., van der Putten, W.H., Birkhofer, K., Hemerik, L., de Vries, F.T., Bardgett, R.D., Brady, M.V., Bjornlund, L., Jørgensen, H.B., Christensen, S., Hertefeldt, T.D., Hotes, S., Gera Hol, W.H., Frouz, J., Liiri, M., Mortimer, S.R., Setälä, H., Tzanopoulos, J., Uteseny, K., Pižl, V., Stary, J., Wolters, V. und Hedlund, K. (2015): Intensive agriculture reduces soil biodiversity across Europe. Global Change Biology 21, S. 973-985. 
Tuck, S.L., Winqvist, C., Mota, F., Ahnström, J., Turnbull, L.A. und Bengtsson, J. (2014): Landuse intensity and the effects of organic farming on biodiversity: a hierarchical meta-analysis. The Journal of applied ecology, 51, S. 746-755.

Tuomisto, H.L., Hodge, I.D., Riordan, P. und Macdonald, D.W. (2012): Does organic farming reduce environmental impacts? - A meta-analysis of European research. Journal of Environmental Management, 112, S. 309-320.

Turnhout, E., Van Bommel, S. und Aarts, N. (2010): How Participation Creates Citizens: Participatory Governance as Performative Practice. Ecology and Society, 15, S. 26.

Ulber, L., Klimek, S., Steinmann, H.H., Isselstein, J. und Groth, M. (2011): Implementing and evaluating the effectiveness of a payment scheme for environmental services from agricultural land. Environmental Conservation, 38, S. 464-472.

Umweltbundesamt (2010a): Daten zur Umwelt - Umwelt und Landwirtschaft, Dessau-Roßlau.

Umweltbundesamt (2010b): Gewässerschutz mit der Landwirtschaft, Dessau-Roßlau.

Umweltbundesamt (2010c): Wasserwirtschaft in Deutschland. Teil 2: Gewässergüte, Dessau-Roßlau.

Umweltbundesamt (2011a): Daten zur Umwelt 2011: Umwelt und Landwirtschaft, Dessau-Roßlau.

Umweltbundesamt (2011b): Wasserwirtschaft in Deutschland: Wasserversorgung und Abwasserbeseitigung, Dessau-Roßlau.

Umweltbundesamt (2012a): Daten zur Umwelt - Organische Umweltchemikalien und Pflanzenschutzmittel in Fließgewässern. Abrufbar unter: http://www.umweltbundesamt-daten-zur-umwelt.de/umweltdaten/public/theme.do?nodeIdent=3170, zuletzt abgefragt: 15.05.2016.

Umweltbundesamt (2012b): Klärschlammentsorgung in der Bundesrepublik Deutschland, Dessau-Roßlau.

Umweltbundesamt (2012c): Organische Umweltchemikalien und Pflanzenschutzmittel in Fließgewässern. Dessau-Roßlau.

Umweltbundesamt (2013a): Critical Loads für Schwermetalle, Dessau-Roßlau. Abrufbar unter: https:// www.umweltbundesamt.de/themen/luft/wirkungen-von-luftschadstoffen/wirkungen-auf-oekosysteme/critical-loads-fuer-schwermetalle\#textpart-1, zuletzt abgefragt: 27.10.2016.

Umweltbundesamt (2013b): Landwirtschaftliche Emissionen. Texte des Umweltbundesamtes, Dessau-Roßlau.

Umweltbundesamt (2014a): Arzneimittel in der Umwelt: Eintrag und Vorkommen, Dessau-Roßlau. Abrufbar unter: http://www.umweltbundesamt.de/themen/chemikalien/arzneimittel/arzneimittel-umwelt, zuletzt abgefragt: 27.06.2016.

Umweltbundesamt (2014b): Berichterstattung unter der Klimarahmenkonvention der Vereinten Nationen und dem Kyoto-Protokoll 2014: Nationaler Inventarbericht zum Deutschen Treibhausgasinventar 1990-2012, Dessau-Roßlau.

Umweltbundesamt (2015a): Daten zur Umwelt 2015: Umwelttrends in Deutschland, Dessau-Roßlau.

Umweltbundesamt (2015b): Überschreitung der Belastungsgrenzen für Eutrophierung. Abrufbar unter: http://www.umweltbundesamt.de/daten/bodenbelastung-land-oekosysteme/ueberschreitung-der-belastungsgrenzen-fuer-0, zuletzt abgefragt: 13.06.2016.

Umweltbundesamt (2015c): Überschreitung der Belastungsgrenzen für Versauerung, Dessau-Roßlau. Abrufbar unter: https://www.umweltbundesamt.de/daten/flaeche-boden-land-oekosysteme/land-oekosysteme/ueberschreitung-der-belastungsgrenzen-fuer, zuletzt abgefragt: 13.06.2016.

Umweltbundesamt (2016): Luftschadstoffe im Überblick, Dessau-Roßlau. Abrufbar unter: https:// www.umweltbundesamt.de/themen/luft/luftschadstoffe-im-ueberblick, zuletzt abgefragt: 15.03.2016.

Utermann, J., Duijnisveld, W.H.M., Godbersen, L. und Fuchs, M. (2009): Uran in Böden und Sickerwässern - gibt es Indizien für eine Phosphordünger-bürtige Uran-Anreicherung? Tagungsbeitrag zur Jahrestagung der DBG: Böden - eine endliche Ressource, Bonn: DBG. Abrufbar unter: https://www.researchgate.net/publication/267943439_Uran_in_Boden_und_Sickerwas- 
sern_-_gibt_es_Indizien_fur_eine_Phosphordunger-_burtige_Uran-Anreicherung, zuletzt abgefragt: 15.01.2017.

Uthes, S. und Matzdorf, B. (2013): Studies on agri-environmental measures: A survey of the literature. Environmental Management, 51, S. 251-266.

Valeeva, N.I., Lam, T.J.G.M. und Hogeveen, H. (2007): Motivation of Dairy Farmers to Improve Mastitis Management. Journal of Dairy Science 90, S. 4466-4477.

van Ast, J.A. und Boot, S.P. (2003): Participation in European water policy. Physics and Chemistry of the Earth, Parts A/B/C, 28, S. 555-562.

van Bers, C., Ortmann, A. und Rotter, S. (2011): Involving Stakeholders (Chapter 5). In: M. Støvring Harbo, J. Pedersen, R. Johnsen und K. Petersen (Hrsg.): Groundwater in a Future Climate - The CLIWAT Handbook. Aarhus: Central Denmark Region.

van Capelle, C., Schrader, S. und Brunotte, J. (2012): Tillage-induced changes in the functional diversity of soil biota - A review with a focus on German data. European Journal of Soil Biology, 50, S. 165-181.

van der Zee, E. (2016): Regulatory structure of standards underlying sustainability labels. Laying the foundations for effective intervention strategies to increase consumer confidence in sustainability labels. Manuscript under review.

van Dijk, W.F.A., Lokhorst, A.M., Berendse, F. und de Snoo, G.R. (2015): Collective agri-environment schemes: How can regional environmental cooperatives enhance farmers' intentions for agri-environment schemes? Land Use Policy, 42, S. 759-766.

van Haaren, C. und Bathke, M. (2008): Integrated landscape planning and remuneration of agri-environmental services - Results of a case study in the Fuhrberg region of Germany. Journal of Environmental Management, 89, S. 209-221.

Van Herzele, A., Gobin, A., Van Gossum, P., Acosta, L., Waas, T., Dendoncker, N. und de Frahan, B.H. (2013): Effort for money? Farmers' rationale for participation in agri-environment measures with different implementation complexity. Journal of Environmental Management, 131, S. $110-120$.

van Swaay, C., van Strien, A., Harpke, A., Fontaine, B., Stefanescu, C., Roy, D., Kühn, E., Õunap, E., Regan, E., Švitra, G., Prokofev, I., Heliölä, J., Settele, J., Pettersson, L., Botham, M., Musche, M., Titeux, N., Cornish, N., Leopold, P., Goloshchapova, S., Julliard, R., Verovnik, R., Öberg, S., Popov, S., Collins, S., Roth, T., Brereton, T. und Warren, M. (2013): The European grassland butterfly indicator: 1990-2011. EEA Technical report No 11/2013, Copenhagen: European Environmental Agency. Abrufbar unter: https://www.eea.europa.eu/publications/ the-european-grassland-butterfly-indicator-19902011, zuletzt abgefragt: 17.11.2016.

VDLUFA (2015): Phosphordüngung nach Bodenuntersuchung: Anpassung der Richtwerte für die Gehaltsklassen ist geboten und notwendig, Speyer: Verband Deutscher Landwirtschaftlicher Untersuchungs- und Forschungsanstalten. Abrufbar unter: https://www.vdlufa.de/Dokumente/ Veroeffentlichungen/Standpunkte/2018_Standpunkt_P-Duengung.pdf, zuletzt abgefragt: 12.01.2017.

Veres, A., Petit, S., Conord, C. und Lavigne, C. (2013): Does landscape composition affect pest abundance and their control by natural enemies? A review. Agriculture, Ecosystems \& Environment, 166, S. 110-117.

Vogel, D. (2010): The private regulation of global corporate conduct achievements and limitations. Business \& Society, 49, S. 68-89.

Vogel, S. und Inauen, N. (2013): Handlungsfeld Landwirtschaft des Aktionsplans Strategie Biodiversität Schweiz, Bern: Eidgenössisches Departement für Wirtschaft, Bildung und Forschung; Bundesamt für Landwirtschaft, Fachbereich Ökologie. Abrufbar unter: https://www.blw. admin.ch/dam/blw/de/dokumente/Nachhaltige\%20Produktion/Umwelt/Biodiversitaet\%20 und $\% 20$ Landschaft/Handlungsfeld $\% 20$ Landwirtschaft $\% 20 \mathrm{des} \% 20$ Aktionsplans $\% 20$ Strategie\%20Biodiversit\%C3\%A4t\%20Schweiz.pdf, zuletzt abgefragt: 25.11.2016.

von Meyer-Höfer, M. und Spiller, A. (2014): Characteristics and barriers of sustainable food consumption in Germany. Jahrbuch der Österreichischen Gesellschaft für Agrarökonomie, 23, S. 231-240. 
Wahlen, S. und Laamanen, M. (2015): Consumption, lifestyle and social movements. International Journal of Consumer Studies, 39, S. 397-403.

Walker, B., Holling, C.S., Carpenter, S. und Kinzig, A. (2004): Resilience, adaptability and transformability in social-ecological systems. Ecology and society, 9, S. 5.

Wätzold, F. und Drechsler, M. (2014): Agglomeration payment, agglomeration bonus or homogeneous payment? Resource and Energy Economics, 37, S. 85-101.

Wätzold, F. und Schwerdtner, K. (2005): Why be wasteful when preserving a valuable resource? A review article on the cost-effectiveness of European biodiversity conservation policy. Biological Conservation, 123, S. 327-338.

Weber, R. (2005): Strukturverbesserungen 2004. Informationen aus dem Bundesamt für Landwirtschaft, Abteilung Strukturverbesserungen (BLW/ASV). Geomatik Schweiz: Geoinformation und Landmanagement, S. 400-402.

WEFWI (2011): Water security: The water-food-energy-climate nexus: The World Economic Forum water initiative, Washington: Island Press. Abrufbar unter: http://www3.weforum.org/ docs/WEF_WI_WaterSecurity_WaterFoodEnergyClimateNexus_2011.pdf, zuletzt abgefragt: 15.04.2017.

Wegener, J., Lücke, W. und Heinzemann, J. (2006): Analyse und Bewertung landwirtschaftlicher Treibhausgasemissionen in Deutschland. Agrartechnische Forschung, 12, S. 103-114.

Wewer, G. (1998): Leitbilder und Verwaltungskultur. In: S. von Bandemer, B. Blanke, F. Nullmeier und G. Wewer (Hrsg.): Handbuch zur Verwaltungsreform. Opladen: Leske + Budrich, S. $141-150$.

Whitfield, J. (2006): How Green Was My Subsidy. Nature, 439, S. 908-909.

Whittingham, M.J. (2007): Will agri-environment schemes deliver substantial biodiversity gain, and if not why not? Journal of Applied Ecology, 44, S. 1-5.

Willer, H. und Lernoud, J. (Hrsg.) (2014): The World of Organic Agriculture. Statistics and Emerging Trends 2014. FiBL-IFOAM Report. Revised version, Frick and Bonn: Research Institute of Organic Agriculture (FiBL) and International Federation of Organic Agriculture Movements (IFOAM).

Wilson, A., Vickery, J. und Pendlebury, C. (2007): Agri-environment schemes as a tool for reversing declining populations of grassland waders: Mixed benefits from Environmentally Sensitive Areas in England. Biological Conservation, 136, S. 128-135.

Wilson, G. und Hart, K. (2000): Financial imperative of conservation concern? EU farmers' motivations for participation in non-subsidized agri-environmental schemes. Environment and Planning A, 32, S. 2161-2185.

Wissenschaftlicher Beirat für Agrarpolitik beim BMEL (2015): Wege zu einer gesellschaftlich akzeptierten Nutztierhaltung. Gutachten, Berlin: BMEL. Abrufbar unter: http://www.bmel.de/ SharedDocs/Downloads/Ministerium/Beiraete/Agrarpolitik/GutachtenNutztierhaltung.pdf?_ blob=publicationFile, zuletzt abgefragt: 11.12.2016.

Wissenschaftlicher Beirat für Agrarpolitik beim BMELV (2010): EU-Agrarpolitik nach 2013 Plädoyer für eine neue Politik für Ernährung, Landwirtschaft und ländliche Räume, Berlin: BMELV.

Wissenschaftlicher Beirat für Biodiversität und Genetische Ressourcen beim BMELV (2007): Agrobiodiversität erhalten, Potenziale der Land-, Forst- und Fischereiwirtschaft erschließen und nachhaltig nutzen, Bonn.

Wissenschaftlicher Beirat für Biodiversität und Genetische Ressourcen beim BMELV (2011): Chancen für die biologische Vielfalt in der Landwirtschaft nutzen - 10 Schlüsselthemen für die Agrobiodiversität in der Agrarpolitik, Berlin.

Wissenschaftlicher Beirat für Biodiversität und Genetische Ressourcen beim BMELV (2018): Für eine gemeinsame Agrarpolitik, die konsequent zum Erhalt der biologischen Vielfalt beiträgt, Berlin: Bundesministerium für Landwirtschaft und Ernährung.

Wrage, N., Strodthoff, J., Cuchillo, H.M., Isselstein, J. und Kayser, M. (2011): Phytodiversity of temperate permanent grasslands: ecosystem services for agriculture and livestock management for diversity conservation. Biodiversity and Conservation, 20, S. 3317-3339. 
Wünscher, T. und Engel, S. (2012): International payments for biodiversity services: Review and evaluation of conservation targeting approaches. Biological Conservation, 152, S. 222-230.

Wünscher, T., Engel, S. und Wunder, S. (2006): Payments for environmental services in Costa Rica: Increasing efficiency through spatial differentiation. Quarterly Journal of International Agriculture, 45, S. 319-337.

Wünscher, T., Engel, S. und Wunder, S. (2008): Spatial targeting of payments for environmental services: A tool for boosting conservation benefits. Ecological Economics, 65, S. 822-833.

Wynne-Jones, S. (2013): Connecting payments for ecosystem services and agri-environment regulation: An analysis of the Welsh Glastir Scheme. Journal of Rural Studies, 31, S. 77-86.

Young, A.R. (2014): Europe as a global regulator? The limits of EU influence in international food safety standards. Journal of European Public Policy, 21, S. 904-922.

Zabel, A. und Roe, B. (2009): Optimal design of pro-conservation incentives. Ecological Economics, 69, S. 126-134.

Zander, K. und Hamm, U. (2010): Consumer preferences for additional ethical attributes of organic food. Food Quality and Preference, 21, S. 495-503.

Zanias, G.P. (2002): The Distribution of CAP Benefits among Member States and the Impact of a Partial Re-nationalisation: A Note. Journal of Agricultural Economics, 53, S. 108-112.

Zaradic, P.A. und Pergams, O.R. (2007): Videophilia: Implications for childhood development and conservation. Journal of Developmental Processes, 2, S. 130-144.

Zehetmeier, M., Baudracco, J., Hoffmann, H. und Heissenhuber, A. (2012): Does increasing milk yield per cow reduce greenhouse gas emissions? A system approach. Animal: an international journal of animal bioscience, 6, S. 154-166. 